\title{
Determination of Elastic Twist in Horizontal Axis Wind Turbines (HAWTs)
}

Final Report: August 1989

F. Stoddard, V. Nelson, K. Starcher, B. Andrews Alternative Energy Institute - West Texas State University Canyon, Texas

Subcontract Report NREL/SR-500-12087 June 2006 


\section{Determination of Elastic Twist in Horizontal Axis Wind Turbines (HAWTs)}

Final Report: August 1989

F. Stoddard, V. Nelson, K. Starcher, B. Andrews Alternative Energy Institute - West Texas State University Canyon, Texas

NREL Technical Monitor:

Prepared under Subcontract No RL-6-06013

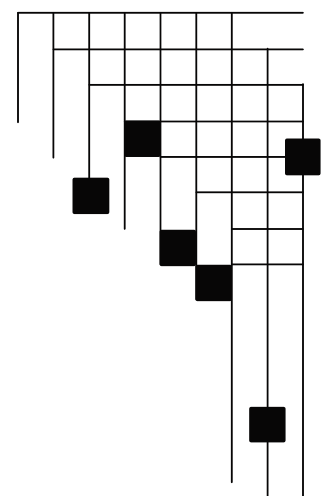


This publication was reproduced from the best available copy Submitted by the subcontractor and received no editorial review at NREL

\section{NOTICE}

This report was prepared as an account of work sponsored by an agency of the United States government. Neither the United States government nor any agency thereof, nor any of their employees, makes any warranty, express or implied, or assumes any legal liability or responsibility for the accuracy, completeness, or usefulness of any information, apparatus, product, or process disclosed, or represents that its use would not infringe privately owned rights. Reference herein to any specific commercial product, process, or service by trade name, trademark, manufacturer, or otherwise does not necessarily constitute or imply its endorsement, recommendation, or favoring by the United States government or any agency thereof. The views and opinions of authors expressed herein do not necessarily state or reflect those of the United States government or any agency thereof.

Available electronically at http://www.osti.gov/bridge

Available for a processing fee to U.S. Department of Energy and its contractors, in paper, from:

U.S. Department of Energy

Office of Scientific and Technical Information

P.O. Box 62

Oak Ridge, TN 37831-0062

phone: 865.576 .8401

fax: 865.576 .5728

email: mailto:reports@adonis.osti.gov

Available for sale to the public, in paper, from:

U.S. Department of Commerce

National Technical Information Service

5285 Port Royal Road

Springfield, VA 22161

phone: 800.553 .6847

fax: 703.605.6900

email: orders@ntis.fedworld.gov

online ordering: http://www.ntis.gov/ordering.htm 


\section{SUMMARY}

This report presents the results of a project at the Alternative Energy Institute (AEI) which measured and calculated the elastic twist of three representative composite horizontal-axis blades: Carter 300 , Gougeon ESI 54, and UTRC $8 \mathrm{~kW}$. The report covers the experimental method used to inspect and test the blades, the theory and computer programs used to develop semi-empirical analytical blade models, calculation of the steady elastic twist of the three blades in simulated operation, and comparison with actual field performance of the wind turbines.

The experimental method employed is suitable for normal engineering blade testing, including quality control, verification of theoretical results, and iteration of design parameters. The computer codes developed under this project are suitable for engineering design of composite wind turbine blades. Suitable instructions for use of the programs are given.

Theory developed under this project can calculate a practical aeroelastic model of geometrically and structurally complex composite wind turbine blades. Such blades show great promise for reducing the weight and cost of future wind turbines; however, they are usually highly twisted and tapered, have variation in mass and stiffness properties in both spanwise and chordwise directions, and employ various forms and orientations of reinforcement, all of which present significant uncertainties in determining the engineering stiffness constants. The method developed here aliows straightforward derivation of the blade section properties (without resorting to difficult-to-implement and error-sensitive finite element computer codes) by combining classical beam analysis with laboratory test results in a semi-empirical process here termed "mass-elastic trianqulation." The aeroelastic blade model thus achieved has the same static deflection and the same fundamental frequencies of vibration, in flapping, lead-lag, and torsion, as the actual blade, and can thus be used in aeroelastic calculations.

Linear beam theory with a simple unidirectional stress state is assumed. The analysis calculates area-weighted flexural and torsional moduli for the elastic beam, determines the beam properties relative to the principal axes, and then performs coordinate transformations to develop blade axis sectional properties. Deflections are calculated by referring the applied blade section loading and moments to the aerodynamic center (quarterchord), the mass center, the bending centroid (weighted area centroid), or the shear center (elastic axis) and transforming the deflections by superposition. Section geometry and moduli are verified by a series of laboratory bench tests including straightforward static load measurements on the actual blade, close blade inspection, and optional blade sectioning and coupon testing, which are also covered in this report. The section moduli and mass moments were verified by measuring experimental fundamental frequencies and modes of vibration.

Elastic twist results are presented for the three test blades: Carter 300 glass-epoxy, Gougeon ESI 54 wood-epoxy, and UTRC $8 \mathrm{~kW}$ pultrusion, illustrating the wide variation in the section properties predicted by (1) composite analysis alone, (2) static deflection empirical tests alone, (3) natural frequency tests alone, and (4) the present method, which combines all three.

Elastic twist of the UTRC blade varied from 3.3 degrees nose down at $4.5 \mathrm{~m} / \mathrm{s}(10 \mathrm{mph}, 0 \mathrm{~kW})$ to 0.25 degrees nose up at $13.4 \mathrm{~m} / \mathrm{s}(30 \mathrm{mph}, 13 \mathrm{~kW})$; the section torsion was dominated by the lift offset from the shear center and most of the twist occurred in the flexbeam. This was seen to greatly affect the performance and helped explain the actual vs. calculated UTRC $8 \mathrm{~kW}$ power curve.

The ESI blade, for both the 77 and $90 \mathrm{rpm}$ production versions, would have had less than 0.1 degree of elastic twist for all conditions if no tip flaps were present, due to its very high torsional stiffness. However, the elastic twist increased to about 0.33 degrees nose up for the standard flat plate tip, and 0.41 degrees nose down for the larger "whisper tip." Still, the effects of this live twist on the ESI performance were negligible.

The Carter blade has substantial blade leading edge ballast weight, which was seen to dominate the section torsion, resulting in over two degrees nose down live twist at high wind speeds. The ballast produced a very beneficial reduction of blade tip deflection, and also counteracted the large nose up elastic moment due to aerodynamic lift offset. Neither the ESI nor the Carter power curve test vs. theory discrepancies could be explained by elastic twist effects. The most significant factors affecting elastic twist were found to be shear center location, flap bending deflection, and added ballast weight. 


\section{TABLE OF CONTENTS}

Background.

1.0 Introduction

Composite Blade Sources of Uncertainty..

Sources of Elastic Twisting Moments.

\subsection{Project Scope and Methodology}

Goal of the Project.

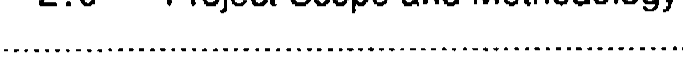

\subsection{Experimental Method}

General

Initial Blade Inspection

Blade Control Axis Location and Twist $\ldots$

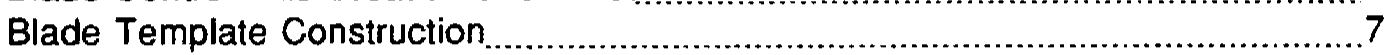

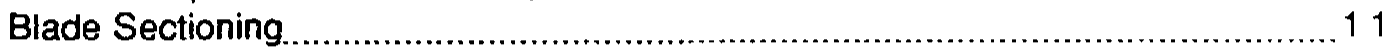

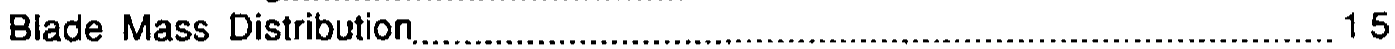

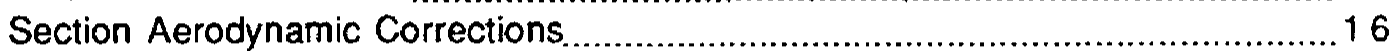

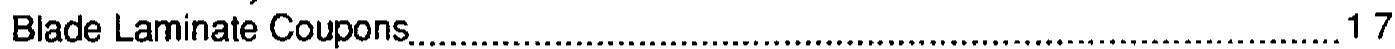

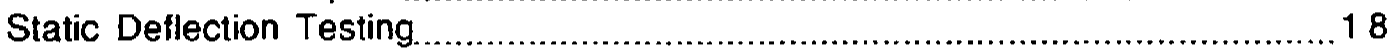

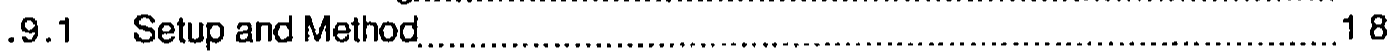

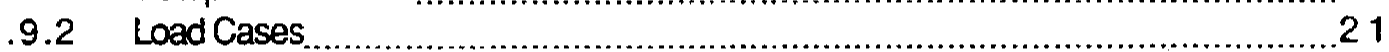

9.3 Deflection Measurement Method

.9 .4 Deflection Corrections to Quarterchord .............................................. 23

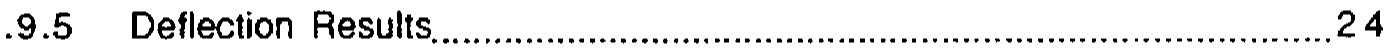

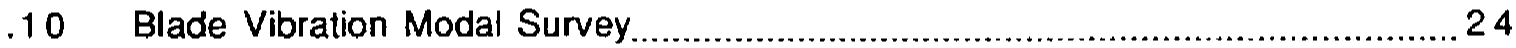

.1 General

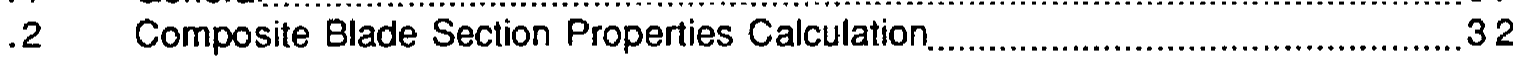

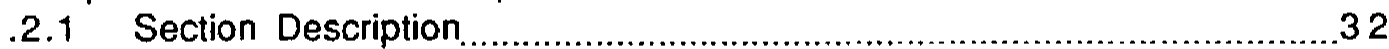

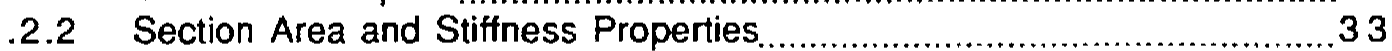

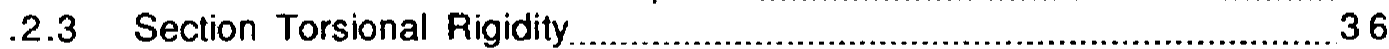

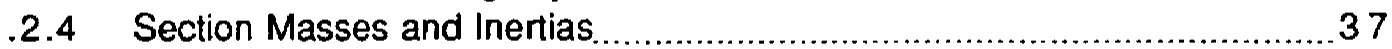

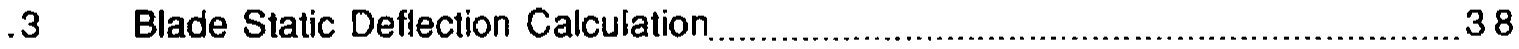

$.4 \quad$ Blade Natural Bending Frequencies and Modes .................................................4 41

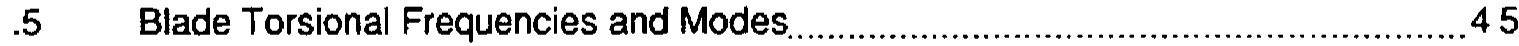

5.0 Development of Blade Mass/Elastic Models

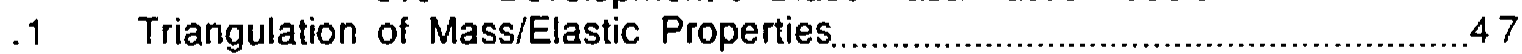

.2 Uniform FRP Blade Example: UTRC Blade..................................................... 47

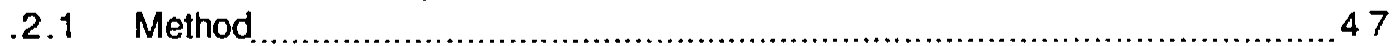

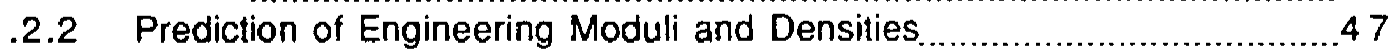

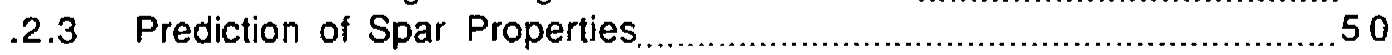

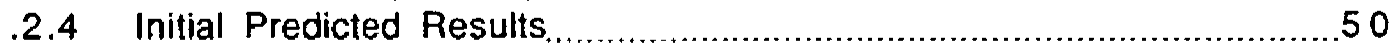

.2.5 Specification Verification by Blade Sectioning ....................................5 50

.2.6 Verification by Deflection Tests........................................................5 51

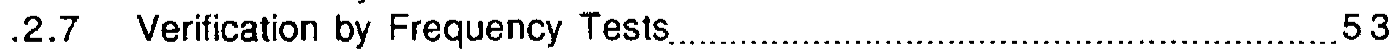

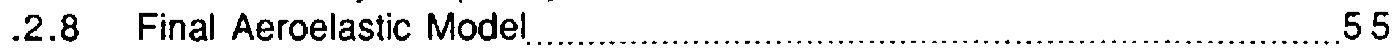




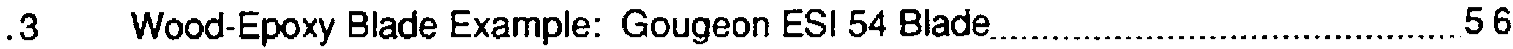

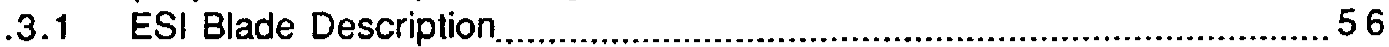

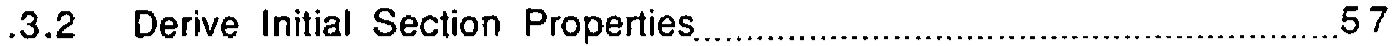

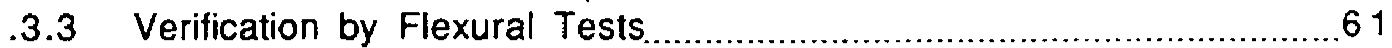

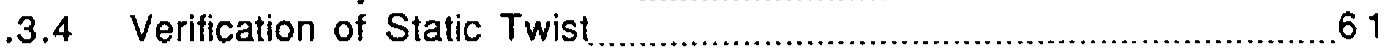

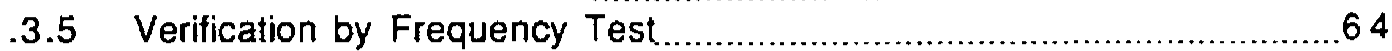

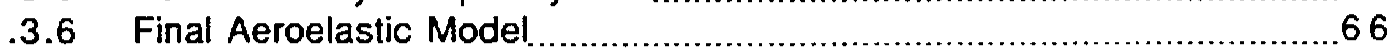

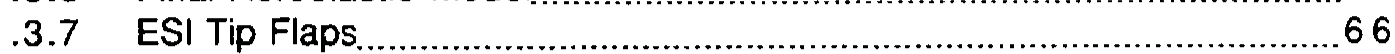

.4 Complex Composite FRP Example: Carter 300 Blade.

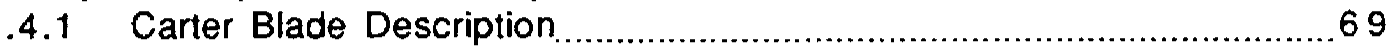

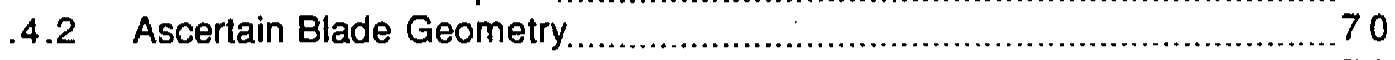

.4 .3 Ascertain Ballast and Weight Distribution .................................... 73

.4 .4 Derive Carter Design Composite Values ............................................. 75

.4 .5 Derive Contribution of I-Beam ........................................................... 79

.4 AEI Measurements for Composite section Properties ............................ 80

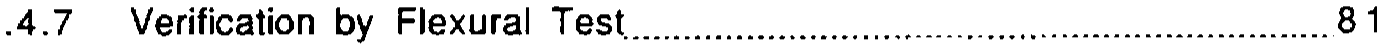

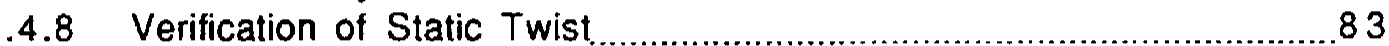

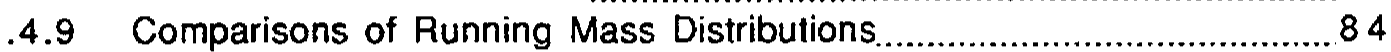

.4 .10 Verification by Frequency of Vibration Tests ...................................... 86

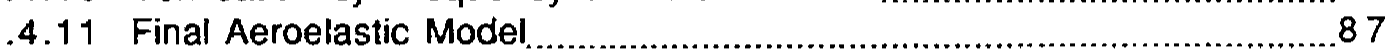

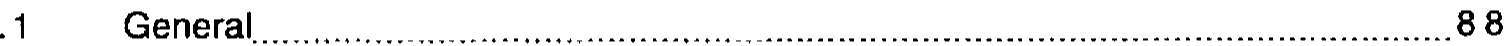

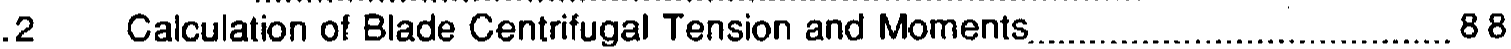

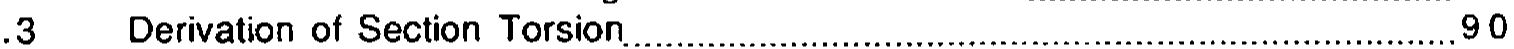

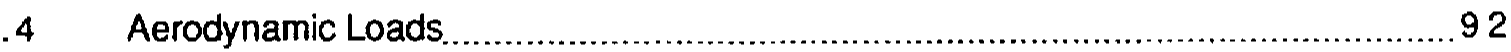

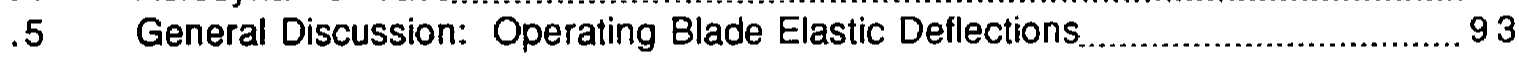

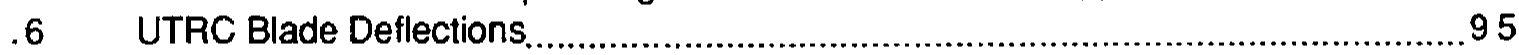

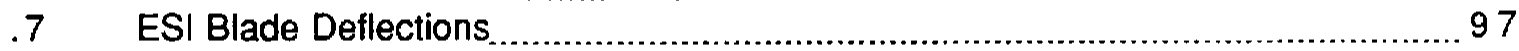

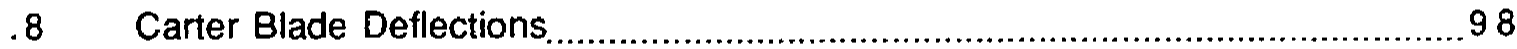

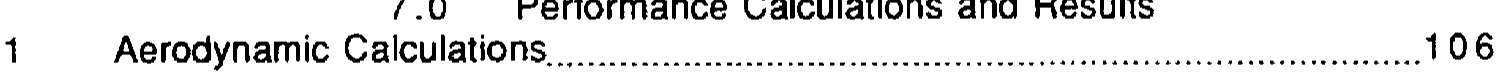

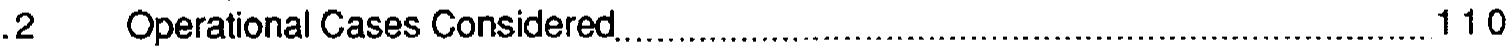

.3 Turbine Power Curve Performance Results...................................................110

.3.1 UTRC $8 \mathrm{~kW}$ Blade

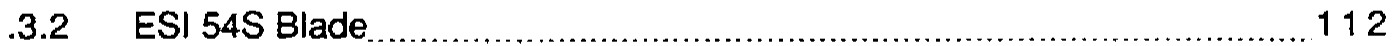

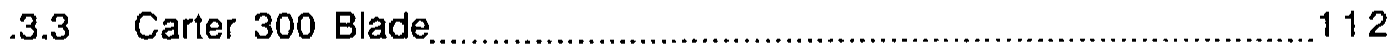

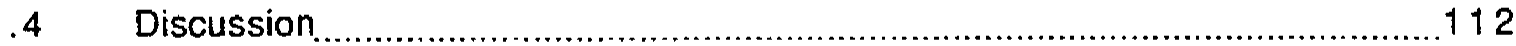

8.0 Conclusions

Comparison of Predicted and Experimental Results...................................114

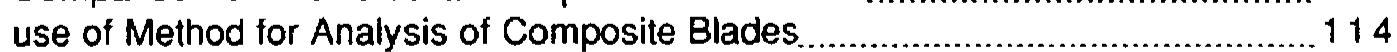

use of Method for Blade Quality Control for Manufacturers .............................114

Design Implications of Results........................................................... 114

Recommendations for Further Work and HAWT Improvement

9.0 References 
10.0 Appendices

.1 Computer Code: Determination of Composite Blade Section Properties ............118

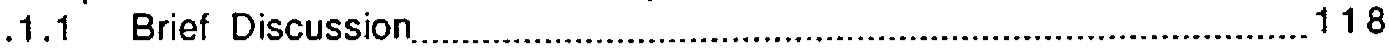

1.2 Program Listing, STRESSM ..................................................... 118

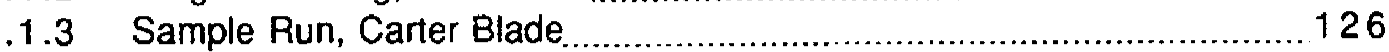

.1.4 Sample Run, ESI Blade. No Tips or Ballast .....................................130

.2 Computer Code: Blade Deflections and Stresses Under Loading ........................ 134

.2 .1 Brief Discussion

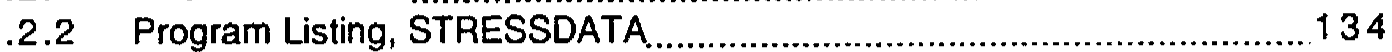

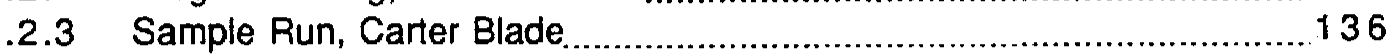

.2 .4 Sample Run, ESl Blade, With Tip Weight......................................138

.3 Computer Code: Composite Blade Bending Frequencies of Vibration.................139

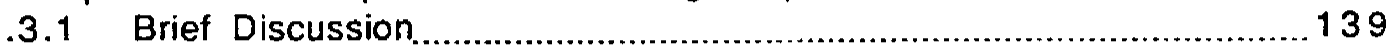

.3 .2 Program Listing, VIBRATE......................................................... 140

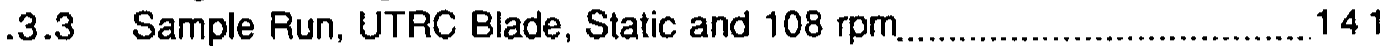

.3.4 Program Listing, VIBETIP, (Tip Weight Version) ..........................143

.4 Computer Code: Composite Blade Torsional Frequencies of Vibration ................143

.4 .1 Summary ............ 143

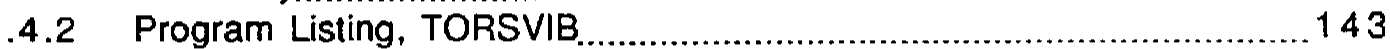

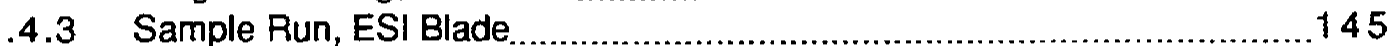

.4 .4 Program Listing, TVIBTIP, (ESI Blade With Tip Inertia)....................145

.4 .5 Sample Run, ESI Blade with Tip Inertia .......................................146

.5 Computer Code: Steady Elastic Twist Calculation.......................................146

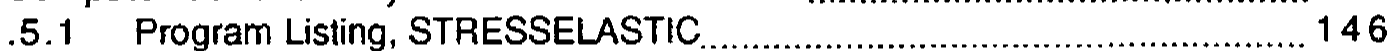

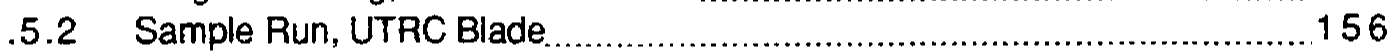

.6 Calculation of Drag Polars for Eight Wind Turbine Blade Sections .................. 158

.6 .1 Procedure and Analysis .................................................................... 158

.6.2 Planform Data Files and Drag Polars ........................................... 158

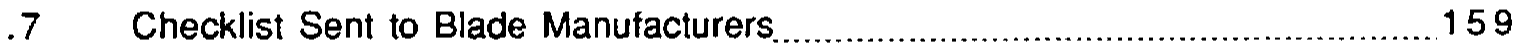




\section{LIST OF FIGURES}

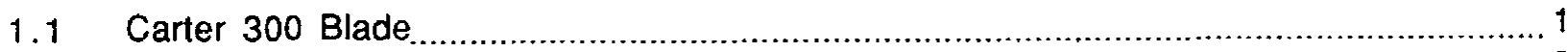

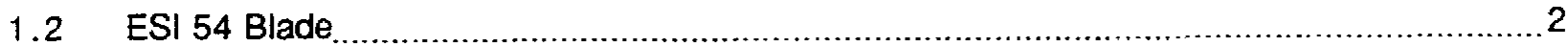

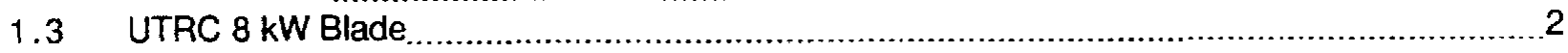

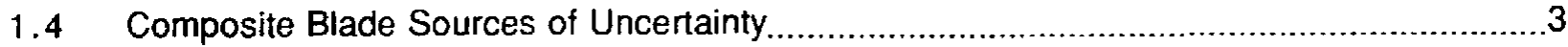

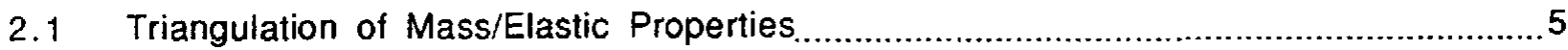

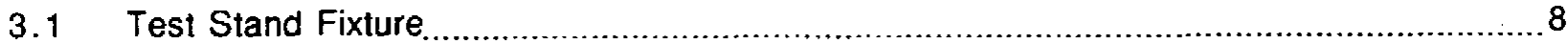

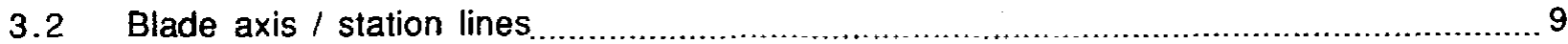

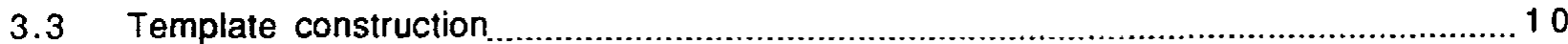

3.4 Twist Measurement by Mechanical Clinometer ..................................................... 11

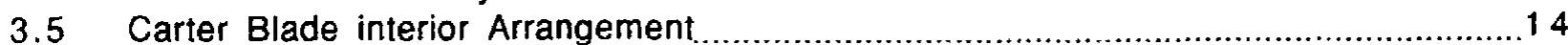

3.6 Lift-Drag Polars Compared (Specified vs. Measured / EPPLER Code) ..........................18

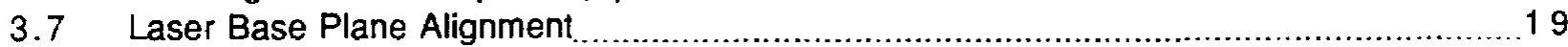

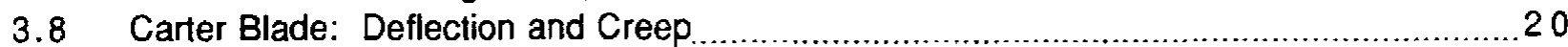

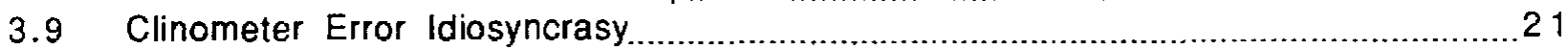

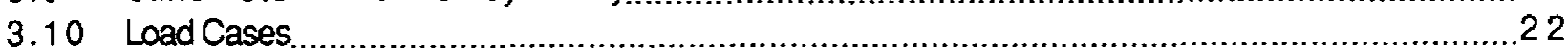

3.11 Test Table

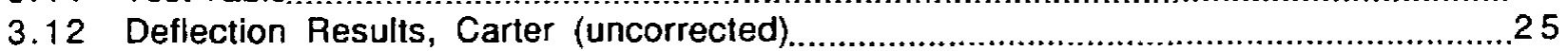

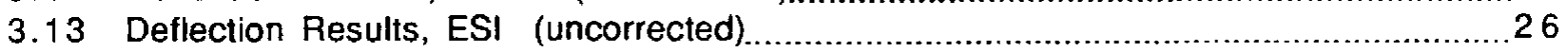

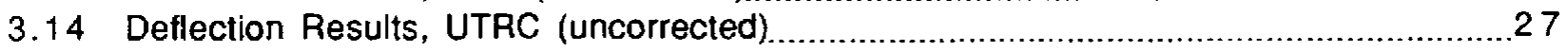

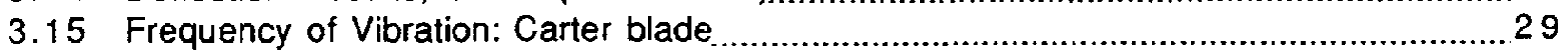

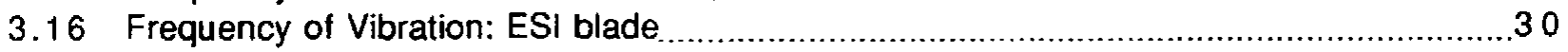

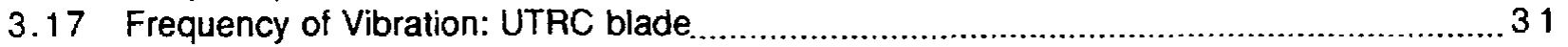

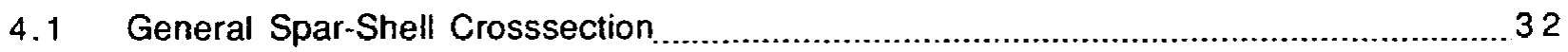

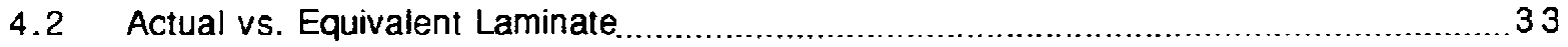

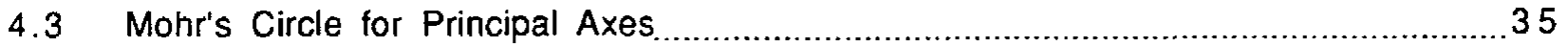

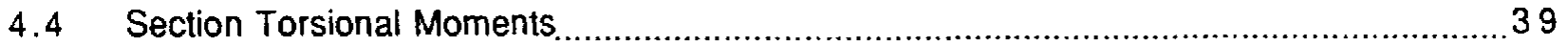

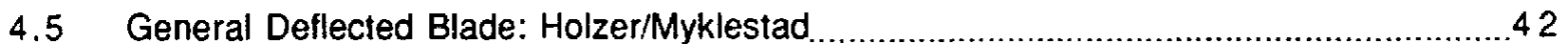

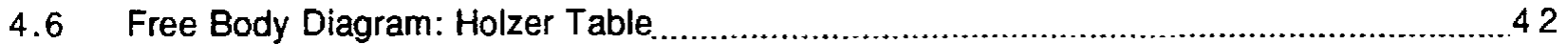

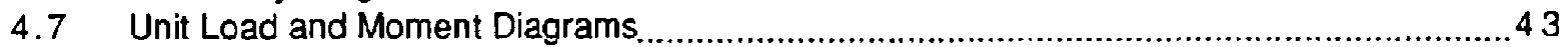

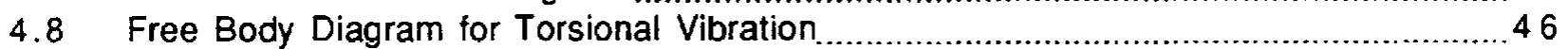

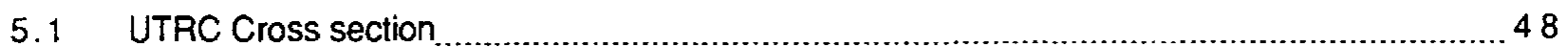

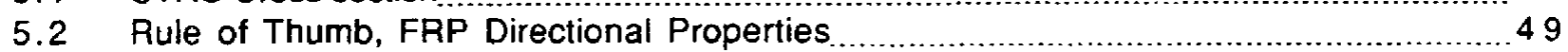

5.3 UTRC Flap Deflection, Test vs. Calculation vs. Specifications ..................................... 51

5.4 UTRC Vibration Modes, Test (modal survey)......................................................5 3

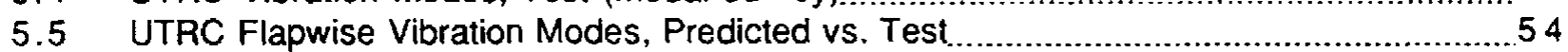

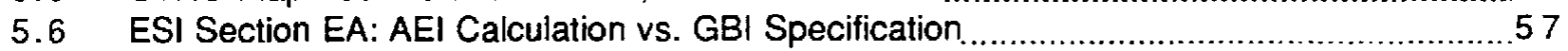

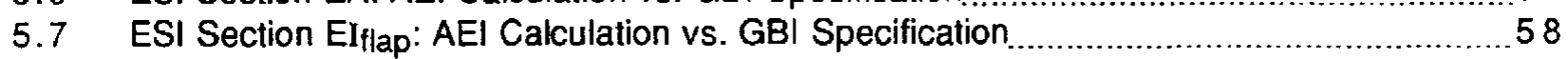

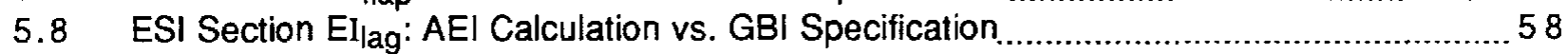

5.9 ESI Blade Running Mass: AEI Calculation vs. GBI Specification ................................... 59

5.10 ESI Section Shear Center: AEI Calculation vs. GBI Specification ....................................59

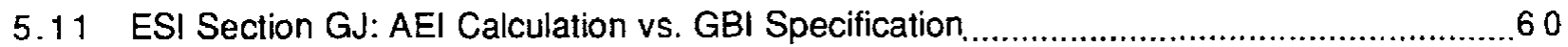

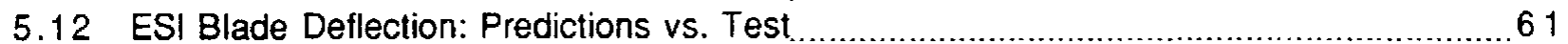

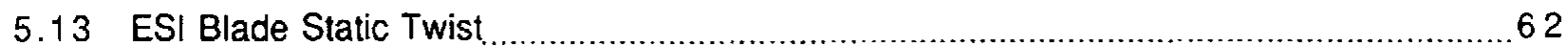

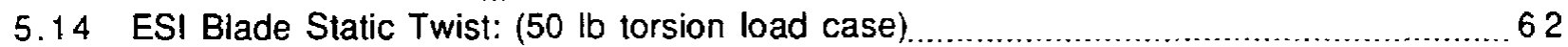




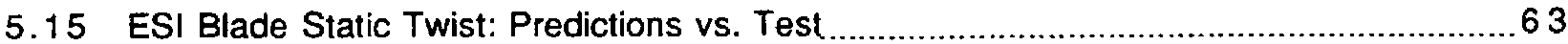

5.16 ESI Blade Twist: Predictions vs. Test (flexure load case) .........................................63

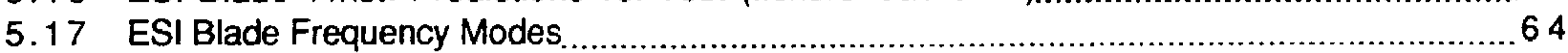

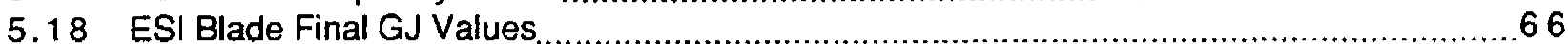

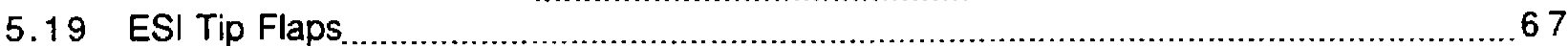

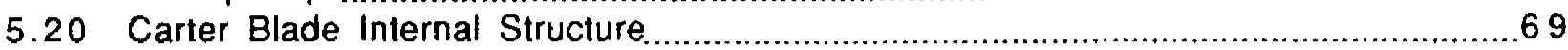

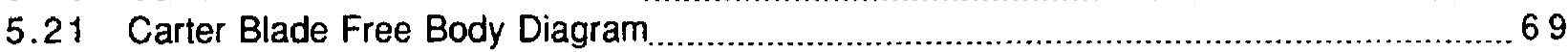

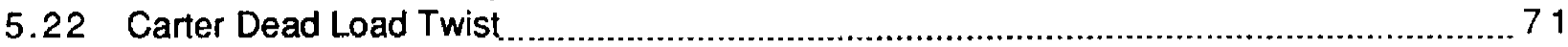

5.23 Carter Blade Twist from Measurement............................................................... 71

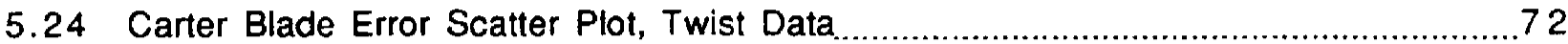

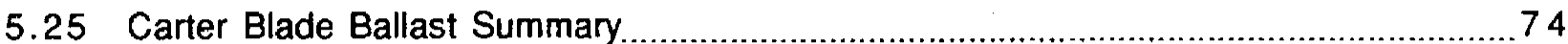

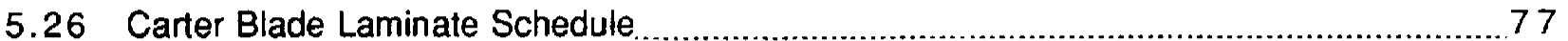

5.27 Carter Spanwise Weight Density (Spar Portion) ................................................... 78

5.28 Carter Static Deflection: Three Load Cases (Corrected) ........................................... 82

5.29 Carter Static Deflection: AEI Calculation vs. Measured (Corrected) ........................... 82

5.30 Carter Measured Twist: (225 lb test) Predicted vs. AEl Calculation

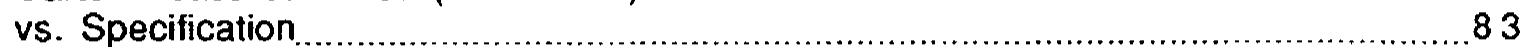

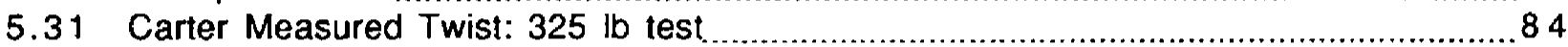

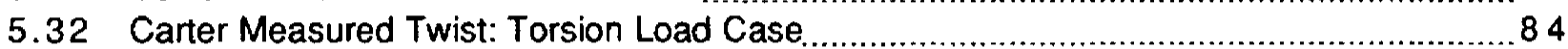

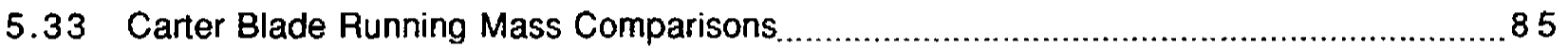

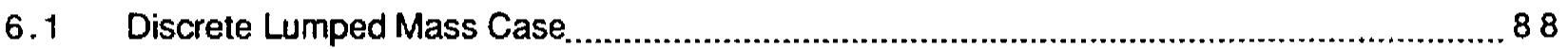

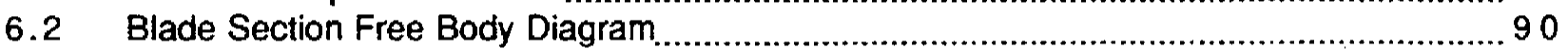

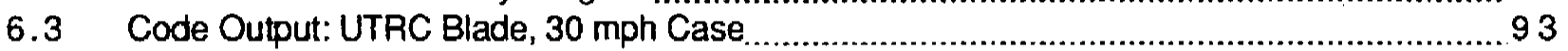

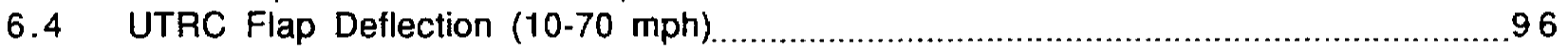

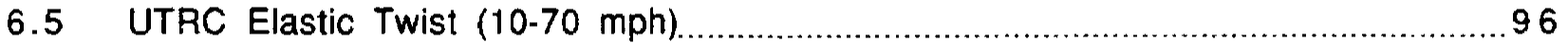

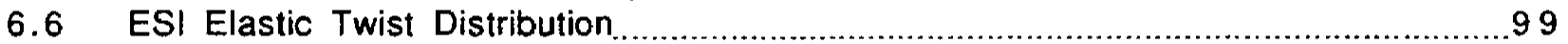

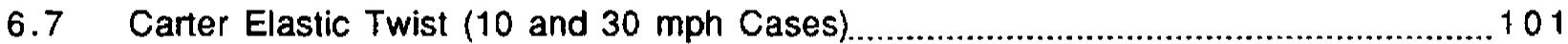

6.8 Carter Pitching Moment Distribution (10 and 30 mph Cases) .................................. 102

6.9 Carter Section Free Body Diagrams ............................................................ 103

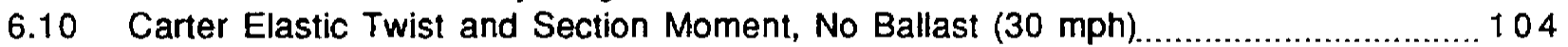

7.1 2-D Section Data Comparison: Standard vs. Viterna vs. Tangler ...........................10

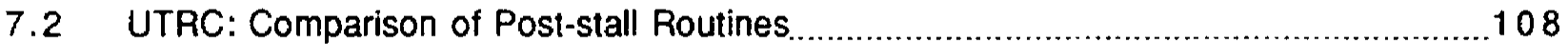

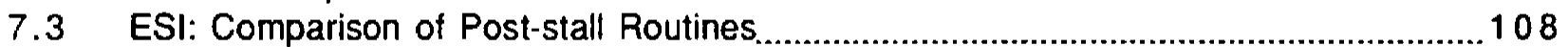

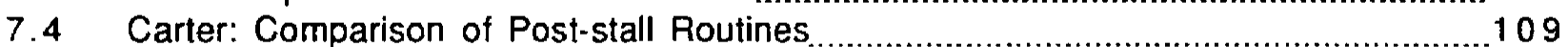

7.5 UTRC Power Output: Comparison of Test, Predictions, and Elastic Twist......................110

7.6 ESI 54 Power Output: Comparison of Data, Predictions, and Elastic Twist................... 110

7.7 Carter 300 Power Output: Comparison of Data, Predictions, and Elastic Twist...........110 


\section{LIST OF TABLES}

3.1 Internal Structural Measurements: Carter blade ................................................ 12

3.2 Internal Structural Measurements: ESI blade ....................................................13

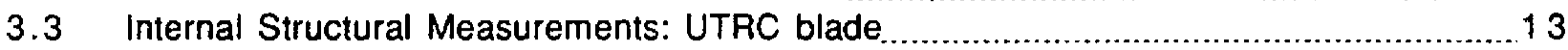

3.4 Sectioned $c g$ and Mass Data: Carter blade ......................................................... 15

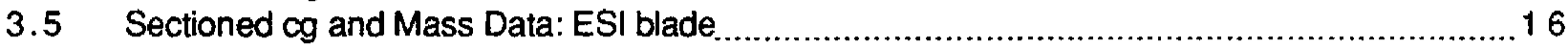

3.6 Sectioned $\mathrm{cg}$ and Mass Data: UTRC blade ......................................................... 16

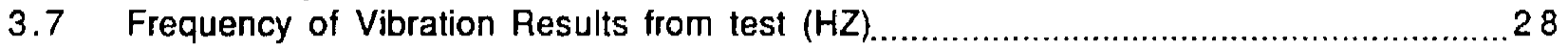

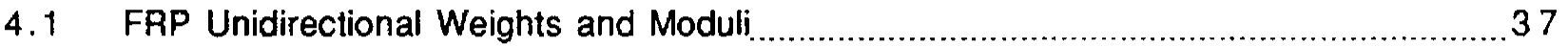

5.1 UTRC blade Section Properties Compared to Manufacturers'

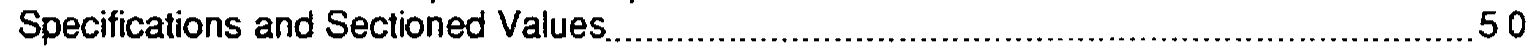

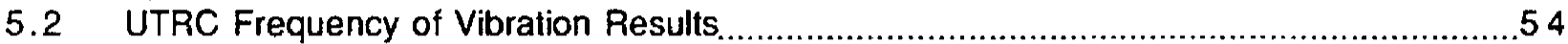

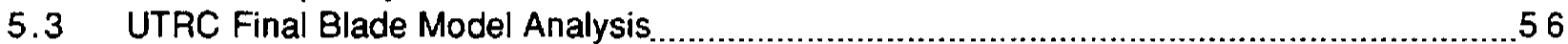

5.4 ESI Blade Frequency of Vibration: Predictions vs. Test ........................................65

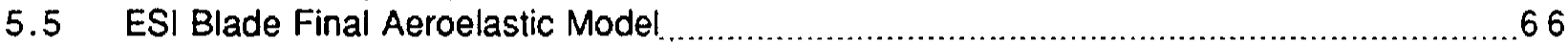

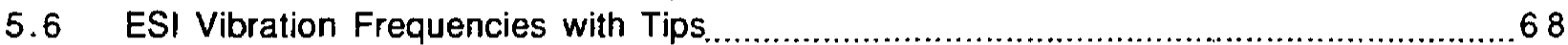

5.7 Carter Blade Blade Geometry, Measured vs. Carter Specifications ................................. 73

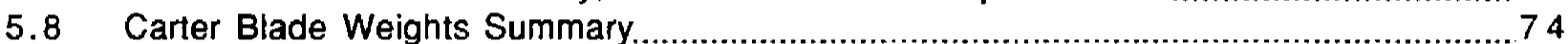

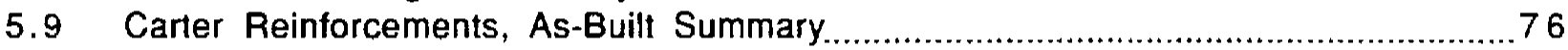

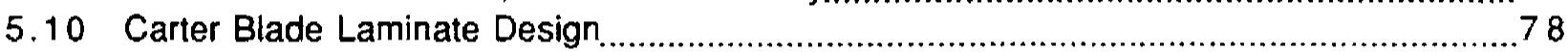

5.11 Carter I-Beam Section Data

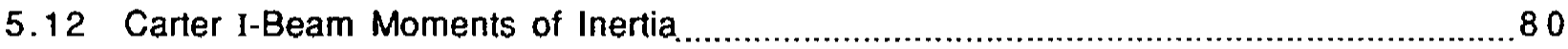

5.13 Carter Blade Section Measurements vs. Carter Specifications.................................... 80

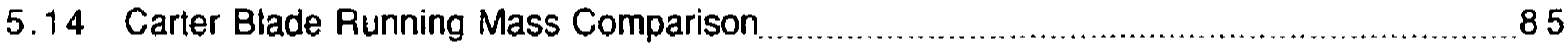

5.15 Carter Blade Flapwise Frequency Comparison.................................................8 86

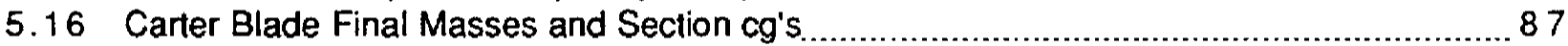

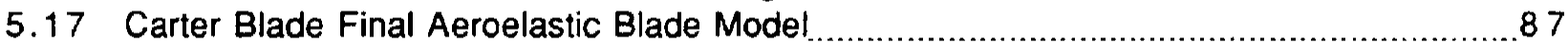

6.1 UTRC Flap Deflection

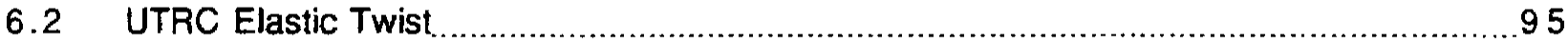

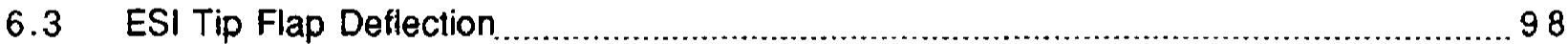

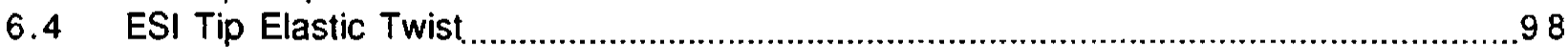

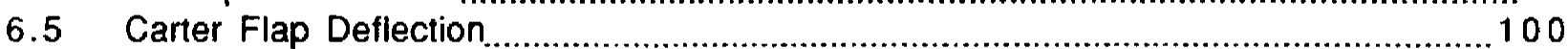

6.6 Carter Lead-Lag Deflection

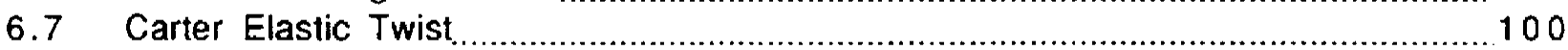

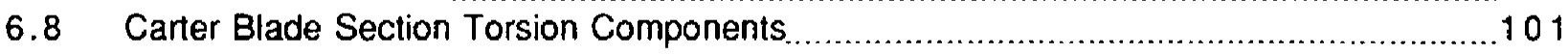

7.1 Airfoil Lift and Drag Data Input to PROPPC 


\subsection{INTRODUCTION}

\section{1: $\quad$ BACKGROUND}

High performance wind turbine rotors in the future may have light, flexible blades to increase cost competitiveness. Blades which are elastic in torsion will experience small pitch changes during operation which will cause large changes in aerodynamic loading leading to errors in wind turbine performance, especially near rated power conditions where blade loading and thrust are high. New airfoils for wind turbines have been and are being developed in order to attenuate the real-world detrimental effects of surface degradation, manufacturing tolerance, and unsteady aerodynamics in turbulent winds (Ref. 1).

Certain wind turbine rotors now in use have demonstrated aeroelastic instabilities and blade flexibility problems in operation (Ref. 2). Invariably the primary cause of flutter and divergence is blade torsional flexibility. In stall-regulated (fixed pitch) rotor systems an added potential flexibility problem is stall flutter. In pitch-regulated (controlled pitch) rotor systems, blade elastic twist in operation can lead to major increases in pitching moment and the attendant pitching system power required. Elastic blade twist near rated conditions, if nose-down or towards reducing angle of attack, causes delay of stall and negates the desired load alleviation, thereby causing potential rotor overloading. Elastic blade twist if nose-up or toward increasing angle of attack. causes premature blade stall. the potential for stall flutter and loss of power. The same arguments, though less significant, apply at operating conditions below rated or stalled conditions.

Up to now, the problem of calculating the blade elastic twist in operation has not been addressed except as qualitative or grossly estimated minor effects. Certainly that approach is defensible for heavy, massive blades which have little bending deflection and virtually no torsional deflection. For wind turbines to be cost-competitive in the future, however, the blade and rotor weights must be low, leading to unavoidably increased blade flexibility which must be accurately estimated. This project analyzed in a quantitative, practical, and reproducible way the amount of elastic twist for three representative wind turbines (Carter 300, ESI 54, and UTRC, Figs. 1.1 - 1.3), the origins of the elastic twist, and the likely changes in the wind turbine field performance.

\begin{tabular}{|c|c|c|c|c|c|c|c|c|c|c|c|c|}
\hline STATION & 0 & 0.1 & 0.2 & 0.3 & 0.4 & 0.5 & 0.6 & 0.7 & 0.8 & 0.9 & 0.97 & 1.0 \\
\hline RADIUS & 0 & 40.28 & 80.55 & 120.83 & 161.10 & 201.38 & 241.65 & 281.90 & 322.20 & 362.48 & 390.67 & 402.75 \\
\hline CHORO & & 66.75 & 52.01 & 42.50 & 34.56 & 28.97 & 25.56 & 23.44 & 22.19 & 21.41 & 21.08 & \\
\hline
\end{tabular}

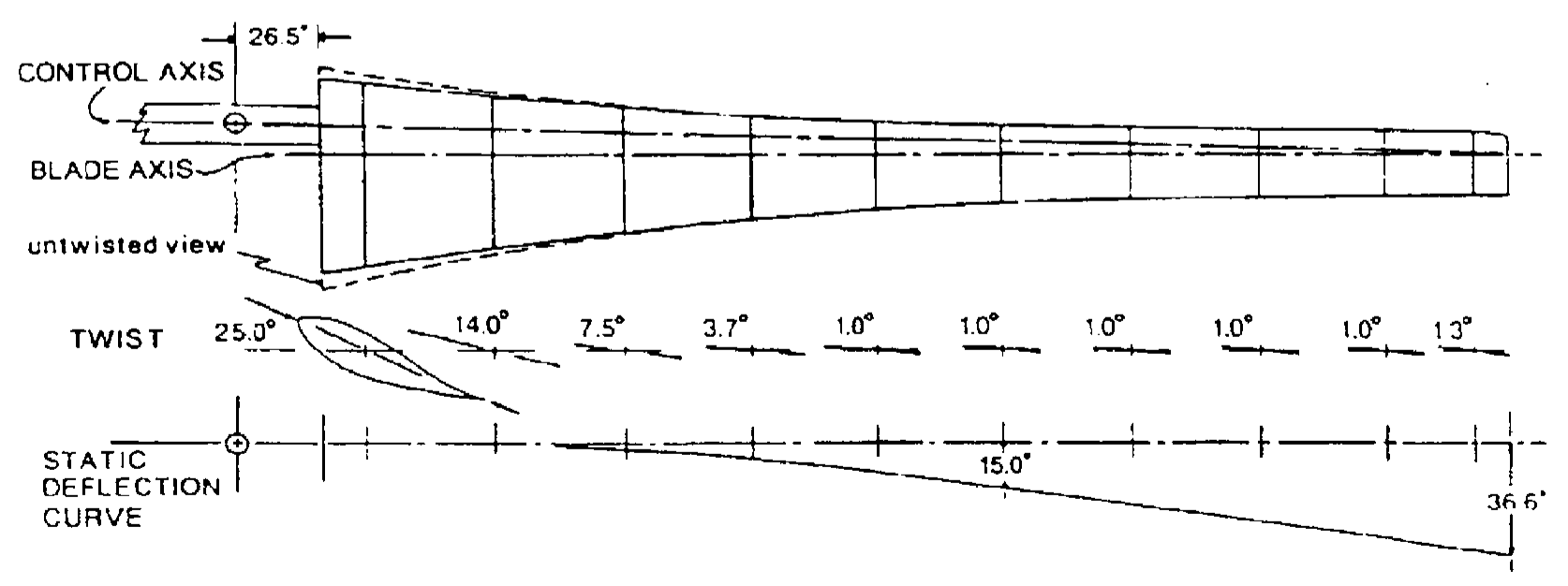

FIG 1.1 CARTER 300 BLADE 


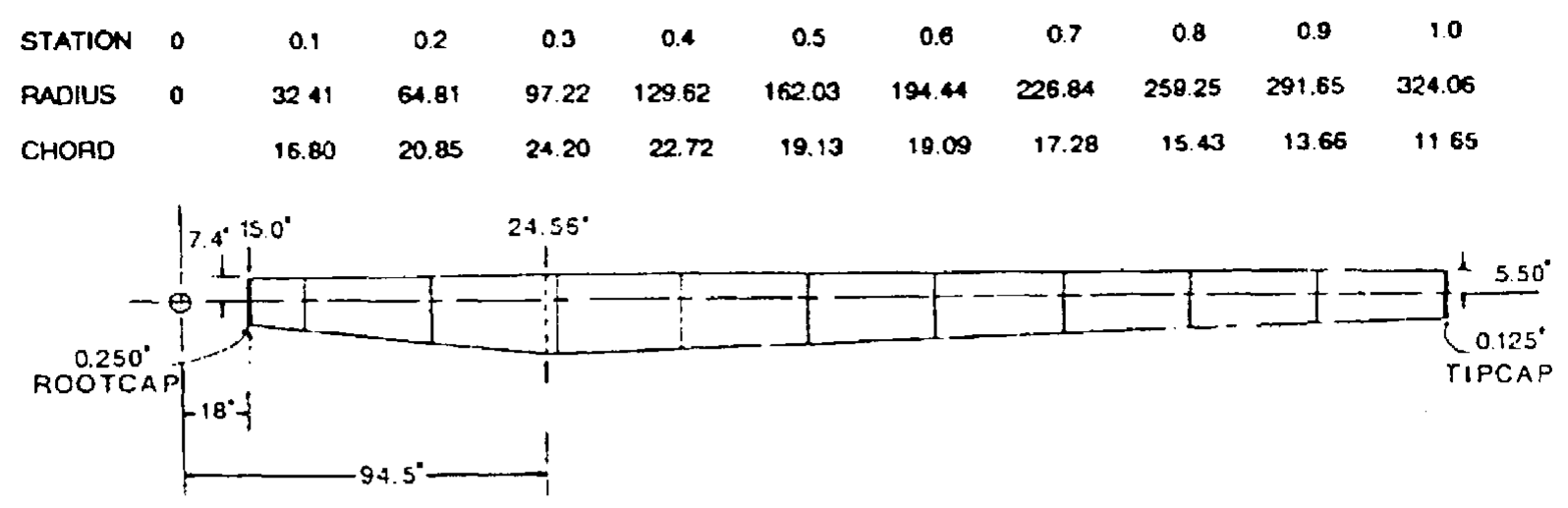

FIG. 1.2 ESI 54 BLADE

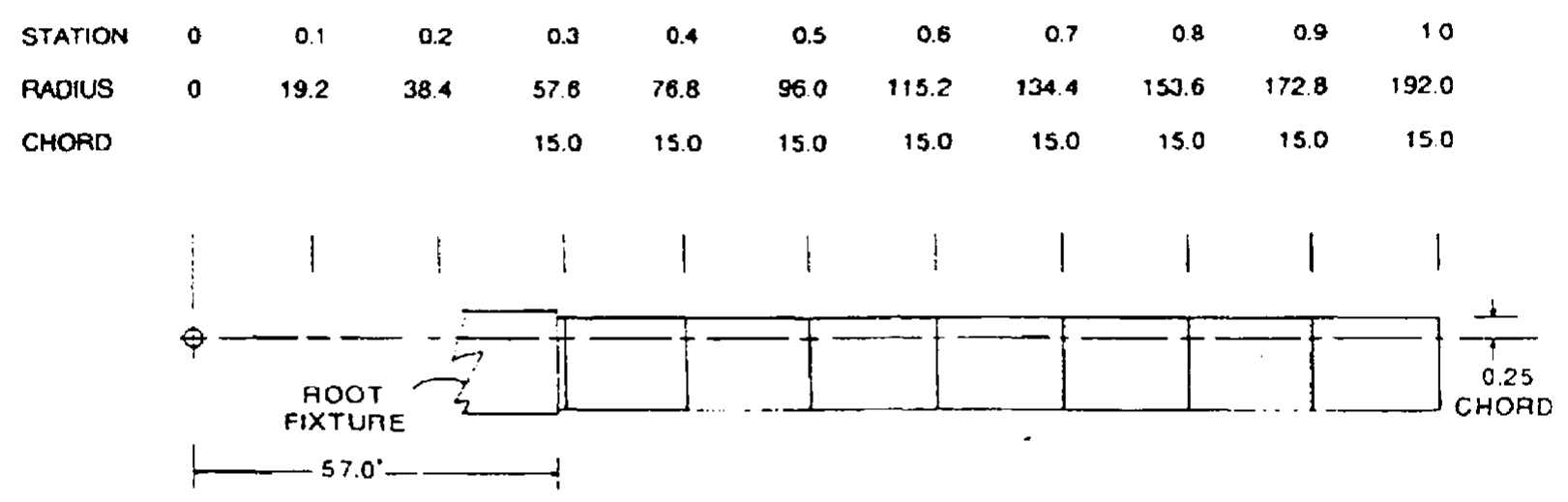

FIG. 1.3 UTRC $8 \mathrm{~kW}$ BLADE

\section{2: $\quad$ COMPOSITE BLADE SOURCES OF UNCERTAINTY}

The combined bending and torsion of composite structures, especially high aspect ratio (span-towidth) HAWT blades, is difficult to analyze, characterize, and predict (Ref. 3). The degree of structural anisotropy of a composite laminate in general extends to a stiffness matrix of 64 possible engineering constants. The methodology of isotropic strength of materials does not work since the molecular and physical structures of composites are radically different from metallic crystal structures (Ref. 4, 5, 6). Therefore, simplifying assumptions were made. For the unidirectional bending plus torsion indicative of wind turbine (and helicopter) blades a simple stress state was assumed, and the laminates were considered to be bidirectional, needing only four elasticity constants for engineering analysis: primary and transverse tensile and shear moduli. It was originally thought that these four could be verified in coupon tests, but that was found to be too difficult for the scope of this project.

In addition to the modulus uncertainties referred to above, manufactured composite blades, particularly those which are molded by hand (Carter, ESI) have significant geometric and internal structural sources of uncertainty (Fig. 1.4). These generally boil down to geometric errors (twist, taper, location of control axis), mass location errors (structural weight distribution, ballast weights), and the unavoidable nonuniformities in the individual laminates or plies (section errors). 


\section{Section Errors:}

Airfoil Shape

Chord

Thickness

Web Placement

Skin Thickness

Spar Thickness
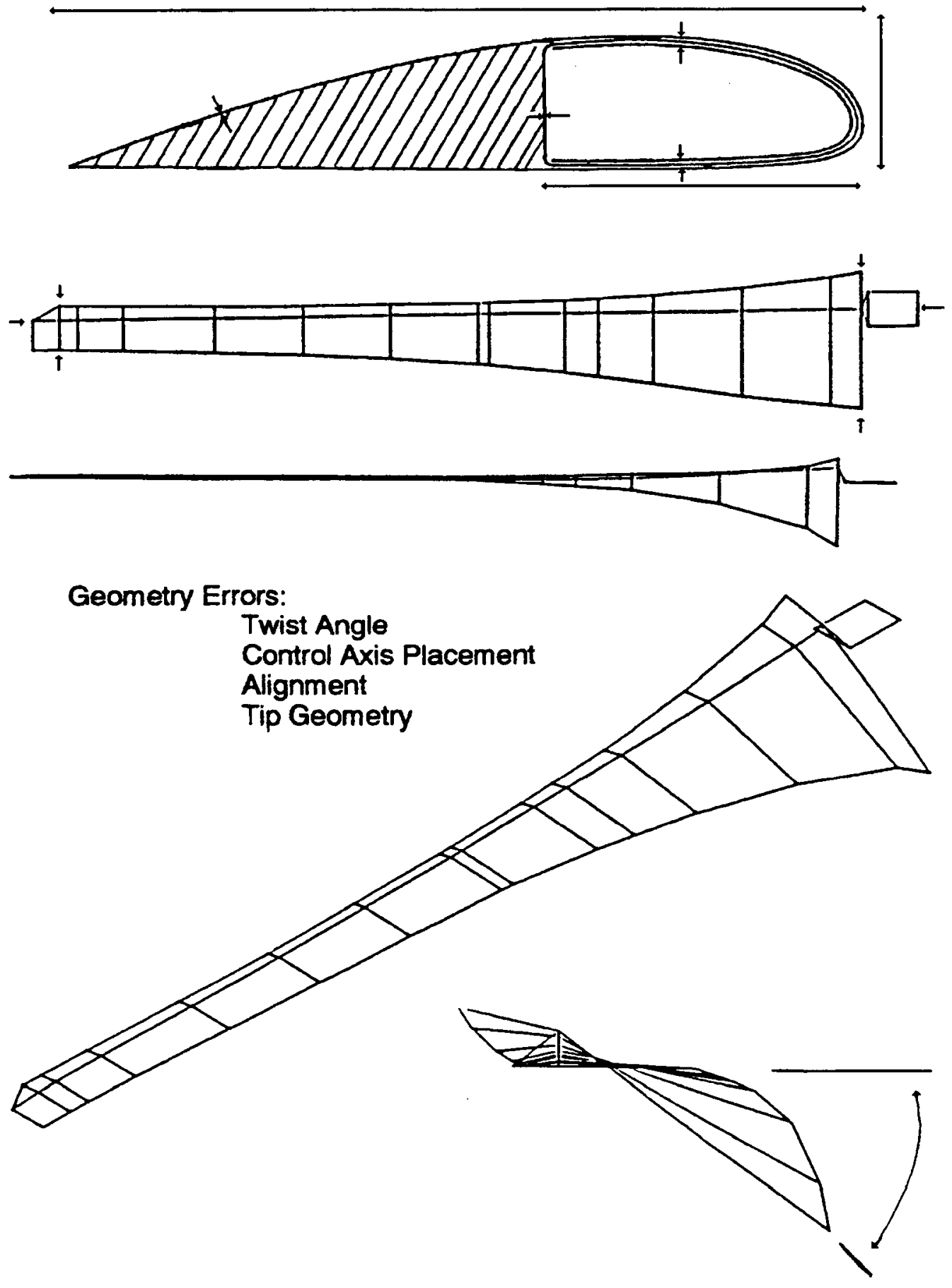

FIG. 1.4 COMPOSITE BLADE SOURCES OF UNCERTAINTY 


\section{3: $\quad$ SOURCES OF ELASTIC TWISTING MOMENTS}

The elastic twist of interest is the steady state twist due to steady forces and pitching moments on the blade. These produce the torsion on the blade which is resisted by the elastic blade structural sections. Additional elastic twist is due to structural coupling between bending and torsion. There are two major sources of the torsional moments: aerodynamic and inertial; that is, the moments due to aerodynamic pressure distributions and the moments due to centrifugal forces. The analysis of these moments is complicated by the difficult blade aerodynamics, which depends on historical 2-D data which does not model the true post stall and unsteady aerodynamic behavior in wind turbulence, and is further aggravated by manufacturing errors in blade airfoil post stall and section geometry. The analysis is also complicated by the typical composite wind turbine blade structure, which has complex geometry and is composed of fiber-reinforced resin, both of which introduce nonuniformities and structural coupling. A further complication is the blade-hub attachment. Sott attachments, such as the Carter "snubber," can cause large elastic pitch errors at the hub, which then add to the elastic twist, as verified during this testing program. Elastic twist introduced by the flexbeam and the pendulum weights for the UTRC was treated as an assumed root twisting moment. 


\subsection{PROJECT SCOPE AND METHODOLOGY}

\section{1: $\quad$ GOAL OF THE PROJECT}

The major goal of this study was to ascertain the elastic blade twist due to wind turbine operation which leads to errors in the prediction of loads and performance for three promising and representative wind turbine rotors. A secondary goal was to develop, verify, and present a practical engineering methodology for the estimation of this elastic twist on other wind turbine rotors.

\section{2: SEMI-EMPIRICAL APPROACH}

This program was divided into two parts of equal importance, experimental and theoretical. There were not enough resources in the program to permit a sophisticated engineering analysis in either area, so a practical engineering approach was taken (Fig. 2.1), and has proved efficient and effective. This allowed the derivation of semi-empirical blade aeroelastic models with which to calculate the steady elastic twist.

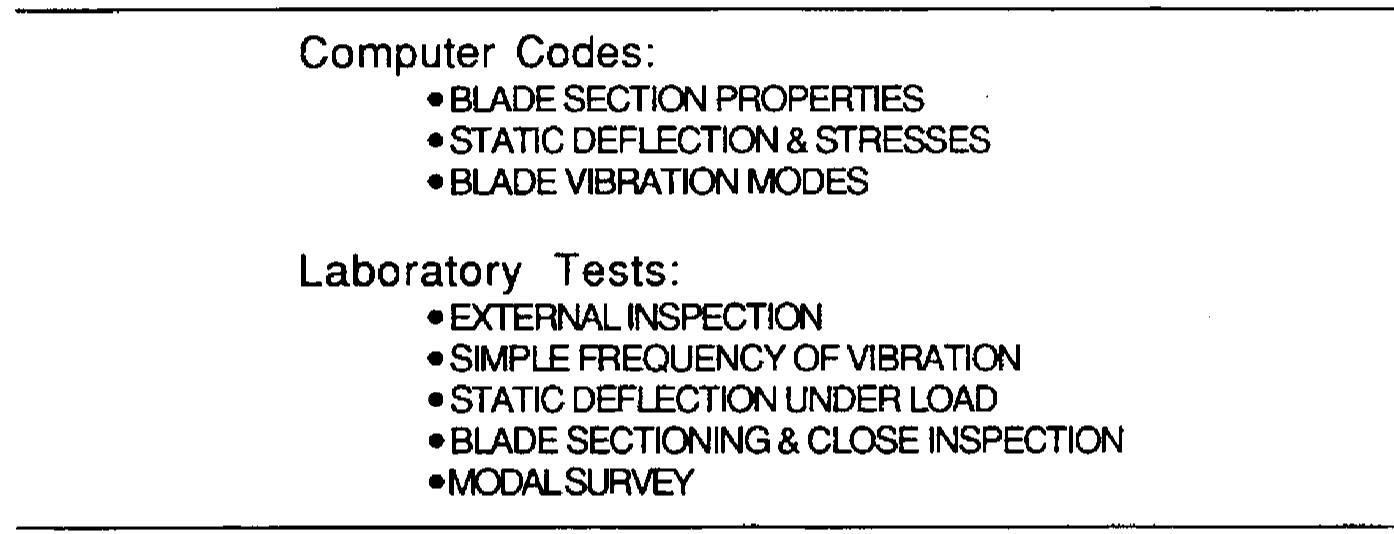

FIG. 2.1 TRIANGULATION OF MASS / ELASTIC PROPERTIES

The structural analysis programs employ practical and verified engineering techniques for the analysis of complex composite structure beams (Ref. 3). The wind turbine performance programs were developed by Solar Energy Research Institute (SERI), and are the standard aerodynamic codes in use in the industry (Ref. 7). The blade tests were performed at the Agricultural Research Service, U.S. Department of Agriculture (USDA) facility in Bushland, TX, under the cooperative agreement with AEI. The test equipment and testing techniques employed are standard laboratory items and methods generally available, with the exception of the Fast Fourier Transform (FFT) modal survey equipment provided by SERI, which was more sophisticated (Ref. 8).

The experimental testing program consisted of inspection, static loading, and frequency of vibration tests. Practical engineering laboratory techniques were used, which required only modest testing equipment (e.g., strip chart recorder, laboratory optical laser, laboratory strain gages), and permitted full-scale bending and torsion tests of the blades cantilevered from floor weldments. Simple mechanical measurement techniques were used at the cost of great accuracy (e.g., section twist measurements were to \pm 10 minutes of arc), but provided a simpler physical feel for the experiments.

The theoretical program used a mainframe computer (PRIME) for the calculation of blade structural parameters, blade aero- and inertial elastic torsion and loading, blade bending and twist, and frequencies of vibration. The mainframe computer was also used for the calculation of aerodynamic loading and wind turbine rotor performance. However, all the programs could be compiled and run on personal computers. Again as in the testing program, a practical engineering approach was taken for the analysis of the blade structure, insofar that a beam analysis program was used that does not calculate the complex shear stress and shear flow, but instead assumes a principal stress state, unidirectional component 
laminates which are representable by only four (empirical) engineering constants, and linear beam theory (plane sections remain plane). Again, the practical approach allowed many more calculations and iterations to be made, and permitted a better and more transferable physical feel for the structural effects.

\section{3: $\quad$ BLADES CHOSEN FOR THIS PROJECT}

The blades selected for this project (Carter 300, Gougeon ESI 54, and UTRC $8 \mathrm{~kW}$ ) represent a wide variety of promising blade characteristics: hub retention, airfoils, rotor diameters, aerodynamic geometries, and structural arrangements. The choice was limited somewhat by these major constraints (1) available field test data, (2) verified manufacturing quality control of mechanical properties, (3) enough flexibility for potential aero- and inertial elastic effects to be seen, and (4) cost and time constraints. These blades were also chosen to represent a range of composite construction and manufacturing techniques which are all potential weight- and cost-savers for future wind turbines. 


\subsection{EXPERIMENTAL METHOD}

\section{1: $\quad$ GENERAL}

The Carter and Gougeon blades (two blades of each) was procured directly from the manufacturer. One was returned for credit after static testing and the second was kept for sectioning. The UTRC blades were already the property of AEI. Additionally, a Carter hub was acquired for mounting its blade and ascertaining root compliance. ESI tip flaps were also acquired to determine their weights and predict their moments of inertia. The UTRC blade overspeed mechanism, the flexbeam/pendulum, was not studied in detail, only the outboard pultrusion.

In addition to the blades and rotor hardware, manufacturers were asked to provide specific data for the rotors (Appendix 10.7). The Gougeon blade was the only well-documented blade in manufacturer's correspondence and in the open literature. The Carter blade required considerable effort to ascertain internal structure, arrangement, and geometry.

\section{2: INITIAL BLADE INSPECTION}

The first requirement was to determine the actual blade external geometry and airfoil shape (measured at .75 and $.95 \mathrm{span}$ ). These were used as the basis for the aerodynamic loading on the blade. The internal structure including laminate thickness and geometry, and their spanwise distributions also had to be found at each tenth station (STA) for input to the structural model. Blade manufacturers had been asked to provide the specification for blade external geometry and structural geometry, and the mechanical properties of the individual laminates used, but this job ultimately rested on the test/inspection program. The hub attachment had to be measured in the same way for its input to the structural model.

The blade geometric parameters, structural details, and airfoil were determined first. A test stand fixture was constructed for each blade (Fig. 3.1) which allowed the individual blades to be cantilevered above the laboratory floor. Each blade was either bolt-on or clamped mounted, or in the case of the Carter, mounted in its own rotor hub.

\section{3: $\quad$ BLADE CONTROL AXIS LOCATION AND TWIST}

A blade "control axis" is defined as the pitch axis for a pitching blade, hence "feathering axis" (e.g. as in the case of the Carter blade), or as the centroid of the root fixation for a rigid blade (ESI). Linear spanwise and chordwise measurements were taken to determine the blade taper and radius. Blade control, or feathering axis, was determined and then inscribed on the blades along with 0.1 radius section lines (Fig. 3.2). Control axis was assumed to be the root centroid axis for the ESI blade; it was the quarterchord axis for the other two. The blade control axis was used as the primary blade reference axis. Twist was first measured on the cantilevered blades by using a laboratory laser, optical bench, and mirrors mounted on the templates. This laser/mirror method was discarded since on the cantilevered blades a precise clinometer gave the same accuracy and was much easier to use. Blade static gravity deflection was zeroed by taking twist measurements for $0^{\circ}$ and $180^{\circ}$ pitch orientation. Root pitch alignment was also measured for the blade/hub attachment. There were minor discrepancies between the measured values and the manufacturers' specifications (see Section 5.0).

\section{4: BLADE TEMPLATE CONSTRUCTION}

Template construction was done by two methods: the first was nondestructive, the second was from the sectioned blade. The former method consisted of pine blanks (Fig. 3.3), which were rough cut on the inside, leaving a small gap between template and blade, to fit both the upper and lower surfaces of the test airfoils at each 0.1 radii (tenth station). The surface of the blade was coated with a thin layer of floor wax and then auto body filler was used to bridge the gap between template and blade surface, with care taken to 

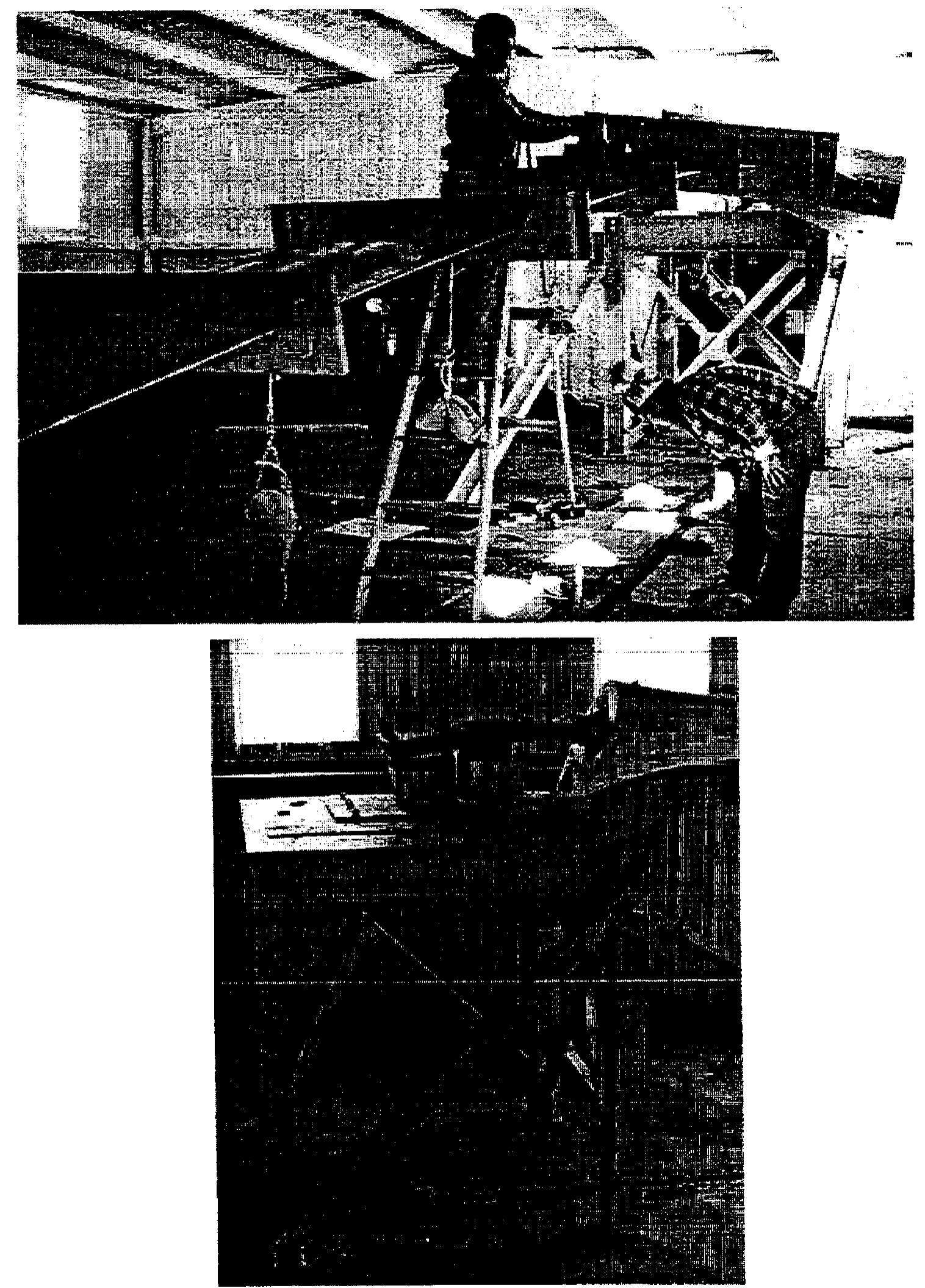

FIG. 3.1 TEST STAND FIXTURE 


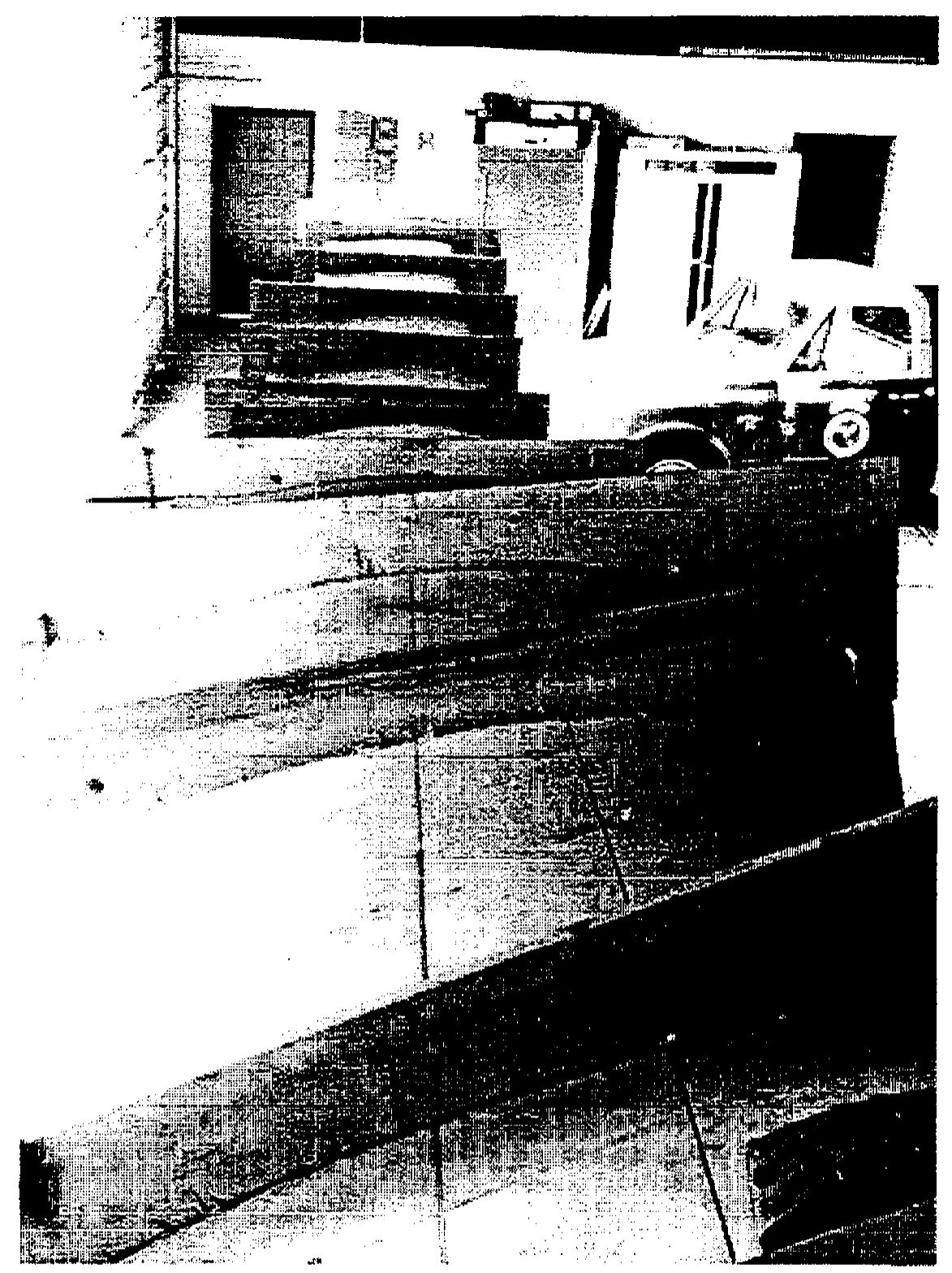

FIG. 3.2 BLADE AXIS / STATION LINES

preserve the chordline at the separation line between upper and lower template halves. Holes were drilled in the leading and trailing edges of the upper and lower parts of each stations' template and then dowel rods were inserted. Rubber bands and strapping tape were used to secure the templates together for inspection and installation on the blades. The clinometer was placed on top of the templates above the control line for measuring twist angles (Fig. 3.4). The template surfaces that butted together were located at the relative chordline for the section at that radius. The floor wax allowed easy removal of the hardened templates after the tests were completed. The squared outer edges of the templates were used as the reference lines for determining the relative twist at each radii using the clinometer. The problems with this method were establishing an accurate chordline and achieving accurate airfoil shape. 

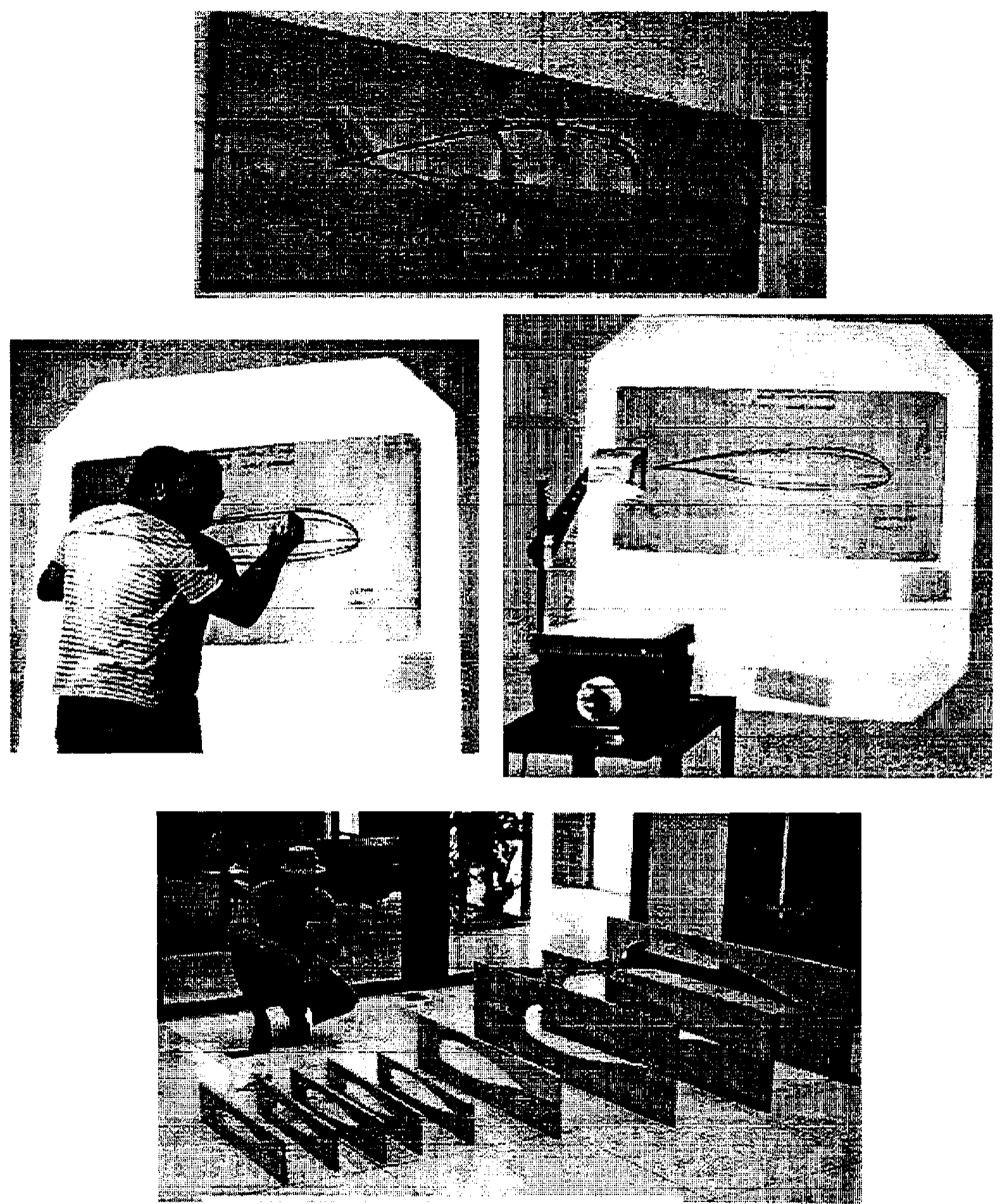

FIG. 3.3 TEMPLATE CONSTRUCTION 


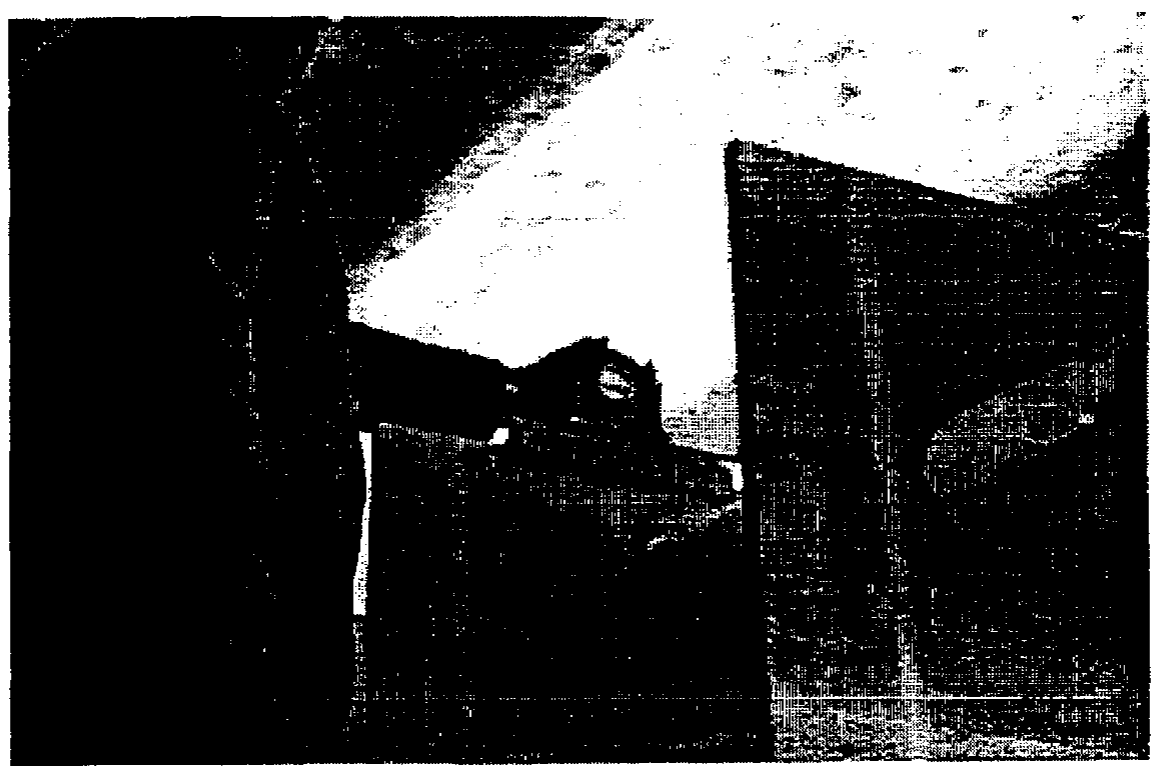

FIG. 3.4 TWIST MEASUREMENT BY MECHANICAL CLINOMETER

The latter method was much easier but depended on the sectioned (cut up) blade portions. The sections were traced onto craft paper and the chordlines marked on the tracing using a projection technique (Fig 3.3). The paper tracings were hung on a wall and transparencies of the members of the airfoil family were projected onto the tracing and judgment used in the location of the chordline. The chordline was then marked on the tracing. The various airfoils were also marked on the tracing for qualitative analysis of the cut sections, which included sending the tracings for EPPLER Code analysis (Sec. 3.7 below).

To construct the templates, the sections were placed on the above tracings with the chordline marked. The section then was placed on the two pine blanks with the chord line split between upper and lower surface. The section was then traced onto the wood for the construction of female templates for each station. The wood was finally cut and filed to closely approximate airfoil shape. Additional female templates were made at stations .75 and .95 for the aerodynamic evaluation.

\section{5: BLADE SECTIONING}

The internal structural geometry could only be accurately determined by cutting a blade into sections. A blade from each set was cut into sections corresponding to its 10 spanwise stations. For each station internal structural measurements were taken to verify the manufacturer's specification (Tables 3.1, 3.2, and 3.3). This allowed internal arrangement verification of ballast and inserts; this was especially important for the Carter blade (Fig. 3.5). Another reason for sectioning the blade was to determine the chordwise center of gravity (cg) position (Sec. 3.6).

An accurate mass axis was drawn from the spanwise $\mathrm{cg}$ positions; this turned out to be a very sensitive indicator of actual laminate thickness, reinforcement/resin ratio, and weighted area centroid. The lumped mass model of the blade, which was used in the frequency of vibration program, was readily determined from sectioned data.

The actual airfoil section geometry was determined accurately from the sectioned blade. in the Carter blade, it was thought that shear relief could modify the airfoil shape when the section was cut, so the .75 and .95 airfoils were determined both before and after sectioning (Sec. 3.7). 
TABLE 3.1: INTERNAL STRUCTURAL MEASUREMENTS: CARTER BLADE (in $\mathrm{cm}$ )
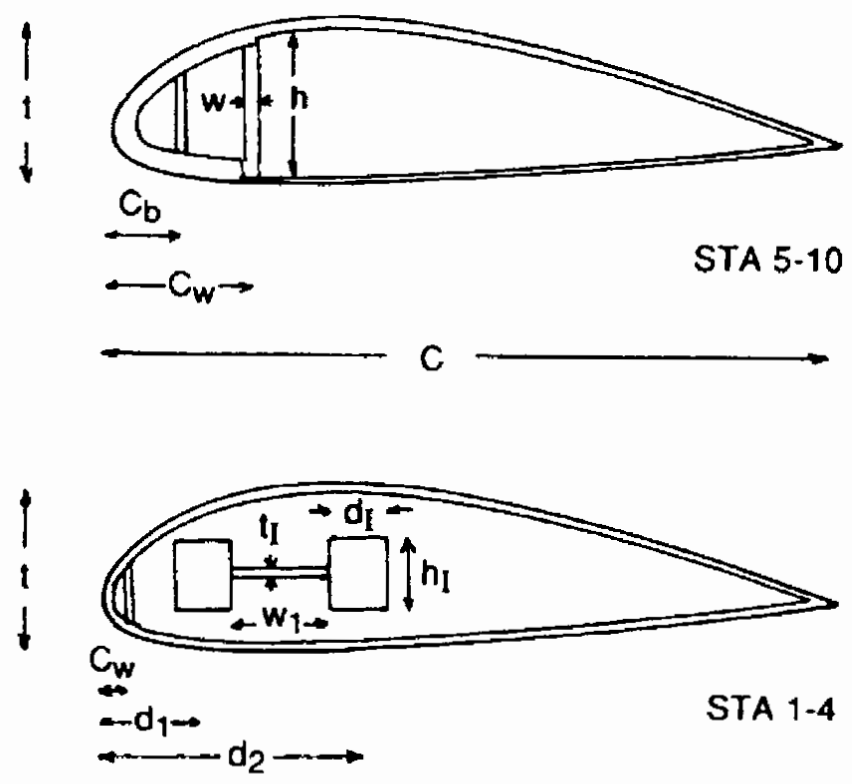

\begin{tabular}{lrrrrrrrrrr} 
STATION & 1 & 2 & \multicolumn{1}{c}{3} & \multicolumn{1}{c}{4} & \multicolumn{1}{c}{5} & \multicolumn{1}{c}{6} & 7 & 8 & 9 & 9.5 \\
\hline $\mathrm{c}$ & 169.55 & 134.14 & 107.95 & 87.78 & 73.58 & 64.92 & 59.54 & 56.36 & 53.98 & 53.19 \\
$\mathrm{t}$ & 35.71 & 28.09 & 20.80 & 13.34 & 9.68 & 8.33 & 7.54 & 7.06 & 6.83 & 6.58 \\
$\mathrm{C}_{\mathrm{b}}$ & & - & - & - & 6.35 & 6.50 & 6.35 & 6.99 & 6.35 & 7.32 \\
$\mathrm{C}_{\mathrm{w}}$ & 13.34 & 8.74 & 4.78 & - & 17.30 & 16.99 & 14.30 & 12.22 & - & - \\
$\mathrm{h}$ & 22.23 & 15.88 & - & - & 6.35 & 5.41 & 4.29 & 4.45 & - & - \\
$\mathrm{w}$ & 1.12 & 1.12 & - & - & .97 & .97 & .97 & .97 & - & - \\
$\mathrm{d}_{1}$ & - & - & 9.22 & 6.20 & & & & & & \\
$\mathrm{~d}_{2}$ & - & - & 35.89 & 27.15 & & & & & & \\
$\mathrm{w}_{1}$ & 25.10 & 27.00 & 21.92 & 16.84 & & & & & & \\
$\mathrm{t}_{1}$ & .64 & .64 & .64 & .64 & & & & & & \\
$\mathrm{~h}_{1}$ & 6.35 & 6.05 & 6.05 & 5.00 & & & & & & \\
$\mathrm{~d}_{1}$ & 6.99 & 3.89 & 3.96 & 3.96 & & & & & & \\
\hline
\end{tabular}


TABLE 3.2: INTERNAL STRUCTURAL MEASUREMENTS: ESI BLADE (in cm)

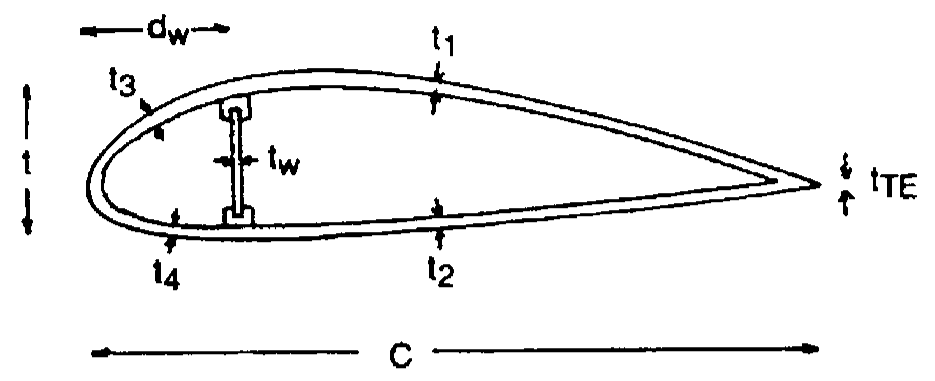

\begin{tabular}{|c|c|c|c|c|c|c|c|c|c|}
\hline STATI & 1 & 2 & 3 & 4 & 5 & 6 & 7 & 8 & 9 \\
\hline $\begin{array}{l}c \\
t \\
d_{w}\end{array}$ & $\begin{array}{c}42.67 \\
23.10 \\
-\end{array}$ & $\begin{array}{l}52.96 \\
21.00 \\
20.70\end{array}$ & $\begin{array}{l}62.00 \\
19.10 \\
20.25\end{array}$ & $\begin{array}{l}57.70 \\
16.70 \\
18.60\end{array}$ & $\begin{array}{l}48.60 \\
14.55 \\
17.30\end{array}$ & $\begin{array}{l}48.50 \\
12.49 \\
16.00\end{array}$ & $\begin{array}{l}43.80 \\
12.56 \\
14.30\end{array}$ & $\begin{array}{c}39.20 \\
8.43 \\
12.45\end{array}$ & $\begin{array}{c}34.70 \\
6.44 \\
10.96\end{array}$ \\
\hline$t_{w}$ & - & 0.67 & 0.67 & 0.66 & 0.68 & 0.68 & 0.66 & 0.65 & 0.68 \\
\hline tTE & 6.28 & 2.70 & 2.56 & 1.98 & 1.69 & 1.78 & 1.43 & 1.17 & 1.19 \\
\hline$t_{1}$ & 6.37 & 2.80 & 2.45 & 1.99 & 1.73 & 1.79 & 1.42 & 1.24 & 1.20 \\
\hline$t_{2}$ & 6.20 & 2.72 & 2.49 & 1.98 & 1.71 & 1.73 & 1.43 & 1.19 & 1.20 \\
\hline$t_{3}$ & 6.43 & 2.72 & 2.49 & 1.99 & 1.79 & 1.68 & 1.38 & 1.23 & 1.16 \\
\hline$t_{4}$ & 6.23 & 2.75 & 2.54 & 1.98 & 1.73 & 1.70 & 1.44 & 1.17 & 1.20 \\
\hline plies & 24 & 10 & 9 & 7 & 6 & 6 & 5 & 4 & 4 \\
\hline
\end{tabular}

TABLE 3.3: INTERNAL STRUCTURAL MEASUREMENTS: UTRC BLADE (in $\mathrm{cm}$ )

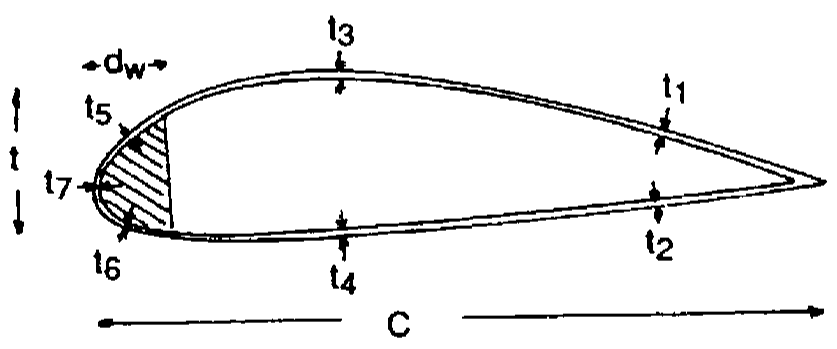

\begin{tabular}{lrrrrrrr} 
STATION & (Flexbeam) & \multicolumn{1}{c}{4} & \multicolumn{1}{c}{5} & \multicolumn{1}{c}{6} & 7 & \multicolumn{1}{c}{8} & \multicolumn{1}{c}{9} \\
\hline $\mathrm{C}$ & 37.96 & 37.82 & 37.42 & 37.90 & 37.95 & 37.90 & 37.90 \\
$\mathrm{t}$ & - & 5.00 & 4.96 & 4.93 & 4.88 & 4.96 & 4.96 \\
$\mathrm{~d}_{\mathrm{w}}$ & 6.50 & 6.50 & 6.50 & 6.50 & 6.50 & 6.50 & 6.50 \\
$\mathrm{t}_{1}$ & .33 & .27 & .29 & .31 & .28 & .38 & .30 \\
$\mathrm{t}_{2}$ & .33 & .27 & .29 & .31 & .28 & .38 & .30 \\
$\mathrm{t}_{3}$ & .29 & .27 & .23 & .26 & .23 & .27 & .28 \\
$\mathrm{t}_{4}$ & - & .23 & .22 & .24 & .32 & .27 & .32 \\
$\mathrm{t}_{5}$ & .27 & .24 & .25 & .26 & .29 & .29 & .29 \\
$\mathrm{t}_{6}$ & .23 & .25 & .23 & .24 & .24 & .23 & .22 \\
$\mathrm{t}_{7}$ & .36 & .39 & .41 & .43 & .41 & .40 & .40 \\
\hline
\end{tabular}




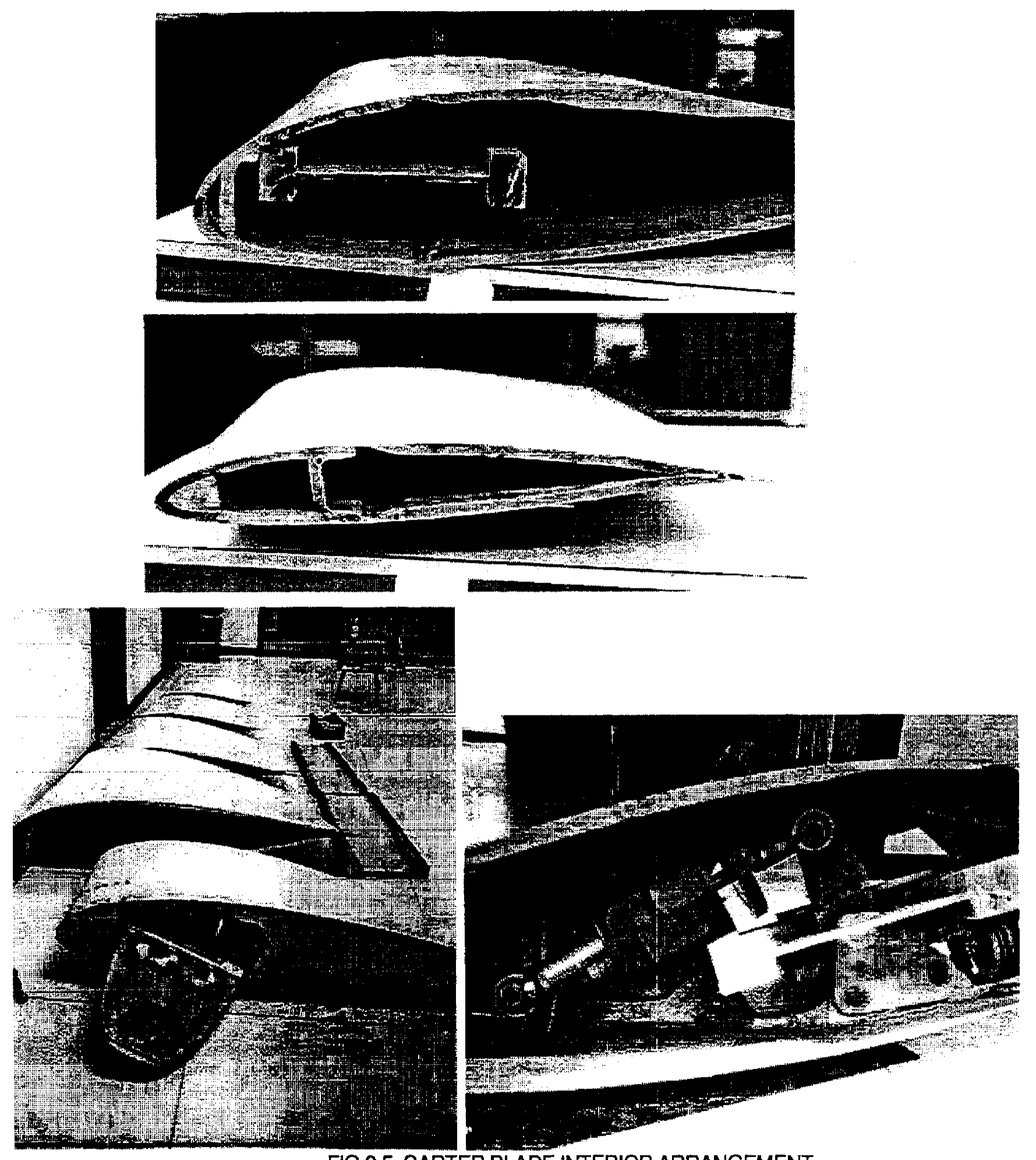

FIG 3.5 CARTER BLADE INTERIOR ARRANGEMENT 


\section{6: $\quad$ BLADE MASS DISTRIBUTION}

The actual mass distribution of the individual blades was determined by measuring the mass of the individual sections on a balance scale and locating the local $\mathrm{cg}$ of each section using an edge balancing beam. The individual sections were placed on a sharp edge (angle iron) and the balance point was determined and the line marked on the section; this was done spanwise, chordwise and at a diagonal. The intersection of these lines was taken as the point mass location and was converted to $X, Y, Z$ coordinates using the local quarterchord point as a 0,0 point ( $X, Y$ origin), and the center of rotation as the $Z$ origin. These points and masses are summarized in Tables 3.4,3.5, and 3.6, and were included as input data points for the computer models.

TABLE 3.4: SECTIONED cg AND MASS DATA: CARTER BLADE

\begin{tabular}{|c|c|c|c|c|c|c|c|c|c|c|}
\hline \multirow{3}{*}{ STA } & \multirow{3}{*}{ PANEL } & 1 & \multirow{2}{*}{\multicolumn{2}{|c|}{$\propto$ POSITION $(\mathrm{cm})$}} & \multirow{2}{*}{1} & \multirow{2}{*}{\multicolumn{2}{|c|}{ BLADE SHEU }} & \multirow{3}{*}{1} & \multicolumn{2}{|c|}{ MASS (kg) } \\
\hline & & i & & & & & & & \multirow{2}{*}{ I-BEAM } & \multirow{2}{*}{ SHEL } \\
\hline & & i & SPAN W & CHORD W & i & SPANW & CHORD W & & & \\
\hline $0-1$ & 1 & 1 & - & - & 1 & - & - & 1 & - & - \\
\hline $1 \cdot 2$ & 2 & 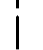 & 45.2 & 19.0 & & 47.8 & 55.8 & $i$ & 15.3 & 26.3 \\
\hline $2-3$ & 3 & 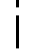 & 50.8 & 16.5 & 1 & 48.8 & 43.0 & $i$ & 12.7 & 21.7 \\
\hline $3-4$ & 4 & 1 & & & & 47.0 & 27.6 & $i$ & (4) & 34.1 \\
\hline $4-5$ & 5 & 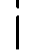 & & & & 49.2 & 21.4 & $i$ & & 30.3 \\
\hline $5-6$ & 6 & 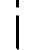 & (see & 4) & & 48.3 & 16.6 & $i$ & & 25.3 \\
\hline $6-7$ & 7 & 1 & & & & 52.2 & 13.7 & $i$ & & 23.1 \\
\hline $7-8$ & 8 & i & & & 1 & 45.7 & 12.2 & $i$ & & 20.6 \\
\hline $8-9$ & 9 & 1 & & & & 55.4 & 15.2 & 1 & & 12.5 \\
\hline $9-10$ & 10 & $i$ & & & 1 & 44.3 & 11.6 & 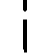 & & 19.4 \\
\hline
\end{tabular}

NOTES:

(1) BLADE WAS SECTIONED ON THE TENTH STATION LINES.

(2) PANEL NUMBER REFERS TO SPANWISE SEQUENCE.

(3) PANELS 4-6 CONTAIN SOME EMBEDDED I-BEAM AND POTTING RESIN.

(4) ROOT RIB AND SNUBBER MECHANISMS ARE IN PANEL 1 PORTION.

(5) PANEL 10 (BLADE TIP) CONTAINS BALANCE WEIGHT.

(6) PANELS 9 \&10 FROM CARTER BLADE \#29B, OTHER PANELS FROM CARTER \#47A. 
TABLE 3.5: SECTIONED cg AND MASS DATA: ESI BLADE

\begin{tabular}{cc|c|c|c|c} 
STATION & PANEL & & \multicolumn{2}{c}{ Cg POSITION $(\mathrm{cm})$} & MASS $(\mathrm{kg})$ \\
\hline $0-1$ & 1 & SPANWISE & CHORDWISE & \\
\hline $1-2$ & 2 & 18.4 & 4.8 & 18.6 & 12.36 \\
$2-3$ & 3 & 41.7 & 21.0 & 19.85 \\
$3-4$ & 4 & 43.4 & 25.4 & 18.03 \\
$4-5$ & 5 & 42.8 & 27.5 & 16.10 \\
$5-6$ & 6 & 41.5 & 24.6 & 12.81 \\
$6-7$ & 7 & 42.0 & 22.5 & 11.23 \\
$7-8$ & 8 & 43.5 & 21.0 & 9.07 \\
$8-9$ & 9 & 41.8 & 18.5 & 7.60 \\
$9-10$ & 10 & 40.7 & 15.8 & 6.01 \\
\hline
\end{tabular}

NOTES:

(1) PANEL 1 INCLUDED THE BLADE ROOT BUT NO STEEL STUDS.

(2) NO TIP WEIGHT OR MECHANISMS INSTALLED.

TABLE 3.6: SECTIONED $\subset$ G AND MASS DATA: UTRC BLADE

\begin{tabular}{|c|c|c|c|c|c|c|}
\hline \multirow[b]{2}{*}{ STATION } & \multirow[b]{2}{*}{ PANEL } & 1 & \multicolumn{2}{|c|}{$\operatorname{cg}$ POSITION $(\mathrm{cm})$} & | & \multirow[t]{2}{*}{ MASS $(\mathrm{kg})$} \\
\hline & & 1 & SPANWISE & CHORDWISE & $i$ & \\
\hline $0-1$ & 1 & 1 & - & - & 1 & 2.50 \\
\hline $1-2$ & 2 & i & - & - & i & 2.50 \\
\hline $2 \cdot 3$ & 3 & i & - & - & i & 18.24 \\
\hline $3-4$ & 4 & i & 23.0 & 12.1 & i & 3.75 \\
\hline $4-5$ & 5 & 1 & 25.2 & 12.0 & 1 & 3.64 \\
\hline $5-6$ & 6 & i & 24.4 & 11.8 & i & 3.64 \\
\hline $6-7$ & 7 & $i$ & 24.5 & 11.6 & i & 3.64 \\
\hline $7-8$ & 8 & | & 25.0 & 11.9 & 1 & 3.64 \\
\hline $8-9$ & 9 & 1 & 24.2 & $\$ 1.6$ & i & 3.70 \\
\hline $9-10$ & 10 & 1 & 24.6 & 12.2 & i & 3.70 \\
\hline
\end{tabular}

NOTES:

(1) STATIONS 0 - 3 COMPRISED THE FLEXBEAM/PENDULUM.

(2) STATIONS 4 - 10 COMPRISED THE PULTRUSION.

(3) PANEL 3 CONTAINED THE ATTACHMENT HARDWARE TO THE FLEXBEAM.

\section{7: $\quad$ SECTION AERODYNAMIC CORRECTIONS}

The airfoil shape manufacturing errors (for STA 0.75 and 0.95 ) were characterized in two methods (Appendix 10.6). The first method was to trace the airfoil shape from the cut edge of the sectioned blade. The second method used the undisturbed airfoil and auto body putty; a fine layer of wax was applied and the putty was placed around the entire airfoil at the desired station. The putty was allowed to harden and then was removed with breaks at the leading and trailing edges. The putty was reassembled and then finished so that one side was flat, then a Xerox copy was made to show the airfoil shape. The shapes were then digitized and smoothed by Prof. Stan Miley, Texas A\&M University, who was the aerodynamics consultant for the project. EPPLER code calculation determined a $C_{L}$ at various angles of attack up to stall 
(Appendix 10.6). The zero lift angle and the lift at 90 degrees were also computed. The differences between the blade specifications and the computer lift/drag polar were compared (Fig. 3.6).

\section{8: $\quad$ BLADE LAMINATE COUPONS}

It was originally thought that the blade material engineering constants could be determined from laboratory bench tests on coupons obtained from samples cut from the discarded blade sections, for each laminate (Ref. 9, 10). The four modulus values needed were tensile (longitudinal and transverse) and shear (longitudinal and transverse). Samples measuring roughly 1 in. by 14 in. and .25 in. thick can be readily used with standard tensile testing equipment to give bulk modulus results. These results are accurate to about $5 \%$ with fiberglass, provided that the sample is truly planar and without residual strain. This turned out to be impossible with coupons from these blades, although the methodology could still work under properly controlled conditions. If time and resources permit, at least three representative samples should be tested for each laminate. Four graph points with test weights or torsion springs would allow adequate accuracy. It is helpful to verify the actual composite engineering constants since the static deflections and frequencies of vibration are very sensitive to them; however, a design range of values can be adopted and used, as was done in this case.

\section{9: $\quad$ STATIC DEFLECTION TESTING}

\subsection{1: SETUP AND METHOD}

The building at USDA, Bushland, TX, was large enough to allow all three blades to be separately tested from the same platform. The test stand was constructed from the leftover base of a $100 \mathrm{~kW}$ vertical axis wind turbine. This stand was bolted to the concrete floor and braced with two 8-inch I-beams. The mounting connection for the Carter 300 blade was the spare hub borrowed from Carter Wind Systems. Mounts for the ESI and UTRC blades were manufactured in house. The most important part of the test rig was the establishing of a baseline using a laser. The line was defined by mounting the laser under the test stand and shining the light through a small peep hole and aligning the laser dot on the far wall (Fig. 3.7). The light line was leveled using a transit and placing a mark on the wall.

When there was no load, the Carter blade had a large deflection just due to its weight. Therefore it was supported at the tip between tests. The template halves were secured together on the blade with strapping tape and rubber bands. When the blade deflected, the cross sectional area (blade shape) changed at stations 2-4. At station 3 the template came apart with a gap of $1 \mathrm{~cm}$ the first time the blade was loaded and then template 3 was loose and had to be repositioned several times during the different load cases. 


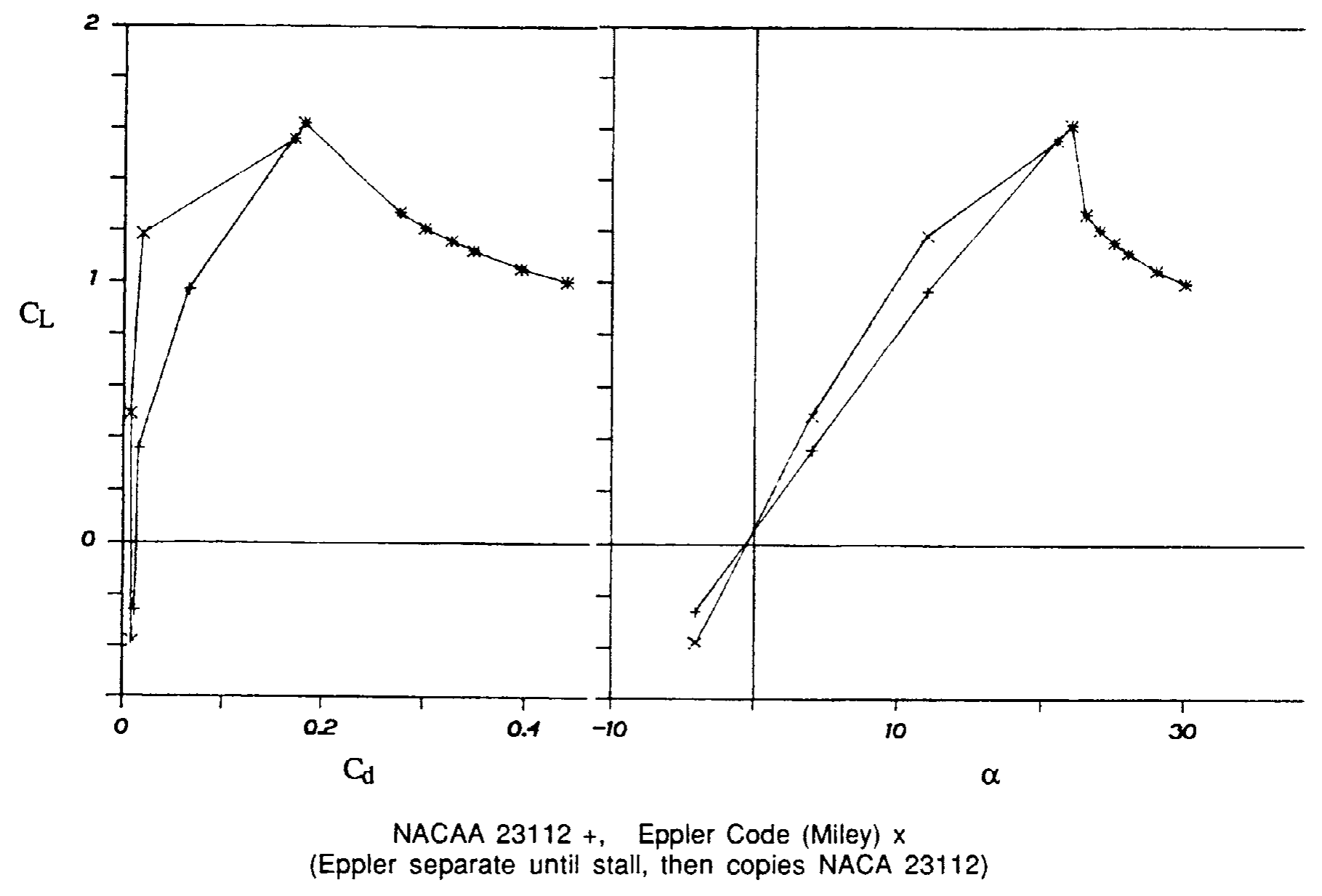

FIG. 3.6 LIFT-DRAG POLARS COMPARED (SPECIFIED VS. MEASURED / EPPLER CODE)

The static deflection tests were performed by using the setup shown in Figure 3.7. Lead-weighted bags were suspended from taped hooks located at the control axis of the blade sections. Lead shot was used for accuracy and tape was used to simulate suction (Sec. 3.9.2 below and Fig. 3.11). Experience with this type of test has shown that temporary creep is a problem with composites (Fig. 3.8 illustrates the Carter blade); therefore, the linear measurements were made relative to a relaxed datum, and averages of several trials were made. The elastic twist was measured in two ways: first, by optical laser and template mirrors providing accuracy to \pm 10 minutes of arc and second by the clinometer. The Fowler clinometer was acquired to take ideal local inclination measurements to within 1 minute accuracy. The base of the unit is beveled so that it can sit on a rounded edge and still give an accurate reading of inclination. The unit was able to read degrees of deviation from $0^{\circ}$ (horizontal) to $\pm 180^{\circ}$. The major problem with this unit was in reading minor angles in the $(-)$ direction. The unit has a manual dial calipered gauge and the negative readings must be read as subtraction's from the displayed degrees. That is, a reading of 45 minutes was 45 minutes when the angle was positive (nose up) but that it was meant to be read as 15 minutes subtracted from the next higher degree reading if the angle was negative. This stet idiosyncrasy of the unit resulted in operator misreading data when very close to $0^{\circ}$ and negative angle. The graph presented Figure 3.9 shows this in the Carter 300 data twist under load vs. Station in stations $4-7$. 


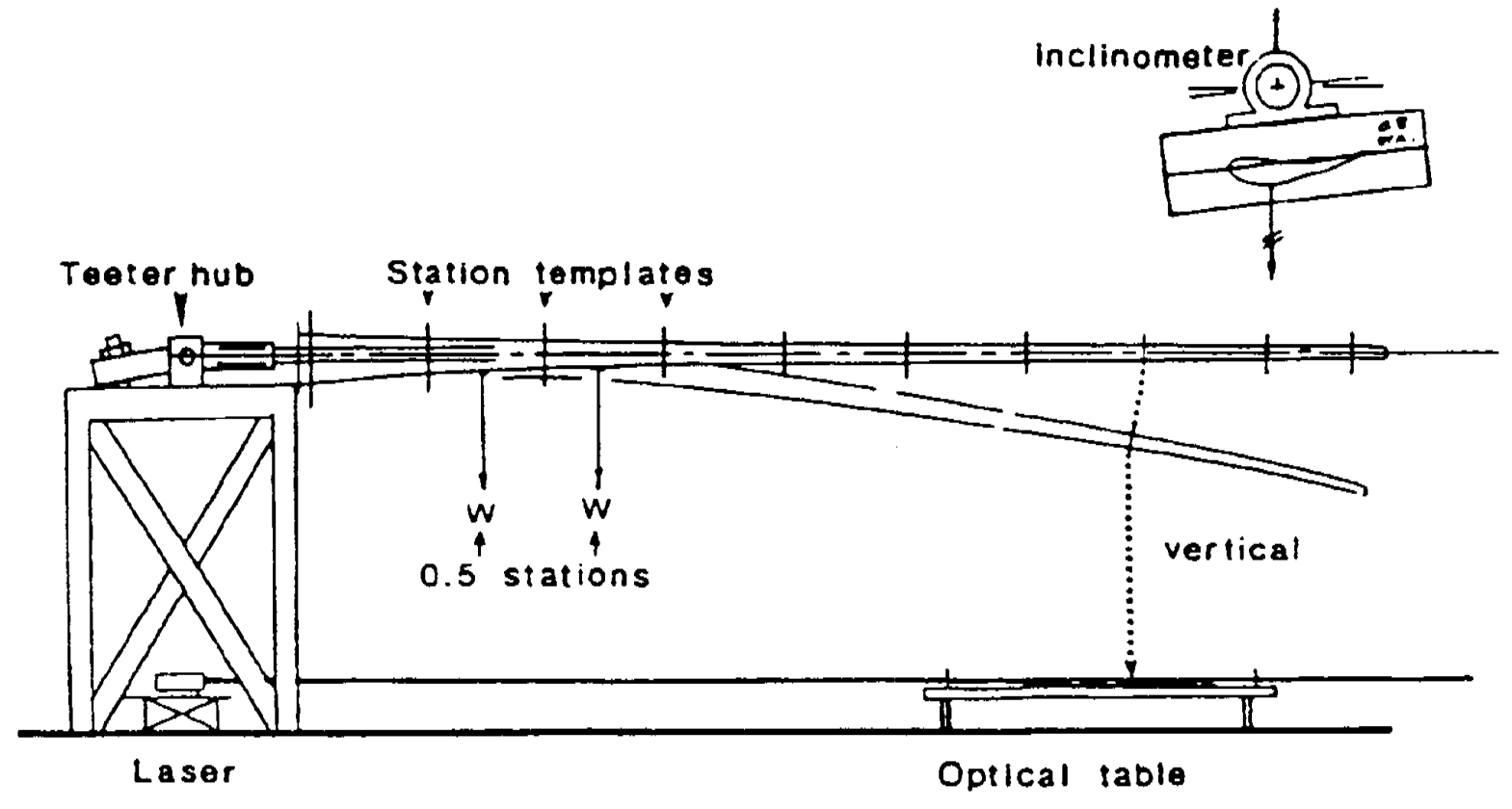

FIG. 3.7 LASER BASE PLANE ALIGNMENT 


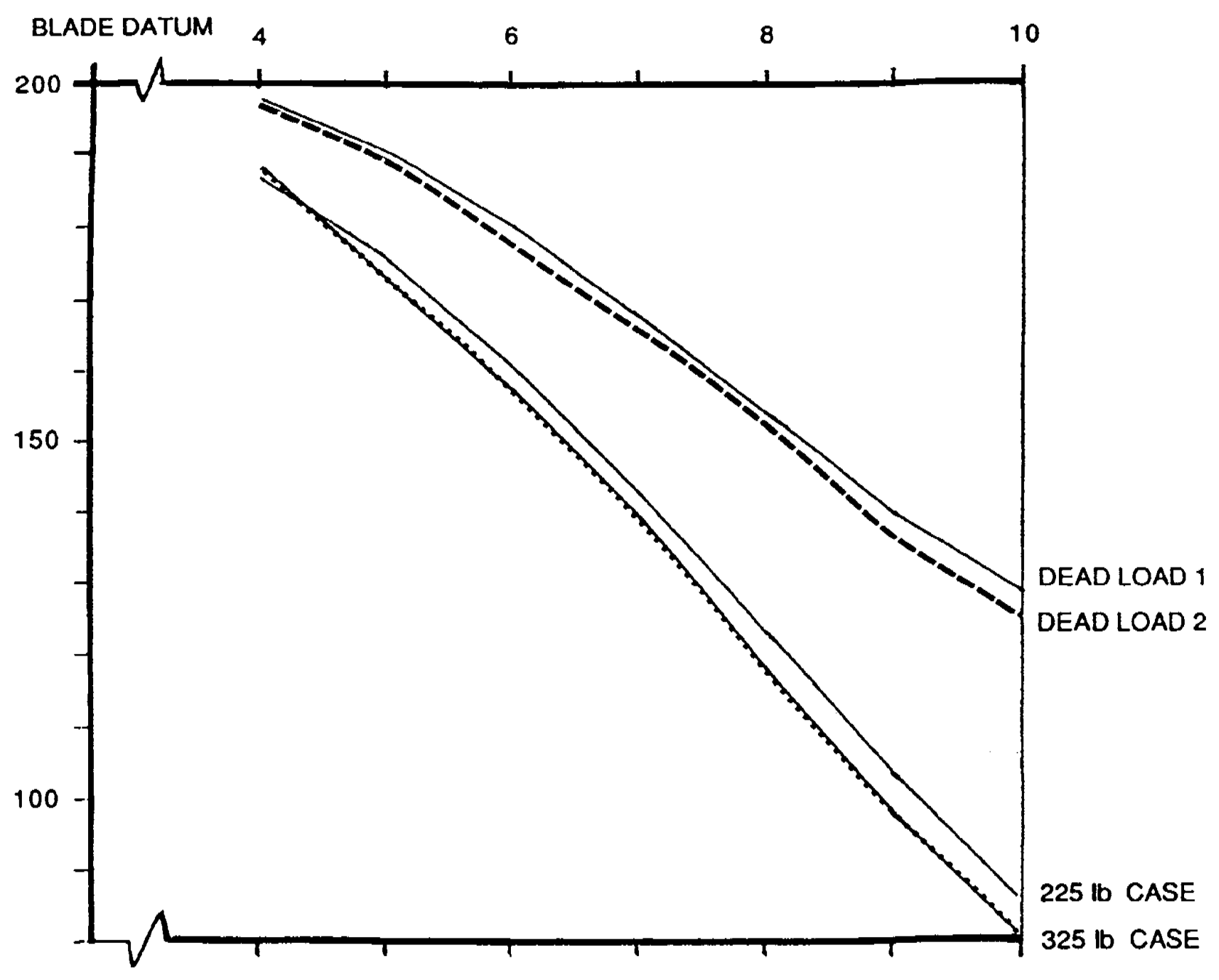

BASELINE

NOTE: Difference between Dead Loads 1 (initial) and 2 (final) represents temporary creep ( 2 hour test run, $11 / 9 / 86,50^{\circ} \mathrm{F}$ )

FIG. 3.8 CARTER BLADE: DEFLECTION AND CREEP 


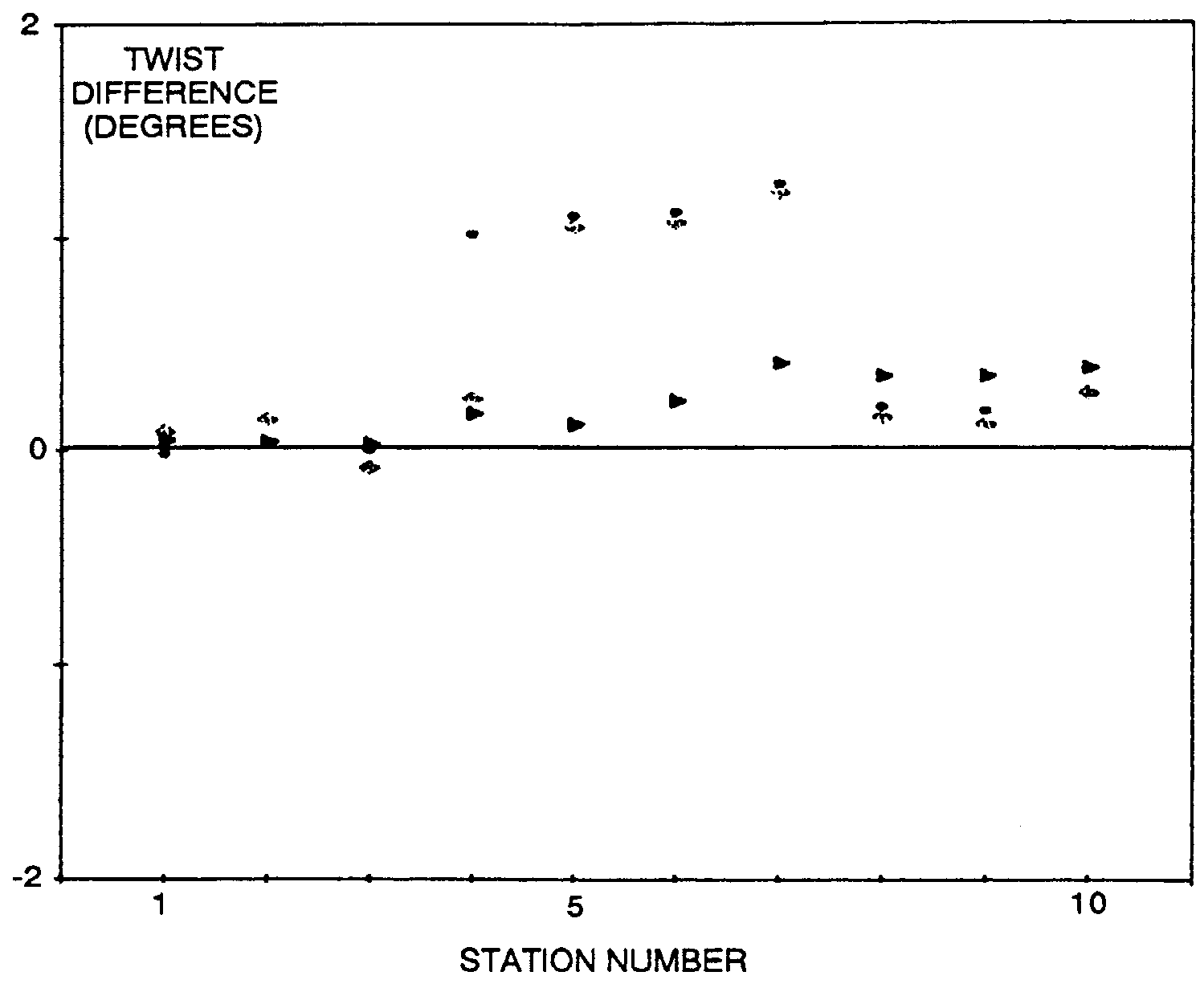

FIG. 3.9 CLINOMETER ERROR IDIOSYNCRASY

\subsection{2: LOAD CASES}

Load cases used for all blades were (1) no load (right side up and up side down), (2) $25 \mathrm{lb}$ hung from the quarterchord line at each mid station [225 lb total], (3) $50 \mathrm{lb}$ producing torsion at 0.70 span on the Carter blade and 0.90 span on the other two blades (with a zero torsion control case), and (4) $50 \mathrm{lb}$ at each station as possible [up to $375 \mathrm{lb}$ total]. Other load cases were tried as well with the Carter blade, totaling 19 different case loads (Fig. 3.10). These cases were chosen not to proof test the actual loads experienced in operation (which would have been greater and much more difficult to reproduce in the lab, e.g., including tension) but to establish the elastic properties of the blades. Hence, it was decided to opt for small linear deflections and the greater measurement accuracy thus required in testing.

The Carter blade was the first blade tested and the initial trial and error learning was done with this blade. The placement of the test weights was attempted in three methods: (1) placement on the blade surface at the quarterchord line, (2) hung from eyebolts attached to a wooden bar attached to the blade, and (3) using wire rods formed into eyelets taped to the blade with an S-hook to support the bags of lead shot. The latter was determined to be the best method and was used on the rest of the blades. Concentrated loads were adequately supported by using the rope/eyelet method, and furthermore allowed placement of the bags directiy on the quarterchord lines. The UTRC blade was not able to support a high load since the root began to delaminate at the $25 \mathrm{lb}$ per station load. 
STATION
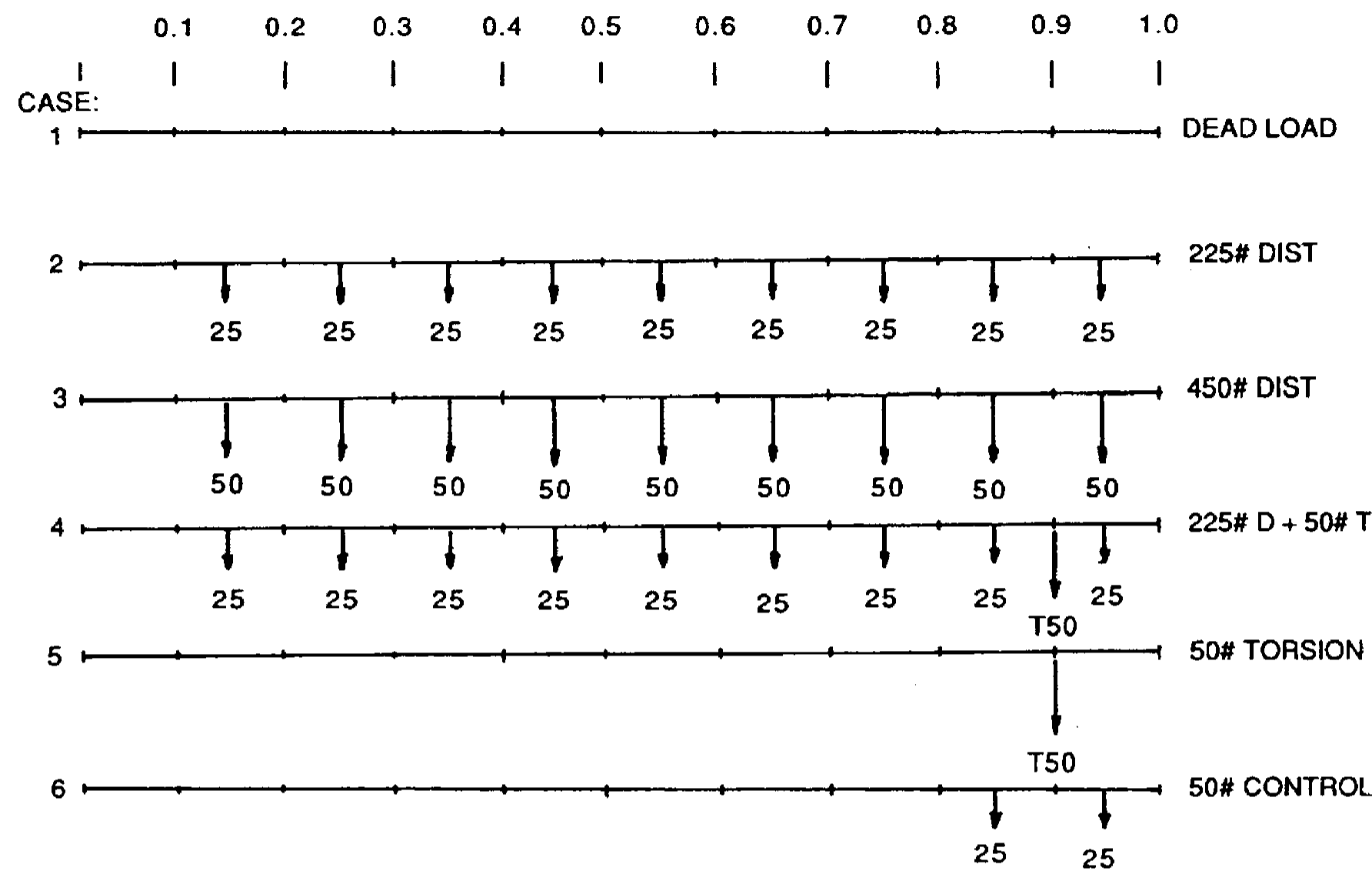

NOTES: 1. All weights were lead shot in bags hung from s-hooks taped to blade at control axis

2. Measurements were made on templates at each station

3. Torsion weights (cases 4 and 5) were hung on templates

4. Carter $325 \mathrm{lb}$. case same as case 3 with $25 \mathrm{lb}$. from STA .55 to tip

FIG. 3.10 LOAD CASES

\subsection{3: DEFLECTION MEASUREMENT METHOD}

The blades were loaded and the data collected for each station in turn for a known set of loads. The test table (base plane register) was moved from station to station and the loads repeated. The first station was routinely remeasured at the end of the test run to check for creep in the blade or a shifting of the blade mount. The portable base plane was constructed from an oak door and three pipe legs, one threaded (Fig. 3.11). The pipe mounts were placed so that two were in line with the established laser line, and the third centered between these and placed at the opposite end of the table. The laser line ran over a massive 20foot I-beam trough that was relatively flat. This trough held two legs of the table. Level adjustment was made by moving a nut up and down the all-thread rod on the third leg. Two pin holes were mounted on an optical bench on the table and placed outside of and in line with the two legs in the trough. The base plane could be raised and lowered by adjusting the nuts until the laser light could pass through both holes of the leveled optical bench. A large bubble level at right angles to the laser line edge and the third leg of the table was used to level the plane of the table relative to the laser line. Droop data then could be recorded from the tip side of each template relative to a registration point on the template (nail). A monofilament line with a construction plumb bob was used to find the relative $X, Y$ position of the nail projected onto the laser base plane. A tape measure was then hung from the nail and the total droop distance from the nail to the 
laser plane was taken. In this way $(X, Y, Z)$ measurements could all be taken to an accuracy of \pm 0.1 in. $(2.5 \mathrm{~mm})$.

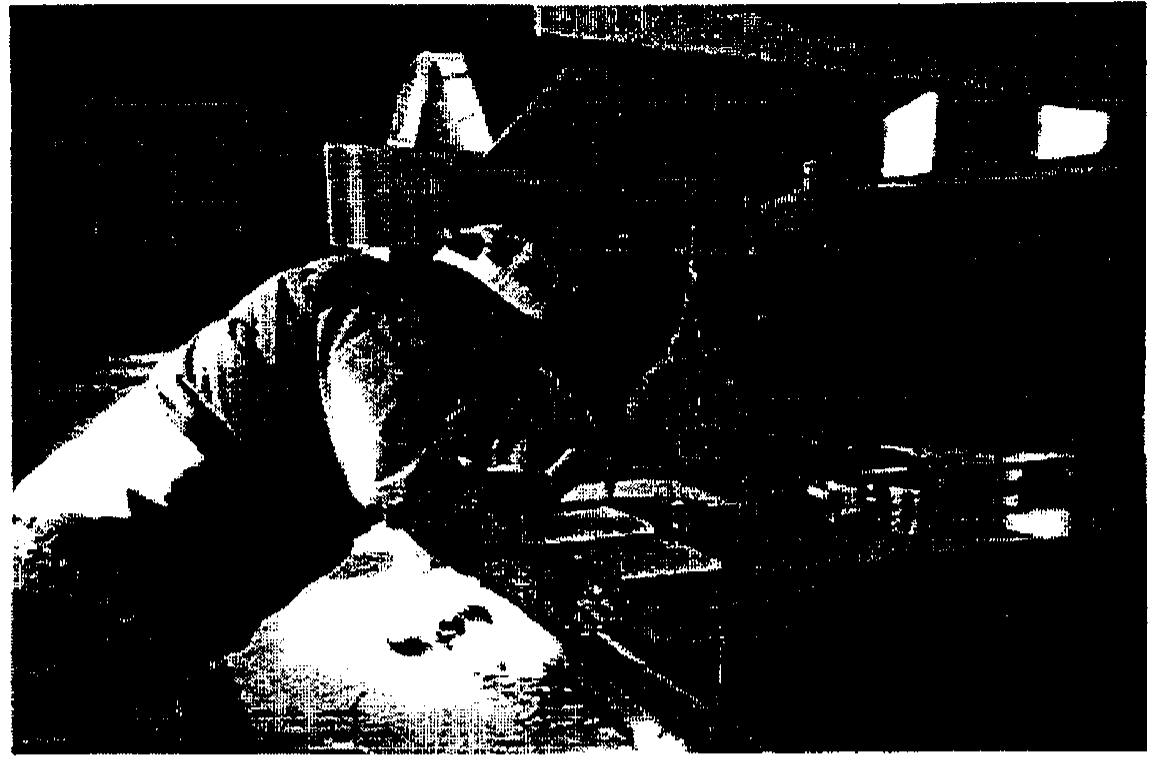

FIG. 3.11 TEST TABLE

\subsection{4: DEFLECTION CORRECTIONS TO QUARTERCHORD}

The procedure for the correction of the droop deflection measurements to the quarterchord point is described here. First, the original data (i.e., template nail to base plane) were summarized in a computer data file for each load case. These data consisted of the station position (station number), load case number, $X$ direction deflection (relative to laser baseline), $Y$ deflection (relative to a moving origin based on the laser table), $Z$ droop measured relative to the laser plane, angular displacement of the chord in degrees and minutes, and the flapwise root fixture bending in degrees and minutes. During the collection of these data a clinometer convention was adopted: positive was defined as airfoil nose down (i.e., tendency for the nose of the airfoil to go into the wind).

The method to calculate the coordinate shifts is described here. In the opening lines of each program were the measured data of the plumb bob support arm (nail) positions by station relative to the quarterchord point. These values were determined from the templates. The nails were already as close as possible to the quarterchord position. Both upper and lower (low and high pressure) sides of the templates were equipped with registration nails. These nail positions were related to the actual quarterchord by their angular deflection and offset $(\Theta$ and $L)$. $V$, the vertical offset, was measured as depicted in the sketch below:

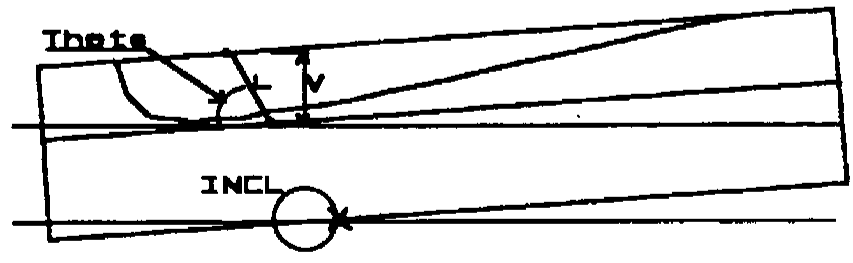


The following sketches show the four cases of nail position that were accommodated: the nail ahead and behind the quarterchord point, and with positive and negative local pitch.

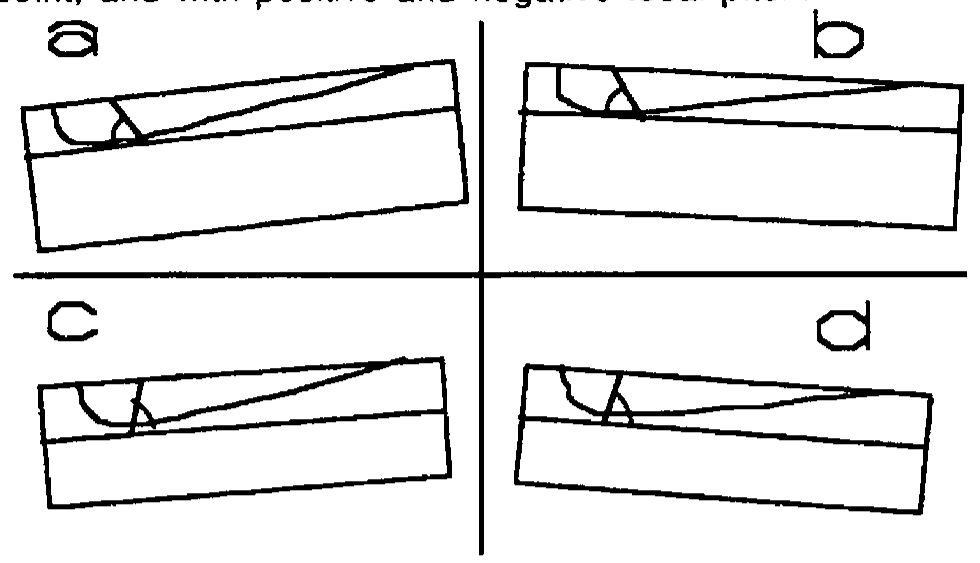

The input data and the constants $(\Theta$ and $L)$ were used to compute three new values: $T$, which is the radian equivalent of the degree measurements; $P$, the radians of the angle $\Theta$; and the calculated $Z$ droop deflection based on measured local station twist and relative nail position:

$$
\begin{aligned}
& V=L\{\sin [(P \text { SGN(P) })+(T C)]\} \\
& V=\text { vertical correction to droop } \\
& L=\text { distance from nail to quarterchord } \\
& P=\text { radian angle measure from nail to quarterchord } \\
& S G N=\text { sign tunction [returns the sign of the variable where }-1 \text { is } \\
& \text { returned for negative numbers and } 1 \text { is returned for positive } \\
& \text { numbers ( } 0 \text { is considered positive)] } \\
& T=\text { radian measure of local station twist } \\
& C=\text { correction sign based on if the nail position is ahead (noseward) or } \\
& \text { behind the quarterchord point. }
\end{aligned}
$$

\subsection{5: DEFLECTION RESULTS}

The deflection results under load and no-load conditions are best seen in the graphs of deflection versus station number (Figs. 3.12 to 3.14) for various load cases (Fig 3.10). These curves are very smooth even with varying loading methods. Of course, this is to be expected since the blades were purposely kept inside their linear limits of elasticity.

\subsection{0: BLADE VIBRATION MODAL SURVEY}

The laboratory frequency-of-vibration tests were performed in the same test fixtures. It was originally thought that the fundamental frequencies of vibration could be obtained simply with an accelerometer and strip chart recorder with a frequency response higher than the expected frequency. It was expected that the required frequency response would be about $100 \mathrm{~Hz}$. However, it became clear that particularly for the Carter blade, no simple fundamental bending modes could be easily identified. Also, the torsional frequencies turned out to be higher than expected for the ESI $(40.5 \mathrm{~Hz})$ and UTRC (29 Hz) blades. Therefore, SERI was asked to perform a more rigorous modal survey on the blades. The method and results are fully presented in Reference 8 , and summarized here. 


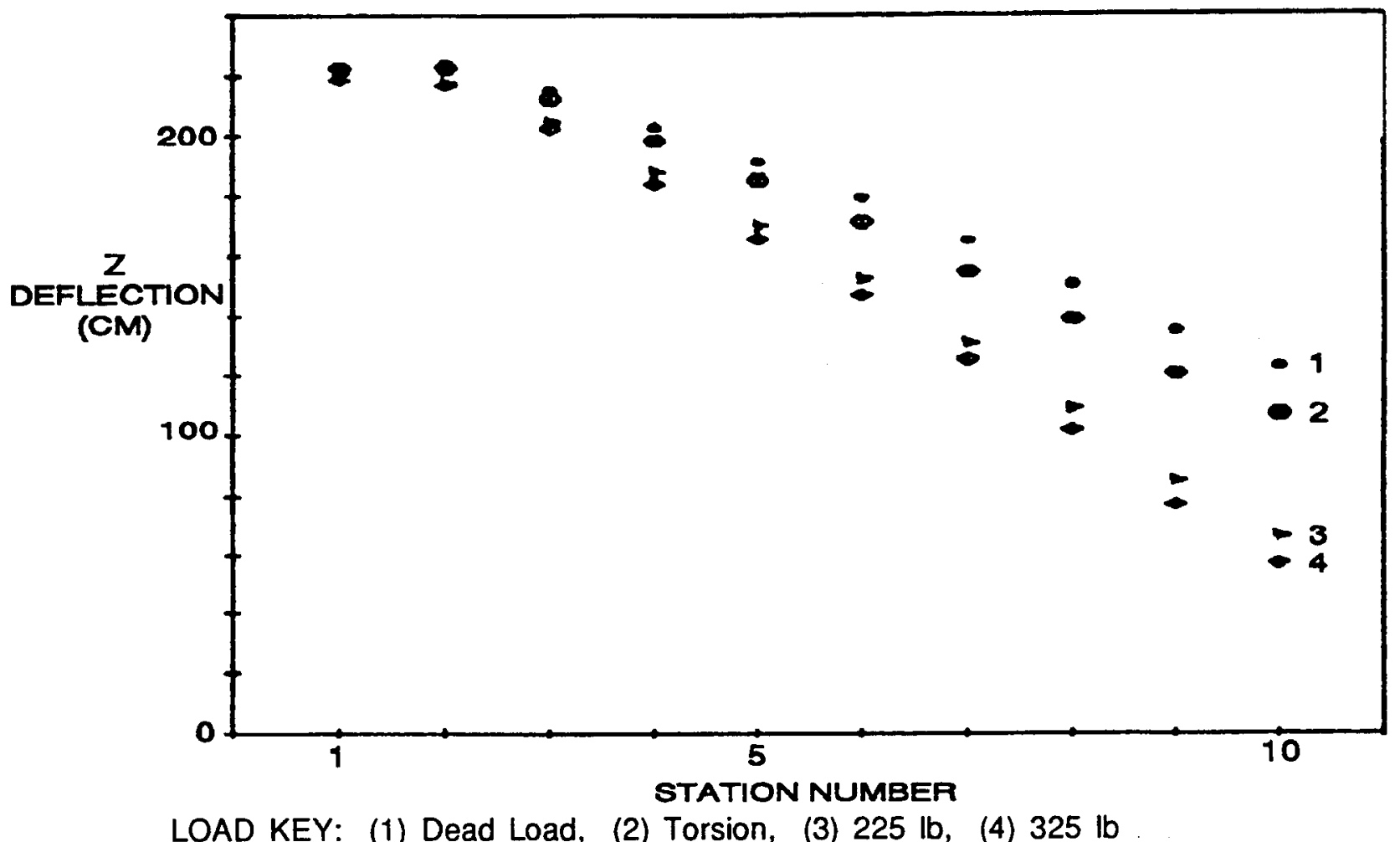

FIG. 3.12 DEFLECTION RESULTS, CARTER (UNCORRECTED) 


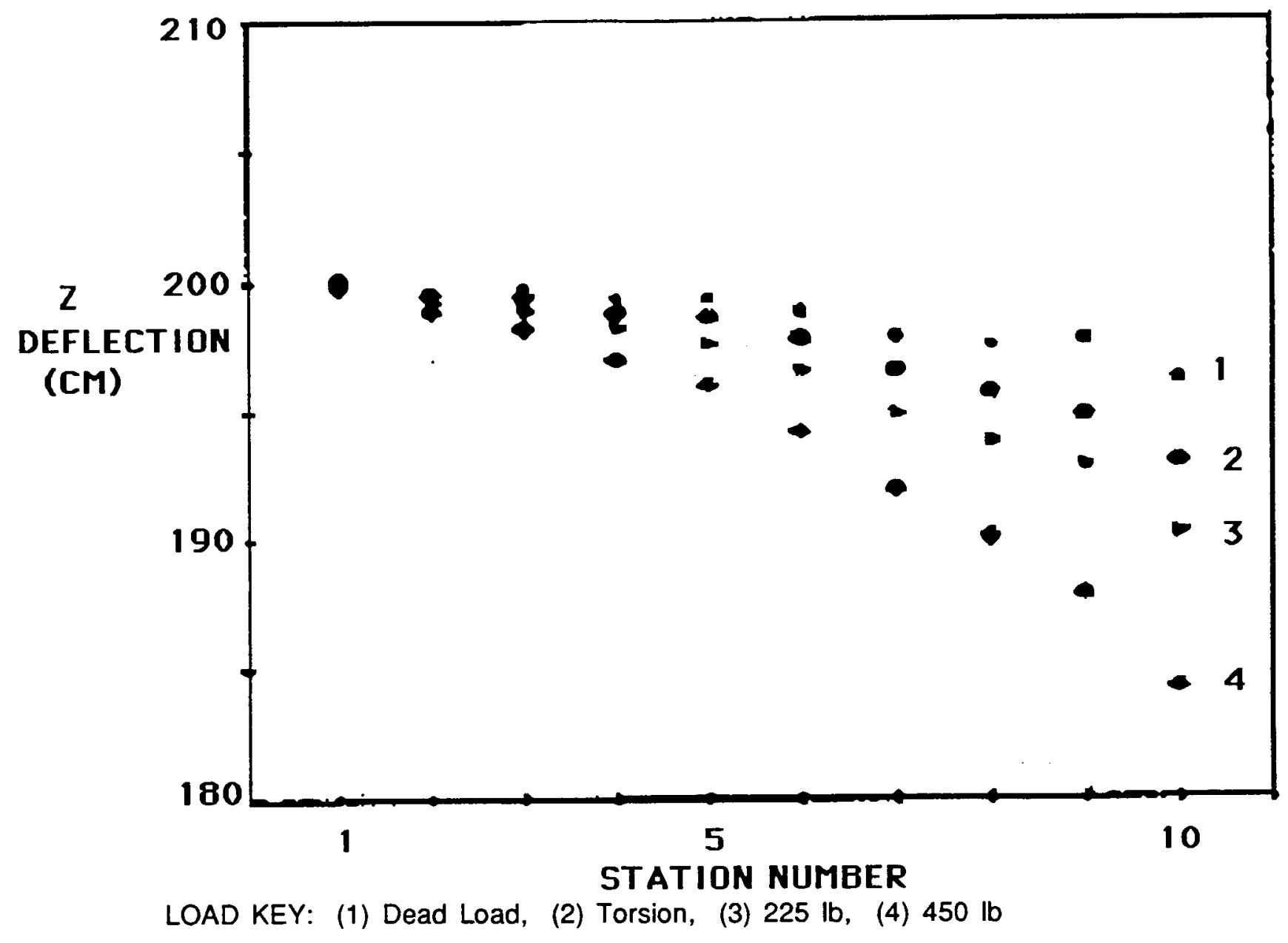

FIG. 3.13 DEFLECTION RESULTS, ESI (UNCORRECTED) 


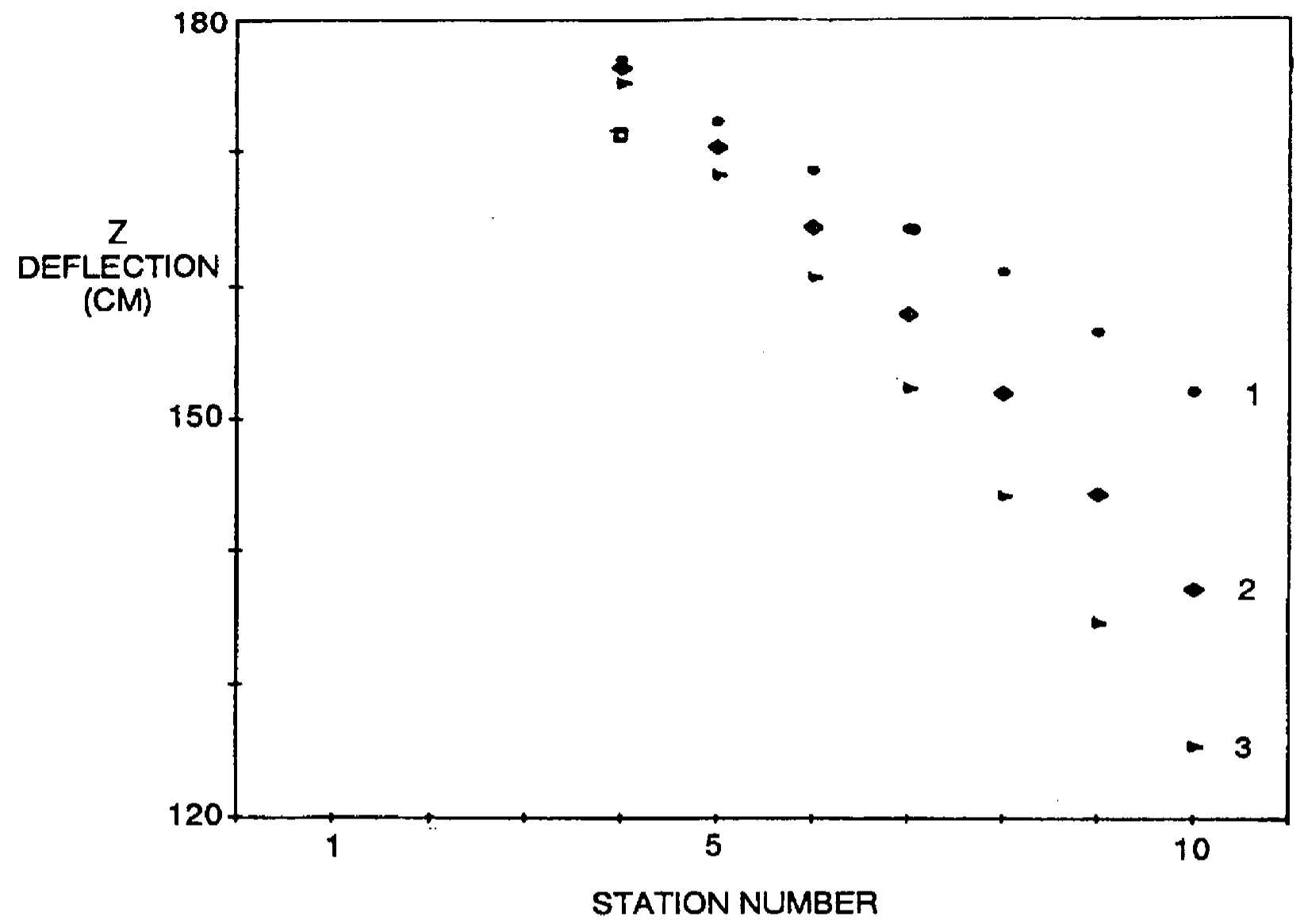

LOAD KEY: (1) Dead Load, (2) Torsion, (3) $175 \mathrm{lb}$

FIG. 3.14 DEFLECTION RESULTS, UTRC (UNCORRECTED)

Modal data collection primarily consists of the judicious application of a known input force and then measurement of the structure's response. In general, the dynamic response to an external excitation is a superposition of all the normal vibration modes of the structure. This collection of large amounts of data and the extensive harmonic analysis can be done with available electronic equipment and desktop computers, as in this project. The modal survey equipment sent by SERI included a Hewlett Packard HP 5420A two-channel spectrum analyzer and an HP 9825 desktop calculator, calibrated impact hammer with charge amplifier, and piezoresistive accelerometers and bridge amplifiers. The outputs from the hammer (forcing function) and the amplified accelerometer signal (response function) were the inputs to the spectrum analyzer (S.A.). The S.A. computed frequency response functions from the impact testing which were then stored for later analysis on the desktop calculator. The comprehensive software for modal survey (Fast Fourier Transform) and the particular hardware system had been used by SERI on previous projects. The impulse method was used since the shakers available from SERI did not have sufficient mass/amplitude for large blades. The shake test method would have given better mode resolution and would have been easier to install in addition to enabling the discovery of hidden modes with less effort. The zerocrossing (peak-picking) method was used to determine mode frequencies.

Frequencies and mode shapes (Table 3.7 and Figs. 3.15 - 3.17) were determined. The Carter 300 was the most complicated blade; it had a dashpot snubber in the root that had to be replaced with a rigid member to eliminate the interference of the snubber (pitching) action. The first flap bending mode turned out to be $0.59 \mathrm{~Hz}$ and the first torsion $13.25 \mathrm{~Hz}$. Above the second flap bending the modes were more complex due to the complicated internal structure and ballast weights. Near $40 \mathrm{~Hz}$, the modal frequencies 
were very highty coupled. The ESI was much stiffer and had a simpler internal structure. The vibration modes were well separated and classic in shape. First flap bending was at $3.5 \mathrm{~Hz}$ and first torsion at 40.5 $\mathrm{Hz}$. The UTRC blade was the simplest: constant chord and no twist. The first flap bending was $2.1 \mathrm{~Hz}$ and the first torsion $29 \mathrm{~Hz}$. The modal survey results compare favorably with the results from the theory of elasticity (see Ref. 11, for example).

TABLE 3.7: FREQUENCY OF VIBRATION RESULTS FROM TEST (HZ)

\begin{tabular}{cccc} 
MODE & UTRC & ESI & CARTER \\
& & & \\
\hline 1ST FLAP & 2.1 & 3.5 & 0.59 \\
2ND FLAP & 13.0 & 11.0 & 2.73 \\
3RD FLAP & 35.0 & 25.0 & 7.38 \\
& & & \\
1ST LAG & 13.0 & 8.0 & 3.0 \\
2ND LAG & 78.0 & 33.0 & 16.0 \\
3RD LAG & 200.0 & 80.0 & 40.4 \\
1ST TORSION & 29.0 & 40.5 & $* 13.25$ \\
2ND TORSION & 84.0 & $* 68.0$ & $* 19.73$ \\
\hline
\end{tabular}

NOTES

(1) ALL BLADES TESTED IN FLYING CONDITION EXCEPT: CARTER SNUBBER LOCKED, NO ESI TIPS.

(2) ROOT FIXITY IDENTICAL TO STATIC TESTS.

(3) ESI 2ND TORSION IS COUPLED WITH 5TH FLAP.

(4) CARTER 1ST TORSION IS I-BEAM ROOT FLEXURE ONLY.

(5) CARTER 2ND TORSION IS OUTBOARD TORSION ONLY. 

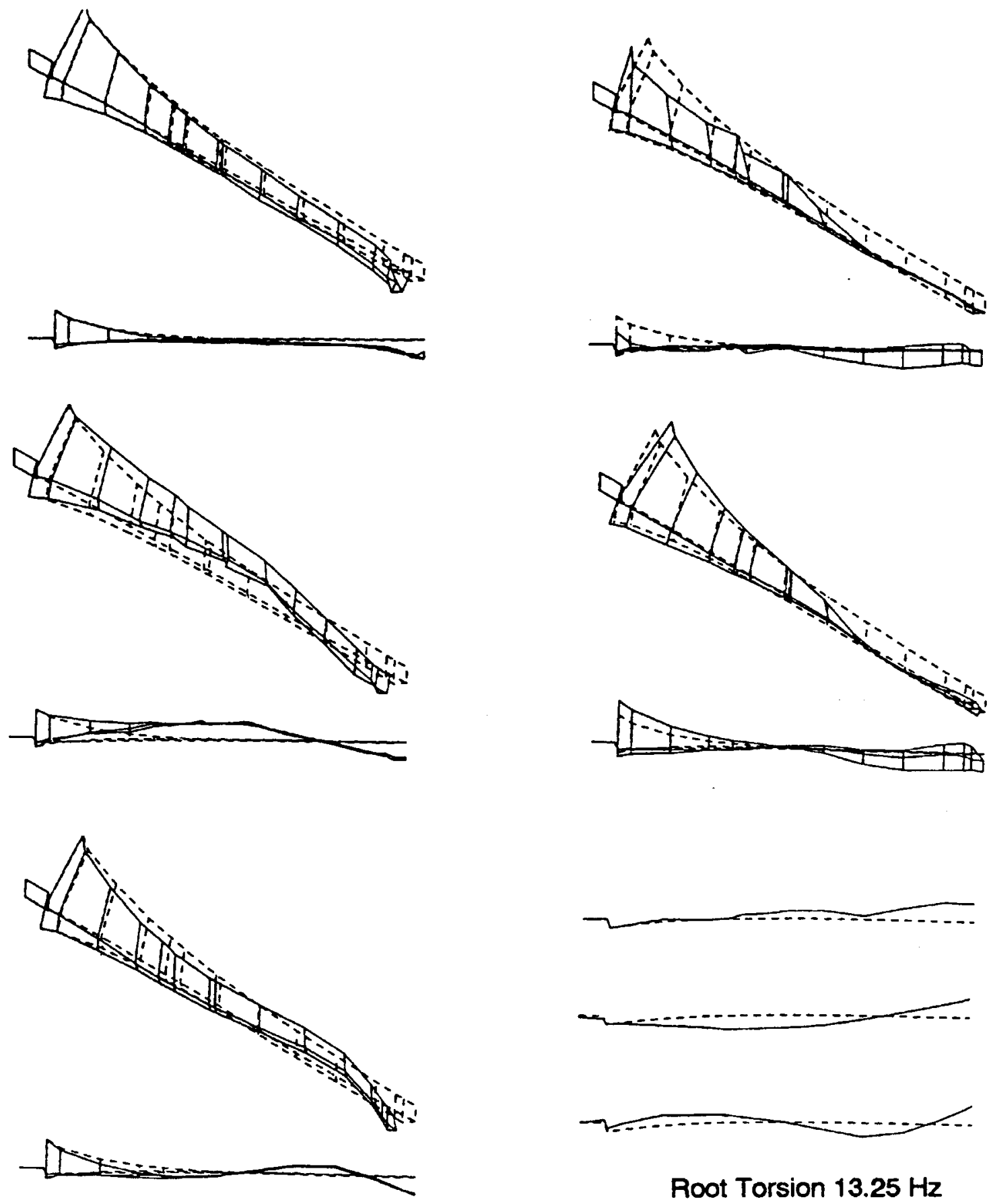

1st Flap $0.59 \mathrm{~Hz}$ 2nd Flap $2.73 \mathrm{~Hz}$ 3rd Flap $7.42 \mathrm{~Hz}$

Root Torsion $13.25 \mathrm{~Hz}$ Outboard Torsion $19.73 \mathrm{~Hz}$ 1st Edgowise $3.0 \mathrm{~Hz}$ 2nd Edgewise $16.0 \mathrm{~Hz}$ 3rd Edgewise $40.4 \mathrm{~Hz}$

FIG. 3.15 FREQUENCY OF VIBRATION: CARTER BLADE 

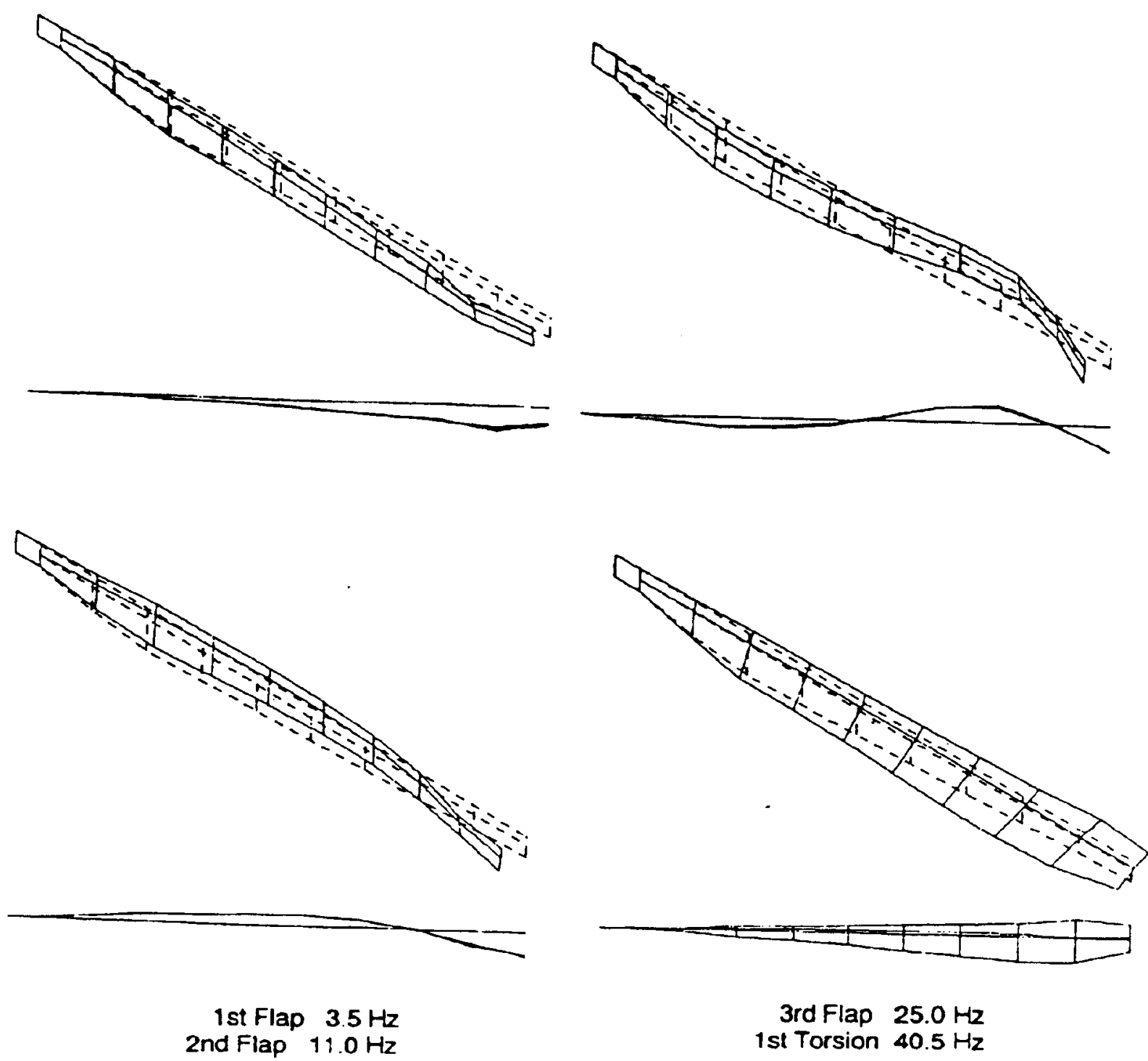

FIG. 3.16 FREQUENCY OF VIBRATION: ESI BLADE 

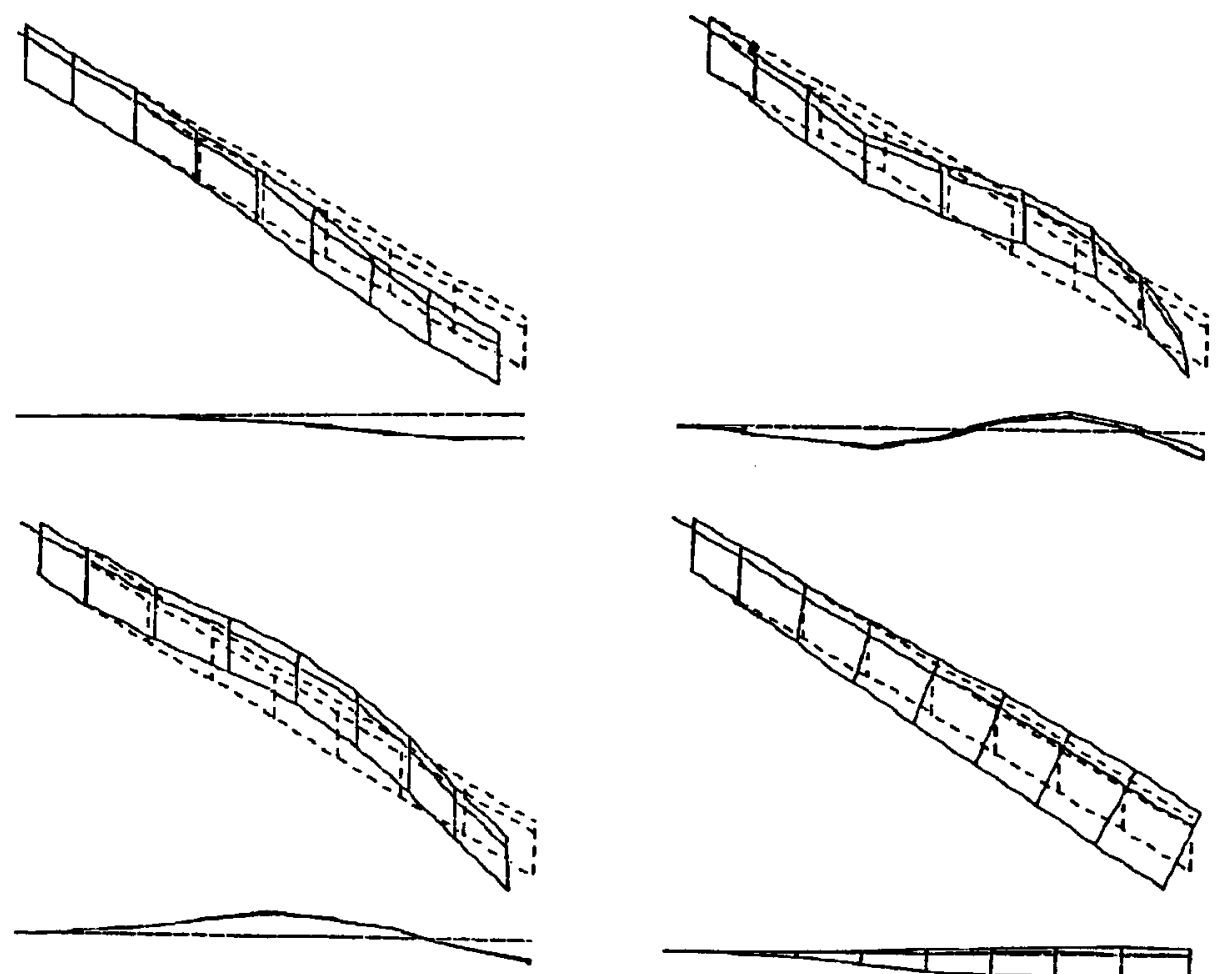

1st Flap $2.1 \mathrm{~Hz}$ 2nd Flap $13.0 \mathrm{~Hz}$

3rd Flap $35.0 \mathrm{~Hz}$ 1st Torsion $29.0 \mathrm{~Hz}$

- calculated ano test values -

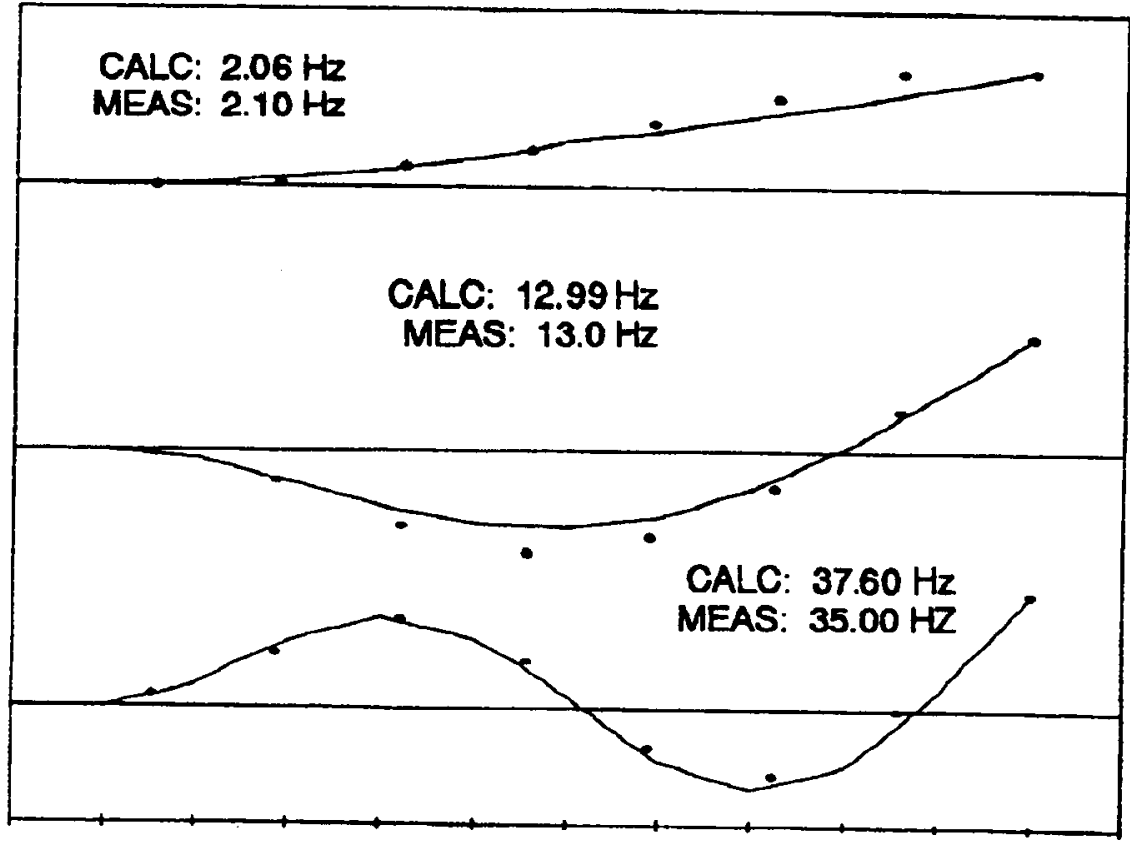

FIG.3.17 FREQUENCY OF VIBRATION: UTRC BLADE 


\subsection{THEORETICAL METHOD}

\section{1: GENERAL}

An arbitrary cross-section, 3-dimensional beam subject to arbitrary distributed loading in bending and torsion is a formidable mathematical problem. It is usually accomplished in the aerospace industry by employing expensive finite-element structural programs (e.g. NASTRAN). This study did not have sufficient resources to employ such a state-of-the-art model, nor was it warranted, since the static bending and frequency of vibration results are very sensitive to the material mechanical properties, which were composite laminates for this effort. In using such computer programs a lot of effort can be wasted needlessly if these engineering constants are not known to a high degree of accuracy.

In view of this, this project used a simpler elastic beam approach which modeled the composite blade sections by calculating area-weighted flexural and torsional moduli, calculated the beam properties relative to the principal axes, and then performed coordinate transformation to develop blade axis sectional properties. Arbitrary cross section including blade skin, spar, shear webs, and afterbodies are all handled in the above approach. The deflections were found, in a likewise manner, by referring the applied loading and moments to the aerodynamic center (quarterchord), the mass center (blade section $\mathrm{cg}$ ), the bending centroid (weighted area centroid), and the shear center (elastic axis), and transforming the deflections by superposition. The deflections, when compared to the test results, then provide a reiteration error of the original assumed laminate constants, hence the term "mass/elastic triangulation."

\section{2: $\quad$ COMPOSITE BLADE SECTION PROPERTIES CALCULATION}

\subsection{1: SECTION DESCRIPTION}

This section describes the beam section properties calculations (Ref. 3). The Bernoulli-Euler theory of bending ("engineers' theory of bending") calculation can be followed in any thorough strength of materials text (e.g., Ref. 12, 13). The key assumptions are 1) deflections are small and linear and 2) plane sections remain plane and normal to the beam axis. These assumptions make it possible to determine the deflections of any point on the beam in terms of points on the axis of the beam. Figure 4.1 shows a general blade cross section for a spar-shell composite blade.

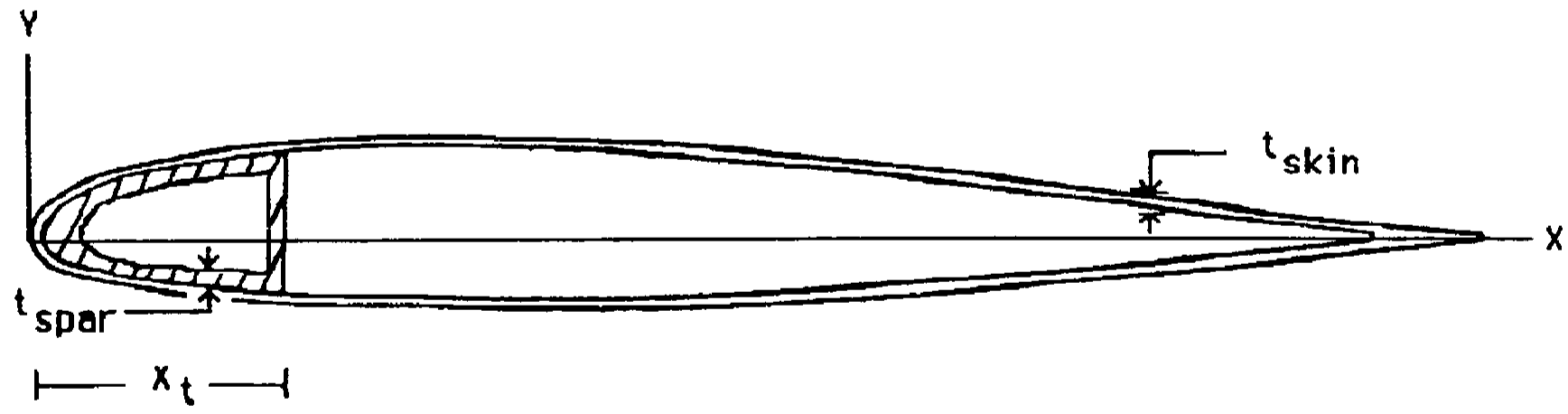

\section{FIG. 4.1 GENERAL SPAR-SHELL CROSS SECTION}

The spar is shown as a constant thickness D-spar and a web at $X_{t}$ in the nose, and the skin is shown also as constant thickness. Section axes have origin at the nose and abscissa along the airfoil chordline. In general the spar and skin will have entirely different constituents (laminate schedules). The spar is usually the principal bending structure, and the skin the torsion structure, also providing a shear load path for aerodynamic pressure forces into the spar. More complicated versions will have more webs and variation in the laminate within the skin or the spar. For now we assume the skin and spar to be uniform.

In general, the spar and skin constituents have directional properties; that is, the elastic constants vary depending on the orientation ( 0 degrees reference is spanwise and 90 degrees is chordwise). For 
simple bending only the longitudinal $\left(0^{\circ}\right)$ values are used. This gives four engineering constants which describe the longitudinal and shear (torsion) behavior of the section: $E_{\text {skin }}$ G $_{\text {skin. }} E_{\text {spar. and }} G_{\text {spar. }}$ or elastic and shear moduli of elasticity. These engineering moduli can be calculated for a given laminate by the area-weighting method. For instance, suppose a laminate is composed of various layers as shown in Figure 4.2.

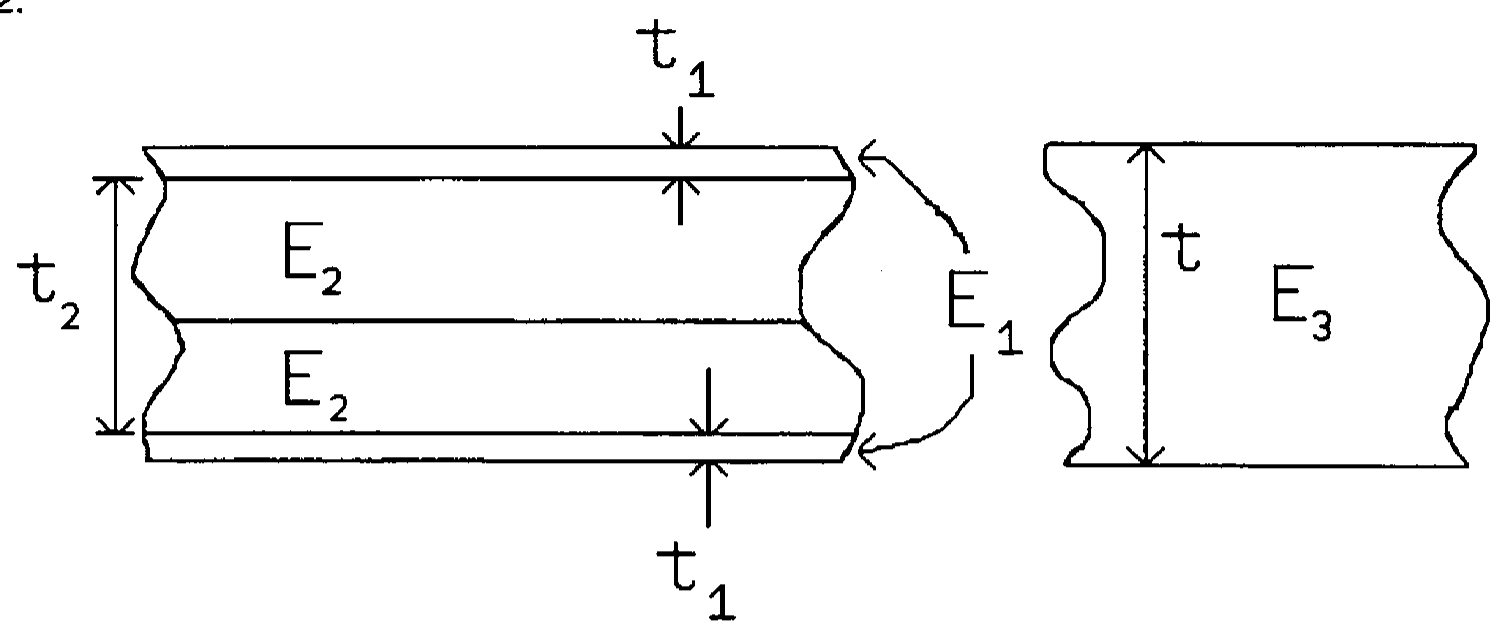

FIG. 4.2 ACTUAL VS. EQUIVALENT LAMINATE

Then the equivalent laminate has the same actual thickness as the aggregate $\left(t=2 t_{1}+t_{2}\right)$, and an elastic modulus based on area ratios is

$$
E_{3}=\frac{\sum E_{n} t_{n}}{\sum t_{n}}=\frac{\left(2 E_{1} t_{1}+E_{2} t_{2}\right)}{\left(2 t_{1}+t_{2}\right)}
$$

Clearly the complicated laminate on the lett can only be approximately represented by the bulk laminate on the right; internal shear stresses (interlaminar shear), for instance, could be calculated also for each layer interface. This detail can be left for the end of the engineering process, after the overall beam deflection and bending stresses have been calculated. Then these residual internal stresses can be checked. In this application the actual thicknesses of the layers are maintained since these will also be used to calculate weights and moments of inertia, and will also be checked with measurements. Alternative approaches may use modulus-weighted thicknesses to simplify calculations.

\subsection{2: $\quad$ SECTION AREA AND STIFFNESS PROPERTIES}

Section area properties are found by simple integration. The airfoil shape $(X, Y)$ is first calculated based on a specific table of offsets and a chordline, and using a simple second-order curve-fit subroutine through each series of three points. The contribution of each differential skin and spar segment is calculated and summed around the airfoil, starting at the nose and also ending there. These integrals are simply

$\mathrm{A}_{\mathrm{t}} \quad=$ Total area $=\int \mathrm{dA}$

$M_{X} \quad=1$ st moment of the area about the $X$ axis $=\int y d A$

$M_{y}=1$ st moment of the area about the $Y$ axis $=\int x d A$

$I_{X} \quad=$ Moment of inertia about the $X$ axis $=\int y^{2} d A$

Iy $\quad=$ Moment of inertia about the $Y$ axis $=\int x^{2} d A$

$I_{x y}=$ Product of inertia $=\int x y d A$ 


$$
\begin{array}{lll}
\bar{X}_{c} \quad=\text { Bending centroid, chordwise position }= & \frac{M_{y}}{A_{t}} \\
\bar{Y}_{c} \quad=\text { Bending centroid, flapwise position }= & \frac{M_{x}}{A_{t}}
\end{array}
$$

The program corrects for shell thickness by using the skin (or spar) segment centers to define the moment arm.

Section stiffness properties are found by the area-weighting method, combining the contributions from the skin and spar, and adding the spar web as a separate item for simplicity. This approach allows the addition of other shear webs or structural elements in the total composite section values. The spar web and further elements are simply described by their area properties:

$$
\begin{aligned}
& A_{W}=\text { Spar web area } \\
& I_{X W}=\text { Spar web moments of inertia } \\
& I_{y W}=\text { Spar web moments of inertia } \\
& I_{X Y W}=\text { Spar web product of inertia } \\
& (X, Y)_{W}=\text { Centroid }
\end{aligned}
$$

Bending values relative to the $(X, Y)$ axis system origin are

$$
E A=E_{\text {skin }} A_{\text {skin }}+E_{\text {spar }} A_{\text {spar }}+E_{\text {web }} A_{\text {web }}
$$

Bending centroids for total section are

$$
\begin{aligned}
& \bar{X}_{T}=\frac{1}{E A} \quad\left[E_{\text {skin }} M_{y \text { skin }}+E_{\text {spar }} M_{y \text { spar }}+E_{\text {web }} M_{y} \text { web }\right] \\
& \bar{Y}_{T}=\frac{1}{E A} \quad\left[E_{\text {skin }} M_{x \text { skin }}+E_{\text {spar }} M_{x \text { spar }}+E_{\text {web }} M_{X \text { web }}\right]
\end{aligned}
$$

Now the bending stiffness for the section is found, but is referenced to the centroid of the composite section. This makes the calculation simpler, and correctly assesses the stiffness contribution of each element by superposition. The parallel axis theorem is used to move each $(X, Y)$ moment of inertia value to the section centroid $\left(X_{T}, Y_{T}\right)$; an intermediate step moves the point to its own centroid. For example, for the skin only, the $X$-moment of inertia about the total composite section centroid is

$$
E_{X} \text { skin }=E_{\text {skin }}\left\{\left[I_{X \text { skin }}-A_{\text {skin }} \bar{Y}^{2} \text { skin }\right]+A_{\text {skin }}\left[\bar{Y}_{\text {skin }}-\bar{Y}_{Y}\right]^{2}\right\}
$$

Therefore, the section stiffnesses are simply the sums of the contributions :

$E I_{X}$ total structure $=\sum E I_{X} \quad$ (where $I_{X}$ is referred to the bending centroid in each case.)

$$
\begin{aligned}
& =E_{\text {skin }}\left\{\left[I_{X} \text { skin }-A_{\text {skin }} \bar{Y}^{2} \text { skin }\right]+A_{\text {skin }}\left[\bar{Y}_{\text {skin }}-\bar{Y}_{T}\right]^{2}\right\} \\
& +E_{\text {spar }}\left\{\left[I_{X \text { spar }}-A_{\text {spar }} \bar{Y}^{2} \text { spar }\right]+A_{\text {spar }}\left[\bar{Y}_{\text {spar }}-\bar{Y}_{T}\right]^{2}\right\} \\
& +E_{\text {web }}\left\{I_{X} \text { web }+A_{\text {web }}\left[\bar{Y}_{\text {web }}-\bar{Y}_{T}\right]^{2}\right\}
\end{aligned}
$$


$E_{y}$ total structure $=E_{\text {skin }}\left\{\left[I_{y}\right.\right.$ skin $-A_{\text {skin }} \bar{X}^{2}$ skin $\left.]+A_{\text {skin }}\left[\bar{X}_{\text {skin }}-\bar{X}_{T}\right]^{2}\right\}$

$+E_{\text {spar }}\left\{\left[I_{y}\right.\right.$ spar $-A_{\text {spar }} \bar{X}^{2}$ spar $\left.]+A_{\text {spar }}\left[\bar{X}_{\text {spar }} \cdot \bar{X}_{T}\right]^{2}\right\}$

$+E_{w e b}\left\{I_{y}\right.$ web $\left.+A_{w e b}\left[\bar{X}_{w e b}-\bar{X}_{T}\right]^{2}\right\}$

$\mathrm{EI}_{\mathrm{xy}}$ total structure $=\Sigma \mathrm{EI}_{\mathbf{x y}}$

$=E_{\text {skin }}\left\{\left[I_{x y \text { skin }}-A_{\text {skin }} \bar{X} \bar{Y}_{\text {skin }}\right]+A_{\text {skin }}\left[\bar{Y}-\bar{Y}_{T}\right]_{\text {skin }}\left[\bar{X}-\bar{X}_{T}\right]_{s k i n}\right\}$

$+E_{\text {spar }}\left\{\left[I_{\text {XY spar }}-A_{\text {spar }} \bar{X} \bar{Y}_{\text {spar }}\right]+A_{\text {spar }}\left[\bar{Y}-\bar{Y}_{T}\right]_{\text {spar }}\left[\bar{X}-\bar{X}_{T}\right]_{\text {spar }}\right\}$

$+E_{\text {web }}\left\{I_{x y}\right.$ web $\left.+A_{\text {web }}\left[\bar{Y} \cdot \bar{Y}_{T}\right]_{\text {web }}\left[\bar{X} \cdot \bar{X}_{T}\right]_{\text {web }}\right\}$

The last step is the calculation of the section principal axis values, which are used later to calculate blade deflections. The principal axes of inertia are found in the program from a Mohr's circle representation depicted here in Figure 4.3:

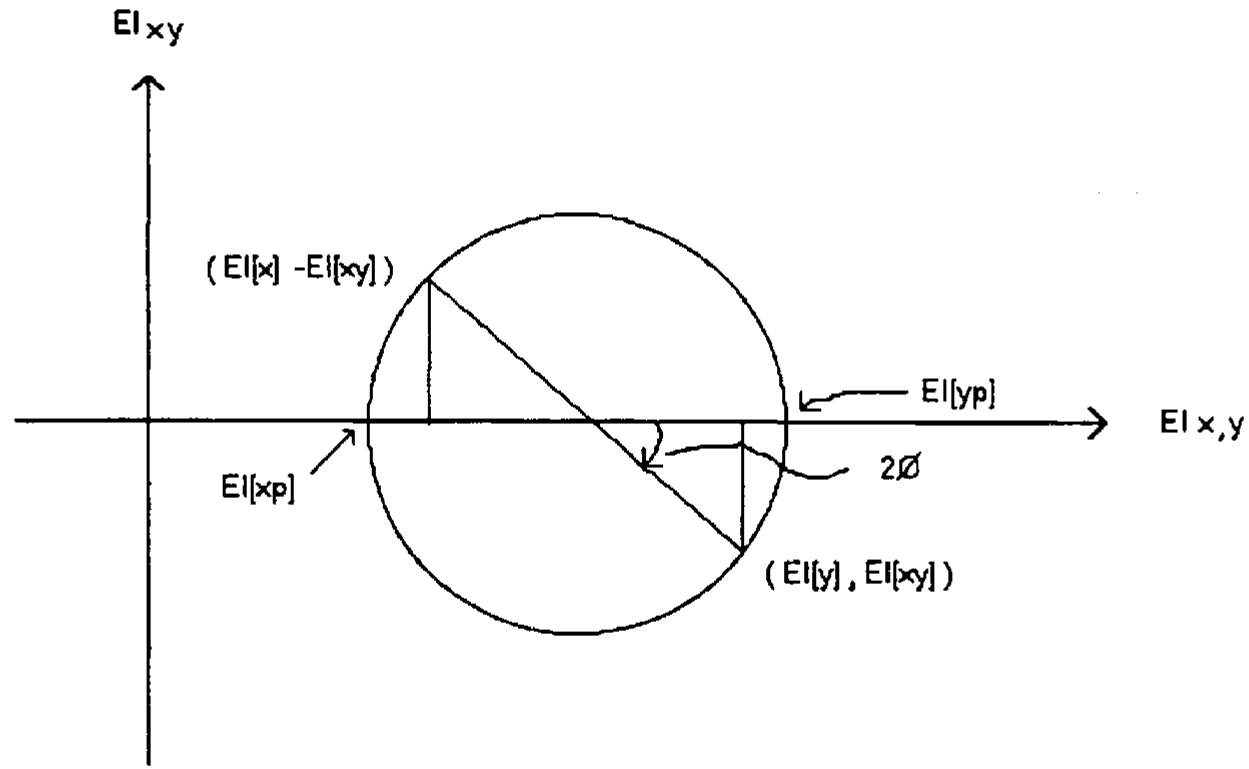

where $ø=$ principal axis angle

$E I[x p], E I[y p]=$ sectional stiffnesses in the principal axes

FIG. 4.3 MOHR'S CIRCLE FOR PRINCIPAL AXES

These principal moments of inertia (stiffnesses) are the maximum and minimum values for this section. For the usual airfoil shapes, these are close to the original $(X, Y)$ directions: chordwise and flapwise. This step simplifies the bending deflection calculation by eliminating the products of inertia. 


\subsection{3: $\quad$ SECTION TORSIONAL RIGIDITY}

To calculate section torsional rigidity also requires care in referencing the contributions to the total torsional centroid (shear center), which in general is different from the bending centroid since the $(E / G)$ ratio of the components are not the same.

$$
G A=G_{\text {skin }} A_{\text {skin }}+G_{\text {spar }} A_{\text {spar }}+G_{\text {web }} A_{\text {web }}
$$

The torsional centroid (shear center) is then

$$
\begin{aligned}
& \bar{Y}_{T T}=[1 / G A]\left\{G_{\text {skin }} M_{x \text { skin }}+G_{\text {spar }} M_{x \text { spar }}+G_{\text {web }} M_{x \text { web }}\right\} \\
& \bar{X}_{T T}=[1 / G A]\left\{G_{\text {skin }} M_{y} \text { skin }+G_{\text {spar }} M_{y \text { spar }}+G_{\text {web }} M_{y} \text { web }\right\}
\end{aligned}
$$

And the torsional rigidity about the shear center is found by using the parallel axis theorem in sequence as before for the bending stiffness:

$$
\begin{aligned}
\text { GJ }= & G_{\text {skin }}\left\{\left[I_{X} \text { skin }-A_{\text {skin }} \bar{Y}^{2} \text { skin }\right]+A_{\text {skin }}\left[\bar{Y}_{\text {skin }}-\bar{Y}_{T T}\right]^{2}\right\} \\
& +G_{\text {spar }}\left\{\left[I_{X} \text { spar }-A_{\text {spar }} \bar{Y}^{2} \text { spar }\right]+A_{\text {spar }}\left[\bar{Y}_{\text {spar }}-\bar{Y}_{T T}\right]^{2}\right\} \\
& +G_{\text {web }}\left\{I_{X} \text { web }+A_{\text {web }}\left[\bar{Y}_{\text {web }}-\bar{Y}_{T T}\right]^{2}\right\} \\
& +G_{\text {skin }}\left\{\left[I_{y} \text { skin }-A_{\text {skin }} \bar{X}^{2} \text { skin }\right]+A_{\text {skin }}\left[\bar{X}_{\text {skin }}-\bar{X}_{T T}\right]^{2}\right\} \\
& +G_{\text {spar }}\left\{\left[I_{y} \text { spar }-A_{\text {spar }} \bar{X}^{2} \text { spar }\right]+A_{\text {spar }}\left[\bar{X}_{\text {spar }}-\bar{X}_{T T}\right]^{2}\right\} \\
& +G_{\text {web }}\left\{I_{y} \text { web }+A_{\text {web }}\left[\bar{X}_{\text {web }}-\bar{X}_{T T}\right]^{2}\right\}
\end{aligned}
$$

Having derived the above expression we now reluctantly relate that this value of $\mathrm{GJ}$ is not usually practical for thin shells, since skin warping greatly reduces the stiffness. For example, in a usual thinskin airfoil with a D-spar, the above calculation is approximately four times the actual values of GJ from experiment. Therefore, the AEI program uses a shear flow approach in calculating $\mathrm{GJ}$ :

$$
\begin{aligned}
& G J=\frac{4 A^{2} G}{\int d s / t} \\
& \text { where: } \quad A=\text { cross-sectional area } \\
& \mathrm{t}=\text { skin thickness } \\
& d s=\text { differential skin length }
\end{aligned}
$$

In the above calculation the thicknesses (hence areas) are modulus-weighted to the skin value of shear modulus and the contribution of the spar web is ignored. The GJ calculated from the above expression has proven to be accurate enough for practical purposes. Further refinement of this calculation could be done for more complex section geometries. [Note: From later calculations, the major error introduced by dropping the web contribution is to incorrectly locate the shear center. For the ESI blade, which has a thick skin and substantial web, this error amounted to $40 \%$ chord (calculated) vs. $25 \%$ chord (test)]. 


\subsection{4: $\quad$ SECTION MASSES AND INERTIAS}

At this point the required stiffness properties in bending and torsion have been calculated for each section of the composite beam. It remains to calculate the masses and inertias. As for the elasticity constants, the blade skin and spar will have different mass densities based on the percentage of reinforcement to resin volume. For the simplest unidirectional laminate with glass fiber reinforcement, the relationship between density and modulus is straightforward (Table 4.1).

TABLE 4.1: FRP UNIDIRECTIONAL WEIGHTS AND MODULI

\begin{tabular}{ccccc}
$\%$ Resin Volume & $\%$ Fiber Volume & $\%$ Resin Weight & Density (lb/cu in.) & $E(k s i)$ \\
\hline 100 & 0 & 100 & .04340 & 0 \\
90 & 10 & 80 & .04890 & 1 \\
80 & 20 & 64 & .05440 & 2 \\
70 & 30 & 51 & .05990 & 3 \\
60 & 40 & 40 & .06593 & 4 \\
50 & 50 & 31 & .07089 & 5 \\
40 & 60 & 23 & .07639 & 6 \\
30 & 70 & 16 & .08189 & 7 \\
20 & 80 & 10 & .08738 & 8 \\
10 & 90 & 5 & .09288 & 9 \\
0 & 100 & 0 & .09838 & 10 \\
\hline
\end{tabular}

where resin $=.0434 \mathrm{lb} / \mathrm{cu}$ in. and glass $=.09838 \mathrm{lb} / \mathrm{cu}$ in.

Practical laminates lie between these extremes in modulus and weight.

The section mass integrals are formed in the same manner as the stiffness integrals above, by summing the individual components using the paraliel axis theorem:

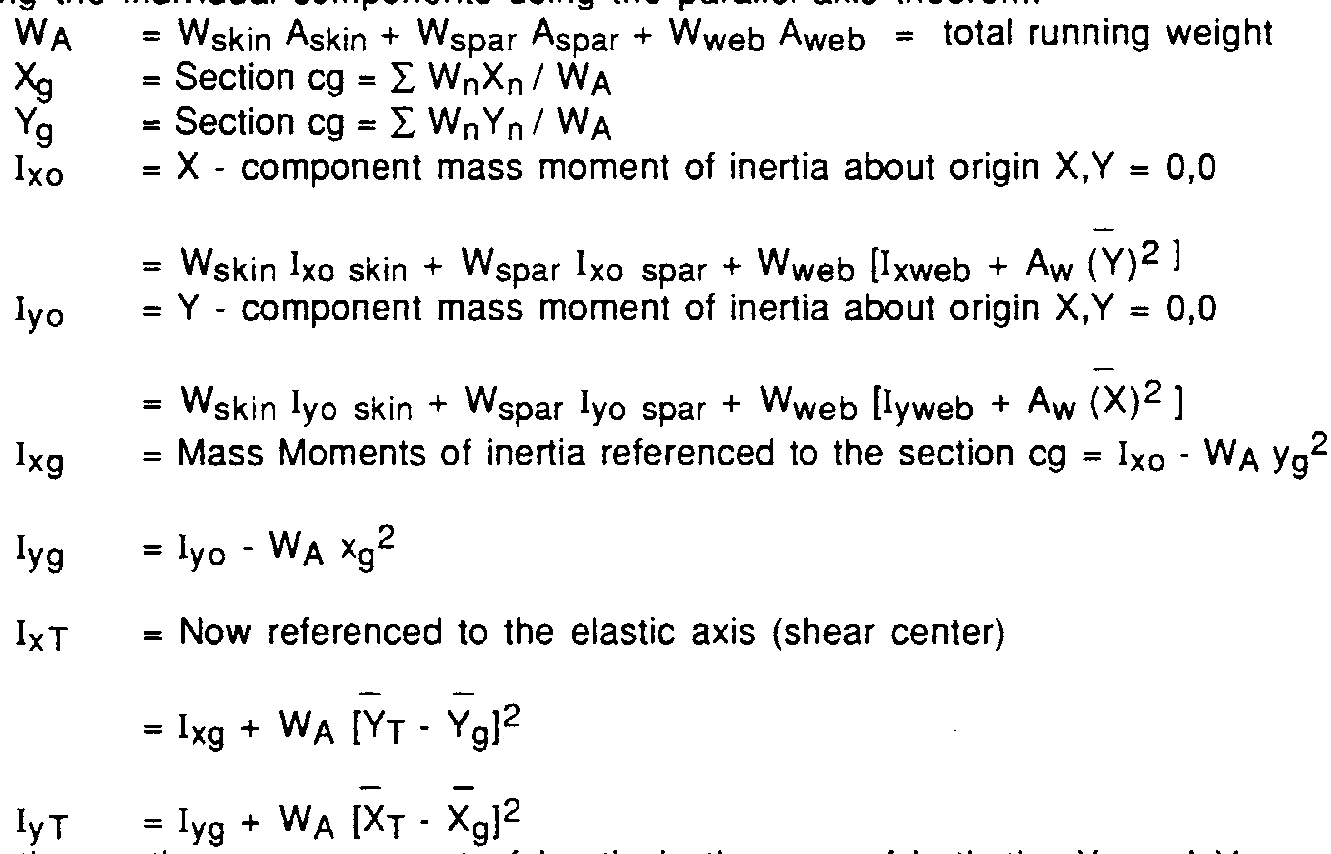

Finally the section mass moment of inertia is the sum of both the $X$-and $Y$-components, for both the section $\mathrm{cg}$ and the shear center:

IT = IXT + IYT and $\quad I_{g} \quad=I X g+I Y g$

The mass moment of inertia about the $\mathrm{cg}, \mathrm{Ig}$, is the minimum value for the section. 
Lastly, the program adds any ballast weight contribution to the mass integrals. In some blades, lead ballast is added to move the basic section $\mathrm{cg}$ position; in others the "ballast" contribution might refer to nonstandard elements embedded in the blade which change the masses but not the stiffness (e.g. tip control mechanism). These ballast contributions are again added by using the parallel axis theorem, as above.

\section{3: $\quad$ BLADE STATIC DEFLECTION CALCULATIONS}

The Bernoulli-Euler theory of bending of slender beams ("engineer's theory of bending") is used. The key assumptions are flexural deflections are small and linear, and plane sections remain plane and normal to the beam (centroidal) axis as the blade deforms. Stresses in the transverse direction are also neglected. This gives a uniaxial stress-strain relationship and satisfies the equilibrium condition since the stress resultants are in equilibrium with the applied forces. These assumptions also make it possible to determine the deflection of any point on the blade in terms of the deflections of the points on the centroidal axis of the blade since strain distribution is linear across the cross section.

The cross products of inertia are dropped out by the adoption of the principal axes:

$$
\varepsilon_{i}=\frac{M_{j} Y}{E I_{i}} \text { (simple flexure formula) }
$$

and the total strain (or stress) can be found by superposition :

$$
\sigma=\sigma_{y}+\sigma_{x}=\frac{M_{z p} Y_{p}}{I_{z p}}-\frac{M_{y p} z_{p}}{I_{y p}}
$$

where $y_{p}$ and $z_{p}$ are now the principal axes of the beam sections, and $x$ is the axis down the spar of the blade. The 3-D coordinate system is now

$X$-axis: lies along the (undeflected) axis of the blade, with the origin at the blade root.

$Y$-axis: lies in the lagging direction (i.e., toward the trailing edge of the airfoil).

Z-axis: lies in the flapwise direction (i.e., in the direction of the free stream wind).

The construction of the 3-D blade model now includes the manufactured blade twist angle at each station. Clearly this affects the principal axes for highly twisted sections, and has the effect of "softening" the blade in the lag direction. As seen below in Figure 4.4, the elastic twist must also be added to the twist before the final equilibrium principal axes and the final equilibrium blade deflections can be found.

The applied loads, $L_{y}$ and $L_{z}$, are assumed to be applied at the airfoil quarterchord (aerodynamic center) as would be true for airloading. For a bench test the applied loading would have to be carefully placed at the quarterchord to satisfy this requirement. Alternatively, the (arbitrary) applied load can be established as a load at the quarterchord plus a moment (couple) about the quarterchord. This way a twisting moment can be applied in the bench test and accurately reflected in the beam calculations. For example, a concentrated weight at .75 span, located on a jig forward of the blade leading edge, produces a load and a substantial moment about the .75 station quarterchord. This was the method used to produce primary blade torsion in the tests.

The total quarterchord moment $\left(M_{x}\right)$ and loading $\left(L_{y}, L_{z}\right)$ are calculated by the program. Then the twisting moment is calculated; this is the section moment about the shear center, which is the center of twisting. Then, using the blade torsional rigidity (GJ) values calculated above via the shear flow method, the blade twist is found. This must be first integrated from the blade tip inward, and then summed from the root to the tip, to accumulate all the differential twist of the blade. Finally the program finds the "elastic twist," which is simply the differential section twist at each station. This will usually be a maximum at the tip, for all practical cases. The blade twist calculation is simpler than the flexural calculation since 
principal axes are not needed. However, the twist must be solved first since it affects the actual resulting principal axes location for each station. The moment about the shear center is simply

$$
Q_{c}=\left[L_{y} \cos \emptyset+L_{z} \sin \varnothing\right] Z_{T}+\left[L_{y} \sin \varnothing-L_{z} \cos \varnothing\right]\left[Y_{T}-.25 C\right]+M_{x}
$$

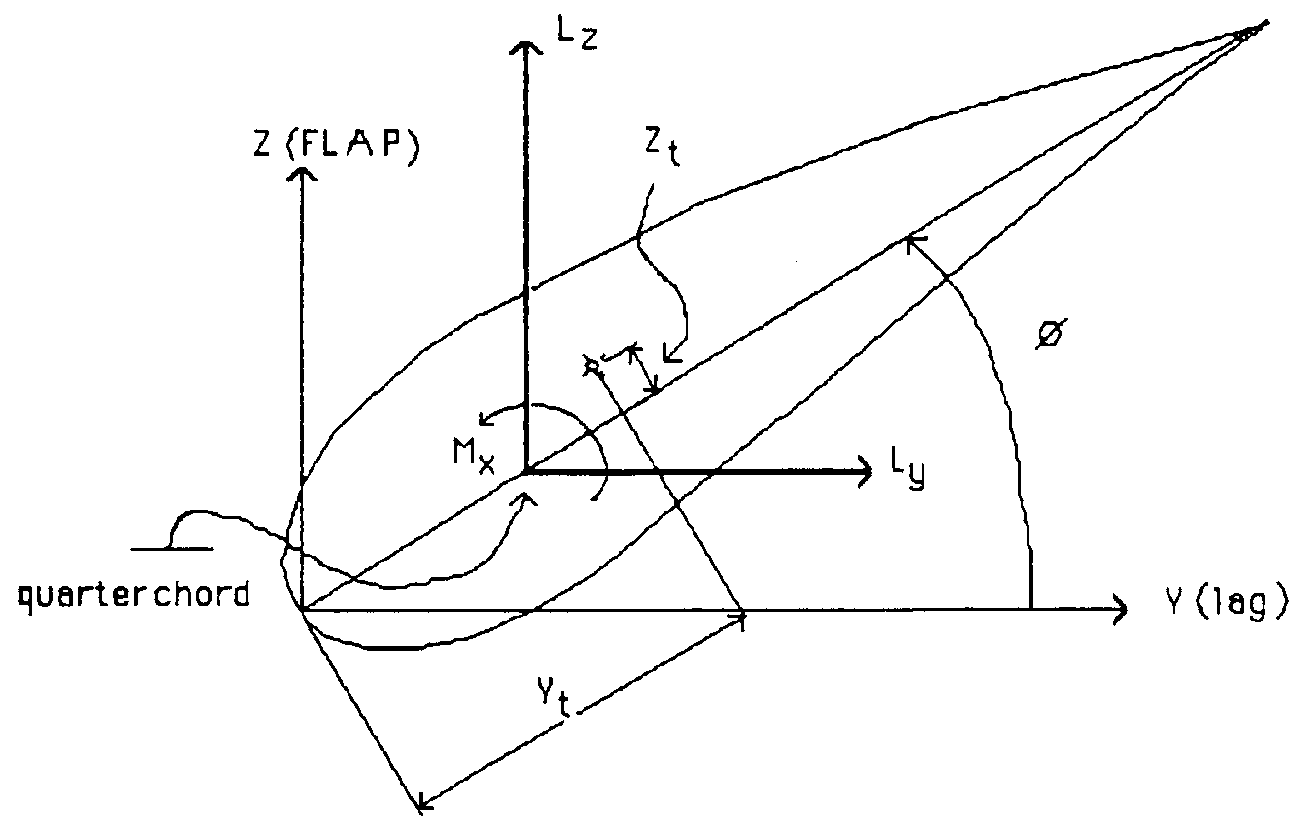

where:

$$
\begin{array}{ll}
C & =\text { blade chord } \\
\sigma & =\text { principal axis angle }+ \text { twist angle }+ \text { elastic twist } \\
L_{z} & =\text { applied flapping load (quarterchord) } \\
L_{y} & =\text { applied lag load (quarterchord) } \\
M_{x} & =\text { applied pitching moment (nose down) } \\
(Y, Z)_{t} & =\text { shear center }
\end{array}
$$

\section{FIG. 4.4 SECTION TORSIONAL MOMENTS}

With the applied loading in the $Y$ and $Z$ directions $\left(L_{y}, L_{z}\right)$ given, as for a static bench test, the blade shears and moments, in the lag and flap directions, and then in the principal axes directions, can be calculated. When the moments in the principal axes have been calculated, the blade strains can be found directly from the simple flexure formula above for each section of the blade.

$$
\begin{aligned}
& V_{y}=\text { inplane shear }=\int_{\text {root }}^{\text {tip }} L_{y} d x \\
& V_{z}=\text { flapping shear }=\int_{\text {root }}^{\text {tip }} L_{z} d x
\end{aligned}
$$




$$
\begin{aligned}
& M_{Z}=\text { inplane moment (moment about the } Z \text { axis) }=\int v_{y} d x \\
& \text { root } \\
& M_{y}=\text { flapping moment (moment about the } Y \text { axis) }=\int_{\text {tip }}^{\text {tip }} V_{z} d x
\end{aligned}
$$

The root shears are important because they combine with other blade root shears to yield hub shears. Likewise, the inplane moment combines with the other blade moments also to form the rotor torque.

Applied moments in the principal axes are

$$
\begin{aligned}
& M_{y p}=M_{y} \cos \emptyset-M_{z} \sin \emptyset \\
& M_{z p}=M_{z} \cos \emptyset+M_{y} \sin \emptyset
\end{aligned}
$$

where $ø$ is the principal axis angle.

Therefore, the bending strains at any given point on the airfoil are given simply by the $(y, z)_{p}$ contributions

$$
\varepsilon(x, y)_{i}=\frac{M_{z p} y_{p i}}{E_{z p}} \cdot \frac{M_{y p} z_{p i}}{E_{\text {Iyp }}}
$$

In the program the selected points on the airfoil ("stress offsets") are calculated first, and appear

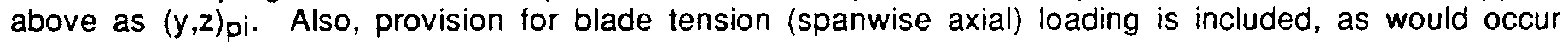
with centrifugal tension for a rotating blade.

The linear flexural deflections of the blade are found simply by integrating the moments, now expressed in the true flap and lag $(y, z)$ directions:

$$
\begin{aligned}
& \frac{M}{E I_{y}}=\frac{M}{E I_{y p}} \cos \theta+\frac{M}{E I_{z p}} \sin \emptyset \\
& \frac{M}{E I_{z}}=\frac{M}{E I_{z p}} \cos \theta-\frac{M}{E I_{y p}} \sin \emptyset \\
& \frac{d^{2} z}{d x^{2}}=\frac{M}{E I_{y}}: \frac{d^{2} y}{d x^{2}}=\frac{M}{E I_{z}}
\end{aligned}
$$

The $z$ and $y$ deflection curves are simply the flap and lag deflections of the blade axis.

In this calculation, a cantilevered blade has been assumed, as would be true for a rigid hub, or for a cantilevered blade bench test. Computationally, this is expressed as boundary conditions of the slope and deflection at the root $(x=0)$ being zero:

$$
z_{0}=y_{0}=0 \quad \text { and } \quad \frac{d z_{0}}{d x}=\frac{d y_{0}}{d x}=0
$$


Analytically from St. Venant torsion theory, statically (Ref. 11):

$$
\tau(x)=G J(x) \frac{d \theta(x)}{d x}
$$

where:

$$
\begin{aligned}
& \tau(x)=\text { applied torque (at the elastic axis) } \\
& G J(x)=\text { distribution of torsional rigidity }
\end{aligned}
$$

$$
\frac{d \theta(x)}{d x}=\text { angle of twist }
$$

The general torsional equation of motion is

$$
\frac{\partial}{\partial x}\left[G J(x) \frac{\partial \theta}{\partial x}\right]-I_{0} \theta-I_{0} \Omega^{2} \theta=\frac{\partial \tau(x)}{\partial x}
$$

where $\mathrm{I}_{\mathrm{o}}=$ torsional mass moment of inertia (polar moment of inertia) per unit length.

\section{4: BLADE NATURAL BENDING FREQUENCIES AND MODES}

The static analysis so far has produced a beam model that has distributed elasticity (section properties) and masses (section masses) from a complex geometry composite blade. Bench tests can be expected to verify (and refine) the elastic constants, and blade sectioning will verify the masses. It remains to verify the dynamics of the blade model, which can only be done by calculating the natural frequencies of vibration (and mode shapes) in flexure and torsion, and checking these also with tests. The static deflection is most sensitive to the stiffness properties, but the frequencies of vibration are most sensitive to the masses.

In the spirit of practical engineering calculation, the flexural frequencies and modes are considered to be uncoupled, flapwise from chordwise. This assumption is less valid for very highly twisted blades, but still accurate enough for present purposes.

The method for solution was suggested by Holzer (Ref. 14) for the purpose of finding natural torsional frequencies of crankshafts, and was then modified by Myklestad (Ref. 15) for the bending vibrations of nonlinear beams. Later work for propellers included the centrifugal effect of rotation (Refs. $16,17)$. The continuous blade (beam) is represented by a number of discrete segments; analytically speaking, the partial differential equation for bending is thus replaced by a set of ordinary differential equations, one for each segment. The free body diagrams for each segment are easy to analyze, and lead to a direct physical knowledge of the vibrating beam; 10 segments are used here. The deflected blade is shown in Figure 4.5. The blade is segmented into ten equal lengths and the mass of each length is equally divided into lumped masses at either end. The elastic properties ( $E I$ and $G J$ ) are assumed to be constant over the beam element. 


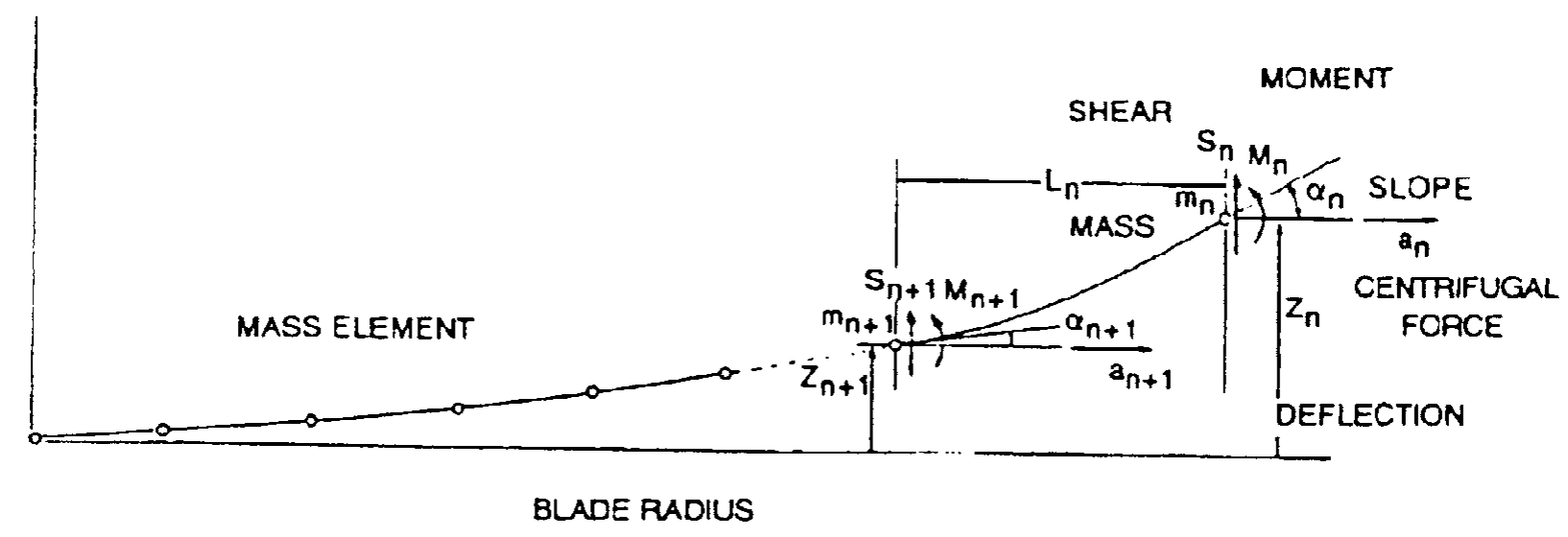

FIG. 4.5 GENERAL DEFLECTED BLADE: HOLZER/MYKLESTAD

The free body diagram for the nth element is shown in Figure 4.6.

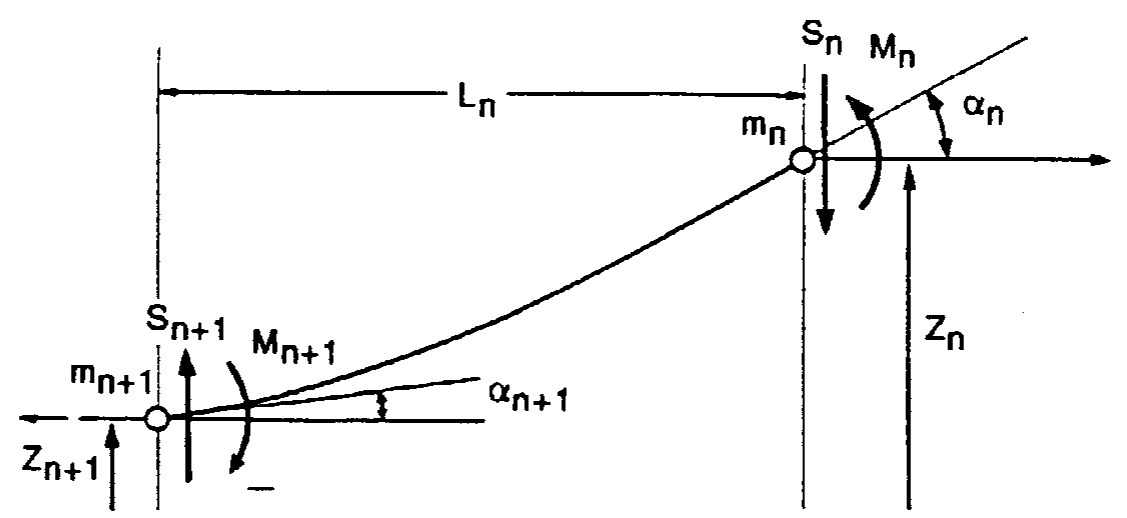

where:

FIG. 4.6 FREE BODY DIAGRAM: HOLZER TABLE

$$
\begin{aligned}
& m_{n}=n \text {th lumped mass } \\
& \alpha_{n}=n \text {th slope } \\
& a_{n}=n \text {th tension (centrifugal force) } \\
& M_{n}=n \text {th bending moment } \\
& L_{n}=n \text {th segment length } \\
& S_{n}=n \text {th shear } \\
& Z_{n}=n \text {th bending deflection }
\end{aligned}
$$

The partial differential equation of bending of the beam is fourth order; therefore, four coefficients are needed to express unit load effects due to an applied moment and an applied force. The unit load and moment coefficients are derived from a simple cantilever with a unit end load, $F$, or a unit moment, $M$ (Figure 4.7). 

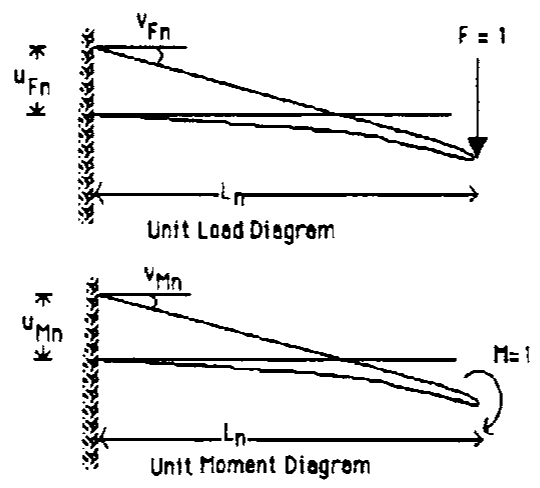

FIG. 4.7 UNIT LOAD AND MOMENT DIAGRAMS

The resulting coefficients are

$u_{F n} \quad=$ deflection due to unit force $=L_{n}{ }^{3} / 3(E I)_{n}$

$V_{F n} \quad=$ slope due to unit force $=L_{n}{ }^{2} / 2(E I)_{n}$

$u_{M n}=$ deflection due to unit moment $=L_{n}{ }^{2} / 2(E I)_{n}$

$V_{M n} \quad=$ slope due to unit moment $=L_{n} /(E I)_{n}$

Note: the above coefficients are sometimes written in non-unit form as

$$
\begin{aligned}
& u_{F n}=U_{F}=F L^{3} /(6 E I) \\
& v_{F n}=\theta_{F}=F L^{2} /(2 E I) \\
& u_{M n}=u_{M}=M L^{2} /(2 E I) \\
& v_{M n}=\theta_{M}=M L /(E I)
\end{aligned}
$$

Also, the Maxwell Reciprocal Relationship "the $u$ due to the $M=\theta$ due to the $F$ " is easily seen above. Now the segmented blade, rotating at frequency $\Omega$, is assumed to vibrate at frequency $\omega$. An additional inertial force $\left(M_{n+1} \omega^{2} z_{n+1}\right)$ is added to the free body diagram above. Force and moment equilibrium then yield

$\begin{array}{llll}\text { tension } & =a_{n+1} & =a_{n}+m_{n+1} \Omega^{2} r_{n+1} \\ \text { shear } & =S_{n+1} & =S_{n}+m_{n+1} \omega^{2} z_{n+1} \\ \text { moment } & =M_{n+1} & =M_{n}-S_{n} L_{n}+a_{n}\left(z_{n}-z_{n+1}\right) \\ \text { slope } & =\alpha_{n+1} & =\alpha_{n}\left(1+a_{n} v_{F n}\right)-S_{n} v_{F n}+M_{n} v_{M n} \\ \text { displacement } & =z_{n+1} & =z_{n}-\left(L_{n}+U F_{n} a_{n}\right) \alpha_{n}+U F_{n} S_{n}-u_{M n} M_{n}\end{array}$

The tension at the nth segment can be rewritten as a sum of tensions:

$$
a_{n}=\sum_{i=1}^{n} m_{i} \Omega^{2} r_{i}
$$

And the deflection can be substituted into the moment expression to give

$$
M_{n+1}=M_{n}\left(1+u M_{n} a_{n}\right)-S_{n}\left(L_{n}+u F_{n} a_{n}\right)+\alpha_{n} a_{n}\left(L_{n}+u_{F n} a_{n}\right)
$$

These recurrence formulas could be solved by successive substitution, working from the tip of the blade to the root. Shear, moment, slope, and deflection would be calculated for each segment, and resultant distributions would be known. The slope is assumed to be an arbitrary angle, $\theta$, and the deflection a unit quantity. Then, trial values for frequency (or $\omega^{2}$ ) are introduced. For the trial $\omega$ to be the correct frequency, the slope at the root and the deflection must correspond to the appropriate root boundary condition; viz: 


\section{Cantilevered:}

Slope at root $=$ deflection at root $=0$

Hinged:

Moment at root $=$ deflection at root $=0$

Myklestad applied this to systematic computation by making judicious new definitions and substitutions, as follows. The load and moment coefficients and centrifugal forces can be combined via the "Myklestad definitions," which simplify the above expressions:

$$
\begin{aligned}
& A_{n}=1+v_{F n} a_{n} \\
& B_{n}=1+U_{M n} a_{n} \\
& C_{n}=L_{n}+U_{F n} a_{n} \\
& D_{n}=C_{n} a_{n}=\left(L_{n}+U F_{n} a_{n}\right) a_{n}
\end{aligned}
$$

Then, a set of assumed linear functions is substituted in the set of four recurrence formulas for shear, moment, slope, and deflection. The assumed slope at the tip, $\varphi$, is carried as the independent variable; the assumed Myklestad amplitude coefficients are

$$
\begin{array}{ll}
\text { Shear: } & S_{n}=-G_{\varphi n \varphi}+G_{n} \\
\text { Moment: } & M_{n}=H_{\varphi n \varphi} \cdot H_{n} \\
\text { Slope: } & \alpha_{n}=h_{\varphi n \varphi} \cdot h_{n} \\
\text { Deflection: } & z_{n}=-g_{\varphi n \varphi}+g_{n}
\end{array}
$$

Substituting these linear functions into the four original recurrence formulas and equating coefficients yields simple recurrence formulas for the amplitude coefficients now suitable for systematic computation. [Note: The classical Myklestad derivation is used here to avoid the ambiguity, which is seen in sign definitions in later works.]

$$
\begin{array}{ll}
G_{\varphi(n+1)} & =G_{\varphi n}+M_{n+1} \omega^{2} g_{\varphi(n+1)} \\
G_{(n+1)} & =G_{n}+M_{(n+1)} \omega^{2} g_{(n+1)} \\
H_{\varphi(n+1)} & =B_{n} H_{\varphi n}+D_{n} h_{\varphi n}+C_{n} G_{\varphi n} \\
H_{(n+1)} & =B_{n} H_{n}+D_{n} h_{n}+C_{n} G_{n} \\
h_{\varphi(n+1)} & =A_{n} h_{\varphi n}+v_{M n} H_{\varphi n}+v_{F n} G_{\varphi n} \\
h_{(n+1)} & =A_{n} h_{n}+v_{M n} H_{n}+v F n G_{n} \\
g_{\varphi(n+1)} & =g_{\varphi n}+C_{n} h_{\varphi n}+u M n H_{\varphi n}+u F_{n} G_{\varphi n} \\
g_{(n+1)} & =g_{n}+C_{n} h_{n}+u_{M n} H_{n}+U F n G_{n}
\end{array}
$$

For the free tip case, the initial values are at the tip:

$$
S_{1}=M_{1} \omega^{2} \quad M_{1}=0 \quad \alpha_{1}=\varphi \quad z_{1}=1.0
$$

This yields the initial values of the amplitude coefficients:

$$
\begin{array}{ll}
G_{\varphi 1}=0 & G_{1}=M_{1} \omega^{2} \\
H_{\varphi 1}=0 & H_{1}=0 \\
h_{\varphi 1}=1.0 & h_{1}=0 \\
g_{\varphi 1}=0 & g_{1}=1.0
\end{array}
$$


Then the root, which is cantilevered, gives the condition (from deflection $=0$ )

$$
\varphi=h_{\text {root }} / h_{\varphi r o o t}
$$

The remainder in the program is the root deflection, which is the trial value; e.g., when the remainder is zero, the trial value of $\omega^{2}$ was correct and a solution has been found:

$$
Z_{\text {root }}=\text { remainder }=-g_{\varphi r o o t} \varphi+g_{\text {root }}=0 \quad \text { (for a root of the equations) }
$$

In general, this iterative method will give as many roots (frequencies) as there are lumped masses. The corresponding shear, moment, and deflection mode shapes are found for each root by simple substitution in the recurrence formulas. For most wind turbine blade dynamics, the lowest or fundamental frequency and mode shape are most important since they dominate the dynamic response (flap and leadlag).

The computer program uses for input data the running mass of the blade and the section stiffness (for either flap or lead-lag) at the operating rpm. Another version of the program also allows the addition of a concentrated tip mass to assess the effect of a tip weight or mechanism (Appendix 10.3, 10.4).

\section{5: $\quad$ BLADE TORSIONAL FREQUENCIES AND MODES}

The blade torsional frequencies are also calculated using the Holzer table approach. The model assumed a shaft fixed at the root, with flywheels $\left(\mathrm{I}_{i}\right)$ at each station representing the lumped masses, and torsional stiffness $\left(k_{i}\right)$ derived from the GJs:

$$
\begin{aligned}
& k=\begin{array}{l}
\text { torsional stiffness }=G J / L=G J / \Delta r \\
\text { where } \Delta r \text { is the segment length }
\end{array} \\
& I=\text { mass moment of inertia }=\iiint r^{2} d m=\iint(\rho d x) r^{2} d A \\
& \text { where: } \quad \begin{aligned}
r & =\text { chordwise coordinate } \\
x & =\text { spanwise coordinate } \\
\rho & =\text { mass density }
\end{aligned}
\end{aligned}
$$

[Note: the polar moment of inertia of a section is the $\left(r^{2} d A\right)$ portion.]

The $\rho r^{2} d A$ integration must be performed in the section properties calculations since the density varies along the composite beam. Also, ballast weight must be assessed since it changes the section mass moment.

The question arises as to which value of section inertia to use, the minimum value (which occurs about the section $\mathrm{cg}$ ), the value referenced to the shear center (about which section twisting occurs), or the value referred to the blade geometric axis (blade axis). In this treatment the elastic axis (shear center) is used as reference as explained here:

- For a uniform blade with a symmetrical cross section and uniform properties spanwise, the geometric, elastic, and mass axes coincide. Therefore, twisting in this case will occur in pure shear (twist) with the geometric center as its "axis of twisting."

- If the geometric axis is offset (chordwise) from the support at the root, the blade torsional motion still occurs on the mass/elastic axis of the blade. The offset support has just added another resultant degree of freedom: that of rigid body flexure and twisting about the support. [Note: this can be easily visualized by making a sketch.]

- Next for an unsymmetrical weight on the shaft (ballast), the new twisting motion will occur about the (old) elastic axis as before, even though it is not the geometric (from above) or mass axis anymore. Finally, for a complex geometry, the action of pure twisting is as close as possible to the elastic axis of the blade, even though this may not be a straight line. [Comment: This line of 
reasoning, admittedly qualitative, was empirically verified in the modal analysis testing of the UTRC blade. The FFT software allowed computer graphics animation of the fundamental torsional motion, which clearly showed the twisting occurring about the elastic axis and not the mass axis.]

If the blade contains nonstructural or ballast weights, the mass centers are relocated off the elastic axis. This adds

1) torsional coupling to the bending modes (flutter),

2) bending to the torsional modes,

3) increasing mass moment of inertia to the section; thus lowering the natural torsional frequencies.

The solution is found using the same approach as before. The free body diagram is shown in Figure 4.8.

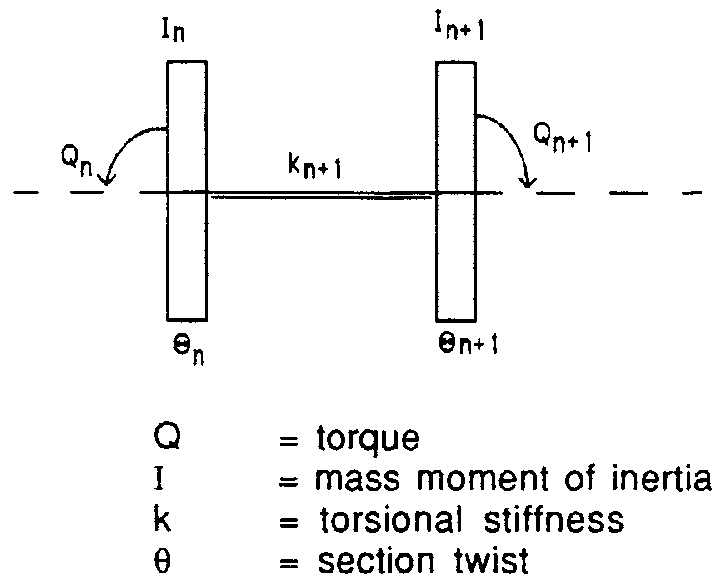

FIG. 4.8 FREE BODY DIAGRAM FOR TORSIONAL VIBRATION

The governing equations (recurrence formulas) are much simpler than before:

$$
\begin{aligned}
& Q_{n+1}=Q_{n}+I_{n+1} \omega^{2}\left(\theta_{n+1}-\theta_{n}\right) \\
& \theta_{n+1}=\theta_{n}+\left(Q_{n+1}-Q_{n}\right) /\left(k_{n+1}\right)
\end{aligned}
$$

The boundary condition at the tip $(n=1)$ is $\theta=1$; the remainder is the twist $(\theta)$ at the root which must equal zero for a solution. Actually this is precisely the method used by Holzer to calculate crankshaft frequencies by hand.

Again, there is provision in one version of this program to assess tip weights (inertias) on torsional frequency (Appendix 10.4). 


\subsection{DEVELOPMENT OF BLADE MASS/ELASTIC MODELS}

\section{1: $\quad$ TRIANGULATION OF MASS/ELASTIC PROPERTIES}

The primary need in this phase was to verify the accuracy of the engineering constants used in the beam analysis. This involved comparing the static bending and frequency of vibration results from the experiments with the predictions based on assumed engineering constants. The static bending and frequency tests "bracket" the error bounds on uncertainty of the engineering constants and masses, since the static deflection results are more sensitive to the moduli, and the frequency results to the masses. Discrepancies must be evaluated and solutions iterated until a satisfactory semi-empirical set of engineering constants exists for each blade that satisfies both the theory and the tests (static and vibration). Additionally, the blade root attachments were evaluated to obtain the bending and torsional compliance of the attachments, primarily by referencing the root restraint view in the modal survey and by baseline spanwise inclinometer readings in the static tests. At the end of this phase a practical, accurate, and workable mathematical model existed for each blade/hub combination of the study.

\section{2: UNIFORM FRP BLADE EXAMPLE: UTRC BLADE}

The UTRC blade was the simplest structurally since it was a uniform pultrusion. The flexbeam/pendulum mechanism was not tested since the twisting kinematics were considered to be beyond the scope of this work. Clearly, the majority of the elastic twist of the flexbeam rotor is occurring in that portion of the blade that could be easily modeled elastically, which is proved in the results. This blade was a good choice for this study since:

a. Blade is uniform pultrusion construction, which is representative of many rotor designs, and offers good cost reduction potential.

b. Blade section properties are uniform and straightforward.

c. Rotor has shown performance and flutter problems in the past due to blade flexibility (these were likely due to the flexbeam, however).

d. Rotor performance was documented by AEI and SERI.

The UTRC blade is shown in Figure 1.3, at its full radius of 192 in., and with the root flexbeam portion comprising the inner 0.3 of the radius. The pultrusion was manufactured by Morrison Molded, and the flexbeam by UTRC. The pendulum weight and flexstrap pitching mechanism is not shown. The blade tested in this report was the outboard pultrusion, which has a cross-section as shown in Figure 5.1, from Ref. 18. This is a simple blade section, formed of a uniform laminate (constant thickness) skin with the airfoil shape and a solid spar in the leading edge, and a foam-filled aft section. The flexbeam portion was not tested but was actually very easily modeled since it was a simple rectangular cross section. This part of the report describes the development of a suitable aeroelastic model for this blade.

\subsection{1: METHOD}

To illustrate the derivation of purely theoretical section properties for a design, this discussion will begin by not including the test data, just the geometries and constituents. The airfoil shape NACA 23112 is determined by a table of offsets. The standard for the AEI program is 33 pairs of points $(X, Y)$ beginning at the nose, proceeding around the upper surface to the tail, and returning to the nose on the lower surface. This is the data set format used in the program.

\subsection{2: PREDICTION OF ENGINEERING MODULI AND DENSITIES}

The skin laminate was comprised of four layers of reinforcement of two types: unidirectional $\left(0^{\circ}\right)$ and balanced $\left( \pm 45^{\circ}\right)$, with two layers of each type. In this case we are not told the reinforcement cloth makeup; instead we have the thicknesses, which are

$.040^{\prime \prime}$ per layer for $\pm 45^{\circ}$ and

$.025^{\prime \prime}$ per layer for $0^{\circ}$

This gives a total (specified) skin thickness of .130 in. (Ref. 1). It remains to reduce this skin laminate to

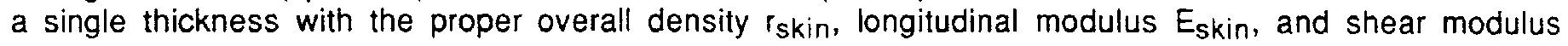
Gskin. The same must be done for the spar later. 
NACA 23112 AIRFOIL

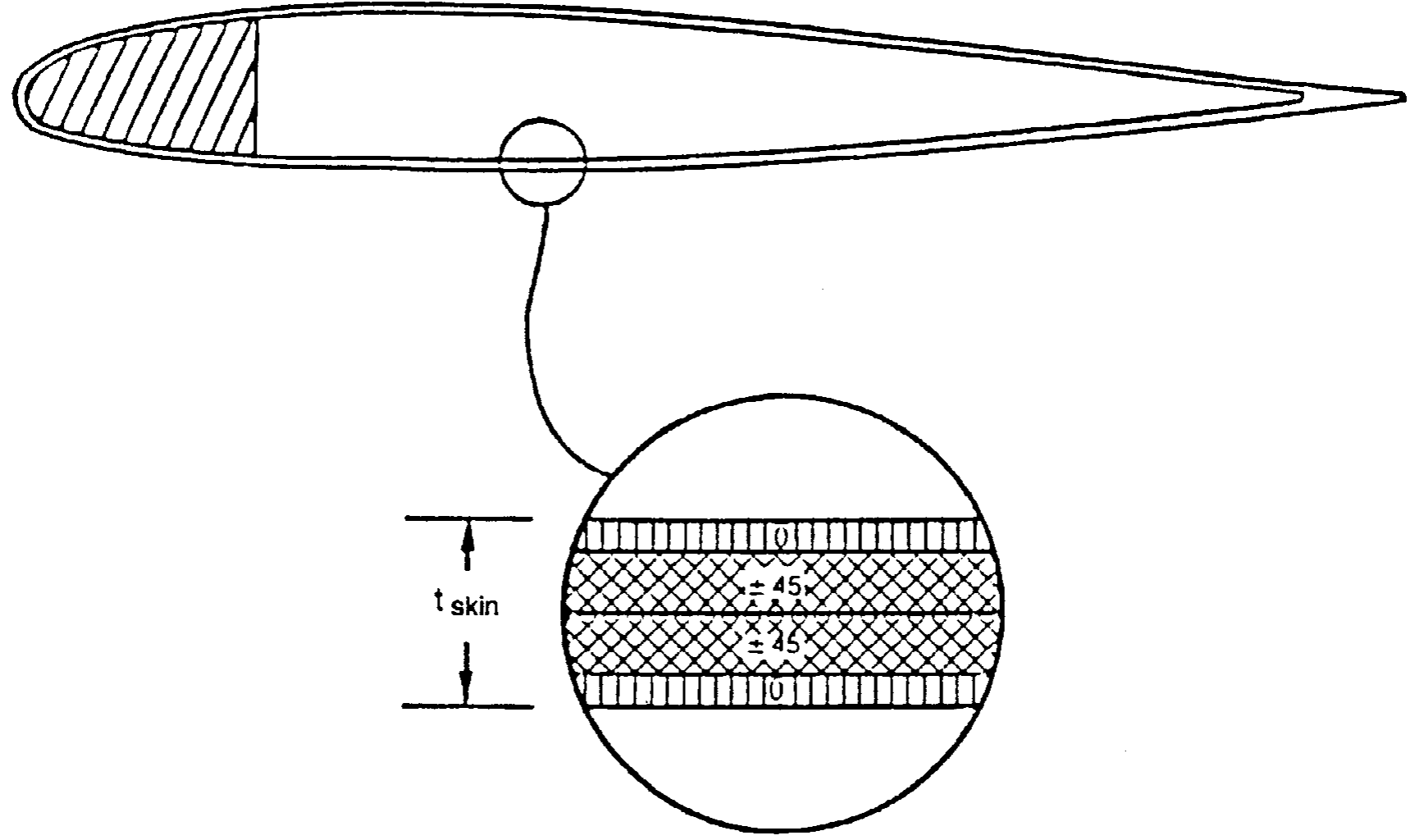

FIG. 5.1 UTRC CROSS SECTION

To calculate these values for the skin, first look at this unidirectional layer:

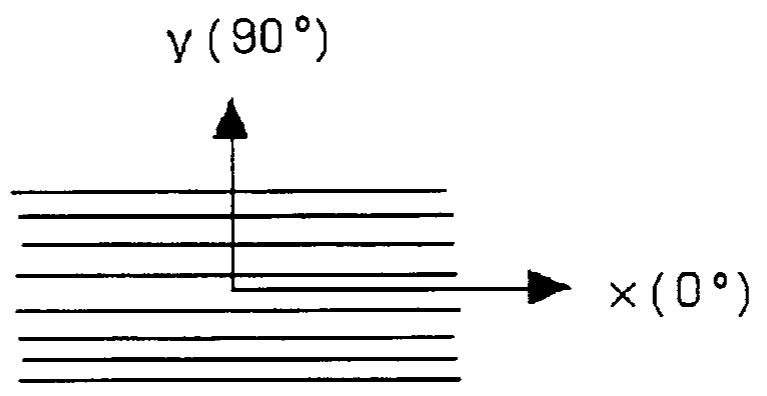

The properties of a unidirectional laminate are shown in Table 4.1, for glass reinforcement and epoxy or polyester resin matrix. If $50 \%$ volume is glass, the E-modulus is $5^{*} 10^{6}$ psi and the density $.0678 \mathrm{lb} / \mathrm{in}^{3}$, from first principles (see Table 4.1). For the transverse $\mathrm{E}$-modulus, zero is normally assumed, as it is only the resin matrix. Practically speaking the G-modulus is also zero. We will estimate $50 \%$ fiber volume as representative of this pultrusion process, as it is for hand lay-up. This fiber ratio (or reinforcement to resin ratio) is very important in composites, as can be seen in this simple example. Fortunately, this ratio is easily found in an ASTM "burn-off" test, which simply burns off the resin under controlled conditions in a crucible to get an actual weight and thus a volume percentage. 
For the $\pm 45^{\circ}$ layer we assume half the fibers run at $0^{\circ}$ and the other half at $90^{\circ}$. This changes the above modulus value by cutting it in half, since half are now at $90^{\circ}$. This gives the $0 \% 90^{\circ}$ properties, again based on a $50 \%$ volume, as

$$
\begin{aligned}
E\left(0^{\circ} / 90^{\circ}\right) & =2.5^{*} 10^{6} \mathrm{psi} \\
\rho & =.0678 \mathrm{~b} / \mathrm{in}^{3}
\end{aligned}
$$

Since this layer is not oriented at $0^{\circ}$ (but $\pm 45^{\circ}$ ), we must decrease the longitudinal E-values by $50 \%$ (because the fibers are not aligned with the E-direction), and also increase the G-value by $50 \%$ (because at $45^{\circ}$, the fibers are now along the principal transverse shear or twisting direction). This is shown in the "rule of thumb" directional properties engineering guide of Figure 5.2.

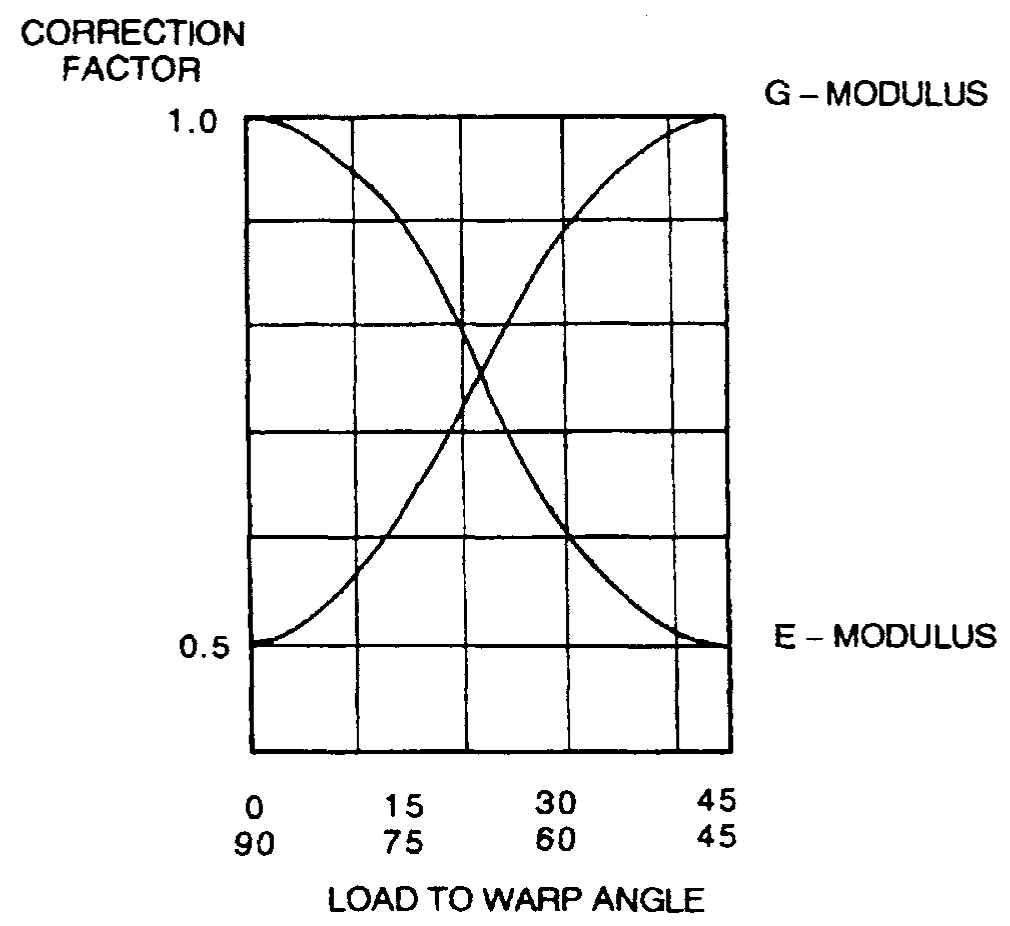

FIG. 5.2 RULE OF THUMB, FRP DIRECTIONAL PROPERTIES

Now we can calculate the total skin values of $E$ and $G$ by using the rule of mixtures. Analytically:

Elaminate $=$

$E_{1} t_{1}+E_{2} t_{2}+E_{3} t_{3}+\ldots$

$$
\text { total }
$$

This gives for the trial values:

$$
\begin{aligned}
& E_{\text {skin }}=\frac{5 * 10^{6} * .050+1.25 * 10^{6} * .080}{.130}=2.69 * 10^{6} \mathrm{psi} \\
& G_{\text {skin }}=\frac{0.050+1.80^{*} 10^{6}+.080}{130}=1.10 * 10^{6} \mathrm{psi}
\end{aligned}
$$

Going on, the spar is a unidirectional bundle of rovings (fibers) in a compact resin matrix. We can expect a higher fiber volume (say $60 \%$ ) and thus higher values than the less compact skin above: 


$$
\begin{aligned}
& E_{\text {spar }}=6 \cdot 10^{6} \mathrm{psi} \\
& \rho_{\text {spar }}=.0727 \mathrm{lb} / \mathrm{in} .3 \\
& G_{\text {spar }}=1.10 * 10^{6} \mathrm{psi} \text { (for simplicity) }
\end{aligned}
$$

\subsection{3: PREDICTION OF SPAR PROPERTIES}

The skin contribution will be based on the composite values of $E, G$, and $\rho$ determined above, integrated around the airfoil shape as input via the offsets. Also the spar values of $E, G$, and $\rho$ have been estimated, but not the geometry. The program has the capability of assessing a D-spar in the leading edge and further spars or webs. Here we do not have a D-shape, so that calculation is eliminated. We treat this "spar" as a "web," so must describe it as a collection of moments of inertia and weights at its centroid. The program then adds its contribution simply by using the parallel-axis theorem. A graphic calculation of the spar yields

$$
\begin{aligned}
A_{\text {spar }} & =2.7076 \text { in. } 2 \\
I_{x} & =0.3831 \text { in. } 4 \\
I_{y} & =0.9887 \text { in. } 4 \\
I_{x y} & \equiv 0 \\
(x, y)_{\text {centroid }} & =(1.4796,0.1843) \text { in. }
\end{aligned}
$$

The contribution of the foam is ignored.

\subsection{4: INITIAL PREDICTED RESULTS}

\begin{tabular}{|c|c|c|c|c|c|c|c|c|c|c|c|c|}
\hline & \multicolumn{3}{|c|}{ Geometry (in.) } & \multirow[b]{2}{*}{$d_{\text {spar }}$} & \multirow[b]{2}{*}{ Im } & \multirow[b]{2}{*}{ (lb/in.) } & \multirow[b]{2}{*}{ Elflap } & \multicolumn{3}{|c|}{ Mass and Stiffness } & \multirow[b]{2}{*}{$E A(\% c)$} & \multirow[b]{2}{*}{ ) } \\
\hline & $c$ & tAF & tskin & & & & & Ellag & GJ & $y_{c g}(\% c)$ & & \\
\hline $\begin{array}{l}\text { Ifr's Spec } \\
\text { it. Pred. }\end{array}$ & $\begin{array}{r}15 \\
(15\end{array}$ & $\begin{array}{l}1.8 \\
1.8\end{array}$ & $\begin{array}{l}.130 \\
(.130)\end{array}$ & & & & & & & & & $\begin{array}{l}6.14 \\
8.59\end{array}$ \\
\hline heasured & 14.9 & 1.95 & .118 & 2.56 & & 22 & $\cdots$ & $\therefore$ & $\ldots$ & 31.6 & -.- & $\cdots$ \\
\hline Final Model & 15 & 1.95 & .118 & 2.56 & 1 & 22 & 5.87 & 32.4 & 5.58 & 31.3 & 46.2 & 8.38 \\
\hline
\end{tabular}

The above predictions yield section properties which we can only expect to be in "the ballpark." These are listed in Table 5.1 which also shows the 1) manufacturer's specifications (which have been used up to now), 2) the values from the blade sectioning, and 3) the final semi-empirical values after they have been verified by both testing and predictions. Obviously it is wise in most cases to perform the tests, including blade sectioning, because the blade model is much more accurate, as can be seen in the table.

\section{TABLE 5.1: UTRC BLADE SECTION PROPERTIES COMPARED TO MANUFACTURERS' SPECIFICATIONS AND SECTIONAL VALUES}

\subsection{5: SPECIFICATION VERIFICATION BY BLADE SECTIONING}

By close blade inspection, and specifically by cutting a blade into sections, some of the predictions of the above approach can be much more accurate. Referring to Table 5.1, the specified skin thickness of $0.130 \mathrm{in}$. turned out to be $0.118 \mathrm{in}$. and the running mass went from $0.352 \mathrm{lb} / \mathrm{in}$. to $0.422 \mathrm{lb} / \mathrm{in}$. Clearly the manufacturer's specifications were wrong; thus the initial predictions above gave section properties that were also wrong; (see Table 5.1). At this stage, though, no direct measurement of EI and GJ can be made, nor have the mass and section inertia values been verified.

What can be concluded is that blade inspection is important to verify a manufacturer's claims. Here all the structural and mass properties are different. The aerodynamic properties as well are different; with an actual section thickness of $13 \%(1.95 / 15)$, this airfoil will behave differently than the specified $12 \%$ (NACA 23112). 


\subsection{6: $\quad$ VERIFICATION BY DEFLECTION TESTS}

The key load case was $175 \mathrm{lb}$ total load, evenly distributed along the span applied to the quarter chord. Figure 5.3 shows the predicted flapwise deflection of the UTRC blade for the El's from both the initial calculation (above) and for the corrected semi-empirical values of EI. The test data are also shown. When the correct values (e.g., of skin thickness, airfoil thickness), have been determined from sectioning, the E-modulus in this case is simply varied until the static deflection matches the theoretical prediction for test, as shown.

There is more to this part, however. A test load case will generally produce flapwise, chordwise, and torsional deflections, and they all must be correctly predicted by the theoretical model. Therefore, it is not enough to match just the flapwise deflection; the chordwise deflection and twist must also match. In the AEI approach the loading is applied at the blade quarterchord. If the principal axes of the section are not exactly horizontal in the laboratory, some chordwise deflection will also occur. Also, if the section shear center (elastic axis) is off the quarterchord, twist will occur. For this load case (175 lb) $10.47 \mathrm{in}$. flapwise and 0.08 in. chordwise defiection, and roughly $0.1^{\circ}$ (nose up) twist were measured. (Note: the blade was mounted lifting surface down to mimic airload.)

DEFLECTION (in)

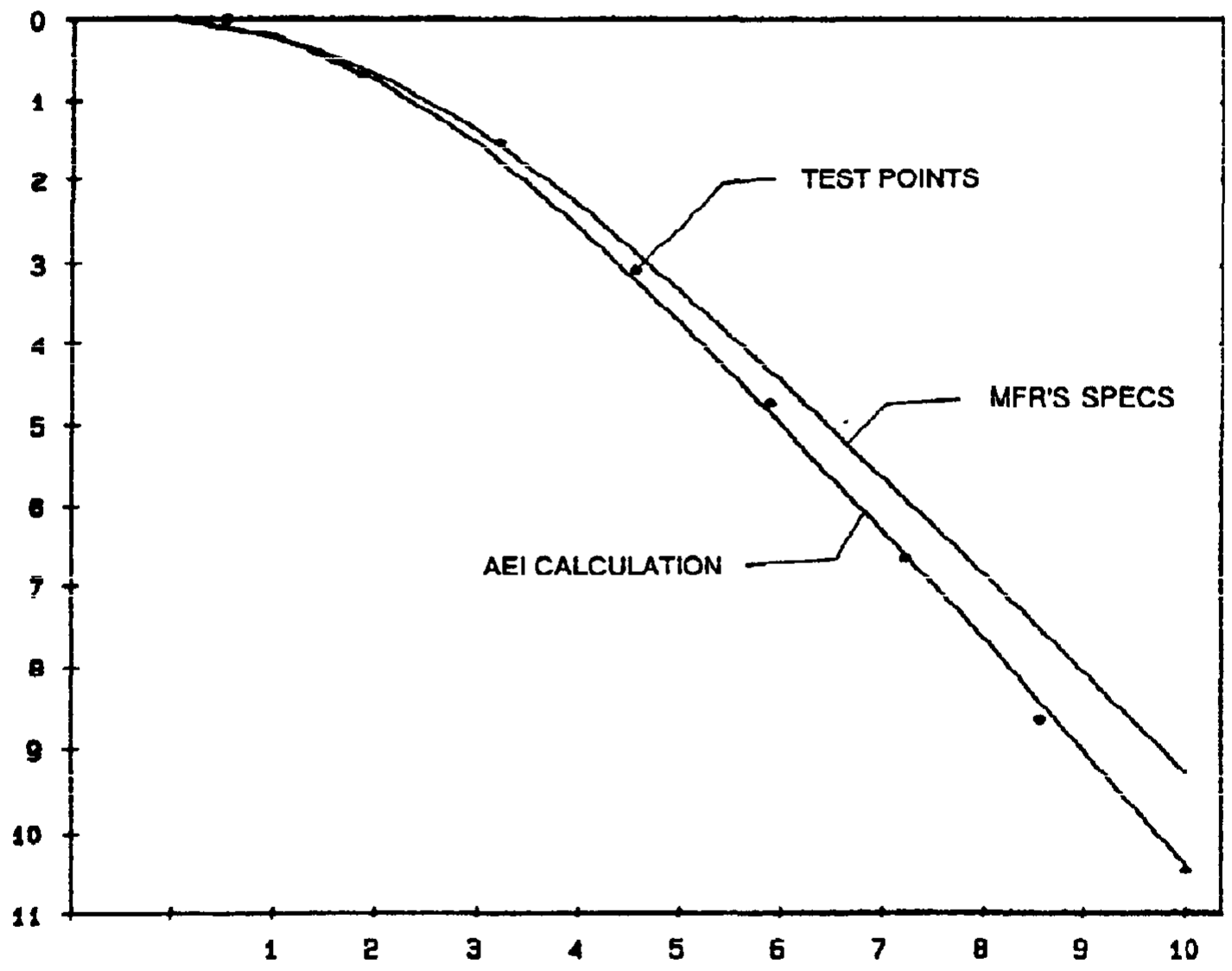

FIG. 5.3 UTRC FLAP DEFLECTION, TEST VS. CALCULATION VS. SPECIFICATIONS

Looking at the section properties, the principal axes of the blade are $0.42^{\circ}$ (nose up). This is the effect which causes the inplane deflection. When this value of $0.42^{\circ}$ is used as a simple pitch input in the calculation, the flap and lead-lag deflections are reproduced accurately. The resulting values of modulus are

$$
\begin{array}{lll}
E_{\text {skin }}=2.05 * 10^{6} \mathrm{psi} & & \begin{array}{l}
\text { initial values } \\
\left(2.67 * 10^{6}\right)
\end{array} \\
E_{\text {spar }}=5.70 * 10^{6} \mathrm{psi} & \left(6.0 * 10^{6}\right) \\
\mathrm{G}_{\text {skin }}=1.34 * 10^{6} \mathrm{psi} & \left(1.1 * 10^{6}\right) \\
\mathrm{G}_{\text {spar }}=0.50 * 10^{6} \mathrm{psi} & \left(1.1 * 10^{6}\right)
\end{array}
$$


The initial guesses are also shown. So far the values of EI (flap and lag) have been verified, but the value of GJ has not, since there was so little elastic twist in this $175 \mathrm{lb}$ load case. Also, the masses and moments of inertia have not been verified; these remain for the frequency tests. Using the torsional test case $(23 \mathrm{ft}$ Ib @ STA 0.9) the predicted twist is $0.24^{\circ}$ (at the tip) compared to the test value of $0.37^{\circ}$. Either the GJ is too high or the elastic axis is slightly off. Rather than modify the G's to achieve congruence at this point, which could be done easily by reducing $G_{\text {skin }}$ and $G_{s p a r}$ equally until $0.37^{\circ}$ is reached, we will depend on the frequency test still to come.

Note: A blade analysis could stop at this point, depending on the static weights for mass distribution and the information gained so far for section $\mathrm{cg}$ and section inertia. However, these parameters are crucial to the elastic twist on a "flying" blade, which has far more centrifugal force than the loads that can be conveniently applied in the lab, so further verification is warranted. 


\subsection{7: VERIFICATION BY FREQUENCY TESTS}

The flapwise and first torsional frequencies and modes are shown in Figures 5.4 and 5.5 .
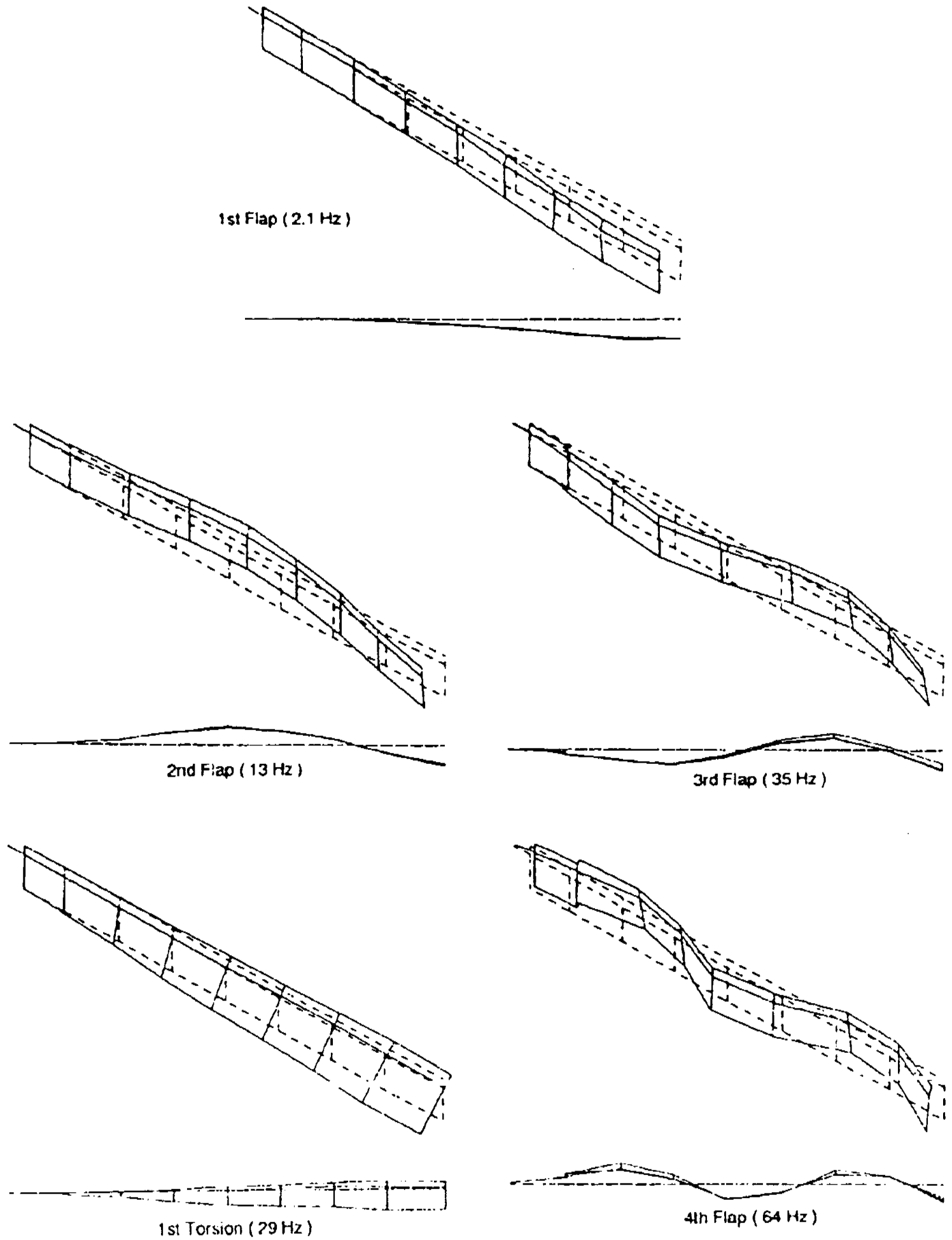

FIG. 5.4 UTRC VIBRATION MODES, TEST (MODAL SURVEY) 


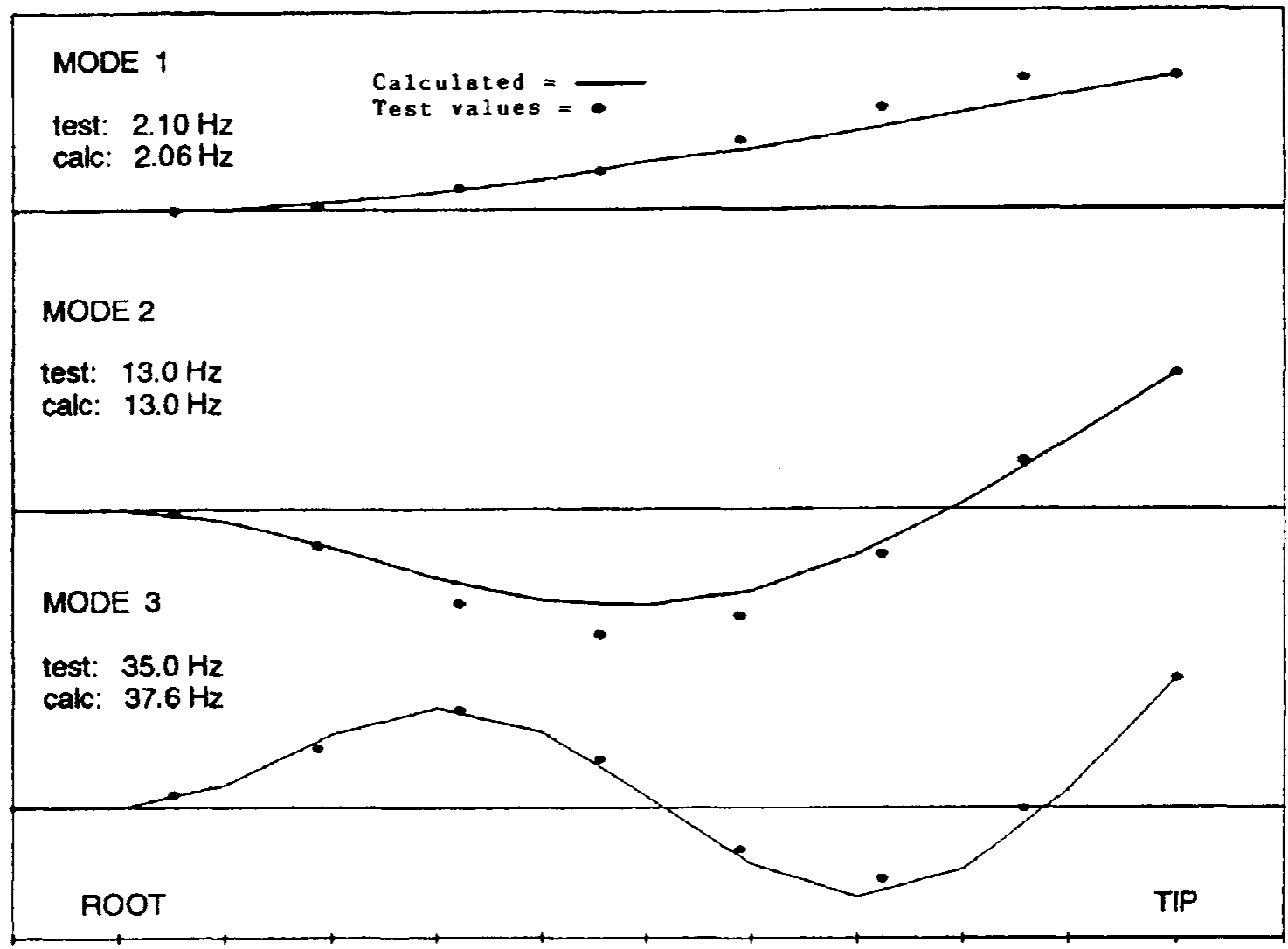

FIG. 5.5 UTRC FLAPWISE VIBRATION MODES, PREDICTED VS. TEST

The frequency program results are shown in Table 5.2 below:

\begin{tabular}{|c|c|c|}
\hline $\begin{array}{l}\text { Mode } \\
\text { 1st flap } \\
\text { 2nd flap } \\
\text { 3rd flap }\end{array}$ & $\begin{array}{c}\text { Test } \\
2.1 \mathrm{~Hz} \\
13.0 \mathrm{~Hz} \\
35.0 \mathrm{~Hz}\end{array}$ & $\begin{array}{r}\text { Prediction } \\
2.06 \mathrm{~Hz} \\
12.99 \mathrm{~Hz} \\
37.60 \mathrm{~Hz}\end{array}$ \\
\hline $\begin{array}{l}\text { 1st lag } \\
\text { 2nd lag }\end{array}$ & $\begin{array}{l}13.0 \mathrm{~Hz} \\
78.0 \mathrm{~Hz}\end{array}$ & $\begin{array}{r}15.22 \mathrm{~Hz} \\
96.0 \mathrm{~Hz}\end{array}$ \\
\hline
\end{tabular}

The principal axis stiffnesses were used since they are the decoupled modes on a uniform blade. It can be seen that the flapwise values are in excellent agreement, verifying the Elflap and masses. The lag frequencies are different from the test presumably because the root fixity softened the cantilever. thus driving the lag frequencies down. In a sense the lag value is inconsequential anyway, because it is much higher than the first flap frequency.

The torsional values are shown below. Clearly the test data have confirmed the blade values of GJ and I; final GJ is $6.2^{*} 10^{6}$ psi with $\mathrm{G}_{\mathrm{spar}}=0.1^{*} 10^{6}$ and $\mathrm{G}_{\mathrm{spar}} 0.9^{*} 10^{6}$.

$\begin{array}{ccc}\text { Mode } & \text { Test } & \text { Prediction } \\ \text { 1st Torsion } & 29.0 \mathrm{~Hz} & 28.39 \mathrm{~Hz} \\ \text { 2nd Torsion } & 84.0 \mathrm{~Hz} & 84.71 \mathrm{~Hz}\end{array}$

In the torsional frequency calculation the blade is assumed to oscillate about its elastic axis (see 4.5 under Theoretical Method), which has a larger mass moment of inertia than about the section cg (10.51 vs. 8.38). 
Going back to the flexure tests will "close the loop" on the GJ. The G's have been matched by the frequency tests, and the predicted static torsion of the load test above (23 ft-lb @ STA .9) now matches the test value of $0.37^{\circ}$. This is a good indication that the blade masses and stiffness are correct since they match static and dynamic test values simultaneously.

\subsection{8: FINAL AEROELASTIC MODEL}

At this stage the pultruded UTRC blade has been successfully modeled; the following conditions have been met by the predictions matching the tests:

1) total blade weight

2) blade flexural deflection, flap and lag

3) blade static torsional deflection

4) blade flapwise frequencies and modes

5) blade lag frequencies and modes

6) blade torsional frequencies and modes

It remains to construct the total aeroelastic model by adding the flexbeam portion and creating the appropriate blade properties file for the elastic twist calculations.

The flexbeam is attached at STA 0.3 and has the rectangular geometry shown below from inspection:

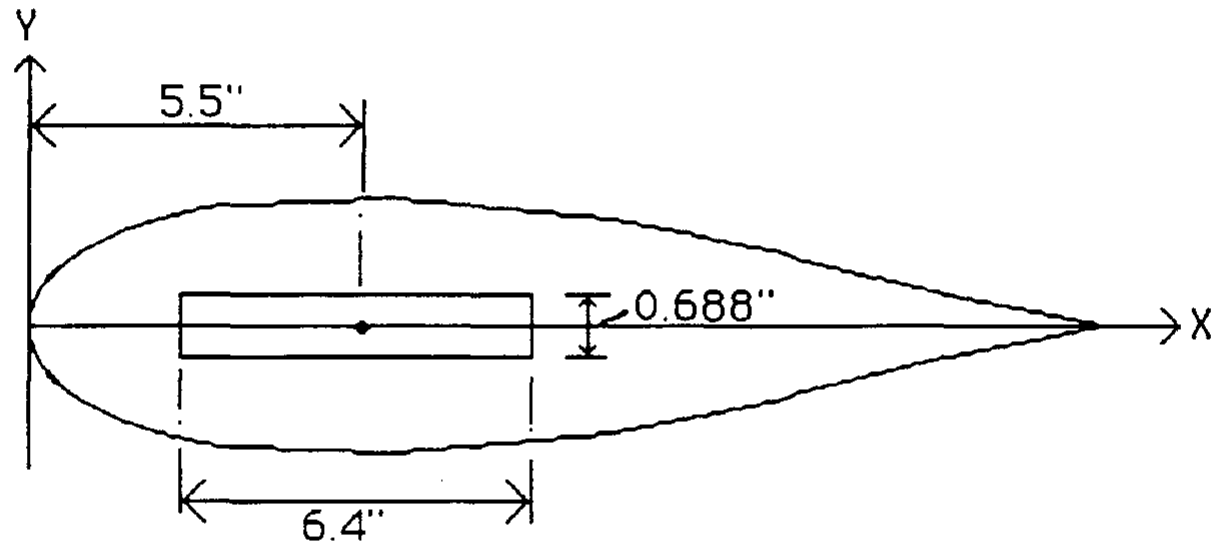

The input data for the flexbeam are derived from the manufacturer's specifications and the inspection values (the stiffnesses were not tested):

also

$\begin{array}{ll}\text { Al-beam } & =4.4032 \mathrm{in.}^{2} \\ I_{x} & =24.04 \mathrm{in.}^{4} \\ I_{y} & =0.2404 \mathrm{in.}^{4} \\ I_{x y} & \equiv 0.0 \\ (x, y)_{c} & =(5.5,0.0)\end{array}$

$\begin{array}{ll}\text { EI }_{\text {flap }} & =1.37 * 10^{6} \\ \text { EI lag }_{\text {Gl }} & =137 * 10^{6} \\ \text { Gl } & =0.24 * 10^{6}\end{array}$


The final values for the UTRC aeroelastic blade model are shown in Table 5.3.

TABLE 5.3: FINAL UTRC BLADE MODEL ANALYSIS

\begin{tabular}{|c|c|c|c|c|c|c|c|c|c|c|}
\hline & STA & $r / R$ & $\underset{r}{(i n .)}$ & $\underset{m_{0}}{(\mid \mathrm{b} / \mathrm{in} .)}$ & $\begin{array}{c}\left(10^{6}\right) \\
\text { Elflap }\end{array}$ & $\begin{array}{c}\left(10^{6}\right) \\
\text { Ellag }\end{array}$ & $\left(\begin{array}{c}* 10^{6} \\
G\end{array}\right)$ & $\begin{array}{l}\text { (in.) } \\
Y_{0 g}\end{array}$ & $\begin{array}{c}(\text { in. }) \\
\text { YEA }\end{array}$ & $\log$ \\
\hline Root & $\begin{array}{r}11 \\
10 \\
9 \\
8 \\
7 \\
6 \\
5 \\
4 \\
3 \\
2 \\
1\end{array}$ & $\begin{array}{l}0 \\
.1 \\
.2 \\
.3 \\
.4 \\
.5 \\
.6 \\
.7 \\
.8 \\
.9 \\
1.0\end{array}$ & $\begin{array}{c}0 \\
19.2 \\
38.4 \\
57.6 \\
76.8 \\
96.0 \\
115.2 \\
134.4 \\
153.6 \\
172.8 \\
192.0\end{array}$ & $\begin{array}{l}.32 \\
.32 \\
.32 \\
.426 \\
.426 \\
.426 \\
.426 \\
.426 \\
.426 \\
.426 \\
.426\end{array}$ & $\begin{array}{l}1.37 \\
1.37 \\
1.37 \\
5.867 \\
5.867 \\
5.867 \\
5.867 \\
5.867 \\
5.867 \\
5.867 \\
5.867\end{array}$ & $\begin{array}{l}137 \\
137 \\
137 \\
324 \\
324 \\
324 \\
324 \\
324 \\
324 \\
324 \\
324\end{array}$ & $\begin{array}{l}0.24 \\
0.24 \\
0.24 \\
5.58 \\
5.58 \\
5.58 \\
5.58 \\
5.58 \\
5.58 \\
5.58 \\
5.58\end{array}$ & $\begin{array}{l}5.50 \\
5.50 \\
5.50 \\
4.69 \\
4.69 \\
4.69 \\
4.69 \\
4.69 \\
4.69 \\
4.69 \\
4.69\end{array}$ & $\begin{array}{l}5.50 \\
5.50 \\
5.50 \\
6.93 \\
6.93 \\
6.93 \\
6.93 \\
6.93 \\
6.93 \\
6.93 \\
6.93\end{array}$ & $\begin{array}{l}1.74 \\
1.74 \\
1.74 \\
8.38 \\
8.38 \\
8.38 \\
8.38 \\
8.38 \\
8.38 \\
8.38 \\
8.38\end{array}$ \\
\hline
\end{tabular}

\section{3: $\quad$ WOOD-EPOXY BLADE EXAMPLE: GOUGEON ESI 54 BLADE}

The ESI/Gougeon blade was chosen for this study for the following reasons:

a. The blade is of composite wood construction, with good material and arrangement quality control and very good documentation by the manufacturer.

b. The airfoil is NACA 230XX series, used for helicopter rotors and other wind turbines, and has substantial available test data.

c. Field test data were available and accurate.

d. Compared to other composite wood blades, this blade has relatively high torsional stiffness but still measurable elastic twist.

e. The root attachment is indicative of a standard teetering approach with high stiffness and potential for pitch/flap coupling.

f. The blade radius is midway between the 10- and 20-meter diameters of the other blades in the test program.

g. The blade planform represents a "tip speed ratio tolerant" design, with slight taper and twist, indicative of most constant RPM wind turbines.

h. Tip brake/weight complicates the loads analysis for both structural and aerodynamic loads and was thought to be responsible for possible elastic twist observed in the field.

i. Because this blade uses a low pitching moment airfoil, inertial elastic twist (especially from the tip brake) is likely to dominate the results.

Note: this prediction turned out to be true.

\subsection{1: ESI BLADE DESCRIPTION}

The ESI 54 blade, which is manufactured by Gougeon Brothers inc. (Bay City, Mich.) is shown in Fig 1.2. It is of a wood-epoxy construction, and uses a web-reinforced shell with constant skin thickness at each span station and no D-spar. Also, it is not highly twisted and has no ballast weights or mechanisms. And probably most important, it has no structural discontinuities as do both the UTRC and Carter 300 blades. The root fixture is an oval bolted-stud pattern of relatively large dimension, so the root fixity in the test rig was relatively rigid. The blade was much stiffer then the Carter, and there was consequently much less static gravity deflection, on the order of a few inches only, which simplified the testing. The blade was tested and analyzed bare without the tip flaps which were used in the ESI 54 turbine. The elastic twist due to the tip mechanisms, as seen later dominates this blade's elastic twist.

The manufacturer, GBI, was very cooperative, and provided a complete engineering description of this blade, along with drawings, section coordinates, section properties, and analyses (Ref. 21). These data are held proprietary to $\mathrm{GBI}$, but the comparisons of the section properties are presented since they are 
important. Clearly this test program verified both AEl's and GBI's section analysis programs, with the exception of the shear center calculation(see below).

\subsection{2: DERIVE INITIAL SECTION PROPERTIES}

The section property code input file for this blade is much easier than for a glass-reinforced composite blade. Geometrically, the only values needed for each station are the offsets (airfoil shape), skin thickness, and web values. This blade uses a modified NACA 230XX airfoil, so the standard profiles could not be used. Instead the GBI specifications were verified by sectioning, and these actual values were used.

The engineering moduli for Douglas fir vary over a rather wide range; however, the constituents and methods used by $\mathrm{GBI}$ are reliable and repeatable. Therefore, we had high confidence in the initial design spec:

$$
\begin{array}{ll}
E_{\text {grain }} & =1.57 \cdot 10^{6} \mathrm{psi} \\
\mathrm{G} & =0.15 \cdot 10^{6} \mathrm{psi} \\
\rho & =0.025 \mathrm{lb} / \mathrm{in}^{3}
\end{array}
$$

It remains to find the skin thickness and web location for each blade station, either from the specs or from the tests, and then to calculate the section properties. The much more effective engineering approach factors in the test results from the very beginning, as illustrated here.

The blade inspection (sectioning) yielded measured values of running mass, section $\mathrm{Cg}$ location, shear web location, and skin thickness. These values plus the estimated moduli and density (above) provided a set of section properties calculated by AEI (Figs. 5.6 to 5.11). As can be seen, the two predictions ( $A E I$ and $G B I)$ are in close agreement, with the notable exceptions of the shear center and GJ (which are discussed further below). Clearly the GBI program has included the contribution of the plywood web differently (and as shown below, more accurately) than the AEI program. It remains to validate all the section data by comparison with flexural and dynamic test data.

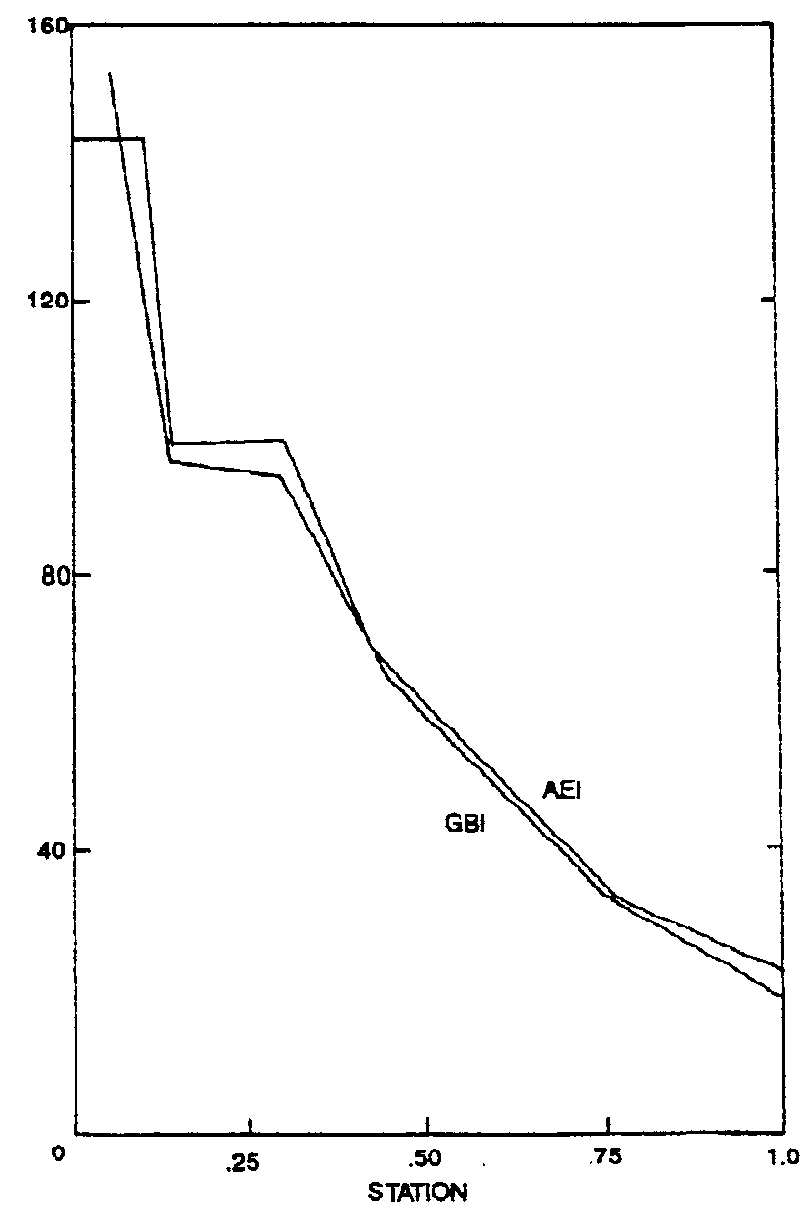

FIG. 5.6 ESI SECTION EA: AEI CALCULATION VS. GBI SPECIFICATION 


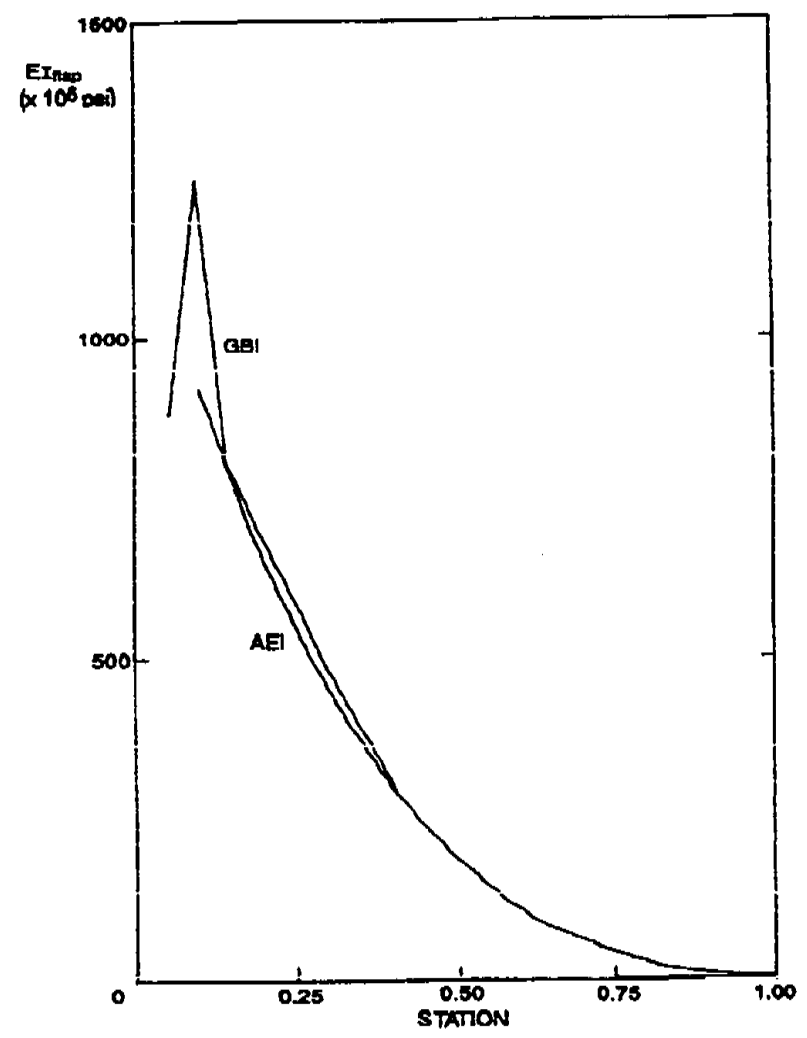

FIG. 5.7 ESI SECTION EIFLAP: AEI CALCULATION VS. GBI SPECIFICATION

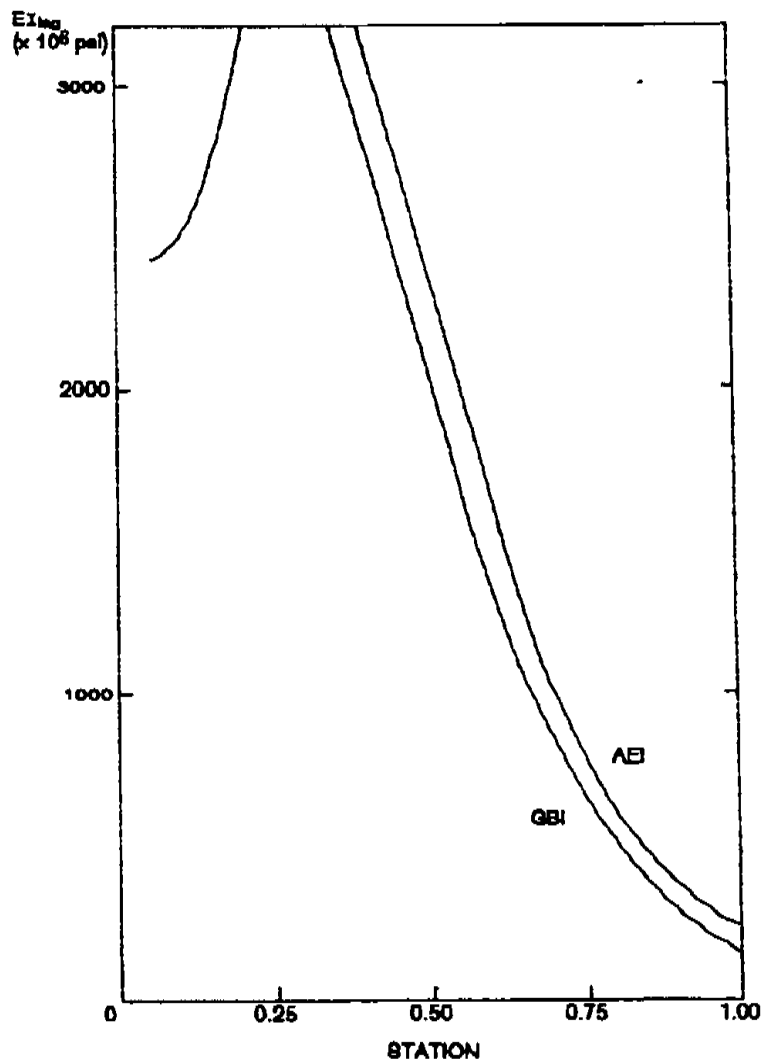

FIG. 5.8 ESI SECTION EILAG,: AEI CALCULATION VS. GBI SPECIFICATION 


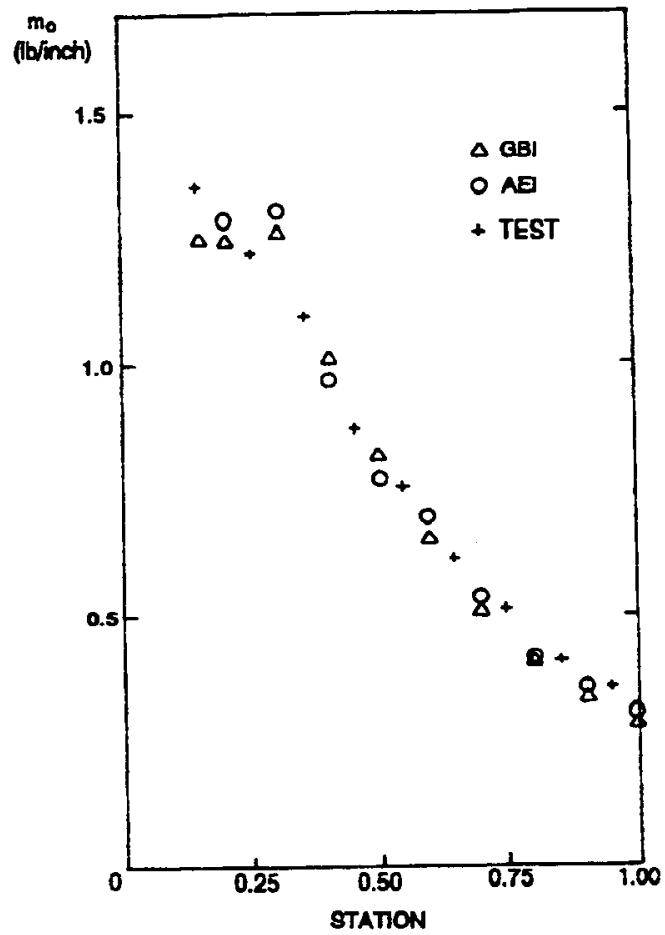

FIG. 5.9 ESI BLADE RUNNING MASS: AEI CALCULATION VS. GBI SPECIFICATION

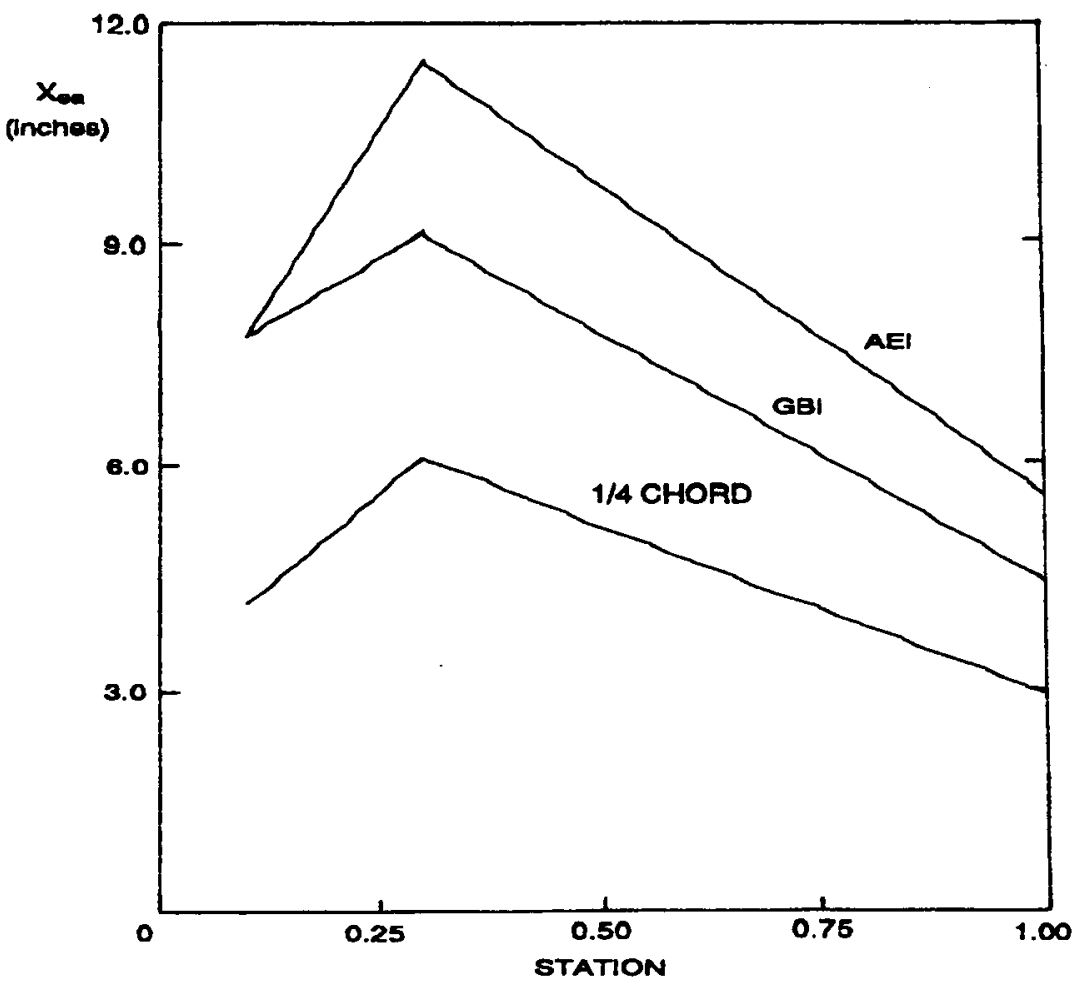

FIG. 5.10 ESI SECTION SHEAR CENTER: AEI CALCULATION VS. GBI SPECIFICATION 


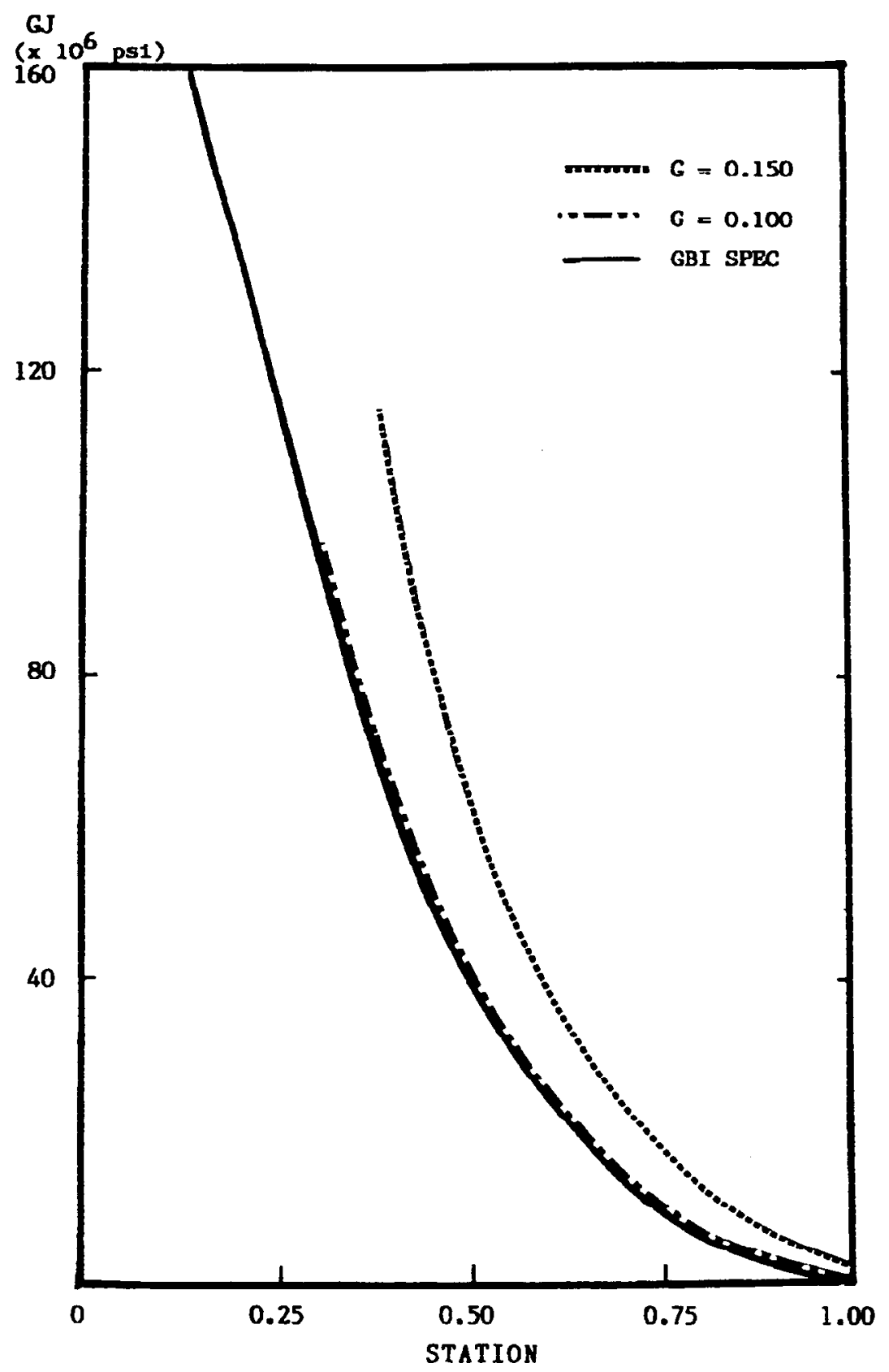

FIG. 5.11 ESI SECTION GJ: AEI CALCULATION VS. GBI SPECIFICATION 


\subsection{3: VERIFICATION BY FLEXURAL TESTS}

The ESI blade flexural data (e.g., deflections vs. load) were different than for the UTRC blade. First, the ESI blade was so stiff in torsion, owing to the large wall thickness for the wood epoxy blade, that practically no measurable $\left( \pm 6^{\prime}\right.$ arc) elastic twist resulted from the bending, even for $450 \mathrm{lb}$. of distributed load (tip deflection of 5 in.). Second, the flap deflection values had to be corrected for the root elasticity; that is, when the blade was loaded, the steel root studs stretched allowing the blade root slope to change. This slope change amounted to 7 minutes of arc at $225 \mathrm{lb}$ load and 16 minutes of arc at $450 \mathrm{lb}$. (Note: this introduced substantial tip errors of $0.66 \mathrm{in.}$ and $1.51 \mathrm{in}$. in the raw distributions.)

The corrected (and uncorrected) blade deflection is compared to both the AEI and GBI section predictions in Figure 5.12. The agreement is very good especially at the tip where it is most important. Therefore, the original input values were considered to be correct for this blade in flexure.

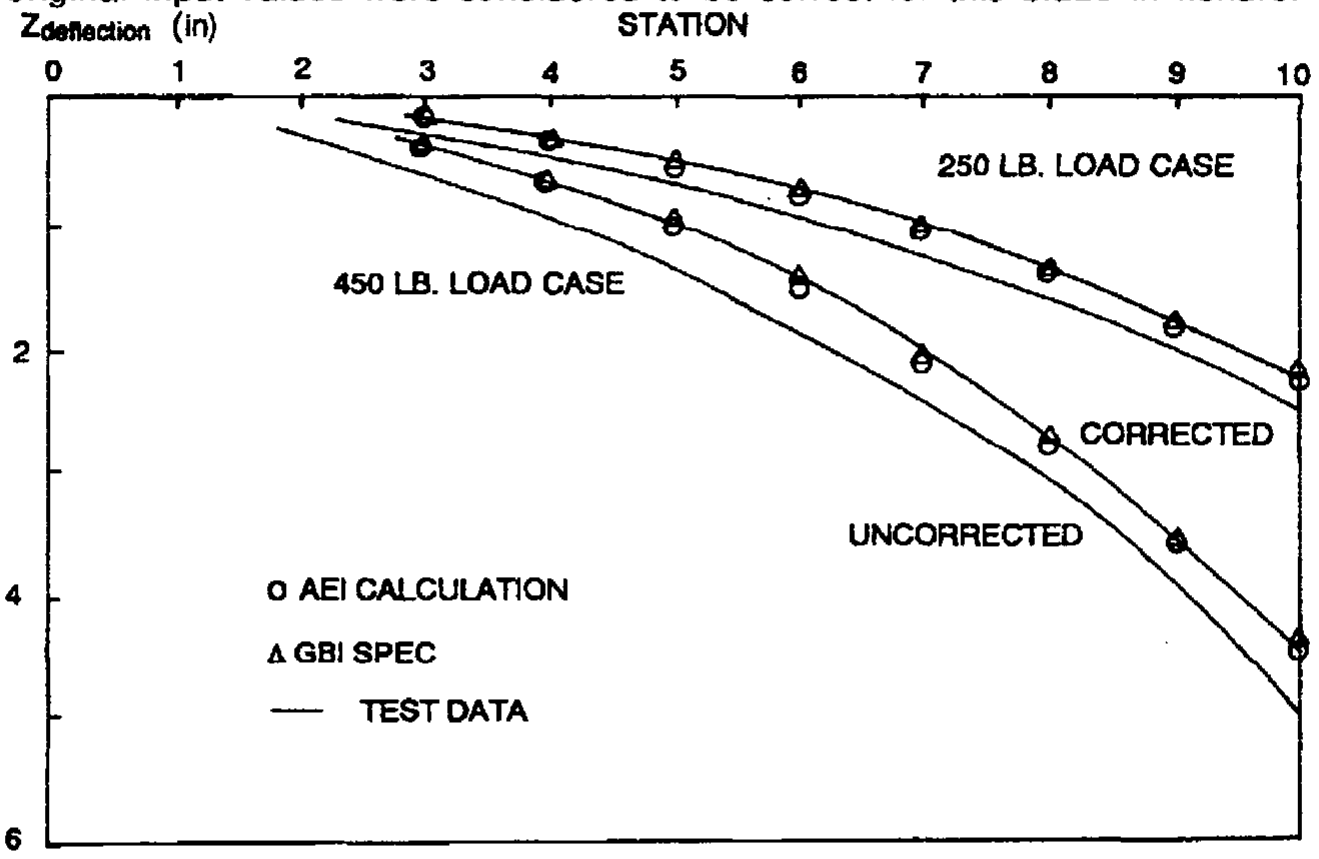

FIG. 5.12 ESI BLADE DEFLECTION: PREDICTIONS VS. TEST

\subsection{4: VERIFICATION OF STATIC TWIST}

The static twist of the distributed load cases $(225 \mathrm{lb}, 450 \mathrm{lb}$ ) was too small to be useful (see Fig 5.13). The torsion load case applied $50 \mathrm{lb}$. concentrated load at STA 0.9 of the test blade, offset 12.4" forward of the blade quarterchord. The test result is shown in Figure 5.14; it is a small but very believable distribution with an accuracy at least as good as $0.1 \mathrm{deg}\left(6^{\prime}\right.$ of arc). Therefore, there was good confidence in both test results, even though the amplitudes were very low. 


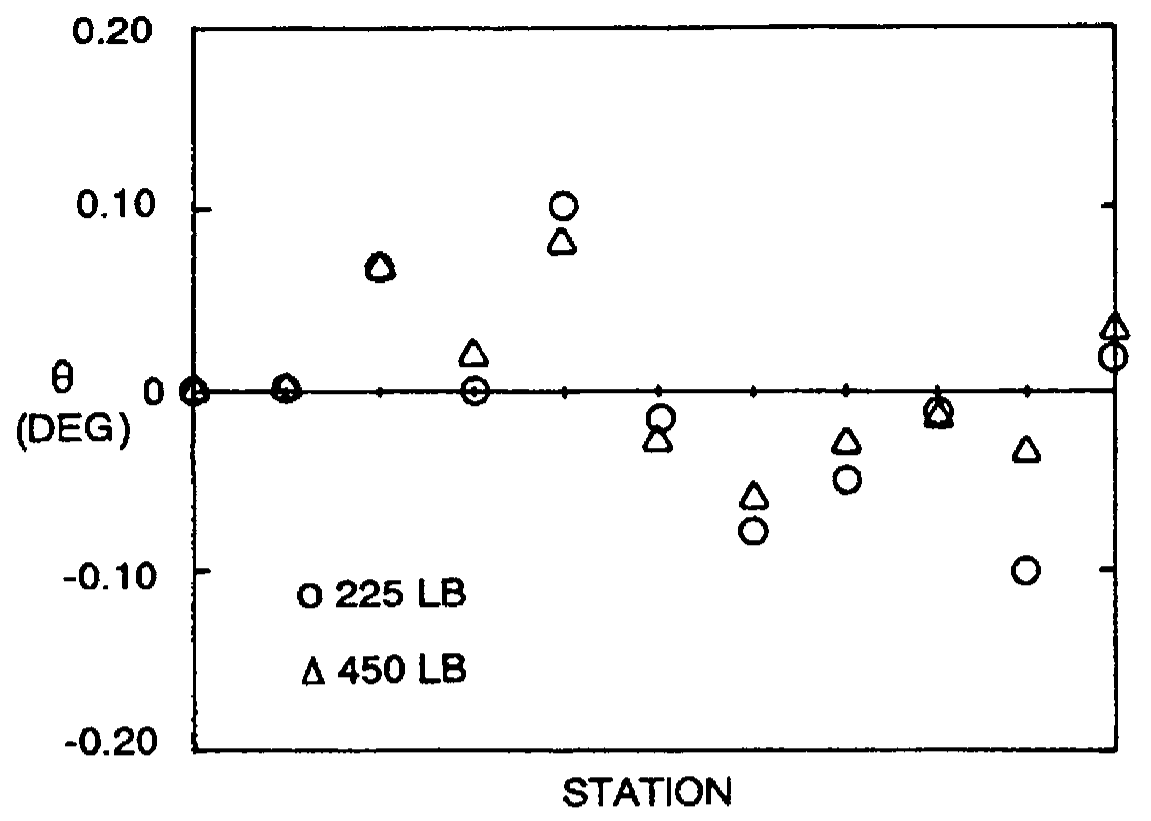

FIG. 5.13 ESI BLADE STATIC TWIST

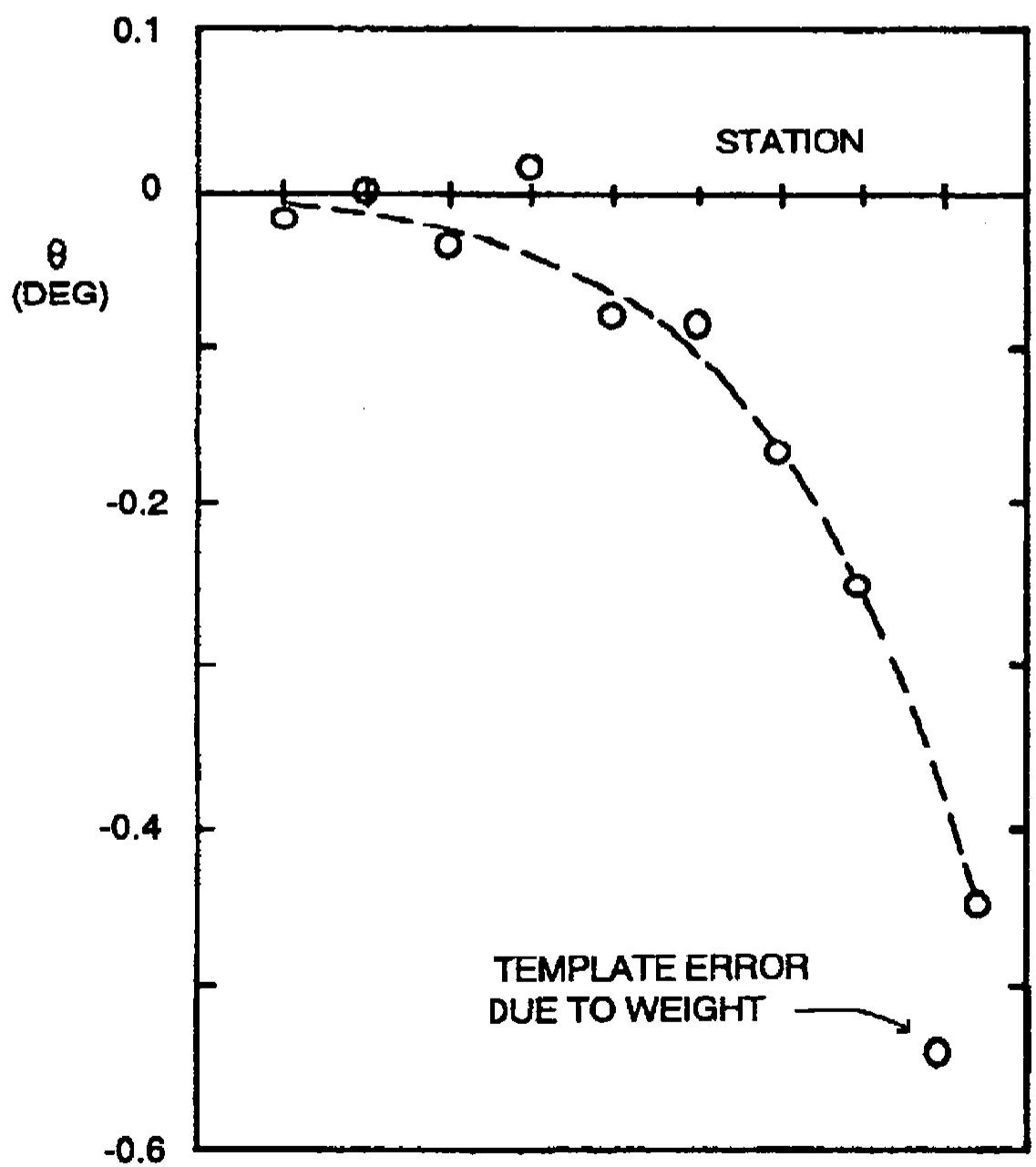

FIG. 5.14 ESI BLADE STATIC TWIST (50 lb TORSION LOAD CASE)

The original distributions are shown in the GJ calculation in Figure 5.11; the AEI prediction used $G=0.15$. Lowering $G$ to 0.10 gives the final GJ, which agrees well with the GBI specification. However, when these two distributions are used to predict the static torsion above, it can be seen in Figure 5.15 that the tip twist varies by about $15 \%$, even though both distributions are close to the test values. The blade GJ can not 
be considered to be verified yet though, since the load case was only a single static moment applied to the quarterchord. [As seen before for the UTRC blade, the torsional frequency is a much more accurate determinant of GJ].

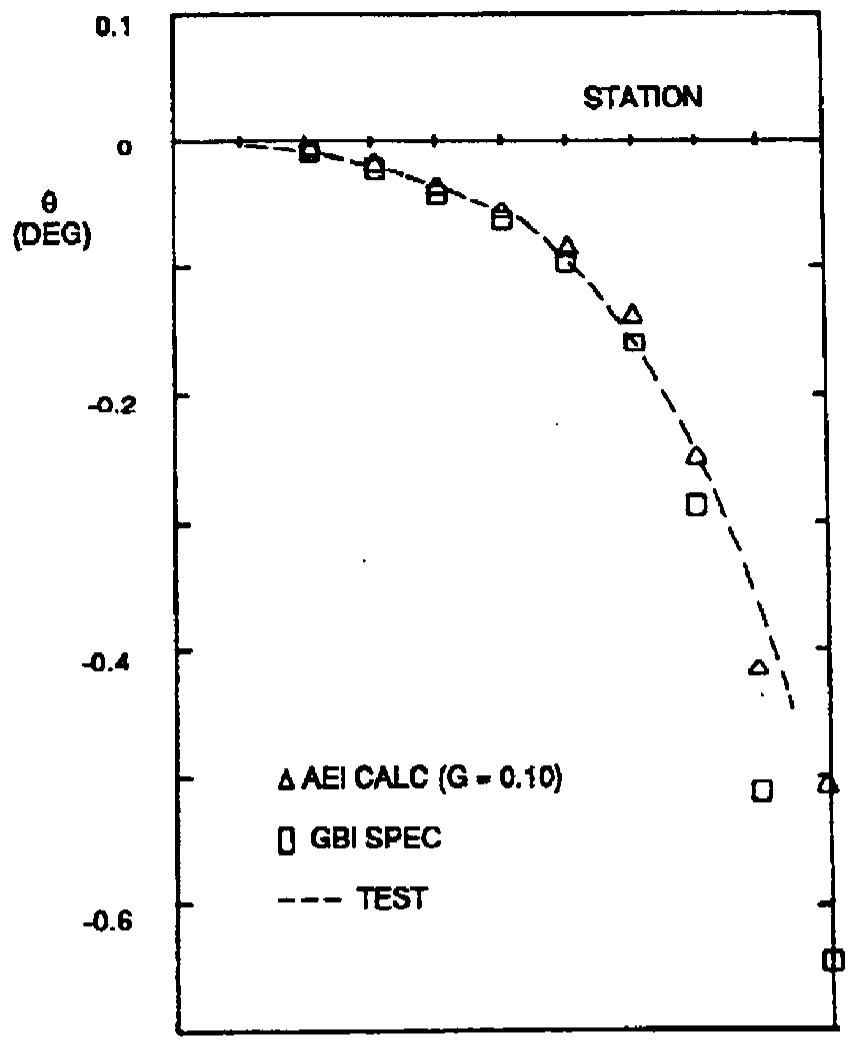

FIG. 5.15 ESI BLADE STATIC TWIST: PREDICTIONS VS. TEST

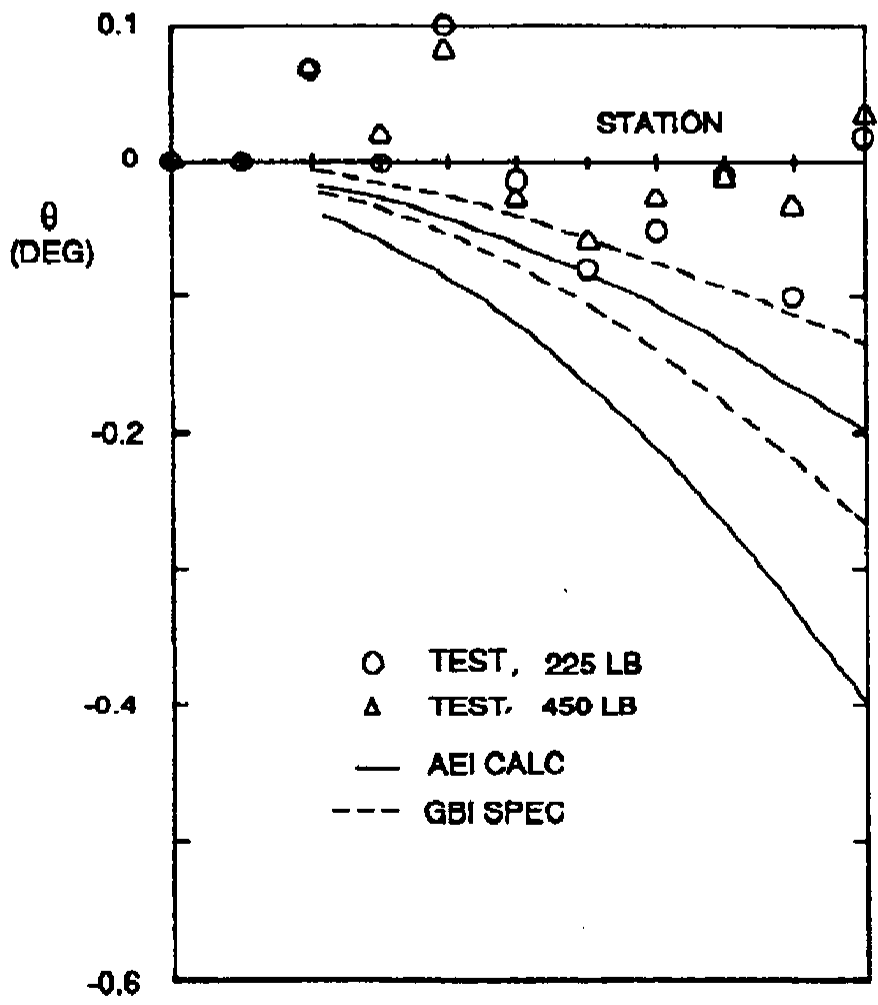

FIG. 5.16 ESI BLADE TWIST: PREDICTIONS VS. TEST (FLEXURE LOAD CASES) 
A further check on twist can be made, to compare the twist of the dead load cases and "bracket" the solution. These are shown overlaid with the test results in Figure 5.16; clearly, they do not match the test data. Since there is high confidence in the above range $(0.1-0.15)$ of $\mathrm{GJ}$, the error must be in the predictions of shear center (both $\mathrm{AEl}$ and $\mathrm{GBI}$ ). These predictions are for an aft shear center (see Fig 5.10) which produces a section moment when loaded at the quarterchord. The error in the predicted twist in Figure 5.16 is attributable to the difference in shear center prediction in Figure 5.10, as described here:

The test data clearly show no effective elastic twist of the ESI blade when loaded at the quarterchord (See Fig 5.16). This is the requirement for the definition of shear center. Therefore, it must be concluded that the actual shear center of the blade is the quarterchord and both predictions are in slight error. Thus for the blade aeroelastic model, an empirical value for shear center must be used: when this value is input for the two high load cases the resulting twist is zero, as in the test data. This illustrates the necessity of doing both static tests; flexural and torsional, otherwise this important result would have been overlooked.

\subsection{5: VERIFICATION BY FREQUENCY TEST}

The total blade weight was measured and predicted to be $260 \mathrm{lb}$., which lends credence to the calculated mass distributions. The first three flap bending modes and the first torsion mode are shown in Figure 5.17. The frequency results are shown in Table 5.4 for the test, the GBI specifications, and the AEI calculation.
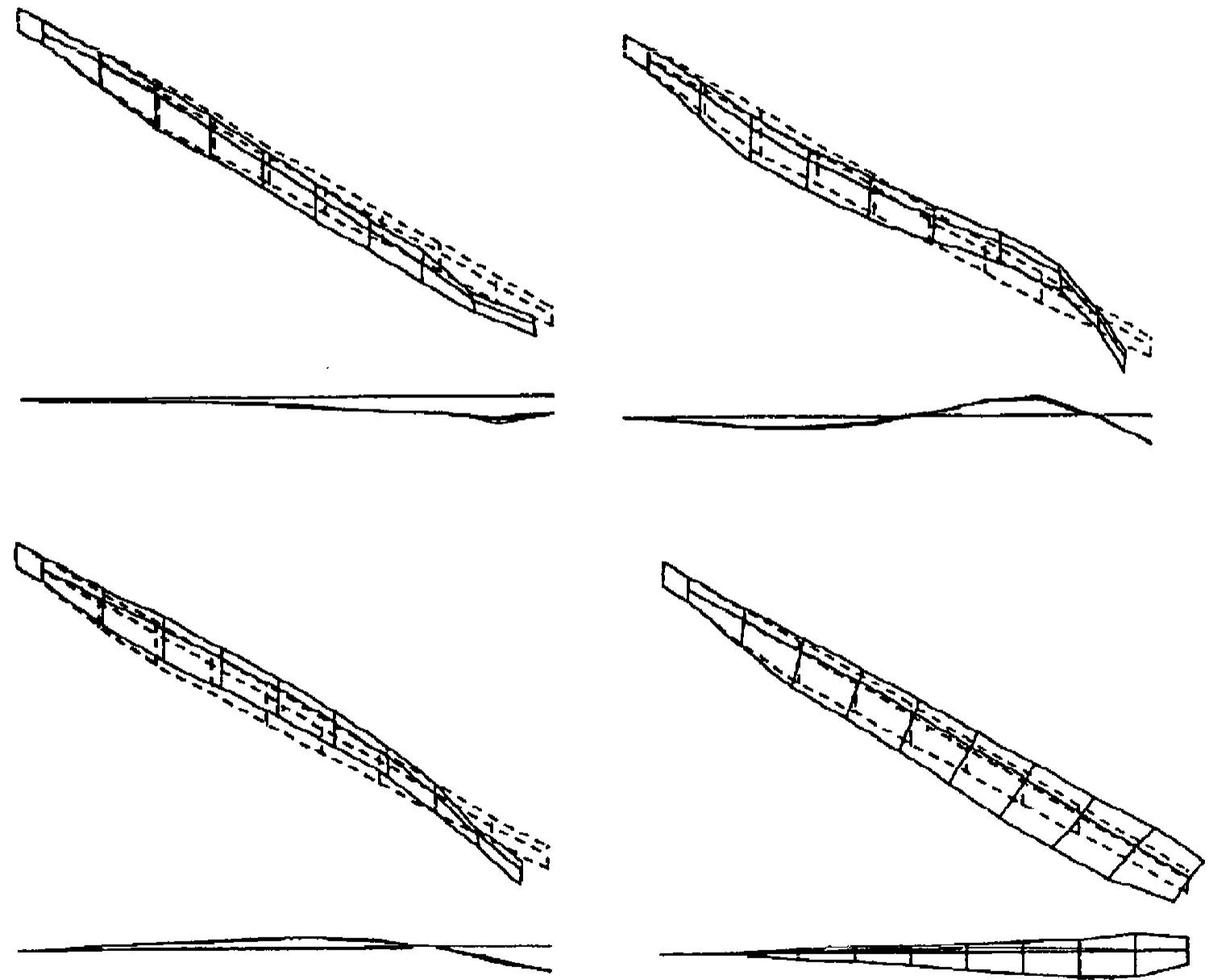

$\begin{array}{lr}\text { 1st FLAP } & 3.5 \mathrm{~Hz} \\ \text { 2nd FLAP } & 11.0 \mathrm{~Hz}\end{array}$

3rd FLAP $\quad 25.0 \mathrm{~Hz}$ 1st TORSION $40.5 \mathrm{~Hz}$

FIG.5.17 ESI BLADE FREQUENCY MODES 


\section{TABLE 5.4 ESI BLADE FREQUENCIES OF VIBRATION: PREDICTION VS. TEST}

\begin{tabular}{|c|c|c|c|}
\hline Mode & $\begin{array}{c}\text { Test } \\
\text { Frequency }\end{array}$ & $\begin{array}{c}A E I \\
\text { Prediction }\end{array}$ & $\begin{array}{c}\text { GBI } \\
\text { Prediction }\end{array}$ \\
\hline $\begin{array}{l}\text { 1st flap } \\
\text { 2nd flap } \\
\text { 3rd flap }\end{array}$ & $\begin{array}{l}3.5 \mathrm{~Hz} \\
11.0 \\
25.0\end{array}$ & $\begin{array}{c}3.2-4.0 \mathrm{~Hz} \\
10.2-12.4 \\
22.8-27.5\end{array}$ & $\begin{array}{l}3.4 \mathrm{~Hz} \\
10.2 \\
23.0\end{array}$ \\
\hline $\begin{array}{l}1 \text { st lag } \\
\text { 2nd lag } \\
3 \text { rd lag }\end{array}$ & $\begin{array}{r}8.0 \\
33.0 \\
80.0\end{array}$ & $\begin{array}{r}9.2 \\
35.0 \\
88.0\end{array}$ & $\begin{array}{r}8.2 \\
31.4 \\
81.0\end{array}$ \\
\hline $\begin{array}{l}\text { 1st torsion } \\
2 \text { nd torsion }\end{array}$ & $\begin{array}{l}40.5 \\
68.0\end{array}$ & $\begin{array}{l}35.1(40.5) \\
60.8(70.0)\end{array}$ & $\begin{array}{l}32.6 \\
54.0\end{array}$ \\
\hline
\end{tabular}

NOTES: (1) The range of the AEI predictions indicates the limits from the true flap and lag directions to the principal axis direction.

(2) Torsional frequencies in parentheses indicate $G$ modification $\left(0.100\right.$ to $\left.0.133{ }^{*} 10^{6}\right)$.

The range in the AEI predictions derives from taking the EI values in the true lag and flap directions or in the principal axis direction which yield a softer "flapwise" frequency. The blade is not highly twisted (8 deg) so the principal axes are definable in blade terms. Also from Table 5.4, since the flap test frequencies are higher than the GBl frequencies, it can be concluded that the GBI values are conservative. For lead-lag the same is probably true since non-ideal root fixity usually depresses the lag frequency test data. This effect is clear with the AEI predictions, but not the GBI values. This also illustrates how sensitive the frequencies are to EI values; Figure 5.7 would not lead one to suspect such a large variation in frequency.

The torsion values clearly show the predicted GJ's to be too low. Recall that these GJ's were based on the static twist results $(G=0.10)$ and very low values of twist $(<18$ minutes of arc). To bring the calculated frequencies up to the test values (shown in parentheses), the value of $G$ was increased from 0.100 to 0.133 . This is understandable since the original prediction was 0.150 . The resulting final GJ is shown in Figure 5.18. This value of GJ matches the static data less well (but still within the experimental accuracy of $\pm 0.1^{\circ}$ ), but the dynamic data are matched exactly; this is more believable since fixity problems are eliminated. 


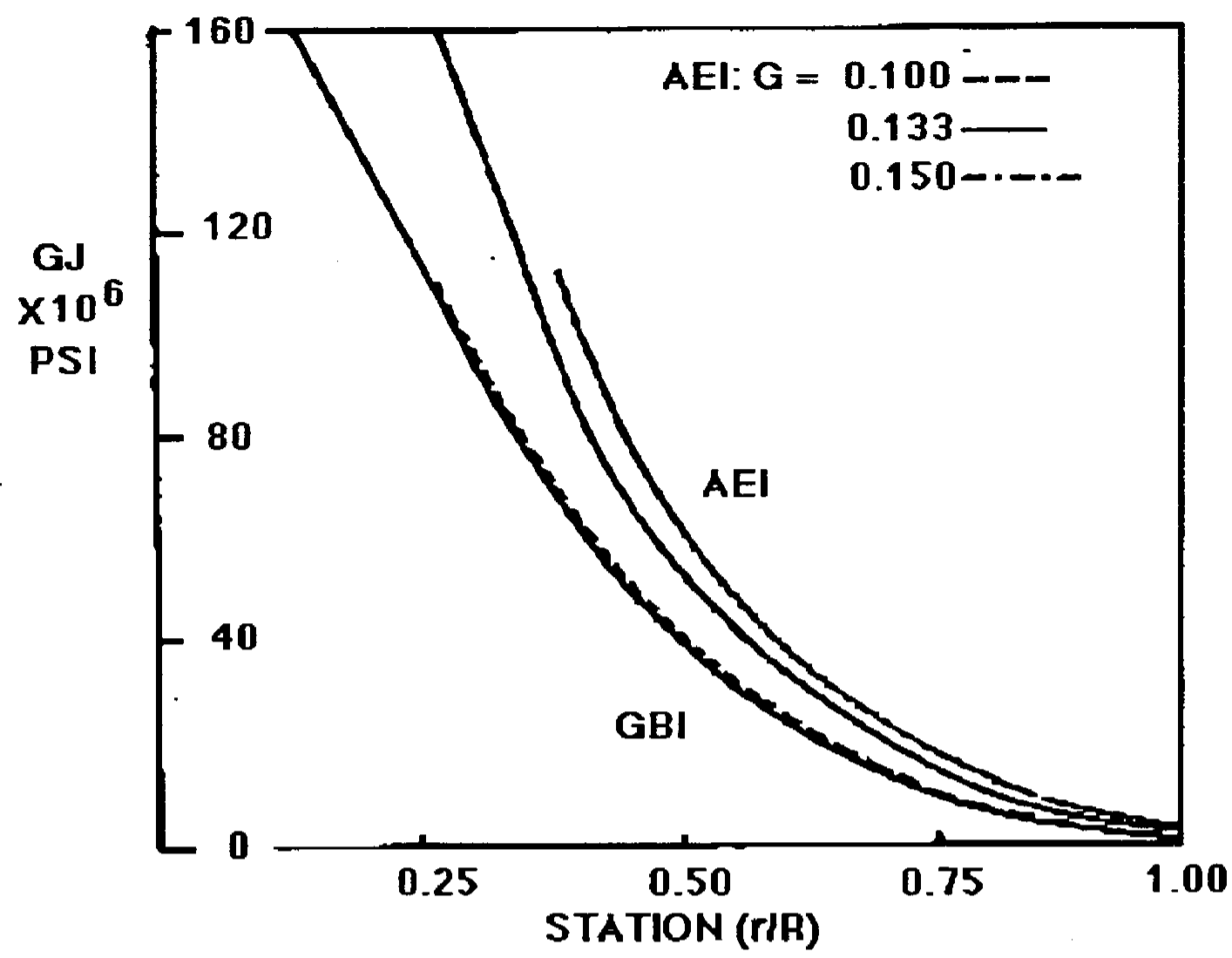

FIG. 5.18 ESI BLADE: FINAL GJ VALUES

\subsection{6: FINAL AEROELASTIC MODEL}

The final values of the ESI aeroelastic blade model are shown in Table 5.5. The blade twist was determined by geometric calculation and verified by measurement.

TABLE 5.5: ESI BLADE: FINAL AEROELASTIC MODEL

\begin{tabular}{|c|c|c|c|c|c|c|c|c|c|c|c|}
\hline & STA & $r / R$ & $\begin{array}{c}\text { (in.) } \\
\text { r }\end{array}$ & $\begin{array}{c}(\mathrm{deg}) \\
\beta_{0}\end{array}$ & $\begin{array}{c}\text { (slugs/in.) } \\
m_{0}\end{array}$ & $\begin{array}{l}10^{6} \text { psi } \\
\text { El flap }\end{array}$ & $\begin{array}{c}10^{6} \text { psi } \\
\text { Ellag }\end{array}$ & $\begin{array}{c}10^{6} \mathrm{psi} \\
\mathrm{GJ}\end{array}$ & $\underset{Y_{\mathscr{g}}}{(i n .)}$ & $\begin{array}{l}(\text { in.) } \\
\text { YEA }_{\text {EA }}\end{array}$ & $\operatorname{Iog}$ \\
\hline Root & 11 & 0 & 0 & 0 & .07823 & 941.0 & 4259 & 349.3 & 7.63 & 4.20 & 67.76 \\
\hline & 10 & .1 & 32.4 & 2.13 & .07823 & 941.0 & 4259 & 349.3 & 7.63 & 4.20 & 67.76 \\
\hline & 9 & .2 & 64.8 & 2.13 & .04012 & 607.4 & 3736 & 166.8 & 9.67 & 5.21 & 56.6 \\
\hline & 8 & .3 & 97.2 & 2.13 & .04084 & 520.4 & 5204 & 152.3 & 11.48 & 6.10 & 74.6 \\
\hline & 7 & .4 & 129.6 & 2.45 & .03021 & 303.7 & 3327 & 86.8 & 10.70 & 5.68 & 47.3 \\
\hline & 6 & .5 & 162.0 & 2.87 & .02417 & 185.0 & 2233 & 53.3 & 9.85 & 5.22 & 31.5 \\
\hline & 5 & .6 & 194.4 & 3.38 & .02175 & 116.8 & 1673 & 35.9 & 9.06 & 4.77 & 23.3 \\
\hline & 4 & .7 & 226.8 & 4.04 & .01679 & 81.0 & 1036 & 23.4 & 8.09 & 4.32 & 14.6 \\
\hline & 3 & .8 & 259.2 & 4.94 & .01291 & 30.4 & 614 & 9.4 & 7.21 & 3.86 & 8.4 \\
\hline & 2 & .9 & 291.7 & 6.12 & .01115 & 13.5 & 416 & 4.9 & 6.45 & 3.42 & 5.60 \\
\hline Tip & 1 & 1.0 & 324.1 & 8.18 & .00956 & 4.9 & 252 & 2.1 & 5.54 & 2.90 & 3.34 \\
\hline
\end{tabular}

\subsection{7: ESI TIP FLAPS}

The operational ESI blade had tip flaps that were attached to a mount (see Fig. 5.19 ) and had the function of centrifugal overspeed brake. There were two versions of the tips: the "regular tip" and the "whisper tip," the latter incorporating a fiberglass-foam aerodynamic fairing. From tests and inspections of the two tips, the weights and Cg's of the assemblies (e.g., brackets, bolts, swing arms, springs, and bash 
plates) were analyzed to correctly account for the added weight and inertia. Given here are free-body diagrams and the resulting values of the equipollent system, which gives the correct mass locations.
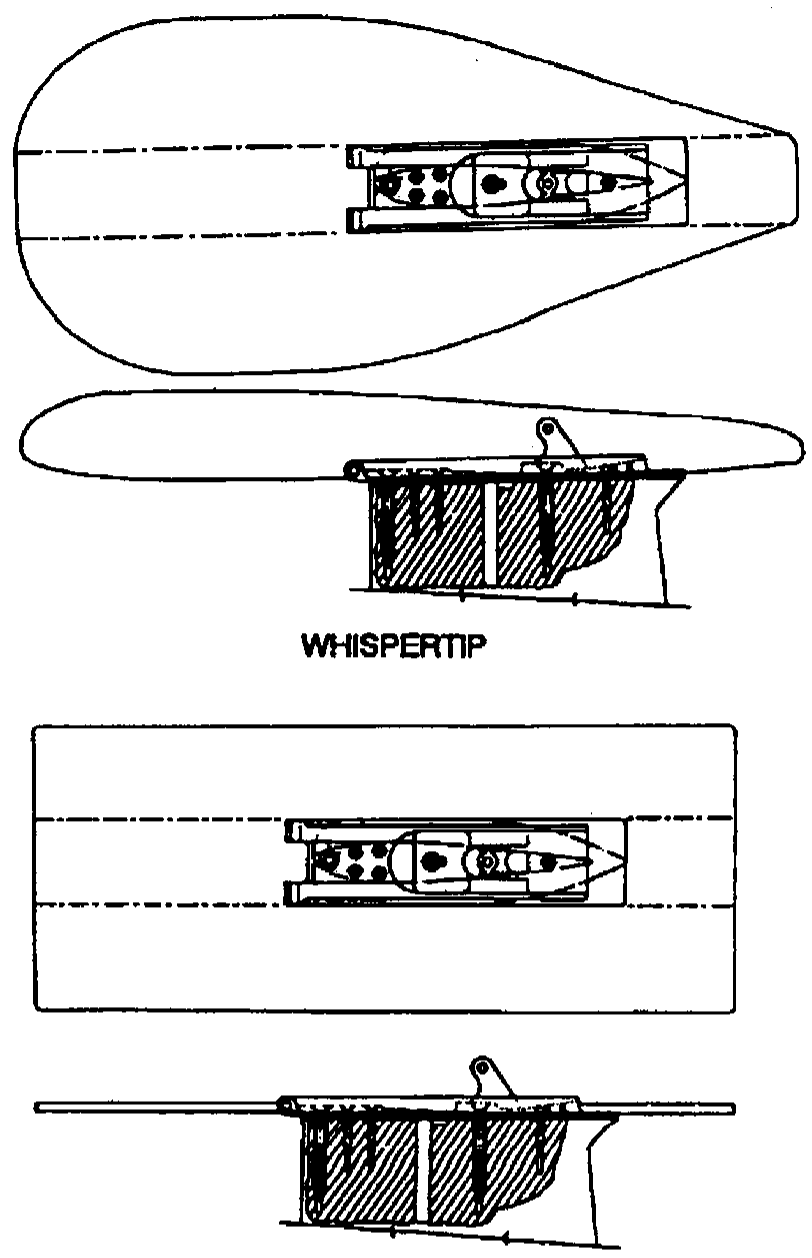

REGULAR TIP

FIG. 5.19 ESI TIP FLAPS 
Reqular Tip:
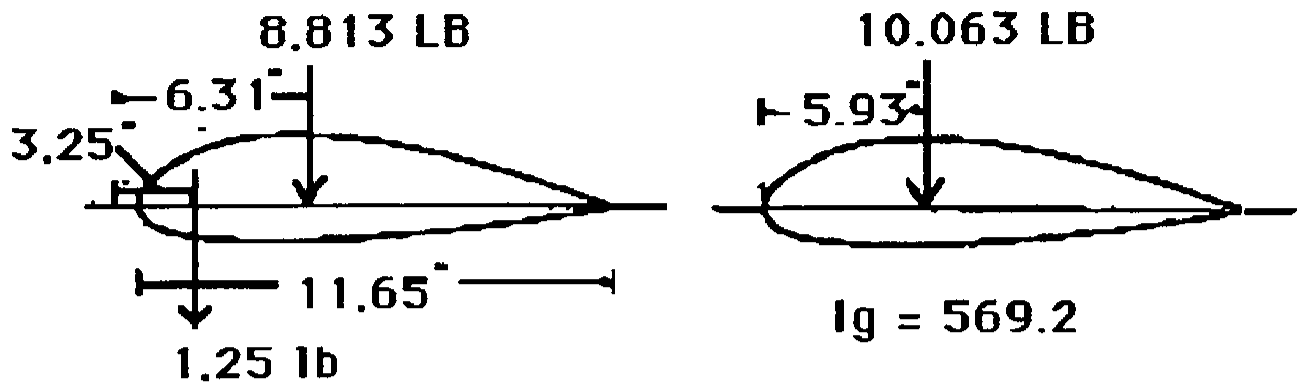

Whisper Tip:

\section{ACTUAL TIPS}

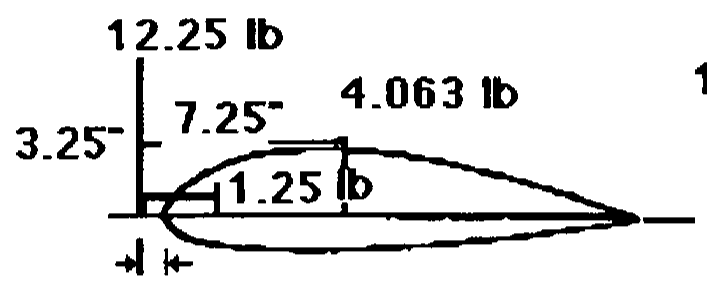

EQUIPOLLENT SYSTEM

$17.438 \mathrm{lb}$

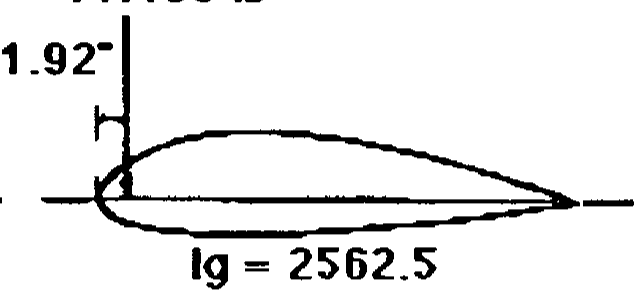

These are treated as tip ballast in the calculations of section properties in the elastic twist computer runs. These values were verified with some (unpublished) test data taken by ESI on the blades in the field and made available for this report (Ret. 26); Table 5.6 shows how the added tip weight decreases the flap frequency, and the added tip inertia greatly decreases the torsional frequency.

TABLE 5.6: ESI VIBRATION FREQUENCIES WITH TIPS (HZ)

\begin{tabular}{|c|c|c|c|c|}
\hline Mode & $\begin{array}{c}\text { No Tip } \\
\text { (Prediction) }\end{array}$ & $\begin{array}{c}\text { Regutar Tip } \\
\text { (Prediction) }\end{array}$ & $\begin{array}{l}\text { Whisper } \\
\text { Prediction }\end{array}$ & $\begin{array}{l}\text { Tip } \\
\text { Test }\end{array}$ \\
\hline $\begin{array}{l}\text { 1st flap } \\
\text { 1st torsion }\end{array}$ & $\begin{array}{l}3.5 \\
40.5\end{array}$ & $\begin{array}{r}2.69 \\
23.60\end{array}$ & $\begin{array}{r}2.41 \\
12.51\end{array}$ & $\begin{array}{r}2.40 \\
12.50\end{array}$ \\
\hline
\end{tabular}

\section{4: COMPLEX COMPOSITE FRP EXAMPLE: CARTER 300 BLADE}

The t0-meter FRP Carter blade was a good candidate for this study for the following reasons:

a. The relatively high tip speed, blade radius, and chord give high Reynolds number and power output.

b. The good quality control of the laminate molding and arrangement by the manufacturer yielded consistent material properties.

c. The relatively low bending stiffness led to measurable structural twist coupling (due to elastic axis offset from loading axis).

d. The large ballast mass added to the outboard blade caused substantial elastic twist.

e. The highly twisted, tapered planform is indicative of high-performance wind turbine rotors.

f. The LS(1) series airfoil was used; it is indicative of new wind turbine airfoil families, and possesses large aerodynamic pitching moment.

g. The root attachment is indicative of underslung, non-pitch/flap coupled teetering hub. 


\subsection{1: CARTER BLADE DESCRIPTION}

The Carter blade was manufactured by Carter Wind Systems (Burkburnett, Tex.); it has a composite shell with a reinforced leading edge (D-spar) plus webs (see Figs. 1.1 and 5.20 ). It has a radius of over $10 \mathrm{~m}(33.6 \mathrm{ft})$ and in a teetering rotor (2-bladed) configuration powers the Carter 300 $\mathrm{kW}$ wind turbine.

If the entire blade had a structure as in Figure 5.20 the structural analysis would proceed in the usual manner as for the above blades. However, the Carter blade incorporates an innovative internal pitching mechanism ("pitch-up snubber") which provides overspeed protection for the wind turbine by causing a pitch change (of roughly $14 \mathrm{deg}$ nose up) past airfoil stall, by the action of an electrically released toggle (see Figs. 3.5 and 5.21). The release of the toggle allows a preloaded overcenter spring in the root to produce a large mechanical control moment between the (moving) blade shell and the (stationary) root I-beam. The I-beam is a high quality (high glass/resin ratio) unidirectional beam comprising the principal loading path in the inner $35 \%$ of the blade as shown. By its I-beam section, it has relatively stiff bending properties and relatively soft torsional rigidity; thus it acts as a torsional spring against the control actuator. In operation, once the blade is pitched up, it is gradually returned to the running pitch position by a spring/damper actuator. This mechanism, unmodified, looks like a one-way spring damper to torsional motion of the blade, thereby drastically altering the fundamental torsional frequency of the blade and the torsional static deflections (via the relatively soft spring/damper). Consequently, for the testing this control arrangement was replaced by a rigid turnbuckle, so the blade could be set at the correct (run) pitch angle and the true blade structural statics and dynamics could be measured.

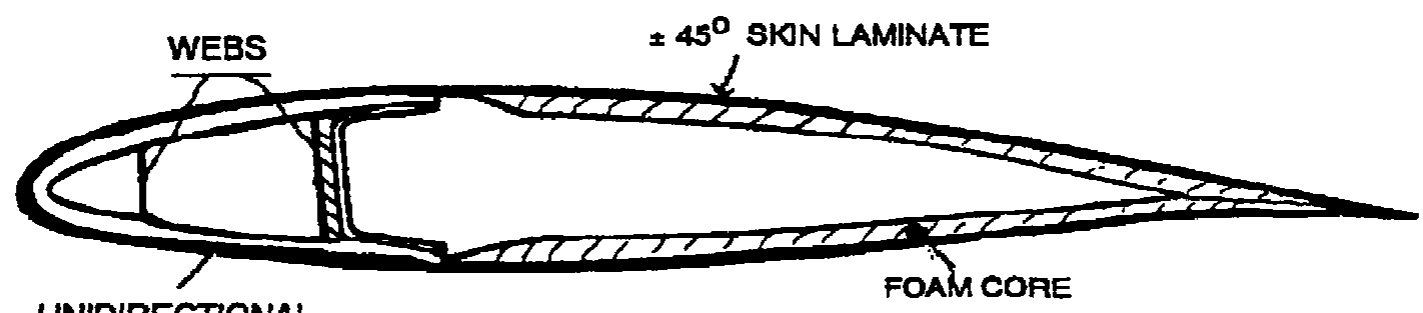

UNIDIRECTIONAL

REINFORCEMENT

FIG. 5.20 CARTER BLADE INTERNAL STRUCTURE
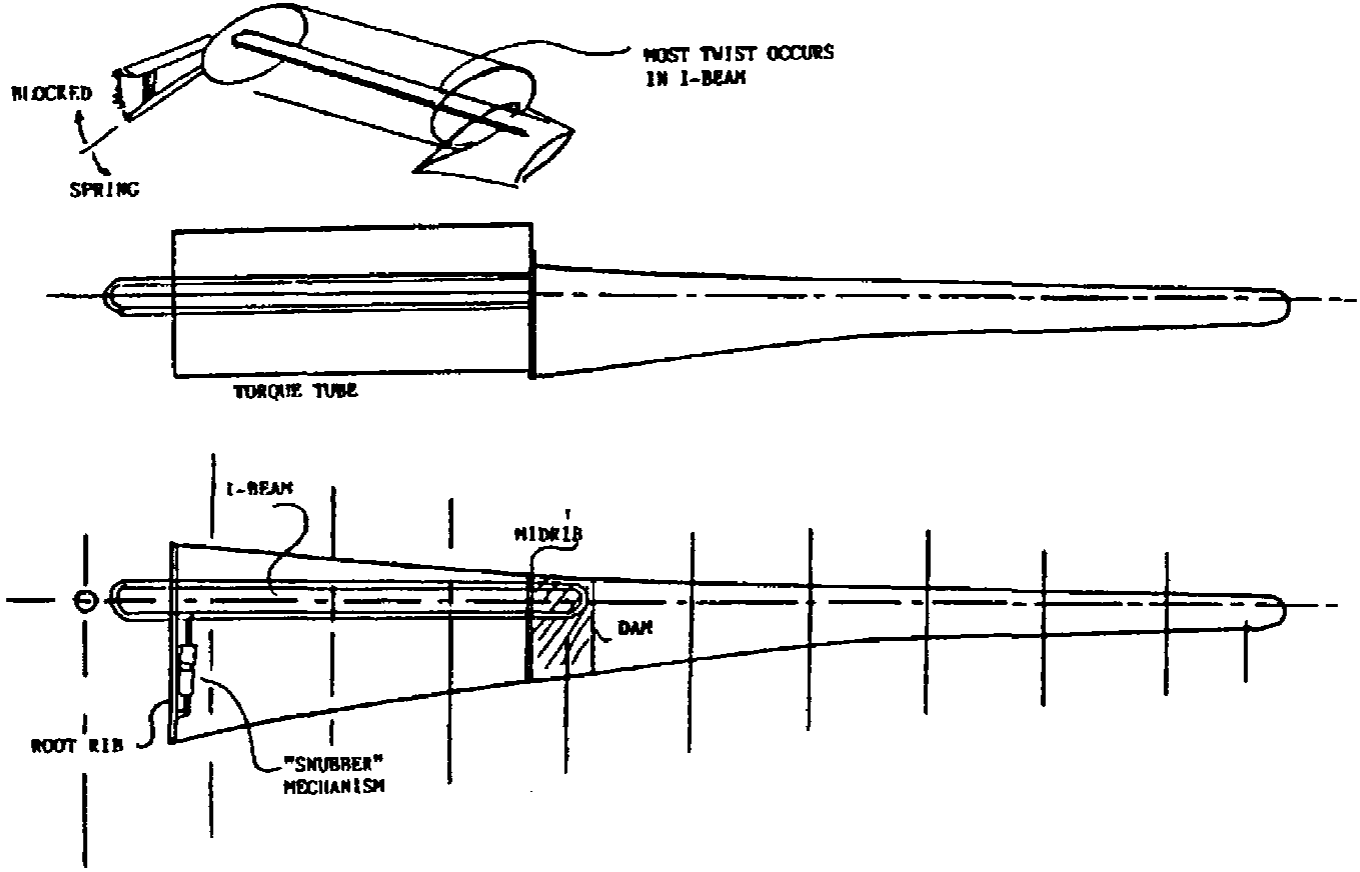

FIG. 5.21 CARTER BLADE FREE BODY DIAGRAM 
The free body diagram in torsion of this blade is also shown in Figure 5.21. The block/spring was replaced by the turnbuckle during testing. The inboard blade consists of a shell or "torque-tube" fixed around the I-beam, which is attached to the hub and a midrib at STA 0.37 radius, and "potted" there in an epoxy resin compound. When the blade is pitched, the twist occurs primarily in the (torsionally soft) Ibeam and very little in the torque tube. It was the goal of this project to establish the torsional and flexural properties of both working in concert. This mechanical arrangement, of course, greatly complicated the testing and analysis, but could be adequately handled in the computer programs as seen below.

The blade was further complicated by the addition of $138.8 \mathrm{lb}$ of lead weight ballast to the leading edge for mass balancing on the outboard third of the blade. This added weight (the basic unballasted blade with mechanisms was only $700 \mathrm{lb}$ ) caused a large static gravity deflection of about 30" when cantilevered, and also created a large section mass moment of inertia outboard. The latter doesn't affect the static elastic twist but does drastically modify the torsional frequencies and modes, as well as the flexural frequencies.

Further structural coupling occurs due to the geometry of the blade. Figure 1.4 illustrates in three views the control and blade axes. The blade shell is geometrically constructed (e.g., twisted and tapered) about the blade axis which is at about $40 \%$ chord; but the blade is fixed (root) and acts about the control axis which is at the quarterchord, since the I-beam is placed there. The mass axis (not shown) is also roughly at or ahead of the quarterchord, and is definitely not a straight line. The intermodal coupling is best illustrated in the frequency mode shapes in the modal survey testing part (Sec 5.4.10).

\subsection{2: $\quad$ ASCERTAIN BLADE GEOMETRY}

The blade data furnished by Carter Wind Systems were meager in comparison to that furnished by $\mathrm{GBI}$, in view of the information needed for this complex blade. The first task was to ascertain the actual blade twist, taper, and arrangement geometry.

The blade radius and chord lengths were found accurately by inspection, as was the thickness distribution. Finding the twist was complicated by the gravity component due to the ballast weight. This effect was eliminated by measuring the dead load twist for the blade in normal position and inverted position. The six individual dead load cases are given in Figure 5.22, and the resulting twist distribution in Figure 5.23; the Carter specification is also shown. Clearly the STA 0.8 template was about $1^{\circ}$ off (which could have been the operator error referred to in 3.9.1); but otherwise the twist measurements were very repeatable. Figure 5.24 is an error scatter plot and shows the variation in cases to be subject to only $0.1 \%$ experimental error.

The final blade geometry is given in Table 5.7 compared to the specifications. The actual blade length was only 0.75 "longer than the spec. (376.25 vs. 375.50 in.). As can be seen, the values agree extremely well, verifying the tooling and assembly method of the Carter 300 blade. [Note: blade sectioning is not actually required for this task, but is a great help; it is required for later verifications.] 


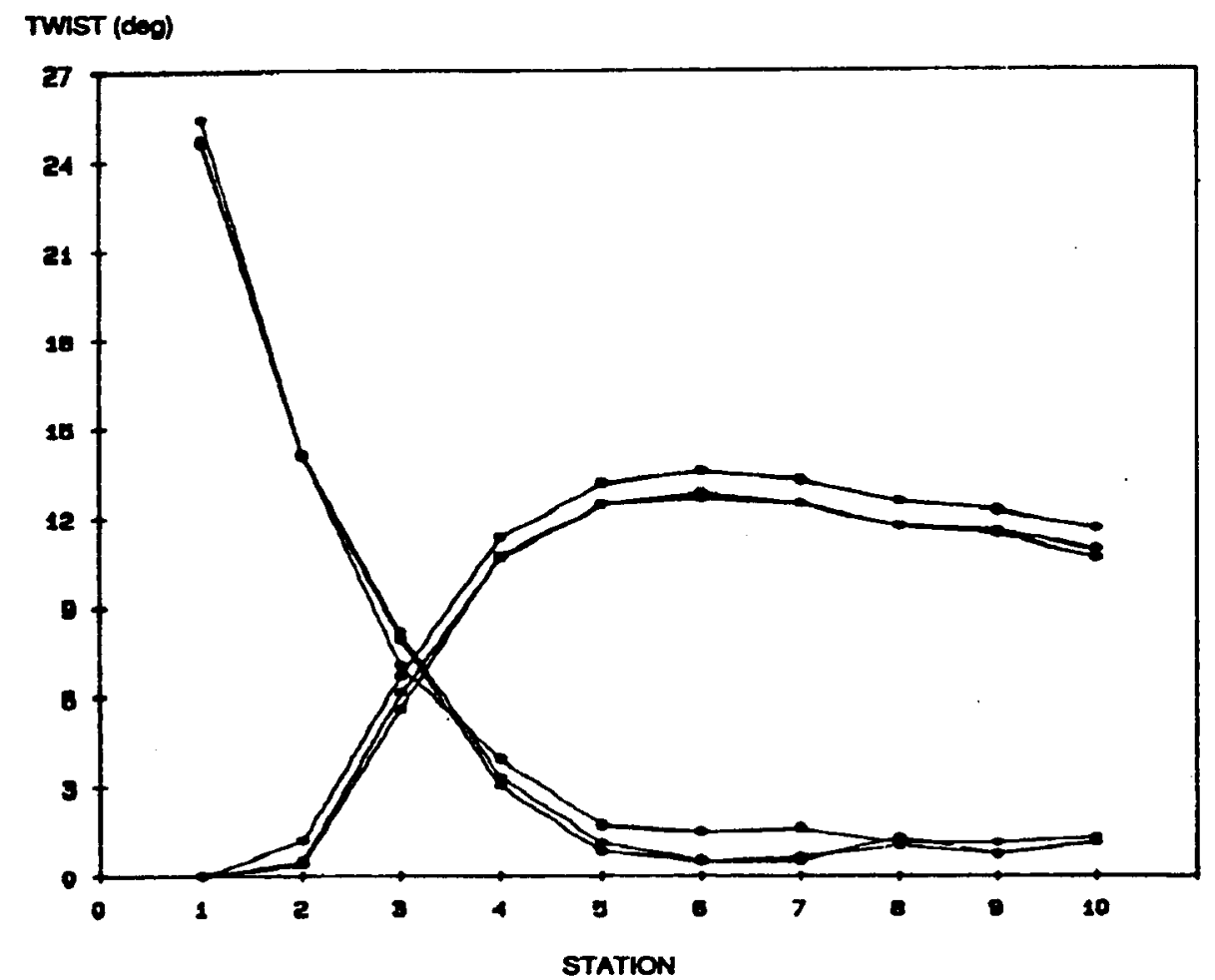

FIG. 5.22 CARTER DEAD LOAD TWIST

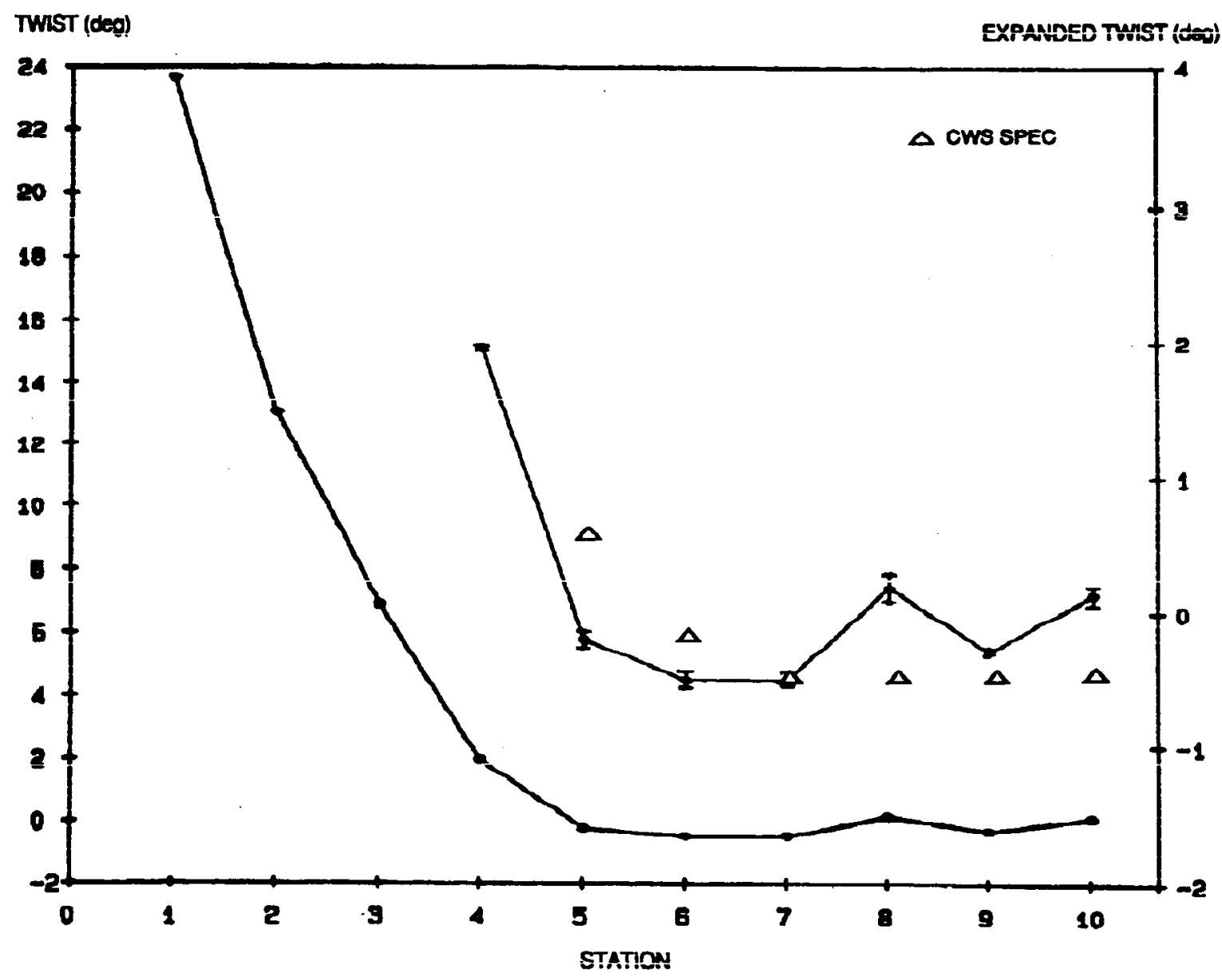

FIG. 5.23 CARTER BLADE: TWIST FROM MEASUREMENT 


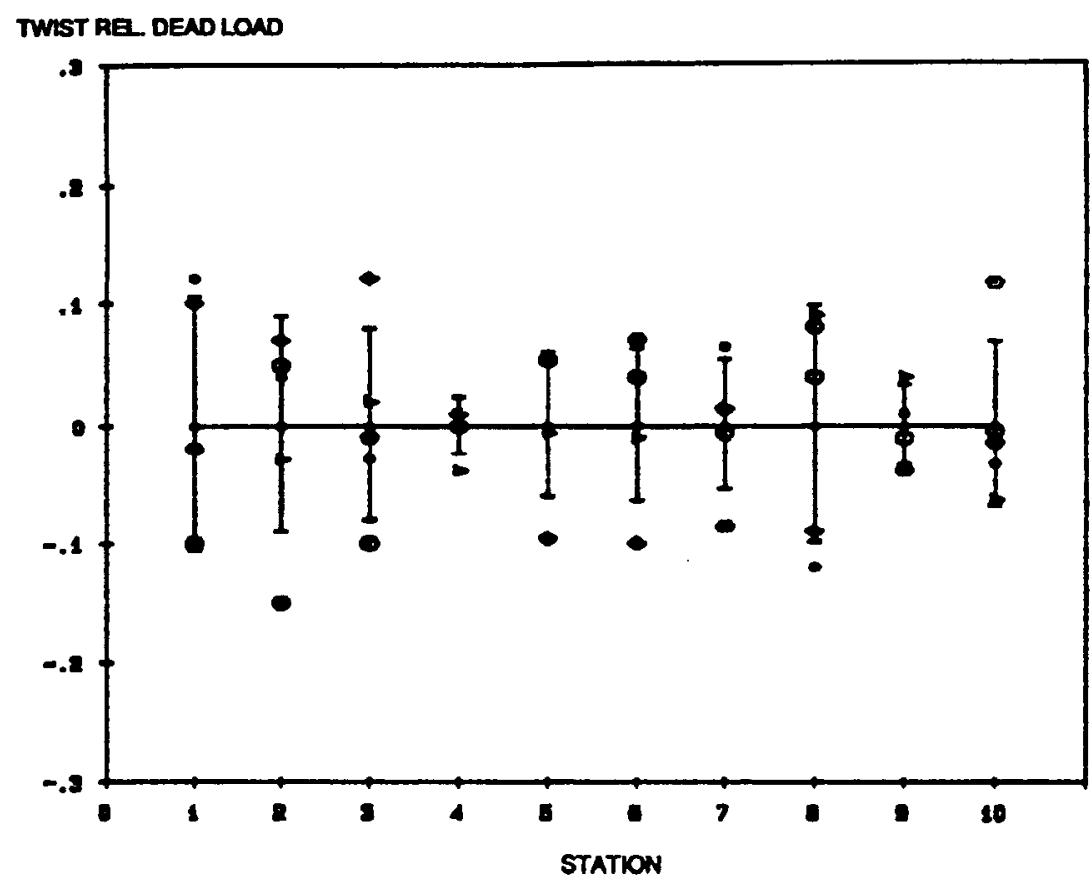

FIG. 5.24 CARTER BLADE: ERROR SCATTER PLOT, TWIST DATA 
TABLE 5.7: CARTER BLADE GEOMETRY, MEASURED VS. CARTER SPECIFICATIONS

\begin{tabular}{|c|c|c|c|c|c|c|c|c|c|c|c|c|}
\hline & STA & $r / R$ & $r$ (in.) & | & chor & (in.) & 1 & thickn & ss (in.) & $t w$ & $\left({ }^{\circ}\right)$ & I \\
\hline & & & & & meas. & spec. & i & meas. & spec. & meas. & spec. & i \\
\hline root & 11 & 0 & 0 & 1 & - - & - - & | & - - & - - & & - - & \\
\hline & 10 & .1 & 40.28 & 1 & 66.75 & 66.80 & 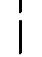 & 14.06 & 14.25 & 26.1 & 27.09 & \\
\hline & 9 & .2 & 80.55 & 1 & 52.81 & 52.80 & 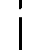 & 11.06 & 11.40 & 15.5 & 15.96 & \\
\hline & 8 & .3 & 120.83 & 1 & 42.50 & 42.88 & 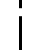 & 8.19 & 8.56 & 9.4 & 9.7 & \\
\hline & 7 & .4 & 161.10 & 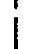 & 34.56 & 34.99 & 1 & 5.25 & 5.70 & 4.5 & 5.67 & \\
\hline & 6 & .5 & 201.38 & 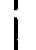 & 28.97 & 29.50 & 1 & 3.81 & 4.00 & 2.3 & 2.93 & \\
\hline & 5 & .6 & 241.65 & 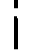 & 25.56 & 26.00 & 1 & 3.28 & 3.39 & 2.0 & 2.19 & \\
\hline & 4 & .7 & 281.93 & 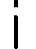 & 23.44 & 23.73 & 1 & 2.97 & 3.09 & 2.0 & 2.01 & \\
\hline & 3 & .8 & 322.20 & i & 22.19 & 22.45 & 1 & 2.78 & 2.92 & 2.0 & 2.00 & \\
\hline & 2 & .9 & 362.48 & & 21.41 & 21.65 & 1 & $\ldots$ & 2.82 & 2.2 & 2.00 & \\
\hline tip & 1 & 1.0 & 402.75 & 1 & 21.00 & 21.00 & 1 & $\ldots$ & 2.73 & 2.6 & 2.00 & \\
\hline
\end{tabular}

\subsection{3: $\quad$ ASCERTAIN BALLAST AND WEIGHT DISTRIBUTION}

Ballast weight distribution information was furnished by Carter, as requested (see App. 10). Still, all the weights and ballast locations had to be accurately measured and verified, since mass distribution in the blade is crucial to its properties.

The AEI sectioned blade actually consisted of portions of two separate blades (Carter \#29B and \#47A), identical in makeup except for the amount and locations of the lead ballast. There were two types of CARTER 300 blade: "mass-balanced" and "non-mass-balanced." The AEI flexural test blade (Carter \#51B), was "mass-balanced," and represented the majority of blades in service, so that configuration was chosen for the analysis. The ballast weights consist of molded lead inserts for the leading edge, with mass of $0.8636 \mathrm{lb} / \mathrm{in}$. and of various lengths. The nondestructive test necessary for finding the ballast locations in the test blade successfully used a standard metal detector. Results are given in Figure 5.25. The "massbalanced" blade is very close to the Carter specification, but the "non mass-balanced" series does not match the specification, and has further additional weight at the tip. The chordwise ballast $\mathrm{cg}$ location was determined by measurement. The weight distribution summary is given in Table 5.8 for the Carter "mass balanced" $300 \mathrm{~kW}$ blade. 

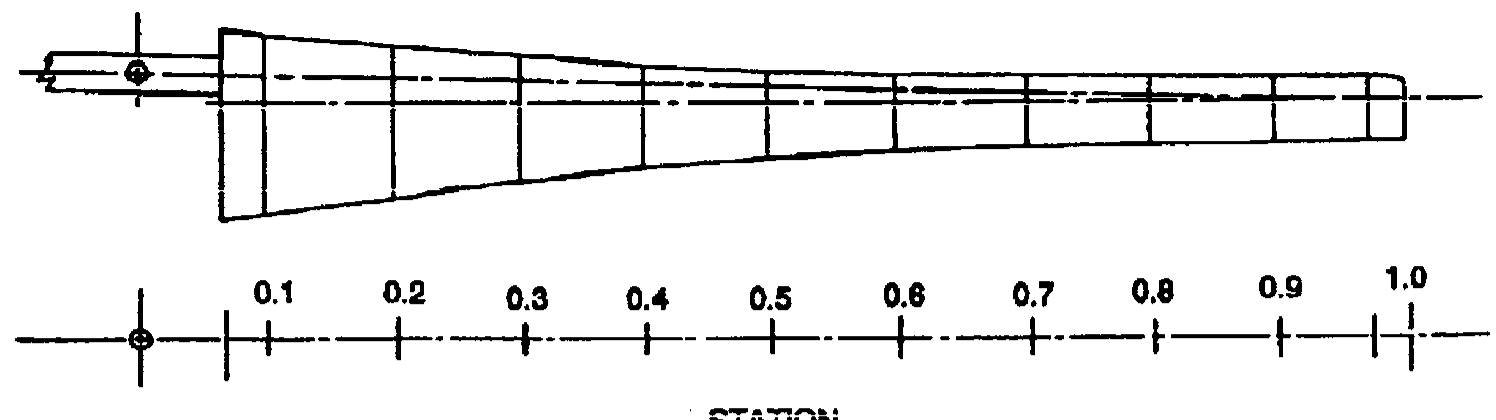

STATION

THON MASS-BALANCED

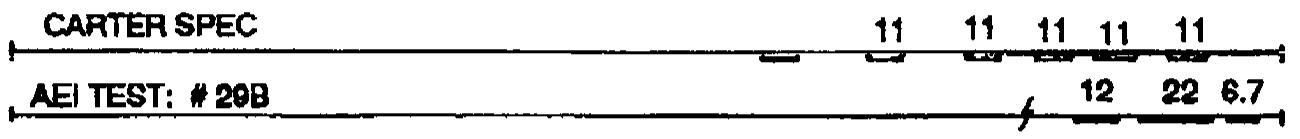

MASSRALANCED"

AEI TEST: $\$ 47 A$

NOTES: (1) Ballast weight is $0.836 \mathrm{lb} / \mathrm{in},(2)$ ballast length indicated in inches.

FIG. 5.25 CARTER BLADE BALLAST SUMMARY

TABLE 5.8: CARTER BLADE WEIGHTS SUMMARY

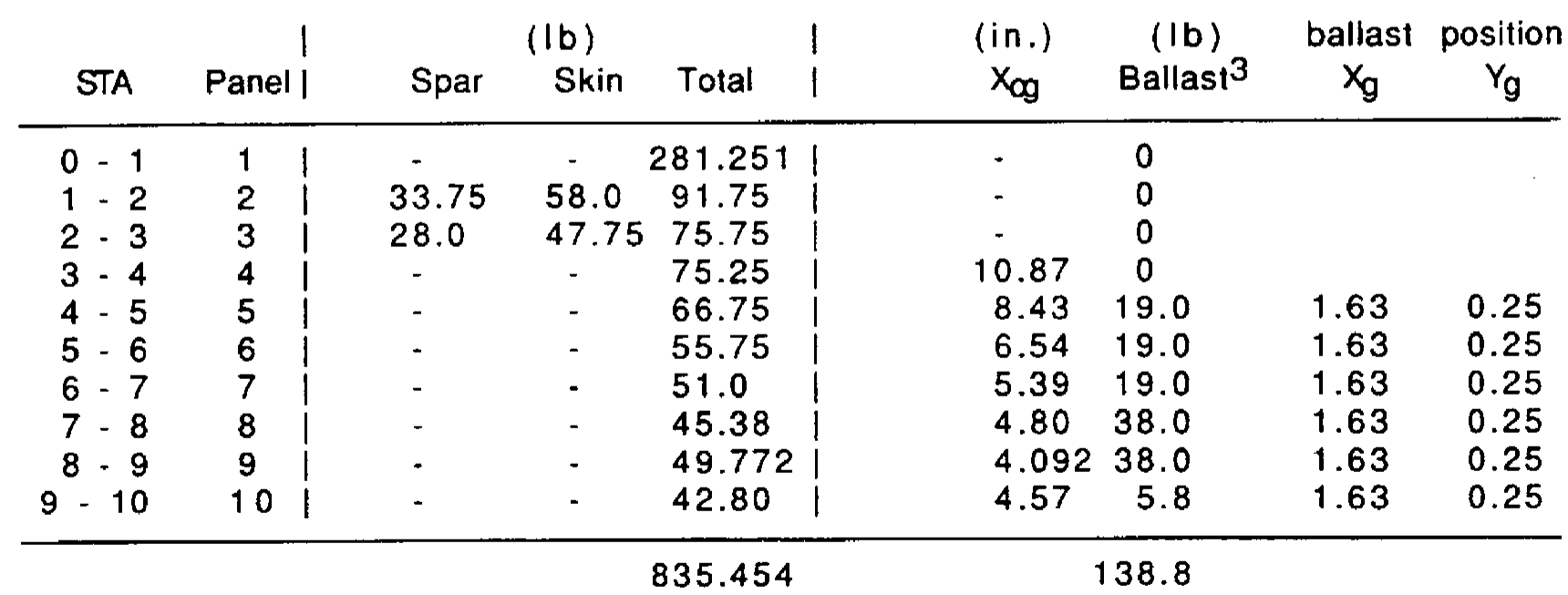

NOTES:

1. includes root rib and mechanisms

$$
\frac{(27.5 * 5.98)+(22.27 \cdot 1.75)}{47.77}=4.09 \text { in. }
$$

2. adjusted to "mass balanced blade":

3. the ballast is centered at the station (i.e., 1/2 contribution for each adjacent panel) and slightly adjusted spanwise for convenience.

4. Carter Wind Systems estimate $700 \mathrm{lb}$. 


\subsection{4: DERIVE CARTER DESIGNED COMPOSITE VALUES}

Carter Wind Systems did not perform a stiffness analysis for this blade design; they relied on allowable ultimate strength values for a specified laminate and load, and performed spot checks on the blade, using prescribed loads (unknown cases) and the trapezoidal approximation to section moment of inertia, (Ref. 27). The AEl study required a stiffness analysis and section properties; therefore, a blade section analysis had to be done based on the manufacturer's specified laminate design. This section covers the derivation of the code input.

The Carter laminate, even though the blade is quite complex, consists of only four types of glass reinforcement; derivations of their expected as-built properties follows.

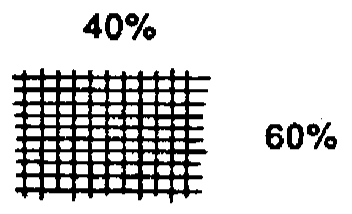

1) Style $60 / 40,7.50 z \cdot / \mathrm{yd}^{2}$ cloth:

This woven cloth reinforcement has $40 \%$ of the glass fibers on the $0^{\circ}$ direction $(y)$ and $60 \%$ in the $90^{\circ}(\mathrm{x})$. Assuming a layer (ply) thickness of $0.015^{\prime \prime}$ (CARTER estimate), a 1-yard square has a volume of $(36)^{*}(36)^{*}(.015)=19.44$ in. $^{3}$. The glass in the $X$-direction amounts to $60 \%$ of $7.5 \mathrm{oz} .=4.5 \mathrm{oz}$. Taking the density of E-glass of $0.09838 \mathrm{lb} / \mathrm{in} .{ }^{3}$ (or $1.57408 \mathrm{oz} / \mathrm{in} .^{3}$ ) and the modulus of $10^{*} 10^{6} \mathrm{psi}$ gives 2.8588 in.$^{3}$ of E-glass in the X-direction ( $90^{\circ}$ orientation) for our "standard" square yard. The Emodulus of the laminate is easily found by the volume percentage of glass in that direction:

$\begin{array}{lll} & \text { glass } & \text { per } y d^{2} \\ 90^{\circ} \text { or } x \text { direction: } & 4.5 \mathrm{oz}=2.8588 \text { in. } .^{3} & E_{1}=10^{*} 10^{6}[2.8588 / 19.44]=1.47^{*} 10^{6} \\ 0^{\circ} \text { or } y \text { direction: } & 3.0 \mathrm{oz}=1.9059 \text { in. }{ }^{3} & E_{2}=10^{*} 10^{6}[1.9059 / 19.44]=0.98^{*} 10^{6}\end{array}$

The glass content of this ply is:

glass content by volume $=$

$$
\frac{4.7647 \text { in. }^{3} \text { (glass) }}{19.49 \text { in. }^{3} \text { (total) }}=.2451 \%
$$

From the chart (Table 4.1 ) this gives a ply weight density of $0.05715 \mathrm{lb} / \mathrm{in}^{3}$. Lastly the Gmodulus is estimated from the engineering chart (Fig. 5.2) and rules of thumb:

$$
\mathrm{G} \equiv 1.47^{\star} 10^{6} \cdot 0.6 \text { (conversion factor for orientation) }=0.882-10^{6} \mathrm{psi}
$$

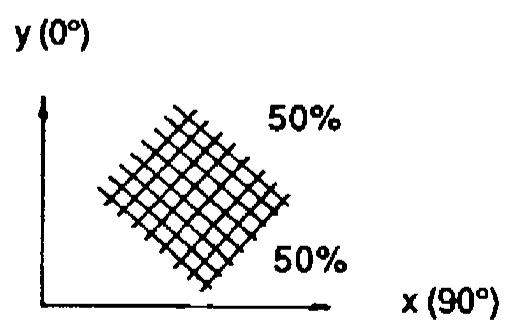

2) Style $\pm 45^{\circ}, 10 \mathrm{oz}$. cloth:

This bonded ply fiber reinforcement ("Knytex")has balanced orthogonal properties oriented $\pm 45^{\circ}$. Carter assumed a layer thickness of $0.020 "$ for this reinforcement. The calculation proceeds as before, except the orientation factor decreases the E-modulus by $50 \%$ and increases the G-modulus by 2 : 


$$
\text { Volume }=(36)(36)(.020)=25.92 \text { in. }^{3}
$$

glass Der yard 2

3.1765

$90^{\circ}$ or $x$ direction: $5 \mathrm{oz} .=3.1765 \mathrm{in}^{3} \quad E_{1,2}=\frac{}{25.92}\left(10^{*} 10^{6}\right)(.5)=0.613^{*} 10^{6}$

$0^{\circ}$ or $y$ direction: $\quad 5 \mathrm{oz} .=3.1765 \mathrm{in}^{3} \quad E_{1,2}=\frac{3.1765}{25.92} \quad\left(10^{*} 10^{6}\right)(.5)=0.613^{*} 10^{6}$

where .5 is the orientation correction factor $( \pm 45 \mathrm{deg})$

glass content by volume $=\frac{6.353}{25.92}=.2451 \%$

weight density (from chart) $=.05715 \mathrm{lb} / \mathrm{in.}^{3}$

$\mathrm{G} 45^{\circ} \equiv\left(0.613 * 10^{6}\right)(1 / 0.5)(0.6)=0.7356 * 10^{6} \mathrm{psi}$

$\mathrm{GO}^{\circ} \equiv\left(.7356 * 10^{6}\right)(1 / 0.5)=1.4712 \cdot 10^{6} \mathrm{psi}$

where 0.6 and 0.5 are the G-correction factor and the orientation factor (Fig. 5.2), respectively.

3) Unidirectional Tape, 13 oz. (Knytex A-130):

This ply is the principal spanwise reinforcement in the blade; this is a convenient way to mold unidirectional properties in the hand lay-up process. This reinforcement weighs $13 \mathrm{oz} . / \mathrm{square}$ yard, and Carter assumed a ply thickness of $0.015 \mathrm{in}$. The properties are calculated easily in the above manner.

4) Style woven rovina. 24 oz:

Woven roving consists of E-glass rovings (yarns) in an open weave pattern, with balanced orthogonal fibers, e.g., 50/50. Carter estimated a ply thickness of 0.026 in. for this reinforcement.

The summary predicted properties for the as-built plies are given in Table 5.9.

TABLE 5.9: CARTER REINFORCEMENTS, AS-BUILT SUMMARY

\begin{tabular}{lccccc} 
STYE & $\begin{array}{c}\text { (in.) } \\
1\end{array}$ & $\begin{array}{c}\left.* 10^{6} \mathrm{psi}\right) \\
\mathrm{E}_{0}\end{array}$ & $\begin{array}{c}\left.* 10^{6} \mathrm{psi}\right) \\
\mathrm{G}\end{array}$ & $\begin{array}{c}\left(\mathrm{lb} / \mathrm{in} .{ }^{3}\right) \\
\rho\end{array}$ & glass\% \\
\hline $60 / 40$ & .015 & 1.47 & 0.882 & .05715 & 24.5 \\
\pm 45 & .020 & 0.613 & 1.471 & .05715 & 24.5 \\
Uni & .015 & 4.25 & 0.400 & .06677 & 42.5 \\
WR & .026 & 2.263 & 1.358 & .06814 & 45.2 \\
\hline
\end{tabular}




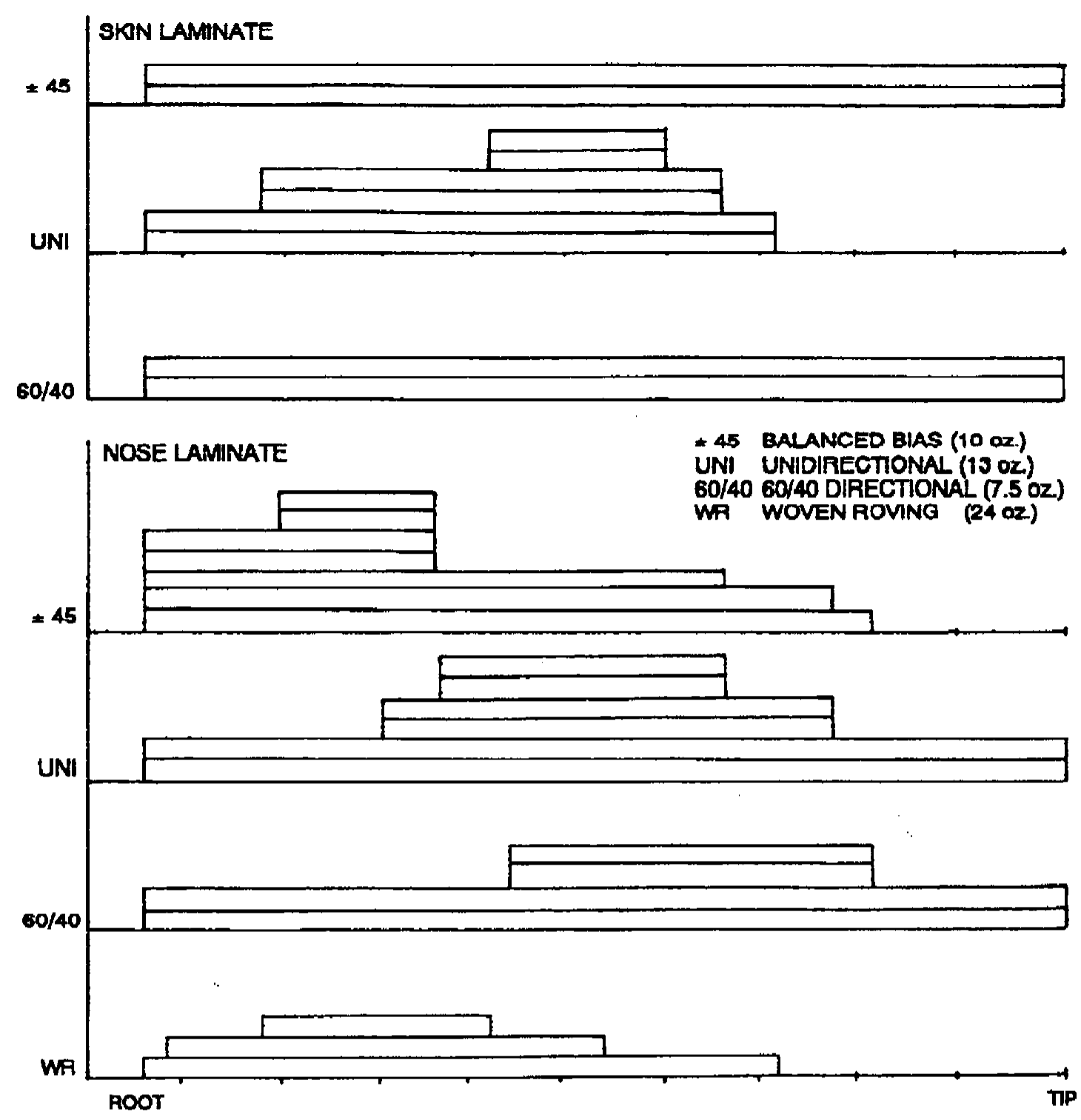

FIG. 5.26 CARTER BLADE LAMINATE SCHEDULE

Now given the laminate schedule for the Carter blade (Fig. 5.26), an estimate can be made for the properties, thicknesses, and other inputs to the section calculation code. For this blade the PVC foam coring (in the aft upper and lower skin panels and the spar web) was ignored, and the laminates at each section were assembled ply by ply and then reduced to skin, D-spar, and web contributions.

Clearly, as the laminate makeup changes, so also do the aggregate properties $E, G$ and $\rho$. Therefore, an average value for the section properties code had to be chosen. One refinement to the section properties code would allow separate engineering values for each station. An example of this variation is shown in Figure 5.27 which plots the weight density distribution for the spar portion. Table 5.10 gives the skin and spar input values for the Carter design schedule. 


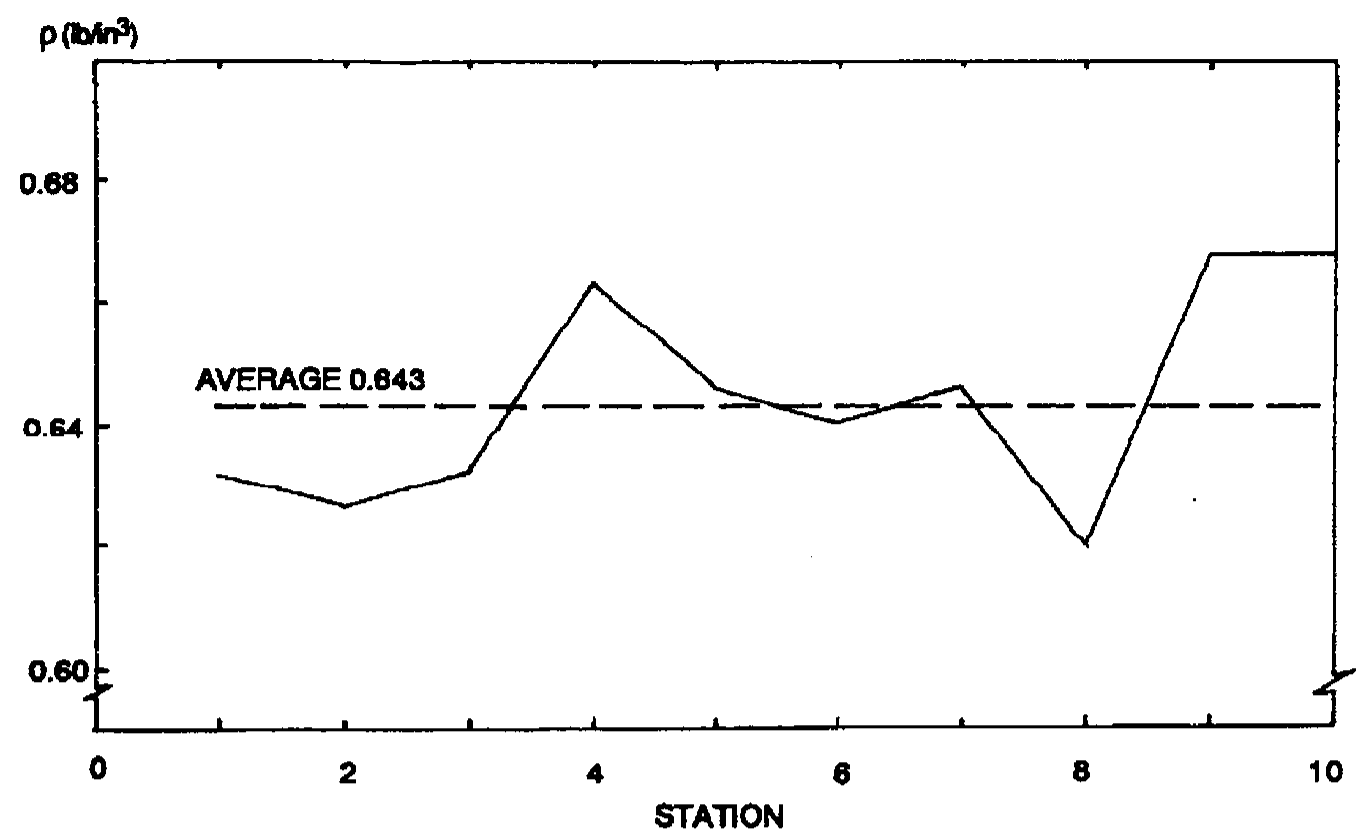

FIG. 5.27 CARTER SPANWISE WEIGHT DENSITY (SPAR PORTION)

The unidirectional reinforcement in the skin and the nose webs were included separately. These values for each station were estimated as tilted rectangles $\left(I=b^{3} / 12\right)$ and summed using the parallel axis theorem. This resulted in the web input given in Table 5.10. Another refinement to the code would integrate these multi-"webs" as it does for the skin and spar rather than assume rectangles.

TABLE 5.10: CARTER BLADE LAMINATE DESIGN SUMMARY

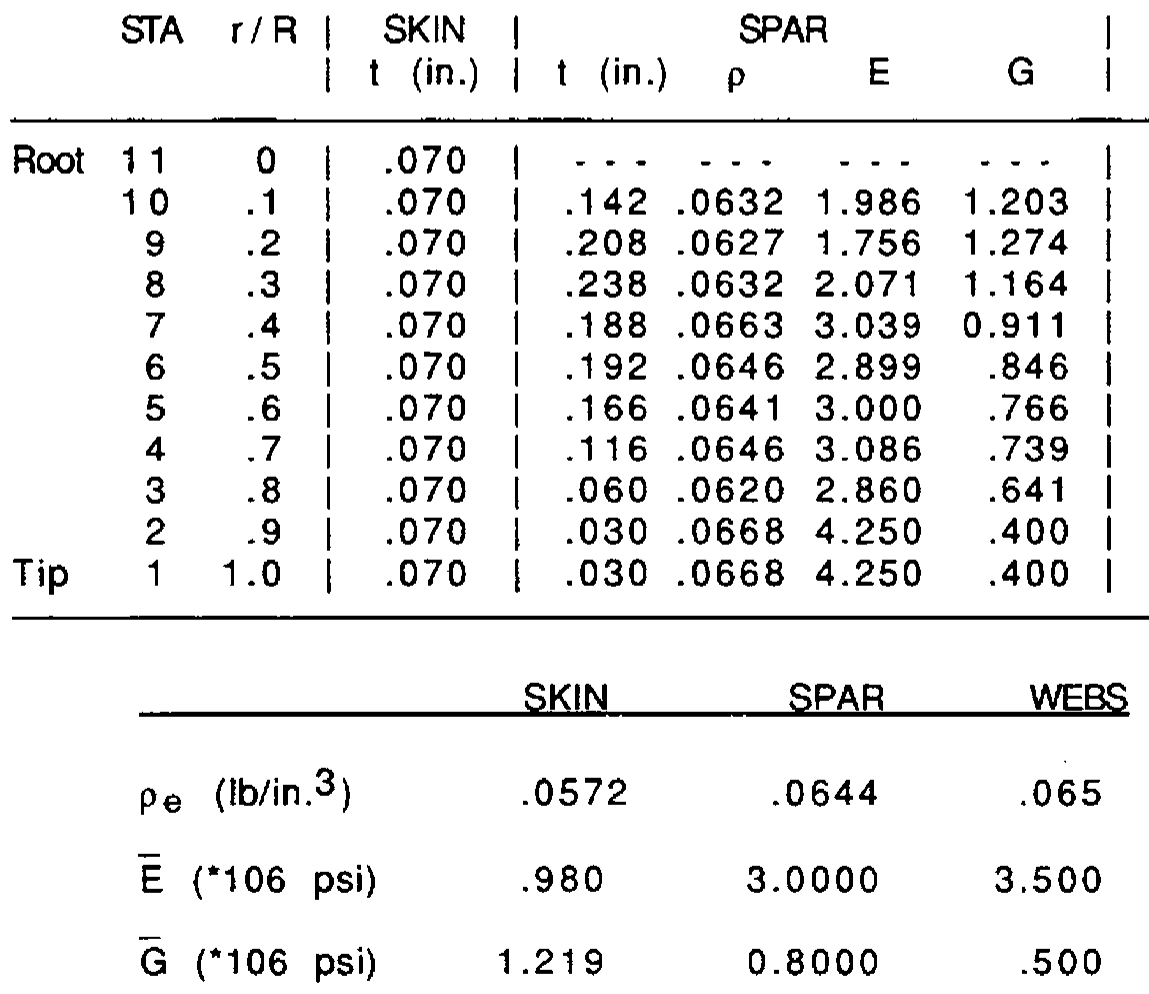




\subsection{5: DERIVE CONTRIBUTION OF I-BEAM}

Lastly, the I-beam must be accounted for in the section properties code. The Carter I-beam cross section is shown below in Table 5.11; the beam portion is highly compacted filament-wound rovings, so it should have a very high glass ratio (in fact, the highest readily achievable in any glass-resin laminate) of $90 \%$. The test data and geometric data must be used to find the area, weight, and moments of inertia for the I-beam.

\section{TABLE 5.11: CARTER I-BEAM SECTION DATA FROM TEST}

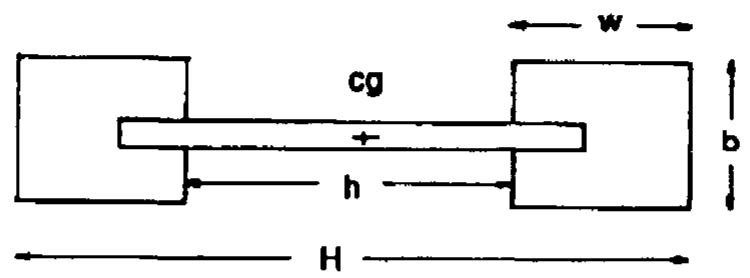

\begin{tabular}{cc|ccc|cccc} 
& & & $(1 / 4$ & & & & (in.) \\
STA & $(r / R)$ & $X_{g}$ & $Y_{g}$ & $H$ & $h$ & $b$ & $w$ \\
\hline 1 & .1 & 16.69 & 0 & & (data lost) & & 1.562 \\
2 & .2 & 13.20 & 0 & 13.88 & 10.56 & 2.38 & 1.562 \\
3 & .3 & 10.63 & 0 & 11.76 & 8.63 & 2.38 & 1.562 \\
4 & .4 & 8.64 & 0 & 9.76 & 6.63 & 1.97 & 1.562 \\
\hline
\end{tabular}

From Carter data, the reinforcement of the unidirectional beams consists of 135 wraps of 40 strand rovings of E-glass. Therefore, the total number of ends are $135^{\star} 40=5400$; so in each beam there are 5400 rovings. The measured beam area is $1.562^{\star} 2.380=3.718$ in. ${ }^{2}$. Taking the standard weight of roving as 450 yards/lb allows a calculation of glass percentage,

For 450 yards $/ \mathrm{lb}$ roving the yarn size is $=0.62765^{*} 10^{3}$ in. ${ }^{2} /$ roving (from $\rho \mathrm{E}$-glass $=0.09838 \mathrm{lb} / \mathrm{in} .^{3}$ ) then: $A(5400$ ends $)=(5400)\left(.62765^{\star} 10^{3}\right)=3.389$ in. $^{2}$ so: glass content $=3.389 / 3.718=91.15 \%$

This verifies the original assumption of $90 \%$ glass in the I-beam. The engineering values for this laminate are, then, from Figure 4.1:

$$
\begin{aligned}
& E_{\text {uni }}=9.115 * 10^{6} \mathrm{psi} \\
& G_{\text {uni }}=0.200 * 10^{6} \mathrm{psi} \\
& \rho_{\text {uni }}=0.09351 \mathrm{lb} / \mathrm{in} .{ }^{3}
\end{aligned}
$$

Adding the small shear web contribution, consisting of mainly $\pm 45^{\circ}$ plies, changes the aggregate values of the I-beam to

$$
\begin{array}{ll}
\mathrm{E}_{\mathrm{I} \text {-beam }} & =9.0 \cdot 10^{6} \mathrm{psi} \\
\mathrm{G}_{\text {I-beam }} & =0.420 \cdot 10^{6} \mathrm{psi} \\
\rho_{\text {I-beam }} & =0.0935 \mathrm{lb} / \mathrm{in}^{3}
\end{array}
$$

At this point it is a good idea to make a quick check on this value from a weight comparison.

I-beam length $=194$ in.

weight of uni spars $=2 * 194 * .09351=135 \mathrm{lb}$.

weight of $\pm 45^{\circ} \mathrm{web}=194^{*} 6 * .260^{*} .05715=\underline{17.3 \mathrm{lb}}$.

total weight

$152.3 \mathrm{lb}$.

This compares favorably with the measured value of $165 \mathrm{lb}$, allowing for gussets and fill at the root end. 
Next the moments of inertia must be found, since the I-beam principal axis is not placed on the chord line because the blade's twisted. The chordline values of I are simply determined from the principal axis values of $I_{\max }$ and $I_{\min }$, using a standard Mohr's circle solution (Ref. 12). This gives the moments of inertia (Table 5.12) of the I-beam at each station, in the section (X-Y or airfoil) coordinate system.

TABLE 5.12: CARTER I-BEAM MOMENTS OF INERTIA

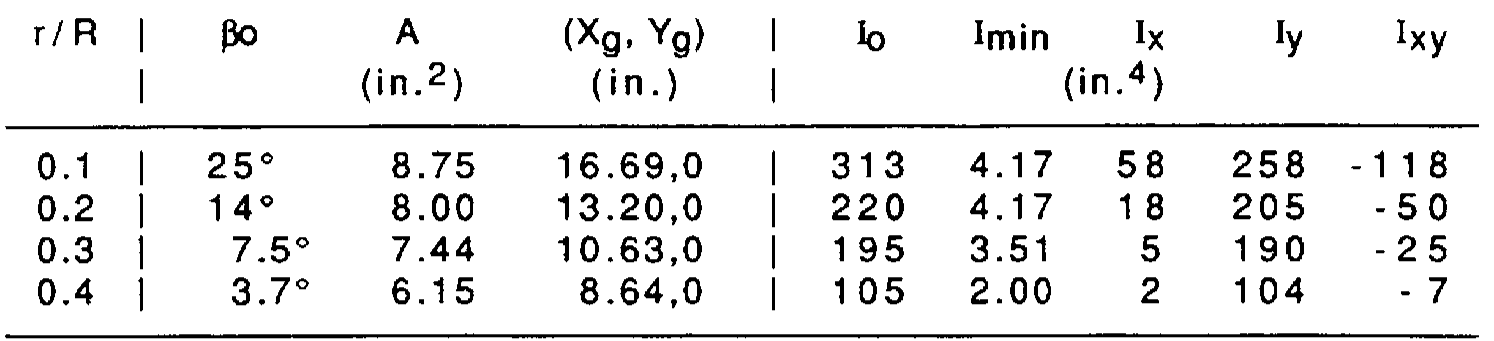

\subsection{6: AEI MEASUREMENTS FOR COMPOSITE SECTION PROPERTIES}

Along with the theoretical design input files developed above from composite engineering analysis, an additional input file was constructed on the basis of the blade sectioning measurements. Practically speaking, the sectioning allows an independent check of the above predictions (for example, $\mathrm{cg}$, laminate thickness, weights) but also will quickly indicate where the actual blade differed from the design (e.g., more plies than specified, presence of defects). The section inspection values are listed in Table 5.13 along with the specified design values.

TABLE 5. 3: CARTER BLADE: SECTION MEASUREMENTS VS. CARTER SPECIFICATIONS (INCHES)

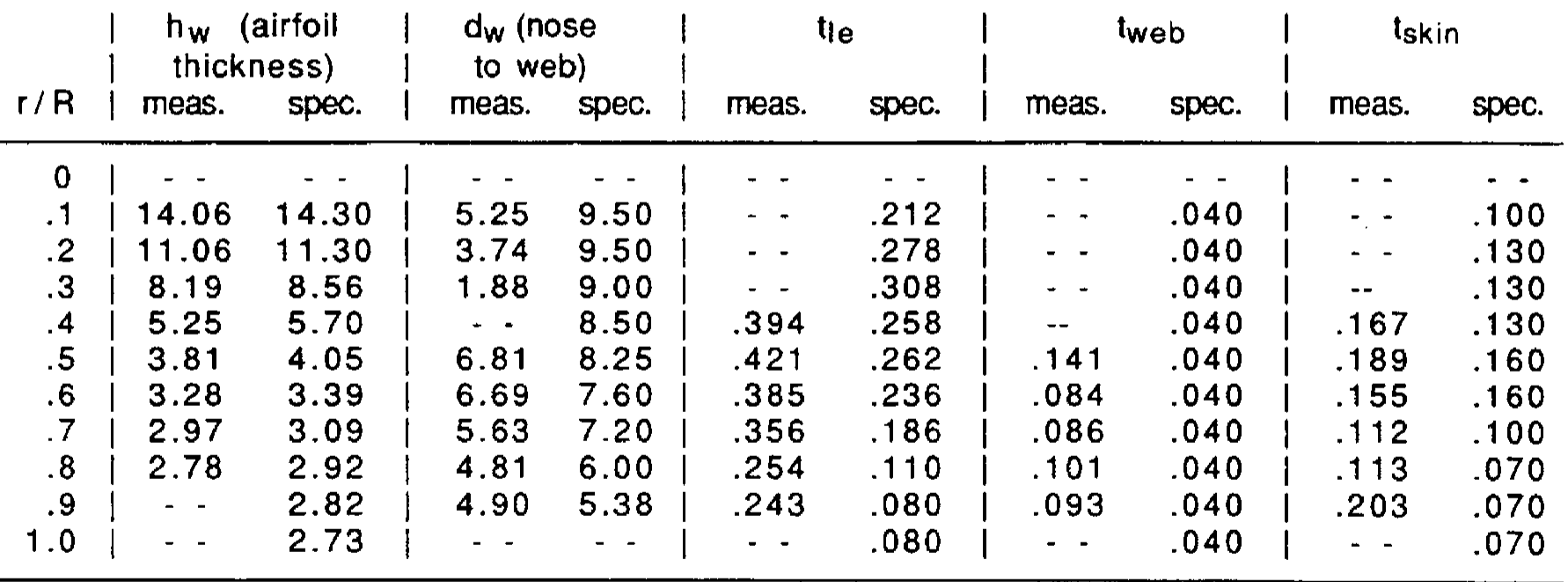

Note: Blanks are missing data or missing component (e.g., web $\equiv 0.1$ )

Conclusions that can be drawn from Table 5.13 are

(1) Difference in $d_{w}$ is evident since Carter assumed a distance from junction of the nose to the skin, and the actual web distance is inset somewhat.

(2) Difference in leading edge thickness is because actual blade has a much higher resin content than predicted, since it was formed in a deep concave mold with no vacuum-bagging or other compacting.

(3) The actual spar web is twice as thick (less PVC core) since the number of $\pm 45^{\circ}$ plies was apparently doubled over the design value.

(4) The airfoil thickness is very close to the prediction, indicating good tooling and assembly.

(5) The skin thickness is very close to the prediction, since it was vacuum-bagged (pressure formed). 
This information now allows the blade section input values to be ascertained from the test values:

(1) The new thicknesses and centroid locations (web) are the test values.

(2) The new $E$ - and G- modulus will be volume weighted to the new thickness since the reinforcement hasn't changed but the resin has.

(3) The resin weight densities are volume-weighted to the measured values of glass content.

In conclusion, the specified laminate schedule is probably close to the actual blade laminate, but there is no way to tell for sure until both $A E I$ and Carter section properties are calculated and the deflections compared with the test values. The I-beam contribution is the same, since measured and predicted values agreed (see above).

The initial (trial) values for the AEI blade model are

$$
\begin{array}{ll}
E_{\text {skin }}=4.0^{*} 10^{6} \mathrm{psi} & E_{\text {spar }}=4.0^{*} 10^{6} \mathrm{psi} \\
\mathrm{G}_{\text {skin }}=0.5^{*} 10^{6} \mathrm{psi} & \mathrm{G}_{\text {spar }}=0.75^{*} 10^{6} \mathrm{psi} \\
\rho_{\text {skin }}=0.05 \mathrm{lb} / \mathrm{in} .3 & \rho_{\text {spar }}=0.05 \mathrm{~b} / \mathrm{in} .{ }^{3}
\end{array}
$$

The initial values for the inboard I-beam portion are

$\begin{array}{llll}\text { STA } & \text { EI }(x p) & \frac{\text { El }}{1}(y p) & \beta \\ 0.0 & 37.53 * 106 & 2817 * 106 & 25^{\circ} \\ 0.1 & 37.53 & 2817 & 25^{\circ} \\ 0.2 & 37.53 & 1980 & 14^{\circ} \\ 0.3 & 31.59 & 1755 & 7.5^{\circ}\end{array}$

where:

(1) $E_{\text {I-beam }}=9 * 10^{6} \mathrm{psi}$

(2) The skin is assumed to contribute no bending stiffness from the root to 0.35 STA, by Carter design intent.

(3) The I-beam values are carried to the origin.

(4) The twist angle will affect the values in the true chordwise $X-Y$ system, but the flexural tests are really in the principle axes of the I-beam.

\subsection{7: VERIFICATION BY FLEXURAL TEST}

The three bending tests used represent two distributed load cases (total load $=225 \mathrm{lb}$ and $325 \mathrm{lb}$ ), and one torsional load case (50 lb @ STA 0.9 in torsion). This section discusses the bending tests only. Again, the test loads were placed on the blade quarterchord. The major deflection was in the flapping direction. As seen in Appendix 6, the inplane (chordwise) deflections were very small, indicating very little principal axis coupling. However, elastic twists were substantial, indicating the elastic axis was not on the quarterchord.

Two corrections had to be made in the (flapwise) data:

1) the dead load droop had to be eliminated, and

2) the root compliance (teeter angle) had to be considered.

The dead load droop was easily found from (averaged) dead load cases. The root angle was measured with the clinometer, with a repeatability better then three minutes of arc. The three flapwise distributions and the corresponding elastic teeter values are shown in Figure 5.28. 


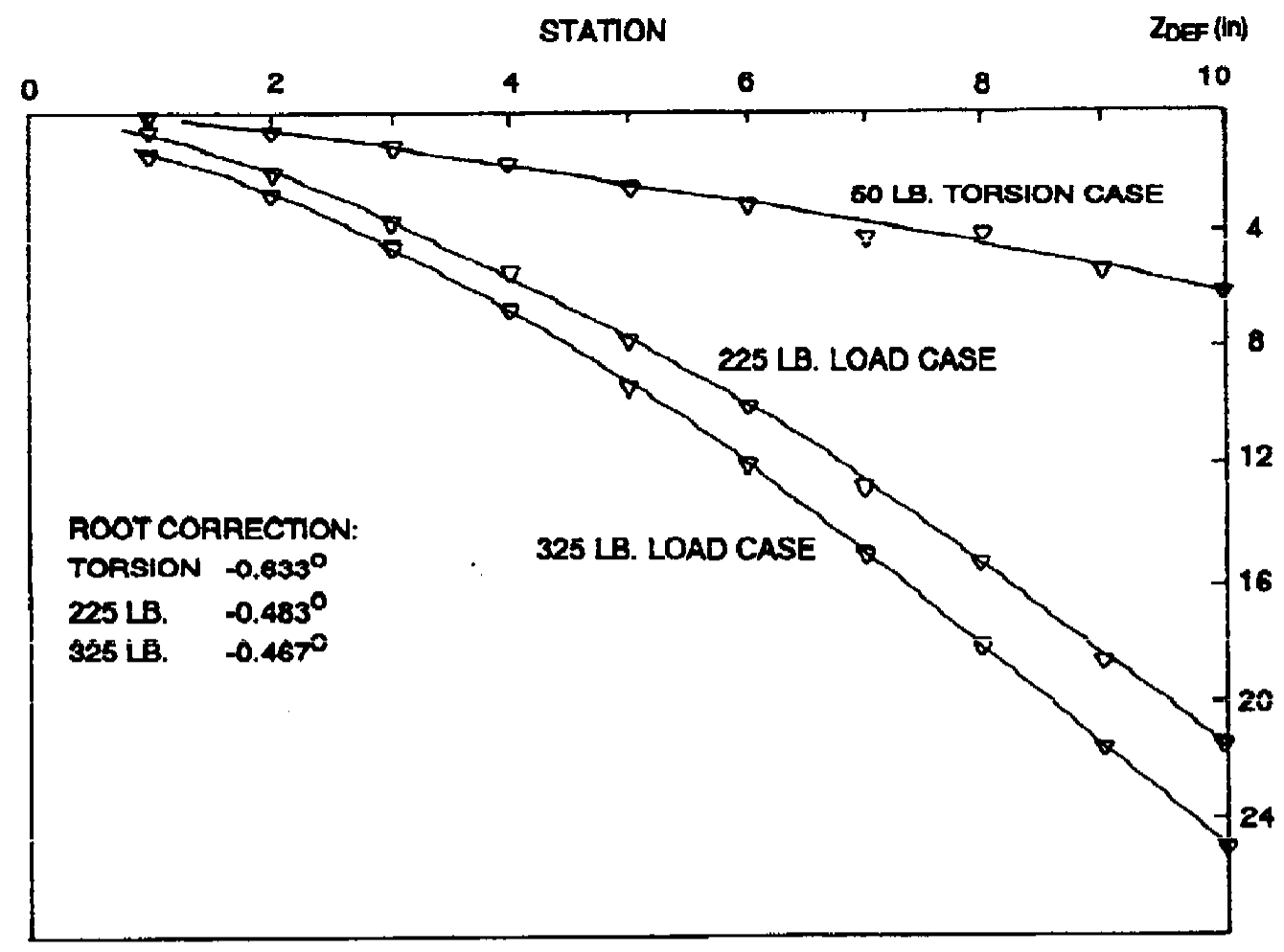

FIG. 5.28 CARTER STATIC DEFLECTION: 3 LOAD CASES (CORRECTED)

As stated above, the flexural stiffness inboard was initially assumed to be provided only by the Ibeam, as conceived by the Carter Wind Systems design. This resulted in predicted blade deflections that were much too large. However, by increasing the inboard section modulus in the flapwise direction by a "fudge-factor" of 2.67, all three bending cases matched the test data, as can be clearly seen in Figure 5.29.

Zoef (in)

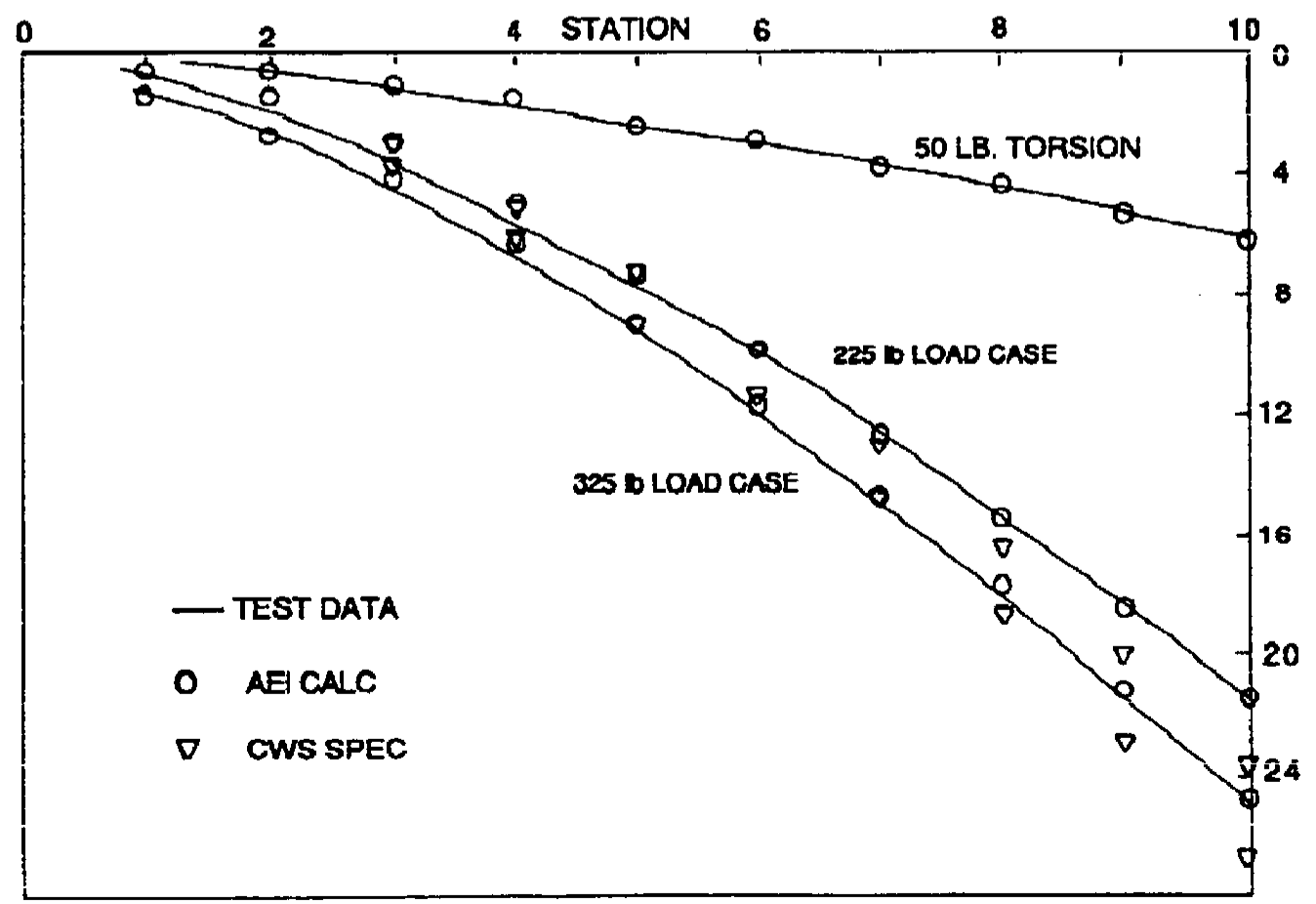

FIG. 5.29 CARTER STATIC DEFLECTION: AEI CALCULATION VS. MEASURED (CORRECTED) 
This is a very interesting result, and indicates that the blade shell is contributing after all to the flexural stiffness inboard, actually more than doubling the stiffness of the I-beam alone. This is evidently caused by some transfer of bending moment through the root rib I-beam attachment, allowing some of the moment to be carried in the skin. For point of reference, if the root rib were rigidly attached to the skin and the I-beam, the flexural stiffness would have been on the order of 50 times the I-beam stiffness, rather than only 2.67. In any case, the root rib joint has to be designed with this additional load in mind.

Another conclusion from Figure 5.29 is that the Carter design laminate and AEI observed laminate curves are very close, verifying the overall Carter laminate specification.

\subsection{8: VERIFICATION OF STATIC TWIST}

The corresponding static twist associated with the two bending cases (225 $\mathrm{lb}$ and $325 \mathrm{lb}$ ) above are shown in Figures 5.30 and 5.31, along with the Carter and $A E I$ laminate predictions. Note the increased scatter of the test data inboard, where the skin profiles were being warped by shear lag, causing the templates to drift. However, the match is still very good, verifying the AEl and Carter laminate models.

A further static torsion test case was also used. The results are shown in Figure 5.32. In this test load a point load at 0.70 STA was used, offset from the quarterchord to produce a point moment. This figure shows the marked difference in the AEl and Carter design laminate, whereas the distributed load cases above really did not. Clearly the AEI laminate values are more accurate than the Carter specs. Note the divergence of the test point right on the loaded template, presumably due to local warping amounting to six minutes of arc.

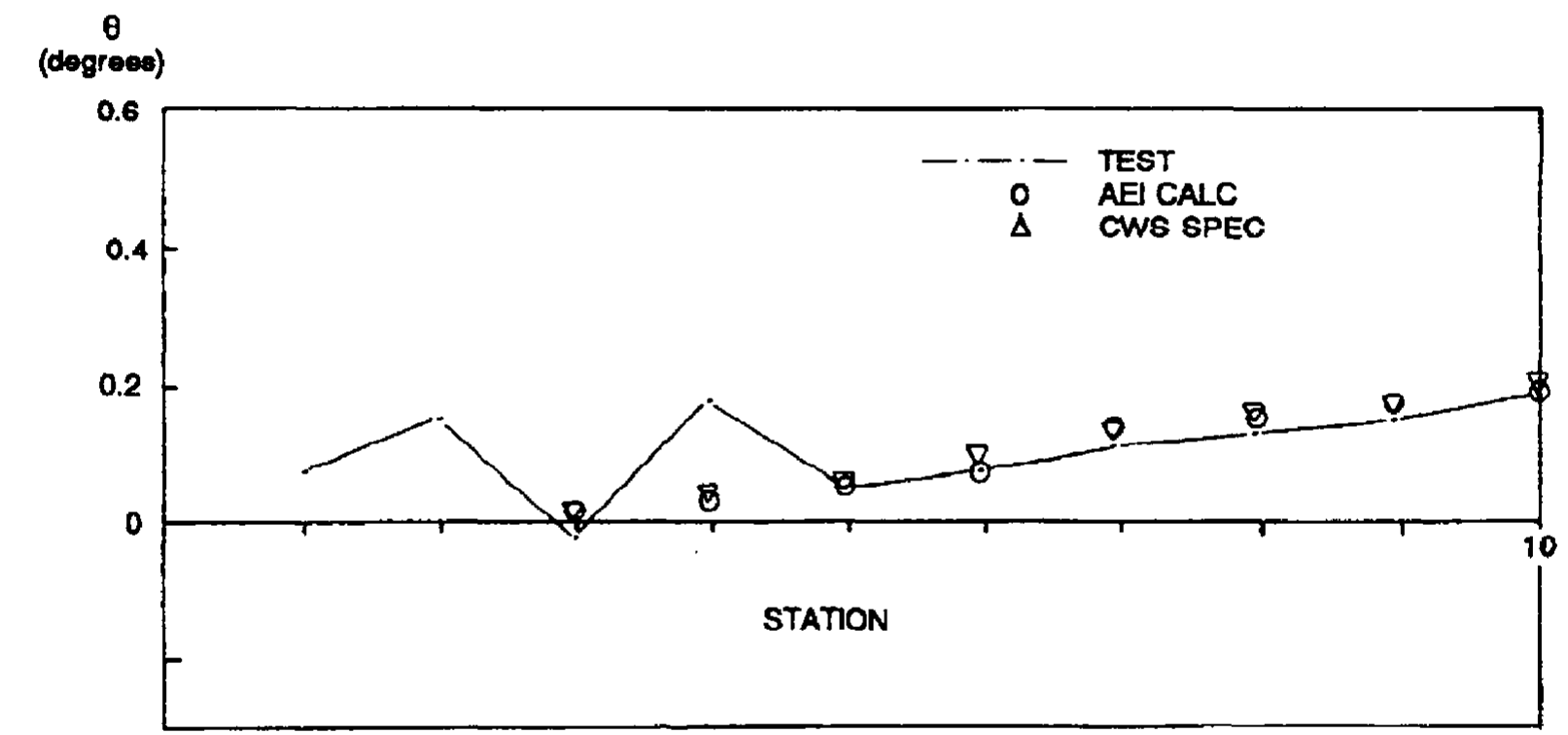

FIG. 5.30 CARTER MEASURED TWIST: (225 POUND TEST) PREDICTED VS. AEI CALCULATION VS. SPECIFICATION 


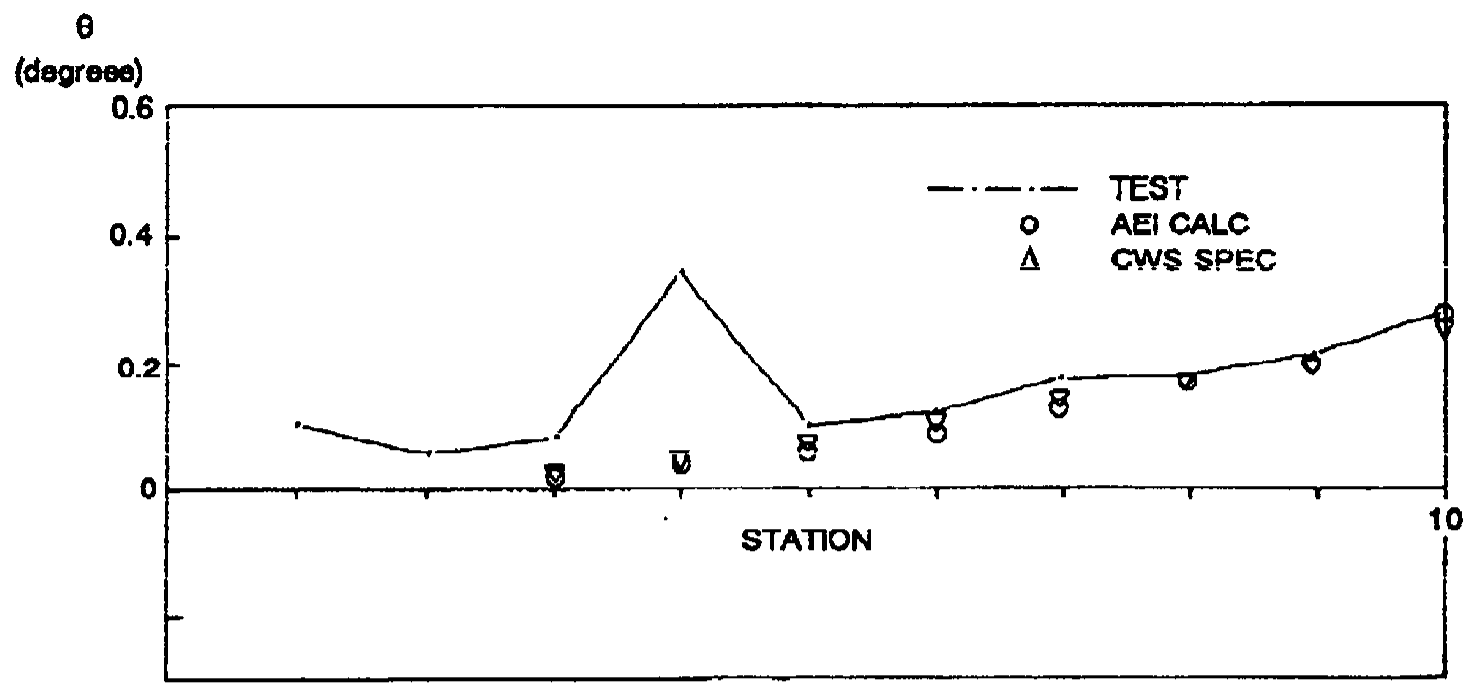

FIG. 5.31 CARTER MEASURED TWIST: 325 POUND TEST

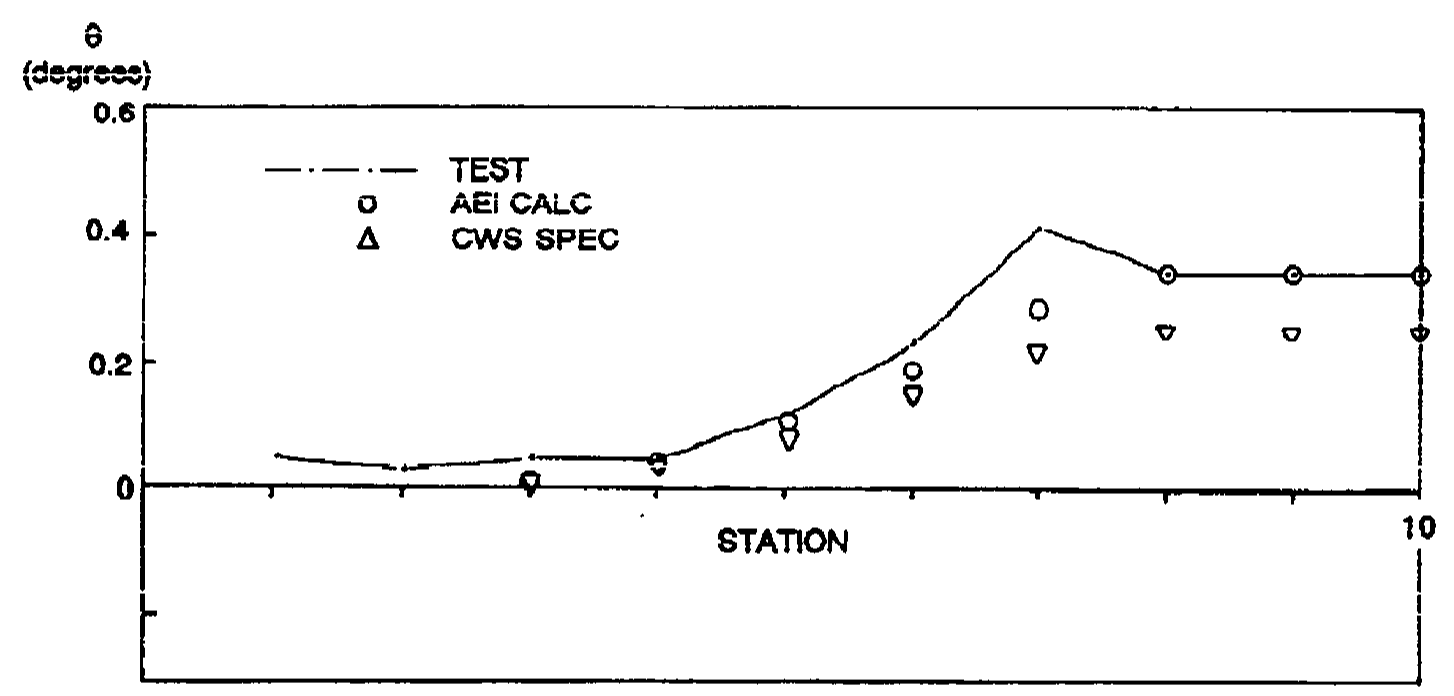

FIG. 5.32 CARTER MEASURED TWIST: TORSION LOAD CASE

\subsection{9: $\quad$ COMPARISONS OF RUNNING MASS DISTRIBUTIONS}

With the estimated weight densities above, the blade running mass distributions for the Carter design laminate and $A E I$ measured laminate are compared with the actual running masses from the sectioning tests (Table 5.14 and Figure 5.33). 
TABLE 5.14: CARTER BLADE RUNNING MASS COMPARISON (LB)

\begin{tabular}{ccccc} 
& STA & AEI Calculation & CARTER desion laminate & AEl Test \\
\hline 0.0 & 2.11 & 1.74 & $0.838^{*}$ \\
0.1 & 2.11 & 1.74 & $6.983^{*}$ \\
0.2 & 1.69 & 1.72 & $2.278^{*}$ \\
0.3 & 1.42 & 1.50 & $1.868^{*}$ \\
0.4 & 1.14 & 1.16 & $1.868^{*}$ \\
0.5 & 1.27 & 1.19 & 1.657 \\
0.6 & 1.07 & 1.08 & 1.384 \\
0.7 & 0.93 & 0.86 & 1.266 \\
& 0.8 & 1.30 & 1.19 & 1.127 \\
& 0.9 & 1.42 & 1.16 & 1.236 \\
& 1.0 & 0.61 & 0.35 & 1.063 \\
\hline Total & $559.9 \mathrm{lb}$ & $512.7 \mathrm{lb}$ & $835.4 \mathrm{lb}$
\end{tabular}

NOTES:

1) The total blade weight of $835.4 \mathrm{lb}$ is from test; the (") items contain mechanisms, counterweights, root rib, mid-rib, an amount of potting resin to attach the I-beam, and an unknown amount of undocumented extra laminate, all adding up to over $200 \mathrm{lb}$ of weight.

2 ) The two calculations contain the outboard ballast weights, but not the inboard ballast weights, which were unknown and impossible to determine nondestructively.

3) The two calculations have weights from STA 0.0 to 0.4 which are artificially low since no weight density correction was used for the (much denser) I-beam portion.

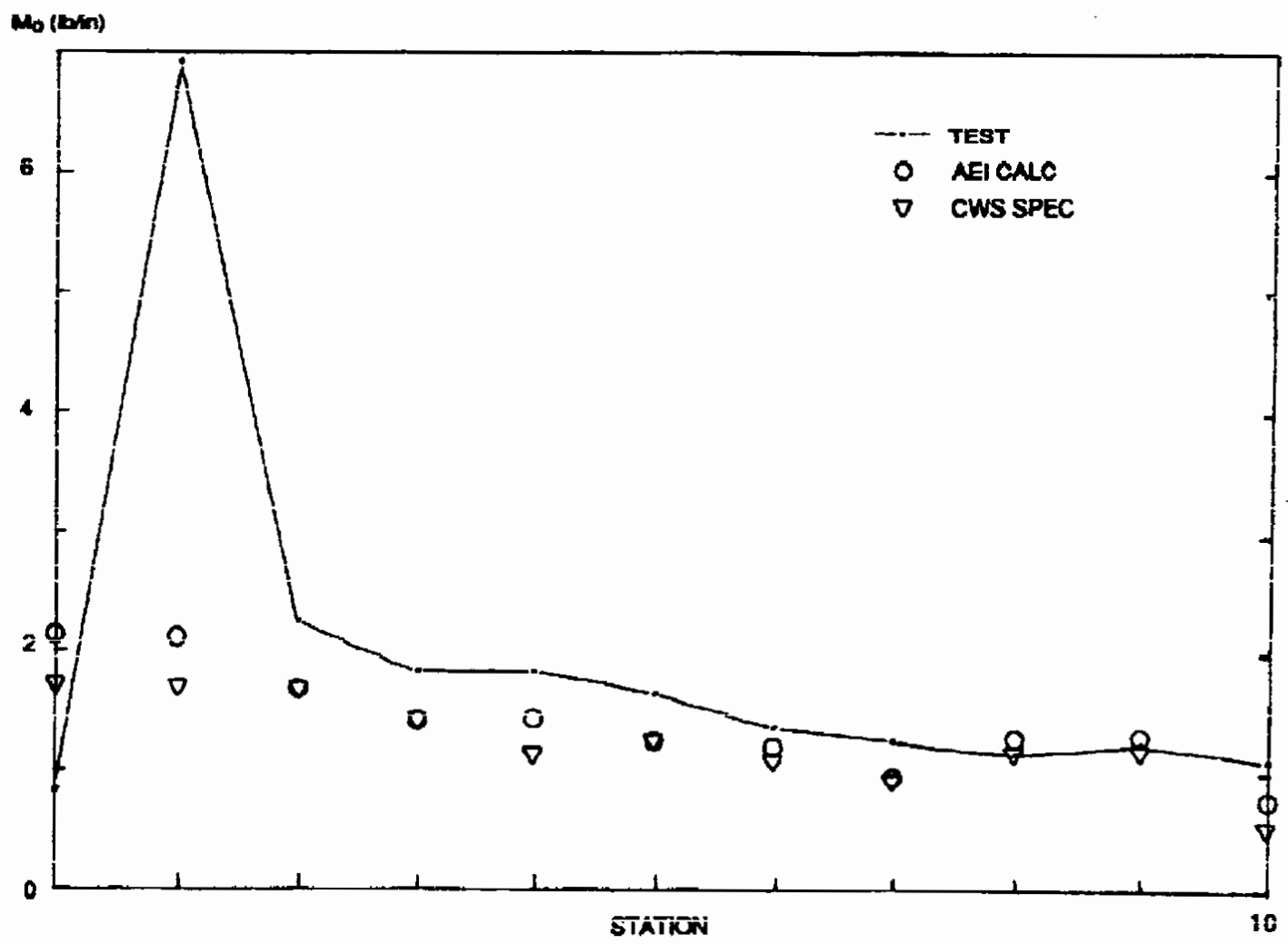

FIG. 5.33 CARTER BLADE RUNNING MASS COMPARISONS

However, the only accurate method for determining mass accuracy is in the frequency test comparisons below. 


\subsubsection{0: VERIFICATION BY FREQUENCY OF VIBRATION TESTS}

The frequency of vibration tests resulted in the first three fundamental modes in flap and lead-lag, well-separated; two fundamental torsional modes were also produced: one called "root torsion" in which the blade performs as a rigid body about the I-beam root segment at the root rib, and the next called "outboard torsion" in which the outboard blade oscillates about the midblade, under the major influence of the outboard ballast weights (See Figure 3.15).

The frequency comparisons are given in Table 5.15 for

1) The Carter design laminate and weights.

2) The AEI calculated value.

3) The AEI calculated value with no ballast.

4 ) The AEI calculated laminate and measured weights.

5) The test results.

As can be seen, the following conclusions can be made about the flap values:

1) All the calculated values had lower masses than the actual blade and thus had higher frequencies of vibration.

2) The calculated value with ballast weight omitted ("AEI-No ballast") had the highest frequencies of all, as expected; this illustrates the degradation of blade natural frequency by adding ballast weights.

3) With the correct (measured) mass distribution, which includes over-specified resin, mechanisms, and so on the flap frequencies are correctly predicted.

TABLE 5.15: CARTER BLADE FLAPWISE FREQUENCY COMPARISON

\begin{tabular}{lccccc} 
Mode & Test & $\begin{array}{c}\text { CARTER } \\
\text { design }\end{array}$ & $\begin{array}{c}\text { AEI } \\
\text { calc }\end{array}$ & $\begin{array}{c}\text { AEI } \\
\text { no ballast }\end{array}$ & $\begin{array}{c}\text { AEI } \\
\text { test weights }\end{array}$ \\
\hline 1st flap & 0.59 & 0.76 & 0.63 & 0.98 & 0.60 \\
2nd flap & 2.73 & 3.13 & 2.90 & 5.54 & 2.59 \\
3rd flap & 7.40 & 8.43 & 8.49 & 13.45 & 7.42 \\
1st lag & 3.00 & 3.08 & 3.16 & - & 3.03 \\
2nd lag & 16.0 & 12.63 & 19.79 & - & 17.56 \\
3rd lag & 40.4 & 33.01 & 51.78 & - & 43.74 \\
root torsion only & 13.25 & - & - & - & - \\
1st outboard torsion & 19.74 & 19.76 & 15.3 & - & 14.89 \\
\hline
\end{tabular}

The conclusions about the lead-lag values are similar; the test weights correctly predict the frequencies.

For the torsional frequencies, these conclusions can be drawn:

1) The root torsion (rigid body mode) was not predicted since root torsional rigidity GJ was not reduced to the I-beam value in the code. This was not considered realistic for the blade study since this motion is really a change in root pitch and not an elastic twist. However, this frequency (degree of freedom) with the Carter dashpot snubber in place will cause the root pitch to change slightly when the blade is in operation. Actually this vibration mode directly affects the root pitch setting and the operation of the pitch-up snubber mechanism in the field, and is bound to be troublesome to the turbine on both counts.

2) The torsional frequency is correctly predicted by the Carter design specification, but not by the actual blade weights. The error is in the actual placement of the "extra mass" seen in the section test weights. They were assumed to be added to the section $\mathrm{cg}$, but in actuality were close to the elastic axis, thus decreasing section mass moment of inertia. This is not considered to be a large mismatch or error since all the other torsional values do check with the data. A refinement in the Carter 300 model would iterate with the "extra masses" to find a new $\mathrm{cg}$ (and section mass moment of inertia) to raise the torsional frequency slightly. 

5.16 .

The final blade weights (cumulative), section running mass, and section cg's are given in Table

TABLE 5.16: CARTER BLADE: FINAL MASSES AND SECTION CG'S

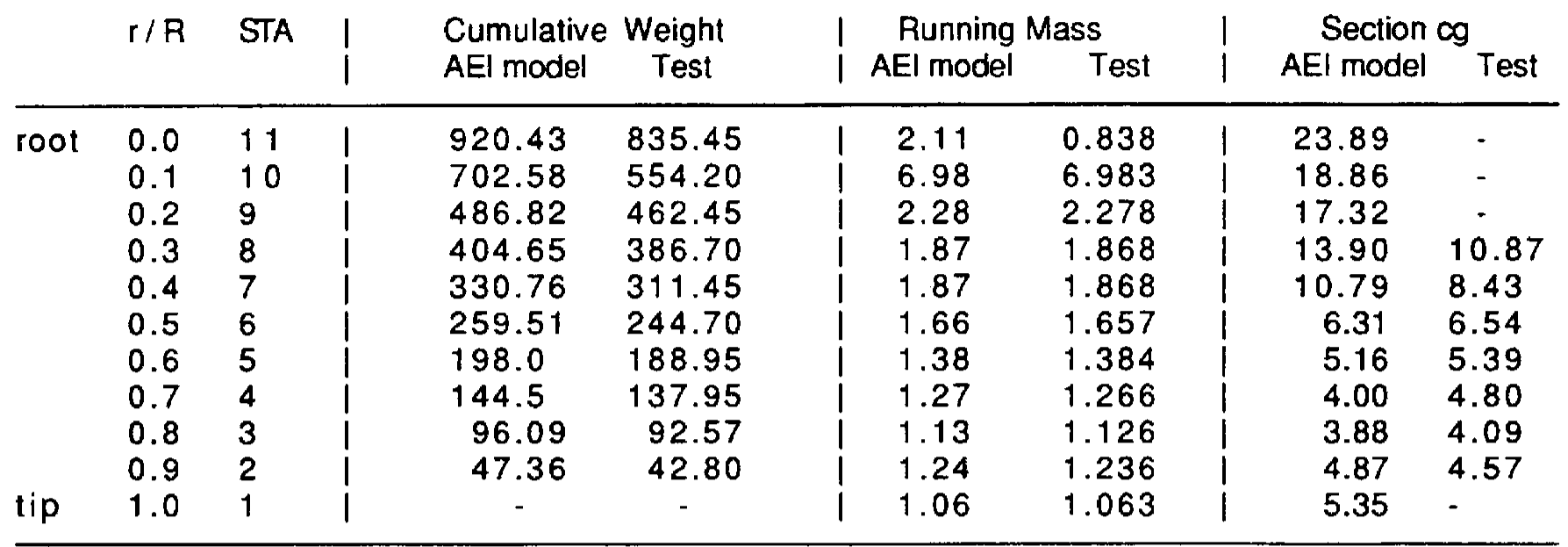

5.4.11: FINAL AEROELASTIC MODEL

The final values of the Carter 300 aeroelastic blade model are shown in Table 5.17.

TABLE 5.17: CARTER BLADE: FINAL AEROELASTIC BLADE MODEL

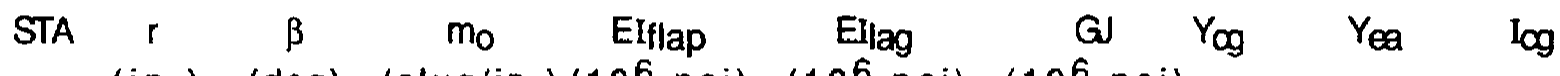
(in.) (deg) (slug/in.) $\left(10^{6} \mathrm{psi}\right)\left(10^{6} \mathrm{psi}\right)\left(10^{6} \mathrm{psi}\right)$

\begin{tabular}{lrrrrrrrrrr}
\hline root & 11 & 0 & - & 2.11 & 100.10 & 2817 & 518.30 & 23.89 & 21.92 & 704.6 \\
& 10 & 40.3 & 26.1 & 6.98 & 100.10 & 2817 & 518.30 & 18.86 & 21.92 & 781.9 \\
& 9 & 80.6 & 15.5 & 2.28 & 100.10 & 1980 & 251.80 & 17.32 & 17.20 & 366.8 \\
& 8 & 120.8 & 9.4 & 1.87 & 84.24 & 1756 & 128.40 & 13.90 & 13.71 & 194.9 \\
& 7 & 161.1 & 4.5 & 1.87 & 781.10 & 7846 & 63.50 & 10.79 & 11.16 & 106.4 \\
& 6 & 201.4 & 2.3 & 1.66 & 260.70 & 4608 & 41.01 & 6.31 & 10.48 & 98.3 \\
& 5 & 241.7 & 2.0 & 1.38 & 140.70 & 2699 & 23.34 & 5.16 & 8.91 & 56.9 \\
& 4 & 281.9 & 2.0 & 1.27 & 89.14 & 1650 & 13.36 & 4.00 & 7.41 & 33.8 \\
& 3 & 322.2 & 2.0 & 1.13 & 63.98 & 1244 & 10.76 & 3.88 & 8.02 & 28.2 \\
& 2 & 362.5 & 2.2 & 1.24 & 78.42 & 1527 & 15.20 & 4.87 & 9.82 & 40.3 \\
tip & 1 & 402.8 & 2.6 & 1.06 & 87.98 & 1506 & 14.98 & 5.35 & 9.81 & 37.8 \\
\hline
\end{tabular}




\subsection{ELASTIC TWIST CALCULATION}

\section{1: $\quad$ GENERAL}

After the blade structural models were developed, the next step was to calculate the expected elastic twist under normal operating wind turbine conditions. This was assumed to be steady-state (e.g., gravity and cyclic loads were not considered). This task consisted of calculating the blade section pitching moments and forces, and then calculating the elastic twist due to those applied loads. These loads are both inertial and aerodynamic. This was the primary theoretical goal of the project and had to be theoretical since no flight testing was done. Briefly, the inertial and aerodynamic loads were used with the structural deflection program described above to obtain the elastic twist due to each component of loading in order to ascertain design significance; that is, to point out where most of the elastic twist in flight originates.

\section{2: CALCULATION OF BLADE CENTRIFUGAL TENSION AND MOMENTS}

The inertial loads on the blade sections are caused by centrifugal forces (and moments) acting on the mass elements. This section derives the centrifugal loads on a flying blade (see also Ref. 28, for example). Up to now the analysis has been static, with beam mass and stiffness properties expressed, calculated and verified. Now the mass properties are combined with the operating condition to give the inertial blade loads.

Consider the discrete lumped mass blade shown in Figure 6.1, which is the easiest way to express centrifugal forces. The figure shows an undeflected lumped mass blade at precone angle $\beta p c$, rotating at angular speed $\Omega$ about the z-axis. The root reactions are $F_{0}$ and $M_{0}$, or centrifugal force (tension) and moment. Mass elements at radius $r_{n}$ and flap deflection $z_{n}$ have an inertial centrifugal force of $m_{n} r_{n} \Omega^{2}$, as shown.

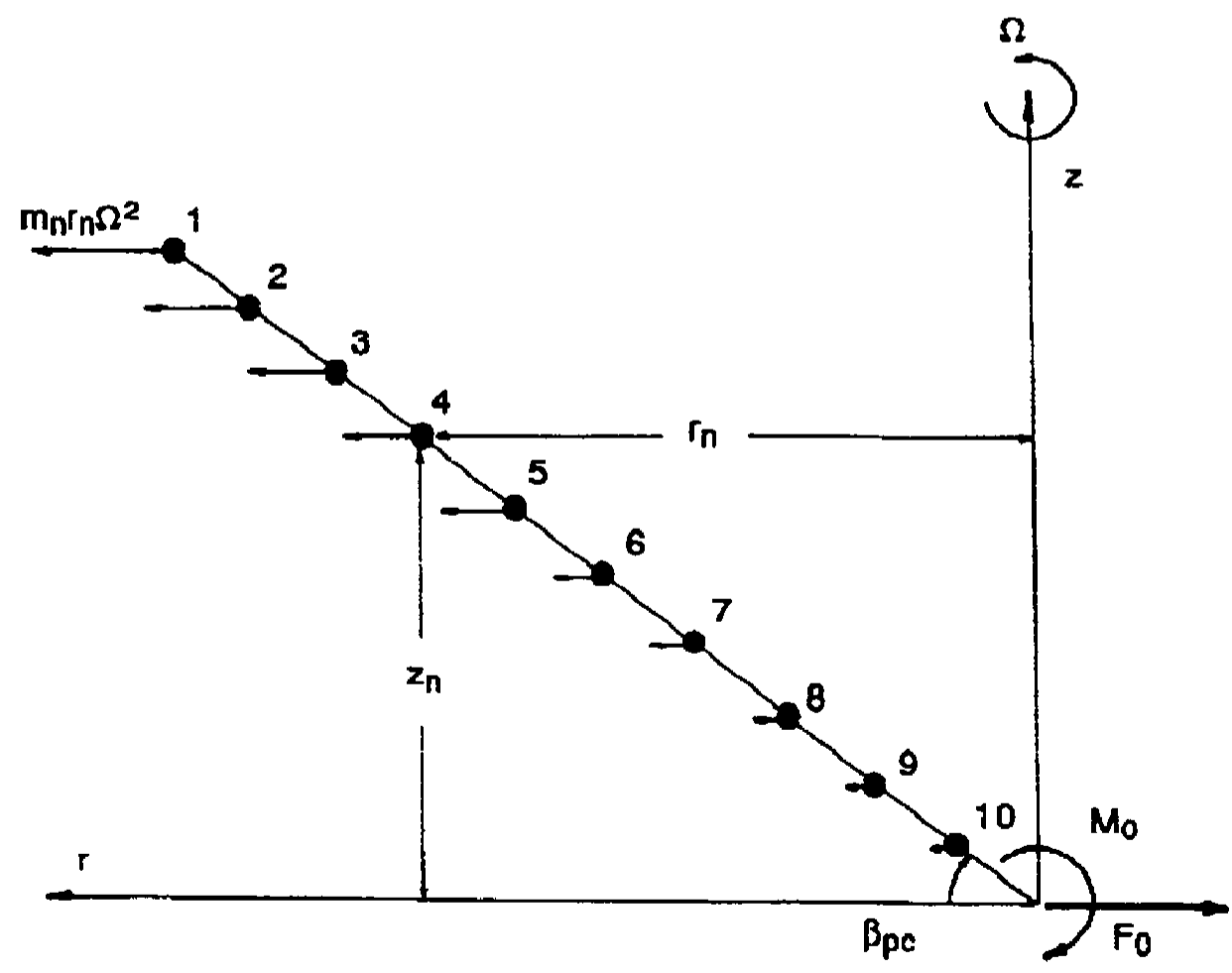

FIG. 6.1 DISCRETE LUMPED MASS CASE 
Basic static theory states

$$
\begin{aligned}
& F_{\text {centrifugal } i=m_{i}} r_{i} \Omega^{2} \\
& F_{0}=\sum_{n=1}^{N} m_{n} r_{n} \Omega^{2} \\
& M_{0}^{N}=\sum_{n=1}^{N} m_{n} r_{n} z_{n} \Omega^{2}
\end{aligned}
$$

and the centrifugal moment at any mass element $n$ is

$$
\begin{aligned}
& M_{n}=\sum_{i=1}^{n-1} F_{\text {cent }} i\left[z_{i}-z_{n}\right] \\
& \quad n-1 \\
& \quad=\sum_{i=1}^{n} F_{\text {cent }} i z_{i}-\sum_{i=1}^{n-1} F_{\text {cent } i} z_{n}
\end{aligned}
$$

These expressions can be generalized to a continuum form, just by imagining many lumped masses, (which in the limit are differential elements) and by starting the integration at the blade tip rather than the root. Note: here $x=R-r$ and is zero at the tip.)

$$
\begin{aligned}
& \text { Blade Tension at } x=\int_{0}^{x} m r \Omega^{2} d x \\
& \text { Blade Moment at } x=\int_{0}^{x} m r \Omega^{2} z d x-z(x) * \text { [Blade Tension] }
\end{aligned}
$$

The above expressions can be easily checked by hand with a simple blade, such as the uniform UTRC blade.

Referring to Figure 6.1 , it can be seen that the calculation algorithm does not change if the blade shape is curved, as it will be when under the combined action of section forces and moments. The calculation program starts with an undeflected (straight) blade and then allows it to deflect in flap, lag, and iwist until a loop convergence is reached (Appendix 10.5). 


\section{3: DERIVATION OF SECTION TORSION}

There are, in general, five significant points on the blade section:

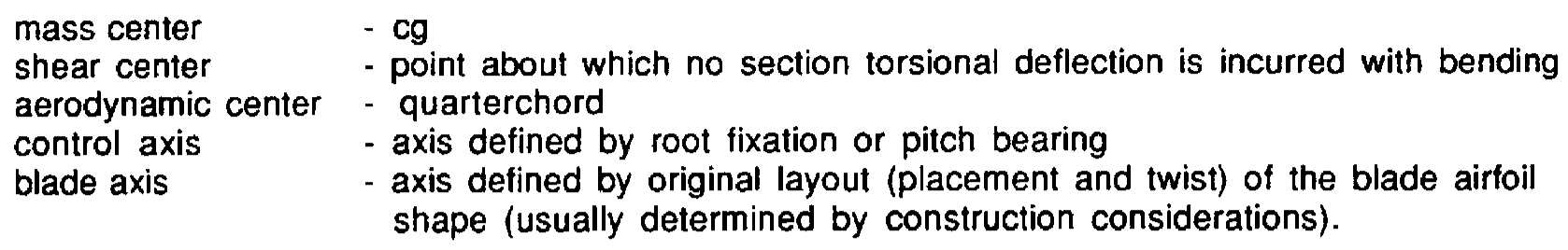

Clearly the first three above are not necessarily straight line loci on the blade, but the last two are (Fig. 1.4). For purposes of calculating the elastic twist the control axis was used. It is defined by the centroid of the blade root fixture as it would be if a pitching bearing were used. Then the elastic twist can be directly added or subtracted from the pitch angle changes. Thus, the four points that must be known or determined for each blade section are $\mathrm{cg}$, shear center, aerodynamic center, and control axis. The cg's and shear centers are calculated in the section program above. The aerodynamic center is placed at the quarterchord, and the control axis is specified by the blade fixture. The section airfoil offsets, which locate these centers relative to each other are also input to the program.

The section torsion that causes elastic twist is most easily calculated relative to the shear center of the section. This would be the "elastic axis" of a uniform blade. The above is true because torsion can be produced by a section force only if the line of action of the force does not pass through the shear center. Section moments then are all added together and must be resisted by an equal and opposite elastic moment at that section's shear center. The calculation then proceeds, introducing the section torsional stiffness GJ, which then gives the differential elastic twist for that section. To determine the total elastic twist, these section values must be integrated starting at the blade root.

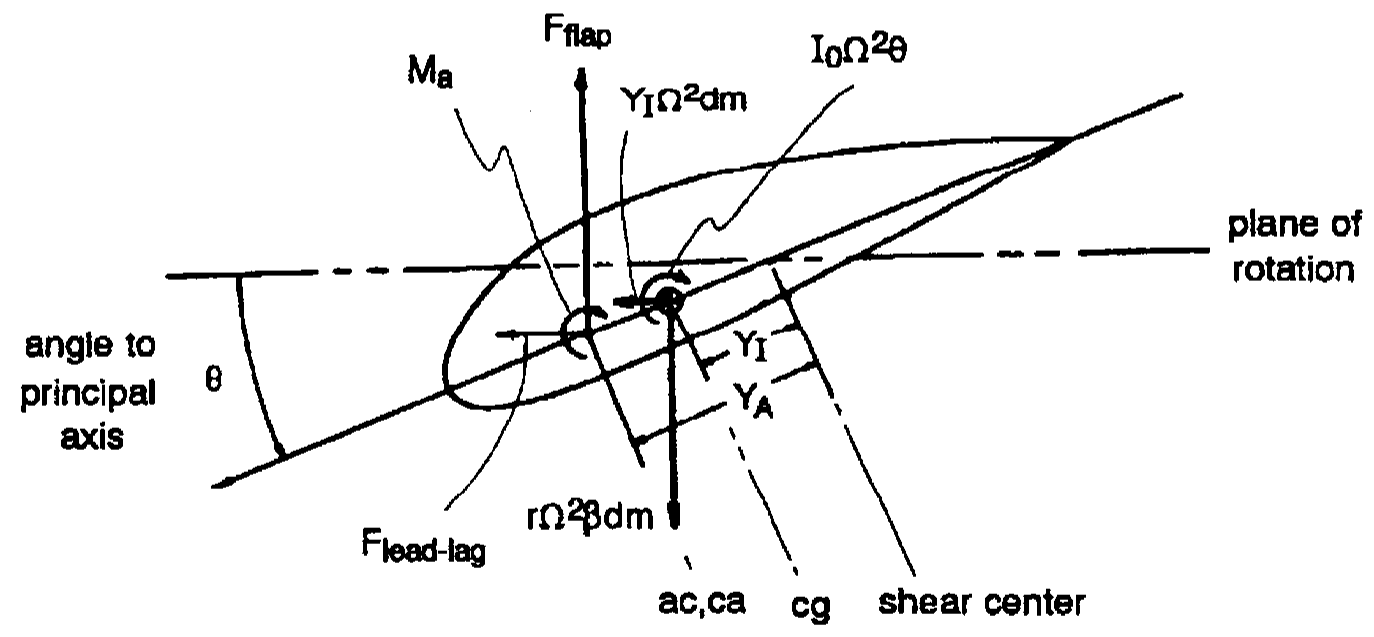

FIG. 6.2 BLADE SECTION FREE BODY DIAGRAM

The differential section steady torsional moments are shown in Figure 6.2, the section free body diagram (see Ref. 2 for a complete derivation): (Note: the offsets $Y_{I}$ and $Y_{A}$ have been simplified for clarity.)

1. Aerodynamic forces in flapping ( $\left.F_{f l a p}\right)$ and lead-lag (Flead-lag) act at the aerodynamic center, with a moment arm to the shear center, $Y_{A} \cos \theta$.

2. Aerodynamic moment $\left(\mathrm{M}_{\mathrm{a}}\right)$ acts at the aerodynamic center and is directly translated to the shear center. 
3. The primary centrifugal moment is due to the vertical force component (flapping deflection) and is simply the product of the section force $r \Omega^{2} \beta \mathrm{dm}$ times its moment arm from the shear center $Y_{I} \cos \theta$.

4. The secondary centrifugal moment is due to the horizontal centrifugal force component, and comes from the vertical distribution of mass around the elastic axis; this is shown as $Y_{I} \Omega^{2}$ $\mathrm{dm}$ in the free body diagram. This force is depicted in the following sketch:

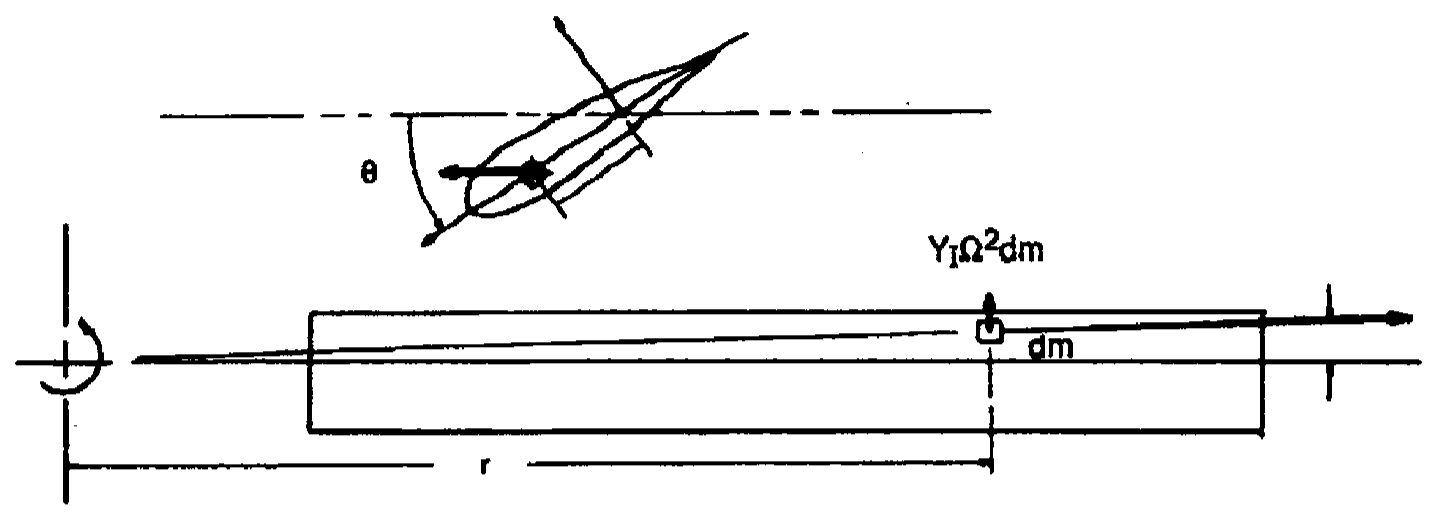

5. The third part of the centrifugal moment is the "tennis racket" or propeller moment, $\mathrm{I}_{0} \Omega^{2}$ $\theta$, where $I_{O}$ is the section pitching mass moment of inertia about the $\mathrm{cg}$ and $\theta$ is the angle from the plane of rotation to the section's principal mass axis. Tennis racket moment is simply caused by a pitch (or a twist) rotation of the section mass away from the plane of rotation, which produces a centrifugal force couple on the mass elements above and below the plane of rotation tending to reduce pitch. This moment is zero when $\theta=0$, or when the plane of rotation passes through the principal (major) mass axis of the blade (which is also referred to as the section principal axis of inertia). See the following sketch:

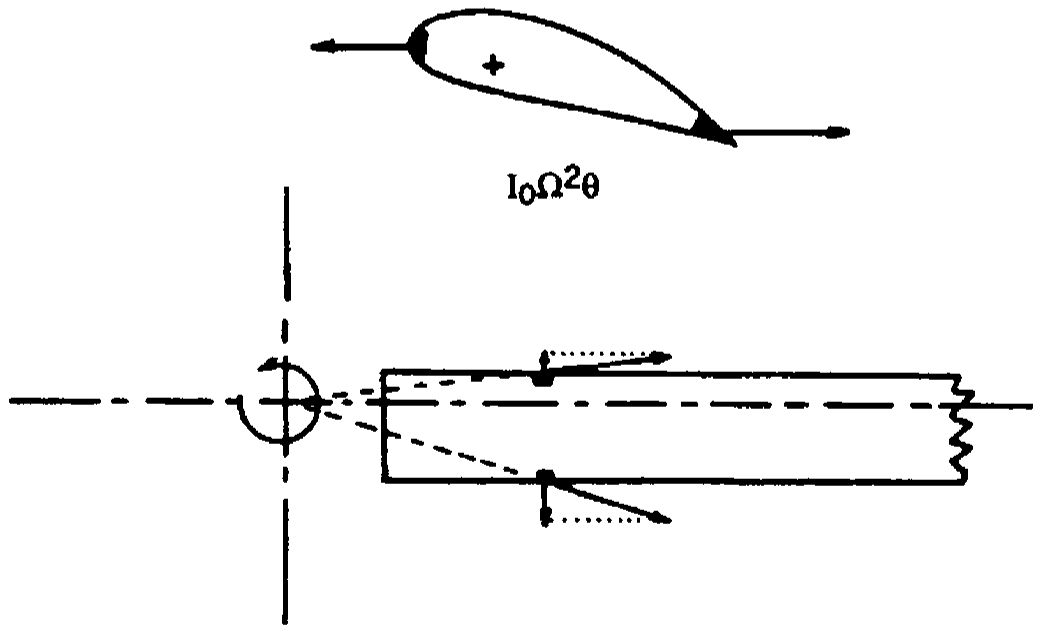

These are the steady torsional moments acting on the section to produce local twist. These torsional moments are calculated in the second part of the STRESS ELASTIC program, and the deflections are then found and integrated to obtain the elastic twist distribution of the blade. The simplified moment arms $Y_{I}$ and $Y_{A}$ from Figure 6.2 are not used in the program, which includes instead all the section moment arm contributions (e.g. $x, y$ components). 
The section ballast weight contributions are done as separate calculations in the program to allow separate assessment of their effects by the blade designer. A ballast weight in the leading edge, for instance, adds to the tennis racket moment and has both the horizontal and vertical centrifugal components as above, again on their (new) moment arms to the shear center. [The basic tennis racket moment, if transposed to the shear center using the parallel axis theorem, confirms the horizontal centrifugal component described above (see Ref. 2).]

Each elastic twist contribution is listed in the program output to assess its individual significance:

1. Aerodynamic Contribution includes torsion due to lift force, inplane aero force, and aerodynamic moment.

2. Centrifugal Contribution

3. Tennis Racket Contribution

4. Ballast Contribution includes the basic section torsion due to horizontal and vertical centrifugal force (less ballast).

includes the basic section tennis racket torsion (less ballast). includes all the contributions of ballast weight, including centrifugal (horizontal and vertical) and additional tennis racket moment.

\section{4: $\quad$ AERODYNAMIC LOADS}

The aerodynamic section loads were calculated with a strip theory similar to the PROPPC code (Ref. 7 and next section) since PROPPC has no section pitching moment calculation. The loads were output (expressed) as distributed load at the aerodynamic center (e.g. Ib/in. or in.-lb/in.) along the span. The airfoil $C_{L} / C_{d}$ section data were corrected by the aerodynamic consultant (S.J. Miley) to reflect airfoil deviations noted in the laboratory inspection of the blade airfoil section (Appendix 10.6). At and above stall no reliable theory exists; SERI had anticipated this and specified post-stall routines for the code (see Sec. 7.1). 


\subsection{GENERAL DISCUSSION: OPERATING BLADE ELASTIC DEFLECTIONS}

Figure 6.3 shows the output of a calculation for the UTRC blade, giving all the flexural output. URR. RABC,FUS.FLEXEAM.1-21-88 UTRC.BLADE. 30NPH.LOAD. 1-21-88

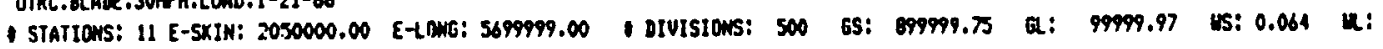

\begin{tabular}{|c|c|c|c|c|c|c|c|c|c|c|}
\hline STATION & $\begin{array}{c}\text { TIP } \\
1\end{array}$ & 2 & 3 & 4 & 5 & 6 & 7 & $B$ & 9 & 10 \\
\hline STA POS & 0.00 & 19.20 & 38.40 & 57.60 & 76.80 & 96.00 & 115.20 & 134.40 & 153.60 & 172.80 \\
\hline$x \cdot \cos$ & 0.00 & 0.00 & 0.00 & 0.00 & 0.00 & 0.00 & 0.00 & 0.00 & 0.00 & 0.00 \\
\hline$y-\angle B A D$ & -0.87 & -0.89 & -0.72 & -0.44 & -0.34 & -0.18 & -0.04 & 0.03 & 0.04 & 0.03 \\
\hline $2-20 n$ & 5.17 & 4.51 & 4.00 & 2.99 & 2.17 & 1.57 & 1.13 & 0.81 & 0.61 & 0.47 \\
\hline$P$ - WONEKT & 5.48 & 3.97 & 3.09 & 3.03 & 2.49 & 2.06 & 1.70 & 1.85 & 1.50 & 1.49 \\
\hline
\end{tabular}

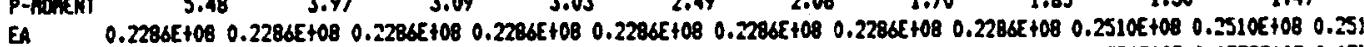

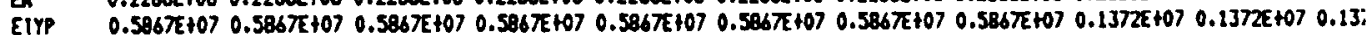

EIIP $0.3240 E+090.3240 E+090.3240 E+090.3240 E+090.3240 E+090.3240 E+09 \quad 0.3240 E+090.3240 E+090.1370 E+090.1370 E+090.13 i$

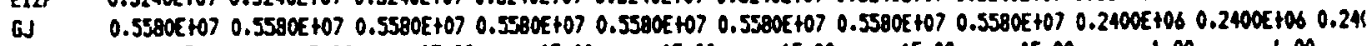

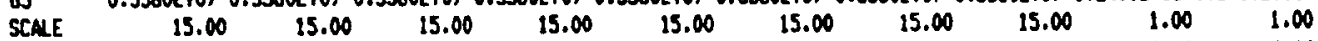

\begin{tabular}{llllllllll} 
ISCAL & 1.00 & 1.00 & 1.00 & 1.00 & 1.00 & 1.00 & 1.00 & 1.00 & 0.10 \\
\hline
\end{tabular}

$\begin{array}{lllllllllll}\text { Y-BCENT } & 3.40 & 3.40 & 3.40 & 3.40 & 3.40 & 3.40 & 3.40 & 3.40 & 5.50 & 5.50\end{array}$

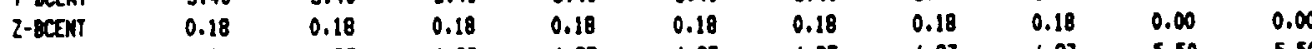

$\begin{array}{lllllllllll}\text { r-siorir } & 6.93 & 6.93 & 6.93 & 6.93 & 6.93 & 6.93 & 6.93 & 6.93 & 5.50 & 5.50\end{array}$

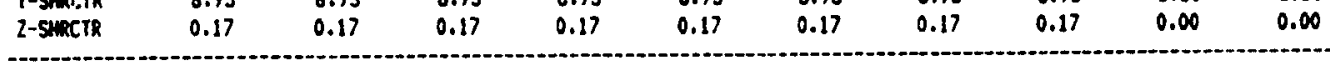

\begin{tabular}{|c|c|c|c|c|c|c|c|c|c|c|}
\hline $\begin{array}{l}y-C 0 \\
z-c 6\end{array}$ & $\begin{array}{l}1.69 \\
0.18\end{array}$ & $\begin{array}{l}4.69 \\
0.18\end{array}$ & $\begin{array}{l}4.69 \\
0.18\end{array}$ & $\begin{array}{l}9.69 \\
0.18\end{array}$ & $\begin{array}{l}4.69 \\
0.18\end{array}$ & $\begin{array}{l}9.67 \\
0.18\end{array}$ & $\begin{array}{l}4.64 \\
0.18\end{array}$ & 0.18 & 0.00 & 0.00 \\
\hline $\begin{array}{l}z-C G \\
1-E A \\
I-C G\end{array}$ & 10.51 & 10.51 & $\begin{array}{r}10.51 \\
8.38\end{array}$ & $\begin{array}{r}10.51 \\
8.78\end{array}$ & $\begin{array}{r}10.51 \\
8.36\end{array}$ & $\begin{array}{r}10.51 \\
8.38\end{array}$ & $\begin{array}{r}10.51 \\
8.39\end{array}$ & $\begin{array}{r}10.51 \\
8.38\end{array}$ & $\begin{array}{l}1.74 \\
1.74\end{array}$ & $\begin{array}{l}1.74 \\
1.74\end{array}$ \\
\hline $\begin{array}{l}\text { BALLAST } \\
\text { TOTA UT }\end{array}$ & $\begin{array}{l}0.00 \\
0.13\end{array}$ & $\begin{array}{l}0.00 \\
0.43\end{array}$ & $\begin{array}{l}0.00 \\
0.43\end{array}$ & $\begin{array}{l}0.00 \\
0.43\end{array}$ & $\begin{array}{l}0.00 \\
0.43\end{array}$ & $\begin{array}{l}0.00 \\
0.43\end{array}$ & $\begin{array}{l}0.00 \\
0.13\end{array}$ & $\begin{array}{l}0.00 \\
0.43\end{array}$ & $\begin{array}{l}0.00 \\
0.32\end{array}$ & $\begin{array}{l}0.00 \\
0.32\end{array}$ \\
\hline $\mathrm{EEU} Y-\mathrm{Cg}$ & 4.69 & 4.69 & 4.69 & 1.69 & 4.69 & 4.69 & 4.69 & 4.69 & 5.50 & 5.50 \\
\hline $\begin{array}{l}\text { WeV } t-c 6 \\
\text { WEU } 1-c 6\end{array}$ & $\begin{array}{l}0.18 \\
8.38\end{array}$ & $\begin{array}{l}0.18 \\
8.38\end{array}$ & $\begin{array}{l}0.18 \\
8.38\end{array}$ & $\begin{array}{l}0.18 \\
8.38\end{array}$ & $\begin{array}{l}0.18 \\
8.38\end{array}$ & $\begin{array}{l}0.18 \\
8.38\end{array}$ & $\begin{array}{l}0.18 \\
8.38\end{array}$ & $\begin{array}{l}0.18 \\
8.38\end{array}$ & $\begin{array}{l}0.00 \\
1.74\end{array}$ & $\begin{array}{l}0.00 \\
1.74\end{array}$ \\
\hline RDO-WCHT & 0.00 & B. 18 & 16.36 & 24.54 & 32.72 & 40.80 & 49.08 & 57.44 & 64.74 & 70.80 \\
\hline V-ARO & 0.00 & 92.68 & 174.18 & 241.06 & 290.36 & 325.99 & 351.64 & 370.06 & 383.43 & 394.27 \\
\hline B-ACEO & 0.00 & 907.62 & 3487.33 & 7501.78 & 12631.62 & 18564.69 & 23095.99 & 32022.73 & 39264.74 & 46740.13 \\
\hline$Y$-AEROD & -0.20 & -0.19 & -0.18 & -0.18 & -0.16 & -0.15 & -0.12 & -0.09 & -0.04 & -0.01 \\
\hline 2-AEROD & 292.85 & 257.55 & 222.31 & 187.30 & 152.77 & 119.03 & 86.46 & 55.13 & 26.49 & 6.96 \\
\hline$z$-conting & 16.73 & 15.06 & 13.39 & 11.71 & 10.04 & 8.37 & 8.69 & 5.02 & 3.35 & 1.67 \\
\hline SECTI & Basion & TO: $1 t$ & mose bu & & & & & & & \\
\hline P-morictit & 5.48 & 3.97 & 3.09 & 3.03 & 2.49 & 2.06 & 1.70 & 1.65 & 1.50 & 1.49 \\
\hline $\begin{array}{l}\text { LIFT } \\
\text { TOT-AERO }\end{array}$ & $\begin{array}{l}-16.54 \\
-11.06\end{array}$ & $\begin{array}{l}-14.46 \\
-10.49\end{array}$ & $\begin{array}{r}-12.82 \\
-9.73\end{array}$ & $\begin{array}{l}-9.58 \\
-6.55\end{array}$ & $\begin{array}{l}-6.95 \\
-4.46\end{array}$ & $\begin{array}{l}-5.01 \\
-2.95\end{array}$ & $\begin{array}{l}-3.60 \\
-1.90\end{array}$ & $\begin{array}{l}-2.56 \\
-0.91\end{array}$ & $\begin{array}{l}-3.18 \\
-1.68\end{array}$ & $\begin{array}{l}-2.46 \\
-0.97\end{array}$ \\
\hline
\end{tabular}

\begin{tabular}{|c|c|c|c|c|c|c|c|c|c|c|}
\hline \multicolumn{11}{|c|}{ SECTIOM ALOXE: } \\
\hline mis-Rext & 0.05 & 0.05 & 0.05 & 0.05 & 0.05 & 0.05 & 0.05 & 0.05 & 0.00 & 0.00 \\
\hline FLAP-LEF & 9.59 & 8.52 & 7.45 & 6.39 & 5.33 & 4.29 & 3.28 & 2.31 & 0.00 & 0.00 \\
\hline LD-LAG & 0.01 & 0.01 & 0.01 & 0.01 & 0.01 & 0.01 & 0.01 & 0.01 & 0.00 & 0.00 \\
\hline TOIAL & 9.65 & 8.58 & 7.51 & 6.44 & 5.38 & 4.35 & 3.33 & 2.37 & 0.00 & 0.00 \\
\hline \multicolumn{11}{|c|}{ BALLAST ALOAE: } \\
\hline TWS-RCXT & 0.00 & 0.00 & 0.00 & 0.00 & 0.00 & 0.00 & 0.00 & 0.00 & 0.00 & 0.00 \\
\hline CENTRIF & 0.00 & 0.00 & 0.00 & 0.00 & $0 . \infty$ & 0.00 & 0.00 & 0.00 & 0.00 & 0.00 \\
\hline TOTAL & $0 . \infty$ & 0.00 & $0 . \infty$ & 0.00 & 0.00 & 0.00 & 0.00 & 0.00 & 0.00 & 0.00 \\
\hline
\end{tabular}

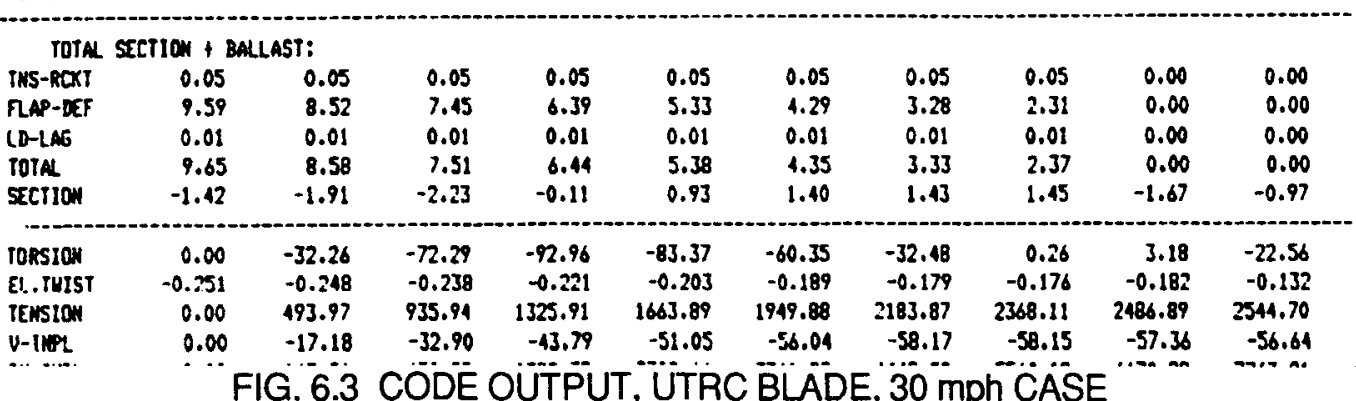

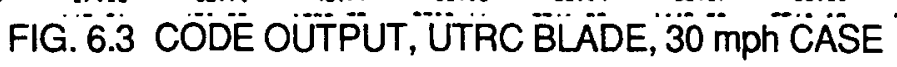


This is shown as an example to describe the format:

Heading- $\quad$ "UTRC blade@ 30 mph load"

rotor speed $(\Omega)=11.31 \mathrm{rad} / \mathrm{sec}=108 \mathrm{rpm}$

pitch angle $=0$ degrees

coning angle $=5$ degrees

The output by station is listed from tip to root, station 1 to 11 ; this divides the blade into 10 equal segments. The top line shows the radius value measured from the tip to each station (thus the root value is the radius of the rotor: $192 \mathrm{in.}$ or $16 \mathrm{ft}$ ).

Blade section aerodynamic load is listed next, in $X, Y$, and $Z$ directions (tension, lead-lag and flap) and pitching moment, all referenced to the quarterchord. These loads are the output of the aerodynamic strip theory. The remainder of this part gives the section stiffness and geometric properties. Bending and shear centers (elastic axis) are given next. It can be seen that this is a uniform blade; the values are constant for the outer $70 \%$ of the blade, which represents the pultrusion portion. The inner $30 \%$, for the UTRC rotor, consists of a flexbeam with different properties. Weights, cg's, and moments of inertia are followed by a ballast weight section (for this blade the ballast is zero, as can be seen). Finally, the running "blade weight" line ends with the total blade weight $(76.9 \mathrm{lb})$, which was verified in the bench test.

The aerodynamic flapping shear and bending moments are given next. [Note: these must be integrated from the tip, as shown.] The root values are the aerodynamic hub shear (400 $\mathrm{lb})$ and bending moment $(54,367$ inch-lb) for this condition. Next, the simple blade deflection is given, that would occur with the aerodynamic load alone (e.g., -0.2 in. inplane and 292 in. in flap) to compare with actual blade deflections. The final line of this part gives the flap deflection due to coning angle (e.g. 16.7 in. at the tip). These are the initial trial values of blade flap deflection. [Note: In this case the trial value is not realistic: 292 in. of flap deflection is twice the rotor radius! The linear beam assumptions have clearly been violated by the large airload at $30 \mathrm{mph}$; as seen below, the aeroelastic moment is largely counteracted by the centrifugal moment bringing the algorithm back into its assumption bounds.]

The next four parts of the output list all the blade section pitching moments (in in.-ib, positive nose-down):

1 - Aerodynamic pitching moment.

2 - Aerodynamic moment due to lift offset from shear center (total aerodynamic moment).

3 - Inertial centrifugal moment due to tennis racket effect.

4 - Inertial moment due to flap deflection.

5 - Inertial moment due to lead-lag deflection (total section inertial moment).

6 - 7 - Inertial moments due to ballast.

$8-10$ - Total section inertial moments due to tennis racket, flap deflection, lead-lag deflection.

11 - Total inertial moment.

12 - Total section moment, or the sum of the inertial and aerodynamic contributions

[Note: on this blade at the tip, the aerodynamic moment is 11 in.-lb nose up and the inertial moment 9.65 in.-lb nose down, yielding 1.4 in.-lb nose up net section moment.]

The torsion is just the integration of the section moment distribution and appears next. Following is the elastic twist in degrees. [Note: this case, $30 \mathrm{mph}$, has only 0.25 degrees, nose up, elastic twist at the tip.]

The following five lines give the blade structural reactions at each station:

tension (lb)

lead-lag shear (lb)

lead-lag bending moment (in.-lb)

flap shear (lb)

flap bending moment (in.-lb) 
The root values constitute the steady hub loading specification for this operating condition. This example shows the UTRC hub experiencing $2564 \mathrm{lb}$ of centrifugal force (tension), $56 \mathrm{lb}$ of lead-lag shear, 8847 in. $\mathrm{lb}$ of torque, $400 \mathrm{lb}$ of flapping shear, and $4880 \mathrm{in}$.- lb of flap bending moment.

Following the principal axis angle and the M/EI values, the blade flexural deflections are given in inches, for lead-lag and flapping. This blade has $0.52 \mathrm{in.} \mathrm{of} \mathrm{inplane} \mathrm{deflection} \mathrm{and} 30 \mathrm{in}$. of flap deflection.

The elastic twist denotes the torsional deflection of this blade. It can be seen that the bulk of the twist occurs in the softer flexbeam portion. This is more obvious in the other UTRC cases that experience greater elastic twist.

Clearly, the centrifugal moments of this blade have reduced the tip deflection via centrifugal relief, which was the design intent. It should be noted that the flap deflection is not a straight line, since it represents the equilibrium position of a complex blade operating under complex aerodynamic and centrifugal loads.

\subsection{UTRC BLADE DEFLECTIONS}

The simplest blade is a uniform pultrusion connected to a torsionally flexible beam at its root $(.30$ STA). The shear centers (elastic axis) and mass centers are collinear and likewise the inertial terms. The flap deflection and elastic twist for the UTRC blade are given in the Tables 6.1 and 6.2 below and plotted in Figures 6.4 and 6.5 for wind speeds from $10-70 \mathrm{mph}$ (pitch $=0$ degrees, coning $=5$ degrees).

TABLE 6.1: UTRC FLAP DEFLECTION (in.)

\begin{tabular}{cccccccccccc} 
Vo(mph) & Root &. &. &. &. &. &. &. &. &. & Tip \\
\hline 10 & 0 & 1.5 & 2.6 & 3.4 & 4.1 & 4.8 & 5.5 & 6.2 & 6.8 & 7.5 & 8.2 \\
20 & 0 & 1.9 & 4.0 & 6.2 & 8.4 & 10.5 & 12.6 & 14.6 & 16.6 & 18.6 & 20.6 \\
30 & 0 & 2.2 & 5.0 & 8.0 & 11.1 & 14.2 & 17.3 & 20.5 & 23.6 & 26.8 & 30.0 \\
40 & 0 & 2.3 & 5.3 & 8.6 & 12.0 & 15.4 & 18.8 & 22.3 & 25.8 & 29.3 & 32.8 \\
50 & 0 & 2.4 & 5.6 & 9.1 & 12.7 & 16.3 & 19.9 & 23.5 & 27.1 & 30.7 & 34.4 \\
60 & 0 & 2.6 & 6.1 & 9.9 & 13.8 & 17.8 & 21.9 & 26.0 & 30.1 & 34.3 & 38.5 \\
70 & 0 & 2.8 & 6.6 & 11.0 & 15.5 & 20.0 & 24.7 & 29.3 & 34.0 & 38.7 & 43.4 \\
\hline
\end{tabular}

TABLE 6.2: UTRC ELASTIC TWIST (DEGREES)

\begin{tabular}{rrrrrrrrrrrr}
$V_{0}(\mathrm{mph})$ Root & \multicolumn{1}{c}{.} & \multicolumn{1}{c}{.} & \multicolumn{1}{c}{.} & \multicolumn{1}{c}{.} &. & \multicolumn{1}{c}{ Tip } \\
\hline 10 & 0 & 1.25 & 2.53 & 3.10 & 3.06 & 3.11 & 3.16 & 3.21 & 3.25 & 3.27 & 3.28 \\
20 & 0 & .25 & .55 & .70 & .70 & .70 & .71 & .73 & .75 & .77 & .78 \\
30 & 0 & -.13 & -.18 & -.18 & -.18 & -.19 & -.20 & -.22 & -.24 & -.25 & -.25 \\
40 & 0 & .10 & .32 & .46 & .44 & .45 & .44 & .43 & .42 & .40 & .39 \\
50 & 0 & .47 & 1.11 & 1.45 & 1.41 & 1.44 & 1.45 & 1.45 & 1.45 & 1.45 & 1.45 \\
60 & 0 & .90 & 2.03 & 2.61 & 2.55 & 2.60 & 2.63 & 2.65 & 2.65 & 2.66 & 2.66 \\
70 & 0 & 1.52 & 3.34 & 4.23 & 4.15 & 4.22 & 4.28 & 4.31 & 4.34 & 4.35 & 4.35 \\
\hline
\end{tabular}



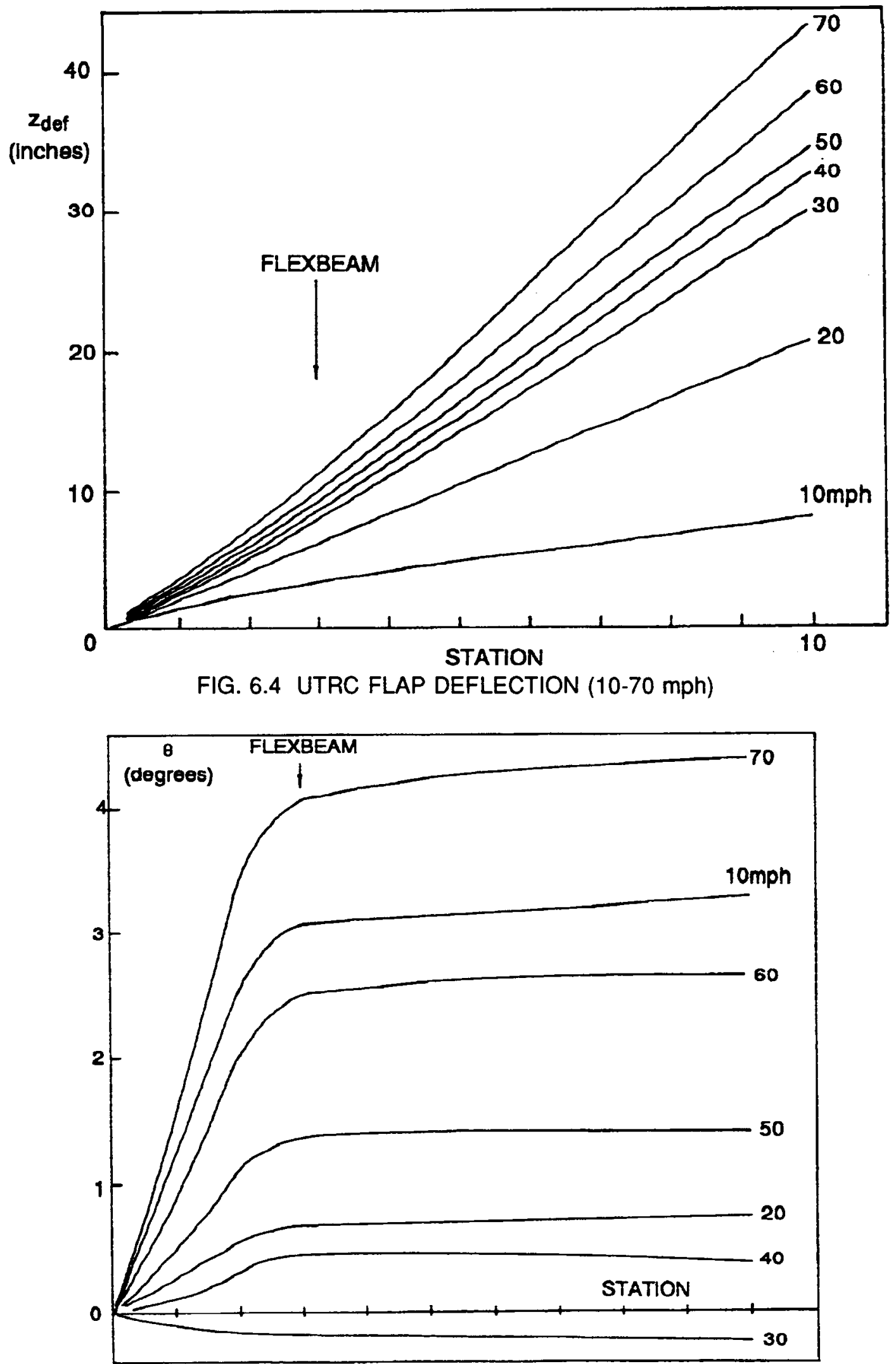

FIG. 6.5 UTRC ELASTIC TWIST (10-70 mph) 
The flap deflections (and lead-lag deflections not shown) are easy to understand. The aerodynamic bending moment increases with increasing wind speed, and the centrifugal moment does not. Therefore, the tip deflection increases as the aerodynamic moment increases. The static tip deflection due to straight coning angle is 16.7 inches (5 degrees); the $10 \mathrm{mph}$ values above are less than this, indicating the centrifugal moment is dominating the aerodynamic moment at $10 \mathrm{mph}$. This is borne out by examining the root flap moment for these cases, reproduced here from the printouts:

\begin{tabular}{cc}
$V_{0}(\mathrm{mph})$ & Root Flap Moment (in-lb) \\
\cline { 2 - 2 } 10 & -1843 \\
20 & 1380 \\
30 & 4280 \\
40 & 5460 \\
50 & 6670 \\
60 & 8790 \\
70 & 11190
\end{tabular}

The elastic twist is more complex. Not only do the gradients change along the blade, but also with wind speed; the tip elastic twist is large at 10 and $70 \mathrm{mph}$, but nearly nothing at $30 \mathrm{mph}$. To discover the sources of this twist, each section moment must be examined over the range $10-70 \mathrm{mph}$. Referring to Tables 6.1 and 6.2 :

\section{at $10 \mathrm{mph}:$}

The 16.7 in. of static flap coning are reduced to 8.2 in. by centrifugal relief. Section twisting moments are dominated by the airfoil pitching moment (NACA 23012) and the centrifugal moment due to flapping, both nose down. About one-third of this is offset by a nose-up lift moment due to aerodynamic center offset. The other section moments are negligible (tennis racket, lead-lag). The net nose-down moment amounts to $290 \mathrm{in}$.-lb of torsion at 0.30 STA, twisting the flexbeam about 3.1 degrees. An additional 0.2 degrees of twist occurs in the much stiffer pultrusion portion of the blade.

at $30 \mathrm{mph}$ : (see Fig. 6.3):

The airfoil pitching moment has not changed appreciably, but the lift offset moment is much greater since lift is much greater. Thus the net aerodynamic moment is now large and nose up. This is nearly offset by a large nose-down centrifugal moment, now made larger than above since the flap deflection is four times as great. Tennis racket and lead-lag are again negligible. The net elastic twist is slightly ( 0.25 degrees) nose up.

at $60 \mathrm{mph}$ :

The aerodynamic pitching moments have not changed drastically from above. But, the centrifugal flap-deflection moment has increased since there is greater flap bending. Now the net moment is again nose down, amounting to 290 in.-lb at 0.30 STA and yielding about 2.6 degrees of elastic twist. The distributions are not linear in any case since the net torsion is the sum of individual nonlinear values (e.g., lift, pitching moment, flap bending).

\subsection{ESI BLADE DEFLECTIONS}

The ESI blade was of vacuum-bagged, wood (Douglas fir), veneer/epoxy internal construction and was easily the stiffest blade tested. This rotor blade was in service at two operating speeds: 77 and 90 $\mathrm{rpm}$, corresponding to 54 and $85 \mathrm{~kW}$ rating; the rpm difference greatly affects the deflections via the inertial and aerodynamic loads. No ballast was used in either approach; however, two versions of the overspeed tip mechanism (tip flap) were used. These are depicted in Figure 5.19: the "regular tip" was a 
simple steel alloy flat plate on a hinged spring/release mechanism, and the "whisper tip" was a much larger fiberglass fairing over a larger baseplate and release mechanism. The regular tip weighed $10.06 \mathrm{lb}$ and the whisper tip $17.44 \mathrm{lb}$, with the $\mathrm{cg}$ of the latter being forward of the former (1.22 in. vs. 5.93 in. aft of LE; see Fig. 5.19).

TABLE 6.3: ESI TIP FLAP DEFLECTION

\begin{tabular}{llccccccc} 
Vo(mph) & & 10 & 20 & 30 & 40 & 50 & 60 & 70 \\
\hline $77 \mathrm{rpm}:$ & no tip & 26.7 & 31.5 & 35.8 & 38.8 & 40.5 & 41.1 & 41.9 \\
& regular tip & 25.5 & 29.9 & 33.9 & 36.7 & 38.3 & 38.9 & 39.6 \\
& whisper tip & 24.7 & 28.9 & 32.7 & 35.4 & 36.9 & 37.4 & 38.1 \\
& & & & & & & \\
$90 \mathrm{rpm}:$ & no tip & 25.4 & 30.7 & 35.7 & 39.9 & 42.7 & 44.3 & 44.9 \\
& regular tip & 24.0 & 28.8 & 33.4 & 37.2 & 39.7 & 41.1 & 41.7 \\
& whisper tip & 23.3 & 27.6 & 31.9 & 35.5 & 37.9 & 39.2 & 39.7 \\
\hline
\end{tabular}

TABLE 6.4: ESI TIP ELASTIC TWIST

\begin{tabular}{llrrrrrrr} 
Vo(mph) & & 10 & 20 & 30 & 40 & 50 & 60 & 70 \\
\hline $77 \mathrm{rpm}:$ & no tip & .11 & .06 & .00 & -.03 & -.02 & .10 & .20 \\
& regular tip & .00 & -.08 & -.15 & -.20 & -.18 & -.07 & .03 \\
& whisper tip & .25 & .22 & .19 & .17 & .19 & .31 & .42 \\
& & & & & & & \\
$90 \mathrm{rpm}:$ & no tip & .17 & .10 & .02 & -.05 & -.10 & -.05 & .09 \\
& regular tip & .02 & -.08 & -.18 & -.27 & -.33 & -.29 & -.15 \\
& whisper tip & .35 & .31 & .27 & .24 & .21 & .27 & .41 \\
\hline
\end{tabular}

No Tip: $\quad$ This case was run to separate the tip weight effects on the deflections. The static coning of $5^{\circ}$ gave 28.2 in. of flap deflection; at $10 \mathrm{mph}$ the centrifugal relief again dominates, giving a negative root bending moment and a tip deflection less than the coned value. As wind speed increases, aerodynamic load increases, faster for the $90 \mathrm{rpm}$ case. The flap deflections bear this out. The $90 \mathrm{rpm}$ case has lower angles of attack at $10-30 \mathrm{mph}$, and as wind speed increases, the $77 \mathrm{rpm}$ version stalls first and sheds airload. Thus the $90 \mathrm{rpm}$ version has a higher eventual tip deflection (44.9 in.). However, this is still a small number since almost half of this value is due to static coning. The root bending moment for this 70 rpm case is a huge $257,000 \mathrm{in}$. -lb (i.e. this is a $2067 \mathrm{lb}$ flap shear load). This certainly must be a very stiff blade; for this highest load case, the root laminate strain is less than 1400 microstrain! The root tension (centrifugal force) is $8100 \mathrm{lb}$ at $90 \mathrm{rpm}$. This is a further indication that the blade is not heavy.

The elastic twist is always negligible for this blade and is dominated by the simple airfoil pitching moment (NACA 230XX variation airfoil). As would be expected the twist increases rapidly in stall, which can be clearly seen in the $77 \mathrm{rpm}$ case at $60.70 \mathrm{mph}$. [Note: these twists are so small that a difference of only $1 \mathrm{in}$. of tip deflection reduces it by $70 \%$; compare the 77 and $90 \mathrm{rpm}, 10-\mathrm{mph}$ case]

Reqular Tip: Adding only $10 \mathrm{lb}$ to the tip at a chordwise position of $5.93 \mathrm{in}$. and some section moment of inertia are the only differences from the above values. As would be expected the centrifugal force at 90 $\mathrm{rpm}$ (root tension) goes up from 8100 to $8840 \mathrm{lb}$ and the root bending moments decrease (e.g. 232,000 vs. $257,000 \mathrm{in.}-(\mathrm{b})$. Again the flap deflections are always less than the runs without tip weight. [Note: this is the traditional reason to add tip weight to helicopter blades .. to reduce tip deflection.] The root laminate strains are also less than before since the flexure is less than with no tip weight. 
The elastic twist is more complex. First, adding the weight to the tip is only part of the story; the additional section moment of inertia also depends on the chordwise distribution of the weights (e.g., tip parts; see Sec. 5.3.7). So each mechanism part had to be calculated independently to get the "ballast" moment of inertia. The elastic twist values are now clearly dominated by the tip ballast via centrifugal twisting moments. As can be seen in Figure 6.6, the elastic twist occurs almost entirely in the outer $20 \%$ of the blade. This is because the "ballast" has been added as a point load at the tip. This means that the tip twist is only an approximate indication of the overall blade effect, even though the highest airload occurs at the blade tip. Still these twists are small (0.33 degree or less).

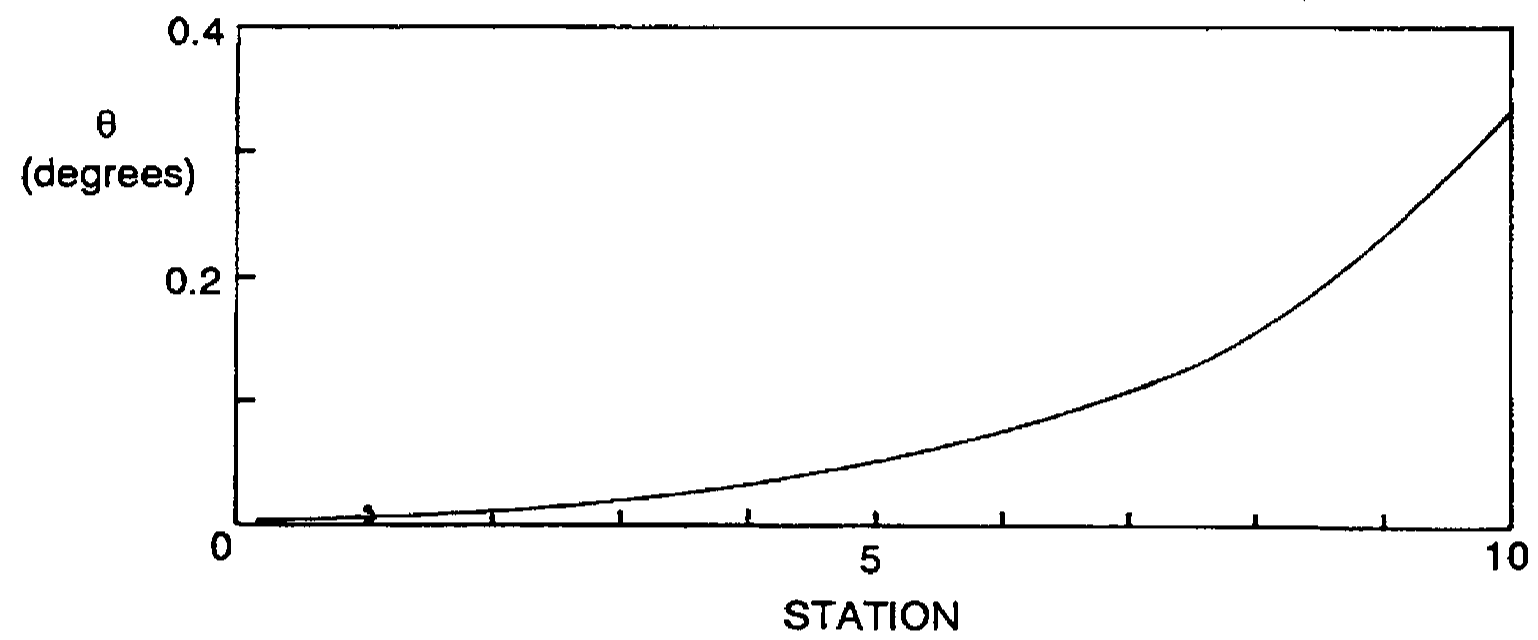

FIG. 6.6 ESI ELASTIC TWIST DISTRIBUTION

The effect of stall can also be seen, as the $77 \mathrm{rpm}$ (more stalled) blade at $60-70 \mathrm{mph}$ has a nosedown twist compared to the $90 \mathrm{rpm}$ (less stalled) blade.

Whisper Tip: The whisper tip is about $7.5 \mathrm{lb}$ heavier than the regular tip. This increased tip weight reduces the flap deflection even more, and increases the root tension slightly $(8100 \mathrm{lb}$ no tip, $8840 \mathrm{lb}$ regular, $9390 \mathrm{lb}$ whisper tip, at $90 \mathrm{rpm})$. The root bending moments are also reduced by the various tip weights $(171,000$ to 148,000 to 133,000 in.-lb).

The other important difference is the $\mathrm{cg}$ location of the whisper tip. The additional weight is forward of the section $\mathrm{cg}$ and shear center; in fact it is almost on the leading edge (1.92 in. from LE). One would expect a far-forward tip mass to produce a large nose-down pitching moment with flap deflection. This can be easily seen to be the case by looking at the elastic twist results in Table 6.4. All the twist is now nose down, and amounts to about 0.33 degree. Still this is a small value and would not be expected to affect performance very much. It does, however, produce section torsion which increases the laminate (veneer) shear strain.

Some unpublished flight test strip chant data were available from ESI tests on the $90 \mathrm{rpm}$, whisper tip version (Ref. 26). They showed an average torsion at 0.90 STA to be about $50 \mathrm{ft}-\mathrm{lb}(600 \mathrm{in}$.-lb) nose-down at 20-25 mph. The calculations show for this condition: $90 \mathrm{rpm}$, whisper tip, 0.90 STA, 20 $\mathrm{mph}$, a blade torsion of $36.9 \mathrm{ft}-\mathrm{lb}$ (443 in.-lb) nose-down, which compares very favorably with the data.

\section{8: $\quad$ CARTER BLADE DEFLECTIONS}

The Carter blade was by far the most complicated of the test series. This blade was made up of various laminates of vacuum-bagged glass reinforcements in an epoxy matrix. The makeup and detailed structural arrangement of the blade are fully described in Section 4.4. The flap and lag deflections and elastic twist for wind speeds of $10-70 \mathrm{mph}$ are given below in Tables $6.5,6.6$ and 6.7 for the operating 
value of $2.0^{\circ}$. [Note: all deflections were corrected to the control axis (quarterchord) of the blade. Also the 50,60 , and $70 \mathrm{mph}$ cases must be considered somewhat academic since the turbine does not operate at those wind speeds.]

The clearest result seen is the increase of blade bending, both flapwise and edgewise, with increasing wind speed. The negative inplane deflections denote forward bending, i.e. positive torque on the rotor shaft. Realistically, with tip deflections of $6 \mathrm{ft}$, the blade can no longer be considered an actuator disk (e.g. as in PROPPC code) and some aerodynamic load relief due to flap bending must be occurring, This probably tends to reduce angles of attack, inplane loads, and the power output. So the power output calculations must all be suspect for this highly flexible blade above $30 \mathrm{mph}$. This discussion will, therefore, focus on the design and elasticity of the structure rather than the power output.

TABLE 6.5: CARTER FLAP DEFLECTION (IN.)

$V_{0}(m p h)$ Root

\begin{tabular}{rlrrrrrrrrrr}
\hline 10 & 0 & 2.4 & 4.3 & 5.9 & 7.3 & 8.7 & 10.0 & 11.3 & 12.6 & 13.8 & 15.1 \\
20 & 0 & 3.1 & 6.5 & 10.2 & 14.1 & 18.1 & 22.1 & 26.1 & 30.0 & 33.9 & 37.8 \\
30 & 0 & 3.7 & 8.7 & 14.4 & 20.6 & 27.1 & 33.8 & 40.6 & 47.4 & 54.2 & 61.0 \\
40 & 0 & 4.0 & 9.7 & 16.5 & 24.0 & 31.9 & 40.2 & 48.7 & 57.4 & 66.2 & 75.0 \\
50 & 0 & 4.1 & 10.2 & 17.4 & 25.4 & 33.9 & 43.0 & 52.5 & 62.4 & 72.4 & 82.6 \\
60 & 0 & 4.1 & 10.2 & 17.4 & 25.3 & 33.8 & 42.7 & 52.1 & 61.9 & 71.9 & 82.0 \\
70 & 0 & 4.2 & 10.3 & 17.6 & 25.6 & 34.1 & 42.9 & 52.2 & 61.9 & 71.7 & 81.6 \\
\hline
\end{tabular}

TABLE 6.6: CARTER LEAD-LAG DEFLECTION (IN.)

$V_{0}(m p h)$ Root

\begin{tabular}{rllllllllllrr}
\hline 10 & 0 & 0.0 & 0.0 & 0.0 & 0.1 & 0.1 & 0.1 & 0.1 & 0.2 & 0.2 & 0.2 \\
20 & 0 & 0.0 & -.1 & -.2 & -.3 & -.5 & -.6 & -.8 & -.9 & -1.1 & -1.2 \\
30 & 0 & -.1 & -.3 & -.8 & -1.3 & -1.9 & -2.6 & -3.2 & -3.9 & -4.6 & -5.3 \\
40 & 0 & -.2 & -.6 & -1.3 & -2.3 & -3.4 & -4.6 & -5.8 & -7.1 & -8.4 & -9.7 \\
50 & 0 & -.2 & -.8 & -1.7 & -3.0 & -4.4 & -5.9 & -7.5 & -9.1 & -10.8 & -12.6 \\
60 & 0 & -.2 & -.8 & -1.8 & -3.1 & -4.7 & -6.3 & -8.0 & -9.8 & -11.7 & -13.6 \\
70 & 0 & -.2 & -.8 & -1.8 & -3.1 & -4.6 & -6.1 & -7.8 & -9.6 & -11.4 & -13.3 \\
\hline
\end{tabular}

TABLE 6.7: CARTER ELASTIC TWIST (DEGREES)

$V_{0}(m p h)$ Root

. . . . Tip

\begin{tabular}{rlllllllllll}
\hline 10 & 0 & .03 & .07 & .14 & .28 & .49 & .79 & 1.23 & 1.71 & 1.98 & 2.05 \\
20 & 0 & .02 & .06 & .12 & .25 & .46 & .76 & 1.22 & 1.69 & 1.96 & 2.02 \\
30 & 0 & .01 & .03 & .08 & .19 & .38 & .66 & 1.08 & 1.50 & 1.71 & 1.75 \\
40 & 0 & .01 & .03 & .08 & .18 & .35 & .59 & .92 & 1.22 & 1.35 & 1.37 \\
50 & 0 & .01 & .04 & .09 & .21 & .38 & .61 & .87 & 1.04 & 1.08 & 1.08 \\
60 & 0 & .02 & .06 & .13 & .27 & .48 & .74 & 1.05 & 1.27 & 1.33 & 1.33 \\
70 & 0 & .04 & .09 & .19 & .36 & .62 & .94 & 1.33 & 1.64 & 1.75 & 1.76 \\
\hline
\end{tabular}


Figure 6.7 shows the spanwise elastic twist and Figure 6.8 the pitching moment distributions of this blade for 10 and $30 \mathrm{mph}$; the corresponding distributions of section moment and the major constituents are also given in Table 6.8 .

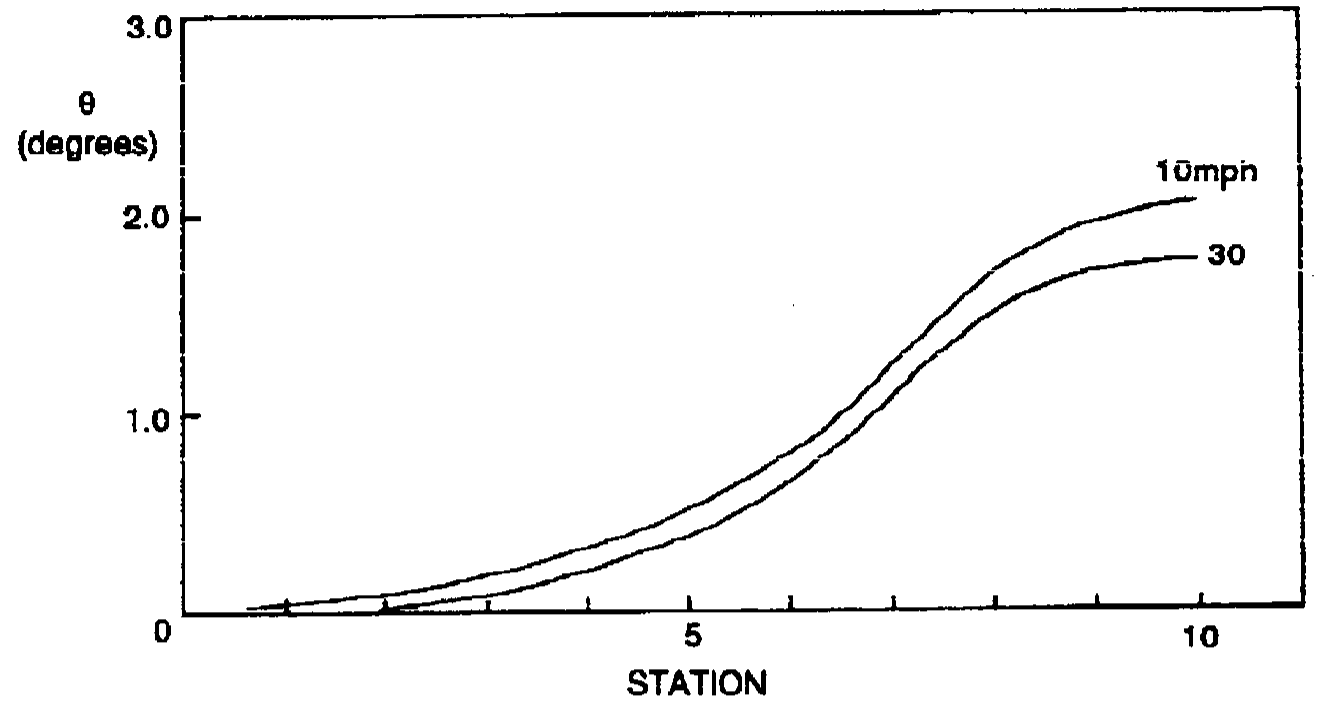

FIG. 6.7 CARTER ELASTIC TWIST (10 AND $30 \mathrm{mph}$ CASES)

TABLE 6.8: CARTER BLADE SECTION TORSION COMPONENTS

$V_{0}=10 \mathrm{mph}$ Root $\cdot$ - Tip

\begin{tabular}{|c|c|c|c|c|c|c|c|c|c|c|c|}
\hline $\begin{array}{l}\text { AERO PITCHING MOMENT } \\
\text { LIFT OFFSET } \\
\text { TENNIS RACKET } \\
\text { FLAP DEFLECTION }\end{array}$ & $\begin{array}{l}0 \\
0 \\
0 \\
0\end{array}$ & $\begin{array}{l}7.5 \\
1.9 \\
-.1 \\
6.4\end{array}$ & $\begin{array}{r}11.3 \\
-.2 \\
-.1 \\
-.2\end{array}$ & $\begin{array}{r}13.3 \\
-2.3 \\
-.1 \\
-.4\end{array}$ & $\begin{array}{r}13.3 \\
-1.6 \\
-1.3 \\
.7\end{array}$ & $\begin{array}{r}14.5 \\
-4.2 \\
-.6 \\
9.1\end{array}$ & $\begin{array}{r}15.2 \\
-3.8 \\
-.4 \\
7.8\end{array}$ & $\begin{array}{r}16.6 \\
-2.4 \\
-.3 \\
7.4\end{array}$ & $\begin{array}{r}18.9 \\
-4.1 \\
-.2 \\
8.9\end{array}$ & $\begin{array}{r}21.6 \\
-8.7 \\
-.4 \\
12.8\end{array}$ & $\begin{array}{r}21.6 \\
-8.9 \\
-.4 \\
10.8\end{array}$ \\
\hline TOTALS: & - & 9.8 & 10.8 & 10.6 & 11.2 & 18.4 & 18.7 & 21.1 & 23.3 & 24.9 & 22.9 \\
\hline
\end{tabular}

$V_{0}=30 \mathrm{mph} \quad$ Root

\begin{tabular}{lcccccccccrr}
\hline AERO PITCHING MOMENT & 0 & 14.5 & 14.3 & 14.3 & 14.0 & 13.8 & 14.5 & 16.9 & 19.5 & 22.1 & 22.1 \\
UFT OFFSET & 0 & -26.5 & -23.9 & -21.8 & -20.2 & -29.7 & -25.2 & -17.4 & -26.9 & -52.4 & -50.0 \\
TENNIS RACKET & 0 & 0 & 0 & 0 & -1.3 & -.6 & -.4 & -.3 & -.2 & -.4 & -.4 \\
FLAP DEFLECTION & 0 & 9.7 & -.5 & -.8 & 2.1 & 28.2 & 26.4 & 26.3 & 33.4 & 50.2 & 43.8 \\
\hline TOTALS: & - & -8.4 & 10.2 & -8.4 & -5.4 & 11.3 & 15.1 & 25.4 & 25.5 & 19.2 & 15.3
\end{tabular}

10 mph Case: The tip deflections are small for this case; the flap value is 15.1 in., which is less than the 28 in. from coning, so the flap root moment is negative. The lead-lag moment is positive, or aft, indicating negative power output.

Surprisingly, the elastic twist is the highest for any wind speed case: over $2^{\circ}$ at the tip. Referring to Table 6.8 allows a closer look at the moment components. The angles of attack are in the lifting range, and there is substantial (nose-down) aeroelastic pitching moment all along the blade, since its large root chord and twist keep the aerodynamic values high. This indicates a well-designed blade, or one where all the blade sections operate close to the same angles of attack (i.e. "work together"). The next line in the table is moment due to lift offset, or quarterchord to elastic axis. This offset is appreciable, with the shear 
center being aft of the quarterchord (see Sec. 4.4). However this moment is small at $10 \mathrm{mph}$ owing to a relatively small lift value.

Inertially (centrifugally) speaking, the only appreciable moment arises from flap coupling. Tennis racket and inplane coupling are small and can be neglected here (see line 3). The fourth line in Table 6.8 shows that the flap coupling term is slightly nose-up inboard; this is due to the section cg being aft of the shear centers inboard. Outboard the situation is drastically reversed, since substantial lead ballast has been added to the leading edge (see Sec. 4.4). This has brought the $\mathrm{cg}$ forward of the shear center, which creates a nose-down flap coupling moment. The net result of all the terms is a substantial nose-down moment, which causes substantial elastic nose-down elastic twist.

All the section centers and the forces and moments are shown as free body diagrams in Figure 6.8 for station 0.90 . Referring to the figure: the small lift $(2.0 \mathrm{lb})$ acts through the aeroelastic offset (4.4 in.) but is not enough to counter the aeroelastic pitching moment (21.6 in.- $\mathrm{lb}$ ) and the centrifugal flap coupling moment (12.8 in.-1b), both of which are nose-down. The net result is a relatively smooth moment distribution which is always nose down (Fig. 6.9).
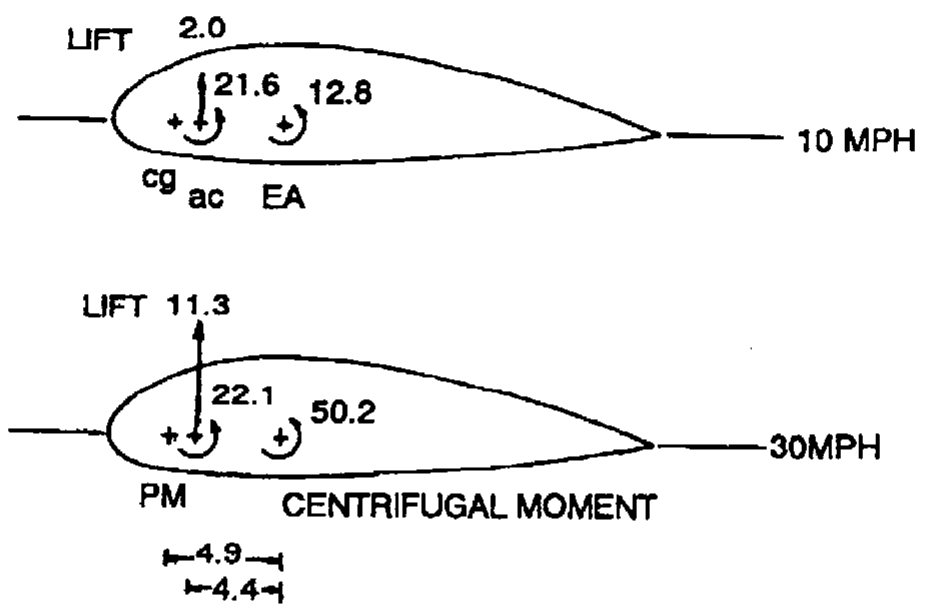

FIG. 6.8 CARTER SECTION FREE BODY DIAGRAMS 
NET MOMENT (NCH-LB/INCH)

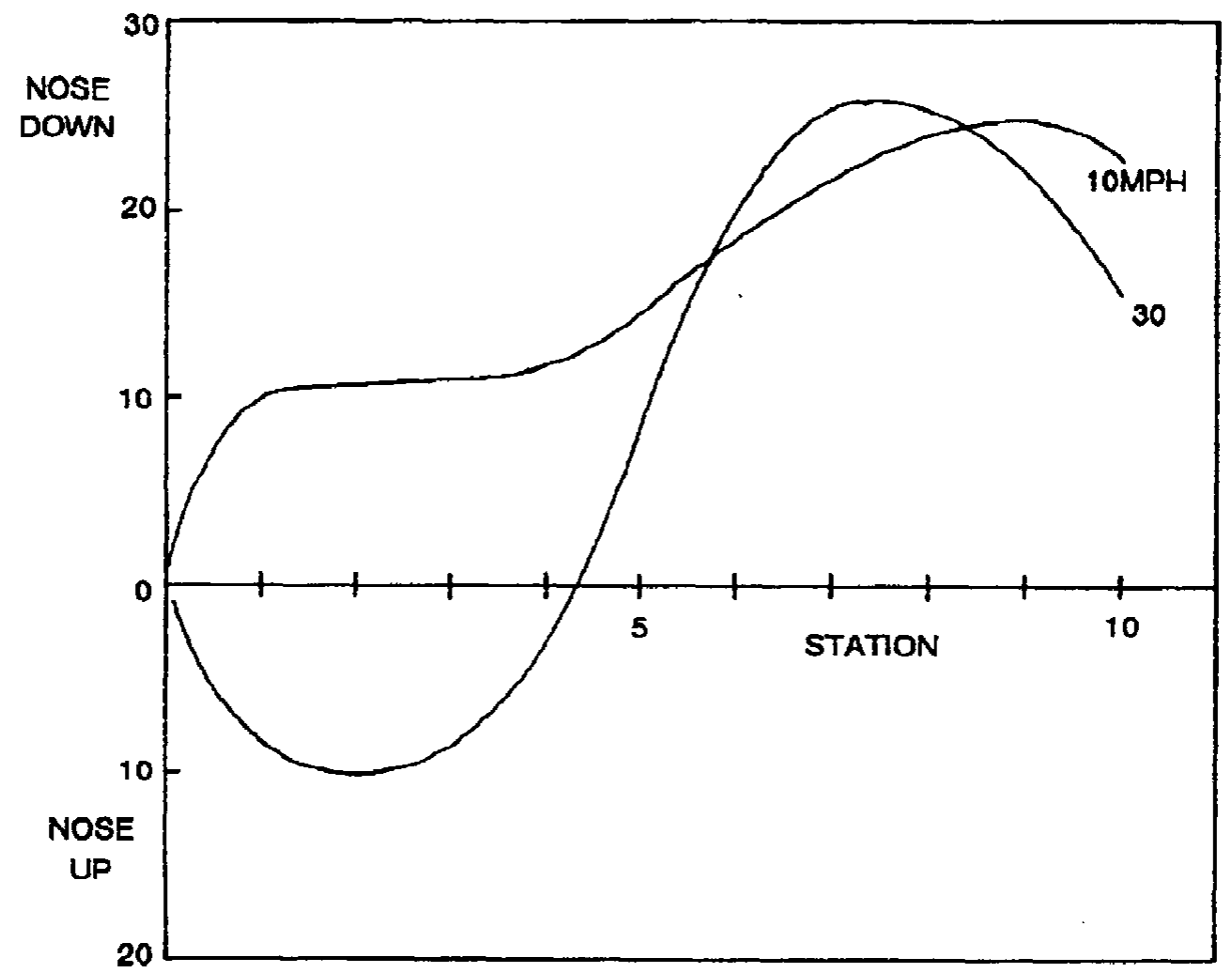

FIG. 6.9 CARTER PITCHING MOMENT DISTRIBUTION(10 AND 30 mph CASES)

30 mph Case: For this case the tip deflections are much greater; this is a load case near the rated output of the turbine, and the tip deflection is over 5 feet. The root flap moment is $10,558 \mathrm{ft}$ - Ib; the flap shear is $2990 \mathrm{lb}$. The root lead-lag shear is $1824 \mathrm{lb}$, and the inplane moment (which gives rotor torque) is about twice the size of the flap moment. Here is an illustration of the advantage of a flexible blade: this blade is flexible in the flap direction, so the loading is largely relieved by centrifugal force; the inplane stiffness is high, so the inplane deflection is low. Very little root load is relieved.

Surprisingly, the elastic twist for this case is less than at $10 \mathrm{mph}$. The aerodynamic loads were much less. The reason can be seen in Figure 6.8, the lift force is much greater, thus the lift offset term is large. The flap coupling term is also larger (50.2 in.-lb) but it is practically canceled by the offset term. The net moment distribution can be seen in Fig 6.9; the moment relief due to lift is easily seen.

Effect of Ballast: The effect of removing the ballast on the elastic twist can be seen in Figure 6.10, which shows the net section moment and elastic twist for the same blade but now with no lead ballast outboard. The elastic twist is now nose up rather than nose down as above. 


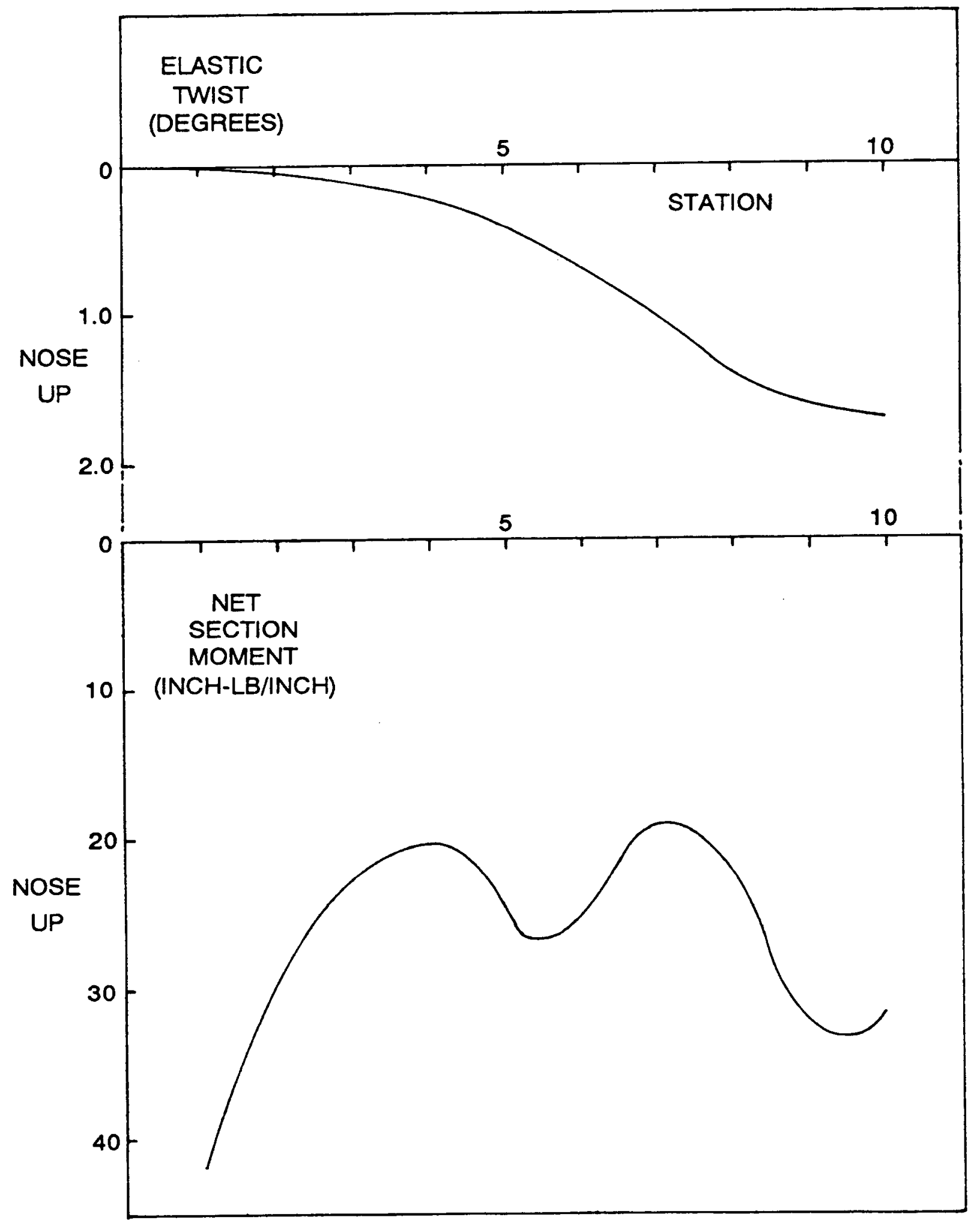

FIG. 6.10 CARTER ELASTIC TWIST AND SECTION MOMENT, NO BALLAST ( $30 \mathrm{mph}$ ) 
This is because the centrifugal moment is now much less because the weight is less and the $\mathrm{cg}$ is much farther aft (closer to the shear center). Referring to Figure 6.8, the free body diagram for $30 \mathrm{mph}$ looks the same except now the centrifugal moment is only $2.9 \mathrm{in}$. $\mathrm{lb} / \mathrm{in}$. rather than $50.2 \mathrm{in}$. $\mathrm{lb} / \mathrm{in}$. (with ballast). With no ballast the nose-up lift offset dominates and the result is nose-up elastic twist.

It is clear that the chordwise position of the ballast can be adjusted (slightly aft) to completely zero the elastic twist, if that becomes the wish of the designer. [Note: this would only be strictly true at one wind speed, since the lift vector changes and the centrifugal moment does not (except for flap deflection contribution)]

Finally, the major designer's reason for the lead ballast can be easily seen in the flap deflection results. Without the ballast this flexible blade, under the $30 \mathrm{mph}$ loading, would have a tip deflection of over 10 feet; by adding the ballast this tip deflection is reduced to 61 in. A secondary effect of reducing the flap deflection is to also reduce the flap coupling term above. 


\subsection{PERFORMANCE CALCULATIONS AND RESULTS}

The last phase of the project used the adjusted twist distributions of the blades to determine the adjusted blade aerodynamic loading and wind turbine performance. We used the current PROPPC.FOR computer code and airfoil $C_{L} / C_{d}$ data sets specified by $S E R I$ and the consultation on aerodynamic corrections due to airfoil shape errors documented in the first phase. Adjusted rotor performance and loads were compared to unadjusted loads, and the aerodynamic loading set used to generate the elastic twist was adequately verified.

\section{1: $\quad$ AERODYNAMIC CALCULATIONS}

This phase finally applied the elastic twist results to rotor performance calculations to modify the wind turbine performance and assess the effect of elastic twist. Four post-stall airfoil $C_{L} / C_{d}$ characteristic options were used, as specified by SERI (Fig 7.1 and Refs. 29-31):

A. Standard 2-D table data with flat plate stall algorithm.

B. Standard 2-D table data with NACA 0012 stall values from wind tunnel test.

C. Viterna post-stall synthesization routine.

D. Viterna/Tangler post-stall routine.

Each operating case was run for each of these, for each rotor, to determine the baseline performance. The cases were then rerun using the adjusted elastic twist results. Comparison of the post-stall synthesization routines can be seen in Figures 7.2, 7.3, and 7.4. The experimental 2-D lift and drag data which were input to PROPPC are shown in Table 7.1

\section{TABLE 7.1: AIRFOIL LIFT AND DRAG DATA INPUT TO PROPPC}

Carter 300: STA 1-5 NACA LS(1)-0417

ESI:

UTRC 8: $\quad$ STA 3-10 NACA23112
STA 6-10 NACA LS(1)-0413

STA 8 NACA 23021 STA 9 NACA 23018

(most of STA 3 is part of the flexbeam)

STA 10 NACA 23015 

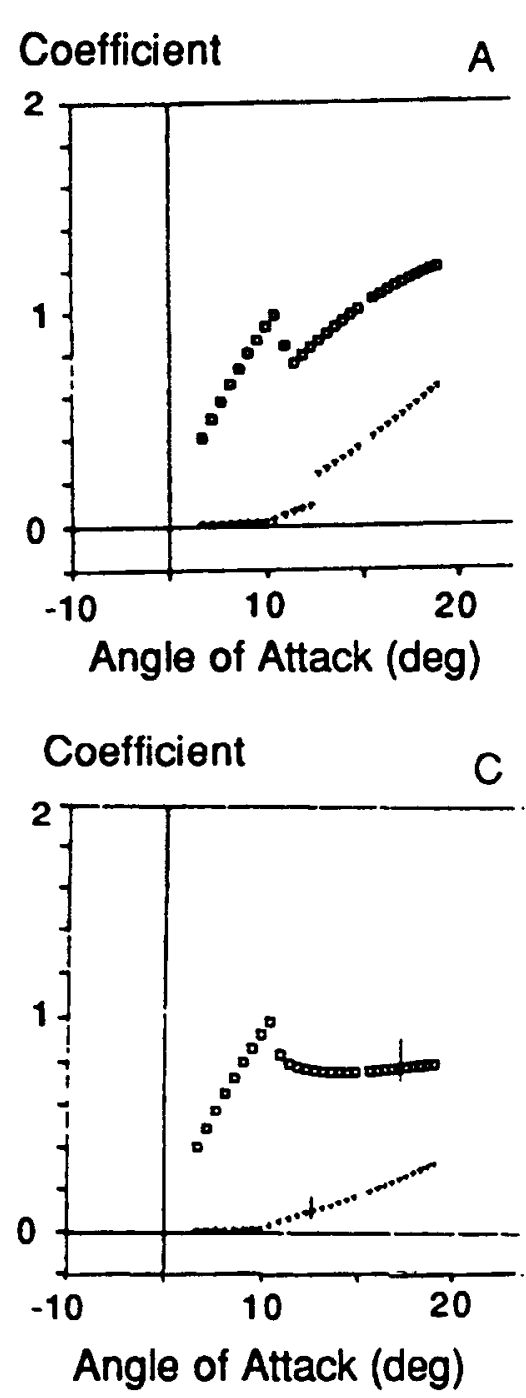
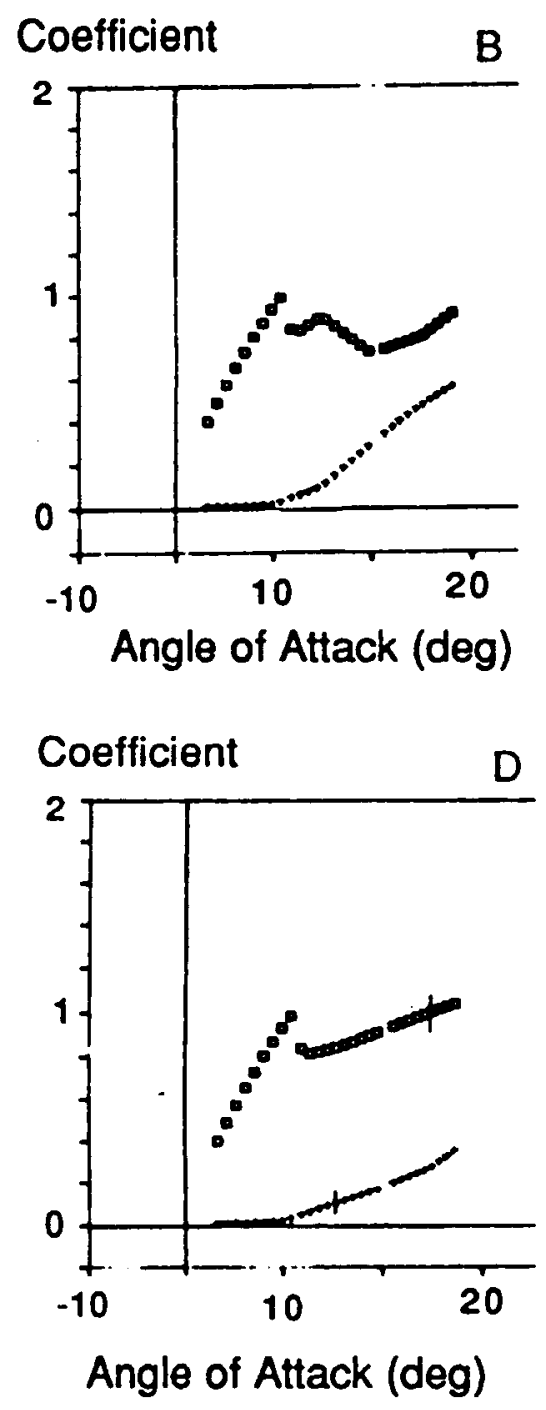

- Drag Coefficient

FIG. 7.1 2-D SECTION DATA COMPARISON: STANDARD VS. VITERNA VS. TANGLER 


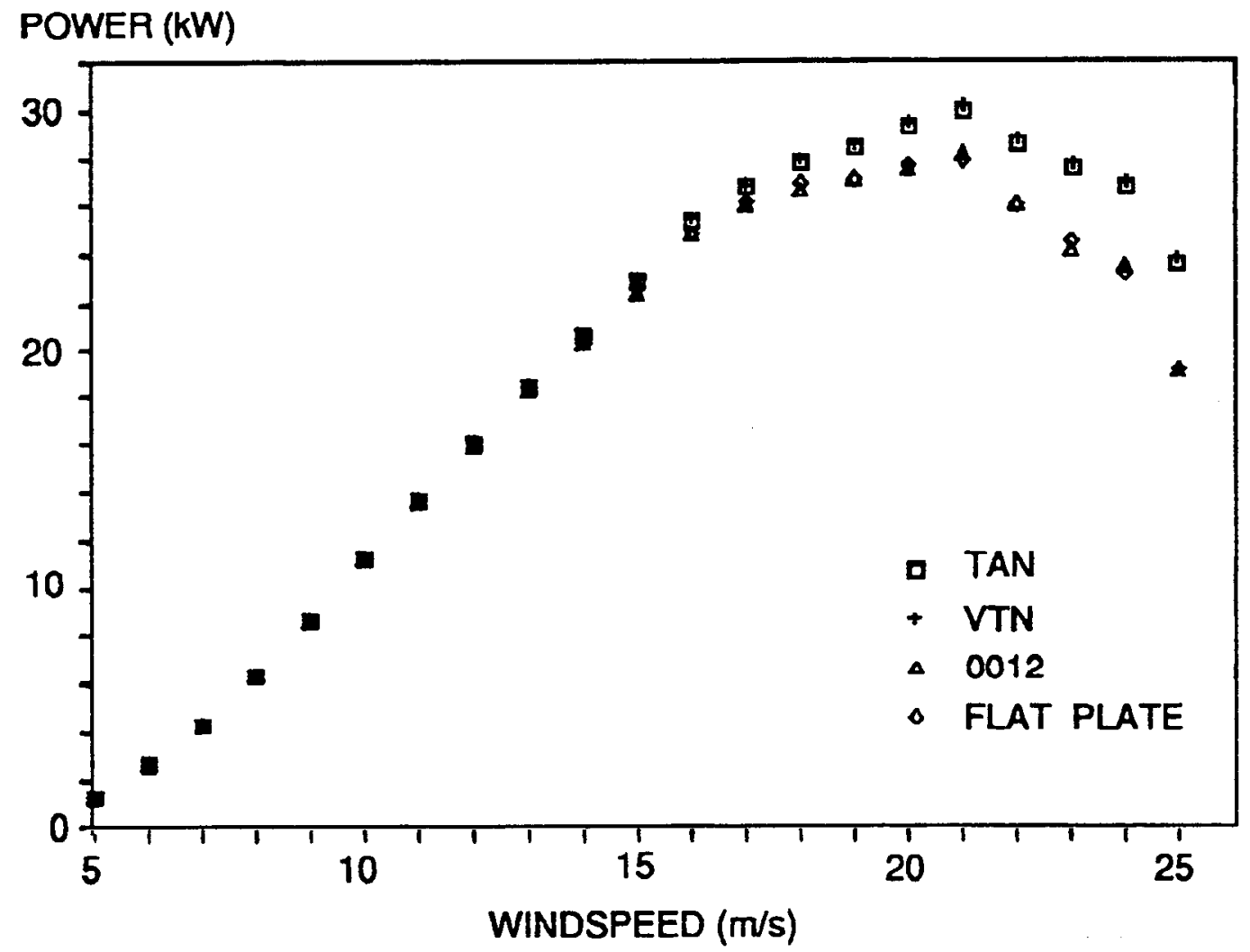

FIG. 7.2 UTRC: COMPARISON OF POST-STALL ROUTINES

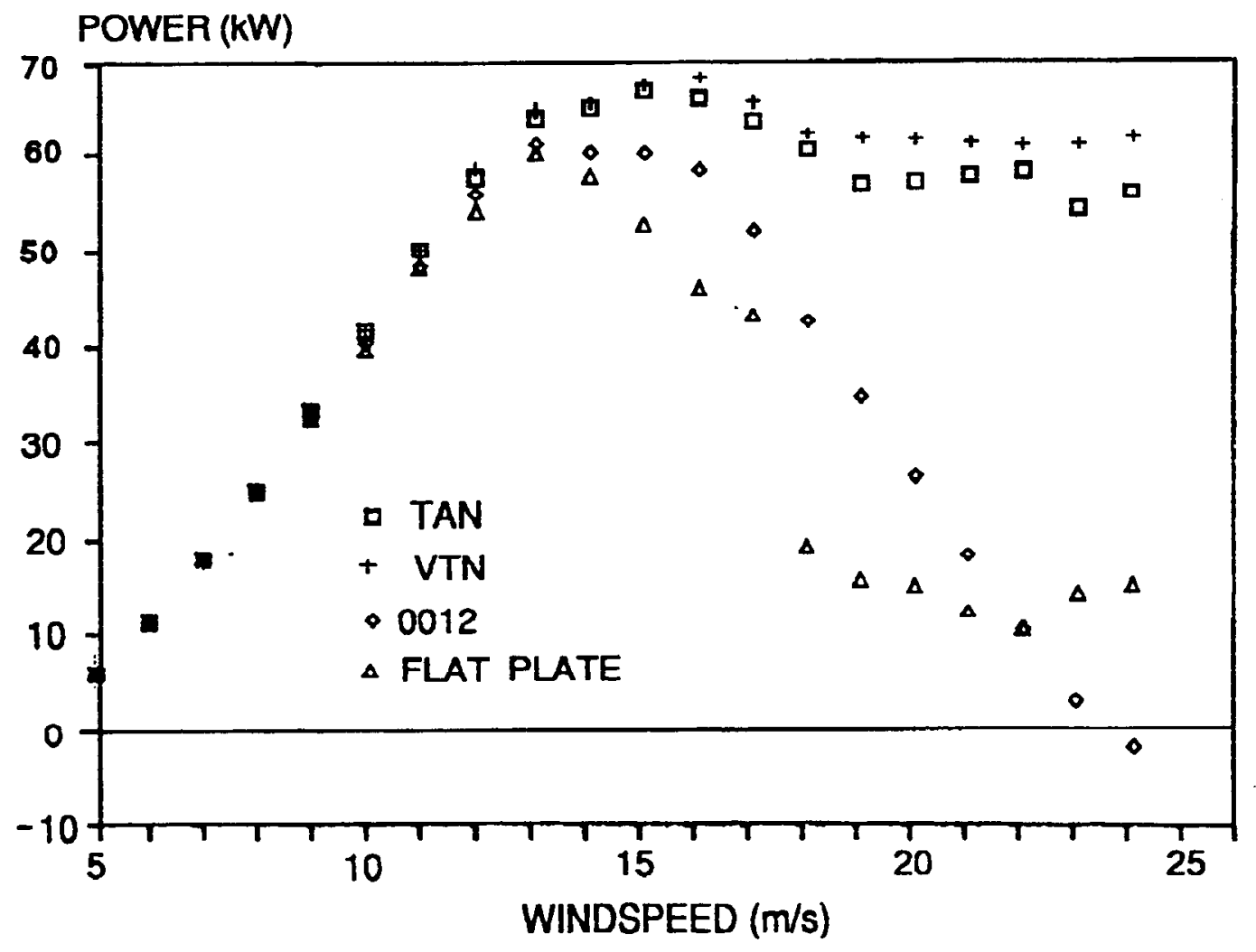

FIG. 7.3 ESI: COMPARISON OF POST-STALL ROUTINES 


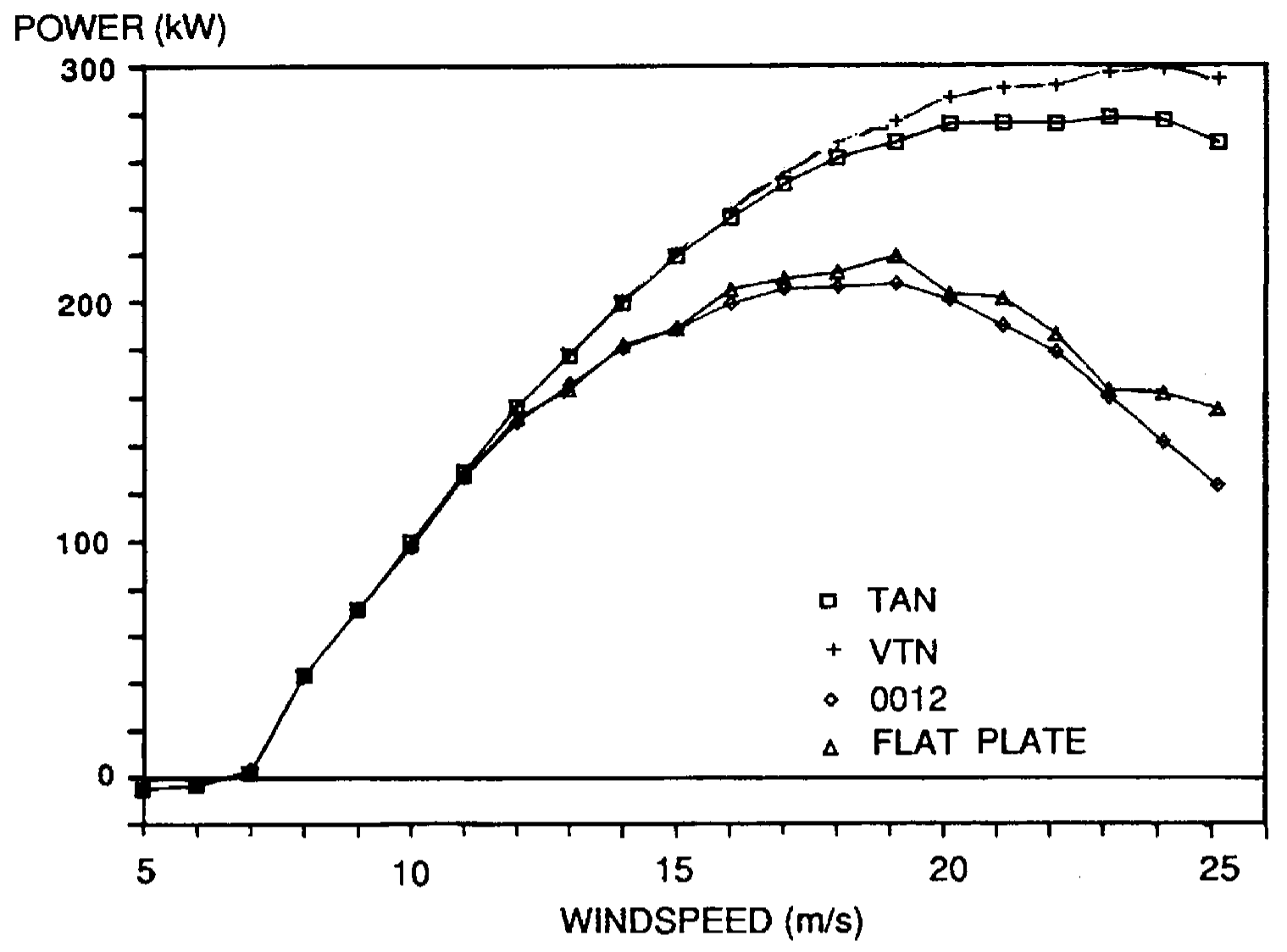

FIG. 7.4 CARTER: COMPARISON OF POST-STALL ROUTINES

For the Carter 300, lift and drag data for the LS(1)-0417 and - 0413 were used to approximate the sections, which varied from -0421 at the root to -0413 at the tip. The ESI 54 has a different airfoil (NACA $230 \times X$ ) at each station, and was approximated by four airfoils. The problem here was that the inboard sections had up to $44 \%$ thickness and no experimental data were available; the thickest airfoil in the available literature, $24 \%$, was used. Since the UTRC 8 was a constant chord, zero twist blade (NACA 23112), the lift and drag data were simpler. When the lift and drag data determined by Miley were used, section data from STA 6-10 were replaced for the Carter 300, 7-10 for the ESI, and all sections for the UTRC 8. Lift and drag coefficients from the static experimental data were used corresponding to the Reynolds numbers for all those stations. Tip and hub losses were included. All units were operated at essentially constant rpm.

For each wind turbine, predicted power curves were obtained with the four different post-stall routines: flat plate, Viterna, Tangler, and 0012 experimental data. The output at each station (angles of attack, lift and drag coefficients, thrust, torque moments and power) were obtained for $5,10,15,20$, and, $25 \mathrm{~m} / \mathrm{s}$.

The Tangler and Viterna methods gave similar power outputs at high wind speeds for all three blades. Both methods predicted higher power than the flat plate and attached 0012 data, which is consistent with power curves obtained in the field. Even though the flat plate and attached 0012 data are included in the graphs, the data should be considered suspect in the post-stall region. None of the curves is smooth at high wind speeds because of the finite number of blade sections allowed in PROPPC. Twenty sections were used for the UTRC 8 and ten for the Carter 300 and ESI 54. 


\section{2: OPERATIONAL CASES CONSIDERED}

The performance results of the elastic twist calculations were finally compared with available field test results. These data were acquired from published sources and manufacturers' literature (see below). The field data were selected by reviewing key accuracy elements in field test such as

a. Anemometer locations and type (cup vs. prop vane)

b. Wind turbulence measurements.

c. Power or load transducers used.

d. Binning and summing techniques.

e. Averaging time.

f. Number of readings taken.

g. Drivetrain and generator loss model.

The UTRC data came from two sources, a final report from Rocky Flats testing (Ref. 18) and the cumulative data collected at the AEl/Borger Stripper Well site (Ref. 32). The Carter data came from a manufacturer's bulletin describing the Carter 300 (Ref. 33). The ESI data were extracted from a final report by Pacific Wind Energy, Inc. describing the Whiskey Run Windfarm (Refs. 34, 35). The data were all power curves with a known anemometer height and corrected to a standard atmospheric density. All curves were converted to metric units for both wind speed and power output.

\section{3: TURBINE POWER CURVE PERFORMANCE RESULTS}

Comparisons of field data and the computer models are given in Figures 7.5 - 7.7.

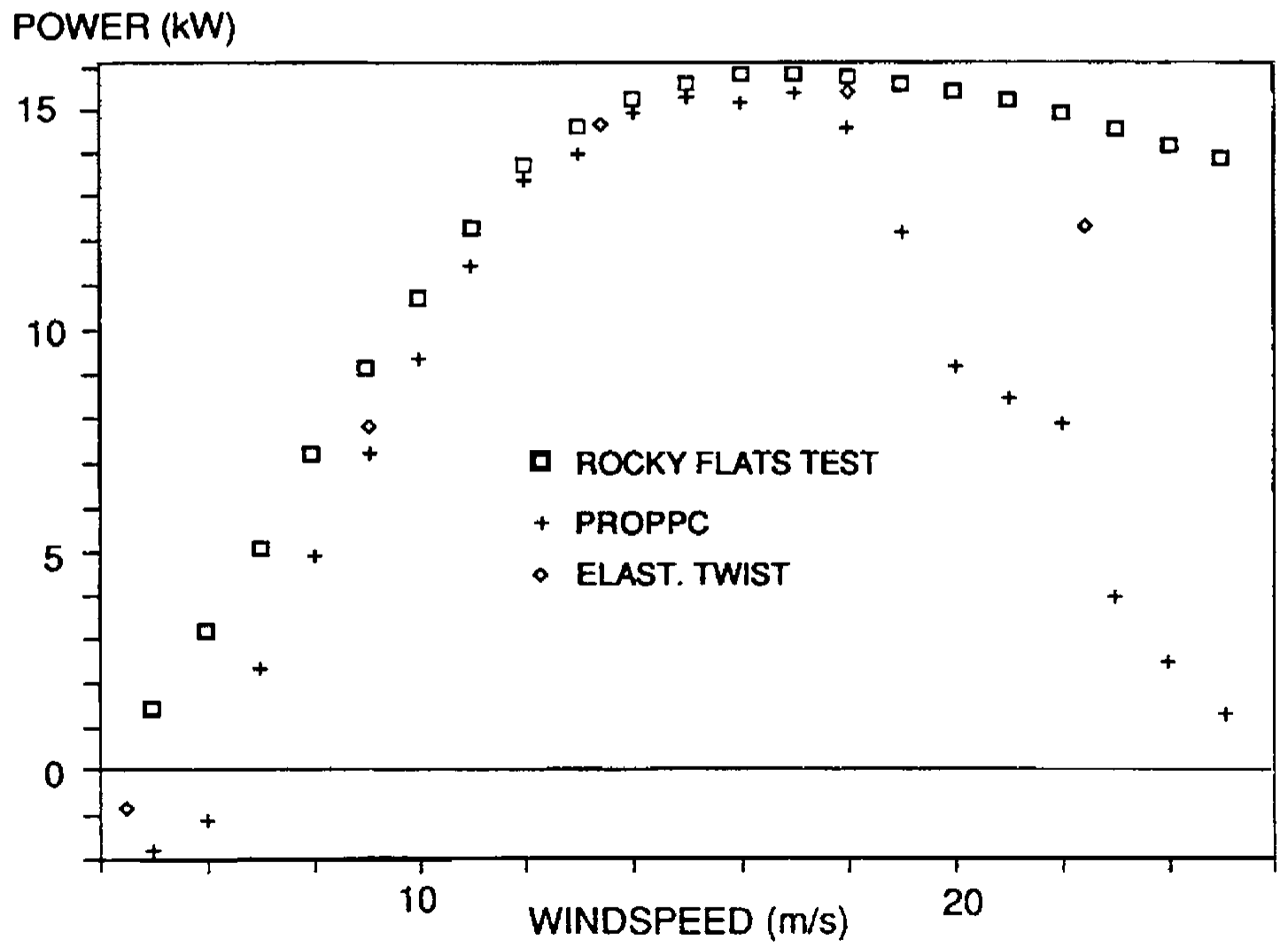

FIG. 7.5 UTRC POWER OUTPUT: COMPARISON OF FIELD TEST, PROPPC PREDICTIONS, AND ELASTIC TWIST 


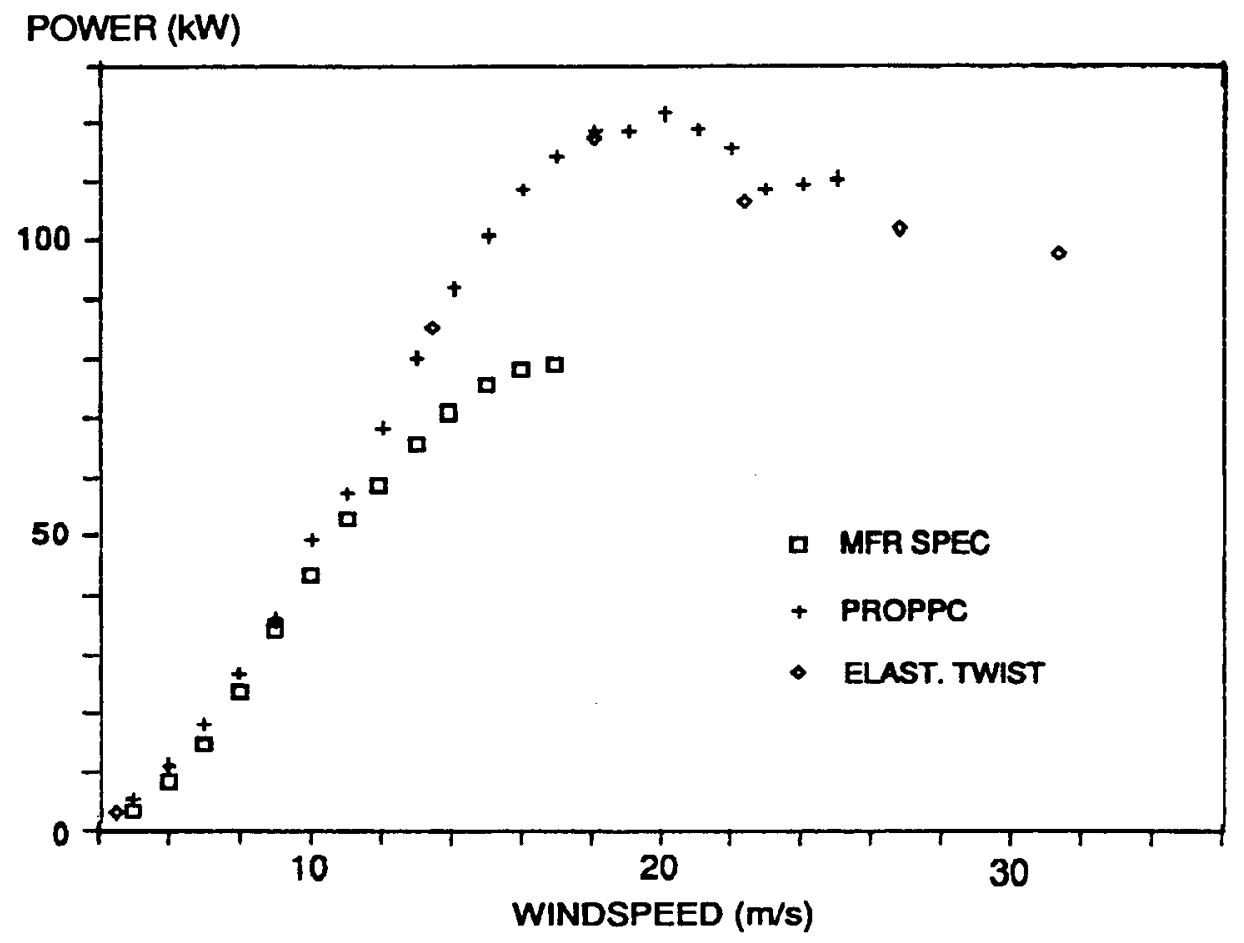

FIG. 7.6 ESI 54 POWER OUTPUT: COMPARISON OF MANUFACTURER DATA, PROPPC PREDICTION, AND ELASTIC TWIST

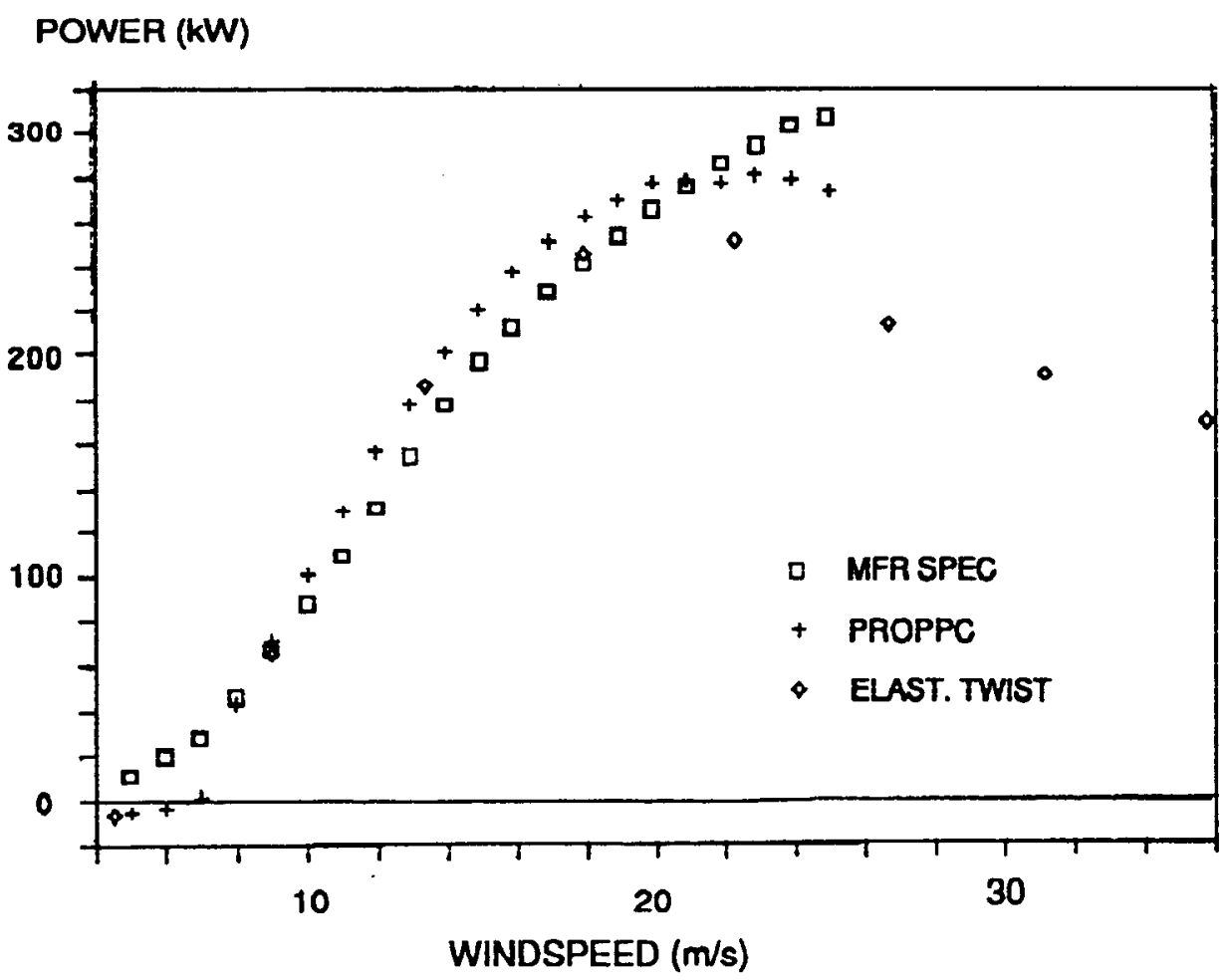

FIG. 7.7 CARTER 300 POWER OUTPUT: COMPARISON OF MANUFACTURER DATA, PROPPC PREDICTION, AND ELASTIC TWIST 


\subsection{1: UTRC $8 \mathrm{~kW}$ BLADE}

There is a large difference at high wind speeds for the UTRC 8 as predicted power output is around $30 \mathrm{~kW}$ (Fig. 7.5). Also notice that the Viterna and Tangler post-stall calculations predict decreasing power at high wind speeds, in contrast to the fairly flat power curve from atmospheric testing. The lift and drag would have to be changed drastically to reduce the peak power to $15 \mathrm{~kW}$, or the pitch needs to be changed. The lift and drag from the EPPLER Code (Appendix 10.6) over predicts the power output due to optimistic lift curve slopes at medium angles of attack, even at low wind speeds.

Clearly the elastic twist of the UTRC 8 blade affects the power curve at wind speeds above $12 \mathrm{~m} / \mathrm{s}$ (27 mph), which helps to explain the discrepancy between tests and prediction. The elastic twist is nose down (Table 6.2), reducing angle of attack. The pendulum/flexstrap root attachment was modeled in the study as a constant root pitch of $5^{\circ}$ nose up, which is its operating equilibrium at rpm.

\subsection{2: $\quad$ ESI 54 S BLADE}

Power curves were obtained for two rotor speeds, 77 and $90 \mathrm{rpm}$ (ESI 54S). The power curve from field data for the ESI $54 S(90 \mathrm{rpm})$ is below the predicted output. Data obtained from the EPPLER Code (Appendix 10.6) also over predicts the power output. The lift and drag data were used for STA 7.8 (.75 radius) and STA 9-10 (.95 radius), while STA $1-6$ were left unchanged. The large discrepancy at 16 $\mathrm{m} / \mathrm{s}$ in the smoothness of the Miley curve is from the artificial connection of EPPLER Code data through angles of attack of $-4^{\circ}$ to $16^{\circ}$, then the experimental data to $20^{\circ}$ (same as before), and then the Tangler post-stall method.

Figure 7.6 shows the ESI data for $90 \mathrm{rpm}$ with the regular tip mechanism. The discrepancy between the PROPPC predictions and test data is still quite large. The effect of the elastic twist is negligible among all the cases of 77 and $90 \mathrm{rpm}$ and the two tip mechanisms. Part, but by no means all, of the difference can be attributed to drivetrain and tip drag losses, which were not accounted for in this case.

\subsection{3: $\quad$ CARTER 300 BLADE}

Predicted power vs. wind speed (Fig. 7.4) shows the characteristic leveling off at high wind speeds for the Tangler and Viterna methods. When the Tangler-predicted output is compared to the manufacturer's data, the field data show higher power output at low and high wind speeds. The predicted power using the lift and drag coefficients obtained from the EPPLER Code agree quite well with the calculated power curve using the experimental data. The Carter 300 blades closely matched the LS (1) airfoil offset specifications.

Figure 7.7 shows the Carter 300 power curve comparisons, the manufacturer's test data, the PROPPC prediction (zero live twist), the prediction with elastic twist, and the power curve that would be obtained with zero ballast weight but including elastic twist. With no ballast weight the elastic twist is large and nose-up (e.g. $2.75^{\circ}$ at $27 \mathrm{~m} / \mathrm{s}$ [60 mph]) since the blade shear center (elastic axis) is at roughly $48 \%$ chord, outboard. This results in a large nose-up section moment due to quarterchord (aeroelastic lift) offset. Adding the ballast weight counteracts this with inertial moments resulting in $1.3^{\circ}$ nose-down (Table 6.7). The effect of this much smaller elastic twist on the Carter power curve is to reduce the angle of attack at higher wind speeds. Still, in Figure 7.7, this effect cannot be clearly seen against the test curve. Again the drivetrain losses have not been accounted for, and presumably the predictions still do not match the test because of some other aerodynamic effect that is delaying stall and/or enhancing the output near the airfoil 2-D stall angles.

\section{4: DISCUSSION}

The PROPPC Code output by station shows how much each part of the blade contributes to the performance. All the blades stall progressively from the root as the wind speed increases, even the Carter 300 which has a large twist (approaching a Glauert approximation). Looking more closely at the poststall methods, some observations can be made. 
First, for a given airfoil, different sections (blades) have a range of angles of attack depending on the wind speed. Combining these into a power curve, a smooth curve of lift and drag coefficients is obtained over a wide range of angles of attack, except for the flat plate method.

Second, the lift and drag data are static data; the post-stall modifications are based on an averaging process. If those routines are attached at different terminal angles of attack of the static data, then the predicted power output changes. Both the Tangler and Viterna modeis should be investigated from angles of attack at the onset of stall rather than only at the end of the data input, which may be a fairer way of comparing stall.

The PROPPC Code is sensitive to the input parameters. Lift and drag data were not available for all the airfoils for the range of Reynolds numbers needed, and also for the thick airfoils at the root. If the actual airfoil is different from the airfoil specified, and/or the pitch or twist is incorrect, then the predicted output will be different from the measured field data, even for the pre-stall region of windspeeds. At $10 \mathrm{~m} / \mathrm{s}$, the differences between the predicted (Tangler post-stall) and the atmospheric power are $13 \%$ for the Carter $300,7 \%$ for the ESI 54 S, and $10 \%$ for the UTRC 8 . Even if the input data are consistent with the actual blade characteristics, the prediction of power still must be considered suspect in the post-stall region. 


\subsection{CONCLUSIONS}

\section{1: COMPARISON OF PREDICTED AND EXPERIMENTAL RESULTS}

Power output and blade loading are affected by the live "elastic twist" on flexible wind turbine blades. The aeroelastic analysis method developed during this project predicts substantial live twist which helps explain discrepancies observed between calculated and measured power curves. Still the discrepancies between predictions and test curves on stall-regulated turbines are significant, and presumably are due to some other aerodynamic phenomenon.

\section{2: USE OF METHOD FOR ANALYSIS OF COMPOSITE BLADES}

A secondary goal of this project was to develop a practical method of analyzing composite blades: one within the means and abilities of most wind turbine blade manufacturers. This necessarily means appropriately practical theoretical methods and complementary bench tests for verification, refinement, and quality control. This process was not straightforward since most composite blades have complex geometry (twist and taper), internal structure (laminate reinforcement and spars), and root attachment.

\section{3: USE OF METHOD FOR BLADE QUALITY CONTROL FOR MANUFACTURERS}

The experimental method developed at AEI has been presented in detail in this report. A conventional cantilever stand was used for all blades, and linear measurements were referenced to a simple laser-defined baseplane. Section angles were taken with a sensitive but readily obtainable mechanical clinometer, and results indicate a routine achievable accuracy of 0.1 degree. A key part of the method was sectioning a blade to check and verify the internal laminate geometry and ballast locations. For a properly constructed blade, the design specifications may be very close to the bench test. Frequencies and modes of vibration were measured on the test stand with SERI assistance, but even this could have been done in house with better instrumentation.

In summary, the experimental methods developed in the project allow for practical verification of the predicted mass and stiffness properties of the blade, including the static bending deflections, static elastic twist, fundamental frequencies, and modes of vibration in flapping, lead-lag, and torsion. The aeroelastic model thus has the same static deflections, twist, modes, and frequencies of vibration as the actual blade. The blade testing procedure and codes permit practical standardized quality control, verification of blade design codes, and assessment of the static effects of ballast and tip mechanisms. For example, the actual mass and stiffness properties of the UTRC blade differed from the manufacturer's specifications by $21 \%$ (on running mass) to $49 \%$ (on torsional rigidity), for the ESI and Carter blades the differences were generally less than $10 \%$.

\section{4: DESIGN IMPLICATIONS OF RESULTS}

The elastic twist is greatly affected, via centrifugal moments, by the addition of ballast or tip weights. However, these effects may be beneficial if used correctly. The methodology used in this study enables designers of composite blades to assess the effect of adding structural elements, ballast, and tip mechanisms. Ultimately this could allow the tailoring of longitudinal and torsional section properties to exploit live twist to either enhance performance (e.g., delay stall) or reduce loading (e.g., promote stall). Also, the elastic axis of a composite blade can be changed dramatically by varying the type and amount of reinforcement, thereby changing the ratio of longitudinal modulus to shear modulus. These features may be used to advantage by FRP blade designers. Progress is being made in Great Britain in power limiting of vertical axis wind turbines by using "mirror" or "spiral" patterns of glass reinforcement (GRP) in just this way (Ref. 36). 


\section{5: RECOMMENDATIONS FOR FURTHER WORK AND HAWT IMPROVEMENT}

Future beneficial work on elastic twist would "close the loop" by recalculating the aerodynamic loads with the elastic twist corrections in place, measuring the actual torsion on operating blades, and parametrically investigating the beneficial effects of live twist, particularly for the Carter-type blade. Other work could examine the calculated laminate flexural and shear strains, which must not be allowed to exceed allowable limits for a specified fatigue life, and which are particularly important at joints of dissimilar structural elements like root hubs. Finally, dynamic elastic twist and its effect on transient loads could be studied. 


\subsection{REFERENCES}

1 J. L. Tangler and D. M. Somers, "Status of the Special-Purpose Airfoil Families", Proceedings, AWEA Conference, "Windpower '87", San Francisco, CA, Oct. 1987.

2 D. M. Eggleston and F. S. Stoddard, Wind Turbine Engineering Desian, Van Nostrand Reinhold, New York, 1987.

3 F. S. Stoddard, "Structural Dynamics, Stability and Control of High Aspect Ratio Wind Turbines", UMass Alternative Energy Program, NTIS RFP-3027/67925/3533/79-7, Amherst, MA, 1979.

4 L. J. Broutman and H. Kroch, Eds., Modern Composite Materials, Addison-Wesley, New York, 1967.

5 G. Lubin, Ed., Handbook of Composites, Van Nostrand Reinhold, New York, 1982.

6 Y. Stavsky and N. J. Hoff, "Mechanics of Composite Structures", in Composite Engineering Laminates, A.G.H. Dietz, Ed., MIT Press, Cambridge, MA, 1969.

7 J. L. Tangler, "A HAWT Performance Prediction Code for PCs: PROPPC", User's Guide, SERI, Golden, CO, Jan., 1987.

8 K. L. Starcher, et al, "Modal Analysis of Six HAWT Blades", Alternative Energy Institute, WTSU, AEI 87-2, Canyon, TX, Sept. 1987.

9 L. J. Broutman, "Mechanical Behavior of Fiber-Reinforced Plastics", in Composite Engineering Laminates, op. cit.

10 S. Jarosch, "Fatigue Properties and Test Procedures of GRP Plastic Rotor Blades", Proceedings, American Helicopter Society 25th Annual Forum, Washington D.C., May, 1969.

11 S. P. Timoshenko and J. M. Goodier, Theory of Elasticity, McGraw-Hill, New York, 1951.

12 J. P. Den Hartog, Advanced Strength of Materials, McGraw-Hill, New York, 1952.

13 R. Rivelio, Theory and Analysis of Flight Structures, Addison-Wesley, New York, 1975.

14 J. P. Den Hartog, Mechanical Vibrations, 4th Ed., McGraw-Hill, New York, 1956.

15 N. O. Myklestad, "A New Method of Calculating Natural Modes of Uncoupled Bending Vibrations of Airplane Wings and other Types of Beams", Journal of Aeronautical Sciences, 15:5, 1944.

16 W. T. Thomsen, Vibration Theory and Applications, Prentice-Hall, 1965.

17 M. A. Prohl, "A General Method For Calculating Critical Speeds of Flexible Rotors", ASME Transactions, A-142, Sept. 1945.

18 R. B. Taylor and M. C. Cheney, "UTRC 8KW Wind System: Phase II Test Technical Report", USDOE/Rocky Flats RFP-3232/2, Golden, CO, Feb. 1981.

19 I. H. Abbott and A. E. Von Doenhoff, Theory of Wing Sections, Dover, New York, 1959. 
20 J. M. Salkind, et al., "Composite Materials: Testing and Design", ASTM STP497, Amer. Soc. Testing and Materials., 1972.

21 R. Monroe, et al., Personal Communications, Calculations and Engineering Drawings for ESI 54 Blade, Gougeon Bros., Inc., Bay City, MI, July 1986.

22 M. D. Zuteck, "Development and Manufacture of Wood Composite Wind Turbine Rotors", Proceedings, DOE/NASA Workshop on Large HAWT, NASA Conf-810752, Cleveland, OH, July 1981.

23 S. Lieblein, et al., "Design and Evaluation of Low-Cost Laminated Wood Composite Blades for Wind Turbines: Blade Design, Fabrication Concept and Cost Analysis", DOE/NASAV101-1, NASA CR165463, Nov. 1982.

24 R. F. Lark, et al., "Fabrication of Low-Cost MOD-OA Wood Composite Wind Turbine Blades", DOE/NASA/20320-45, NASA-TM-83323, Feb. 1983.

25 R. H. Monroe, "Design and Development of Large Wood/Epoxy Wind Turbine Blades", Proceedings, AWEA Conference, "Windpower '87", San Francisco, CA, Oct. 1987.

26 C. P. Butterfield, et al., Personal Communication and Raw Field Test Data, ESI, Inc., Boulder, CO, 1986.

27 J. Carter, Jr., Personal Communications and Engineering Calculations, Carter Wind Systems, Burkburnett, TX, 1987.

28 A.R.S. Bramwell, Helicopter Dynamics, John Wiley and Sons, New York, 1976.

29 L. A. Viterna and R. D. Corrigan, "Fixed Pitch Rotor Performance of Large HAWT", Proceedings, DOE/NASA Workshop on Large HAWT, NASA Conference-810752, Cleveland, OH, July 1981.

30 J. L. Tangler and C. Ostowari, "HAWT Post Stall Airfoil Characteristics Synthesization", DOE No. DE-ACO4-76DP03533, Rocky Flats Test Center, Golden, CO, 1984.

31 S. F. Hoerner, Fluid-Dynamic Drad, Hoerner Fluid Dynamics, P.O. Box 342, Brick Town, NJ, 08723, 1975.

32 E. H. Gilmore, "Windpower to Assist in Stripper (Oil) Well Pumping", AEI/WTSU Report 85-1, Canyon, TX, Feb. 1985.

33 D. M. Prossner, "Carter Wind Systems: Performance Results", Carter Wind Systems Press Release, Burkburnett, TX, May 1985.

34 "ESI 54S Manufacturer's Specifications", ESI Inc., Boulder, CO, Aug. 1983.

35 S. N. Walker and J. E. Wade., "Effects of Precipitation on Wind Turbine Performance", Pacific Wind Energy, SERISTR-217-3287, March 1988.

36 N. M. Karaolis, et al., "Passive Aerodynamic Control Using Composite Blades", Proceedings, BWEA Workshop on use of Composite Materials for Wind Turbines, ETSU-N-109, Harwell, UK, Nov. 1987. 


\subsection{APPENDICES}

\section{1: COMPUTER CODE: DETERMINATION OF COMPOSITE BLADE SECTION PROPERTIES}

\subsection{1: BRIEF DISCUSSION}

The computer program used to calculate the blade section properties uses the algorithms explained in Chapter 4. In summary, the program has the following general characteristics:

Uses unidirectional composite elasticity theory. makeup.

Has provision for arbitrary skin, spar, shell, shear web, and afterbody geometry and laminate

Has provision for addition of ballast weights or other nonstructural elements.

Uses either the actual laminate thickness or the weighted-area method for determining blade section values.

Employs second-order curve-fit routines to calculate 33 differential section element contributions and spanwise integration over 10 span stations.

Provides the following section properties:

1. principal axes.

2. moments and products of inertia.

3. polar moments of inertia.

4. bending centroid (area centroid).

5. torsional centroid (shear center).

6. section bending stiffness.

7. section torsional rigidity.

8. mass center.

\subsubsection{PROGRAM LISTING, STRESSM}

The program STRESSM is listed here in its entirety. This version is intended for static loading conditions such as a laboratory bench test. Part 1 contains the beam section calculations and Part 2 the deflection calculations. Following that is a summary section and the aeroelastic results. The working of the program is fully explained in the comment lines, as are the input and output files, forms and units. In the elastic twist analysis programs, the deflection calculations of Part 2 and the summaries at the end differ; these are explained below in further Appendices (STRESSDATA and STRESSELASTIC).

PROGRAM ELASTIC TWIST

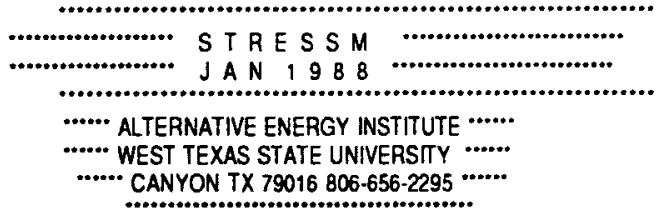

THIS PROGRAM CALCULATES THE SECTION MASS AND STIFFNESS PROPERTIES OF AN ARBITRAAY EXTERNAL GEOMETRY (TWIST, TAPER) AND SPECIFIED INTERNAL SPAR-SHELL STRUCTURAL GEOMETRY, COMPOSTTE WIND TURBINE BLADE, AND THEN FINDS THE DEFLECTIONS AND STRAINS IN THE LAMINATE FOR A SPECIFIED EXTERNAL LOAD.

THIS VERSION OF THE PROGRAM "STRESSM") INCLUDES ALL THE BENDING AND TORSIONAL CALCULATIONS, AND IS INTENDED FOR STATIC LOADING CONDITIONS SUCH AS FOR A LABORATORY BENCH TEST.

THE PROGRAM WAS ORIGINALLY WRITEN BY E.VAN DUSEN (COMPOSITE ENGINEERING, INC., 277 BAKER AVE., CONCORD MA 01742 617-371-3132) FOR COMPOSTIE RACING SHELLS AND HAS BEEN EXTENSIVELY REWRITTEN BY F.S. STODDARD (AEI, BOX 248 WTSU, CANYON TX 79016) FOR WIND TURBINE BLADES.

\section{PROGRAM DESCAIPTION}

PART 1 OF THIS PROGRAM CALCULATES THE SECTION PROPERTIES OF AN ARBITRARY COMPOSITE BEAM. USING EITHER ACTUAL THICKNESSES AND MODULI OF PLIES, OR USING MODULUS-WEIGHTING (THE LATTER ROUTINE HAS BEEN SUPPRESSED FOR THE CALCULATION OF ELASTIC TWIST). THE BEAM (BLADE) IS MODELED BY A SHELL OF CONSTANT THICKNESS WITH ARBITRARY LONGTUDINAL MODULUS AND SHEAR MOOULUS, AND A SPAR CONSISTING OF A NOSE SECTION EMBEDDED NEXT TO THE SHELI IN THE BLADE LEADING EDGE, AND A WEB (OR WEBS) LOCATED INTERNALLY. THE SPAR ALSO HAS CONSTANT THICKNESS AND ARBITRARY LONGIUDINAL AND SHEAR MODULI. THE WEB IS DESCRIBED ONLY BY ITS MOMENTS OF INERTIA AND CENTROID IN THE SECTION AXIS SYSTEM. MULTI-WEB BEAM DESIGNS ARE HANDLED BY INCLUDING ALL THE ACTUAL WEBS INTO THIS ONE MODEL WEB.

THE SECTION AIRFOIL SHAPES ARE DETERMINED BY SCALING AN INPUT TABLE OF OFFSETS. THE CHORD SCALING FACTOR (SCALE) DETERMINES THE AIRFOIL CHORD AND THE THICKNESS SCALING FACTOR (TSCALE) DETERMINES THE SECTION THICKNESS PROPORTIONAL TO THE TABLE OF OFFSETS. ALL THESE INPUT DATA ARE INCLUDED IN A DATAFILE GIVING THE GEOMETRY AND STRUCTURAL DESIGN OF THE BLADE OF INTEREST.

IN PART I PARABOLIC SEGMENTS ARE FITTED THROUGH EACH GROUP OF THREE SECTION OFFSET POINTS. THE I OF THE SKIN+SPAR+WEB IS CALCULATED BY DIVIDING THE GROUP OF POINTS INTO (DIV) STRAIGHT LINE SEGMENTS OF ROUGHLY EQUAL SRE AND SUMMING THE APPROPRIATE VARIABLES.

THE SECTION DETAILS ARE PRINTED FOR EACH STATION, INCLUDING THE ACTUAL AIRFOIL OFFSETS WHICH CAN BE USED FOR TEMPLATE CONSTRUCTION. 
PART 2 OF THE PROGRAM SUBJECTS THE BLADE TO APPLIED LOADING WHICH MIGHT BE DUPLICATED IN A BENCH TEST. THE BLADE IS CANTILEVERED AT ITS ROOT (STATION 11) AND FREE AT THE TIP (STATION 1)

THE PROGRAM THEN CALCULATES THE EQUILIBRIUM DEFLECTIONS AND TWIST OF THE LOADED BLADE, AND THE RESULTING STRAINS IN THE SKIN LAMINATE AT 6 SPECFFEO INPUT LOCATIONS AROUND THE SECTION FOR EACH STATION.

THE DISTRIBUTED (APPLIED) LOADING IS RESOLVED INTO X,Y, AND $Z$ COMPONENTS. X LIES ALONG THE (UNDEFLECTED) AXIS OF THE BLADE WITH THE ORIGIN AT THE BLADE ROOT. Y-AXIS IS IN THE LAGGING DIRECTION AND Z-AXIS IS IN THE FLAPPING DIRECTION. THE FREE STREAM WIND WOULD BE IN THE POSITIVE Z-DIRECTION, AND ROTATION WOULD THUS BE CLOCKWISE LOOKING UPSTREAM FROM AFT OF THE ROTOR.

THE BLADE STATION SPACING AND APPLIED LOADING ARE LISTED IN ANOTHER DATAFILE, WHICH ALSO INCLUDES ROTOR SPEED, BLADE RADIUS, BLADE PITCH ANGLE, AND BLADE CONING ANGLE. THE INPUT DATAFILES ARE COMPLETELY COMPATIBLE BETWEEN VERSIONS OF THIS PROGRAM: STRESSM, STRESSELASTIC, AND STRESSNOTWIST.

THE X.Y PLANE CONSTITUTES THE PLANE OF ROTATION OF THE ROTOR THE Z-DEFLECTIONS ARE THE FLAPPING VALUES AND THE Y-DEFLECTIONS THE LEAD-LAG VALUES. TWIST AND PITCH ARE MEASURED POSITIVE AIRFOIL NOSE-DOWN, OR TENDING TO DECREASE ANGLE-OF-ATTACK

(

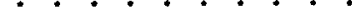

.....-.-INITIALIZE VARIABLES

DIMENSION XC(5000),YC(5000),R(4),XX(33),YY(33)

DIMENSION RX(11),YSO(6),ZSO(6),DFY(11), DFZ(11),BWT(11)

REAL MLAG(11),MFLAP(11),MLAGP(11),MFLAPP(11)

DIMENSION Q(11),OT(11),OS(11),TLAST1(11),TLAST2(11)

+,ELAST1(11),ELAST2(11),TELAST1(11),TELAST2(11)

COMMON $X(33), Y(33)$

DIMENSION STX(11),EIYP(11),EIZP(11),FX(11),PANG(11)

+ VY(11),VZ(11),EA1(11),SCALE(11),AANG(11),TSCALE(11)

+ ,SXTN(11),SX(11),DEFY(11),DEFZ(11),YCB(11)

,,ZCB(11),GJ1(11),YCT(11),ZCT(11),PM(11),TANG(11)

+,GJ2(11),EIFLAP(11),ELAG(11),WB(11),YBAL(11),ZBAL(11)

DIMENSION WA1(11), YGB(11),ZGB(11),WT(11),YGBB(11),ZGBB(11)

REAL IXMO,IYMO, IXMT, IYMT, IXMG, IYMG,IBMT, IBMG, IMBB, IMBG

REAL IZO(11), YYO(11),IZT(11),IYT(11),IZG(11), YG(11)

REAL IMT(11),IMG(11),IMGB(11)

INTEGER STA,IST, NPT, I, J,DIV, ID,KC,JC,IC,J1, NSO,TOGGLE

REAL CY,LX(11),LY(11),LZ(11),MY(11),MZ(11),MPY(11),MPZ(11)

+,NANG(11),MYE(11),MZE(11),MYP(11),MZP(11),LYC(11),LZC(11)

CHARACTER*40 INP1

CHARACTER*40 INP2

CHARACTER* 40 HEADING

CHARACTER 80 HEAD2

C

-.-READ INPUT FILES: INP1 IS THE BLADE SECTION GEOMETRY FILE ---AND INP2 IS THE APPLIED LOADING FILE. BOTH ARE READ FROM -..--THE CURRENT FILE WORKSPACE UNDER THE NAMES INPUT FROM THE

...-KEYBOARD VIA THE FOLLOWING COMMANDS

WRITE $(1,100)$

100 FORMAT(ENTER INPUT FILE NAME FOR SECTION GEOMETRY:") READ $(1,101)$ NP1

101 FORMAT (1A40)

WRTTE $(1,102)$

102 FORMAT(ENTER INPUT FILE NAME FOR APPLIED LOADING:") READ $(1,101)$ NP2

C

OPEN(UNIT $=5$, FILE $=$ INP1)

OPEN(UNT $=6, F I L E=\mid N P 2)$

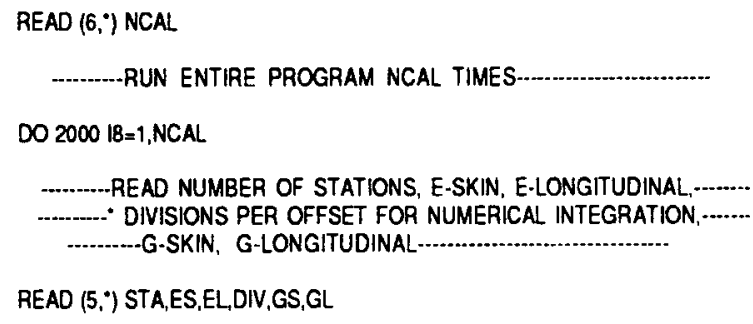

READ (5.") STA,ES,EL,DIV,GS,GL

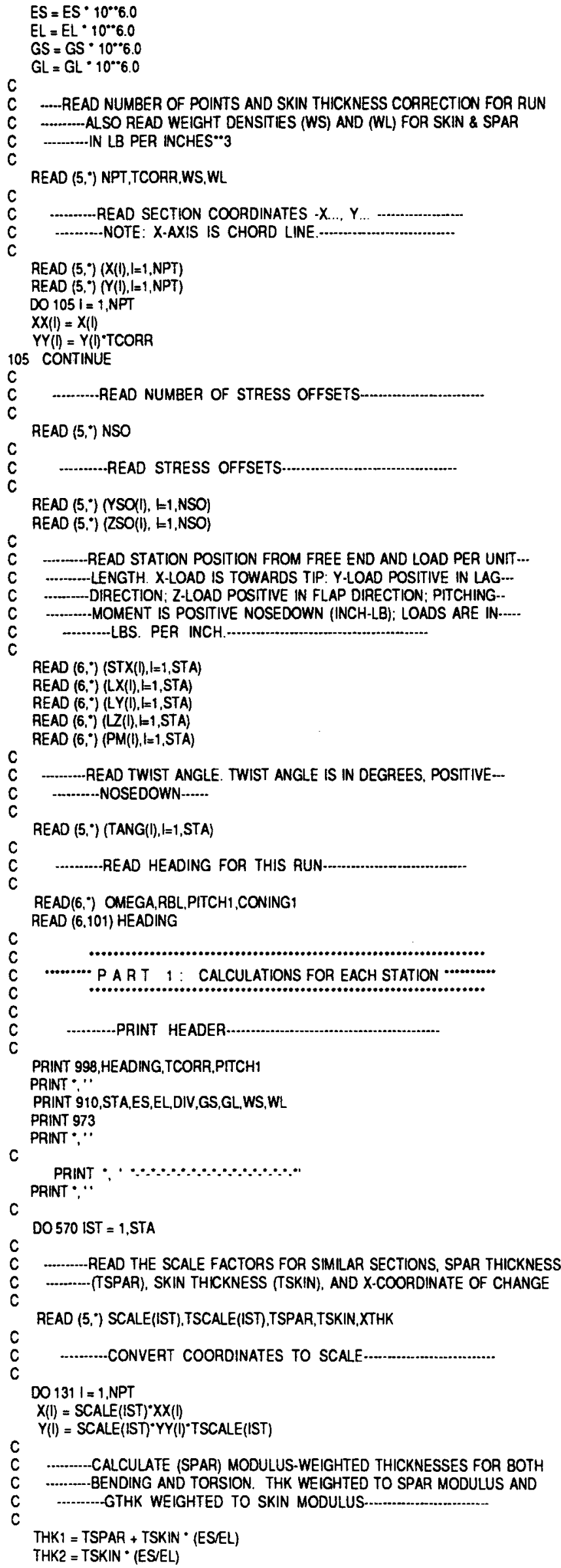




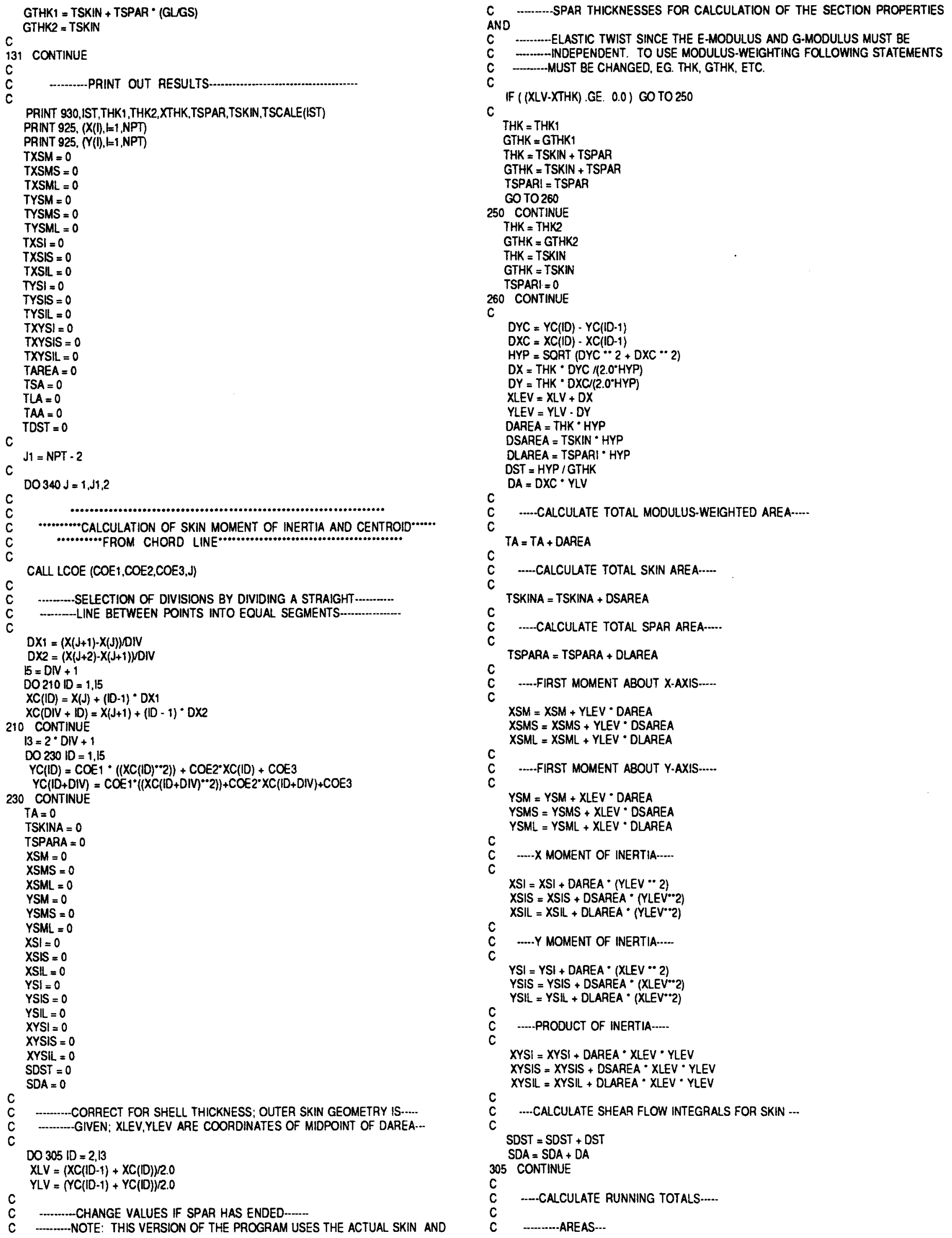


C

TAREA $=$ TAREA + TA

$T S A=T S A+T S K I N A$

c

$T L A=T L A+T S P A R A$

C

TXSM $=T X S M+X S M$

TXSMS $=$ TXSMS + XSMS

$T X S M L=T X S M L+X S M L$

$c$
C
C

C -...-1ST MOMENT OF AREA ABOUT Y-AXIS-..

TYSM $=$ TYSM + YSM

TYSMS $=$ TYSMS + YSMS

TYSML $=$ TYSML + YSML

C ........-X-MOMENT OF INERTIA--

$T X S|=T X S|+X S \mid$

TXSIS = TXSIS + XSIS

$\mathrm{TXSIL}=\mathrm{TXSH}+\mathrm{XSH}$

c -....... Y-MOMENT OF INERTIA-..

$T Y S I=T Y S I+Y S I$

TYSIS = TYSIS + YSIS

TYSIL $=$ TYSH + YSII

C

C -...PRODUCT OF INERTIA-.-

TXYSI $=$ TXYSI + XYSI

TXYSIS $=$ TXYSIS + XYSIS

TXYSIL $=$ TXYSIL + XYSIL

C

$T A A=T A A+S D A$

TDST $=$ TDST + SDST

340 CONTINUE

c •CALCULATE SECTION VALUES BASED ON MODULUS-WEIGHTING $* . . .$.$$
\text { -..-MODULUS-WEIGHTED BENDING CENTROID... }
$$

$S X B=$ TYSM $/$ TAREA

SYB $=$ TXSM $/$ TAREA

C

C -.-MODULUS-WEIGHTED ERS.....

$E X S I=T X S I \cdot E L$

C

$E Y S i=T Y S I \cdot E L$

C …....READ WEB INFORMATION - CROSS-SECTIONAL AREA (AL)......

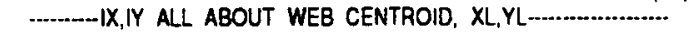

READ $\left(5,{ }^{\circ}\right) A L, X L I, Y L I, X Y L I, X L, Y L$

C

C

C

PRINT 940,AL, XLI,YLI,XYLI,XL,YL

C --1ST AREA MOMENTS OF WEB....

$X L M=A L \cdot Y L$

$Y L M=A L \cdot X L$

C

C

$E X L:=E L * X L I$

$E Y L I=E L \cdot Y L I$

C

C -...-...-CALCULATION OF CENTROID AND EI FOR TOTAL COMPOSITE STRUCTURE

C

$E A=E L \cdot T A R E A+E L \cdot A L$

$E A 1($ IST) $=E A$

C -.-TOTAL COMPOSITE STRUCTURE BENDING CENTROID BASED ON

C -..-MODULUS-WEIGHTING--.-

C

$X B=(E L * T Y S M+E L * Y L M) / E A$

$Y B=(E L \cdot T X S M+E L \cdot X L M) / E A$

$Y C B(\mid S T)=X B$

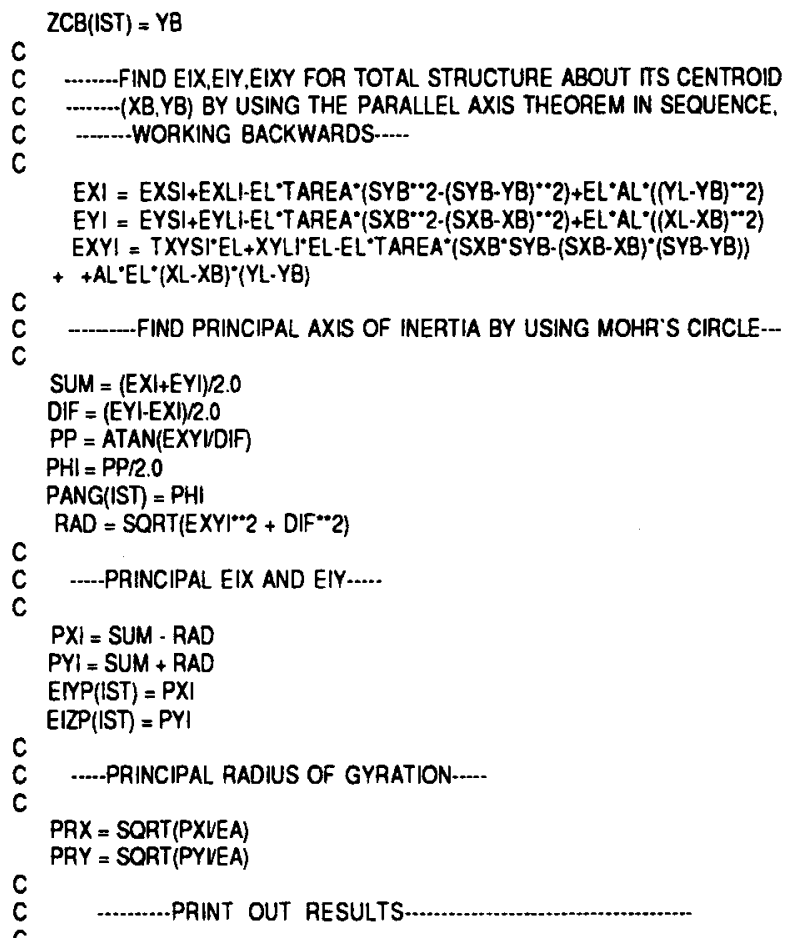

\section{PRINT 945,X9,YB,TAREA,PHI,PXI,PYI,PRX,PRY,EA}

.........CALCULATE POLAR MOMENT OF INERTIA: $J=I X+\mid Y . . . . . .$.

......IF G-SKIN \& E-SKIN ARE DIFFERENT THE TORSIONAL CENTOID. ......-WILL DIFFER FROM THE BENDING CENTROID

$G A=G S * T A R E A *(E S / E L)+G L \cdot A L$

C

-...-CALCULATE SHEAR CENTER (TORSIONAL CENTROID) BASED ON .....MODULUS-WEIGHTED VALUES.....

$X B T=(G S \cdot T Y S M+G L \cdot Y L M) / G A$

$Y B T=(G S \cdot T X S M+G L \cdot X L M) / G A$

$Y C T(I S T)=X B T$

C $Z C T(I S T)=Y B T$

C
C CALCULATE TORSIONAL STIFFNESS

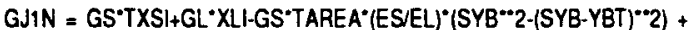

+ GL*AL'(YL-YBT)**2 + GS*TYSI + GL*YLI - GS*TAREA*(ES/EL)*

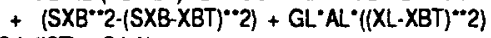

$G J 1(I S T)=G / 1 N$

C ....CALCULATE ALTERNATE TORSIONAL STIFFNESS BASED ON SHEAR FLOW.-

GJ2N $=\mathrm{GS}^{*} 4.0^{\circ}\left(\mathrm{TAA}^{* * 2}\right) /$ TDST

GJ2(IST) $=$ GJ2N

C

C $\quad$-........PRINT FURTHER RESULTS

C PRINT 950,XBT, YBT,GJ1N,GJ2N

C PRINT $\because, \quad \ldots \ldots \ldots \ldots$.......

PRINT,$\cdot \cdot$

C "CALCULATE SECTION VALUES BASED ON ACTUAL THICKNESSES $\ldots . .$.

-...-SKIN AND SPAR BENDING CENTROIDS....

SXBS $=$ TYSMS $/$ TSA

$S X B L=T Y S M L / T L A$

SYBS $=$ TXSMS $/$ TSA

C

$S Y B L=T X S M L / T L A$

C -..-CALCULATION OF CENTROID AND EI FOR TOTAL COMPOSITE STRUCTURE

$E A=E S \cdot T S A+E L \cdot T L A+E L \cdot A L$

$E A 1(I S T)=E A$

C

C ...-TOTAL COMPOSITE STRUCTURE BENDING CENTROID BASED ON

C -...ACTUAL THICKNESSES.... 
$c$

$X B=(E S \cdot T Y S M S+E L \cdot T Y S M L+E L \cdot Y L M) / E A$

$Y B=(E S \cdot T X S M S+E L \cdot T X S M L+E L \cdot X L M) / E A$

$\mathrm{YCB}(\mathrm{IST})=\mathrm{XB}$

$\mathrm{ZCB}(\mathrm{ST})=\mathrm{YB}$

.....FIND EIX,EIY,EIXY FOR TOTAL STRUCTURE ABOUT ITS CENTROID

..-(XB,YB) BY USING THE PARALLEL AXIS THEOREM IN SEOUENCE

....-.-WORKING BACKWARDS, FOR THE SKIN, SPAR, AND WEB -...-

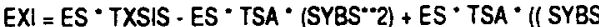

$\left.+\quad-Y B)^{*} \cdot 2\right)+E L \cdot T X S I L-E L \cdot T L A \cdot(S Y B L \cdot * 2)+E L \cdot T L A$

- ((SYBL $-Y B) \cdots 2)+E L \cdot X L I+E L \cdot A L \cdot((Y L-Y B) * 2)$

$E Y \mid=E S^{*}$ TYSIS - ES * TSA * $\left(\right.$ SXBS $\left.^{*} 2\right)+$ ES $^{*}$ TSA ${ }^{*}($ (SXBS

$+\quad-X B)^{* * 2)}+E L \cdot T Y S I L-E L \cdot T L A \cdot(S X B L * 2)+E L+T L A$

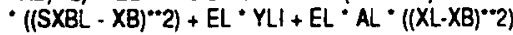

EXYI $=$ ES * TXYSIS $-E S \cdot T S A \cdot S Y B S \cdot S X B S+E S \cdot T S A$

$+\quad$ (SYBS - YB) * (SXBS - XB) + EL - TXYSIL - EL * TLA

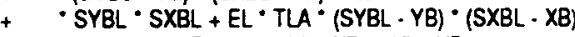

c

$c$

$+E L \cdot X Y L I+E L \cdot A L \cdot(X L-X B) \cdot(Y L-Y B)$

SUM $=(E X|+E Y|) / 2.0$

DIF $=(E Y L E X \mid) / 2.0$

$P P=A T A N(E X Y V D I F)$

$\mathrm{PHI}=\mathrm{PP} / 2.0$

PANG(IST) $=$ PH

RAD $=$ SORT $(E X Y|\cdots 2+D| F \cdots 2)$

C -..PRINCIPAL EIX AND EIY....

$P X I=S U M-R A D$

$P Y I=S U M+R A D$

EIYP(LST) $=P X \mid$

$\mathrm{EIZP}(\mathbf{I S T})=\mathrm{PY} \mid$

C -..PRINCIPAL RADIUS OF GYRATION..--

PRX = SORT (PXIEA)

$P R Y=$ SORT (PYIEA)

-...-PRINT OUT RESULTS

PPINT 946,XB, YB,TSA,TLA, PHI,PXI,PYI,PRX,PRY,EA

CALCULATE POLAR MOMENT OF INERTIA $J=I X+I Y$

-...IF G-SKIN \& E-SKIN ARE DIFFERENT THE TORSIONAL CENTROID. WILL DIFFER FROM THE BENDING CENTROID-

-IT WILL ALSO INCLUDE CONTRIBUTIONS FROM SKIN,SPAR\&WEB-

$G A=G S * T S A+G L \cdot T L A+G L * A L$

-CALCULATE SHEAR CENTER (TORSIONAL CENTROID) BASED ON

-.-ACTUAL THICKNESSES OF LAMINATES--.

$X B T=(G S \cdot T Y S M S+G L \cdot T Y S M L+G L \cdot Y L M) / G A$

$Y B T=(G S \cdot T X S M S+G L \cdot T X S M L+G L \cdot X L M) / G A$

$\mathrm{YCT}($ IST $)=\mathrm{XBT}$ $\mathrm{ZCT}(\mathrm{LST})=\mathrm{YBT}$

-.........CALCULATE TORSIONAL STIFFNESS

GJ1N = GS * TXSIS - GS * TSA $\cdot\left(\right.$ SYBS**2) + GS ${ }^{*}$ TSA ${ }^{*}($ (SYBS

$\left.-Y B T)^{*}=2\right)+G L * T X S I L-G L \cdot T L A \cdot(S Y B L * 2)+G L$

- $T L A \cdot\left((S Y B L+Y B T)^{*} 2\right)+G L \cdot X L I+G L \cdot A L \cdot((Y L$

- YBT)*-2) + GS * TYSIS - GS* TSA* (SXBS*2) + GS

- TSA* ((SXBS - XBT)"-2)+ GL・TYSIL - GL・TLA

- $\left(\mathrm{SXBL} \cdot{ }^{-2}\right)+\mathrm{GL} \cdot \mathrm{TLA} *\left((\mathrm{SXBL}-\mathrm{XBT}) \cdot{ }^{* 2}\right)+\mathrm{GL} \cdot \mathrm{YLI}$

$+G L \cdot A L \cdot((X L-X B T) * 2)$

$\mathrm{Gl}_{1}(\mathrm{IST})=\mathrm{GJ1N}$

C

c

C

PRINT 950,XBT, YBT, GJIN,GJ2N

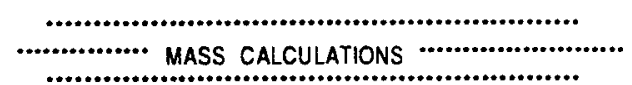

SECTION MASS CALCULATIONS CAN NOW BE DONE USING THE ACTUAL THICKNESSES....

----TOTAL RUNNING WEIGHT IS THE SUM OF ALL COMPONENTS....

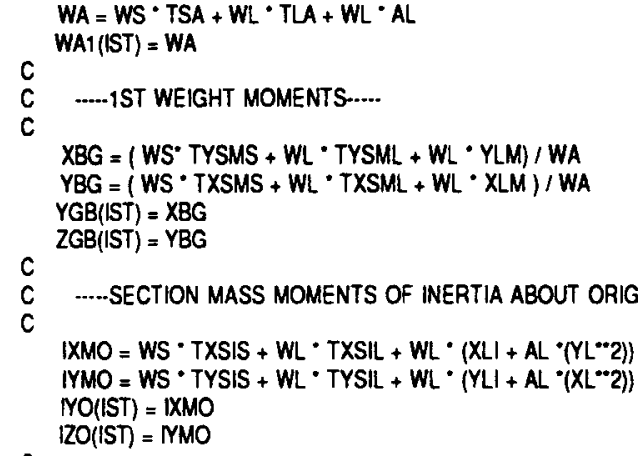




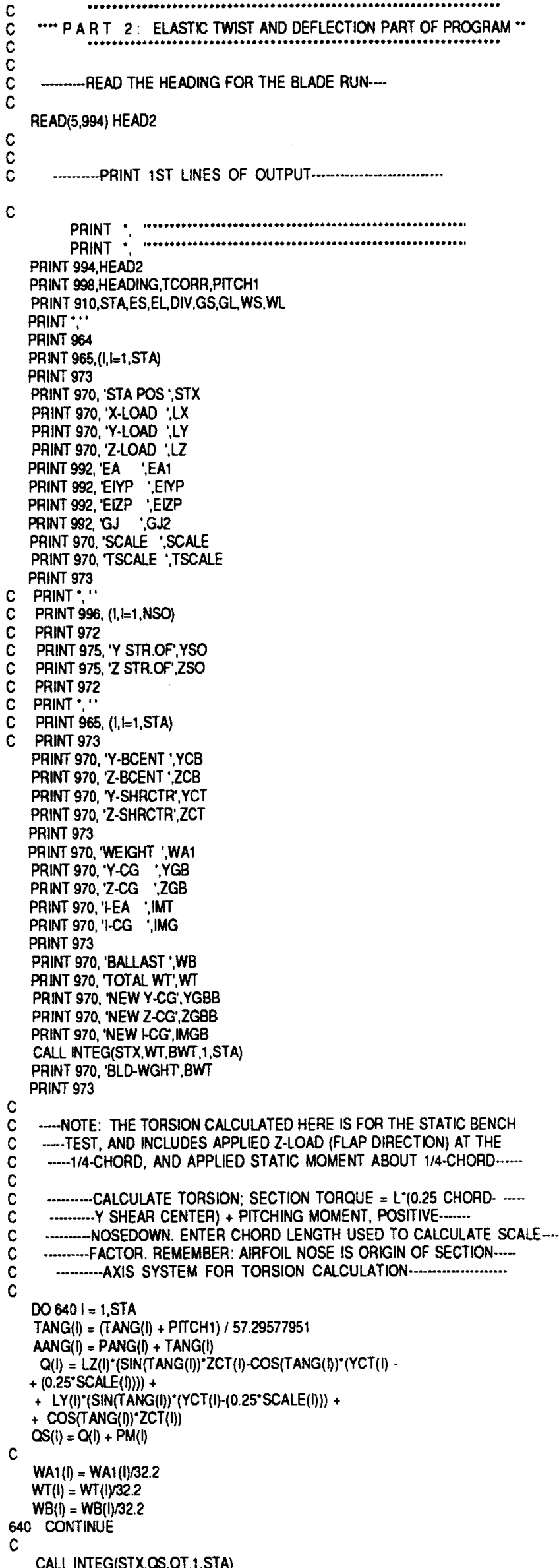

C

PRINT 970, TORSION ';OT

C

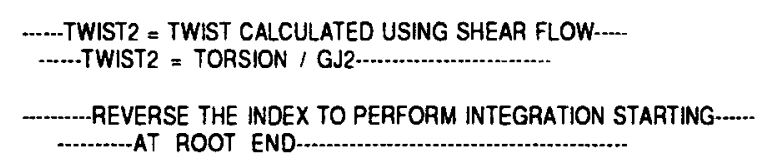

-..-PRINT TWIST, RENUMBERED

C PRINT 993, 'TORSDEF1',TELAST1

DO $800 \mathrm{l}=1$, STA

TELAST2(1)=TELAST2(1) 57.29578

800 CONTINUE

C

c

PRINT 995,'EL TWIST, TELAST2

DO $820 !=1$, STA

TELAST2(1)=TELAST2(1)/57.29578

820 CONTINUE

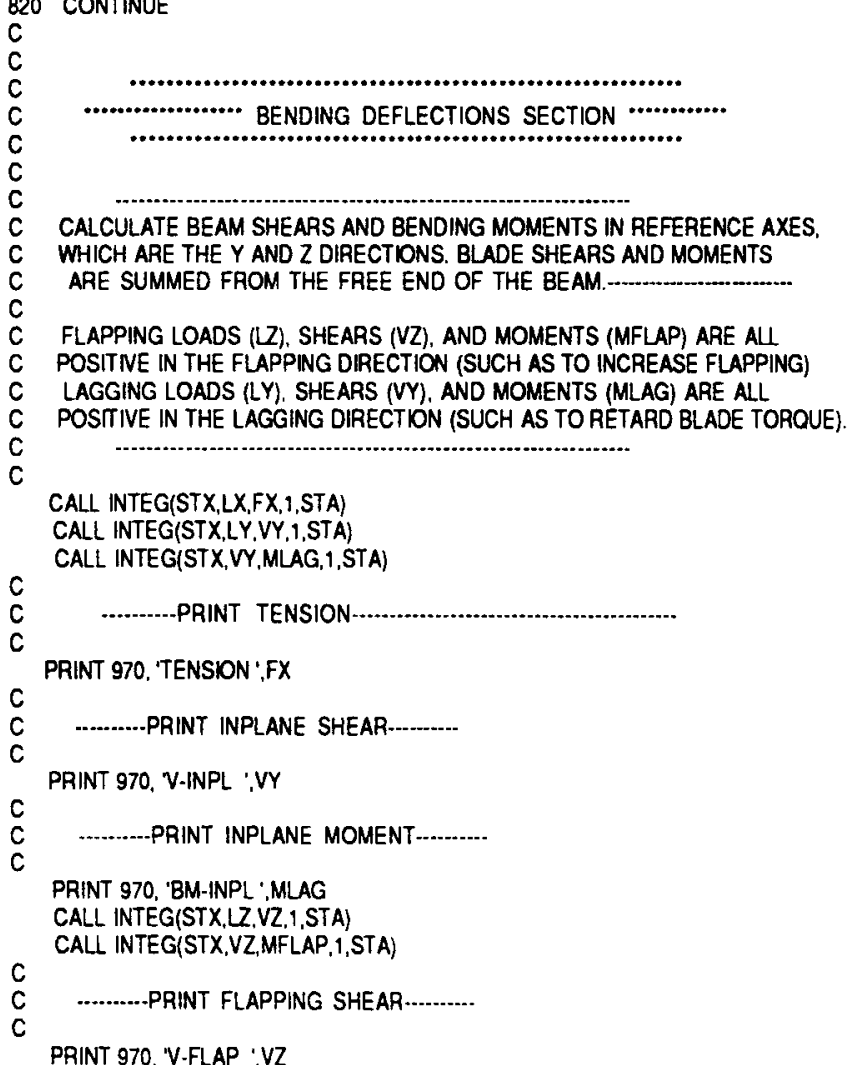


PRINT 970, 'BM-FLAP ',MFLAP

NOTE: THIS PROGRAM DOES NOT PRINT OUT LAMINATE STRESSES.

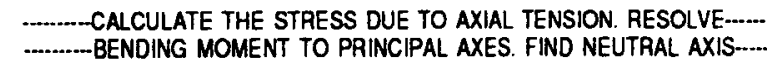

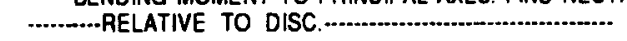

DO 870 L 1 ,STA

TANG $(I)=$ TANG $(i)+$ TELAST2 $(I)$

AANG $(1)=$ PANG $(D)+$ TANG $(1)$

$\operatorname{SXTN}(1)=F X(1) * E S / E A 1(1)$

.........NOTE: ROTATION OF AXES FROM SECTION COORDINATES TO-...

........PRINCIPAL AXES COORDINATES IS THROUGH POSITIVE ANGLE.... -PANG (COUNTERCLOCKWISE)

MFLAPP $(1)=$ MFLAP $(1)^{*} \operatorname{COS}($ AANG $(I))-M L A G(1) \cdot \operatorname{SIN}($ AANG $(1))$ $M L A G P(I)=M L A G(I)^{\circ} \operatorname{COS}(A A N G(I))+M F L A P(I) * S I N(A A N G(I))$

870 CONTINUE

DO $900 \notin 1$, STA

AANG $(I)=$ AANG $(1) \cdot 57.29577951$

900 CONTINUE

C

PRINT 970, 'PR A A :AANG

C

C

DO901 l=1,STA

AANG ()$=$ PANG $(\eta)+$ TANG ()

901 CONTINUE

PRINT 970, 'SX TENS.',SXTN

-......CALCULATE STRESSES DUE TO BENDING. STRESS IS THE SUM-.-...-OF BENDING IN PLANES OF PRINCIPAL AXES.

$D O 1005 K=1, N S O$

DO $885 \mathrm{~J}=1$, STA

........TRANSLATE YSO AND ZSO TO COORDINATES THROUGH CENTROID-

$Y K=Y S O(K)+\operatorname{SCALE}(J) \cdot Y C B(J)$

$Z K=Z S O(K)^{-S C A L E}(J)-Z C B(J)$

\section{-...-...'S ARE DISTANCES TO STRESSED FIBER}

-.--IN PRINCIPAL AXIS SYSTEM, FOR THE SELECTED STRESS OFFSETS -CALCULATED ABOVE

$C Y=Y K * \operatorname{COS}(P A N G(J))+Z K * \operatorname{SIN}(P A N G(J))$

$C Z=Z K{ }^{*} \operatorname{COS}(P A N G(J))-Y K^{*} \operatorname{SIN}(P A N G(J))$

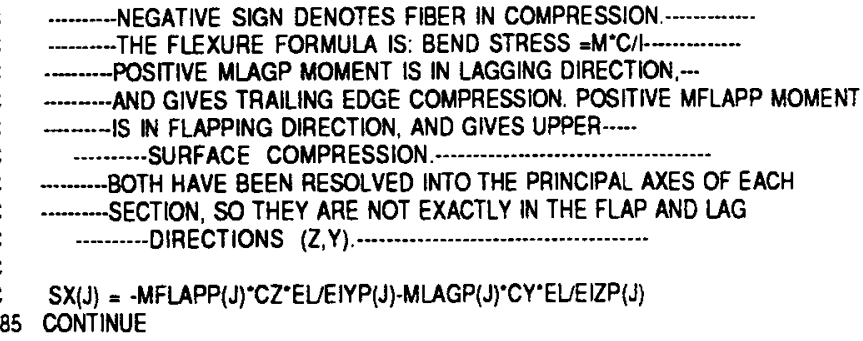

C

c

C

890

C

C

C

1005

$\mathrm{C}$

C

\section{DO $1110 !=1$, STA}

$R=S T A+1-1$

$R X(I R)=S T X(S T A)-S T X(D)$

MYP $(I)=$ MFLAPP $(I) / E \mid Y P(I)$

$M Z P(I)=$ MLAGP(I)/EIZP(I)

$M Y(I R)=\left(M Y P(I){ }^{*} \operatorname{COS}(A A N G(M))+\left(M Z P(1)^{*} \operatorname{SIN}(A A N G(1))\right.\right.$

C

$M Z(I R)=(M Z P(I))^{\circ} \operatorname{COS}(A A N G(I))-\left(M Y P(I)^{*} \operatorname{SIN}(A A N G(I))\right.$

1110 CONTINUE

C

C

DO $1120 \mathrm{l}=1$ STA

IR $=$ STA $+1-1$

$M Y E(l)=M Y(\mathbb{R})$

$M Z E(I)=M Z(\mathbb{R})$

$L Y C(1)=L Y(1)$

C

$L Z C(1)=L Z(D)$

1120 CONTINUE

C .....PRINT THE CURVE M/EI-REVERSE, Y-DIRECTION, INTEGRATED FROM

C -....-THE FIXED END -....

PRINT 992, 'M.EIYP ',MYP

C

C -.-PRINT THE CURVE MEI-REVERSE, Z-DIRECTION, INTEGRATED FROM

C ......THE FIXED END.....

PRINT 992, 'M.ERP ',MZP

PAINT 973

C

C .........FIND DEFLECTION BY INTEGRATION: D2YIDX2 = MEF..........

C ….....WHERE LYC = DYIDX AND LZC = DZOX ARE THE ROOT SLOPES

C -.......NOTE THIS SECTION IS SIMILAR TO THE SUBROUTINE "INTEG"

$\operatorname{lYC}(1)=0.0$

$\operatorname{LZC}(1)=0.0$

$\operatorname{DEFY}(1)=0.0$

DEFZ $(1)=0.0$

$16=$ STA -1

DO $1115 \mathrm{JJ}=1,16,2$

$\mathrm{J}=\mathrm{JJ}$

IF(J.EQ. 6$)\rfloor=\jmath$

CALL LCOE1(C1,C2,C3,J,RX,MZ)

CALL LCOE1(D1,D2,D3,JRX,MY)

IF(JJ.EQ. . 6$) J=J+1$

$C_{4}=L Y C(J)-C 1^{*} \operatorname{RX}(J) \cdot 3 / 3-C 2^{*} \operatorname{RX}(\mathrm{J})^{*} / 2 / 2-\mathrm{C}^{*} \operatorname{RX}(\mathrm{J})$

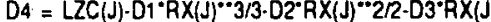

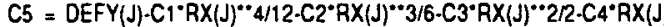

D5 = DEFZ(J)-D1*RX(J)**4/12-D2*AX(J)**3/6-D3*RX(J)**2/2-D4*AX(J)

$K=J+1$

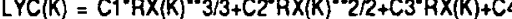

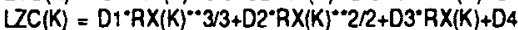

DEFY $(K)=C 1^{*} R X(K)^{* *} 4 / 12+C 2^{*} R X(K)^{* *} 3 / 6+C 3^{*} R X(K)^{* *} 2 / 2+C 4^{*} R X(K)+C 5$

$D E F Z(K)=D 1^{*} R X(K) * 4 / 12+D 2^{*} R X(K)^{* *} 3 / 6+D 3 * R X(K)^{* *} 2 / 2+D 4^{*} R X(K)+D 5$

IF (JJ.EO. 6) GOTO 1115

$K=J+2$

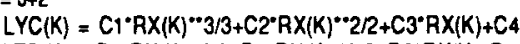

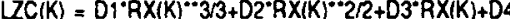

$D E F Y(K)=C 1^{*} R X(K)^{* *} 4 / 12+C 2^{*} R X(K)^{* *} 3 / 6+C 3^{*} A X(K)^{*} 2 / 2+C 4^{*} R X(K)+C 5$

C

$D E F Z(K)=D 1^{\circ} R X(K)^{* *} 4 / 12+D 2^{*} R X(K)^{* *} / 6+D 3^{*} R X(K)^{* *} / 2+D 4^{*} R X(K)+D 5$

1115 CONTINUE

C

..........REVERSE ORDER

DO $1215 \mathrm{l}=1$,STA 


$$
\begin{aligned}
& \mathbb{R}=\mathbf{S T A}+1-1 \\
& \mathrm{DFY}(\mathbb{R})=\mathrm{DEFY}(\mathbb{I})
\end{aligned}
$$$$
\text { C }
$$$$
1215 \text { CONTINUE }
$$

C

C

C

C

C

PRINT 970, 'Z-DEF. ',DFZ

DO $1300 \mathrm{l}=1$, STA

EIFLAP(I) $=$ EIYP $(1)^{\circ} \operatorname{COS}($ AANG $(1))+E I Z P(1)^{*} \operatorname{SIN}($ AANG $(1))$

1300 CONTINUE

C

C

PRINT 992, 'EI-FLAP' 'EIFLAP

C PRINT 992 'ELLAG 'EILAG

PRINT 973

C

C

C FIND STRAINS DUE TO AXIAL TENSION (CENTRIFUGAL FORCE) C AND BENDING MOMENT.

STRAIN DUE TO TENSION (SXTN) IS JUST TENSION AT A C STATION DIVIDED BY THE COMPOSTIE EA OF THE STATION

DO $1500 \quad I=1$, STA

SXTN $($ (I) $=$ FX(I) $/$ EA $1($ I

AANG $(1)=$ PANG $(1)+$ TANG $(1)$

1500 CONTINUE

C

C CALCULATE STRAINS DUE TO BENDING. STRAIN IS THE SUM

C OF THE BENDING STRAIN IN THE PLANES OF THE PRINCIPAL

C AXES.

C

DO $1600 K=1, N S O$

C

C FIRST TRANSLATE Y AND Z STRAIN OFFSETS (YSO,ZSO) GIVEN

C IN UNITY SECTION COORDINATES, TO COORDINATES REFERRED TO

C THE SECTION CENTROID. THIS CALCULATION INCLUDES THE TWO

C SCALE FACTORS FOR THE BLADE SIMILAR SECTIONS (SCALE,

C TSCALE).

$Y K=Y S O(K) \cdot S C A L E(J)-Y C B(J)$

C

$Z K=Z S O K K) \cdot \operatorname{SCALE}(J) \cdot \operatorname{TSCALE}(J)-Z C B(J)$

C THE C'S ARE THE DISTANCES TO STRESSED FIBER IN THE

C PRINCIPAL AXIS SYSTEM, FOR THE SELECTED STRAIN OFFSETS

CALCULATED ABOVE

$C Y=Y K \cdot \operatorname{COS}(-A A N G(J))+Z K \cdot \operatorname{SIN}(-A A N G(J))$

$\mathrm{CZ}=Z K \cdot \operatorname{COS}(-A A N G(J)) \cdot Y K \cdot \operatorname{SIN}(-\operatorname{AANG}(J))$

THE NEGATIVE SIGN DENOTES FIBER IN COMPRESSION. THE FLEXURE FORMULA FOR STRAIN IS: BENDING STRAIN =

$M \cdot C / E I$

NOTE: THE POSITIVE MLAGP MOMENT IS IN THE LAGGING DIRECTION, AND GIVES TRALING EDGE COMPRESSION. POSITIVE MFLAPP MOMENT IS IN THE FLAPPING DIRECTION, AND GNES UPPER SURFACE COMPRESSION. BOTH HAVE BEEN RESOLVED INTO THE PRINCIPAL AXES OF EACH SECTION, SO THEY ARE NOT EXACTLY IN THE FLAP AND LAG DIRECTIONS $(Z, Y)$.

\section{CALCULATE BENDING STRAIN}

$S X(J)=-M F L A P P(J) \cdot C Z E I Y P(J)-M L A G P(J))^{*}$ CY/EIZP(J)

CALCULATE TOTAL STRAIN AND MICROSTRAIN:

$\operatorname{SX}(J)=\operatorname{SX}(J)+\operatorname{SXTN}(J)$

$S X(J)=\operatorname{SX}(J) * 1000000.0$
C

1550 CONTINUE

C

PRINT 971,'M-STRAIN',K,SX

1600 CONTINUE

C

2000 CONTINUE

C

C

905 FORMAT(5X,12,2F6.2,14,2F6.2)

910 FORMAT'" STATIONS: ',I2,2X,'E-SKIN: ',F10.2,2X,E-LONG: ',

+F10.2.' " DIVISIONS: ',14,' GS: ',F10.2.' GL: ',F10.2,

+ WS:'F5.3' WL:',F5.3)

915 FORMAT $(5 X, 12,2 X, F 6.4)$

920 FORMAT(5X,9F6,4,I,5X,8F6.4, $, 5 \times, 8 F 6.4, /, 5 X, 8 F 6.4)$

925 FORMAT(5X,9F8.4,/,3(5X,8F8,4,n)

930 FORMAT' OFFSETS FOR STATION: 'R,' THK1:'F6.4

+' THK2: 'F6.4," XTHK: ',F7.3,1,29X,'TSPAR: ",

+ F6.4: TSKIN : 'F6.4,' TSCALE: 'F6.4

933 FORMAT $(5 \times 6$ F6.4)

935 FORMAT $(5 \times, 4 \mathrm{~F} 6.3)$

940 FORMAT' WEB DATA - AL, IX,IY, IXY,XL, YL', $, 6(3 X, 68.3), n$

945 FORMAT(' POSITION OF CENTROID FROM BASE LINE X: ',F8.2

+' Y:'F8.2,/'SKIN AREA: 'F10.4/l' ANGLE OF PRINCl',

+'PAL AXIS: ',F6.2,' EIX: ',E12.5,' EIY: ',E12.5,I, RX:',

+F6.2,' RY: 'F6.2,' EA: ',E12.5, /n

946 FORMATC POSITION OF CENTROID FROM BASE LINE X: ',F8.2

+' $Y:$ : F8.2, $I^{\prime}$ 'SKIN AREA: ',F10.4,' SPAR AREA: ',F10.4,

+1, ANGLE OF PRINCIPAL AXIS: ',F6.2,' EIX: ',E12.5, EN: :

+E12.5/,'RX:'F6.2,'RY ',F6.2,'EA: 'E12.5,In

950 FORMATPPOSTTION OF TORSIONAL CENTROID X: 'F8.2,' $Y$ : '

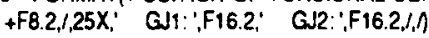

955 FORMATTSECTION MASS INFORMATION WSPAR: :F6.4,' WSKIN: '

+F6.4,10X,'SECTION RUNNING WEIGHT: 'F8.4', XCG: 'F8.2

+' YCG: ',F8.2,/,10X,'ELASTIC AXIS MOMENT OF INERTIA: ',F12.4,/

$+10 X$,'SECTION CG MOMENT OF INERTIA: $; F 12.4, /, / n$

956 FORMAT'SECTION BALLAST INFORMATION WBALLAST: 'F6.4,' XBAL

+ 'F8.2.' YBAL: 'F8.2,10X,'NEW SECTION RUNNING WEIGHT: '

+,F8.4,' XCG: ',F8.2,' YCG: ',F8.2,,10X,'OLD SECTION CG

+MOMENT OF INERTIA: ',F12,4,1,10X,'NEW SECTION CG MOMENT OF + INERTIA: ',F12.4,I!II)

963 FORMAT $(5 X, 13)$

964 FORMAT(19X,'TIP', 106X,'ROOT)

965 FORMAT(STATION :11(9X,12))

970 FORMAT(' AB,1X,11F11.2)

971 FOAMAT( 'AB, R, 11(F10.0,1X))

972

973

FORMAT('-

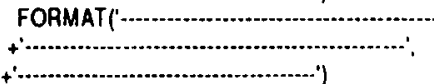

975 FORMAT(' ' A8,3X,6F12.2)

980 FORMAT(5X,8F8.2, $1,5 X, 3 F 8.2$

981 FORMAT(6X,8F8.3,I,6X,3F8.3)

983 FORMAT $7 \times, 6 F 8.4)$

985 FORMAT(', AB, 11, 11F11.2)

990 FORMAT(5X,8F8.4,/,5X,3FB.4)

992 FORMAT(' 'A8,1X,11(E10.4,1X)

993 FORMAT( 'AB, $1 X, 11 F 11.6$

994 FORMAT(1ABO)

995 FORMAT(' AB, $1 \times, 11 F 11.3$ )

996 FORMAT"' STRESS OFFSETS: ", 10, $12,10 \mathrm{X})$

998 FORMATC',1A40,' $\cdots$ TCORA = 'F6.3.' $\cdots$ PITCH = ',F6.3,

C
C

CLOSE(5)

CLOSE $(6)$

C

END 
C THIS ROUTINE INTEGRATES THE AREA UNDER A CURVE OF HEIGHT Y AND

C SPACING X BY FITIING A SECOND ORDER EOUATION THROUGH THE POINTS

C XI IS THE AREA OF EACH SEGMENT. AND XS IS THE SUM UP TO THAT

C POINT. IT STARTS AT $X(I S)$ AND STOPS AT $X(I E)$

C

DIMENSION $X(12), Y(12), X \mid(12), X S(12)$

$X \mid(1)=0.0$

$X S(1)=0.0$

$12=\mathbf{S}+$

C

DO $1070 \mathrm{JJ}=12, \mathrm{IE}, 2$

$\mathrm{J}=\mathrm{JJ}$

IF(JJ.EO. IE) $\mathrm{J}=\mathrm{J}-1$

CALL LCOE $1(\mathrm{CO} 1, \mathrm{CO} 2, \mathrm{CO} 3, \mathrm{~J}-1, X, Y)$

$\mathrm{K}=\mathrm{J}+1$

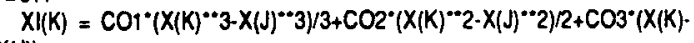
$+X(J)$

IF(JJ .EQ. IE) GO TO 1070

$K=J \cdot 1$ $+X(K)$

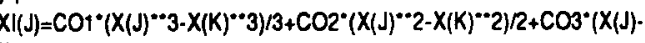

1070 CONTINUE

$X S(I S+1)=X I(I S+1)$

IF(IS .GE. IE-1) GO TO 1310

$15=1 \mathrm{~S}+2$

DO $1305 \mathrm{~J}=15 . \mathrm{JE}$

$X S(J)=X(J)+X S(J-1)$

1305 CONTINUE

1310 CONTINUE

RETURN

END

C

SUBROUTINE LCOE $(\mathrm{CO}, \mathrm{CO}, \mathrm{CO}, \mathrm{J})$

THIS SUBROUTINE FITS A SECOND ORDER CURVE THROUGH EACH SET OF 3

POINTS: STARTING AT $J . X$ IS THE ABSCISSA, AND Y THE ORDINATE.

$V I Z: Y=C^{\circ} 1^{\circ} X^{\circ} \cdot 2+\operatorname{CO}^{\circ} X+C O 3$. (THE PARABOLIC AXIS IS VERTICAL)

COMMON $X(33), Y(33)$

$\mathrm{CO} 1=0.0$

$\mathrm{CO} 2=0.0$

$\mathrm{COB}=0.0$

C1N $=X(J) *(Y(J+2)-Y(J+1))+X(J+1)^{*}(Y(J)-Y(J+2))+X(J+2)^{*}(Y(J+1)-Y(J))$

$C 2 N=\left(X(J)^{\bullet * 2}\right)^{*}(Y(J+1)-Y(J+2))+\left(X(J+1)^{* * 2}\right)^{*}(Y(J+2)-Y(J))+\left(X(J+2)^{*}\right.$ $+\rightarrow(Y(J) \cdot Y(J+1))$

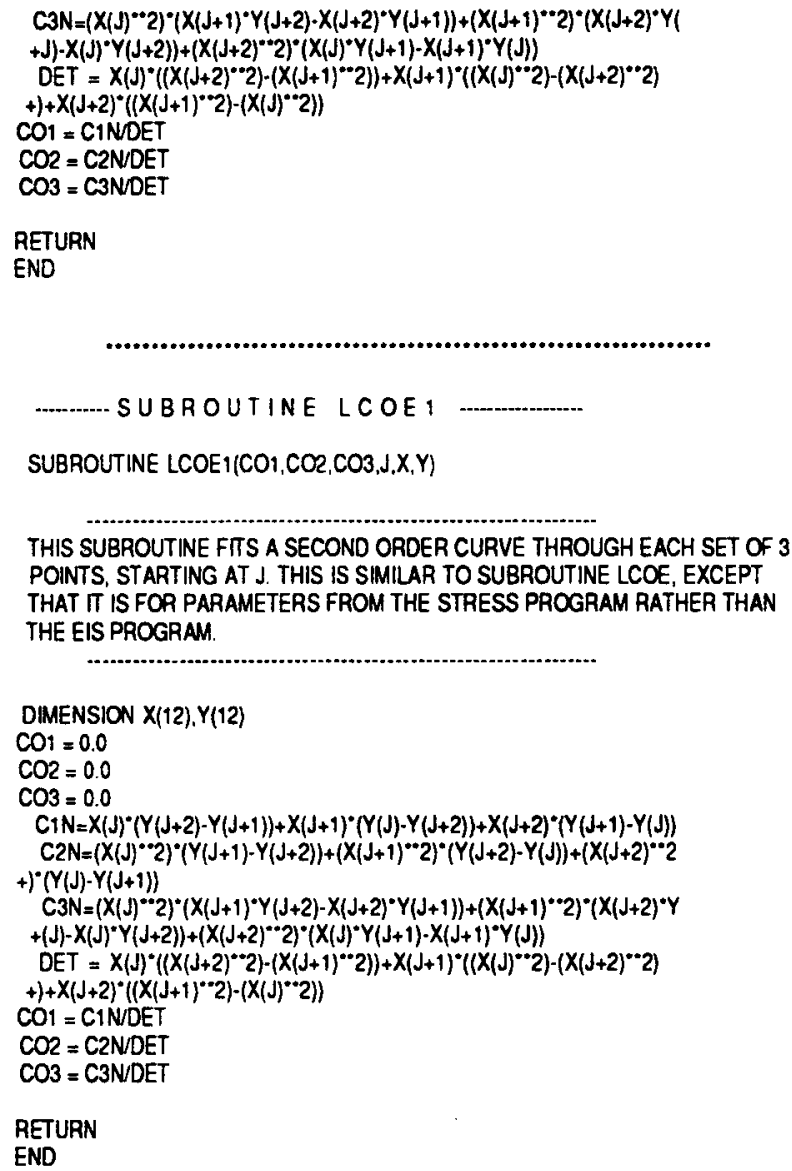

\subsubsection{SAMPLE RUN, CARTER BLADE}

The sample input files are for the Carter blade (CARTBL.AEIFINAL) and zero applied loading (CARTLD.ZERO) to illustrate the calculation of the section values for a complex composite blade. Note: for this run the Carter I-beam was not included, as this complication must be handled by the next version of the code (STRESSDATA) as shown below.

\section{INPUT BLADE FLE: CARTBLAGFNAL}

114.004 .005000 .5000 .750

$33 \quad 1.000 \quad 050 \quad 050$

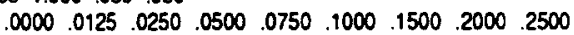

$\begin{array}{llllllllll}.3500 & .5000 & .6000 & .7000 & .8000 & .9000 & .9500 & 1.0000\end{array}$

$\begin{array}{llllllllll}.9500 & 9000 & .8000 & .7000 & .6000 & .5000 & .3500 & .2500\end{array}$

$\begin{array}{llllllllll}.2000 & .1500 .1000 & .0750 & .0500 & .0250 & .0125 & .0000\end{array}$

$\begin{array}{lllllllllll}0000 & 0244 & 0342 & 0476 & 0564 & 0.0629 & 0722 & 0783 & 0822\end{array}$

$\begin{array}{llllllllllll}0850 & 0787 & 0685 & .0542 & 0368 & 0.0174 & 0070 & 00000\end{array}$

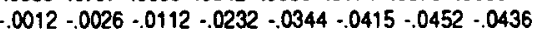

$\begin{array}{llllllll}-.0415 & -.0382 & -.0333 & -.0299 & -.0255 & -.0191 & -.0145 & .0000\end{array}$

006

$\begin{array}{llllllllll}0000 & .2500 & .5000 & 1.0000 & .5000 & .2500\end{array}$

$\begin{array}{lllllllll}.0000 & .3042 & .0787 & .0000 & -.0415 & -.0436\end{array}$

2.62 .22 .02 .02 .02 .3

4.59 .415 .526 .126 .1

$\begin{array}{lllll}21.41 & 0.995 & 0.040 & 0.200 \quad 5.90\end{array}$

$\begin{array}{llllllllll}0.153 & 0.038 & 0.0001 & 0.000 & 4.500 & 0.680\end{array}$

$\begin{array}{llll}0.594 & 1.630 & 0.250\end{array}$ $\begin{array}{lllll}21.41 & 0.995 & 0.040 & 0.203 & 5.90\end{array}$

$\begin{array}{llllllllll}0.158 & 0.038 & 0.0001 & 0.000 & 4.630 & 0.680\end{array}$

$\begin{array}{llll}0.763 & 1.630 & 0.250\end{array}$

$\begin{array}{lllll}22.19 & 0.996 & 0.141 & 0.113 & 6.31\end{array}$

$\begin{array}{llllllllllll}0.177 & 0.045 & 0.0001 & 0.000 & 4.810 & 0.700\end{array}$

$\begin{array}{llllll}0.773 & 1.630 & 0.250\end{array}$

$\begin{array}{llllll}23.44 & 0.997 & 0.244 & 0.112 & 7.00\end{array}$

$\begin{array}{llllllllll}0.145 & 0.035 & 0.0001 & 0.000 & 5.630 & 0.740\end{array}$

$0.812 \quad 1.630 \quad 0.250$

$\begin{array}{lllll}25.56 & 0.999 & 0.230 & 0.155 & 7.75\end{array}$

$\begin{array}{llllllllll}0.179 & 0.068 & 0.0009 & 0.000 & 6.690 & 0.820\end{array}$

$0.782 \quad 1.6300 .250$

$\begin{array}{llllll}28.97 & 1.001 & 0.232 & 0.189 & 8.94\end{array}$

$\begin{array}{lllllllllll}0.353 & 0.184 & 0.0001 & 0.000 & 6.810 & 0.950\end{array}$

$\begin{array}{llll}0.862 & 1.630 & 0.250\end{array}$

$\begin{array}{llllll}34.56 & 1.019 & 0.227 & 0.167 & 10.00\end{array}$

$\begin{array}{lllllll}6.150 & 2.000 & 104.0 & -7.00 & 8.64 & 0.00\end{array}$

$0.7308 .64 \quad 0.00$

$\begin{array}{llllll}42.50 & 1.055 & 0.227 & 0.167 & 13.25\end{array}$

$\begin{array}{llllll}7.440 & 5.000 & 190.0 & -25.00 & 10.63 & 0.00\end{array}$ 
$0.450 \quad 10.63 \quad 0.00$

$\begin{array}{llllllll}52.81 & 1.070 & 0.227 & 0.167 & 16.00\end{array}$

$\begin{array}{llllll}8.000 & 18.00 & 205.0 & -50.00 & 13.20 & 0.00\end{array}$

$.590 \$ 3.20 \quad 0.00$

$66.75 \quad 1.0710 .227 \quad 0.167 \quad 21.88$

$\begin{array}{llllll}8.750 & 58.00 & 258.0 & -118.0 & 16.69 & 0.00\end{array}$

4.8716 .690 .00

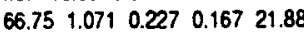

$\begin{array}{lllllll}8.750 & 58.00 & 258.0 & -118.0 & 16.69 & 0.00\end{array}$

0.00016 .690 .00

CARTER.BLADE.AEI.DATA.ALL.TEST.WTS.5-14-88
001

$00.00 \quad 40.28 \quad 80.55 \quad 120.83 \quad 161.10 \quad 201.38 \quad 241.65 \quad 281.93$

$322.20 \quad 362.48 \quad 402.75$

$\begin{array}{llllllllllll}00.00 & \infty 0.00 & 00.00 & 00.00 & 00.00 & 00.00 & 00.00 & 00.00\end{array}$

$00.00 \quad 00.00 \quad 00.00$

$\begin{array}{lllllllll}00.00 & 00.00 & \infty 0.00 & 00.00 & 00.00 & 00.00 & 00.00 & 00.00\end{array}$

$00.00 \quad 00.00 \quad 00.00$

$\begin{array}{lllllllllllllll}00.00 & 00.00 & 00.00 & 00.00 & 00.00 & 00.00 & 00.00 & 00.00\end{array}$

$00.00 \quad 00.00 \quad 00.00$

$\begin{array}{lllllllllllllllllllllll}0.00 & 0.00 & 0.00 & 0.00 & 0.00 & 0.00 & 0.00 & 0.00\end{array}$

$0.00 \quad 0.00 \quad 0.00$

$\begin{array}{llll}0.00 & 402.75 & 0.00 \quad 0.00\end{array}$

CARTER.BLADE.ZERO.LOAD.TEST.12-11-87

\section{INPUT LOAD FLE: CARTLD.ZERO}

[Note: This output gives the values for each blade section, starting with the blade tip. The airfoil shape offsets appear first. These can be used for making templates.]

TERMMNAL INPUT AND OUTPUT: STRESSM

ENTER INPUT FILE NAME FOR SECTION GEOMETAY

CARTBL, AEIFINAL

ENTER INPUT FILE NAME FOR APPLIED LOADING:

CARTLD.ZERO

CARTER.BLADE.ZERO.LOAD.TEST.12-11.87 * TCORR $=1.000 *$ PITCH $=0.000$

\# STATIONS: 11 E-SKIN: 3999999.50 E-LONG: 3999999.50 DIVISIONS: 500 GS: 499999.94 GL: 749999.88 WS: 0.050 WL: 0.050

$\because \cdots \cdots \cdots \cdots \cdots+\cdots$

OFFSETS FOR STATION: 1 THK1: 0.2400 THK2: 0.2000 XTHK: 5.900 TSPAR: 0.0400 TSKIN: 0.2000 TSCALE: 0.9950

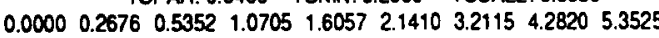
7.493510 .705012 .846014 .987017 .128019 .269020 .339521 .4100 $20.339519 .269017 .128014 .987012 .846010 .7050 \quad 7.4935 \quad 5.3525$

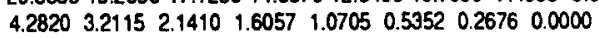

$\begin{array}{llllllllll}0.0000 & 0.5198 & 0.7286 & 1.0140 & 1.2015 & 1.3400 & 1.5381 & 1.6680 & 1.7511\end{array}$

$\begin{array}{llllllllll}1.8108 & 1.6765 & 1.4593 & 1.1546 & 0.7839 & 0.3707 & 0.1491 & 0.0000\end{array}$

$\begin{array}{llllllll}-0.0256 & -0.0554 & -0.2386 & -0.4942 & -0.7328 & -0.8841 & -0.9629 & -0.9288\end{array}$

$-0.8841-0.8138-0.7094-0.6370-0.5432-0.4069-0.30890 .0000$

WEB DATA - AL,IX,IY,IXY,XL, YL

$\begin{array}{llllll}0.153 & 0.038 & 0.000 & 0.000 & 4.500 & 0.680\end{array}$

POSITION OF CENTROID FROM BASE LINE X: $10.04 \quad Y: 0.32$

SKIN AREA: 8.7520 SPAR AREA: 0.5023

ANGLE OF PRINCIPAL AXIS: $-0.01 \mathrm{EIX}: 0.31945 E+08 \mathrm{EIY}: 0.15086 \mathrm{E}+10$

RX: 0.92 RY: $6.33 \mathrm{EA}: 0.37629 \mathrm{E}+08$

POSITION OF TORSIONAL CENTROID X: $\quad 9.81 \mathrm{Y}: \quad 0.32$

GJi: 200538112.00 GJ2: 14980016.00

SECTION MASS INFORMATION WSPAR: 0.0500 WSKIN: 0.0500 SECTION RUNNING WEIGHT: 0.4704 XCG: 10.04 YCG: 0.32 ELASTIC AXIS MOMENT OF INERTIA: 19.2822

SECTION CG MOMENT OF INERTLA: $\quad 19.2574$

SECTION BALLAST INFORMATION WBALLAST: 0.5940 XBAL: 1.63 YBAL: 0.25 NEW SECTION RUNNING WEIGHT: 1.0644 XCG: 5.35 YCG: 0.28 OD SECTION CG MOMENT OF INERTIA: $\quad 61.3052$ NEW SECTION CG MOMENT OF INERTIA: $\quad 37.8391$

$$
\text { - . . . . . . . . }
$$

OFFSETS FOR STATION: 2 THK1: 0.2430 THK2: 0.2030 XTHK: 5.900 TSPAR: 0.0400 TSKIN: 0.2030 TSCALE: 0.9950 $0.0000 \quad 0.2676 \quad 0.5352 \quad 1.0705 \quad 1.6057 \quad 2.1410 \quad 3.2115 \quad 4.2820 \quad 5.3525$ 7.493510 .705012 .846014 .987017 .128019 .269020 .339521 .4100 $20.339519 .269017 .128014 .987012 .846010 .70507 .4935 \quad 5.3525$ $\begin{array}{lllllllll}4.2820 & 3.2115 & 2.1410 & 1.6057 & 1.0705 & 0.5352 & 0.2676 & 0.0000\end{array}$

$\begin{array}{llllllllll}0.0000 & 0.5198 & 0.7286 & 1.0140 & 1.2015 & 1.3400 & 1.5381 & 1.6680 & 1.7511\end{array}$ $\begin{array}{llllllllll}1.8108 & 1.6765 & 1.4593 & 1.1546 & 0.7839 & 0.3707 & 0.1491 & 0.0000\end{array}$ $-0.0256-0.0554-0.2386-0.4942-0.7328-0.8841-0.9629-0.9288$ $-0.8841-0.8138-0.7094-0.6370-0.5432-0.4069-0.30890 .0000$

WEB DATA - AL, IX,IY,IXY,XL,YL

$\begin{array}{llllll}0.158 & 0.038 & 0.000 & 0.000 & 4.630 & 0.680\end{array}$

POSITION OF CENTROID FROM BASE LINE X: $10.05 \mathrm{Y}: \quad 0.32$

SKIN AREA: 8.8833 SPAR AREA: 0.5023

ANGLE OF PRINCIPAL AXIS: -0.01 EIX: $0.32305 E+08$ EIY: $0.15289 E+10$

RX: 0.92 RY: $6.33 \mathrm{EA}: 0.38174 E+08$

POSITION OF TORSIONAL CENTROID X: $\quad 9.82 Y: \quad 0.32$

GJ1: 203117408.00 GJ2: 15195294.00
SECTION MASS INFORMATION WSPAR: 0.0500 WSKIN: 0.0500 SECTION RUNNING WEIGHT: 0.4772 XCG: 10.05 YCG: 0.32 ELASTIC AXIS MOMENT OF INERTIA: 19.5401 SECTION CG MOMENT OF INERTIA: $\quad 19.5155$

SECTION BALLAST INFORMATION WBALLAST: 0.7630 XBAL: $\quad 1.63$ YBAL: $\quad 0.25$ NEW SECTION RUNNING WEIGHT: 1.2402 XCG: 4.87 YCG: 0.28 OLD SECTION CG MOMENT OF INERTIA: 73.6074 NEW SECTION CG MOMENT OF INERTIA: $\quad 40.3281$

OFFSETS FOR STATION: 3 THK1: 0.2540 THK2:0.1130 XTHK: 6.310 TSPAR: 0.1410 TSKIN: 0.1130 TSCALE: 0.9960 $\begin{array}{lllllllll}0.0000 & 0.2774 & 0.5547 & 1.1095 & 1.6642 & 2.2190 & 3.3285 & 4.4380 & 5.5475\end{array}$ 7.766511 .095013 .314015 .533017 .752019 .971021 .080522 .1900 $21.080519 .971017 .752015 .533013 .314011 .0950 \quad 7.7665 \quad 5.5475$ $\begin{array}{llllllll}4.4380 & 3.3285 & 2.2190 & 1.6642 & 1.1095 & 0.5547 & 0.2774 & 0.0000\end{array}$

$\begin{array}{llllllllll}0.0000 & 0.5393 & 0.7559 & 1.0520 & 1.2465 & 1.3902 & 1.5957 & 1.7305 & 1.8167\end{array}$ $\begin{array}{lllllllll}1.8786 & 1.7394 & 1.5139 & 1.1979 & 0.8133 & 0.3846 & 0.1547 & 0.0000\end{array}$

$\begin{array}{lllllllllll}-0.0265 & -0.0575 & -0.2475 & -0.5127 & -0.7603 & -0.9172 & -0.9990 & -0.9636\end{array}$ $\begin{array}{lllllllllll}-0.9172 & -0.8443 & -0.7360 & -0.6608 & -0.5636 & -0.4221 & -0.3205 & 0.0000\end{array}$

WEB DATA - AL, IX,IY,IXY,XL,YL

$\begin{array}{llllll}0.177 & 0.045 & 0.000 & 0.000 & 4.810 & 0.700\end{array}$

POSTIION OF CENTROID FROM BASE LINE X: $8.71 \mathrm{Y}: 0.34$

SKIN AREA: 5.1252 SPAR AREA: 1.8902

ANGLE OF PRINCIPAL AXIS: 0.01 EIX: $0.27781 E+08$ EIY: $0.12456 E+10$

RX: 0.98 RY: 6.58 EA: $0.28770 E+08$

POSITION OF TORSIONAL CENTROID $X: \quad 8.02 Y: \quad 0.34$ GJ1: 175248992.00 GJ2: 10754780.00

SFCTION MASS INFORMATION WSPAR: 0.0500 WSKIN: 0.0500 SECTION RUNNING WEIGHT: 0.3596 XCG: 8.71 YCG: 0.34 ELASTIC AXIS MOMENT OF INERTIA: $\quad 16.0909$ SECTION CG MONENT OF INERTIA: $\quad 15.9172$

SECTION BALLAST INFORMATION WBALLAST: 0.7730 XBAL: 1.63 YBAL: 0.25 NEW SECTION RUNNING WEIGHT: 1.1326 XCG: 3.88 YCG: 0.28 OLD SECTION CG MOMENT OF INERTIA: $\quad \mathbf{5 4 . 6 8 0 5}$ NEW SECTION CG MOMENT OF INERTIA: 28.2251

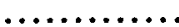

OFFSETS FOR STATION: 4 THK1: 0.3560 THK2:0.1120 XTHK: 7.000 TSPAR: 0.2440 TSKIN: 0.1120 TSCALE: 0.9970 $\begin{array}{lllllllllll}0.0000 & 0.2930 & 0.5860 & 1.1720 & 1.7580 & 2.3440 & 3.5160 & 4.6880 & 5.8600\end{array}$ 8.204011 .720014 .064016 .408018 .752021 .096022 .268023 .4400 $22.268021 .096018 .752016 .408014 .064011 .7200 \quad 8.2040 \quad 5.8600$ $\begin{array}{llllllll}4.6880 & 3.5160 & 2.3440 & 1.7580 & 1.1720 & 0.5860 & 0.2930 & 0.0000\end{array}$ 


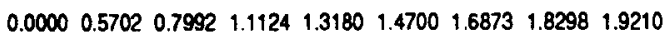
$\begin{array}{llllllllllll}1.9864 & 1.8392 & 1.6008 & 1.2666 & 0.8600 & 0.4066 & 0.1636 & 0.0000\end{array}$ $-0.0280-0.0608-0.2617-0.5422 \cdot 0.8039-0.9698 \cdot 1.0563 \cdot 1.0189$ $\begin{array}{lllllllllllllllllllllll}-0.9698 & -0.8927 & -0.7782 & -0.6988 & -0.5959 & -0.4464 & 0.3389 & 0.0000\end{array}$

WEB DATA - AL, IX,IY,IXY,XL,YL

$\begin{array}{llllll}0.145 & 0.035 & 0.000 & 0.000 & 5.630 & 0.740\end{array}$

POSITION OF CENTROID FROM BASE LINE X: $8.22 \quad \mathrm{Y}: \quad 0.36$

SKIN AREA: 5.3662 SPAR AREA: 3.6181

ANGLE OF PRINCIPAL AXIS: $0.00 \mathrm{EIX}: 0.38537 \mathrm{E}+08 \mathrm{EIY}: 0.16515 E+10$

RX: 1.03 RY: 6.72 EA: $0.36517 E+08$

POSITION OF TORSIONAL CENTROID X: $\quad 7.41 \quad Y: 0.36$ GJ1: 233931296.00 GJ2: 13356832.00

SECTION MASS INFORMATION WSPAR: 0.0500 WSKIN: 0.0500 SECTION RUNNING WEIGHT: 0.4565 XCG: 8.22 YCG: 0.36 ELASTIC AXIS MOMENT OF INERTIA: 21.4275 SECTION CG MOMENT OF INERTIA: 21.1251

SECTION BALLAST INFORMATION WBALLAST: 0.8120 XBAL: $\quad 1.63$ YBAL: 0.25 NEW SECTION RUNNING WEIGHT: 1.2685 XCG: 4.00 YCG: 0.29 OLD SECTION CG MOMENT OF INERTIA: 56.4041 NEW SECTION CG MOMENT OF INERTIA: 33.8205

OFFSETS FOR STATION: 5 THK1: 0.3850 THK2: 0.1550 XTHK: 7.750 TSPAR: 0.2300 TSKIN: 0.1550 TSCALE: 0.9990 $\begin{array}{lllllllll}0.0000 & 0.3195 & 0.6390 & 1.2780 & 1.9170 & 2.5560 & 3.8340 & 5.1120 & 6.3900\end{array}$ 8.946012 .780015 .336017 .892020 .448023 .004024 .282025 .5600 $24.282023 .004020 .448017 .892015 .336012 .7800 \quad 8.9460 \quad 6.3900$

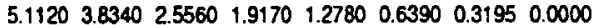

$\begin{array}{lllllllll}0.0000 & 0.6230 & 0.8733 & 1.2154 & 1.4401 & 1.6061 & 1.8436 & 1.9993 & 2.0989\end{array}$ $\begin{array}{llllllllllll}2.1704 & 2.0096 & 1.7491 & 1.3840 & 0.9397 & 0.4443 & 0.1787 & 0.0000\end{array}$

$-0.0306-0.0664-0.2860-0.5924-0.8784-1.0597-1.1542-1.1133$

$\begin{array}{llllllllllllll}-1.0597 & -0.9754 & -0.8503 & -0.7635 & -0.6511 & -0.4877 & -0.3702 & 0.0000\end{array}$

WEB DATA - AL,IX,IY,IXY,XL,YL

$\begin{array}{llllll}0.179 & 0.068 & 0.000 & 0.000 & 6.690 & 0.820\end{array}$

POSITION OF CENTROID FROM BASE LINE X: $\quad 9.74 \quad \mathrm{Y}: \quad 0.39$

SKIN AREA: 8.0987 SPAR AREA: 3.7737

ANGLE OF PRINCIPAL AXIS: -0.01 EIX: $0.60330 E+08$ EIY: $0.27016 E+10$

RX: 1.12 RY: $7.49 \mathrm{EA}: 0.48206 \mathrm{E}+08$

POSITION OF TORSIONAL CENTROID X: $8.91 \mathrm{Y}: \quad 0.39$ GJ1: 381029376.00 GJ2: 23335424.00

SECTION MASS INFORMATION WSPAR: 0.0500 WSKIN: 0.0500 SECTION RUNNING WEIGHT: 0.6026 XCG: 9.74 YCG: 0.39 ELASTIC AXIS MOMENT OF INERTIA: $\quad 34.9366$ SECTION CG MOMENT OF INERTIA: $\quad 34.5241$

SECTION BALAST INFORMATION WBALLAST: 0.7820 XBAL: $\quad 1.63$ YBAL: $\quad 0.25$ NEW SECTION RUNNING WEIGHT: 1.3846 XCG: 5.16 YCG: 0.31 OLDSECTION CG MOMENT OF INERTIA: 85.9536 NEW SECTION CG MOMENT OF INERTIA: $\quad \mathbf{5 6 . 9 0 6 4}$

OFFSETS FOR STATION: 6 THK1: 0.4210 THK2: 0.1890 XTHK: 8.940 TSPAR: 0.2320 TSKIN: 0.1890 TSCALE: 1.0010 $\begin{array}{llllllllllll}0.0000 & 0.3621 & 0.7242 & 1.4485 & 2.1727 & 2.8970 & 4.3455 & 5.7940 & 7.2425\end{array}$ 10.139514 .485017 .382020 .279023 .176026 .073027 .521528 .9700 $27.521526 .073023 .176020 .279017 .382014 .485010 .1395 \quad 7.2425$ $\begin{array}{llllllll}5.7940 & 4.3455 & 2.8970 & 2.1727 & 1.4485 & 0.7242 & 0.3621 & 0.0000\end{array}$

$\begin{array}{llllllllll}0.0000 & 0.7076 & 0.9918 & 1.3804 & 1.6355 & 1.8240 & 2.0937 & 2.2706 & 2.3837\end{array}$ $\begin{array}{llllllllll}2.4649 & 2.2822 & 1.9864 & 1.5717 & 1.0672 & 0.5046 & 0.2030 & 0.0000\end{array}$ $-0.0348-0.0754-0.3248-0.6728-0.9976-1.2035-1.3108 \cdot 1.2644$

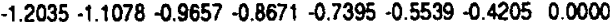

WEB DATA - AL, IX,IY,IXY,XL, YL

$\begin{array}{llllll}0.353 & 0.184 & 0.000 & 0.000 & 6.810 & 0.950\end{array}$

POSITION OF CENTROID FROM BASE LINE X. $11.37 \mathrm{Y} \cdot 0.45$

SKIN AREA: 11.1935 SPAR AREA: 4.3878

ANGLE OF PRINCIPAL AXIS: -0.01 EIX: $0.10277 E+09$ EIY: $0.46142 E+10$

RX: 1.27 RY: $8.51 E A: 0.63737 E+08$

POSTION OF TORSIONAL CENTROID $X: 10.48 \mathrm{Y}: \quad 0.45$

Gl1: 648485632.00 GJ2: $\quad 41007072.00$
SECTION MASS INFORMATION WSPAR: 0.0500 WSKIN: 0.0500 SECTION RUNNING WEIGHT: 0.7967 XCG: 11.37 YCG: 0.45 ELASTIC AXIS MOMENT OF INERTIA: $\quad 59.5947$

SECTION CG MOMENT OF INERTIA: $\quad 58.9623$

SECTION BALLAST INFORMATION WBALLAST: 0.8620 XBAL: $\quad 1.63$ YBAL: 0.25 NEW SECTION RUNNING WEIGHT: 1.6587 XCG: 6.31 YCG: 0.34 OLD SECTION CG MOMENT OF INERTIA: 140.7870 NEW SECTION CG MOMENT OF INERTIA: 98.2643

OFFSETS FOR STATION: 7 THK1: 0.3940 THK2: 0.1670 XTHK: 10.000 TSPAR: 0.2270 TSKIN: 0.1670 TSCALE: 1.0190 $\begin{array}{llllllllllll}0.0000 & 0.4320 & 0.8640 & 1.7280 & 2.5920 & 3.4560 & 5.1840 & 6.9120 & 8.6400\end{array}$ 12.096017 .280020 .736024 .192027 .648031 .104032 .832034 .5600 32.832031 .104027 .648024 .192020 .736017 .280012 .09608 .6400 $\begin{array}{lllllllll}6.9120 & 5.1840 & 3.4560 & 2.5920 & 1.7280 & 0.8640 & 0.4320 & 0.0000\end{array}$

$\begin{array}{lllllllll}0.0000 & 0.8593 & 1.2044 & 1.6763 & 1.9862 & 2.2151 & 2.5426 & 2.7575 & 2.8948\end{array}$ $\begin{array}{llllllllll}2.9934 & 2.7715 & 2.4123 & 1.9087 & 1.2960 & 0.6128 & 0.2465 & 0.0000\end{array}$

$-0.0423-0.0916-0.3944-0.8170-1.2115-1.4615-1.5918-1.5354$

$-1.4615-1.3453-1.1727-1.0530-0.8980-0.6726-0.51060 .0000$

WEB DATA - AL, IX,IY,IXY,XL,YL

$\begin{array}{llllll}6.150 & 2.000 & 104.000 & -7.000 & 8.640 & 0.000\end{array}$

POSITION OF CENTROID FRON BASE LINE X: 12.17 Y: 0.38

SKIN AREA: 11.8070 SPAR AREA: 4.8286

ANGLE OF PRINCIPAL AXIS: $0.00 \mathrm{EIX}: 0.18026 \mathrm{E}+09 \mathrm{EIY}: 0.78827 \mathrm{E}+10$

RX: 1.41 RY: $9.30 E A: 0.91142 E+08$

POSITION OF TORSIONAL CENTROID X: $11.16 \mathrm{Y}: \quad 0.35$ GJ1: 111865395200 GJ2: 63499192.00

SECTION MASS INFORMATION WSPAR: 0.0500 WSKIN: 0.0500 SECTION RUNNING WEIGHT: $t 1393$ XCG: 12.17 YCG: 0.38 ELASTIC AXIS MOMENT OF INERTIA: 101.9601 SECTION CG MOMENT OF INERTIA: $\quad 100.7866$

SECTION BALLAST INFORMATION WBALLAST: 0.7300 XBAL: $\quad 8.64$ YBAL: $\quad 0.00$ NEW SECTION RUNNING WEIGHT: 1.8693 XCG: 10.79 YCG: 0.23 OLD SECTION CG MOMENT OF INERTIA: 110.0087 NEW SECTION CG MOMENT OF INERTIA: 106.4072

$$
\text { ............. }
$$

OFFSETS FOR STATION: 8 THK1: 0.3940 THK2: 0.1670 XTHK: 13.250 TSPAR: 0.2270 TSKIN: 0.1670 TSCALE: 1.0550 $\begin{array}{llllllllll}0.0000 & 0.5312 & 1.0625 & 2.1250 & 3.1875 & 4.2500 & 6.3750 & 8.5000 & 10.6250\end{array}$ 14.875021 .250025 .500029 .750034 .000038 .250040 .375042 .5000 40.375038 .250034 .000029 .750025 .500021 .250014 .875010 .6250 $\begin{array}{llllllll}8.5000 & 6.3750 & 4.2500 & 3.1875 & 2.1250 & 1.0625 & 0.5312 & 0.0000\end{array}$

$0.0000 \quad 1.0940 \quad 1.53342 .1343 \quad 2.52882 .8203 \quad 3.2373 \quad 3.5108 \quad 3.6856$ $\begin{array}{llllllll}3.8112 & 3.5287 & 3.0714 & 2.4302 & 1.6500 & 0.7802 & 0.3139 & 0.0000\end{array}$ $-0.0538-0.1166-0.5022-1.0402-1.5424-1.8608-2.0267-1.9549$ $-1.8608-1.7128-1.4931-1.3406-1.1434-0.8564-0.6501 \quad 0.0000$

WEB DATA - AL,IX,IY,IXY,XL,YL

$\begin{array}{llllll}7.440 & 5.000 & 190.000 & -25.000 & 10.630 & 0.000\end{array}$ POSITION OF CENTROID FROM BASE LINE X: $14.94 \mathrm{Y}: \quad 0.50$

SKIN AREA: 14.5394 SPAR AREA: 6.3910

ANGLE OF PRINCIPAL AXIS: -0.01 EIX: $0.38680 E+09$ EIY: $0.14688 E+11$ RX: 1.85 RY: $11.38 E A: 0.11348 E+09$

POSITION OF TORSIONAL CENTROID X: $13.79 \mathrm{Y}: \quad 0.46$ GJ1: 2091510272.00 GJ2: 128430672.00

SECTION MASS INFORMATION WSPAR: 0.0500 WSKIN: 0.0500 SECTION RUNNING WEIGHT: 1.4185 XCG: 14.94 YCG: 0.50 ELASTIC AXIS MOMENT OF INERTIA: $\quad 190.5984$ SECTION CG MOMENT OF INERTIA: $\quad 188.4384$

SECTION BALLAST INFORMATION WBALLAST: 0.4500 XBAL: 10.63 YBAL: 0.00 NEW SECTION RUNNING WEKGHT: 1.8685 XCG: 13.90 YCG: 0.38 OLD SECTION CG MOMENT OF INERTIA: 196.9089 NEW SECTION CG MOMENT OF INERTIA: 194.8689

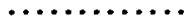

OFFSETS FOR STATION: 9 THK1: 0.3940 THK2: 0.1670 XTHK: 16.000 TSPAR: 0.2270 TSKIN: 0.1670 TSCALE: 1.0700 $0.0000 \quad 0.6601+.3202264053 .9607528107 .921510562013 .2025$ 18.483526 .405031 .686036 .967042 .248047 .529050 .169552 .8100 $50.169547 .5290 \quad 42.248036 .967031 .686026 .405018 .483513 .2025$ $\begin{array}{llllllll}10.5620 & 7.9215 & 5.2810 & 3.9607 & 2.6405 & 1.3202 & 0.6601 & 0.0000\end{array}$ 


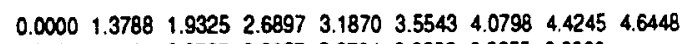
$\begin{array}{lllllllll}4.8031 & 4.4471 & 3.8707 & 3.0627 & 2.0794 & 0.9832 & 0.3955 & 0.0000\end{array}$

$-0.0678-0.1469-0.6329 \cdot 1.3110 \cdot 1.9438 \cdot 2.3450-2.5541-2.4637$

$-2.3450 \cdot 2.1586-1.8817 \cdot 1.6896-1.4409 \cdot 1.0793 \cdot 0.81930 .0000$

WEB DATA - AL,IX,IY,IXY,XL,YL

$\begin{array}{llllll}8.000 & 18.000 & 205.000 & -50.000 & 13.200 & 0.000\end{array}$

POSITION OF CENTROID FROM BASE LNE X: $18.76 \quad \mathrm{Y}: \quad 0.65$

SKIN AREA: 18.0770 SPAR AREA: 7.7412

ANGLE OF PRINCIPAL AXIS: -0.01 EIX: $0.80271 E+09$ EIY: $0.27440 E+11$

RX: 2.44 RY: 14.24 EA: $0.13527 E+09$

POSITION OF TORSIONAL CENTROID $X: \quad 17.20 \mathrm{Y}: \quad 0.60$ GJt: 3895587328.00 GJ2: 251821952.00

SECTION MASS INFORMATION WSPAR: 0.0500 WSKIN: 0.0500 SECTION AUNNING WEIGHT: 1.6909 XCG: 18.76 YCG: 0.65 ELASTIC AXIS MOMENT OF INERTIA: 357.1907 SECTION CG MOMENT OF INERTIA: $\quad 353.0326$

SECTION BALLAST INFORMATION WBALLAST: 0.5900 XBAL: 13.20 YBAL: $\quad 0.00$ NEW SECTION RUNNING WEIGHT: 2.2809 XCG: 17.32 YCG: 0.48 OLD SECTION CG MOMENT OF INERTIA: 371.5366

NEW SECTION CG MOMENT OF INERTIA: 366.7502

\section{$\cdots \cdots \cdots \cdots$}

OFFSETS FOR STATION: 10 THK1: 0.3940 THK2: 0.1670 XTHK: 21.880 TSPAR: 0.2270 TSKIN: 0.1670 TSCALE: 1.0710 $0.0000 \quad 0.8344 \quad 1.6687 \quad 3.3375 \quad 5.0062 \quad 6.675010 .012513 .350016 .6875$ 23.362533 .375040 .050046 .725053 .400060 .075063 .412566 .7500 $63.412560 .075053 .400046 .7250 \quad 40.050033 .375023 .362516 .6875$

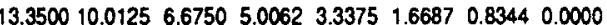

$0.0000 \quad 1.74432 .44493 .4029 \quad 4.0320 \quad 4.4967 \quad 5.1615 \quad 5.5976 \quad 5.8764$ $\begin{array}{llllllllll}6.0766 & 5.6262 & 4.8970 & 3.8747 & 2.6308 & 1.2439 & 0.5004 & 0.0000\end{array}$ $-0.0858-0.1859-0.8007-1.6586-2.4592-2.9668-3.2313-3.1169$ $-2.9668-2.7309-2.3806-2.1375-1.8230-1.3654-1.0366 \quad 0.0000$

WEB DATA - AL,IX,IY,IXY,XL,YL

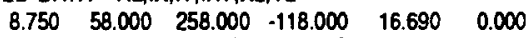
POSITION OF CENTROID FROM BASE LINE X: $23.89 \mathrm{Y}: \quad 0.85$ SKIN AREA: 22.8496 SPAR AREA: 10.5372 ANGLE OF PRINCIPAL AXIS: -0.01 EIX: $0.17931 E+10$ EIY: $0.54572 E+11$ RX: $3.26 \mathrm{RY}: 17.99 \mathrm{EA}: 0.16855 \mathrm{E}+09$
POSITION OF TORSIONAL CENTROID X: $21.92 \mathrm{Y}: \quad 0.81$ GJ1: 7759856640.00 GJ2: 518288448.00

SECTION MASS INFORMATION WSPAR: 0.0500 WSKIN: 0.0500 SECTION RUNNING WEIGHT: 2.1068 XCG: 23.89 YCG: 0.85 ELASTIC AXIS MOMENT OF INERTIA: $\quad 712.7999$ SECTION CG MOMENT OF INERTIA: $\quad 704.5621$

SECTION BALLAST INFORMATION WBALLAST: 4.8700 XBAL: 16.69 YBAL: 0.00 NEW SECTION RUNNING WEIGHT: 6.9768 XCG: 18.86 YCG: 0.26 OLD SECTION CG MOMENT OF INERTIA: $\quad 960.7367$ NEW SECTION CG MOMENT OF INERTIA: 781.9205

OFFSETS FOR STATION: 11 THK1: 0.3940 THK2: 0.1670 XTHK: 21.880 TSPAR: 0.2270 TSKIN: 0.1670 TSCALE: 1.0710

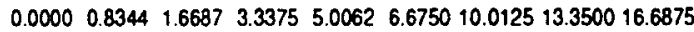
23.362533 .375040 .050046 .725053 .400060 .075063 .412566 .7500 63.412560 .075053 .400046 .725040 .050033 .375023 .362516 .6875 $\begin{array}{llllllll}13.3500 & 10.0125 & 6.6750 & 5.0062 & 3.3375 & 1.6687 & 0.8344 & 0.0000\end{array}$

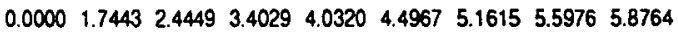
$\begin{array}{llllllll}6.0766 & 5.6262 & 4.8970 & 3.8747 & 2.6308 & 1.2439 & 0.5004 & 0.0000\end{array}$ $-0.0858 \cdot 0.1859-0.8007-1.6586 \cdot 2.4592 \cdot 2.9668 \cdot-3.2313 \cdot 3.1169$ $-2.9668-2.7309-2.3806-2.1375-1.8230-1.3654-1.03660 .0000$

WEB DATA - AL, IX,IY,IXY,XL,YL

$8.750 \quad 58.000 \quad 258.000-118.000 \quad 16.690 \quad 0.000$ POSITION OF CENTROID FROM BASE LINE X: $23.89 \mathrm{Y}: \quad 0.85$ SKIN AREA: 22.8496 SPAR AREA: 10.5372 ANGLE OF PRINCIPAL AXIS: 0.01 EIX: $0.17931 E+10$ EY: $0.54572 E+11$ RX: 3.26 RY: $17.99 \mathrm{EA}: 0.16855 \mathrm{E}+09$

POSITION OF TORSONAL CENTROID X: $21.92 \mathrm{Y}: \quad 0.81$ GJ1: 7759856640.00 GJ2: 518288448.00

SECTION MASS INFORMATION WSPAR: 0.0500 WSKIN: 0.0500 SECTION RUNNING WEIGHT: 2.1068 XCG: 23.89 YCG: 0.85 ELASTIC AXIS MOMENT OF INERTIA: $\quad 712.7999$ SECTION CG MOMENT OF INERTIA: 704.5621

SECTION BALLAST INFORMATION WBALLAST: 0.0000 XBAL: $\quad 16.69$ YBAL: $\quad 0.00$ NEW SECTION RUNNING WEIGHT: 2.1068 XCG: 23.89 YCG: 0.85 OLD SECTION CG MOMENT OF INERTIA: 704.5621 NEW SECTION CG MOMENT OF INERTIA: 704.5620

CARTER.BLADE.AEI.DATA.ALL.TEST.WTS.5-14-8B

CARTER.BLADE.ZERO.LOAD.TEST.12-11-87 $\stackrel{*}{ }$ TCORR $=1.000 *$ PITCH $=0.000$

\# STATIONS: 11 E-SKIN: 3999999.50 E-LONG: 3999999.50 \# DIVISIONS: 500 GS: 499999.94 GL: 749999.88 WS: 0.050 WL: 0.050

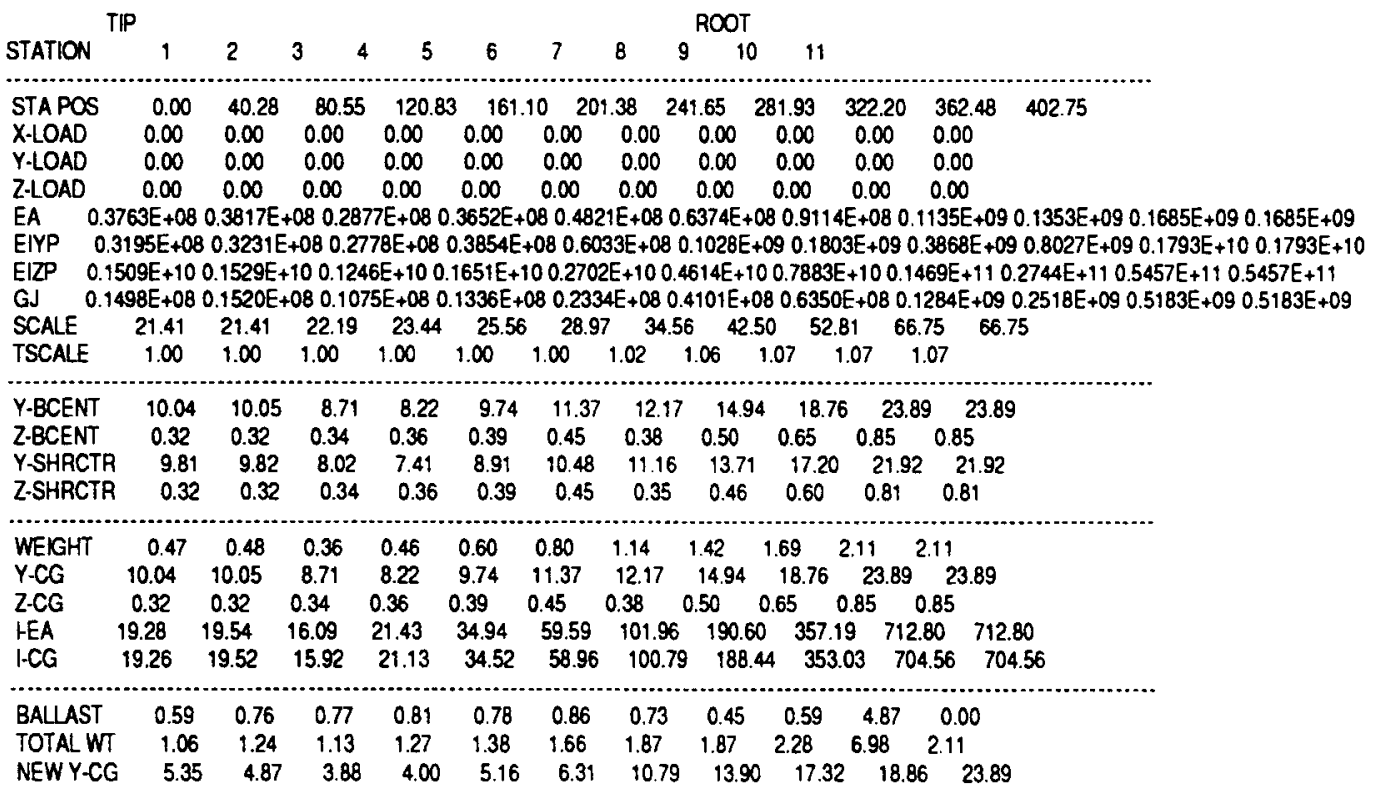




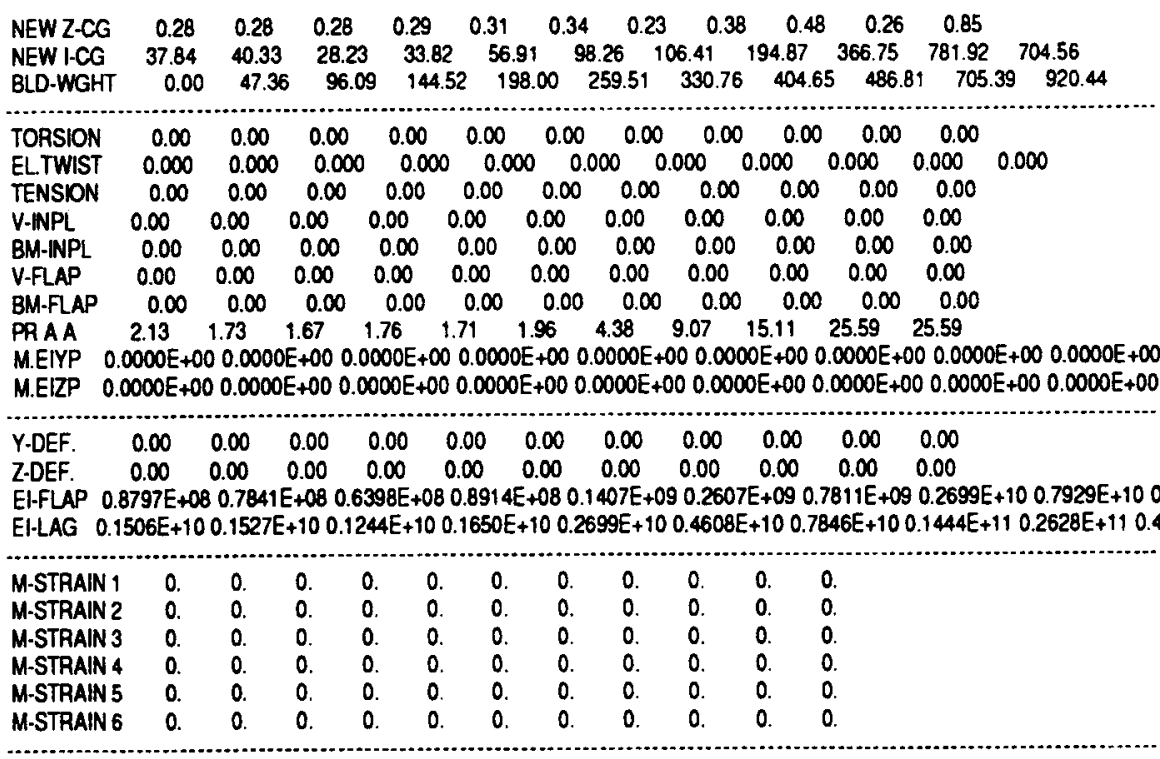

.... STOP

\subsubsection{SAMPLE RUN, ESI BLADE, NO TIPS OR BALLAST}

This sample run is for the ESI blade to illustrate a simpler blade structure, in this case woodepoxy laminated veneers with no ballast or mechanisms.

\section{NPUT BLADE RLE: ESSBL}

$\begin{array}{llllll}11 & 1.88 & 1.88 & 500 & 0.133 & 0.133\end{array}$

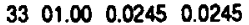

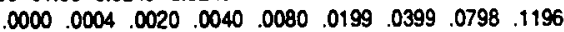

$\begin{array}{lllllll}.1994 .2792 & .3589 & .4786 & .5982 & 7179 & .8375 & 1.0000\end{array}$

$\begin{array}{lllllll}8375 & .7179 .5982 .4786 & .3589 & .2792 .1994 .1196\end{array}$

$\begin{array}{llllllllll}0.0798 & 0399 & 0199 & 0080 & 0040 & 0020 & .0004 & .0000\end{array}$

$\begin{array}{lllllllllll}0000 & 0060 & 0140 & .0211 & .0289 & .0463 & .0636 & .0842 & .0981\end{array}$

.1123 .1173 .1161 .053 .0879 .0664 .0413 .0000

$.0377-.0618-.0828-.0981-.1065-.1067-.1013-.0861$

$.0730-.0534 \cdot .0389-.0257-.0182-.0126 \cdot .0060 \quad .0000$

006

$\begin{array}{llllll}.0000 & .2792 & .4786 & 1,0000 & .4786 & .2792\end{array}$

$\begin{array}{llllll}0000 & 1173 \quad 1053 \quad 0000 & -00981 & -.1067\end{array}$

$\begin{array}{lllllllllll}0.000 & 0.85 & 1.65 & 2.49 & 3.29 & 4.10 & 4.94 & 5.75 & 6.55 & 7.71 & 7.71\end{array}$

$\begin{array}{llll}11.65 & 0.690 .00001 & 0.472 \quad 5.00\end{array}$

$\begin{array}{llllllllllll}1.236 & 0.272 & 0.00 & 0.00 & 4.00 & 0.00\end{array}$

$0.00 \quad 0.00 \quad 0.00$

$\begin{array}{llllll}13.66 & 0.827 & .00001 & 0.472 & 5.00\end{array}$

$\begin{array}{lllllllll}1.236 & 0.272 & 0.00 & 0.00 & 4.31 & 0.00\end{array}$

$0.00 \quad 0.00 \quad 0.00$

$\begin{array}{llll}15.430 .961 & 00001 \quad 0.472 \quad 5.00\end{array}$

$\begin{array}{lllllll}1.653 & 0.769 & 0.00 & 0.00 & 4.90 & 0.00\end{array}$

$0.000 .00 \quad 0.00$

$17.28 \quad 1.192 \quad 00001 \quad 0.555 \quad 5.00$

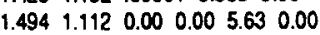

$0.00 \quad 0.00 \quad 0.00$

$19.091 .150 \quad .00001 \quad 0.677 \quad 5.00$

$\begin{array}{llllll}0.964 & 1.040 & 0.00 & 0.00 & 6.30 & 0.00\end{array}$

$0.00 \quad 0.00 \quad 0.00$

$\begin{array}{llllllll}20.87 & 1.226 & .00001 & 0.681 & 5.00\end{array}$

$\begin{array}{lllllllllllllllllll}1.187 & 1.940 & 0.00 & 0.00 & 6.81 & 0.00\end{array}$

$0.00 \quad 0.00 \quad 0.00$

$22.72 \quad 1.291 .00001 \quad 0.7805 .00$

$\begin{array}{lllllllll}1.350 & 2.858 & 0.00 & 0.00 & 7.32 & 0.00\end{array}$

$0.00 \quad 0.00 \quad 0.00$

$24.40 \quad 1.376 \quad .00001 \quad 0.980 \quad 5.00$

$\begin{array}{llllllll}1.503 & 3.943 & 0.00 & 0.00 & 7.97 & 0.00\end{array}$

$\begin{array}{llll}0.00 & 0.00 \quad 0.00\end{array}$

$20.85 \quad 1.771 .00001 \quad 1.0805 .00$

$\begin{array}{lllll}1.641 & 5.124 & 0.00 & 0.00 \quad 8.15 & 0.00\end{array}$

$0.00 \quad 0.00 \quad 0.00$

$\begin{array}{llll}16.80 & 2.416 \quad .00001 & 2.490 \quad 5.00\end{array}$

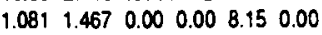

$0.00 \quad 0.00 \quad 0.00$

16.802 .416 .000012 .4905 .00

$1.0811 .467 \quad 0.00 \quad 0.00 \quad 8.150 .00$

$0.00 \quad 0.00 \quad 0.00$

ESI.BLADE.1ST.TRY.MEAS.DATA.1-24-88

\section{WPUT LOAD FLE: ESILDZERO}

001

$00.00 \quad 32.41 \quad 64.8197 .22129 .62162 .03 \quad 194.44226 .84$

259.25291 .65324 .06

$\begin{array}{llllllllll}00.00 & 00.00 & 00.00 & 00.00 & 00.00 & 00.00 \quad 00.00 & 00.00\end{array}$

$00.00 \quad 00.00 \quad 00.00$

$\begin{array}{llllllllllll}00.00 & 00.00 & 00.00 & 00.00 & 00.00 & 00.00 & 00.00 & 00.00\end{array}$

$\infty 0.00 \quad 00.00 \quad 00.00$

$\begin{array}{llll}0.00 & 00.00 \quad 00.00 \quad 00.00 \quad 00.00 \quad 00.00 \quad 00.00 \quad 00.00\end{array}$

$00.00 \quad 00.00 \quad 00.00$

$\begin{array}{lllllll}\infty .00 & 00.00 & 00.00 & 00.00 \quad 00.00 \quad 00.00 \quad 00.00 & 00.00\end{array}$

$\infty .0000 .00 \quad 00.00$

$0.00 \quad 324.06 \quad 0.00 \quad 0.00$

ESI.BLADE.ZERO.LOAD 1-24-88

TERMMAL INPUT AND OUTPUT: STRESSM

ENTER INPUT FILE NAME FOR SECTION GEOMETRY:

ESBBL

ENTER INPUT FILE NAME FOR APPLIED LOADING

ESILDZERO

ESI.BLADE.ZERO.LOAD.1-24.88 $*$ TCORR $=1.000 *$ PITCH $=0.000$

* STATIONS: $1:$ E-SKIN: 1880000.00 E-LONG: 1879999.75 \#IVISIONS: $500 \mathrm{GS}$ 132999.97 GL: 132999.97 WS: 0.025 WL: 0.025

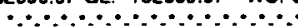

OFFSETS FOR STATION: 1 THK $: 0.4720$ THK2: 0.4720 XTHK: 5.000 TSPAR: 0.0000 TSKIN: 0.4720 TSCALE: 0.6900

$\begin{array}{lllllllllll}0.0000 & 0.0047 & 0.0233 & 0.0466 & 0.0932 & 0.2318 & 0.4648 & 0.9297 & 1.3933\end{array}$

$\begin{array}{llllllll}2.3230 & 3.2527 & 4.1812 & 5.5757 & 6.9690 & 8.3635 & 9.7569 & 11.6500\end{array}$

$\begin{array}{llllllll}9.7569 & 8.3635 & 6.9690 & 5.5757 & 4.1812 & 3.2527 & 2.3230 & 1.3933\end{array}$

$\begin{array}{llllllllll}0.9297 & 0.4648 & 0.2318 & 0.0932 & 0.0466 & 0.0233 & 0.0047 & 0.0000\end{array}$

$\begin{array}{lllllllll}0.0000 & 0.0482 & 0.1125 & 0.1696 & 0.2323 & 0.3722 & 0.5112 & 0.6768 & 0.7886\end{array}$

$\begin{array}{lllllllllll}0.9027 & 0.9429 & 0.9333 & 0.8465 & 0.7066 & 0.5338 & 0.3320 & 0.0000\end{array}$

$\begin{array}{llllllllll}-0.3031 & -0.4968 & -0.6656 & -0.7886 & -0.8561 & -0.8577 & -0.8143 & -0.6921\end{array}$

$-0.5868-0.4293-0.3127-0.2066-0.1463-0.1013-0.0482 \quad 0.0000$

WEB DATA - AL, IX,IY,IXY,XL,YL

$\begin{array}{llllll}1.236 & 0.272 & 0.000 & 0.000 & 4.000 & 0.000\end{array}$

POSTION OF CENTROID FROM BASE LINE X: $5.54 \mathrm{Y}: \quad 0.03$

SKIN AREA: 11.3314 SPAR AREA: 0.0001

ANGLE OF PRINCIPAL AXIS: $0.00 \mathrm{EIX}: 0.49275 \mathrm{E}+07 \mathrm{EIY}: 0.25157 \mathrm{E}+09$

RX: 0.46 RY: 3.26 EA: $0.23627 E+08$ 
POSTION OF TORSIONAL CENTROID $X: \quad 5.54 Y: \quad 0.03$

GJ1: 18146008.00 G.2: 2125619.00

SECTION MASS INFORMATION WSPAR: 0.0245 WSKIN: 0.0245 SECTION RUNNING WEIGHT: 0.3079 XCG: 5.54 YCG: 0.03 ELASTK AXIS MOMENT OF INERTIA: $\quad 3.3427$

SECTDN CG MOMENT OF INERTIA: $\quad 3.3427$

SECTION BALLAST INFOAMATION WBALLAST: $0.0000 \times 8 \mathrm{BAL}: \quad 0.00$ YBAL: 0.00 NEW SECTION RUNNING WEIGHT: 0.3079 XCG: 5.54 YCG: 0.03 OLD SECTION CG MOMENT OF INERTIA: 3.3427 NEW SECTION CG MOMENT OF INERTIA: 3.3427

$$
\text { .......... }
$$

OFFSETS FOR STATION: 2 THK1: 0.4720 THK2: 0.4720 XTHK: 5.000 TSPAR: 0.0000 TSKIN: 0.4720 TSCALE: 0.8270 $\begin{array}{lllllllllllllll}0.0000 & 0.0055 & 0.0273 & 0.0546 & 0.1093 & 0.2718 & 0.5450 & 1.0901 & 1.6337\end{array}$

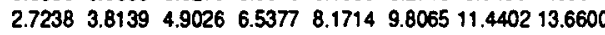

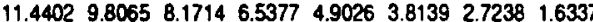
$\begin{array}{lllllllll}1.0901 & 0.5450 & 0.2718 & 0.1093 & 0.0546 & 0.0273 & 0.0055 & 0.0000\end{array}$

$\begin{array}{lllllllll}0.0000 & 0.0678 & 0.1582 & 0.2384 & 0.3265 & 0.5230 & 0.7185 & 0.9512 & 1.1082\end{array}$ $\begin{array}{lllllllllll}1.2686 & 9.3251 & 1.3116 & 1.1896 & 0.9930 & 0.7501 & 0.4666 & 0.0000\end{array}$

$-0.4259-0.6981-0.9354-1.1082-1.2031 \cdot 1.2054-1.1444-0.9727$ $\begin{array}{llllllllllll}-0.8247 & -0.6033 & -0.4394 & -0.2903 & -0.2056 & -0.1423 & -0.0678 & 0.0000\end{array}$

WEB DATA - AL, IX,IY,IXY,XL,YL

$\begin{array}{llllll}1.236 & 0.272 & 0.000 & 0.000 & 4.310 & 0.000\end{array}$

POSITION OF CENTROID FROM BASE LINE X: $\quad 6.45 \quad \mathrm{Y}: \quad 0.04$

SKIN AREA: 13.4188 SPAR AREA: 0.000

ANGLE OF PRINCIPAL AXIS: $0.00 \mathrm{EIX}: 0.13500 \mathrm{E}+08 \mathrm{EIY}: 0.41608 \mathrm{E}+09$

RX: 0.70 RY: $3.89 E A: 0.27551 E+08$

POSITION OF TORSIONAL CENTROID $X: \quad 6.45 Y: \quad 0.04$ GJ1: $\quad 30390596.00 \quad G J 2: \quad 4873743.00$

SECTION MASS INFORMATION WSPAR: 0.0245 WSKIN: 0.0245 SECTION RUNNING WEIGHT: 0.3590 XCG: 6.45 YCG: 0.04 ELASTIC AXIS MOMENT OF INERTIA: $\quad 5.5983$ SECTION CG MOMENT OF INERTIA: $\quad \mathbf{5 . 5 9 8 3}$

SECTION BALLAST INFORMATION WBALLAST: 0.0000 XBAL: $\quad 0.00$ YBAL: $\quad 0.00$ NEW SECTION RUNNING WEIGHT: 0.3590 XCG: 6.45 YCG: 0.04 OLD SECTION CG MOMENT OF INERTIA: $\quad 5.5983$ NEW SECTION CG MOMENT OF INERTIA: 5.5983

OFFSETS FOR STATION: 3 THK1: 0.4720 THK2: 0.4720 XTHK: 5.000 TSPAR: 0.0000 TSKIN: 0.4720 TSCALE: 0.9610 $\begin{array}{lllllllllll}0.0000 & 0.0062 & 0.0309 & 0.0617 & 0.1234 & 0.3071 & 0.6157 & 1.2313 & 1.8454\end{array}$ $3.0767 \quad 4.30815 .5378 \quad 7.38489 .230211 .077212 .922615 .4300$ $12.922611 .0772 \quad 9.2302 \quad 7.3848 \quad 5.5378 \quad 4.3081 \quad 3.0767 \quad 1.8454$ $\begin{array}{lllllllll}1.2313 & 0.6157 & 0.3071 & 0.1234 & 0.0617 & 0.0309 & 0.0062 & 0.0000\end{array}$

$\begin{array}{llllllllll}0.0000 & 0.0890 & 0.2076 & 0.3129 & 0.4285 & 0.6865 & 0.9431 & 1.2485 & 1.4546\end{array}$ $\begin{array}{llllllllll}1.6652 & 1.7394 & 1.7216 & 1.5614 & 1.3034 & 0.9846 & 0.6124 & 0.0000\end{array}$ $-0.5590-0.9164-1.2278-1.4546 \cdot 1.5792-1.5822-1.5021-1.2767$ $\begin{array}{llllllll}-1.0825 & -0.7918 & -0.5768 & -0.3811 & -0.2699 & -0.1868 & -0.0890 & 0.0000\end{array}$

WEB DATA - AL, IX,IY,IXY,XL,YL

$\begin{array}{llllll}1.653 & 0.769 & 0.000 & 0.000 & 4.900 & 0.000\end{array}$

POSITION OF CENTROID FROM BASE LINE X: $7.21 \quad \mathrm{Y}: \quad 0.05$

SKIN AREA: 15.3179 SPAR AREA: 0.0001

ANGLE OF PRINCIPAL AXIS: $0.00 \mathrm{EIX}: 0.30363 \mathrm{E}+08 \mathrm{EIY}: 0.61440 \mathrm{E}+09$

RX: 0.98 RY: $4.39 E A: 0.31905 E+08$

POSITION OF TORSIONAL CENTROID X: 7.21 Y: 0.05

$$
\text { GJ1: } \quad 45613552.00 \text { GJ2: } \quad 9385898.00
$$

SECTION MASS INFORMATION WSPAR: 0.0245 WSKIN: 0.0245 SECTION RUNNING WEIGHT: 0.4158 XCG: 7.21 YCG: 0.05 ELASTK AXIS MOMENT OF INERTIA: $\quad 8.4025$ SECTION CG MOMENT OF INERTIA: $\quad 8.4025$

SECTION BALLAST INFORMATION WBALLAST: 0.0000 XBAL: $\quad 0.00$ YBAL: $\quad 0.00$ NEW SECTION RUNNING WEIGHT: 0.4158 XCG: 7.21 YCG: 0.05 OLD SECTION CG MOMENT OF INERTIA: 8.4025 NEW SECTION CG MOMENT OF INERTIA: 8.4025

- $\ldots \ldots \ldots+\cdots$

OFFSETS FOR STATION: 4 THK1: 0.5550 THK2: 0.5550 XTHK: 5.000 TSPAR: 0.0000 TSKIN: 0.5550 TSCALE: 1.1920 $\begin{array}{llllllllllllll}0.0000 & 0.0069 & 0.0346 & 0.0699 & 0.1382 & 0.3439 & 0.6895 & 1.3789 & 2.0667\end{array}$
$3.4456 \quad 4.8246 \quad 6.2018 \quad 8.270210 .336912 .405314 .472017 .2800$

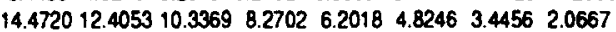

$\begin{array}{lllllllll}1.3789 & 0.6895 & 0.3439 & 0.1382 & 0.0691 & 0.0346 & 0.0069 & 0.0000\end{array}$

$\begin{array}{llllllllll}0.0000 & 0.1236 & 0.2884 & 0.4346 & 0.5953 & 0.9537 & 1.3100 & 1.7343 & 2.0206\end{array}$ $\begin{array}{lllllllll}2.3131 & 2.4161 & 2.3914 & 2.1689 & 1.8105 & 1.3677 & 0.8507 & 0.0000\end{array}$

$-0.7765-1.2729 \cdot 1.7055 \cdot 2.0206-2.1937-2.1978-2.0866 \cdot 1.7735$

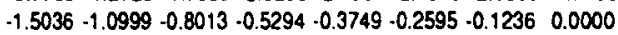

WEB DATA - AL, IX,IY, IXY,XL,YL

$\begin{array}{llllll}1.494 & 1.112 & 0.000 & 0.000 & 5.630 & 0.000\end{array}$

POSTION OF CENTROID FROM BASE LINE X: $8.09 \quad \mathrm{Y}: \quad 0.07$

SKIN AREA: 20.5731 SPAR AREA: 0.0001

ANGLE OF PRINCIPAL AXIS: $-0.01 \mathrm{EIX}: 0.80980 E+08 \mathrm{EIY}: 0.10365 \mathrm{E}+10$

RX: 1.40RY: $5.00 \mathrm{EA}: 0.41486 \mathrm{E}+08$

POSITION OF TORSIONAL CENTROID X: $8.09 \mathrm{Y}: \quad 0.07$ GJ1: 79055488.00 GJ2: 23382744.00

SECTION MASS INFORMATION WSPAR: 0.0245 WSKIN: 0.0245 SECTION RUNNING WEKGHT: 0.5406 XCG: 8.09 YCG: 0.07 ELASTIC AXIS MOMENT OF INERTIA: 14.5629 SECTION CG MOMENT OF INERTIA: $\quad 14.5629$

SECTION BALLAST INFORMATION WBALLAST: 0.0000 XBAL: $\quad 0.00$ YBAL: $\quad 0.00$ NEW SECTION RUNNING WEIGHT: 0.5406 XCG: 8.09 YCG: 0.07 OLD SECTION CG MOMENT OF INERTIA: 14.5629 NEW SECTION CG MOMENT OF INERTIA: 14.5628

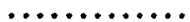

OFFSETS FOR STATION: 5 THK1: 0.6770 THK2: 0.6770 XTHK: 5.000 TSPAR: 0.0000 TSKIN: 0.6770 TSCALE: 1.1500 $\begin{array}{lllllllllllll}0.0000 & 0.0076 & 0.0382 & 0.0764 & 0.1527 & 0.3799 & 0.7617 & 1.5234 & 2.2832\end{array}$ 3.80655 .32996 .85149 .136511 .419613 .704715 .987919 .0900 $15.987913 .704711 .41969 .1365 \quad 6.85145 .3299 \quad 3.8065 \quad 2.2832$ $\begin{array}{llllllll}1.5234 & 0.7617 & 0.3799 & 0.1527 & 0.0764 & 0.0382 & 0.0076 & 0.0000\end{array}$

$0.0000 \quad 0.13170 .30730 .46320 .63451 .0164+39621.84852 .1536$ $\begin{array}{lllllllllll}2.4654 & 2.5751 & 2.5488 & 2.3117 & 1.9297 & 1.4577 & 0.9067 & 0.0000\end{array}$ $-0.8276-1.3567-1.8178-2.1536-2.3380-2.3424-2.2239-1.8902$

$-1.6026-1.1723-0.8540 \cdot 0.5642 \cdot 0.3996-0.2766-0.13170 .0000$

WEB DATA - AL, IX,IY,IXY,XL,YL

$\begin{array}{llllll}0.964 & 1.040 & 0.000 & 0.000 & 6.300 & 0.000\end{array}$

POSTIION OF CENTROID FROM BASE LINE $X: \quad 9.06 \mathrm{Y}: \quad 0.08$

SKIN AREA: 27.6210 SPAR AREA: 0.0001

ANGLE OF PRINCIPAL AXIS: -0.01 EIX: $0.11679 E+09$ EIY: $0.16730 E+10$ RX: 1.47 RY: $5.58 E A: 0.53740 E+08$

POSITION OF TORSIONAL CENTROID X: $\quad 9.06 \quad Y: \quad 0.08$ GJ1: 126615280.00 GJ2: 35927928.00

SECTION MASS INFORMATION WSPAR: 0.0245 WSKIN: 0.0245 SECTION RUNNING WEIGHT: 0.7003 XCG: 9.06 YCG: 0.08 ELASTIC AXIS MOMENT OF INERTIA: 23.3238 SECTION CG MOMENT OF INERTIA: 23.3238

SECTION BALLAST INFORMATION WBALLAST: 0.0000 XBAL: $\quad 0.00$ YBAL: 0.00 NEW SECTION RUNNING WEIGHT: 0.7003 XCG: $\quad 9.06$ YCG: 0.08 OLO SECTION CG MOMENT OF INERTIA: 23.3238 NEW SECTION CG MOMENT OF INERTIA: 23.3238

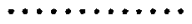

OFFSETS FOR STATION: 6 THK1: 0.6810 THK2:0.6810 XTHK: 5.000 TSPAR: 0.0000 TSKIN: 0.6810 TSCALE: 1.2260

$\begin{array}{lllllllllll}0.0000 & 0.0083 & 0.0417 & 0.0835 & 0.1670 & 0.4153 & 0.8327 & 1.6654 & 2.4961\end{array}$

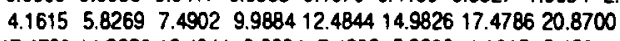

$17.4786 \quad 14.9826 \quad 12.4844 \quad 9.9884 \quad 7.4902 \quad 5.8269 \quad 4.1615 \quad 2.4961$

$\begin{array}{llllllllll}1.6654 & 0.8327 & 0.4153 & 0.1670 & 0.0835 & 0.0417 & 0.0083 & 0.0000\end{array}$

$\begin{array}{llllllllll}0.0000 & 0.1535 & 0.3582 & 0.5399 & 0.7395 & 1.1847 & 1.6273 & 2.1544 & 2.5100\end{array}$

$\begin{array}{llllllll}2.8734 & 3.0013 & 2.9706 & 2.6943 & 2.2491 & 1.6990 & 1.0567 & 0.0000\end{array}$

$-0.9646-1.5813-2.1186-2.5100-2.7250-2.7301-2.5919-2.2030$

$-1.8678-1.3663-0.9953-0.6576-0.4657-0.3224-0.15350 .0000$

WEB DATA - AL, IX, IY, IXY,XL,YL

$\begin{array}{llllll}1.187 & 1.940 & 0.000 & 0.000 & 6.810 & 0.000\end{array}$

POSTIION OF CENTROID FROM BASE LINE X: $\quad 9.85 \quad \mathrm{Y}: \quad 0.09$

SKIN AREA: 30.5815 SPAR AREA: 0.0001

ANGLE OF PRINCIPAL AXIS: -0.01 EIX: $0.18505 E+09$ EIY: $0.22333 E+10$

RX: 1.76 RY: $6.11 \mathrm{EA}: 0.59725 \mathrm{E}+08$

POSITION OF TORSIONAL CENTROIO X: $\quad 9.85 \mathrm{Y} ; \quad 0.09$ GJ1: 171082528.00 GJ2: $\quad 53306408.00$ 
SECTION MASS INFCRMATION WSPAR: 0.0245 WSKIN: 0.0245 SECTION RUNNING WEIGHT: 0.7783 XCG: 9.85 YCG: 0.09 ELASTIC AXIS MOMENT OF INERTIA: $\quad 31.5152$ SECTION CG MOMENT OF INERTIA: $\quad 31.5152$

SECTION BALLAST INFORMATION WBALLAST: 0.0000 XBAL: $\quad 0.00$ YBAL: $\quad 0.00$ NEW SECTION RUNNING WEIGHT: 0.7783 XCG: 9.85 YCG: 0.09 OLD SECTION CG MOMENT OF INERTL: 31.5152 NEW SECTION CG MOMENT OF INERTIA: $\mathbf{3 1 . 5 1 5 2}$

OFFSETS FOR STATION: 7 THK $1: 0.7800$ THK2: 0.7800 XTHK: 5.000 TSPAR: 0.0000 TSKIN: 0.7800 TSCALE: 1.2910 $\begin{array}{llllllllll}0.0000 & 0.0091 & 0.0454 & 0.0909 & 0.1818 & 0.4521 & 0.9065 & 1.8131 & 2.7173\end{array}$ $4.5304 \quad 6.34348 .154210 .873813 .5911 \quad 16.3107 \quad 19.028022 .7200$ $19.028016 .3107 \quad 13.591110 .8738 \quad 8.1542 \quad 6.3434 \quad 4.5304 \quad 2.7173$ $\begin{array}{lllllllll}1.8131 & 0.9065 & 0.4521 & 0.1818 & 0.0909 & 0.0454 & 0.0091 & 0.0000\end{array}$

$\begin{array}{llllllllll}0.0000 & 0.1760 & 0.4106 & 0.6189 & 0.8477 & 1.3580 & 1.8655 & 2.4697 & 2.8774\end{array}$ $\begin{array}{lllllllll}3.2939 & 3.4406 & 3.4054 & 3.0886 & 2.5782 & 1.9476 & 1.2114 & 0.0000\end{array}$

$-1.1058 \cdot 1.8127-2.4286-2.8774-3.1238-3.1297-2.9713-2.5254$

$-2.1412-1.5663-1.1410-0.7538-0.5338-0.3696-0.1760 \quad 0.0000$

WEB DATA - AL, IX,IY,IXY,XL,YL $\begin{array}{llllll}1.350 & 2.858 & 0.000 & 0.000 & 7.320 & 0.000\end{array}$ POSITION OF CENTROID FROM BASE LINE X: $10.70 \mathrm{Y}: \quad 0.10$

SKIN AREA: 38.3593 SPAR AREA: 0.0001 ANGLE OF PRINCIPAL AXIS: -0.01 EIX: $0.30366 E+09$ EIY: $0.33267 E+10$ RX: 2.02 RY: 6.68 EA: $0.74654 E+08$

POSITION OF TORSIONAL CENTROID X: $10.70 \mathrm{Y}: \quad 0.10$ GJ1: 256825120.00 GJ2: $\quad 86833120.00$

SECTION MASS INFORMATION WSPAR: 0.0245 WSKIN: 0.0245 SECTION RUNNING WEIGHT: 0.9729 XCG: 10.70 YCG: 0.10 ELASTK AXIS MOMENT OF INERTIA: $\quad 47.3099$ SECTION CG MOMENT OF INERTLA: $\quad \mathbf{4 7 . 3 0 9 9}$

SECTION BALAST INFORMATION WBALLAST: 0.0000 XBAL: $\quad 0.00$ YBAL: $\quad 0.00$ NEW SECTION RUNNING WEIGHT: 0.9729 XCG: 10.70 YCG: 0.10 OLD SECTION CG MOMENT OF INERTLA: $\quad 47.3099$ NEW SECTION CG MOMENT OF INERTIA: $\quad 47.3099$

(............

OFFSETS FOR STATION: 8 THK1: 0.9800 THK2: 0.9800 XTHK: 5.000 TSPAR: 0.0000 TSKIN: 0.9800 TSCALE: 1.3760 $\begin{array}{llllllllll}0.0000 & 0.0098 & 0.0488 & 0.0976 & 0.1952 & 0.4856 & 0.9736 & 1.9471 & 2.9182\end{array}$ $4.8654 \quad 6.8125 \quad 8.757211 .677814 .596117516820 .435024 .4000$ $20.435017 .5168 \quad 14.5961 \quad 11.6778 \quad 8.7572 \quad 6.8125 \quad 4.8654 \quad 2.9182$ $\begin{array}{lllllllllll}1.9471 & 0.9736 & 0.4856 & 0.1952 & 0.0976 & 0.0488 & 0.0098 & 0.0000\end{array}$

$\begin{array}{llllllllll}0.0000 & 0.2014 & 0.4700 & 0.7084 & 0.9703 & 1.5545 & 2.1353 & 2.8270 & 3.2936\end{array}$ $\begin{array}{llllllllll}3.7704 & 3.9383 & 3.8980 & 3.5354 & 2.9512 & 2.2293 & 1.3866 & 0.0000\end{array}$

$-1.2658-2.0749-2.7800-3.2936-3.5757 \cdot 3.5824-3.4011-2.8908$

$\begin{array}{llllllll}-2.4509 & -1.7929 & -1.3060 & -0.8629 & -0.6111 & -0.4230 & -0.2014 & 0.0000\end{array}$

WEB DATA - AL, IX,IY,IXY,XL,YL

$\begin{array}{llllll}1.503 & 3.943 & 0.000 & 0.000 & 7.970 & 0.000\end{array}$

POSITION OF CENTROID FROM BASE LINE $X: 11.48 \mathrm{Y}: 0.12$

SKIN AREA: 52.1716 SPAR AREA: 0.0001

ANGLE OF PRINCIPAL AXIS: $-0.01 \mathrm{EIX}: 0.52050 \mathrm{E}+09 \mathrm{EIY}: 0.52037 \mathrm{E}+10$

RX: 2.27 RY: $7.18 E A: 0.10091 E+09$

POSITION OF TORSIONAL CENTROID X: $11.48 \mathrm{Y}: \quad 0.12$ GI1: 404957888.00 GJ2: 152297408.00

SECTION MASS INFORMATION WSPAR: 0.0245 WSKIN: 0.0245 SECTION RUNNING WEIGHT: 1.3150 XCG: 11.48 YCG: 0.12 ELASTKC AXIS MOMENT OF INERTIA: 74.5975 SECTON CG MOMENT OF INERTIA: $\quad 74.5975$

SECTION BALLAST INFORMATION WBALLAST: 0.0000 XBAL: $\quad 0.00$ YBAL: $\quad 0.00$ NEW SECTION AUNNING WEIGHT: 1.3150 XCG: 11.48 YCG: 0.12 OLDSCTION CG MOMENT OF INERTIA: 74.5975 NEW SECTION CG MOMENT OF INERTIA: 74.5975

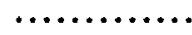

OFFSETS FOR STATION: 9 THK1: 1.0800 THK2: 1.0800 XTHK: 5.000 TSPAR: 0.0000 TSKIN: 1.0800 TSCALE: 17710 $\begin{array}{lllllllllll}0.0000 & 0.0083 & 0.0417 & 0.0834 & 0.1668 & 0.4149 & 0.8319 & 1.6638 & 2.4937\end{array}$ \begin{tabular}{l}
$4.1575 \quad 5.8213 \quad 7.4831 \quad 9.978812 .472514 .968217 .461920 .8500$ \\
\hline
\end{tabular}

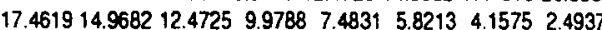

$\begin{array}{llllllll}1.6638 & 0.8319 & 0.4149 & 0.1668 & 0.0834 & 0.0417 & 0.0083 & 0.0000\end{array}$

$\begin{array}{lllllllll}0.0000 & 0.2216 & 0.5170 & 0.7791 & 1.0671 & 1.7096 & 2.3485 & 3.1091 & 3.6224\end{array}$ $\begin{array}{lllllll}4.1467 & 43313 \quad 4.2870 & 3.8882 & 3.2457 & 2.4518 & 1.5250 & 0.0000\end{array}$

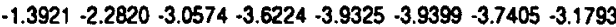

$-2.6956-1.9718-1.4364-0.9490-0.6720-0.4653-0.2216 \quad 0.0000$

WEB DATA - AL, IX,IY,IXY,XL,YL

$\begin{array}{llllll}1.641 & 5.124 & 0.000 & 0.000 & 8.150 & 0.000\end{array}$

POSTION OF CENTROID FROM BASE LINE X: $9.67 \mathrm{Y:} \quad 0.13$

SKIN AREA: 51.0920 SPAR AREA: 0.0001

ANGLE OF PRINCIPAL AXIS: -0.01 EIX: $0.60742 E+09$ EIY: $0.37357 E+10$

RX: 2.48 RY: $6.14 \mathrm{EA}: 0.99138 E+08$

POSITION OF TORSIONAL CENTROID $X: \quad 9.67 \mathrm{Y}: \quad 0.13$ GJ1: 307252352.00 GJ2: 166813920.00

SECTION MASS INFORMATION WSPAR: 0.0245 WSKIN: 0.0245 SECTION RUNNING WEIGHT: 1.2920 XCG: 9.67 YCG: 0.13 ELASTIC AXIS MOMENT OF INERTIA: $\quad 56.5991$ SECTION CG MOMENT OF INERTIA: $\quad 56.5991$

SECTION BALLAST INFORMATION WBALLAST: 0.0000 XBAL: $\quad 0.00$ YBAL: $\quad 0.00$ NEW SECTION RUNNING WEIGHT: 1.2920 XCG: 9.67 YCG: 0.13 OLD SECTION CG MOMENT OF INERTIA: $\quad 56.5991$ NEW SECTION CG MOMENT OF INERTIA: $\quad 56.5990$ $\ldots \ldots \ldots$

OFFSETS FOR STATION: 10 THK1:2,4900 THK2: 2.4900 XTHK: 5.000 TSPAR: 0.0000 TSKIN: 2.4900 TSCALE: 2.4160

$\begin{array}{llllllllllll}0.0000 & 0.0067 & 0.0336 & 0.0672 & 0.1344 & 0.3343 & 0.6703 & 1.3406 & 2.0093\end{array}$

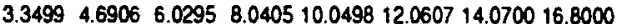

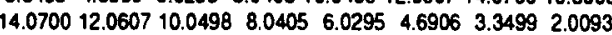

$\begin{array}{llllllll}1.3406 & 0.6703 & 0.3343 & 0.1344 & 0.0572 & 0.0336 & 0.0067 & 0.0000\end{array}$

$\begin{array}{lllllllll}0.0000 & 0.2435 & 0.5682 & 0.8564 & 1.1730 & 1.8793 & 2.5814 & 3.4176 & 3.9818\end{array}$

$\begin{array}{llllllllll}4.5581 & 4.7611 & 4.7124 & 4.2740 & 3.5678 & 2.5951 & 1.6763 & 0.0000\end{array}$

$-1.5302-2.5084 \cdot 3.3608-3.9818-4.3227-4.3308-4.1116-3.4947$

$-2.9630-2.1674-1.5789-1.043 i-0.7387-0.5114-0.2435 \quad 0.0000$

WEB DATA - AL, IX,IY,IXY,XL,YL

$\begin{array}{llllll}1.081 & 1.467 & 0.000 & 0.000 & 8.150 & 0.000\end{array}$

POSITION OF CENTROID FROM BASE LINE X: $7.63 \mathrm{Y}: \quad 0.15$

SKIN AREA: 101.7326 SPAR AREA: 0.0002

ANGLE OF PRINCIPAL AXIS: $-0.02 \mathrm{EIX:} 0.94100 \mathrm{E}+09 \mathrm{EIY}: 0.42585 \mathrm{E}+10$

RX: 2.21 RY: $4.69 E A: 0.19329 E+09$

POSITION OF TORSIONAL CENTROID $X: \quad 7.63 \mathrm{Y}: \quad 0.15$ GJ1: $\quad 367838144.00$ GJ2: $\quad 349336960.00$

SECTION MASS INFORMATION WSPAR: 0.0245 WSKIN: 0.0245 SECTION RUNNING WEIGHT: 2.5189 XCG: 7.63 YCG: 0.15 ELASTIC AXIS MOMENT OF INERTIA: 67.7597 SECTION CG MOMENT OF INERTIA: $\quad 67.7597$

SECTION BALLAST INFORMATION WBALLAST: 0.0000 XBAL: $\quad 0.00$ YBAL: 0.00 NEW SECTION RUNNNG WEIGHT: 2.5189 XCG: 7.63 YCG: 0.15 OLD SECTION CG MOMENT OF INERTIA: $\quad 67.7597$ NEW SECTION CG MOMENT OF INERTIA: 67.7596

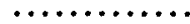

OFFSETSFOR STATION: 11 THK $: 2.4900$ THK2: 2.4900 XTHK: 5.000 TSPAR: 0.0000 TSKIN: 2.4900 TSCALE: 2.4160

$\begin{array}{lllllllllll}0.0000 & 0.0067 & 0.0336 & 0.0672 & 0.1344 & 0.3343 & 0.6703 & 1.3406 & 2.0093\end{array}$ $3.34994 .6906 \quad 6.0295 \quad 8.040510 .049812 .060714 .070016 .8000$ $14.070012 .0607 \quad 10.0498 \quad 8.0405 \quad 6.0295 \quad 4.6906 \quad 3.34992 .0093$ $\begin{array}{lllllllll}1.3406 & 0.6703 & 0.3343 & 0.1344 & 0.0672 & 0.0336 & 0.0067 & 0.0000\end{array}$

$\begin{array}{lllllllll}0.0000 & 0.2435 & 0.5682 & 0.8564 & 1.1730 & 1.8793 & 2.5814 & 3.4176 & 3.9818\end{array}$ $\begin{array}{lllllllll}4.5581 & 4.7611 & 4.7124 & 4.2740 & 3.5678 & 2.6951 & 1.6763 & 0.0000\end{array}$

$-1.5302-2.5084-3.3608-3.9818-4.3227-4.3308-4.1116-3.4947$

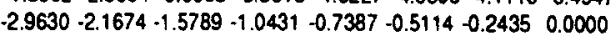

WEB DATA - AL, IX,IY,IXY,XL,YL $\begin{array}{llllll}1.081 & 1.467 & 0.000 & 0.000 & 8.150 & 0.000\end{array}$ POSTIION OF CENTROID FROM BASE LINE X: $7.63 \mathrm{Y}: \quad 0.15$ SKIN AREA: 101.7326 SPAR AREA: 0.0002 ANGLE OF PRINCIPAL AXIS: $\quad 0.02$ EIX: $0.94100 E+09$ EIY: $0.42585 E+10$ RX: 2.21 RY: 4.69 EA: $0.19329 E+09$

POSITION OF TORSIONAL CENTROID $X: \quad 7.63 Y: 0.15$ GJ1: 367838144.00 GJ2: 349336960.00

SECTION MASS INFORMATION WSPAR: 0.0245 WSKIN: 0.0245 
SECTION RUNNING WEIGHT: 2.5189 XCG: 7.63 YCG: $\quad 0.15$ ELASTIC AXIS MOMENT OF INERTIA: $\quad 67.7597$ SECTION CG MOMENT OF INERTIA: $\quad 67.7597$
SECTION BALLAST INFORMATION WBALLAST: 0.0000 XBAL: 0.00 YBAL: 0.00 NEW SECTION RUNNING WEIGHT: 2.5189 XCG: 7.63 YCG: 0.15 OLD SECTION CG MOMENT OF INERTIA: 67.7597 NEW SECTION CG MOMENT OF INERTIA: $\quad 67.7596$

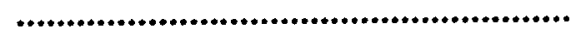

ESI.BLADE.1ST.TAY.MEAS.DATA.1-24-88

ESI.BLADE.ZERO.LOAD.1-24-88 $\cdots$ TCORR $=1.000 \cdots$ PITCH $=0.000$
}

\#STATIONS: 11 E-SKIN: 1880000.00 E-LONG: 1879999.75 \# ONISIONS: 500 GS: 132999.97 GL: 132999.97 WS: 0.025 WL: 0.025

\begin{tabular}{|c|c|c|c|c|c|c|c|c|c|c|c|c|}
\hline \multicolumn{3}{|c|}{ TIP } & \multicolumn{10}{|c|}{ ROOT } \\
\hline STATION & 1 & 2 & 3 & 5 & 6 & 7 & 8 & 91 & 10 & & & \\
\hline $\begin{array}{l}\text { STAPOS } \\
X-L O A D \\
Y-L O A D \\
Z-L O A D\end{array}$ & $\begin{array}{l}0.00 \\
0.00 \\
0.00 \\
0.00\end{array}$ & $\begin{array}{l}32.41 \\
0.00 \\
0.00 \\
0.00\end{array}$ & $\begin{array}{l}64.81 \\
0.00 \\
0.00 \\
0.00\end{array}$ & $\begin{array}{l}97.22 \\
0.00 \\
0.00 \\
0.00\end{array}$ & $\begin{array}{l}129.62 \\
0.00 \\
0.00 \\
0.00\end{array}$ & $\begin{array}{l}2.16 \\
0.00 \\
0.00 \\
0.00\end{array}$ & $\begin{array}{l}03 \\
0.00 \\
0.00 \\
0.00\end{array}$ & $\begin{array}{ll}194.44 \\
0.00 \\
0.00 \\
0.00\end{array}$ & $\begin{array}{r}226.84 \\
0.00 \\
0.00 \\
0.00\end{array}$ & $\begin{array}{c}259.25 \\
0.00 \\
0.00 \\
0.00\end{array}$ & $\begin{array}{l}291.65 \\
0.00 \\
0.00 \\
0.00\end{array}$ & 324.06 \\
\hline
\end{tabular}

2-LOAD $0.2363 E+080.2755 E+080.3191 E+080.4149 E+080.5374 E+080.5973 E+080.7465 E+080.1009 E+090.9914 E+080.1933 E+090.1933 E+09$

ENP $0.4928 E+070.1350 E+08 \quad 0.3036 E+080.8098 E+080.1168 E+090.1850 E+090.3037 E+090.5205 E+090.6074 E+090.9410 E+090.9410 E+09$

EIZP $0.2516 E+090.4161 E+090.6144 E+090.1036 E+100.1673 E+100.2233 E+100.3327 E+100.5204 E+100.3736 E+100.4259 E+100.4259 E+10$

GJ $0.2126 E+070.4874 E+070.9386 E+070.2338 E+080.3593 E+080.5331 E+080.8683 E+080.1523 E+090.1668 E+090.3493 E+090.3493 E+09$

$\begin{array}{llllllllllll}\text { SCALE } & 11.65 & 13.66 & 15.43 & 17.28 & 19.09 & 20.87 & 22.72 & 24.40 & 20.85 & 16.80 & 16.80\end{array}$

$\begin{array}{llllllllllll}\text { TSCALE } & 0.69 & 0.83 & 0.96 & 1.19 & 1.15 & 1.23 & 1.29 & 1.38 & 1.77 & 2.42 & 2.42\end{array}$

\begin{tabular}{llllllllllll}
\hline Y-BCENT & 5.54 & 6.45 & 7.21 & 8.09 & 9.06 & 9.85 & 10.70 & 11.48 & 9.67 & 7.63 & 7.63
\end{tabular}

$\begin{array}{llllllllllll}\text { Z-BCENT } & 0.03 & 0.04 & 0.05 & 0.07 & 0.08 & 0.09 & 0.10 & 0.12 & 0.13 & 0.15 & 0.15\end{array}$

$\begin{array}{llllllllllll}\text { Y-SHRCTR } & 5.54 & 6.45 & 7.21 & 8.09 & 9.06 & 9.85 & 10.70 & 11.48 & 9.67 & 7.63 & 7.63\end{array}$

$\begin{array}{llllllllllll}\text { Z-SHRCTR } & 0.03 & 0.04 & 0.05 & 0.07 & 0.08 & 0.09 & 0.10 & 0.12 & 0.13 & 0.15 & 0.15\end{array}$

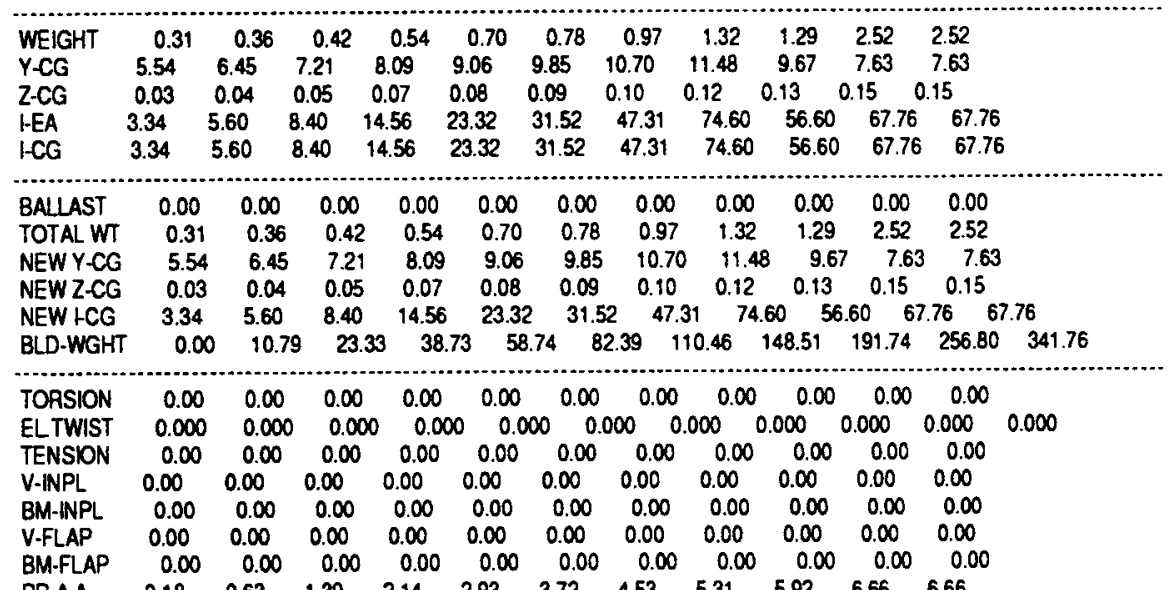

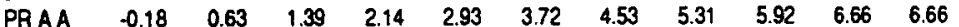

M.EIYP $0.0000 E+00 \quad 0.0000 E+00 \quad 0.0000 E+00 \quad 0.0000 E+\infty 00.0000 E+00 \quad 0.0000 E+000.0000 E++00 \quad 0.0000 E++000.0000 E+000.0000 E+00 \quad 0.0000 E+00$ M.EIZP $0.0000 E+000.0000 E+00 \quad 0.0000 E+00 \quad 0.0000 E+00 \quad 0.0000 E+00 \quad 0.0000 E+000.0000 E+000.0000 E+00 \quad 0.0000 E+000.0000 E+000.0000 E+\infty 0$

$\begin{array}{llllllllllll}\text { Y.DEF. } & 0.00 & 0.00 & 0.00 & 0.00 & 0.00 & 0.00 & 0.00 & 0.00 & 0.00 & 0.00 & 0.00\end{array}$

$\begin{array}{llllllllllll}\text { Z-DEF. } & 0.00 & 0.00 & 0.00 & 0.00 & 0.00 & 0.00 & 0.00 & 0.00 & 0.00 & 0.00 & 0.00\end{array}$

EFFLAP $0.4116 E+070.1805 E+080.4528 E+08 \quad 0.1196 E+090.2023 E+090.3295 E+090.5657 E+090.9995 E+090.9895 E+090.1429 E+100.1429 E+10$ EH-LAG $0.2516 E+090.4159 E+090.6135 E+090.1033 E+100.1665 E+100.2217 E+100.3292 E+100.5133 E+100.3653 E+100.4121 E+100.4121 E+10$

$\begin{array}{llllllllllll}\text { M-STRAIN 1 } & 0 . & 0 . & 0 . & 0 . & 0 . & 0 . & 0 . & 0 . & 0 . & 0 . & 0 . \\ \text { M-STRAIN 2 } & 0 . & 0 . & 0 . & 0 . & 0 . & 0 . & 0 . & 0 . & 0 . & 0 . & 0 . \\ \text { M-STRAIN 3 } & 0 . & 0 . & 0 . & 0 . & 0 . & 0 . & 0 . & 0 . & 0 . & 0 . & 0 . \\ \text { M-STRAIN 4 } & 0 . & 0 . & 0 . & 0 . & 0 . & 0 . & 0 . & 0 . & 0 . & 0 . & 0 . \\ \text { M-STRAIN 5 } & 0 . & 0 . & 0 . & 0 . & 0 . & 0 . & 0 . & 0 . & 0 . & 0 . & 0 . \\ \text { M-STRAIN 6 } & 0 . & 0 . & 0 . & 0 . & 0 . & 0 . & 0 . & 0 . & 0 . & 0 . & 0 .\end{array}$

…...... STOP 


\section{2: COMPUTER CODE: BLADE DEFLECTIONS AND STRESSES UNDER LOADING}

\subsubsection{BRIEF DISCUSSION}

The bending and twisting calculations are performed using the method described in Chapter 4 . The program listed above in Appendix 10.1 (STRESSM) contains in Part 2 the static bending deflection and strain calculations. In summary, this code has the following characteristics:

Uses linear beam theory (plane sections remain plane).

Employs a second order curve-fit routine to integrate spanwise values.

Has provision for full three-dimensional applied loading and torques, although only three axis forces and the torsional moment are applied.

Boundary conditions are for a simple cantilever with arbitrary section properties.

Calculates deflections and twist relative to the local principal axes and shear center and transforms the values to the specified blade feathering (control) axis.

No small deflections are assumed, and the transverse shear strain terms are calculated and applied in the bending calculations.

The program provides simultaneous bending deflections in transverse planes (flapping and leadlag) and torsional deflections (elastic twist).

\subsubsection{PROGRAM LISTING, STRESSDATA}

Program STRESSDATA is listed here. This code has provision for substitution of known (or test) beam section values prior to the deflection calculations. STRESSDATA includes the elastic twist calculations for a flying blade (e.g., including centrifugal force) which STRESSM does not, since it is for a static bench test. Note: portions which are identical to STRESSM (App. 10.1.2) and STRESSELASTIC (App.

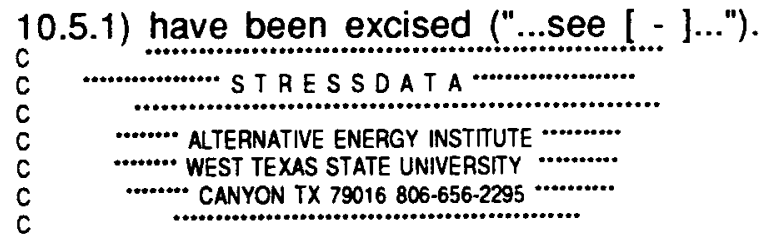

THIS PROGRAM CALCULATES THE SECTION MASS AND STIFFNESS PROPERTIES OF AN ARBITRARY EXTERNAL GEOMETRY (TWIST, TAPER) AND SPECIFIED INTERNAL SPAR-SHELL STRUCTURAL GEOMETRY, COMPOSTIE WIND TURBINE BLADE, AND THEN FNDS THE BENDING DEFLECTIONS, ELASTIC TWIST, AND STRANS IN THE LAMINATE FOR A SPECIFIED EXTERNAL LOAD AND ROTOR ROTATIONAL SPEED.

THIS VERSION OF THE PROGRAM (STRESSDATA) CONTAINS ALL THE ROUTINES AND PAINTS OUT THE TORSIONAL DEFLECTIONS (ELASTIC TWIST) DUE TO ALL INERTIAL AND APPLIED LOADS, AND DUE TO BLADE BENDING.

THIS VERSION CALCULATES THE SECTION MASS AND AREA DATA, BUT USESC ANOTHER

C INPUT FILE FOR THE SECTION STIFFNESS PROPERTIES. THIS ALTERNATE DATA IS ASSUMED TO BE FROM ANOTHER SOURCE FOR CHECKING.

THIS PROGRAM WAS ORIGINALLY WRITTEN BY E.VAN DUSEN (COMPOSITE ENGINEERING, INC., 277 BAKER AVE., CONCORD MA 01742 617.371-3132) IN 1977 FOR COMPOSTTE RACING SHELLS AND HAS BEEN EXTENSIVELY REWRITT BY F.S. STODDARD (AEI, BOX 248 WTSU, CANYON TX 79016 806-656-2295) FOR WIND TURBINE BLADES.

\section{GENERAL PROGRAM DESCRIPTION:}

PART 1 OF THIS PROGRAM CALCULATES THE SECTION PROPERTIES OF AN ARBITRARY COMPOSITE BEAM, USING EITHER ACTUAL THICKNESSES AND MODULI OF PLIES, OR USING MODULUS-WEIGHTING (THE LATTER ROUTINE HAS BEEN SUPPRESSED FOR THE CALCULATION OF ELASTIC TWIST). THE BEAM (BLADE) IS MOOELED BY A SHEL OF CONSTANT THICKNESS WTTH ARBITRARY LONGITUDINAL MODULUS AND SHEAR MODULUS, AND A SPAR CONSISTING OF A NOSE SECTION EMBEDDED NEXT TO THE SHELL IN THE BLADE LEADING EDGE, AND A WEB (OR WEBS) LOCATED INTERNALLY. THE SPAR ALSO HAS CONSTANT THICKNESS AND ARBITRARY LONGITUDINAL AND SHEAR MODULI. THE WEB IS DESCRIBED ONLY BY ITS MOMENTS OF INERTIA AND CENTROID IN THE SECTION AXIS SYSTEM. MULTI-WEB BEAM DESIGNS ARE HANDLED BY INCLUDING ALL THE ACTUAL WEBS INTO THIS ONE MODEL WEB.

THE SECTION AIRFOIL SHAPES ARE DETERMINED BY SCALING AN INPUT C. TABLE OF OFFSETS. THE CHORD SCAL ING FACTOR (SCALE) OETERMINES THE

C AIRFOIL CHORD AND THE THICKNESS SCALING FACTOR (TSCALE) DETERMINES
C THE SECTION THICKNESS PROPORTIONAL TO THE TABLE OF OFFSETS.

C ALL THESE INPUT DATA ARE INCLUDED IN A DATAFILE GIVING THE GEOMETAY C AND STRUCTURAL DESIGN OF THE BLADE OF INTEREST.

IN PART 1 PARABOLIC SEGMENTS ARE FITTED THROUGH EACH GROUP OF THREE SECTION OFFSET POINTS. THE I OF THE SKIN+SPAR+WEB IS CALCULATED BY DIVIDING THE GROUP OF POINTS INTO (DIV) STRAIGHT LINE SEGMENTS OF ROUGHLY EOUAL SIZE AND SUMMING THE APPROPRIATE VARIABLES. THE OUTPUT OF PART 1 IS NOT PRINTED IN THIS VERSION OF THE PROGRAM. SECTION DETALSS ARE GIVEN IN THE PROGRAM VERSION "STRESSM".

PART 2 OF THE PROGRAM SUBJECTS THE BLADE TO APPLIED LOADING AND TO CENTRIFUGAL (INERTIAL) LOADING. THE BLADE IS ASSUMED TO BE CANTILEVERED AT ITS ROOT (STATION 11) AND FREE AT THE TIP (STATION 1). THE PROGRAM THEN CALCULATES THE EOUILIBRIUM DEFLECTIONS OF THE ROTATING BLADE AND THE RESULTING STRAINS IN THE SKIN LAMINATE AT 6 SPECIFIED INPUT LOCATIONS AROUND THE SECTION FOR EACH BLADE STATION.

THE DISTRIBUTED LOADING IS RESOLVED INTO $X, Y$, AND $Z$ COMPONENTS $X$ LIES ALONG THE (UNDEFLECTED) AXIS OF THE BLADE WITH THE ORIGIN AT THE BLADE ROOT. Y.AXIS.IS IN THE LAGGING DIRECTION AND Z-AXIS IS IN THE FLAPPING DIRECTION. THE FREE STREAM WIND IS IN THE POSTIVE Z.DIRECTION. AND ROTATION IS THUS CLOCKWISE LOOKING UPSTREAM FROM AFT OF THE ROTOR.

THE BLADE STATION SPACING AND APPLIED LOADING (WHICH CONSISTS OF PREVIOUSLY CALCULATED AERODYNAMIC LOAD AND ANY OTHER EXTERNAL BLADE

LOAD) ARE LISTED IN ANOTHER INPUT DATAFILE, WHICH ALSO INCLUDES ROTOR SPEED, BLADE RADIUS, BLADE PITCH ANGLE, AND BLADE CONING ANGLE.

THE X-Y PLANE CONSTITUTES THE PLANE OF ROTATION OF THE ROTOR. THE Z-DEFLECTIONS ARE THE FLAPPING VALUES AND THE Y-DEFLECTIONS THE LEAD-LAG VALUES. TWIST AND PITCH ARE MEASURED POSTTIVE AIRFOIL NOSE-DOWN, OR TENDING TO DECREASE ANGLE-OF-ATTACK.

NOTE: IF THE BLADE OF INTEREST HAS A FLEXIBLE ROOT ATTACHMENT (IE FLEXBEAM) THE GJ OF THE ROOT CAN BE SPECIFIED AS THE MINIMUM IN THE GJ2N SECTION OF PART I OF THE PROGRAM. EI'S ARE HANDLED AS THE ROOT WEB.

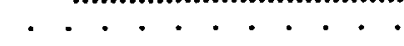

....INITIALIZE VARIABLES-

DIMENSION XC(5000),YC(5000),R(4),XX(33),YY(33),DFZ2(11),DFZC(11) 
DIMENSION RX(11),YSO(6),ZSO(6),DFY(11),DFZ(11),DFY1(11),DFZ1(11) DIMENSION ZTOT(11),OFZT(11),ANGTOT(11),TANGTOT(11),AANG1(11) REAL MLAG(11),MFLAP(11),MLAGP(11),MFLAPP(11),MCENT(11),RCENT(11) REAL MCX(11),MC1(11),MFLAPA(11)

DIMENSION O(11),OT(11),OS(11),TLAST1(11),TLAST2(11),FCENT(11)

+,ELAST1(11),ELAST2(11), TELAST1(11), TELAST2(11),MCENT1(11),BWT(11)

COMMON X(33),Y(33)

DIMENSION G1(11),G2(11),G3(19),G4(11),G5(11),G6(19)

DIMENSION STX(11),EIYP(11),EIZP(11),FX(11),PANG(11)

+,VY(11),VZ(11),EA1(11),SCALE(11),AANG(11),FXT(11),TSCALE(11)

+,SXTN(11),SX(11),DEFY(11),DEFZ(11),YCB(11)

+,ZCB(11),GJ1(11),YCT(11),ZCT(11),PM(11), TANG(11), TANG1(11)

$+, G \cdot 2(11), E I F L A P(11), E I L A G(11)$

DIMENSION WA1(11), YGB(11),ZGB(11),YGBB(11),ZGBB(11),WB(11)

+YBAL(11),ZBAL(11),WT(11),RS(11),RB(11),RBAL(11)

DIMENSION OA(11),OC1(11),OC2(11), OTRM(11),OTI(11),,$C 1 B(11)$.

$+O C 28(11), O T A M B(11)$ OTIB(11) OCB(11),OB(11),OBTR(11),OL(11)

REAL IXMO,IYMO,IXMT,IYMT, IXMG, IYMG, IBMT, IBMG, IMBB, IMBG

REAL IZO(11), YO(11),IZT(11),IYT(11),IZG(11), YG(11)

REAL IMT(11), IMG(11),IMGB(11)

INTEGER STA, IST,NPT, I,J,DIV,ID,KC,JC, IC, J1,NSO,TOGGLE

REAL CY,LX(11),LY(11),LZ(11),MY(11),MZ(11),MPY(11),MPZ(11)

+,NANG(11),MYE(11),MZE(11),MYP(11),MZP(11),LYC(11),LZC(11)

$+L X|(11), L Y|(11), L Z \mid(11)$

CHARACTER*40 INP1

CHARACTER 40 INP2

CHARACTER* 40 INP3

CHARACTER* 40 HEADING

CHARACTER・BO HEAD2

CHARACTER 80 HEAD3

DATA DFZ,DFZ1,DFZ2,ZTOT,DFZT/55०0.0/

DATA ANGTOT,TANGTOT,AANG1/33\%0.0/

C -READ INPUT FILES: INP1 IS THE BLADE SECTION GEOMETAY FILE

c -.-AND INP2 IS THE APPLIED LOADING FILE. BOTH ARE READ FROM

C -.-THE CURRENT FILE WORKSPACE UNDER THE NAMES INPUT FROM THE

-..-KEYBOARD VIA THE FOLLOWING COMMANDS

WRTE $(1,100)$

100 FORMAT(ENTER INPUT FILE NAME FOR SECTION GEOMETRY:')

READ $(1,101)$ INP1

101 FORMAT(1A4O)

WRTE $(1,102)$

102 FORMAT(ENTER INPUT FILE NAME FOR APPLIED LOADING:)

103 FORMAT(1ABO)

C

C -.-.INP3 IS THE FILENAME FOR THE ALTERNATE SECTION STIFFNESS

C -...-DATA TO BE LISTED LATER: FLAP AND LAG STIFFNESSES, TORSIONAL .......STIFFNESS, CHORDWISE SHEAR CENTER, AND EA PRODUCT.

WRITE $(1,104)$

104 FORMAT(ENTER INPUT FILE NAME FOR ALTERNATE SECTION PROPERTIES:)

READ $(1,101)$ INP3

READ; ECORR

GCORR

C

OPEN(UNIT $=5$, FILE $=$ INP1)

OPEN(UNT $=6, F|L E=| N P 2)$

C

OPEN(UNT $=8, F I L E=I N P 3)$

C

READ $\left.(6,)^{\circ}\right)$ NCAL

READ NUMBER OF CALCULATIONS

…...-RUN ENTIRE PROgRAM NCAL tIMES

DO $200018=1$,NCAL

C

-......-READ NUMBER OF STATIONS, E-SKIN, E-LONGITUDINAL,........

. DIVISIONS PER OFFSET FOR NUMERICAL INTEGRATION,-.....

-...---G-SKIN, G-LONGITUDINAL

READ (5,") STA,ES,EL,DIV,GS,GL

$\mathrm{ES}=\mathrm{ES} \cdot 10 * 6.0$

$\mathrm{EL}=\mathrm{EL} * 10 * 6.0$

GS $=$ GS* $10^{* *} 5.0$

C

$\mathrm{GL}=\mathrm{GL} * 10 * 6.0$

READ $\left(80^{\circ}\right)(\mathrm{G} 1(1), 1=1, \mathrm{STA})$

READ $\left(8^{\circ}\right)(G 2(1), I=1$, STA

READ $\left(8^{\circ}\right)(\mathrm{G} 3(1), l=1$, STA

READ $\left(8,0^{\circ}\right)(G 4(\eta), l=1$, STA
$\operatorname{READ}\left(8^{\circ}\right)(G 5(i),=1, \mathrm{STA})$

C

READ $(8,103)$ HEAD3

--.-READ NUMBER OF POINTS AND SKIN THICKNESS CORRECTON FOR RUN

…...ALSO READ WEIGHT DENSITIES (WS) AND (WL) FOR SKIN \& SPAR

$\ldots$......N LB PER INCHES *3

READ (5, ${ }^{\circ}$ NPT,TCORR,WS,WL

c

C

READ (5,") $(X(1), L=1, N P T$

$\operatorname{READ}\left(50^{\circ}\right)(Y(1), l=1, \mathrm{NPT}$

DO $105 !=1$, NPT

$X X(1)=X(1)$

$Y Y(I)=Y(I)^{*}$ TCORR

105 CONTINUE

C

-.-READ NUMBER OF STRESS OFFSETS

READ (5,) NSO

AEAD $(5, ")(Y S O(1), i=1, N S O)$

READ $\left(5^{\circ}\right)(Z S O(1), l=1, N S O)$

........READ STATION POSITION FROM FREE END AND LOAD PER UNIT--.

.........ENGTH. X-LOAD IS TOWARDS TIP: Y-LOAD POSITIVE IN LAG-

......-DIRECTION; Z-LOAD POSITIVE IN FLAP DIRECTION; PITCHING--

.......-MOMENT IS POSITIVE NOSEDOWN (INCH-LB); LOADS ARE IN....

.........-LBS. PER INCH

READ (6.) (STX(1), I =1, STA

READ $\left(6,{ }^{\circ}\right)(L X(1), 1=1, S T A)$

READ $\left(6,{ }^{\circ}\right)(L Y(j), l=1, S T A)$

READ $\left(6,{ }^{\circ}\right)(Z Z(I), I=1$, STA)

READ $\left(6,{ }^{\circ}\right)(P M(\eta), 1=1$, STA

....AEAD ROTATIONAL SPEED OMEGA (RAD/SEC), BLADE RADIUS (IN.)

C

C

PITCH $=$ PITCH1/57.2957795

.......-READ TWIST ANGLE. TWIST ANGLE IS IN DEGREES, POSITNE NOSEDOWN--

C -.-COUNTER-CLOCKWISE, LOOKING DOWN THE BLADE FROM THE TIP.

READ $\left(5,{ }^{\circ}\right)$ (TANGUM, $\}=1$, STA

c

C

READ $(6,101)$ HEADING

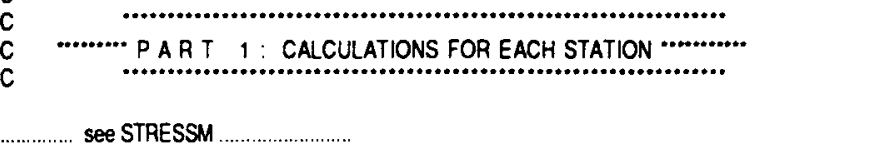

570 CONTINUE

C

C NOTE: THIS SECTION INPUTS MANUFACTURER'S DATA WHICH IS BEING

C SUBSTITUTED FOR THE CALCULATED VALUES OF THE SECTION PROPERTIES

C ABOVE. THE REMAINDER OF THE SECTION PROPERTIES HAVE NOT BEEN

C CHANGED.

DO $600 \mathrm{i}=1, \mathrm{STA}$

EIYP(I) $=G 1(I) \cdot 1000000 . \cdot E C O R R$

$E I Z P(I)=G 2(I) * 1000000 .{ }^{\circ} E C O R A$

$\mathrm{GJ} 2(1)=\mathrm{G} 3(1) \cdot 1000000 \cdot{ }^{\circ} \mathrm{GCORR}$

$Y C T(I)=G 4(I)$

$E A 1(1)=G 5(1) \cdot 1000000 \cdot E C O R R$

600 CONTNUE

C

PRINT 103, HEAD3

C ‥PART 2 : ELASTIC TWIST AND DEFLECTION PART OF PROGRAM

. 


\subsubsection{SAMPLE RUN, CARTER BLADE}

The first sample run uses the Carter blade, this time with the modified I-beam for the inboard stations (see Chap. 5) since this is the way the blade really works in practice. This run is for the actual Carter blade (CARTBL.AEIFINAL), the airloading and operating condition of $30 \mathrm{mph}$ (CARTLD.30MPH), and the actual Carter Wind Systems I-beam which was modified to accurately reflect the bending carried in the blade shell (I-BEAM2). The output summary at the end of the output file gives the equilibrium aeroelastic condition of the blade, including all twist and deflection.

\section{INPUT BLADE FLE: CARTBLAEFNAL}

114.004 .005000 .5000 .750

$331.000 .050 \quad 050$

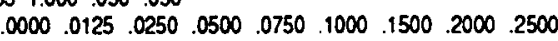

$\begin{array}{llllllll}.3500 & .5000 & .6000 & .7000 & .8000 & .9000 & .9500 & 1.0000\end{array}$

$\begin{array}{lllllll}9500 & 9000 & 8000 & .7000 \quad .6000 & .5000 & .3500 .2500\end{array}$

.2000 .1500 .1000 .0750 .0500 .0250 .0125 .0000

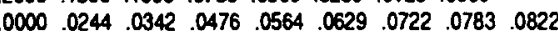

$\begin{array}{llllllll}0050 & 0787 & 0685 & 0542 & 0368 & 0174 & .0070 .0000\end{array}$

$-.0012-.0026 \cdot .0112-.0232 \cdot .0344-.0415 \cdot .0452-.0436$

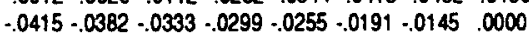

006

$\begin{array}{llllll}.0000 & .2500 & .5000 & 1.0000 & .5000 & .2500\end{array}$

$\begin{array}{lllll}.0000 & .00042 & .0787 & .0000 \cdot .0415 .0436\end{array}$

2.62 .22 .02 .02 .02 .3

$4.59 .4 \quad 15.526 .126 .1$

$21.410 .995 \quad 0.040 \quad 0.200 \quad 5.90$

$\begin{array}{lllllll}0.153 & 0.038 & 0.0001 & 0.000 & 4.500 & 0.680\end{array}$

$0.5941 .630 \quad 0.250$

$\begin{array}{lllll}21.41 & 0.995 & 0.040 & 0.203 & 5.90\end{array}$

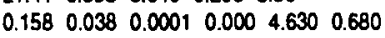

$0.7631 .630 \quad 0.250$

$22.190 .996 \quad 0.141 \quad 0.113 \quad 6.31$

$0.1770 .0450 .00010 .000 \quad 4.810 \quad 0.700$

$0.773 \quad 1.6300 .250$

$23.440 .997 \quad 0.2440 .1127 .00$

$\begin{array}{lllllllll}0.145 & 0.035 & 0.0001 & 0.000 & 5.630 & 0.740\end{array}$

$0.812 \quad 1.630 \quad 0.250$

$25.56 \quad 0.999 \quad 0.230 \quad 0.155 \quad 7.75$

$\begin{array}{lllllllllllllll}0.179 & 0.068 & 0.0001 & 0.000 & 6.690 & 0.820\end{array}$

$0.782 \quad 1.630 \quad 0.250$

$\begin{array}{llllll}28.97 & 1.001 & 0.232 & 0.189 & 8.94\end{array}$

$\begin{array}{llllllllllll}0.353 & 0.184 & 0.0001 & 0.000 & 6.810 & 0.950\end{array}$

$\begin{array}{llll}0.862 & 1.630 & 0.250\end{array}$

$\begin{array}{llll}34.56 & 1.0190 .227 & 0.167 & 10.00\end{array}$

$\begin{array}{lllllllll}6.150 & 2.000 & 104.0 & -7.00 & 8.64 & 0.00\end{array}$

0.7308 .640 .00

$\begin{array}{llllll}42.50 & 1.055 & 0.227 & 0.167 & 13.25\end{array}$

$\begin{array}{lllllll}7.440 & 5.000 & 190.0 & -25.00 & 10.63 & 0.00\end{array}$

$0.45010 .63 \quad 0.00$

$\begin{array}{lllllll}52.81 & 1.070 & 0.227 & 0.167 & 16.00\end{array}$

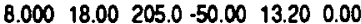

.59013 .200 .00

$\begin{array}{lllll}66.75 & 1.071 & 0.227 & 0.167 & 21.88\end{array}$

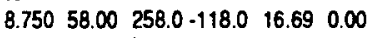

4.8716 .690 .00

$\begin{array}{llllll}66.75 & 1.071 & 0.227 & 0.167 & 21.88\end{array}$

$\begin{array}{lllllllll}8.750 & 58.00 & 258.0 & -118.0 & 16.69 & 0.00\end{array}$

0.00016 .690 .00

CARTER.BLADE.AEI.DATA.ALL.TEST.WTS.5-14-88

\section{NPUT LOAD FLE: CARTLD.3OMPH}

001

$00.00 \quad 40.28 \quad 80.55 \quad 120.83 \quad 161.10 \quad 201.38 \quad 241.65 \quad 281.93$

$322.20 \quad 362.48 \quad 402.75$

$00.00 \quad 00.00 \quad 00.00 \quad 00.00 \quad 00.00 \quad 00.00 \quad 00.0000 .00$

$00.00 \quad 00.00 \quad 00.00$

$0.000-3.579-3.675-4.717-5.055-5.190-5.388-5.553$

$-5.488-5.139 \quad 0.000$

$11.306 \quad 11.306 \quad 10.27610 .027 \quad 9.044 \quad 8.265 \quad 6.913 \quad 5.568$

$4.025 \quad 2.528 \quad 0.000$

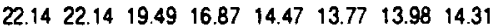

$14.26 \quad 14.46 \quad 0.00$

$7.64 \quad 402.75 \quad 0.00 \quad 4.00$

CART.BLADE.LOADS.30.MPH.12-23-87

WPUT MODIFED SECTION PROPERTIES FLE: I-BEAM2

1.1. 1. 1.1 .1 .5 .339 .3611 .1211 .1211 .12

1.1.1.1.1.1.104.8 195.1220 .0313 .0313 .0

1. 1. 1. 1. 1. 1. $-4.5-9.4-15.5-26.1-26.1$

1.1.1.1.1.1.1.1.1.1.1

ONLY I-BEAM CONTRIBUTES TO BENDING STIFFNESS INBOARD

TERMNAL SESSION AND OUTPUT: STRESSDATA

ENTER INPUT FILE NAME FOR SECTION GEOMETRY

CARTBL.AEIFINAL

ENTER INPUT FILE NAME FOR APPLIED LOADING

CARTLD.3OMPH

ENTER INPUT FILE NAME FOR ALTERNATE SECTION PROPERTIES

1-BEAM2

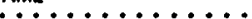

ONLY I-BEAM CONTRIBUTES TO BENDING STIFFNESS INBOARD

CARTER.BLADE.AEI.DATA.ALL.TEST.WTS.5-14-88

CART.BLADE.LOADS.30.MPH.12-23-87 " $\quad$ TCORR $=1.000 *$ OMEGA $=7.640 \cdots$ PICH $=0.00 \cdots$ CONING $=4.00$

\# STATIONS: 11 E-SKIN: 3999999.50 E-LONG: 3999999.50 \#DIVISIONS: 500 GS: 499999.94 GL: 749999.88 WS: 0.050 WL: 0.050

\begin{tabular}{|c|c|c|c|c|c|c|c|c|c|c|c|c|}
\hline \multicolumn{3}{|c|}{ TIP } & \multicolumn{10}{|c|}{ ROOT } \\
\hline STATION & 1 & 2 & 3 & 5 & 6 & 7 & 8 & $9 \quad 10$ & 11 & & & \\
\hline $\begin{array}{l}\text { STA POS } \\
\text { X-LOAD } \\
\text { Y-LOAD } \\
Z-L O A D\end{array}$ & $\begin{array}{c}0.00 \\
0.00 \\
0.00 \\
11.31\end{array}$ & $\begin{array}{l}40.28 \\
0.00 \\
-3.58 \\
11.31\end{array}$ & $\begin{array}{l}80.55 \\
0.00 \\
-3.68 \\
10.28\end{array}$ & $\begin{array}{l}120.83 \\
0.00 \\
-4.72 \\
10.03\end{array}$ & $\begin{array}{c}161.1 \\
0.00 \\
-5.06 \\
9.04\end{array}$ & $\begin{array}{c}0 \quad 201 \\
0.00 \\
-5.19 \\
8.26\end{array}$ & $\begin{array}{c}1.38{ }^{2} \\
0.00 \\
-5.39 \\
6.91\end{array}$ & $\begin{array}{c}241.65 \\
0.00 \\
9 \\
-5.55 \\
5.57\end{array}$ & $\begin{array}{c}281.93 \\
0.00 \\
-5.49 \\
4.02\end{array}$ & $\begin{array}{c}322.20 \\
0.00 \\
-5.14 \\
2.53\end{array}$ & $\begin{array}{l}362.48 \\
0.00 \\
0.00 \\
0.00\end{array}$ & 402.75 \\
\hline
\end{tabular}

$\begin{array}{llllllllllll}\text { P-MOMENT } & 22.14 & 22.14 & 19.49 & 16.87 & 14.47 & 13.77 & 13.98 & 14.31 & 14.26 & 14.46 & 0.00\end{array}$

EA $\quad 0.3763 E+080.3817 E+080.2877 E+080.3652 E+080.4821 E+080.6374 E+080.9114 E+080.1135 E+090.1353 E+090.1685 E+090.1685 E+09$

EIYP $0.3195 E+080.3231 E+080.2778 E+080.3854 E+080.6033 E+080.1028 E+090.1803 E+090.8424 E+080.1001 E+090.1001 E+090.1001 E+09$

EIZP $0.1509 E+100.1529 E+100.1246 E+100.1651 E+100.2702 E+100.4614 E+10 \quad 0.7883 E+100.1756 E+100.1980 E+100.2817 E+100.2817 E+10$

GJ $0.1498 E+080.1520 E+080.1076 E+080.1336 E+080.2334 E+080.4101 E+080.6350 E+080.1284 E+090.2518 E+090.5183 E+090.5183 E+09$

$\begin{array}{llllllllllll}\text { SCALE } & 21.41 & 21.41 & 22.19 & 23.44 & 25.56 & 28.97 & 34.56 & 42.50 & 52.81 & 66.75 & 66.75\end{array}$

$\begin{array}{llllllllllll}\text { TSCALE } & 1.00 & 1.00 & 1.00 & 1.00 & 1.00 & 1.00 & 1.02 & 1.06 & 1.07 & 1.07 & 1.07\end{array}$

$\begin{array}{llllllllllll}\text { Y.BCENT } & 10.04 & 10.05 & 8.71 & 8.22 & 9.74 & 11.37 & 12.17 & 14.94 & 18.76 & 23.89 & 23.89\end{array}$

$\begin{array}{llllllllllll}\text { Z-BCENT } & 0.32 & 0.32 & 0.34 & 0.36 & 0.39 & 0.45 & 0.38 & 0.50 & 0.65 & 0.85 & 0.85\end{array}$

$\begin{array}{lllllllllllll}\text { Y.SHRCTR } & 9.81 & 9.82 & 8.02 & 7.41 & 8.91 & 10.48 & 11.16 & 13.71 & 17.20 & 21.92 & 21.92\end{array}$

$\begin{array}{llllllllllll}\text { Z-SHRCTR } & 0.32 & 0.32 & 0.34 & 0.36 & 0.39 & 0.45 & 0.35 & 0.46 & 0.60 & 0.81 & 0.81\end{array}$

$\begin{array}{llllllllllll}\text { WEIGHT } & 0.47 & 0.48 & 0.36 & 0.46 & 0.60 & 0.80 & 1.14 & 1.42 & 1.69 & 2.11 & 2.11\end{array}$ 


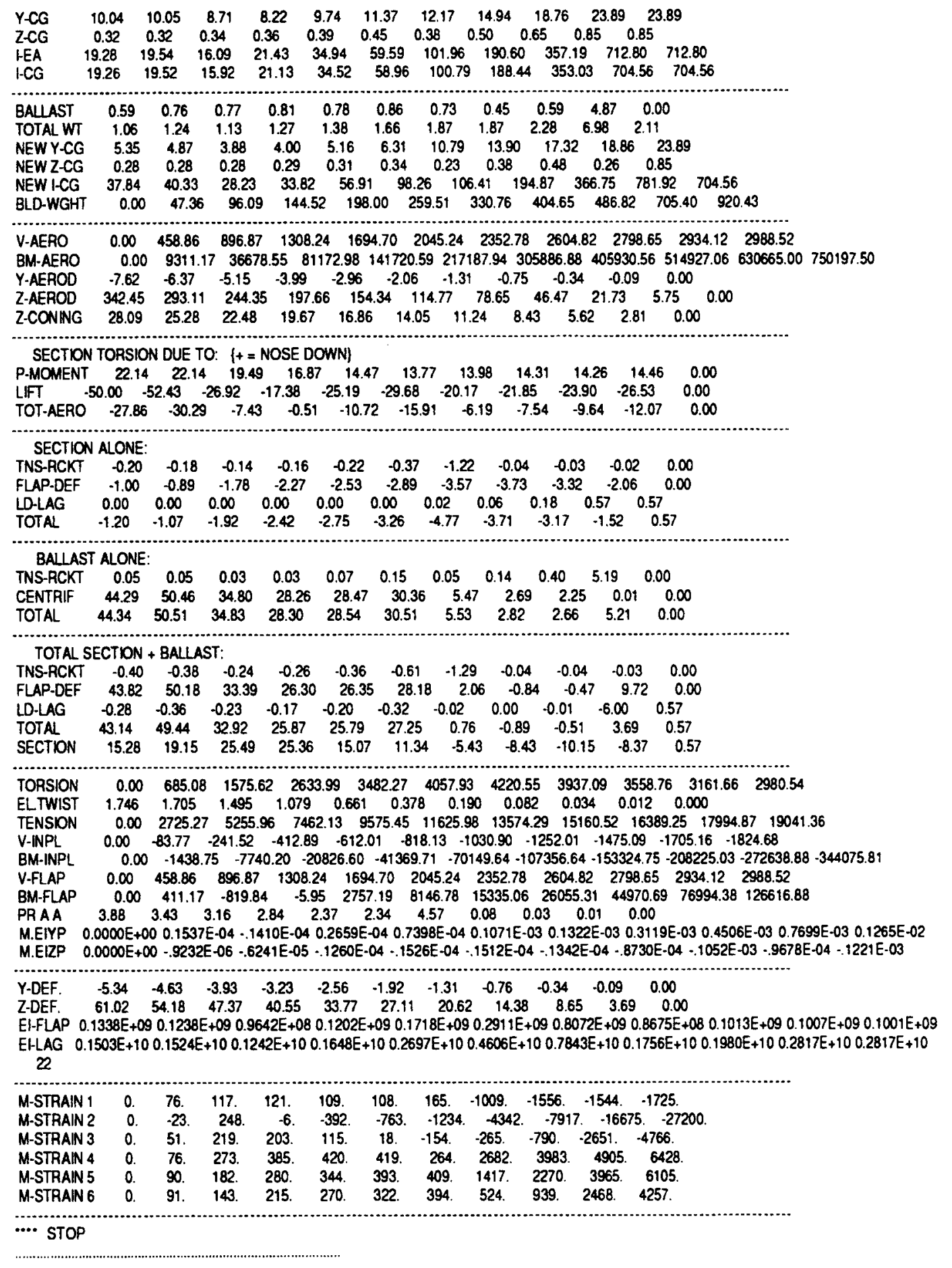




\subsubsection{SAMPLE RUN, ESI BLADE, WITH TIP WEIGHT}

The second sample run uses the ESI blade data. In this case only the location of the shear center had to be changed. This version of the ESI blade had the regular tip weight and ran at $90 \mathrm{rpm}$.

\section{WPUT BLADE RLE: ESBBLREGTIP}

$11 \quad 1.88 \quad 9.88500 \quad 0.133 \quad 0.133$

$3301.00 \quad 0.0245 \quad 0.0245$

$\begin{array}{llllllllll}0000 & .0004 & .0020 & .0040 & 0080 & 0199 & .0399 & .0798 & .1196\end{array}$

$\begin{array}{lllllllll}1994 & 2792 & 3589 & 4786 & 5982 & 7179 & 8375 & 1.0000\end{array}$

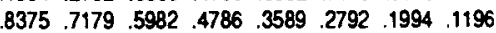

$\begin{array}{lllllllllll}.0798 & .0399 & .0199 & .0080 & .0040 & .0020 & .0004 & .0000\end{array}$

$\begin{array}{llllllllll}0000 & .0060 & .0140 & .0211 & .0289 & .0463 & .0636 & .0842 & .0981\end{array}$

$\begin{array}{lllllllll}1123 & 1173 \quad 1161 \quad 1053 \quad 0879 & 0664 & 0413 \quad 0000\end{array}$

$-.0377-.0618-.0828 \cdot .0981-.1065 \cdot .1067-.1013-.0861$

$-.0730-.0534-.0389 \cdot .0257-.0182-.0126 \cdot .0060 \quad .0000$ 006

$\begin{array}{lllllll}0000 & .2792 & .4786 & 1.0000 & .4786 & .2792\end{array}$

$\begin{array}{lllllll}0000 & .1173 & .47853 & .0000 & -.0981 & -.1067\end{array}$

$0.000 \quad 0.85 \quad 1.65 \quad 2.49 \quad 3.29 \quad 4.10 \quad 4.94 \quad 5.75 \quad 6.55 \quad 7.71 \quad 7.71$

$11.65 \quad 0.690 .00001 \quad 0.472 \quad 5.00$

$\begin{array}{llllllll}1.236 & 0.272 & 0.00 & 0.00 & 4.00 & 0.00\end{array}$

$0.930 \quad 5.73 \quad 0.0$

$\begin{array}{lllllll}13.66 & 0.827 & 00001 & 0.4725 .00\end{array}$

$\begin{array}{lllllllllllllll}1.236 & 0.272 & 0.00 & 0.00 & 4.31 & 0.00\end{array}$

$0.00 \quad 0.00 \quad 0.00$

$\begin{array}{lllll}15.43 & 0.961 \quad .00001 & 0.472 \quad 5.00\end{array}$

$\begin{array}{llllllllll}1.653 & 0.769 & 0.00 & 0.00 & 4.90 & 0.00\end{array}$

$0.00 \quad 0.00 \quad 0.00$

$\begin{array}{llll}17.28 & 1.192 \quad .00001 & 0.555 & 5.00\end{array}$

$\begin{array}{llllll}1.494 & 1.112 & 0.00 & 0.00 & 5.63 & 0.00\end{array}$

$0.00 \quad 0.00 \quad 0.00$

$19.09 \quad 1.150 .00001 \quad 0.6775 .00$

$\begin{array}{llllllllllllll}0.964 & 1.040 & 0.00 & 0.00 & 6.30 & 0.00\end{array}$

$0.00 \quad 0.00 \quad 0.00$

$20.87 \quad 1.226 \quad .00001 \quad 0.6815 .00$

$\begin{array}{llllllll}1.187 & 1.940 & 0.00 & 0.00 & 6.81 & 0.00\end{array}$

$0.00 \quad 0.00 \quad 0.00$

22.721 .291 .000010 .7805 .00

$\begin{array}{llllllll}1.350 & 2.858 & 0.00 & 0.00 & 7.32 & 0.00\end{array}$

$0.00 \quad 0.00 \quad 0.00$

$24.40 \quad 1.376 .000010 .9805 .00$

$\begin{array}{llllll}1.503 & 3.943 & 0.00 & 0.00 & 7.97 & 0.00\end{array}$

$0.00 \quad 0.00 \quad 0.00$

$20.85 \quad 1.771 .00001 \quad 1.0805 .00$

$\begin{array}{lllllll}1.641 & 5.124 & 0.00 & 0.00 & 8.15 & 0.00\end{array}$

$0.00 \quad 0.00 \quad 0.00$
$16.802 .416 .000012 .490 \quad 5.00$

$\begin{array}{lllllllllllll}1.081 & 1.467 & 0.00 & 0.00 & 8.15 & 0.00\end{array}$

$0.00 \quad 0.00 \quad 0.00$

$\begin{array}{llll}16.80 & 2.416 \quad 000012.490 \quad 5.00\end{array}$

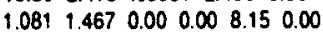

$\begin{array}{llll}0.00 & 0.00 & 0.00\end{array}$

ESI.BLADE.MEAS.DATA.REGULAR.TIP.1-27-88

WPUT LOAD PLE: ESI9OLD.3OMPH

001

$00.00 \quad 32.41 \quad 64.8197 .22129 .62162 .03194 .44226 .84$

259.25291 .65324 .05

$\begin{array}{lllllllllllll}00.00 & 00.00 & 00.00 & 00.00 & 00.00 & 00.00 & 00.00 & 00.00\end{array}$

$00.00 \quad 00.00 \quad 00.00$

$-1.25-1.63-2.30-2.71-2.82 \cdot 1.93+1.16-.42-.030 .00 .0$

4.795 .476 .176 .205 .664 .743 .191 .660 .84 .580 .0

9.9410 .129 .828 .796 .325 .695 .673 .191 .901 .800 .0

$9.425324 .06 \quad 0.05 .0$

ESI.BLADE.90.RPM.30.MPH.LD.1-27-88

WPUT MODIFED SECTION PROPERTES FLE: OUARTERCHORD

3.512 .030 .058 .0111 .0182 .0304 .0487 .0688 .01250 .0880 .0

167.3 400.520. 880. 1360. 2050. 2770. 4396. 3200. 2500. 2400.

652.506 .5013 .324 .339 .863 .199 .2136 .0180 .0144 .5

2.93 .423 .864 .324 .775 .225 .686 .15 .214 .24 .2

19.1125 .3031 .138 .849 .059 .574 .594 .494 .9120 .0152 .0

ONLY THE SHEAR CENTER HAS BEEN CHANGED BELOW TO MATCH TESTDATA

TERMNAL SESSION AND OUTPUT: STRESSDATA

ENTER INPUT FILE NAME FOR SECTION GEOMETRY:

ESIBLREGTIP

ENTER INPUT FILE NAME FOR APPLIED LOADING:

ESI9OLD.30MPH

ENTER INPUT FILE NAME FOR ALTERNATE SECTION PROPERTIES

QUARTERCHORD

$\because \because \because \because \because \because \because \cdots \cdots$

ONLY THE SHEAR CENTER HAS BEEN CHANGED BELOW TO MATCH TESTDATA

ESI.BLADE.MEAS.DATA.REGULAR.TIP. $1-27-88$

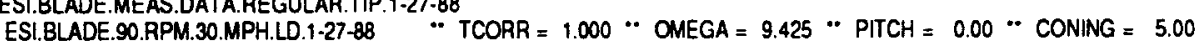

\# STATIONS: 11 E-SKIN: 1880000.00 E-LONG: 1879999.75 DNISIONS: 500 GS: 132999.97 GL: 132999.97 WS: 0.025 WL:0.025

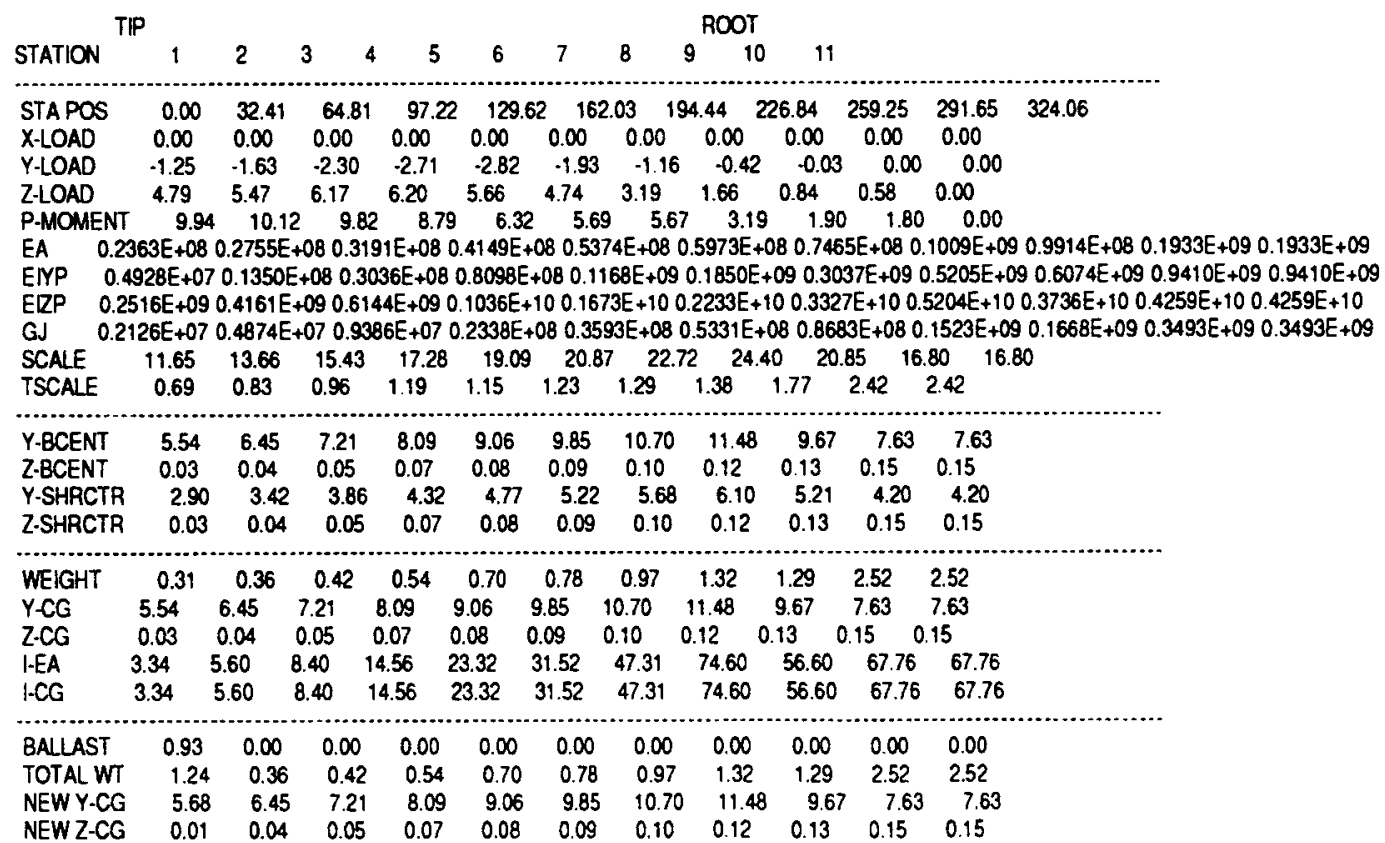




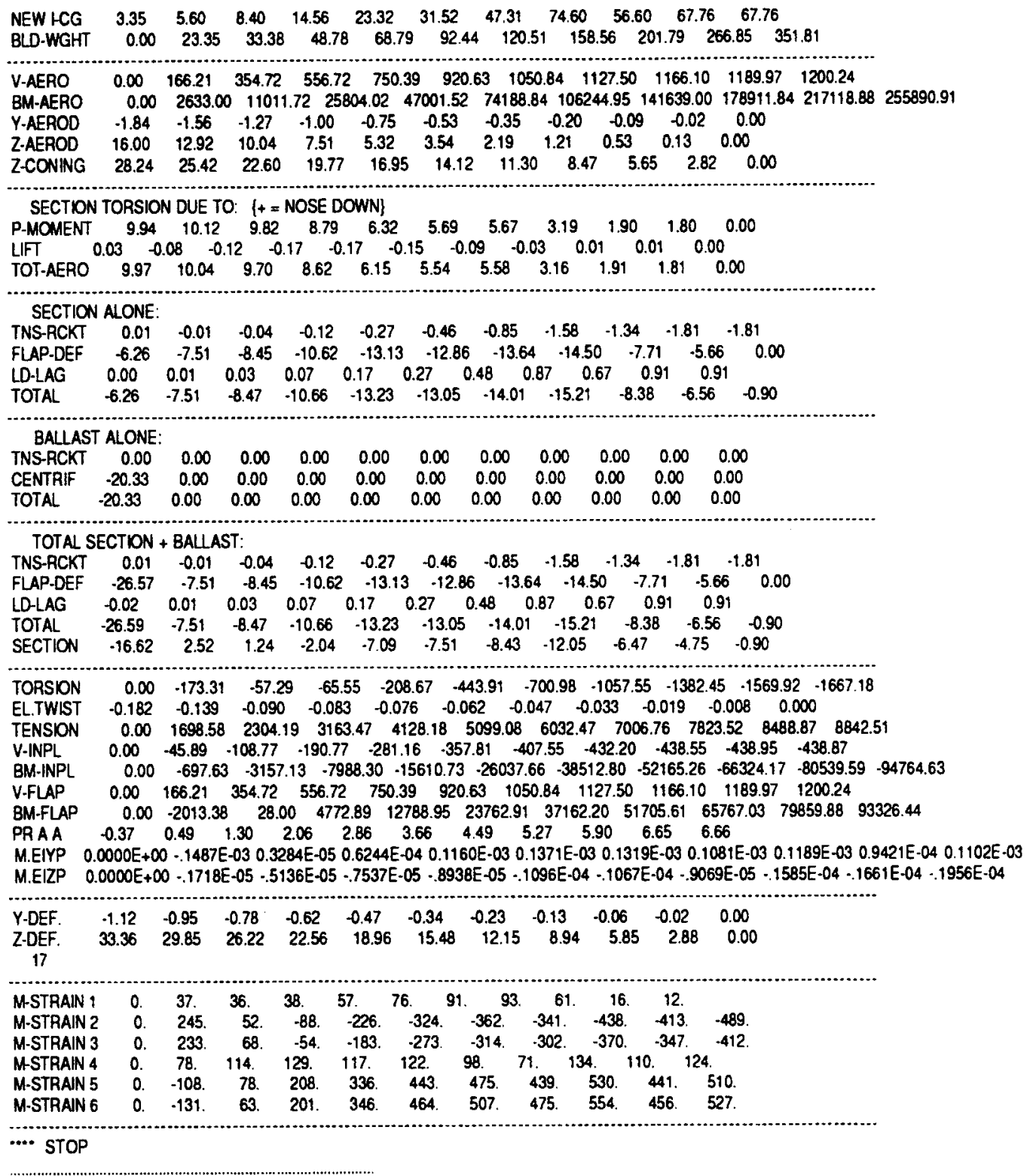

\section{3: COMPUTER CODE: COMPOSITE BLADE BENDING FREQUENCIES OF VIBRATION}

\subsubsection{BRIEF DISCUSSION}

The bending frequency of vibration calculations used the methodology of Chapter 4 (Holzer table). The code has the following general characteristics:

Uses Myklestad-Prohl lumped mass analysis.

Does not assume mode shapes; calculates frequencies and mode shapes from arbitrary section mass and stiffness data.

Assumes uncoupled bending modes in flapping, lead-lag, and torsion; bending mode coupling corrections can be added to the routine if the frequencies of interest coincide.

Can use cantilevered or simply supported beam model.

Program gives frequencies and mode shapes for the principal bending, and gives influence coefficients which can be used in other aeroelastic programs. 


\subsubsection{PROGRAM LISTING, VIBRATE}

The code VIBRATE is listed here. Comment lines explain the logic and input and output parameters

$$
\begin{aligned}
& \text { a } \\
& \text { c } \\
& \text { C } \\
& \text { C } \\
& \text { C } \\
& \text { C } \\
& \text { C } \\
& \text { C } \\
& \text { C } \\
& \text { C } \\
& \text { C } \\
& \text { C } \\
& \text { C }
\end{aligned}
$$$$
\begin{aligned}
& C \\
& C \\
& C \\
& C \\
& C \\
& C \\
& C \\
& C \\
& C
\end{aligned}
$$

\section{PROGRAM VIBRATE}

THIS PROGRAM FINDS THE NATURAL FREQUENCIES

-.....IN BENDING OF A CANTILEVER BEAM BY THE MYKLESTAD METHOD-

-. THE BEAM IS FIXED AT STATION 11. THE BEAM IS DIVIDED -...

--_INTO 11 DISCRETE LUMPED MASSES AND THE BEAM ROTATES-..-

........AT FREOUENCY OMEGA ABOUT THE FIXED END. THE BEAM-......

-...-THE BEAM SEGMENTS ARE OF EOUAL LENGTH

DIMENSION EL(12),RM(12),El(12),UF(12),VF(12),UM(12),VM(12),EM(12) DIMENSION ELRC(12),ELR(12),EMR(12), FCR(12),FC(12),DFC(12),DFCR(12) DIMENSION A(12),B(12),C(12),D(12)

DIMENSION BGP(12),BHP(12),HP(12),GP(12),BG(12),BH(12),H(12),G(12), + DEF(12)

CHARACTER 40 INPI

CHARACTER'BO HEADING

FORMAT(3X,15,3X,F8.4)

FORMAT $(3 X, F 6.2)$

FORMAT $(3 X, 6 F 9.6, /, 3 X, 5 F 9.6)$

FORMAT $(3 X, 6 F 8.4,1,3 \times, 5 F 8.4)$

FORMAT(1ABO)

FORMAT (1A4O)

FORMAT $(, 1,1$ ABO)

-...-READ INPUT FILE: INP1 IS THE FILE WHICH CONTAINS THE

-...-..-BEAM MASS AND STIFFNESS PROPERTIES -...

-FIRST: NO. OF STATIONS AND BEAM LENGTH (INCHES)

-.........-SECOND: BEAM RUNNING MASS (SLUGS PER INCH)

.........THIRD: BEAM EIS (FLAP OR LAG) (LB-IN*2) 10E-6

........FOURTH: RPM OF BLADE

-LAST: HEADING FOR THE DATASET

WRTE $(1,70)$

70 FORMAT(ENTER INPUT FILE NAME FOR BEAM PROPERTIES:)

READ $(1,58)$ INP1$$
\text { C }
$$

.......-READ NUMBER OF STATIONS,LENGTH,RUNNING MASS, AND EIS-.. BEGINNING AT THE TIP

OPEN(UNIT $=5, F H E=$ INP1)

READ $\left(5^{\circ}\right)$ NSTARAD

$\operatorname{READ}\left(5^{\circ}\right)(\mathrm{RM}(\mathrm{N}), \mathrm{N}=1, \mathrm{NST} A)$

C

DOBON $=1$ NSTA

$E I(N)=E I(N) * 10 \cdots 6.0$

$\mathrm{RM}(\mathrm{N})=\mathrm{RM}(\mathrm{N}) / 12.0$

80 CONTINUE

C

C

READ $\left.(5,)^{\circ}\right) \mathrm{RPM}$

READ $(5,57)$ HEADING

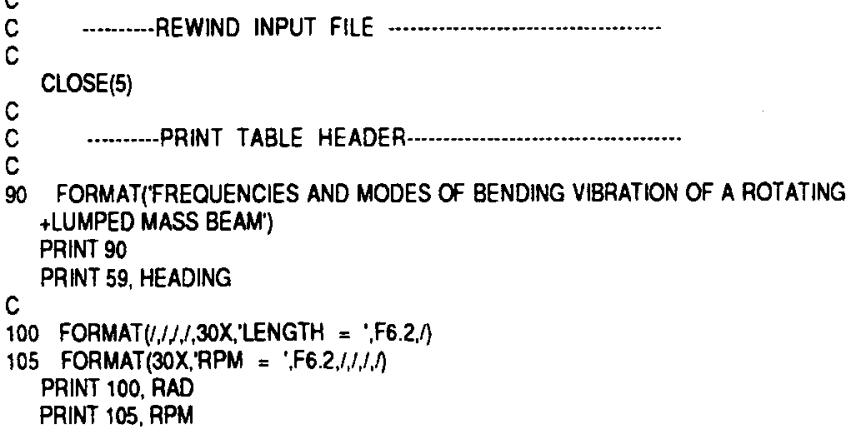

C

110 FORMATCMODE FREQUENCY (TIP)',11X,'STATION',62X,'ROOTY

112 FORMATS RADISEC CYCISEC 1119 PRINT 110

C

PRINT 112, $(N, N=1,11)$

C

OMEGA2 $=($ RPM $\cdot 2.0 \cdot 3.1415926 / 60.0) * 2.0$

C ..........CALCULATE THE INFLUENCE VALUES FROM L'S AND EI'S-.....

DELTR = RAD $/$ FLOAT $(N S T A-1)$

DO $145 \mathrm{~N}=1$, NSTA

$E L(N)=R A D-(F L O A T(N-1))^{*}$ (DELTR $)$

$\mathrm{UF}(\mathrm{N})=(\mathrm{DELTR} \cdot 3.0) /\left(3.0^{\circ} \mathrm{El}(\mathrm{N})\right)$

$V F(N)=(D E L T R \cdots 2.0) /\left(2.0^{\circ}\right.$ EI(N)

$\mathrm{UM}(\mathrm{N})=\mathrm{VF}(\mathrm{N})$

$V M(N)=$ DELTR $/ E I(N)$

145 CONTINUE

C

DO $150 \mathrm{~N}=1, \mathrm{NSTA}$

$E M(N)=0.0$

$A(N)=0.0$

$B(N)=0.0$

$C(N)=0.0$

$D(N)=0.0$

150 CONTINUE

C

$\mathrm{J} \mathrm{J}=0$

c

$E M(1)=(\operatorname{DELTR} / 4.0) \cdot(R M(1)+\operatorname{RM}(2))$

$E M(11)=(D E L T R / 4.0)^{\circ}(R M(10)+R M(11))$

DO $165 \mathrm{~N}=2.10$

$E M(N)=\langle D E L T R / 4.0)^{*}\left(R M(N-1)+\left(2.0^{\circ} R M(N)\right)+R M(N+1)\right)$

165 CONTINUE

C

DO $180 \mathrm{~N}=1, \mathrm{NSTA}$

$\mathrm{DFC}(\mathrm{N})=0.0$

$F C(N)=0.0$

180 CONTINUE

C

$D O 200 N=1$,NSTA

$D F C(N)=E M(N) \cdot E L(N) \cdot O M E G A 2$

$F C(N)=D F C(N)+F C(N-1)$

200 CONTINUE

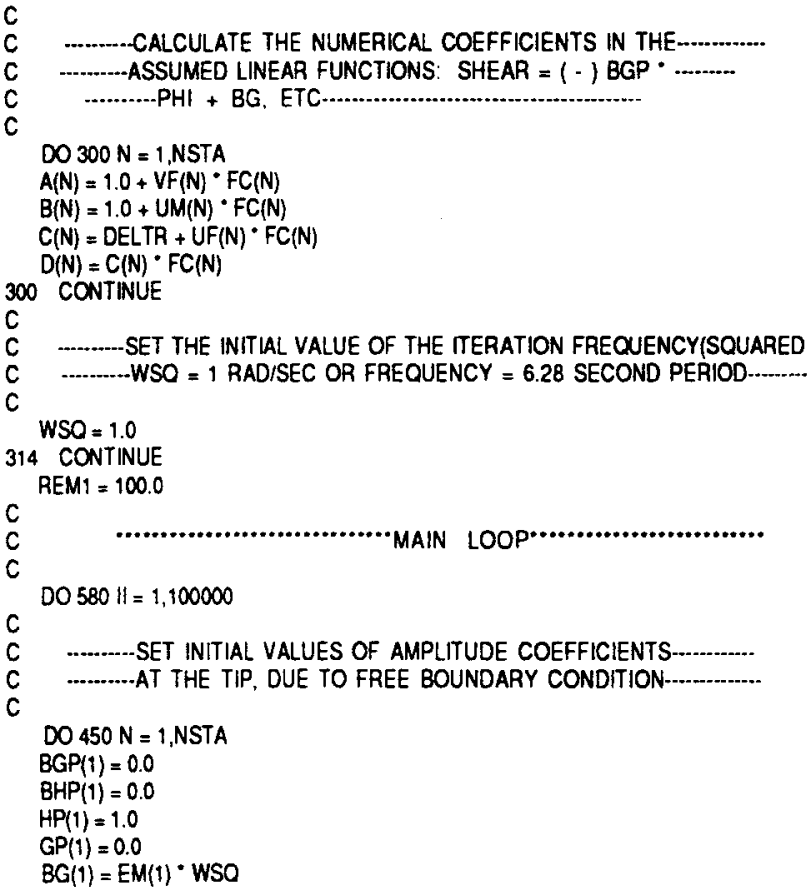



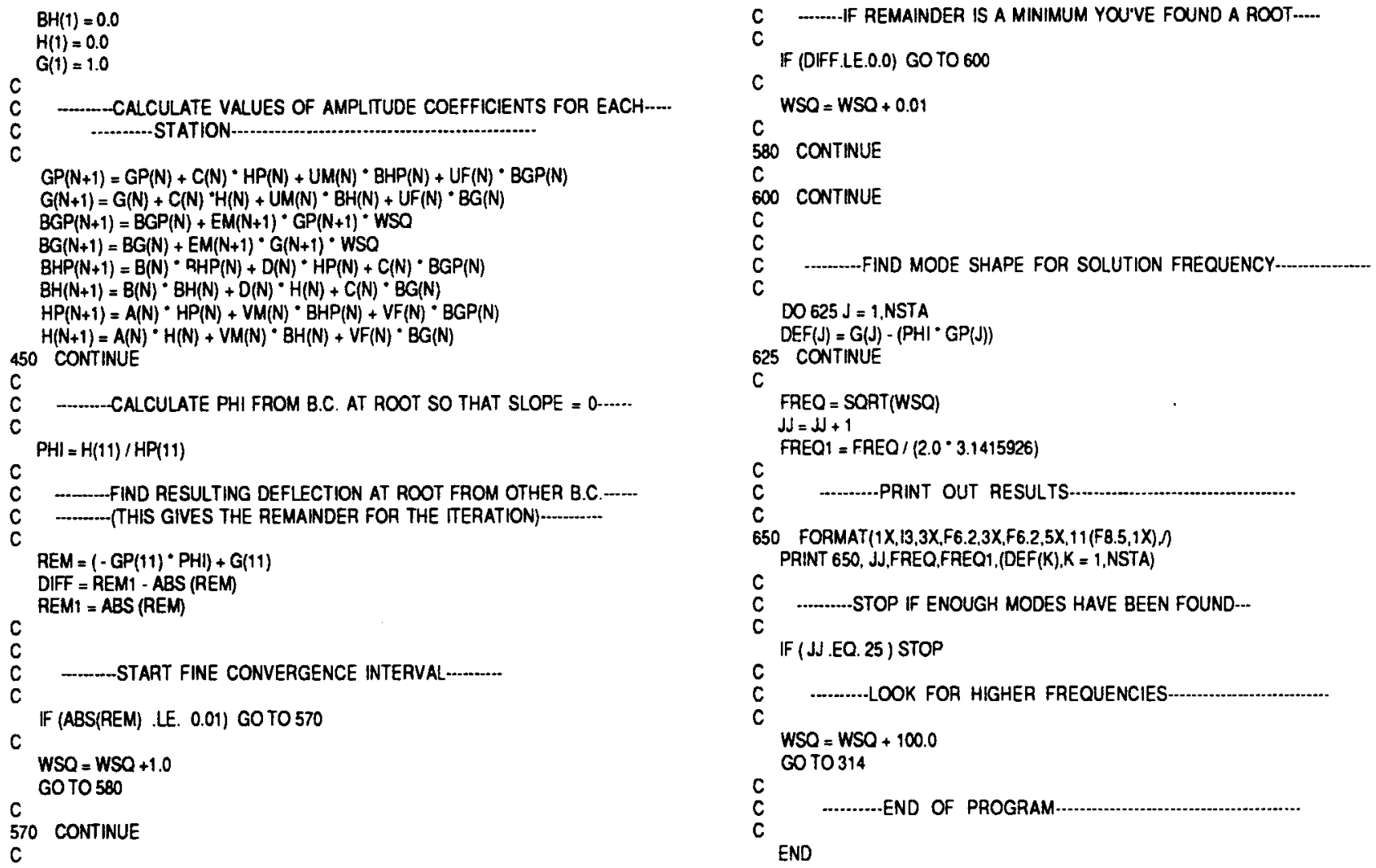

\subsubsection{SAMPLE RUN, UTRC BLADE, STATIC AND 108 RPM}

These sample runs use the uniform pultrusion UTRC blade for simplicity. The input file contains the blade structural properties and the rotor speed. The first run is for static frequencies, and the second for the $108 \mathrm{rpm}$ condition. Note: the output list multiple solutions for each root due to coarse convergence criteria; the best solution is the one with minimum root deflection (e.g., deflection = 0 at STA 11). Also, the program must be manually stopped when all practical roots have been found (the program will continue to find higher and higher roots).

\section{INPUT BLADE FILE: UTRCFLAPVBBE}

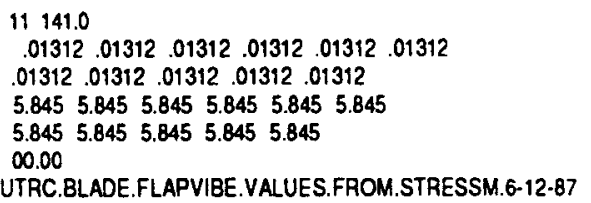

TERAMNAL SESSION AND OUTPUT: VIBE

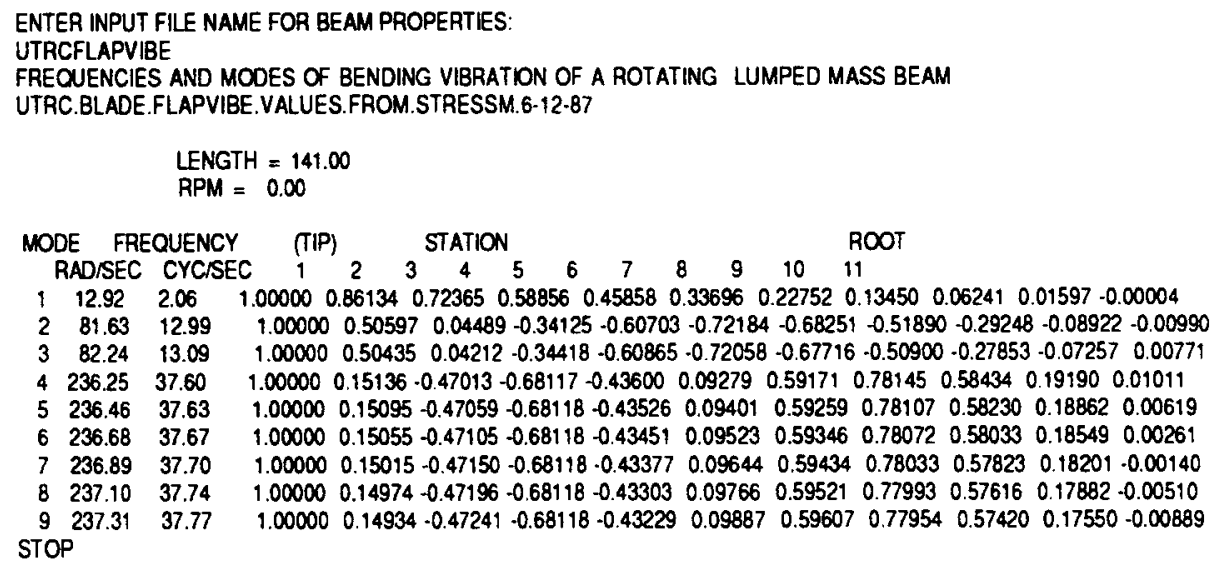


The following run is for the same blade, but at its operating speed of $108 \mathrm{rpm}$.

INPUT BLADE FLE: UTRCFLAPVBE. 108

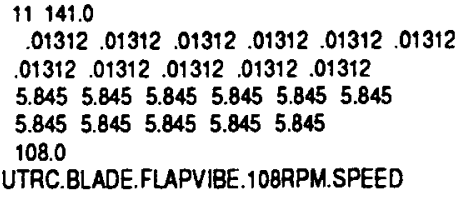




\subsubsection{PROGRAM LISTING, VIBETIP, (TIP WEIGHT VERSION)}

The following version of the vibration program has provision for the addition of a tip mass to the blade from the keyboard.

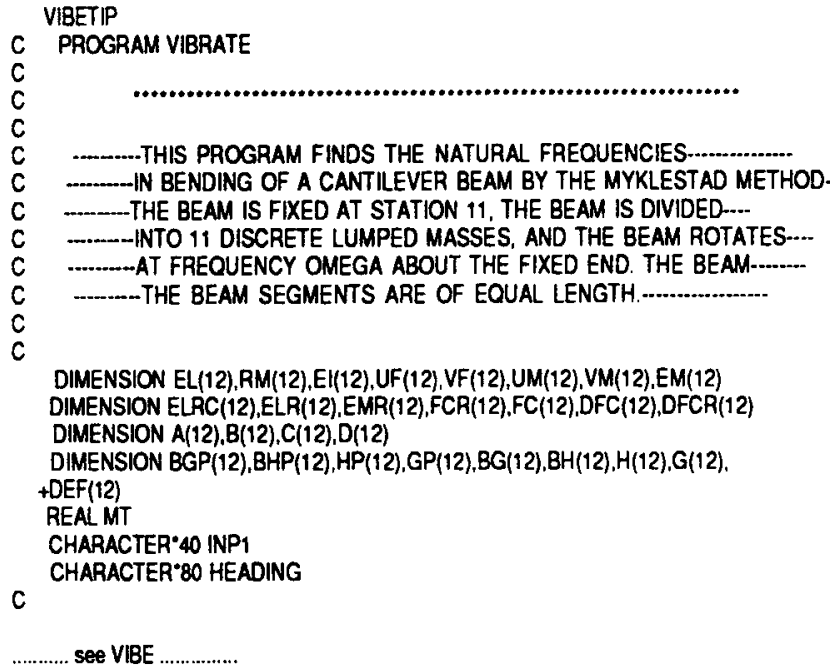

70 FORMAT(ENTER INPUT FILE NAME FOR BEAM PROPERTIES:')

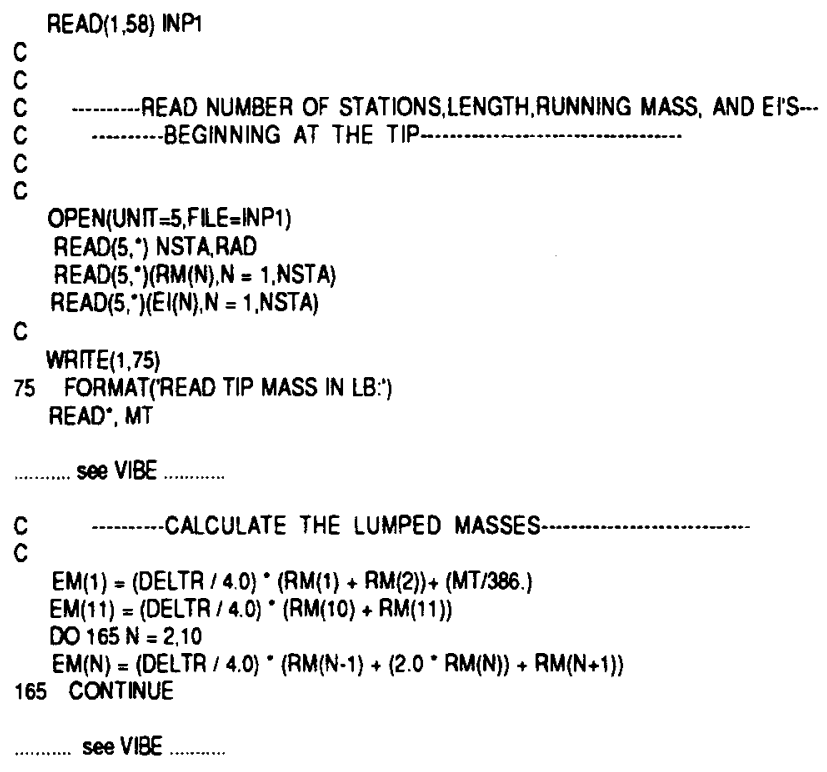

\section{4: COMPUTER CODE: COMPOSITE BLADE TORSIONAL FREQUENCIES OF VIBRATION}

\subsubsection{SUMMARY}

The code that calculates torsional frequencies and mode shapes is also a Holzer table (see Chap. 4). There are two versions of this program: one for general torsional beams (TORSVIB) and the other with provision for added tip moment of inertia (TVIBTIP). This latter program was used to find the frequencies of the blades with tip weights and mechanisms (ESI). The sample runs are for this blade first bare and then with the regular tip (see Chap. 5).

\subsubsection{PROGRAM LISTING, TORSVIB} TORSVIB

PROGRAM VIBAATE

50 FORMAT $(3 \times, 15,3 \times, F 8.4)$

52 FORMAT(3X,F6.2)

55 FORMAT $(3 X, 6 \mathrm{~F} 9.6, l, 3 X, 5 \mathrm{~F} 9.6)$

56 FORMAT $(3 X, 6 F 8.4,1,3 X, 5 F 8.4)$

57 FORMAT $(1 A B O)$

58 FORMAT(TA40)

59 FORMAT(U,I.1ABO)

.........-READ INPUT FILE: INP1 IS THE FILE WHICH CONTAINS THE

.......-BEAM INERTIAS AND TORSIONAL STIFFNESSES

…......FIRST: NO. OF STATIONS AND BEAM LENGTH (INCHES)

...-.-.-SECOND: BEAM SECTION RUNNING MASS MOMENTS OF INERTIA (LB-IN“2 PER INCH) WHICH INCLUDES CONTRIBU. TIONS FROM THE SKIN, SPAR, WEB, AND COUNTER. WEIGHTS. THE MASS MOMENTS ARE REFERRED TO THE AXIS OF TORSION, OR ELASTIC AXIS.

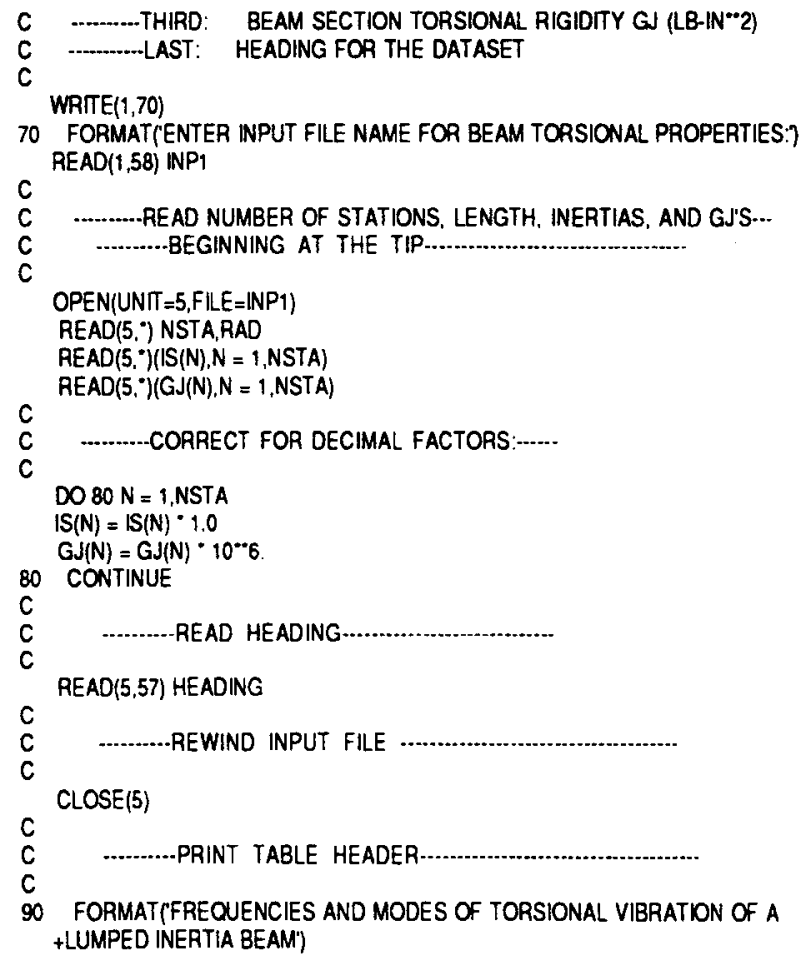




\section{PRINT 90}

C

PRINT 59, HEADING

100 FORMAT $(1,1,30 X$, 'LENGTH $=', F 6.2,1$

PRINT 100, RAD

110 FORMATC MODE FREOUENCY (TIP)',11X,'STATION',62X,'ROOT')

112 FORMATS RADISEC CYCISEC',119)

PRINT 110

PRINT 112, $(\mathbf{N}, \mathbf{N}=1,11)$

$\stackrel{C}{c}$

C...CALCULATE MASS MOMENTS OF INERTIA (LB-IN**2) AND TORSIONAL

C ....STIFFNESS (IN-LB/RADIAN) FOR EACH STATION....

DELTR $=$ RAD / FLOAT (NSTA-1)

$K(1)=G(1) /(D E L T R / 2)$

C

$I(1)=I S(1) *(D E L T R / 2)$

DO $145 \mathrm{~N}=2, \mathrm{NSTA}$

C ...TORSIONAL STIFFNESS FOR THE SEGMENT IS THE AVERAGE OF THE

C -..ADJACENT GJ'S--

$K(N)=(G N(N)+G J(N+1)) /\left(2^{\circ} D E L T R\right)$

C -..-MASS MOMENT OF INERTIA IS THE SECTION VALUE TIMES THE SECTION

-...-LENGTH.--

C

$I(N)=I S(N) \cdot D E L T R$

145 CONTINUE

C

$J J=0$

C -CHANGE UNITS OF MASS MOMENT OF INERTIA FROM (LB-IN*2) TO

-...(LB-IN-SEC**2) BY DIVIDING BY THE ACCELERATION OF GRAVITY

-..-IN THE ENGLISH SYSTEM (= 386 INCHESISEC**2)-...

DO $180 \mathrm{~N}=1, \mathrm{NSTA}$

$I(N)=I(N) / 386$

180 CONTINUE

C

C ........SET THE INITIAL VALUE OF THE ITERATION FREQUENCY (SORD)

C ..........WSO $=1$ RAD/SEC OR FREQUENCY $=6.28$ SECOND PERIOD

$W S Q=1.0$

C

314 CONTINUE

C

...

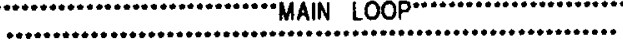

$D O 580 \|=1,100000$

C

C .....SET INITLAL VALUES FOR THE TIP, WHICH IS FREE OF RESTRAINT

C -..-AND HAS (NORMALIRED) TWIST ANGLE AMPLITUDE OF 1.0

-.-TWIST ANGLE, INERTIAL TOROUE, SUMMED TOROUES, AND TWIST ANGLE FOR

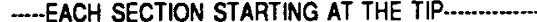

THETA $(1)=1.0$

ON $(1)=l(1) \cdot W S O$

$\operatorname{SON}(1)=\operatorname{CN}(1)$
$\operatorname{DTHETA}(1)=\operatorname{SON}(1) / K(1)$

-...CALCULATE VALUES OF TWIST ANGLE, TOROUE, AND SUMS FOR

-..-EACH STATION-...

DO $450 \mathrm{~N}=2, \mathrm{NSTA}$

C

C --FIND TWIST ANGLE FOR THIS STATION--.-

$\operatorname{THETA}(N)=\operatorname{THETA}(\mathrm{N}-1) \cdot$ DTHETA $(\mathrm{N}-1)$

C -.-.-CALCULATE INERTIAL TOROUE FOR THIS STATION-.--

$\mathrm{ON}(\mathrm{N})=I(N) \cdot W S O \cdot \operatorname{THETA}(N)$

C -...-SUM TOROUES FOR ALL STATIONS SO FAR....

$\operatorname{SON}(N)=\operatorname{SON}(N-1)+\operatorname{ON}(N)$

c

C -...FIND TWIST ANGLE FOR THIS STATION---

$\operatorname{DTHETA}(N)=\operatorname{SON}(N) / K(N)$

C

450 CONTINUE

C

C

THETAR $=$ THETA(10) - DTHETA(10)

THETA(11) = THETAR

C

IF (ABS(THETAR) .LE. 0.001$)$ GOTO 600

C -.-OTHERWISE INCREASE THE WSO VALUE. AND TRY AGAIN-...

C

WSO $=W S O+1.0$

C

580 CONTINUE

600 CONTINUE

C -..-NOW PRINT OUT MODE NUMBER, SOLUTION FAEQUENCY IN RAD/SEC, AND

C -..-CYCLESISEC, AND TORSIONAL MODE SHAPE FOR EACH STATION-...

FREQ $=$ SORT (WSO)

FREO1 $=$ FREO $/(2.0 \cdot 3.1415926)$

$\mathrm{JJ}=\mathrm{J}+1$

C

C .........PRINT OUT RESULTS

650 FORMAT( $1 X, 13,3 X, F 6.2,3 X, F 6.2,5 X, 11(F 8.5,1 X), /)$

PRINT 650, JJ,FREO,FREQ1,(THETA(J), J = 1,NSTA)

C …....IF ALL 10 MODES HAVE BEEN FOUND, END PROGRAM....

IF ( JJ .EQ. 10) STOP '10 MODES COMPLETED'

C

c

$W S O=W S O+100.0$

COTO314

$\mathrm{C}$
$\mathrm{C}$

END 


\subsubsection{SAMPLE RUN,ESI BLADE}

INPUT BLADE FLE: ESTORSVBE.FNAL
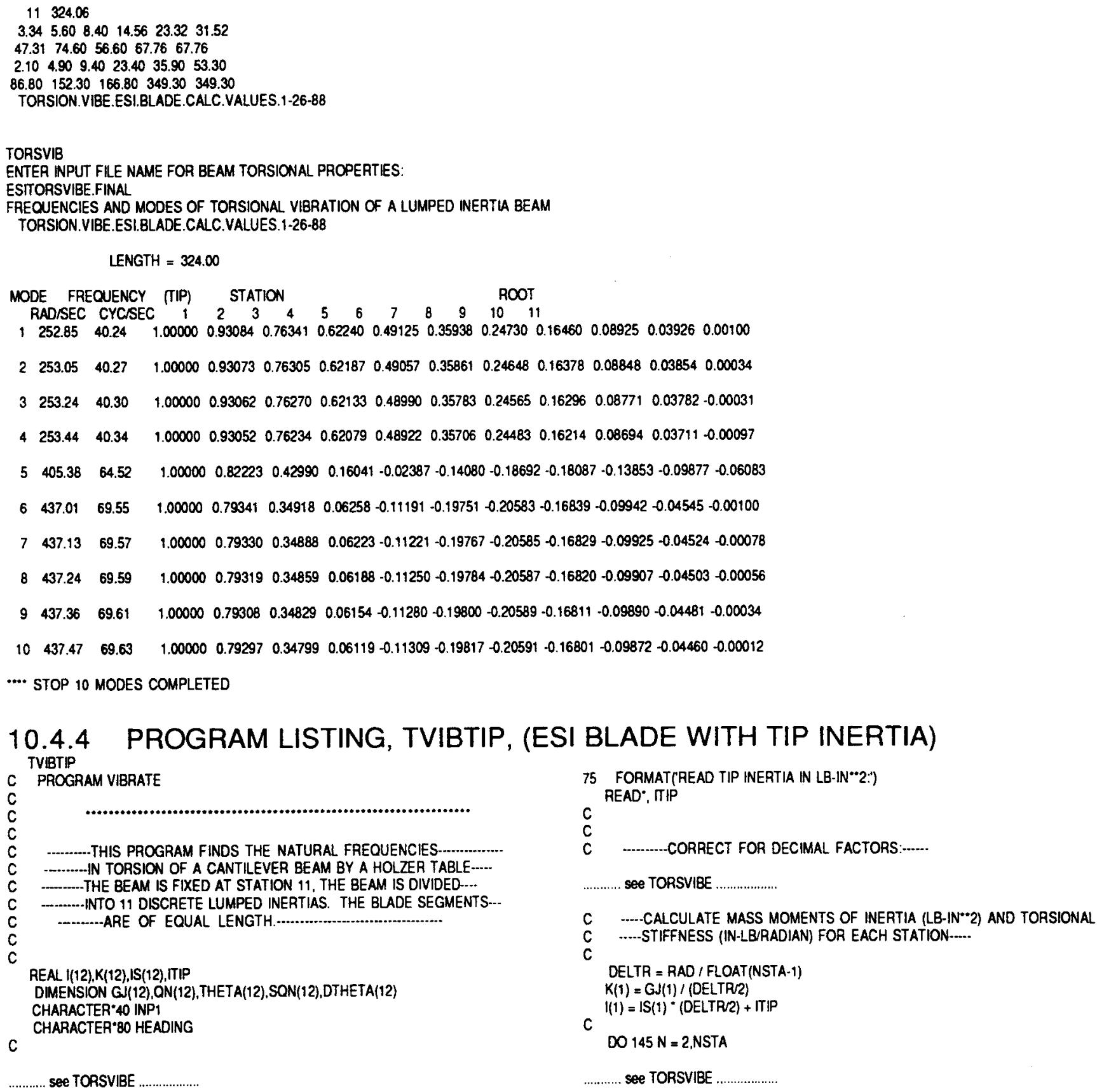


\subsubsection{SAMPLE RUN, ESI BLADE WITH TIP INERTIA}

TVIBTIP

ENTER INPUT FILE NAME FOR BEAM TORSIONAL PROPERTIES

ESTTORSVIBE.FINAL

READ TIP INERTIA IN LB-IN`2:

B31.

FREOUENCIES AND MODES OF TORSIONAL VIBRATION OF A LUMPED INERTIA BEAM

TORSION. VIBE.ESIBLLADE.CALC.VALUES.1-26-88

$$
\text { LENGTH }=324.06
$$

MODE FREQUENCY (TIP) STATION

$\begin{array}{lllllllllllllll}1 & 148.29 & 23.60 & 1.00000 & 0.61094 & 0.35378 & 0.23082 & 0.15603 & 0.10142 & 0.06393 & 0.04045 & 0.02172 & 0.00986 & 0.00098\end{array}$

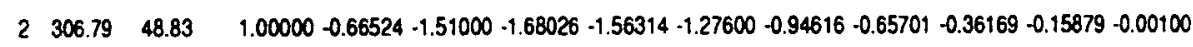

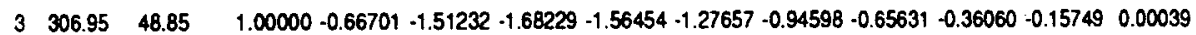

QUIT.

\section{5: COMPUTER CODE: STEADY ELASTIC TWIST CALCULATION}

\subsection{1: PROGRAM LISTING, STRESSELASTIC}

The code STRESSELASTIC is the chief program used for this study. It contains all the calculations required for finding elastic twist and all the bending deflections. A listing is given here in its entirety. 


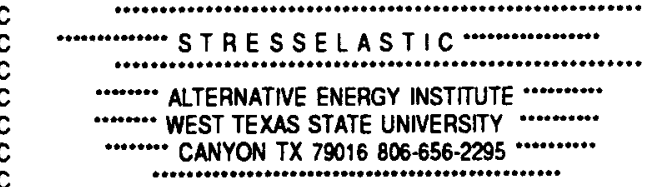

THIS PROGRAM CALCULATES THE SECTION MASS AND STIFFNESS PROPERTIES OF AN ARBITRARY EXTERNAL GEOMETRY (TWIST, TAPER) AND SPECIFIED INTERNAL SPAR.SHELL STRUCTURAL GEOMETRY, COMPOSTE WIND TURBINE BLADE, AND THEN FINDS THE BENDING DEFLECTIONS, ELASTIC TWIST, AND STRAINS IN THE LAMINATE FOR A SPECIFIED EXTERNAL LOAD AND ROTOR ROTATIONAL SPEED.

THIS VERSION OF THE PROGRAM ("STRESSELASTIC") CONTAINS ALL THE ROUTINES AND PRINTS OUT THE TORSIONAL OEFLECTIONS (ELASTIC TWIST) DUE TO ALL INERTIAL AND APPLIED LOADS, AND DUE TO BLADE BENDING.

THIS PROGRAM WAS ORIGINALLY WRITTEN BY E.VAN DUSEN (COMPOSITE ENGINEERING. INC., 277 BAKER AVE., CONCORD MA 01742 617-371-3132) IN 1977 FOR COMPOSTIE RACING SHELLS AND HAS BEEN EXTENSNELY REWRITTEN BY F.S. STODDARD (AEI, BOX 248 WTSU, CANYON TX 79016 806-656-2295) FOR WIND TURBINE BLADES

\section{GENERAL PROGRAM DESCRIPTION:}

PART 1 OF THIS PROGRAM CALCULATES THE SECTION PROPERTIES OF AN ARBITRARY COMPOSITE BEAM, USING EITHER ACTUAL THICKNESSES AND MODULI OF PLIES, OR USING MODULUS-WEIGHTING (THE LATTER ROUTINE HAS BEEN SUPPRESSED FOR THE CALCULATION OF ELASTIC TWIST). THE BEAM (BLADE) IS MOOELED BY A SHEL OF CONSTANT THICKNESS WITH ARBITRARY LONGITUDINAL MODULUS AND SHEAR MODULUS, AND A SPAR CONSISTING OF A NOSE SECTION EMBEDDED NEXT TO THE SHEUL IN THE BLADE LFADING EDGE, AND A WEB IOR WEBS LOCATED INTERNALLY. THE SPAR ALSO HAS CONSTANT THICKNESS AND ARBITRARY LONGITUDINAL AND SHEAR MODULL. THE WEB IS DESCRIBED ONLY BY ITS MOMENTS OF INERTIA AND CENTROID IN THE SECTION AXIS SYSTEM. MULTI-WEB BEAM DESIGNS ARE HANDLED BY INCLUDING ALL THE ACTUAL WEBS INTO THIS ONE MODEL WEB.

THE SECTION AIRFOIL SHAPES ARE DETERMINED BY SCALING AN INPUT TABLE OF OFFSETS. THE CHORD SCALING FACTOR (SCALE) DETERMINES THE AIRFOIL CHORD AND THE THICKNESS SCALING FACTOR (TSCALE) DETERMINES THE SECTION THICKNESS PROPORTIONAL TO THE TABLE OF OFFSETS. ALL THESE INPUT DATA ARE INCLUDED IN A DATAFILE GNING THE GEOMETRY AND STRUCTURAL DESIGN OF THE BLADE OF INTEREST

IN PART I PARABOLIC SEGMENTS ARE FITTED THROUGH EACH GROUP OF THREE SECTION OFFSET POINTS. THE IOF THE SKIN+SPAR+WEB IS CALCULATED BY DIVIDING THE GROUP OF POINTS INTO (DIV) STRAIGHT LINE SEGMENTS OF ROUGHLY EOUAL SIZE AND SUMMING THE APPROPRIATE VARIABLES. THE OUTPUT OF PART 1 IS NOT PRINTED IN THIS VEASION OF THE PROGRAM. SECTION DETALLS ARE GNEN NN THE PROGRAM VERSION "STRESSM"

PART 2 OF THE PROGRAM SUBJECTS THE BLADE TO APPLIED LOADING AND TO CENTRIFUGAL (INERTIAL) LOADING. THE BLADE IS ASSUMED TO BE CANTILEVERED AT ITS ROOT (STATION 11) AND FREE AT THE TIP (STATION 1). THE PROGRAM THEN CALCULATES THE EQULLBBRIUM DEFLECTIONS OF THE ROTATING BLADE AND THE RESULTING STRAINS IN THE SKIN LAMINATE AT 6 SPECIFIED INPUT LOCATIONS AROUND THE SECTION FOR EACH BLADE STATION

THE DISTRIBUTED LOADING IS RESOLVED INTO $X, Y$, AND $Z$ COMPONENTS $X$ LLES ALONG THE (UNDEFLECTED) AXIS OF THE BLADE WITH THE ORIGIN AT THE BLADE ROOT. Y-AXIS IS IN THE LAGGING DIRECTION AND Z-AXIS IS IN THE FLAPPING DIRECTION. THE FREE STREAM WIND IS IN THE POSTTIVE Z-DIRECTION, AND ROTATION IS THUS CLOCKWISE LOOKING UPSTREAM FROM AFT OF THE ROTOR

THE BLADE STATION SPACING AND APPLIED LOADING WWICH CONSISTS OF TADE PREVIOUSLY CALCULATED AERODYNAMIC LOAD AND ANY OTHER EXTERNAL

LOAD) ARE LISTED IN ANOTHER INPUT DATAFILE, WHICH ALSO INCLUDES ROTOR SPEED, BLADE RADIUS, BLADE PITCH ANGLE, AND BLADE CONING ANGLE.

THE X-Y PLANE CONSTITUTES THE PLANE OF ROTATION OF THE ROTOR. THE Z-DEFLECTIONS ARE THE FLAPPING VALUES AND THE Y-DEFLECTIONS THE LEAD-LAG VALUES. TWIST AND PITCH ARE MEASURED POSTTIVE AIRFOIL NOSE-DOWN, OR TENDING TO DECREASE ANGLE-OF-ATTACK

NOTE: IF THE BLADE OF INTEREST HAS A FLEXIBLE ROOT ATTACHMENT (IE FLEXBEAM) THE GJ OF THE ROOT CAN BE SPECIFIED AS THE MINIMUM IN THE GJ2N SECTION OF PART IOF THE PROGRAM. EISS ARE HANDLED AS THE ROOT WEB $c$
$c$
$c$
$c$

DIMENSION XC(5000),YC(5000),R(4),XX(33),YY(33),DFZ2(11),DFZC(11) DIMENSION RX(11),YSO(6),ZSO(6),DFY(11),DFZ(11),DFY1(11),DFZ1(11) DIMENSION ZTOT(11),DFZT(11),ANGTOT(11),TANGTOT(11),AANG1(11) REAL MLAG(11),MFLAP(11),MLAGP(11),MFLAPP(11),MCENT(11),RCENT(11) REAL MCX(11),MC1(11),MFLAPA(11)

DIMENSION O(11),OT(11),OS(11),TLAST1(11),TLAST2(11),FCENT(11)

+,ELAST1(11),ELAST2(11),TELAST1(11),TELAST2(11),MCENT1(11),BWT(11) COMMON X(33), Y(33)

DIMENSION STX(11),ENYP(11),EIZP(11),FX(11),PANG(11)

,+ VY(11),VZ(11),EA1(11),SCALE(11),AANG(11),FXT(11),TSCALE(11)

+,SXTN(11),SX(11),DEFY(11),DEFZ(11),YCB(11)

$+, Z C B(11), G J 1(11), Y C T(11), Z C T(11), P M(11)$, TANG(11),TANGI(11)

+.GJ2(11),EIFLAP(11),EILAG(11)

DIMENSION WA1(11),YGB(11),ZGB(11),YGBB(11),ZGBB(11),WB(11)

+YBAL(11),ZBAL(11),WT(11),RS(11),RB(11),RBAL(11)

DIMENSION OA(11),OC1(11),OC2(11),OTRM(11),OT(111),OC18(11),

+OC2B(11),OTRMB(11), OTIB(11),OCB(11), OB(11),OBTR(11),OL(11)

REAL IXMO,IYMO, IXMT, IYMT, IXMG, IYMG, IBMT, IBMG, IMBB, IMBG

REAL RO(11),IYO(11),IZT (11), $Y T(11), I Z G(11), I Y G(11)$

REAL MT (11),IMG(11),MGB(11)

INTEGER STA IST NPT I, JIV , KD KC,JC IC, J1 NSO TOGGLE

REAL CY,LX(11),LY(11),LZ(11),MY(11),MZ(11),MPY(11),MPZ(11)

+,NANG(11),MYE(11),MZE(11),MYP(11),MZP(11),LYC(11),LZC(11)

$+L X|(11) \cdot L Y|(11), L Z \mid(11)$

CHARACTER*40 INP1

CHARACTER* 40 INP2

CHARACTER 40 HEADING

CHARACTER' 80 HEAD2

DATA DFZ,DFZ1,DFZ2,ZTOT,DFZT/ $/ 55^{\circ} 0.0$

DATA ANGTOT, TANGTOT, AANG1/33०0.0/

c -...-READ INPUT FILES: INP1 IS THE BLADE SECTION GEOMETRY FHLE

C.-.-AND INP2 IS THE APPLIED LOADING FILE. BOTH ARE READ FROM

C -..-THE CURRENT FILE WORKSPACE UNDER THE NAMES INPUT FROM THE

c

WRTE $(1,100)$

100 FORMAT'ENTER INPUT FILE NAME FOR SECTION GEOMETRY:?

AEAD $(1,101)$ INP1

101 FORMAT(1A40)

WRTE $(1,102)$

102 FORMAT(ENTER INPUT FILE NAME FOR APPLIED LOADING:)

READ $(1,101)$ INP2

C

C

OPEN(UNT $=5, F L E=I N P 1)$

c

OPEN(UNIT =6, FILE=INP2)

c

$$
\text { C }
$$

READ NUMBER OF CALCULATIONS

READ $\left(6,0^{\circ}\right)$ NCAL

C

$c$

DO 2000 18=1,NCAL

C ........-AEAD NUMBER OF STATIONS, E-SKIN, E-LONGITUDINAL,.......

C .........- DIVISIONS PER OFFSET FOR NUMERICAL INTEGRATION .......

C

READ $(5, ")$ STA,ES,EL,DIV,GS,GL

$E S=E S * 10 * 6.0$

$E L=E L \cdot 10 * 6.0$

$G S=G S \cdot 10 * 6.0$

$\mathrm{GL}=\mathrm{GL} \cdot 10 \times 60$

C -.-PEAD NUMBER OF POINTS AND SKIN THICKNESS CORRECTION FOR RUN

C........ALSO READ WEIGHT DENSITIES (WS) AND (WL) FOR SKIN \& SPAR

........... L LB PER INCHES**3

READ (5,') NPT, TCORR,WS,WL

C

C

C

$\operatorname{READ}\left(5^{\circ}\right)(X(1), 1=1, \mathrm{NPT}$

READ $\left(50^{\circ}\right)(Y(l), i=1, N P T)$

DO $105 \mathrm{i}=1$,NPT

$X X(1)=X(1)$

$Y Y(I)=Y(I))^{\top} T C O R R$ 
105

C

C

READ $\left(5,{ }^{\circ}\right)(Y S O(1), l=1, N S O)$ AEAD $\left(5,{ }^{\circ}\right)(Z S O(1), l=1, N S O)$

-READ STATION POSITION FROM FREE END AND LOAD PER UNIT-..

-...-LENGTH. X-LOAD IS TOWARDS TIP: Y-LOAD POSITIVE IN LAG-

-.....-DIRECTION; Z-LOAD POSITIVE IN FLAP DIRECTION; PITCHING.-

-...-MOMENT IS POSTIVE NOSEDOWN (INCH.LB); LOADS ARE IN....

-.-. LBS. PER INCH.

READ $\left(6^{\circ}\right)(S T X(1), 1=1, S T A)$

READ $\left(6^{\circ}\right)(L X(1), 1=1, S T A)$

READ $\left(6^{\circ},\right)(L Y(I), I=1, S T A)$

READ $(6, *)(L Z(I), I=1, S T A)$

$\operatorname{READ}\left(6,{ }^{\circ}\right)(\operatorname{PM}(), 1=1, \mathrm{STA})$

-.-READ ROTATIONAL SPEED OMEGA (RAD/SEC), BLADE RADIUS (IN.)

...-NOSEDOWN PITCH ANGLE, AND CONING ANGLE (BOTH IN DEGREES)-...

READ $\left(6,{ }^{\circ}\right)$ OMEGA,RBL,PITCH1,CONING1

c -...-CHANGE ANGLES FROM DEGREES TO RADIANS----

PTCH $=$ PICH1/57.29577951

CONING $=$ CONING1/57.29577951

C

......READ TWIST ANGLE TWIST ANGLE IS IN DEGREES, POSITIVE NOSEDOWN.-COUNTER-CLOCKWISE, LOOKING DOWN THE BLADE FROM THE TIP.

READ $\left(5,{ }^{\circ}\right)$ (TANGI(I),L=1,STA)

AEAD $(6,101)$ HEADING

PAR T 1 : CALCULATIONS FOR EACH STATION

.........PRINT HEADER

PAINT 998,HEADING,TCORR

PRINT,$\cdot "$

PRINT 910, STA,ES,EL,DN,GS,GL,WS,WL

PRINT 973

PRINT *,"

PRINT $\cdots \cdots \because \because \because \cdots \cdots \cdots \cdots \cdots \cdots$

DO 570 IST $=1$, STA

C

READ (5, ) SCALE(IST),TSCALE(IST), TSPAR, TSKIN,XTHK

C

DO $131 i=1$,NPT

$X(I)=$ SCALE $(I S T) \cdot X X(I)$

$Y(l)=$ SCALE(IST) ${ }^{*} Y(1)^{\bullet}$ TSCALE $($ IST

-........CALCULATE (SPAR) MODULUS-WEIGHTED THICKNESSES FOR BOTH ....-BENDING AND TORSION. THK WEIGHTED TO SPAR MODULUS AND

THK1 $=$ TSPAR + TSKIN $\cdot(E S / E L)$

THK2 $=$ TSKIN * (ESIEL)

GTHKI $=$ TSKIN + TSPAR * (GLGS)

C

GTHK2 $=$ TSKIN

131 CONTINUE

C

C

C PRINT 930,IST,THK1,THK2,XTHK,TSPAR,TSKIN,TSCALEIIST)

C PRINT 925, (X(1), l=1,NPT)

PRINT 925, $(Y(1), 1=1, N P T)$

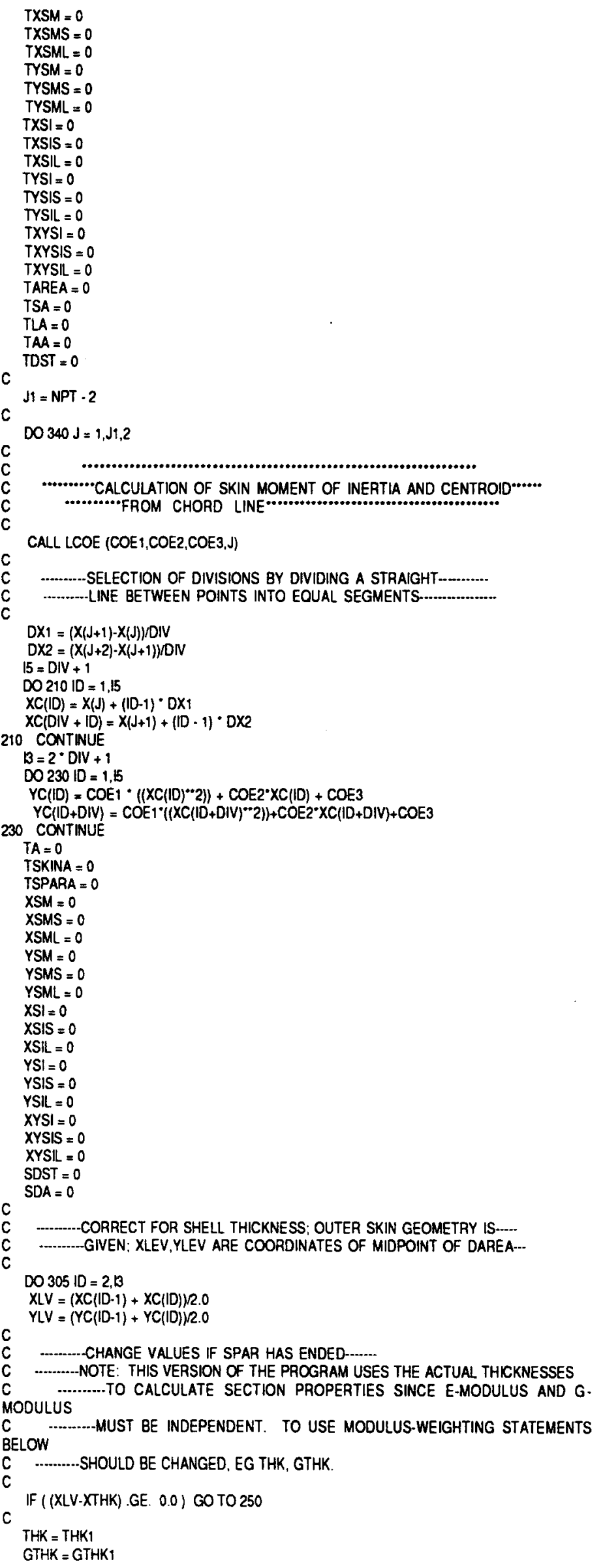




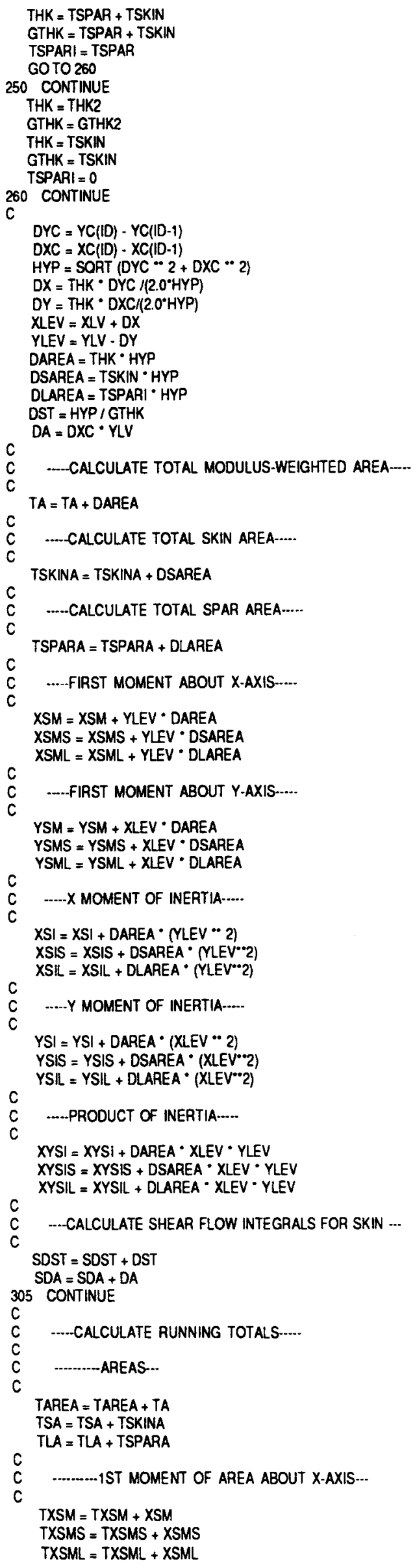

C

$T Y S M=T N S M+Y S M$

TYSMS $=$ TYSMS + YSMS

C

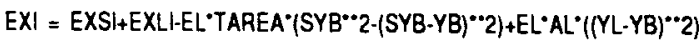
$E Y \mid=E Y S I+E Y L I-E L \cdot T A R E A *\left(S X B^{* *} 2-(S X B-X B)^{* * 2}\right)+E L L^{*} A L^{*}\left((X L-X B)^{* * 2}\right.$

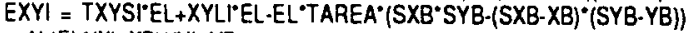


SUM $=(E X|+E Y|) / 2.0$

DIF $=(E Y H E X \mid) R 2.0$

$P P=A T A N(E X Y U D I F)$

$P H I=P P 20.0$

PANG(IST) $=$ PHI

C

RAD $=$ SORT (EXYI"*2 + DIF*2)

$\mathrm{C}$

$\mathrm{PXI}=\mathrm{SUM} \cdot \mathrm{RAD}$

$P Y I=S U M+R A D$

EIYP(IST) $=P X \mid$

$\stackrel{c}{c}$

.....PRINCIPAL RADIUS OF GYRATION..-..

PRX $=$ SORT $(P X I V E A)$

PAY = SORT (PYVEA)

$c$
$C$
$C$
$C$
$C$
$C$
$C$
$C$
$c$
$c$
$C$
$c$
$c$

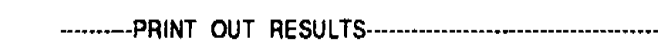

\section{PRINT 945,XB,YB,TAREA, PHI,PXI,PYI,PRX,PRY,EA}

-.....-CALCULATE POLAR MOMENT OF INERTIA: $J=|X+| Y-\ldots$

-..-IF G-SKIN \& E-SKIN ARE DIFFERENT THE TORSIONAL CENTOID-WILL DIFFER FROM THE BENDING CENTROID

$G A=G S *$ TAREA* $(E S E L L)+G L \cdot A L$

.....CALCULATE SHEAR CENTER (TORSIONAL CENTROID) BASED ON -.-MOOULUS-WEIGHTED VALUES--.-

$\mathrm{XBT}=(\mathrm{GS} \cdot \mathrm{TYSM}+\mathrm{GL} \cdot \mathrm{YLM}) / \mathrm{GA}$

$Y B T=(G S \cdot T X S M+G L \cdot X L M) / G A$

$Y C T(I S T)=X B T$

$\mathrm{c}$

$\mathrm{ZCT}(\mathrm{IST})=\mathrm{YBT}$

C -...-CALCULATE TORSIONAL STIFFNESS

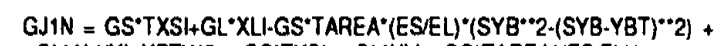

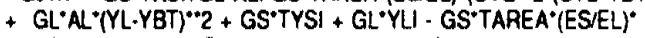
$+($ SXB"*2-(SXB-XBT)**2) + GL'AL"* ((XL-XBT)**2)

$\mathrm{c}$ G. $1(\mathrm{STT})=\mathrm{GJ} 1 \mathrm{~N}$

C --CALCULATE ALTERNATE TORSIONAL STIFFNESS BASED ON SHEAR FLOW--NOTE: IF GJ IS BELOW THE MINIMUM FOR CONVERGENCE, MAKE IT EOUAL C -..-TO THE MINIMUM.....

GJ2N $=G^{*} 4.0 \cdot\left(T_{A A} * 2\right) /$ TDST

IF (GJ2N .LE. 240000) GJ2N $=240000$.

C

$G \sqrt{ } 2(S T)=G \cdot \sqrt{2 N}$

C -...-PRINT FURTHER RESULTS

PRINT 950,XBT, YBT,GJ1N,GJ2N

PRINT *.

PRINT $\therefore$

C

"CALCULATE SECTION VALUES BASED ON ACTUAL THICKNESSES.....

---SKIN AND SPAR BENDING CENTROIDS-...

SXBS $=$ TYSMS $/$ TSA

$S X B L=$ TYSML, TLA

SYBS $=$ TXSMS $/$ TSA

SYBL = TXSML $/$ TLA

C -.-CALCULATION OF CENTROID AND EI FOR TOTAL COMPOSITE STRUCTURE

$E A=E S \cdot T S A+E L \cdot T L A+E L \cdot A L$

$E A 1(I S T)=E A$

C...TOTAL COMPOSITE STRUCTURE BENDING CENTROID BASED ON

-..-ACTUAL THICKNESSES--.-

$X B=(E S \cdot T Y S M S+E L \cdot T Y S M L+E L \cdot Y L M) / E A$

$Y B=(E S \cdot T X S M S+E L \cdot T X S M L+E L \cdot X L M) / E A$

$Y C B(I S T)=X B$

C
C -...-...FIND EIXEIY EIXY FOR TOTAL STRUCTURE ABOUT ITS CENTROID

-...(XB.YB) BY USING THE PARALLEL AXIS THEOREM IN SEOUENCE,

-......WORKING BACKWARDS, FOR THE SKIN, SPAR, AND WEB----

EXI $=$ ES * TXSIS - ES * TSA * (SYBS"*2) + ES * TSA * ( SYBS

$+\quad$ Y YB) $\left.{ }^{\prime \prime} 2\right)+E L \cdot T X S I L-E L \cdot T L A \cdot(S Y B L \cdot * 2)+E L \cdot T L A$

$\left.*\left((S Y B L-Y B)^{*}+2\right)+E L \cdot X L I+E L \cdot A L \cdot(Y Y-Y B)^{*}=2\right)$

EYI × ES * TYSIS - ES * TSA - (SXBS"-2) + ES * TSA - (ISXBS

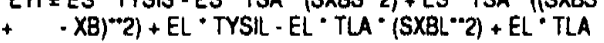

$\cdot\left((\mathrm{SXBL}-\mathrm{XB})^{* * 2}\right)+E \mathrm{EL} \cdot \mathrm{YLI}+\mathrm{EL} \cdot \mathrm{AL} \cdot\left((\mathrm{XI}-\mathrm{XB})^{* * 2}\right)$

$E X Y \mid=E S \cdot T X Y S I S-E S \cdot T S A \cdot S Y B S \cdot S X B S+E S \cdot T S A$

(SYBS - YB) * (SXBS - XB) + EL - TXYSIL - EL * TLA

$+\quad$ SYBL- SXBL +EL * TLA - (SYBL $+Y B) \cdot(S X B L+X B)$

$+\quad+E L \cdot X Y L L+E L \cdot A L \cdot(X L-X B) \cdot(Y L \cdot Y B)$

$\stackrel{c}{c}$

SUM $=(E X I+E Y M) / 2.0$

$\mathrm{DIF}=($ EYI-EXI)/2.

$\mathrm{PP}=$ ATAN(EXYMIF)

$\mathrm{PH}=\mathrm{PP} / 2.0$

PANG(IST) $=$ PHI

C

RAD $=$ SOAT $\left(E X Y{ }^{*}=2+\right.$ DIF*2)

c

$P X I=S U M-A A D$

$P Y I=S U M+A A D$

ENP(IST) $=P X !$

C

c

$P R X=$ SORT(PXUEA)

c

PRY $=$ SORT (PYVEA)

PAINT 946,XB,YB,TSA, TLA,PHI,PXI,PYI,PRX,PRY,EA

-........CALCULATE POLAR MOMENT OF INERTIA: $J=I X+I Y$

.......-...F G-SKIN \& E-SKIN ARE DIFFERENT THE TORSIONAL CENTROID-

-.........WILL DIFFER FROM THE BENDING CENTROID-

---IT WILL ALSO INCLUDE CONTRIBUTIONS FROM SKIN,SPAR\&WEB--

$G A=G S * T S A+G L * T L A+G L \cdot A L$

$\mathrm{C}$

$\mathrm{C}$

c

$X B T=(G S \cdot T Y S M S+G L \cdot T Y S M L+G L \cdot Y L M) / G A$

$Y B T=(G S \cdot T X S M S+G L \cdot T X S M L+G L \cdot X L M) / G A$

$\mathrm{YCT}(\mathrm{IST})=\mathrm{XBT}$

$c$

$Z C T(I S T)=Y B T$

C

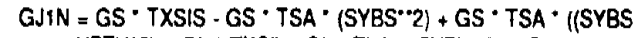

$\left.+\quad-Y B T)^{*} 2\right)+G L \cdot T X S L \cdot G L \cdot T L A \cdot(S Y B L \cdot 2)+G L$

$+\quad \cdot T L A \cdot((S Y B L=Y B T) \cdot * 2)+G L \cdot X L I+G L \cdot A L \cdot((Y L$

$+\quad$ - YBT)“"2) + GS* TYSIS - GS* TSA* (SXBS“2) + GS

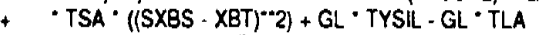

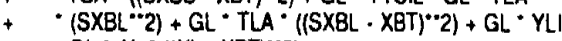

$+\quad+\mathrm{GL} \cdot \mathrm{AL} \cdot\left((\mathrm{XL}-\mathrm{XBT})^{* *} 2\right)$

C

$\mathrm{GJ} 1(\mathrm{IST})=\mathrm{GJIN}$

..........PRINT FUATHER RESULTS

PRINT 950,XBT,YBT,GJTN,GJ2N

MASS CALCULATIONS

-..-SECTION MASS CALCULATIONS CAN NOW BE DONE USING THE ACTUAL -..-.-.-THICKNESSES-...

.....TOTAL RUNNING WEIGHT IS THE SUM OF ALL COMPONENTS-..--

$W A=W S \cdot T S A+W L \cdot T L A+W L \cdot A L$

$W A t(I S T)=W A$

$c$

-.-.-IST WEIGHT MOMENTS--..

$X B G=\left(W S^{*} T Y S M S+W L \cdot T Y S M L+W L \cdot Y L M\right) / W A$ 


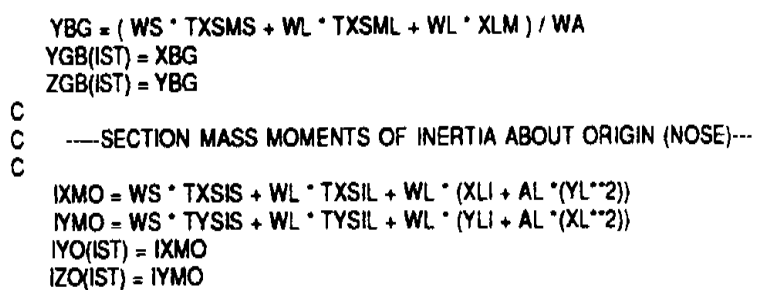

C

READ(5,994) HEAO2

C

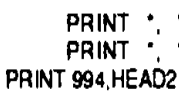

650 CONTINUE 


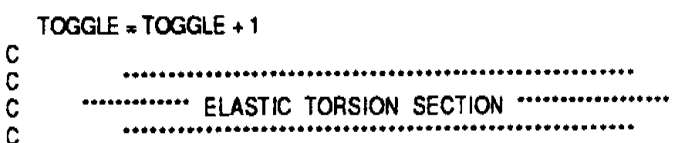

NOTE: WHL ENTER THIS SECTION AFTER INTIAL DEFLECTIONS ARE FOUND (TOGGLE = 1) AND THEN WILL ITERATE

UNTL CONVERGENCE IS AEACHED ON ZDEF (DFZT) INCLUDING CONING

DO $690 \quad \mid=1$, STA

C.-.-CALCULATE TORSION ABOUT THE SHEAR CENTER: THIS WILL

- PRODUCE THE ELASTIC TWIST OF THE SECTION-

-...-..-AERODYNAMIC TORSION DUE TO LIFT (LZ IN THE FLAPPING DIRECTION), DRAG (LY IN THE LAGGING DIRECTION). AND -.PITCHING MOMENT (PM) ALL POSITIVE NOSE-DOWN-...

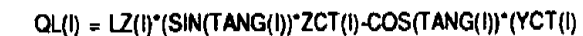

$+(0.25+\operatorname{SCALE}(1)))+$

+ LY(I) (SIN(TANG(I)) $)^{\circ}($ YCT(I)-10.25*SCALE(I))) +

C

+ $\cos (T A N G(D))^{*} Z C T(I)$

$O A(1)=Q L(I)+P M(I)$

C .

- ....NO BALLAST ADDED. NOTE: THIS CAN'T BE CALCULATED

C -...-UNTIL THE TOTAL BEAM DEFLECTIONS ARE CALCULATED FIRST

C.-.-WHICH MUST INCLUDE THE CONING ANGLE DEFLECTION....

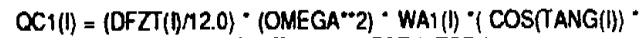

$+\quad(Y C T(I)-Y G B(I))-\operatorname{SIN}(T A N G(I))(Z C T(I)-Z G B(I)))$

c .........CENTRIFUGAL TORSION DUE TO HORIZONTAL (LEAD-LAG)

-COMPONENT OF CENTRIF FORCE AT A VERTICAL MOMENT ARM

C....FROM SHEAR CENTER, AND NO BALLAST ADDED. NOTE: THIS

...-TERM IS A SIMPLE MANIFESTATION OF THE PARALLEL AXIS THM-

$R S(I)=$ SQRT $((Y C T(1)-Y G B(1)) * 2+(Z C T(1)-Z G B(1)) * 2)$

$O C 2(1)=-\operatorname{RS}(1)^{*}\left(\mathrm{OMEGA}^{*}+2\right)^{*}$ WAI $\left(D^{*}(1.0 / 12.0)^{\circ}\right.$

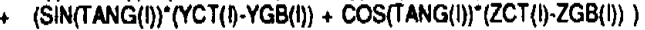

C .........TORSION DUE TO THE TENNIS RACKET MOMENT WHEN PAINCIPAL

C -...-MASS AXIS (HERE ASSUMED SAME AS PRINCIPAL BENDING AXIS)

........F THE SECTION, LESS BALLAST, IS ROTATED AN ANGLE (AANG)

.........FROM THE PLANE OF ROTATION-..

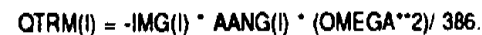

$\mathrm{C}$

C ...-..-TOTAL INERTIAL TORSION IS THE SUM--.-

$\operatorname{OT}(1)=\alpha C 1_{1}(1)+O C_{2}(1)+O \operatorname{TRM}(1)$

C - - NOW FIND TOTAL INERTIAL TORSION INCLUDING BALLAST, INCL

--..THE TWO CENTRIFUGAL TERMS AND THE NEW TENNIS RACKET MOMENT

-........NOTE: RECALL THAT THE BALLAST WEIGHT HAS CHANGED THE

........SECTION MASS, CG LOCATION, AND MASS MOMENT OF INERTIA-...

$R B(I)=\operatorname{SORT}\left((Y C T(I) \cdot Y G B B(I))^{* * 2}+(Z C T(I)-Z G B B(\eta) * 2)\right.$

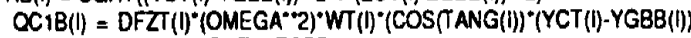

$+\quad$ SIN(TANG(l) )* ZCT(I)-ZGBB(D)) 12.0

$\alpha_{2} B(I)=-R B(1)^{*}\left(O M E G A^{*}{ }^{*}\right){ }^{*} W T(1)^{*}\left(S I N(T A N G(I))^{*}(Y C T(I) \cdot Y G B B(I))\right.$

$\left.+\quad+\operatorname{COS}(T A N G(1))^{*}(Z C T(1)-Z G B B(I))\right) / 12.0$

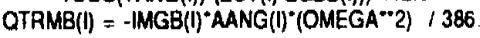

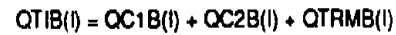

C -...-CENTAIFUGAL TORSION DUE TO ADDED BALLAST WEIGHT, CONSISTS

C -...... OF THE TWO CENTRIFUGAL TERMS DESCRIBED ABOVE-...

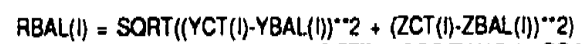

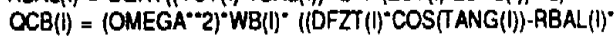

+ $\quad$ SIN(TANG(I) ) - YCT(I)-YBAL(I)) - (ABALL(I) COS(TANG(I)) +

$\left.+\quad \operatorname{DF} Z(1))^{*} \operatorname{SiN}(\operatorname{TANG}(1))\right)^{*}(\mathrm{ZCT}(1)-\mathrm{ZBAL}(\mathrm{D})) / 12.0$

C

C -...-...NOW THE TOTAL CONTRIBUTION OF THE BALLAST WEIGHT-..

$O B(I)=O T(B(I)-O T(\mid)$

$O B T A()=O B(\eta) \cdot O C B(1)$

c -........FINALLY THE TOTAL SECTION TORSION FOR THE ELASTIC TWIST
690 CONTINUE

C

C PRINT 973

C PRINT 999

C PAINT 970, 'P-MOMENT',PM

C PRINT 970, 'LIFT:OL

C PRINT 970. TOT-AERO,OA

C PAINT 973

C PRINT 1000

C PRINT 970 , 'TNS-RCKT, OTRM

C PAINT 970, 'FLAP.DEF'OC1

C PRINT 970, 'LD-LAG ' $O C 2$

C PRINT 970, 'TOTAL 'OTII

C PRINT 973

C PRINT 1010

C PRINT 970, 'TNS-RCKT CBTR

C PRINT 970, CENTAIF:OCB

C PAINT 970, TOTAL :OB

C PRINT 973

C PRINT 1020

C PRINT 970, TNS-RCKT',QTRMB

C PRINT 970, 'FLAP-DEF,CX1B

C PRINT 970, 'LDLAG :OC2B

C PRINT 970, TOTAL :OTIB

C PRINT 970, 'SECTION',OS

C PRINT 973

C ....FIND TOTAL TORSION BY INTEGRATING FROM THE BLAOE TIP..

CALL INTEG(STX,OS,OT, 1,STA)

C

C

c

C

c

C

C

c

C

\section{DO $705 !=1$, STA}

$\mathrm{IR}=\mathrm{STA}+1-\mathrm{I}$

ELAST $1(I)=$ OT $(\mathbb{R}) \cdot T O R K G J 1(\mathbb{R})$

ELAST2(l) $=$ OT(IR) $/$ GJ2(IR)

705 CONTINUE

C

CALL INTEG(STX,ELAST1,TLAST1,1,STA)

CALL INTEG(STX ELAST2,TLAST2,1,STA)

DO $725 \mid=1$.STA

IR $=\mathrm{STA}+1 \cdot 1$

C

TELAST 1 (1) = TLAST1 $($ IR

TELAST2 $(\|)=$ TLAST2 $(\mathbb{R})$

725 CONTINUE

C

C PRINT 993, 'TORSOEF1'.TELAST1

C PRINT 993, TORSDEF2' TELAST

C

DO750 $\quad I=1$,STA

RCENT $(D)=$ RBL $-S T X(1)$

FCENT $(l)=W T(I) \cdot \operatorname{RCENT}(I) \cdot \operatorname{OMEGA} * 2 *(1 . / 12$.

$L X \mid(I)=L X(1)+F C E N T(I)$

$L Z \mid(\mid)=L Z(I)$

$M C X(I)=F C E N T(I) * D F Z T(I)$

750 CONTINUE 
$\mathrm{C}$

CALL INTEG(STX,LXI,FX, 1, STA)

DO $755 \mathrm{~b}=1, \mathrm{STA}$

MCENT(I) $=\operatorname{MC} 1(1) \cdot F X(1) \cdot \operatorname{DFZT}(1)$

755 CONTINUE

$\mathrm{C}$

760 CONTINUE

C CALL INTEG(STX,LXI,FX, 1,STA)

CALL INTEG(STX,LYI,VY,1,STA)

CALL NTEG(STX,VY,MLAG, $1, S T A$ )

CALL INTEG(STX,LZI,VZ,1,STA) CALL INTEG(STX,VZ,MFLAPA, 1,STA)

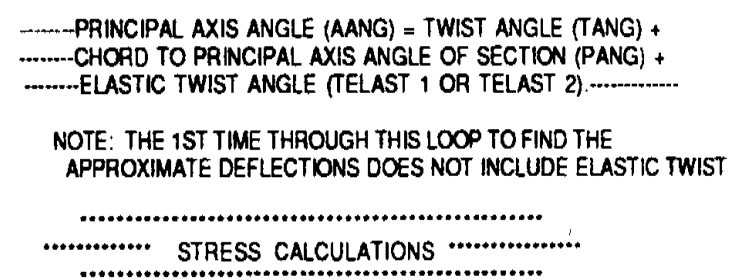




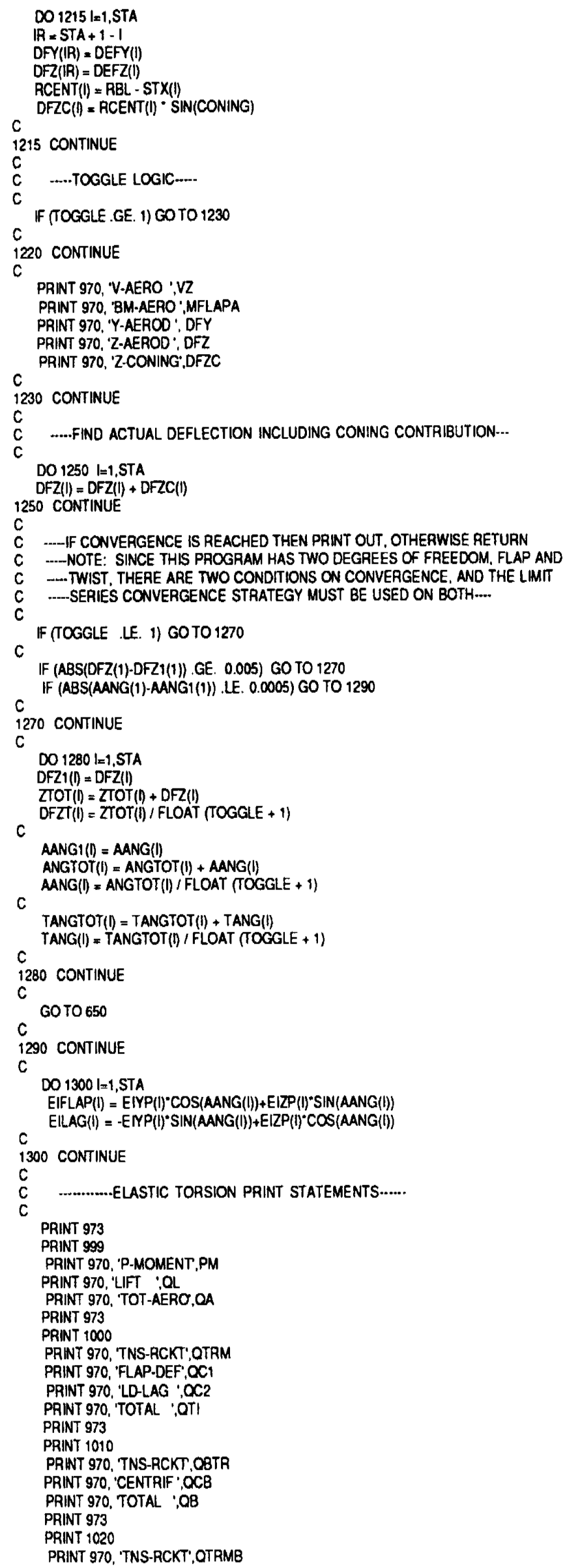

PAINT 970, 'FLAP-DEF', $O C 1 B$

PRINT 970, 'LD-LAG ' OC2B

PRINT 970 'TOTAL 'OTIB

PRINT 970. 'SECTION',OS

PAINT 970. TORSION : OT

C PRINT 993, 'TORSDEF1',TELAST1

DO 1390 I=1,STA

TELAST2(l)=TELAST2(1) 57.29578 1390 CONTINUE

C

PRINT 995, EL TWIST, TELAST2

C

PRINT 970, 'TENSION 'FX

C -......PRINT INPLANE SHEAR...........

PRINT 970, V-INPL :VY

PAINT 970, 'BM-INPL ',MLAG

$\mathrm{C}$

C

PRINT 970, V-FLAP : VZ

c ..........PAINT FLAPPING MOMENT -.......

PRINT 970, 'BM-FLAP :MFLAP

C .......PRINT PRINCIPAL AXIS ANGLE AFTER CONVERTING TO DEGREES-

DO $1400 \mathrm{I}=1$, STA

AANG(I) = AANG(I) $\cdot 57.29577951$

1400 CONTINUE

C

PRINT 970, 'PR A A :AANG$$
\text { C }
$$$$
\text { c }
$$ 


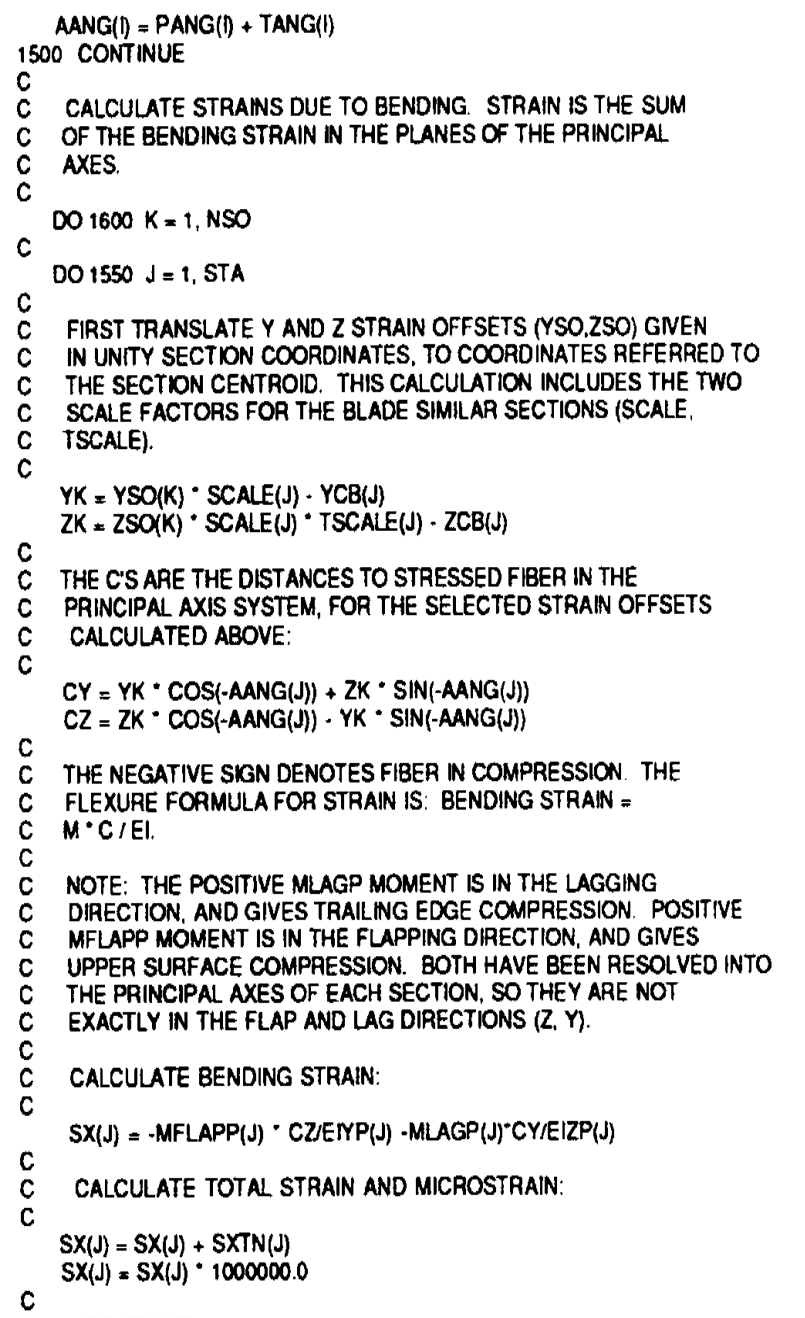

1550 CONTINUE

C PAINT TOTAL STRAIN:
C PRINT 971,'M-STRAIN,K,SX
C PRO CONTINUE
PRINT 973$$
\text { c }
$$

2000 CONTINUE$$
\text { C }
$$$$
905 \text { FORMAT(5X,12,2F6.2,14,2F6.2) }
$$$$
910 \text { FORMAT(' STATIONS: ', 12,1X,'E-SKIN: ',F10.2,2X,'E.LONG: '. }
$$$$
\text { +F10.2,' DNISIONS: ', 4,' GS:',F10.2,' GL: 'F10.2. }
$$$$
\text { +'WS: :F5.3,' WL:',F5.3) }
$$$$
915 \text { FORMAT(5X,12,2X,F6,4) }
$$$$
920 \text { FORMAT }(5 X, 9 F 6.4, /, 5 X, 8 F 6.4, /, 5 X, 8 F 6.4,1,5 X, 8 F 6.4)
$$$$
925 \text { FORMAT }(5 X, 9 \mathrm{FB}, 4,1,3(5 \times, 8 \mathrm{FB}, 4, \eta)
$$

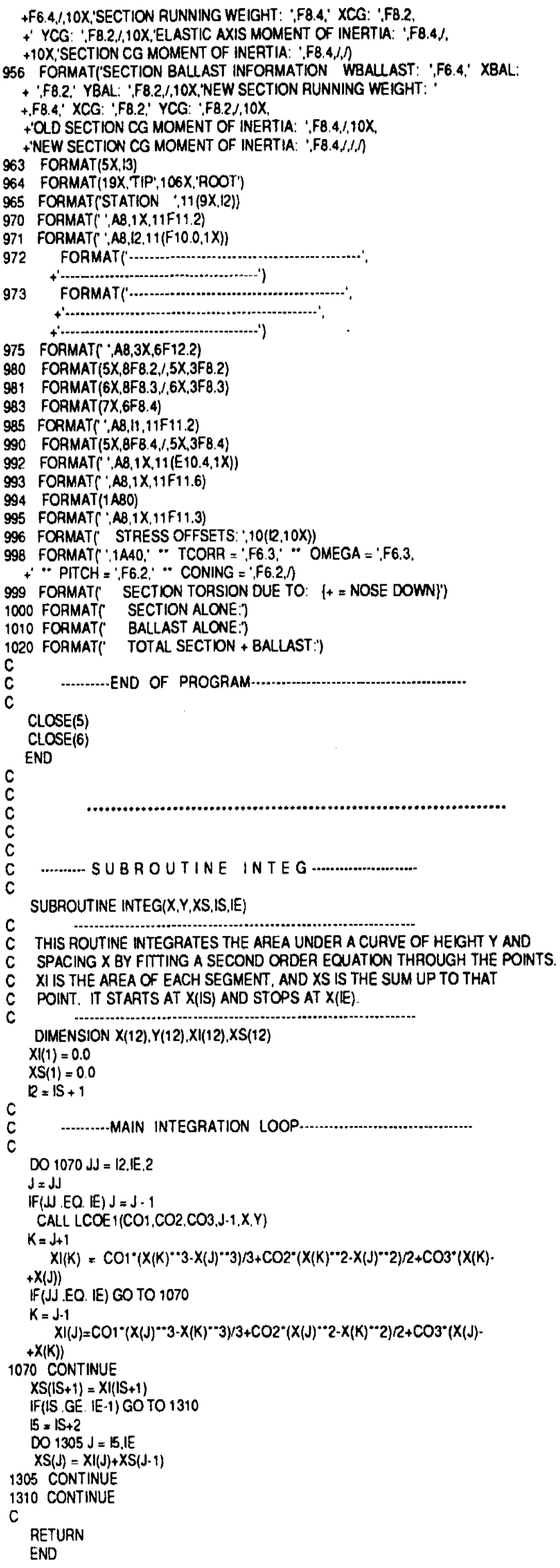



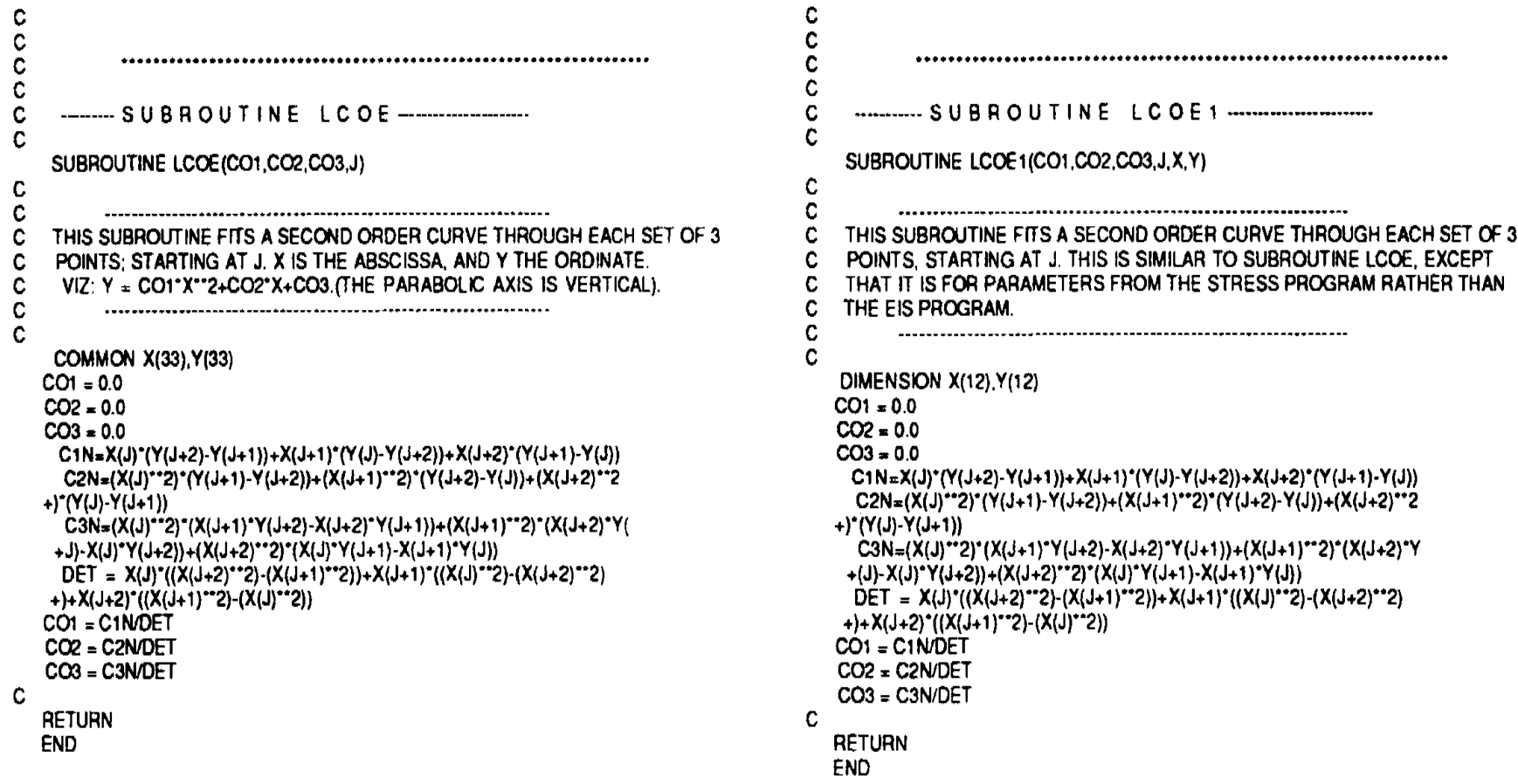

\subsubsection{SAMPLE RUN, UTRC BLADE}

This sample run uses the UTRC blade, with flexbeam attached, operating at $30 \mathrm{mph}, 108 \mathrm{rpm}$, and 5 degrees coning angle. These results are also given in Chap. 5.

\section{WPUT BLADE FLE: FLEXBL.}

331.083 .064 .0717

$.0000 .0125 .0250 \quad .0500 \quad 0750.1000 \quad 1500.2000 \quad .2500$

$.3500 .5000 .6000 .7000 .8000 .9000 .9500 \quad 1.0000$

$.9500 .9000 .8000 .7000 .6000 .5000 .3500 \quad .2500$

$.2000 .1500 .1000 \quad .0750 \quad 0500 \quad 0250.0125 \quad .0000$

.0000 .0267 .0361 .0491 .0580 .0643 .0719 .0750 .0760

$\begin{array}{lllllll}.0755 & 0641.0547 .0436 & 0308 & 0168 & 0092 & .0000\end{array}$

$-.0070-.0123-.0216-.0300-0367-.0417-.0446-.0428$

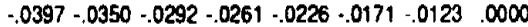

006

$\begin{array}{lllllll}.0000 & 0760 & 0641 & 0000-0417-0428\end{array}$

$.0000 .0000 \quad 0000 \quad 0000 \quad 0000 \quad .0000 \quad 0000 \quad .0000$

.0000 .0000 .0000

$15.000 \quad 1.000 .00001 .1180 \quad 5.000$

$\begin{array}{llllll}2.7076 & 3831.9887 & .0000 & 1.4796 & .0843\end{array}$

0.000 .000 .00

$15.000 \quad 1.000 .00001, \$ 180 \quad 5.000$

$\begin{array}{llllll}2.7076 & 3831 & .9887 & .0000 & 1.4796 & .1843\end{array}$

0.000 .000 .00

t5.000 $1.000 .00001 .1180 \quad 5.000$

0.000 .000 .00

$\$ 5.0001 .000 .00001 \quad 1180 \quad 5.000$

0.000 .000 .00

$15.000 \quad 1.000 \quad 00001.1180 \quad 5.000$

$\begin{array}{lllll}2.7076 & .3831 .9887 & .0000 & 1.4796 & .1843\end{array}$

0.000 .000 .00

$\begin{array}{lllll}15.000 & 1.000 \quad 00001 & .1180 & 5.000\end{array}$

$\begin{array}{lllllll}2.7076 & 3831.9887 & 0000 & 1.4796 & .1843\end{array}$

$0.000 .00 \quad 0.00$

$15.0001 .000 \quad 00001 \quad 1180 \quad 5.000$ $\begin{array}{llllll}.0000 & .2500 & .5000 & 1.0000 & .5000 & .2500\end{array}$

$\begin{array}{llllll}2.7076 & 3831 & .9887 & 0000 & 1.4796 & .1843\end{array}$

$\begin{array}{lllll}2.7076 & 3831.9887 .0000 & 1.4796 & 1843\end{array}$

\author{
$\begin{array}{llllll}2.7076 \quad 3831 & 9887 & 0000 & 1.4796 & .1843\end{array}$ \\ 0.000 .000 .00 \\ $\begin{array}{llll}15.000 \quad 1.000 \quad .00001 & 1180 \quad 5.000\end{array}$ \\ $\begin{array}{llllll}2.7076 & .3831 & 9887 & .0000 & 1.4796 & 1843\end{array}$ \\ $0.00 \quad 0.000 .00$ \\ 1.0 .10 .00001 .000015 .0

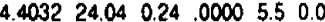 \\ $0.00 \quad 0.00 \quad 0.00$ \\ 1.0 .10 .00001 .000015 .0 \\ $\begin{array}{llll}4.4032 & 24.04 \quad 0.24 \quad 00005.5 \quad 0.0\end{array}$ \\ 0.000 .000 .00 \\ 1.0 .10 .00001 .000015 .0 \\ $4.4032 \quad 24.04 \quad 0.24 \quad 00005.50 .0$ \\ $0.000 .00 \quad 0.00$ \\ UTRC.BLADE.PLUS.FLEXBEAM.1-21-88
}

\section{NPUT LOAD FLE: $10.30 \mathrm{MPH}$}

001

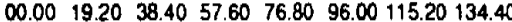

$153.6 \quad 172.80192 .00$

$00.00 \quad 00.00 \quad 00.00 \quad 00.00 \quad 00.00 \quad 00.00 \quad 00.00 \quad 00.00$

$00.00 \quad 00.00 \quad 00.00$

$.870 \cdot .888-719 \cdot .444 \cdot 340 \cdot 183 \cdot .041 .032 .039 .0320 .0$

5.1684 .5114 .0032 .9952 .1711 .5681 .131 .808 .6054680 .0

5.483 .973 .093 .032 .492 .061 .701 .651 .501 .490 .0

11.31192 .00 .0050

UTRC.BLADE. 30MPH. LOAD. :-21-BE

TERMINAL SESSION AND OUTPUT: STRESSELASTIC

ENTER INPUT FUL NAME FOR SECTION GEOMETRY:

FLEXBL

ENTER INPUT FILE NAME FOR APPLIED LOADING:

LD.3OMPH

UTRC.BLADE.PLUS.FLEXBEAM 1-21-B8

UTRC.BLADE.3OMPH.LOAD.1-21-88

* TCORA $=1,083 *$ OMEGA $=11,310^{*}$ PITCH $=0.00 *$ CONING $=5.00$

\# STATIONS: 11 E-SKIN: 2050000.00 E-LONG: 5699999.00 \#DNISIONS: 500 GS: 899999.75 GL: 99999.97 WS: 0.064 WL: 0.072 


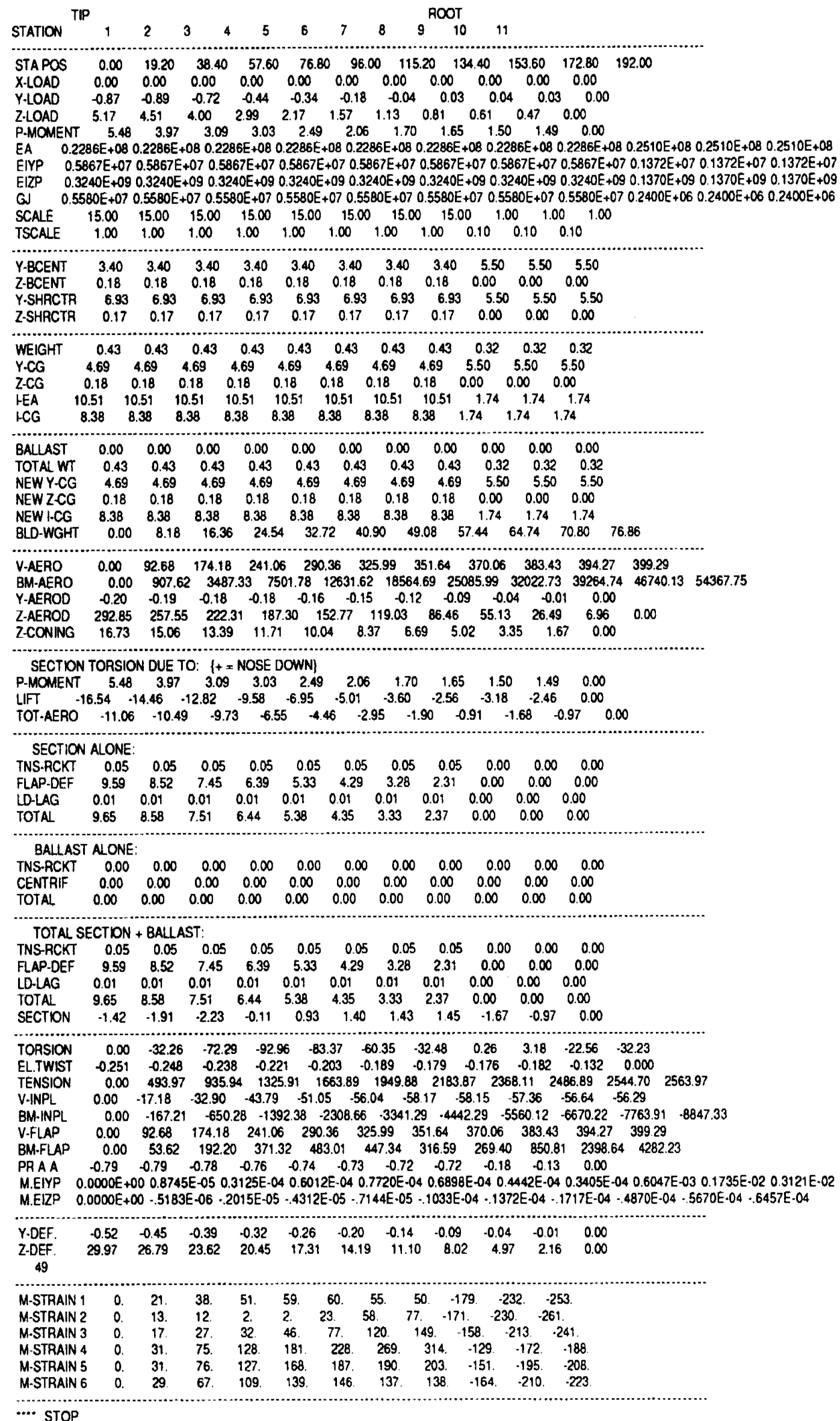




\section{6: CALCULATION OF DRAG POLARS FOR EIGHT WIND TURBINE BLADE SECTIONS}

\subsubsection{PROCEDURE AND ANALYSIS:}

The airfoil templates were digitized with a Houston Instruments Hi-Pad digitizer with a 10 in. $\times 10$ in. working area. Each template was divided into two or three parts as necessary. Reference points were marked on each template before dividing. The individual data files for each template were combined into one file per template. Using coordinate transformations, the complete airfoil was reconstituted into a single coordinate table with the leading edge at $0.0,0.0$ and the trailing edge at 1.0,0.0. The finite trailing edge thickness was maintained.

The coordinate data then were subjected to smoothing to meet the sensitivity requirements of the Eppler Code. Smoothing required the data to be transformed so that the independent variable is monotonically increasing. Neither the $x$ nor $y$ coordinate can be used for this because neither varies in a monotonic fashion. A smoothing procedure utilizing the coordinates used in the Theodorsen Transformation was tried first. The coordinate reference is shifted to the fifty percent chord location so that the leading edge is at $-0.5,0.0$ and the trailing edge is at $0.5,0.0$. The transformation variables are $\theta$ and $\psi$ according to:

$$
x=\cosh \psi^{*} \cos \theta \quad y=\sinh \psi^{*} \sin \theta
$$

The transformation is very sensitive near the leading and trailing edges as $\sin \theta$ goes to zero here. Cubic spline smoothing applied to the $\theta-\psi$ coordinates was unsuccessful. Modifications to the trailing edge geometry occurred that could not be controlled. The problem was traced to the fact that smoothing the $\psi$ variable modified not only the $y$ coordinate, but also the $x$ coordinate. A new transformation was then tried by replacing the $x$ coordinate with $\theta$ so that

$$
x=0.5 \cos \theta_{l} \quad y=y
$$

where $\theta_{i}$ is the ith knot of $n$ knots. This weighted the leading edge, where geometric accuracy is more critical. Each airfoil was smoothed and a coordinate table of 61 points was generated for a range of knot sets increasing up to the maximum allowed of twenty-seven. Each set was tested with the Eppler potential solution. The coordinate table with the highest number of knot points before waves appeared in the velocity distribution was selected. This criterion resulted in the same knot point sets for all airfoils. The airfoil data used were based on twenty-one knot points, ten upper surface, ten lower surface, and one at the leading edge.

All airfoils were then subjected to a drag polar analysis using the Eppler Code. Reynolds number ranges were checked for each, and where there was a significant span, the low and the high ends of the range were used. Only the Carter airfoils required more than one Reynolds number.

The generated data for each airfoil consists of the following: plot of the airfoil and velocity distributions for six angles of attack; plot of the drag polar, lift and pitching moment coefficients versus angle of attack; laminar-turbulent transition and turbulent separation locations for upper and lower surfaces; tabulated values of all plotted data. The transition and separation locations are measured from the trailing edge along the surface contour. In considering the lift and drag values, the separation location should be consulted. Any separation location greater than ten percent (ninety percent chord location) suggests a warning. There is presently no good method for calculating accurate values for large regions of separated flow.

\subsubsection{PLANFORM DATA FILES AND DRAG POLARS}

Tabulated Data Lists:

ESI $\quad 0.75$ radius

ESI 0.95 radius

UTRC 0.75 radius

UTRC 0.95 radius

Carter $\quad 0.75$ radius uncut

Carter $\quad 0.95$ radius uncut

Carter $\quad 0.75$ radius cut

Carter $\quad 0.95$ radius cut

Note: All these data files and drag polar plots can be obtained at AEl. 


\section{7: CHECKLIST SENT TO BLADE MANUFACTURERS}

This information is necessary for the formulation of an accurate computer model of the blade structure for use in aeroelastic and structural dynamic codes. These data will be verified, insofar as possible, by bench tests at AEI using the actual blades mounted in test stands, and from coupon tests and measurements from sectioned blades. Proprietary data furnished by the blade manufacturer will be treated as such if properly marked and/or otherwise specifically indicated. Results of the test program and the computer simulations will not generally be treated as proprietary, and will likely be reported in the public domain.

\section{BLADE SPECIFICATIONS}

\section{Geometric:}

blade dimensions

blade twist distribution

blade taper (chord) distribution

airfoil shape distribution and offsets if not a standard airfoil

location of control (pitch) axis

procedure and benchmark method for setting

blade pitch angle

Note: Any engineering drawings, airfoil lift and drag data, or layout sketches of the rotor/blade will be greatly appreciated.

\section{Structural:}

blade internal geometry

root attachment method

balancing and ballast weights

shell/skin/spar nominal locations and thicknesses

locations of shear webs

structural or internal control attachments

spanwise mass distribution

chordwise mass distribution, or location of mass centers

location of shear centers (elastic axis)

blade natural frequencies (first mode) and mode shapes in bending (flapping and lead-lag) and torsion

Note: Engineering drawings and cross-section detail drawings are particularly needed.

\section{Laminate:}

laminate schedule

individual laminate thicknesses

engineering tensile, compressive, and shear moduli for each laminate layer (i.e., cloth, sandwich, etc.)

laminate flexural modulus, if known

cross sectional views

for cloth and unidirectional plies, the fiber orientation (i.e., 45 degree, etc.)

for wound plies, the tape or filament advance angle

glass (or reinforcement) to resin ratios for the laminates

mass densities for the laminates

mass density for the catalyzed resins

mass density and thickness of surface finish and/or gelcoat

Note: Cross-sectional construction drawings, test results on individual laminates (especially ASTM burnoff and outgassing tests), manufacturers' specifications on glass rovings and woven goods, and particularly any composite modulus (E) and section modulus (EI) data are needed. References already published with such material will be greatly appreciated. 


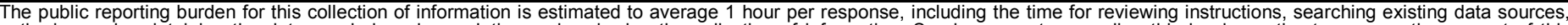

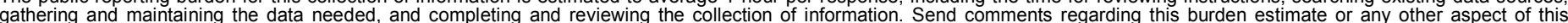

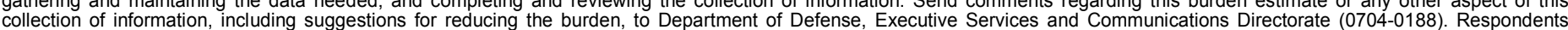

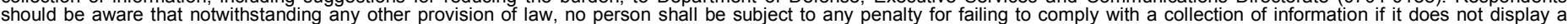

should be aware that notwithstanding

PLEASE DO NOT RETURN YOUR FORM TO THE ABOVE ORGANIZATION.

29-08-2006

subcontract report

4. TITLE AND SUBTITLE

Determination of Elastic Twist in Horizontal Axis Wind Turbines

(HAWTs)

5a. CONTRACT NUMBER

DE-AC36-99-G010337

5b. GRANT NUMBER

5c. PROGRAM ELEMENT NUMBER

6. AUTHOR(S)

F. Stoddard, V. Nelson, K. Starcher, B. Andrews

5d. PROJECT NUMBER

NREL/SR-500-12087

5e. TASK NUMBER

WER6.7502

5f. WORK UNIT NUMBER
7. PERFORMING ORGANIZATION NAME(S) AND ADDRESS(ES)

F. Stoddard, V. Nelson, K. Starcher, B. Andrews, Alternative Energy

Institute, West Texas State University, Canyon, Texas
8. PERFORMING ORGANIZATION REPORT NUMBER

RL-6-06013

9. SPONSORING/MONITORING AGENCY NAME(S) AND ADDRESS(ES)

National Renewable Energy Laboratory

10. SPONSOR/MONITOR'S ACRONYM(S) NREL

1617 Cole Blvd.

Golden, CO 80401-3393

11. SPONSORING/MONITORING AGENCY REPORT NUMBER NREL/SR-500-12087

12. DISTRIBUTION AVAILABILITY STATEMENT

National Technical Information Service

U.S. Department of Commerce

5285 Port Royal Road

Springfield, VA 22161

13. SUPPLEMENTARY NOTES

NREL Technical Monitor: A. Laxson

14. ABSTRACT (Maximum 200 Words)

This report presents the results of a project at the Alternative Energy Institute that measured and calculated the elastic twist of three representative composite horizontal-axis blades. It covers the experimental method used to inspect and test the blades, the theory and computer programs used to develop semi-empirical analytical blade models, calculation of the steady elastic twist of the three blades in simulated operation, and comparison with actual field performance of the wind turbines.

15. SUBJECT TERMS

wind energy; wind turbine; composite wind turbine blades; horizontal-axis blades

\begin{tabular}{l} 
16. SECURITY CLASSIFICATION OF: \\
\begin{tabular}{|l|l|l|}
\hline a. REPORT & b. ABSTRACT & c. THIS PAGE \\
Unclassified & Unclassified & Unclassified \\
\end{tabular} \\
\hline
\end{tabular}

19a. NAME OF RESPONSIBLE PERSON

19b. TELEPHONE NUMBER (Include area code) 